\title{
SOLID STATE DIVISION PROGRESS REPORT \\ for Period Ending September 30, 1990
}

\author{
J. B. Roberto, Director \\ L. A. Boatner, Section Head \\ J. F. Cooke, Section Head \\ B. C. Larson, Section Head \\ R. M. Moon, Section Head \\ D. M. Zehner, Section Head
}

Edited by:

P. H. Green

L. W. Hinton

Date Publiched - March 1991

Prepared for the

Division of Materials Sciences

Prepared by the

OAK RIDGE NATIONAL LABORATORY

Oak Ridge, Tennessee 37831

managed by

MARTIN MARIETTA ENERGY SYST:MS, INC.

for the

DEPARTMENT OF ENERGY 
Reports previously issued in this series are as follows:

ORNL-1095

ORNL-1128

ORNL-1214

ORNL-1261

ORNL-135"

ORNL-1359

ORNL-1429

ORNL-1506

ORNL-1606

ORNL-1677

ORNL-1762

ORNL-1851

ORNL-1852

ORNL-1944

ORNL-1945

ORNL-2051

ORNL-2052

ORNL-2188

ORNL-2189

ORNL-2413

ORNL-2414

ORNL-2614

ORNL-2829

ORNL-3017

ORNL-3213

ORNL-3364

ORNL-3480

ORNL-3676

ORNL-3841

ORNL-3935

ORNL-4098

ORNL-4250

ORNL-4408

ORNL-4526

ORNL-4669

ORNL-4779

ORNL-4861

ORNL-4952

ORNL-5028

ORNL-5135

ORNI.-5328

ORNL-5486

ORNL-5640

ORNL-5850

ORNL-5975

ORNL-6128

ORNL-6306

ORNL-6453

ORNL-6571
Period Ending April 30, 1951

Period Ending July 31, 1951

Period Ending October 31, 1951

Period Ending January 31, 1952

Period Ending May 10, 1952

Period Ending August 10, 1952

Period Ending November 10, 1952

Period Ending February 10, 1953

Prriod Ending Aus : st 30, 1953

Period Ending February 28, 1954

Per od Ending August 31, 1954

Period Ending February 28, 1955

Period Ending February 28, 1955

Period Ending August 31, 1955

Period Ending August 31, 1955

Period Ending February 29, 1956

Period Ending February 29, 1956

Period Ending August 31, 1956

Period Ending August 31, 1956

Period Ending August 31, 1957

Period Ending August 31, 1957

Period Ending August 31, 1958

Period Ending August 31, 1959

Period Ending August 31, 1960

Period Ending August 31, 1961

Period Ending August 31, 1962

Period Ending May 31, 1963

Period Ending May 31, 1964

Period Ending May ?1, 1965

Period Ending December 31, 195

Period Ending December 31, 1966

Period Ending December 31, 1967

Period Ending December 31, 1968

Period Ending December 31, 1969

Period Ending Drcember 31, 1970

Period Ending December 31, 1971

Period Ending December 31, 1972

Period Ending December 31, 1973

Period Ending December 31, 1974

Period Ending December 31, 1975

Period Ending April 30, 1977

Period Ending September 30, 1978

Period Ending February 29, 1980

Period Endirig September 30, 1981

Period Ending March 31, 1983

I'criod Ending September 30, 1984

Period Ending March 31, 1986

Period Ending September 30, 1987

Period Ending, March 31, 1989 


\title{
Special Dedication
}

\author{
Stanley T. Sekula \\ 1927-1989
}

This report is dedicated to the memory of Stanley T. Sekula, a Senior Research Staff Physicist in the Solid State Division and a member of the division from 1959 until his death fom a cerebral hemorrtage on December 5, 1989.

"Stan" Sekula joined the research staff at ORNL immediately after receiving his Ph.D. degree from Cornell University. With the exception of a leave taken during the 1963-1964 academic year to teach at the Middle East University in Ankara, Turkey, he spent his entire professional career at ORNL. He was a distinguished investigator in the field of superconductivity who specialized in electromagnetic characterization studies of the bulk and hysteretic properties of superconducting materials. Stan Sekula was a resourceful and determined scientist who carried out a number of pioneering investigations in the field of superconductivity.

As the leader of the Superconductivity Group in the ORNL Solid State Division, Stan Sekula guided his group's research efforts through the exciting and turbulent times associated with the discovery of high-temperature superconductivity in the metal oxides. He was an Adjunct Professor of Physics at The University of Tennessee in Knoxville and served on the Editorial Board of The Journal of Superconductivity. Stan Sekula was a steadfast colleague and a respected friend and associate to numerous solid state physicists around the world. This report is dedicated to his accomplishments both as a scientist and as a person. 


\section{Contents}

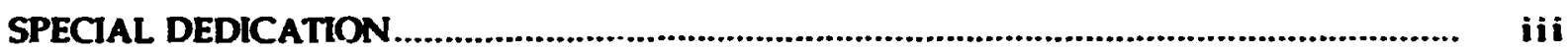

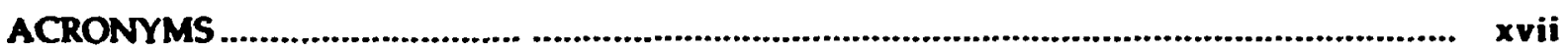

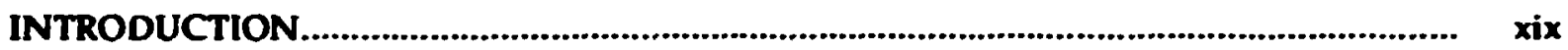

\section{Theoretical Solid State Physics}

\section{SUPERCONDUCTIVITY}

Intralayer Versus Interlayer Pairing in the Copper Oxide Superconductors-R. A. Klemm and S. H. Liu.

Self-Induced Effective Gauge Fields in the Copper-Oxygen Plane of High-T, Perovskites:

A Lattice and a Continuum Formulation-Mark Rasolt and G. Vignale

On the Ordering of Chirality for Many Planes of Anycns-A. G. Rojo and G. S. Canright

First-Principles Hartree-Fock Cluster and Band Calculations for $\mathrm{La}_{2} \mathrm{CuO},-P$. Saalfrank,

R. F. Wood, I. Ladik, M. A. Abdd-Reouf, and C.-M. Liegener.

d9 Spin-Polaron Model of Higin- $T_{c}$ Superconductivity-R. F. Wood, Mark Mostoller,

I. F. Cooke, and M. A. Abdel-Raouf..

Spin Polarons, Hole Filling, and High- $\mathrm{T}_{6}$ Superconductivity in $\mathrm{YBa}_{2} \mathrm{Cu}_{3} \mathrm{O}_{7}$ ard $\mathrm{PrBa}_{2} \mathrm{Cu}_{3} \mathrm{O}_{7}$ Alloys and Layered Films-R. F. Wood.

Kosterlitz-Thouless Transition and Charge Redistribution in the Supstconductivity of YBCO/PBCO Superlattices-Mark Resolt, Taner Edis, and Zlatko Tesanooic

Dielectric Function of Layered Materials -G. D. Mahan ................................................ 7

Lattice Dynamics of High- $T_{c}$ Supercondustors-Mark Mostoller

Effect of Zeeman Splinting and Impurities on Superconductivity in the Quantum Limit of Strong Magnetic Fields $-M$. Rasolt and Z. Tesanović 


\section{ELECTRONIC PROPERTIES AND LATTICE DYNAMICS}

Itinerant-Electron Susceptibility Calculations for Iron and Cobalt-I. F. Cooke,

J. A. Biackman, and K. N. Trahidou

The Screened Anderson Model-H. O. Frote and G. D. Mahan .............................................. 11

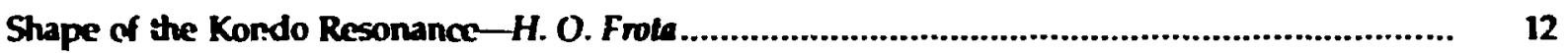

Quantum Fluctuations and Charging Effects in Small Tunnel Junctions-Karsten Flensberg

and $M$. Jonson ...

Charging Effects in Tunnel Junctions: A Four-Level Study-Karsten Flensberg and

H. O. Frota

Indirect lonic Interaction-G. D. Mahan and M. Mostoller.

Phomon Dispersion and Kohn Anomalies in Cun.enAL 16 -Henry Chou, S. M. Shapiro,

S. C. Moss, and Mark Mostoller.

Thermal Boundary Resistance-S. Pettersson and G. D. Mahan.

\section{SURFACES, SAND PILES, AND ION SCATTERING}

Layer-by-Layer Segregation and Deep Multilayer Relaxation in the Random Alloy Jurface Mos5e Re $_{15}(100)-H$. L. Davis, D. M. Zehner, B. Dötsch, D. v. Gemünden, and

K. Müller.

Multilayer Relaxation of the Ru(0001) Surface and Changes Induced by Monolayer

Coverage of $\mathrm{Cu}$ : Structure of a Simple Metallic Interface-H. L. Davis, J. E. Houston,

ard D. G. O'Neil.

Ceometry and Dynamics of Deterministic Sand Piles-S. H. Liu, T. Kuplan, and L. I. Gray ........ 18

Critical Exponents in Sand Piles-Theodore Kaplan, S. H. Liu, and L. J. Gray

The Temporal Development of Collision Cascades in the Binary-Collision

Approximation-Mark T. Robinson. 


\section{Neutron Scattering}

\section{SMALL-ANGLE SCATTERING}

Neutron Small-Angle Scattering Massurement of the Fluxoid Lattice in $\mathrm{Ba}_{0.6} \mathrm{~K}_{0.8} \mathrm{BiO}_{3}$

H. A. Mook, G. D. Wignall, M. I. Norton, and H-Yi Tang

Theory of Correlations in a Binary Fluid Mixture of Spherical Particles with Embedded

Oriented Dipoles-John B. Hayter and Roger Pymn

Differential Deuteration Effects in Block Copolymers as Observed by Small-Angle Neutron Scattering (SANS)-G. D. Wignall, J. H. Rosedale, and F. S. Bates

Gaussian-to-Stretched-Coil Transition in Block Copolymer Melts-K. Almdakd, F. S. Bates,

G. H. Fredrickson, I. H. Rosedale, and G. D. Wigmall.

Sriall-Angle Neutron Scattering Characterization of Polyphenylene Sulfide-

G. B. Caflisch, D. R. Fagerburg, C. Sass, and G. D. Wignall

Sir..ll-Angle Neutron Scattering from Bimodal Polymer Melts-C. Tangari, R. Ullıan,

S. S. King. and G. D. Wignall

Es bmation of Incoherent Backgrounds in Small-Angle Neutron Scattering (SANS) Studies of Polymers-G. D. Wignall, W: S. Dubner, and J. M. Schultz.

Time-Resolved X-Ray Studies on Premelting During Annealing of Gelation Crystallized Ultrahigh Molecular Weight Polyethylene-M. H. Cho, T. Kyu, J. S. lin, K. Saijo, and T. Hashimoto.

Structural Determination of Microstructures in Model Eile-E. W. Kaler, M. Long,

A. Martino, and G. D. Wignall .

Monovalent Counter-Ion Distributions Around Persistence Length DNA Fragments in

Solution-S. L. Chang, S. H. Chen, R. L. Rill, and J. S. Lin.

\section{NEUTRON DIFFRACTION}

Pressure Effects on the Martensitic Transformation in Metallic Lithium-H. C. Smith,

R. Berliner, J. D. Jorgensen, M. Nielsen, and J. Trivisonno.

Pressure Effects on the Martensitic Transformation in Metallic Sodium--H. C. Smith,

R. Berliner, I. D. Jorgensen, and I. Trivisonno.

Atomic Magnetic Moment Spatial Correlations in Fe-13.5\% V-I. W. Cable, B. Cillon,

I. Mirebeau, G. Parette, and $\because$. Nakai.

High-Resolution Powder Diffractometer-J. A. Fernandez-Baca and H. A. Mook. 
Rietveld Analysis of $\mathrm{Bi}_{2} \mathrm{ST}_{2-2} \mathrm{La}_{2} \mathrm{CuO}_{6+\mathrm{y}}$ from Neutron Powder Diffraction Data-

B. C. Chakoumakos, I. A. Fernandez-Baca, B. C. Sales, and H. A. Mook.

Search for Strain Effects in the Spinodal Decomposition of Mn-Cu Alloys-Sicoe Spooner,

J. A. Fermandez-Baca, and Y. Morï

Cotsrent Neutron Scattering Length of ${ }^{*} \mathrm{Ca}-$ R. M. Mcon, J. A. Fernandez-Baca,

and S. Raman.

Neutron Diffraction Study of Residual Stresses in a Multiple-Pass Weld in a Thick

Stainless Steel Plate-T. M. Holden, I. H. Roct, Joyce Goldstone, M. A. M. Bourke,

S. A. Dooid, and Steor Spooner.

\section{INELASTIC NEUTRON DIFFRACTION}

Magnetic Fluctuations in $\mathrm{La}_{1}, \mathrm{X}_{5} \mathrm{CuO}_{4}: \mathrm{X}=\mathrm{Ba}$ or $\mathrm{Sr}-H$. A. Mook, G. Aqpli, S. M. Hayden,

D. Rytz, and Z. Fisk.

Observation of Phonon Softening at the Superconducting Transition in $\mathrm{Bi}_{2} \mathrm{Si}_{2} \mathrm{CaCu}_{2} \mathrm{O}_{6}$

H. A. Mook, M. Mostoller, I. A. Harvey, N. W. Hill, B. C. Chatoumakos, and B. C. Sales......

Magnetic Excitations in Thulium-J. A. Fernandez-Baca, R. M1. Nicklow, Z. Tun,

and I. J. Rhyne.

Magnetic Critical Scattering from Thulium-M. Hagen, H. R. Child,

I. A. Fernandez-Bace, and I. L. Zarestky.

\section{Synthesis and Physical Properties of Materials}

\section{SUPERCONDUCTORS}

Orientation-Dependent Critical Currents in $\mathrm{YBa}_{2} \mathrm{Cu}_{3} \mathrm{O}_{7-\mathrm{z}}$ Epitaxial Thin Films: Evidence for Intrinsic Flux Pinning-D. K. Christen, C. E. Klabunde, R. Feenstra, D. H. Lowndes, D. P. Norton, J. D. Budai, H. R. Kerchner, J. R. Thompson, S. Zhu, and A. D. Marwick.

Defect Independent Flux Creep in As-Grown and Proton-Irradiated Single-Crystal

$\mathrm{YBa}_{2} \mathrm{Cu}_{3} \mathrm{O}_{T}$ I. R. Thompson, D. K. Christen, Yangren Sun, L. Ciznle, A. D. Marwick,

M. W. McElfresh, T. K. Worthington, A. P. Malozemoff, and F. H. Holtzberg.

Magnetic Flux Pinning in $\mathrm{YBa}_{2} \mathrm{Cu}_{3} \mathrm{O}_{7-x}$ Films- $H$. R. Kerchner, $\boldsymbol{\kappa}$. Feenstra, 1 . O. Thomson,

I. R. Thompson, D. K. Christen, S. T. Sekula, and L. A. Boatner.

Dynamic Magnetic Hysteresis of $\mathrm{YBa}_{2} \mathrm{Cu}_{2} \mathrm{O}_{7 .}$ Thin Films-H. R. Kerchner, I. O. Thomson,

$R$. Feenstra, and D. K. Christen 
Superconductivity in Nonsymmetric Epilaxial $\mathrm{YBa}_{2} \mathrm{Cu}_{3} \mathrm{O}_{7-2} / \mathrm{PrB}_{2} \mathrm{Cu}_{3} \mathrm{O}_{7-2}$ Superlattices:

The Superconducting Exhavior of Cu-O Bilayers-Dougles H. Lowndes, Droid P. Norton, and I. D. Budai.

Superconducting Transitions of $\mathrm{YBa}_{2} \mathrm{Cu}_{3} \mathrm{O}_{-2} / \mathrm{PrBa}_{2} \mathrm{Cu}_{5} \mathrm{O}_{3}$ Superlattice Thin Filus H. R. Kerchner, J. O. Thomson, I. R. Thompson, J. Ossendon, D. Norton, and D. H. Lowndes.

Stability of $\mathrm{YBa}_{2} \mathrm{Cu}_{3} \mathrm{O}_{7-2} / \mathrm{PrB}_{2} \mathrm{Cu}_{3} \mathrm{O}_{7-2}$ Superlattices: Effect of Elevated Temperature, Low-Pressure Oxyzen Annealing-D. H. Lowndes, D. P. Norton, R. Feenstru, and J. D. Budai.

Electrical Transport Dissipation Effects in Epitaxial $\mathrm{YBa}_{2} \mathrm{Cu}_{3} \mathrm{O}_{7-x}-D$. K. Christen, C. E. Klabunde, R. Feenstra, D. H. Lownde, D. Norton, H. R. Kerchner, J. R. Thoompson, S. T. Setula, J. D. Budai, L A. Boaluer, J. Nanayn, and R. Singh

Effect of Oxygen Pressure on the Synthesis of $\mathrm{YBa}_{2} \mathrm{Cu}_{3} \mathrm{O}_{2}$ Thin Films by Postdeposition Annealing--R. Feenstro, T. B. Lindemer, I. D. Budai, and M. D. Galloway.

Static and Dynamic Magnetization Studies of $\mathrm{YBa}_{2} \mathrm{Cu}_{3} \mathrm{O}_{7}$ Thin Films-S. T. Selula,

R. Feenstra, I. R. Thompson, D. K. Christen, H. R. Kerchner, L. A. Boatner, and J. Budai.

Effect of Grain Boundaries on Critical Current Density in $\mathrm{YB}_{2} \mathrm{Cu}_{3} \mathrm{O}_{-}$Films on Polycrystalline Zirconia-David P. Norton, Dougles H. Lowndes, J. D. Budai, D. K. Christen, E. C. Jones, K. W. Lay, and I. E. Tkaczyt

Hole Filling and Hole Creation in the Superconducting Compounds $\mathrm{Bi}_{2} \mathrm{Si}_{2-x} \mathrm{RE}_{2} \mathrm{CuO}_{6+\mathrm{y}}$

RE = La, Pr, Nd, and Sm-B. C. Sales and B. C. Chatoumakos.

Temperature-Dependent Magnetic Penetration Depth $\lambda(T)$ in High-Temperature Superconductors-I. R. Thompson, D. K. Christen, Yengren Sun, S. T. Sekula, I. G. Ossandon, H. R. Kerchner, J. Brynestad, B. C. Chakoumakos, B. C. Sales, I. Budai, and E. Sonder

\section{SOLID ELECTROLYTES AND CERAMIC FILMS}

Electrical Properties of $\mathrm{Li}_{2} \mathrm{O}_{-} \mathrm{SiC}_{2}-\mathrm{P}_{2} \mathrm{O}_{5}$ Amorphous Lithium Electrolyte Thin FilmsI. B. Bates, N. I. Dudney, and C. F. Luck.

Segregation of Lithium During RF Magnetron Sputtering of Lithium OrthosilicateN. J. Dudncy, J. B. Bates, and I. D. Robertson.

Fabrication, Composition, and Structure of $\mathrm{Li}_{2} \mathrm{O}: \mathrm{SiO}_{2}: \mathrm{P}_{2} \mathrm{O}_{3}$ Thin Films-I. B. Bates, N. I. Dudney, J. D. Robertson, B. C. Sales, R. A. Zuhr, A. L. Wachs, and C. F. Luck

Sputtering of $\mathrm{Li}_{4} \mathrm{SiO}_{4} \mathrm{Li}_{3} \mathrm{PO}_{4}$ and $\mathrm{Li}_{2} \mathrm{O}-\mathrm{N}$. I. Dudney, I. B. Bates, and J. D. Robertson 62 
Plasma Diagnostic Studies of the Influence of Process Variables on the Atomic and Molecular Species Ejected from (1-x)Li,SiO, $x_{1} i_{3} P O_{4}$ Targets During RF Magnetron Sputtering-A. L. Wachs, J. B. Bates, N. J. Dudney, and C. F. Luck

Microbattery Research-G. R. Gruzalshi, J. B. Bates, N. J. Dudncy, and C. F. Luck

Enhancea lonc Conduction in Silver Halide-Alumina Composites-N. J. Dudney

Interface and Bulk Relaxation in Solid lonic Conductors-I. B. Bates, J. C. Wang.

and Y.T. Chu.

\section{OPTICAL, MAGNETIC, AND PHYSICAL : CHARACTERIZATION OF MATERIALS}

Characterization of Textured Ceramics by Electron Paramagnetic Resor :nce (EPR)

Spectroscopy: Formation and Properties of Textured MgO-J.-L. Boliad O., L. A. Boalnet,

and M. M. Abraham.

Structural Relaxation Dynamics of Phosphate Glasses: The Effects of Network Topology

on The Glass Transition-B. C. Sales.

a-Decay-Induced Condensation of Phosphate Anions in a Mineral-B. C. Chakoumakos,

B. C. Sales, and I.. A. Boalner.

Low-Frequency Relaxation Modes and Structural Disorder in $\mathrm{KTa}_{1-2} \mathrm{Nb}_{2} \mathrm{O}_{3}-J$. P. Sokolof,

L. L. Chase, and L. A. Boainer

Infrared Study of Oxygen Vacancies in $\mathrm{KTaO}_{3}-S$. Jandl, M. Bant rille, P. Dufour,

S. Coulombe, and L. A. Boalner.

Longitudinal Elastic Behavior in the Mixed Ferroelectric KTN-X. M. Wang, I. Toulouse,

and L. A. Boalner

Fuorescence and Ferroelectric Microregions in $\mathrm{KTaO}_{3}-P$. Grenier, G. Bernier, S. Jandl,

B. Salce, and L. A. Boatner.

Study of Protons in Acceptor-Doped $\mathrm{KTaO}_{3}$ Crystals by IR and EPR Techniques-S. Q. Fu, W.-K. Lee, A. S. Nowick, L. A. Boalner, and M. M. Abraham

Determination of the Optical Functions of Transparent Classes Using Spectroscopic

Ellipsometry-C. E. Jellison. Jr., and B. C. Sales.....

Use of the Biased Estimator in the Interpretation if Spectroscopic Ellipsometry Data - -

G. E. Jellison, Jr.

Accurate Calibration of a Photoelastic Modulator in Polarization Modulation

Ellipsometry-G. E. Jellison, Ir., and F. A. Modine. 
Temperature Dependence of Singly and Doubly lonized Acceptor-Hole Recombination in Germanium-L.S. Darken and G. E. Jellison, Ir.

Examination of Thin $\mathrm{SO}_{2}$ Films on Si Using Spectmscopic Polarization Modulation Ellipsometry-G. E. Jellison, Jr.

Trapped-Hole Centers in Alkaline-Earth Oxides-Y. Chen and M. M. Abraham

Prospects for Wavelength Tunable Lasers Based on Vacancy Defects in Alkalina-Earth

Oxides-Y. Chen, V. M. Orern, and R. Gonzalez.

Effect of Substitutional Hydride lons on the Charge States of Oxygen Vacancies in Ther mochemically Reduced $\mathrm{CaO}$ and $\mathrm{MgO}-Y$. Chen, V. M. Oren, R. Conzalez,

R. T. Williams, G. P. Williams, G. H. Rosenblatt, and G. J. Pogetshnik.

Analysis of Solidification Microstructures in Fe-Ni-Cr Single-Crystal Welds-

M. Rappaz, S. A. David, J. M. Vilek, and L. A. Baatner.

Microstructure of Stainless Steel Single-Crystal Electron Beam Welds-S. A. Dawid,

J. M. Vitek, M. Rappaz, and L. A. Boatner.

Anomalously Small 4f-5d Oscillator Strengths and $4 f-4 f$ Electronic Raman Scattering Cross Sections for $\mathrm{Ce}^{3+}$ in Crystals of LuPO,-G. M. Willioms, N. Edelstein, L. A. Boatner, and M. M. Abraham

Excitation Profiles of Resonance Electronic Raman Scattering in ErPO, Crystals-

G. M. Williams, P. C. Becker, N. Edelstain, L. A. Boatner, and M. M. Abraham

Intensities of Electronic Raman Scattering Between Crystal-Field Levels of $\mathrm{Ce}^{3+}$ in LuPO,: Nonresonant and Near-Resonant Excitation-G. M. Williams, P. C. Becker,

I. G. Conway, N. Edelstain, L. A. Boatner, and M. M. Abraham.

A Photovoltaic-Powered Microtransmitter Used for Mating-Habit Studies of Africanized Bees-R. D. Westbro,x, G. E. Jellison, J., P. H. Fleming, D. D. Falter, G. T. Alley, K. G. Falier, and !. M. Rochelle.

\section{Ion Beam and Laser Processing}

\section{ION BEAM PROCESSING}

Oriented Aluminum Films on Silicon by Direct Ion Beam Deposition-R. A. Zuhr and T. E. Haynes.

Ion-Induced Damage Formation by Frenkel Pair Separation-0. W. Holland and

C. W. White.

Mechanisms of Low-Temperature Crystallization in Si-O. W. Holland, C. W. White, M. K. El.Ghor, and I. D. Budai 
Precipitation in Silicon Implanted by Oxygen and Carbon Plus Oxygen-Y. L. Chen,

R. W. Carpenter, and T. P. Sjoreen..

Optical Absorption of $\mathrm{Cu}$ - and Bi-Implanted Silica-R. H. Magruder, R. A. Weeks,

and R. A. Zuhr

Nonlinear Optical Measurements of lon-Implanted Silica-K. Becker, L. Yang,

R. F. Hoglund, R. H. Magruder, R. A. Weets, and R. A. Zuhr.

Ion Implantation fo: Zorrosion Inhibition of Aluminum Alloys in Saline Media-

I. M. Williams, A Gonzales, I. Quintana, I-S. Lee, R. A. Buchanan, F. C. Bums,

R. J. Culbertson, M. Leay, and J. R. Treglio

Optical Waveguide Fabrication by Stoichiometric Implantation of $\mathrm{Ti}$ and $\mathrm{O}$ into $\mathrm{LiNbO}_{3}-$ D. B. Poket ani. W. Xia

Structural Differences Between the Glass State and Ion Beam Amorphized States of Lead Pyrophosphate-B. C. S.'les, I. O. Ramey, J. C. McCallum, and L. A. Boatner..

Ion Irradiation Effects for $\mathrm{MgF}_{2}$ Films on LiF Substrates_-G. C. Farlow and L. A. Evatner............ 97

Electron Cyclotron Resonance Microwave Plasma Source Studies-S. M. Corbatkin,

L. A. Berry, and I. B. Roberto

Electron Cyclotron Resonance Microwave Plasma-Etch Tool Development-L. A. Berry.

S. M. Gorbatkin, G. H. Henkel, and I. B. Roberto.

\section{ION IMPLANTATION AND ANALYSIS}

Dose-Rate Effects on Damage Accumulation in Ion-Implanted Gallium Arsenide-

$T$. E. Haynes and $O$. W. Holland.

Comparative Study of Damage Accumulation in Ion. Implanted Gallium Arscnide and

Germanium-T. E. Haynes, O. W. Holland, and S. I. Pennycook.

Buried Er Silicide Formation by Ion Implantation and Annealing-A. Colanski,

C. W. White, M. D. Galloway, W. H. Christie, H. E. Harmon, J. L. Park, and

S. J. Pennycook.

Manar Channeling for Dopant Site Location in Dual-Implanted Gallium Phosphide-

N. R. Parith, C. T. Keo, D. R. Lee, I. Muse, M. L. Swanson. T. E. Haymes,

$R$. Venkalasubramanian, and $M$. Timmons..

Annealing of Buried Amorphous Layers in MeV-Ion-Implanted Ge-T. P. Sjoreen,

S. I. Pennycook, and O. W. Holland.....

Ion Beam Induced Epitaxial Crystallization of Heavily Doped Si-S. P. Withrow,

O. W. Holland, and S. I. Pennycuok. 
Trapping of D in SiC and Damage Due to Implantation-R. Sigete, S. P. Withrow, J. Roth, and B. M. U. Scherzer.

Localized Conversion of Epitaxial $\mathrm{YBa}_{2} \mathrm{Cu}_{3} \mathrm{O}_{7-2}$ from $\mathrm{C} \perp$ to $\mathrm{a}_{\perp}$ by lon Implantation and Low Oxygen Pressure Annealing-R. Feenstra, S. J. Pennycook, D. P. Norton, D. H. Lowndes, J. D. Budai, and M. D. Galloway.

Effect of $\mathrm{H}_{2} \mathrm{O}$ Vapor on the Epitaxial Recrystallization of Ion Beam Amorphized $\mathrm{SrTiO}_{3}$-I. Rankin, J. C. McCallum, and L A. Boatner.

High-Resolution X-Ray Study of Implanted Sulphur as a Probe of Coimplanted Oxygen in an Oxide Substrate-T. M. Rossed, R. S. Peterson, C. R. Vane, I. P. Young, and R. A. Zukr.

Ion Mixing of Semiconductor Superlattices-W. Xia, S. A. Pappert, C. Cozzolino, I. Zhang, C. W. Tu, P. K. L Yu, S. S. Lau, and D. B. Poker

\section{LASER AND MOLECULAR BEAM PROCESSING OF THIN FILMS}

In Situ Growth of High-Quality Epitaxial $\mathrm{YBa}_{2} \mathrm{Cu}_{3} \mathrm{O}_{7-3}$ Thin Films by Pulsed-Laser Ablation-D. H. Lowndes, D. P. Norton, J. W. McCany, R. Feenstra, J. D. Budai, D. K. Christen, and D. B. Poker

Y-Ba-Cu-O Thin Films Grown on Rigid and Flexible Polycrystalline Yttria-Stabilized Zirconia by Pulsed-Laser Ablation-David P. Norton, Douglas H. Lowndes, J. W. McCamy, J. D. Budai, D. K. Christen, E. C. Jones, Thomas D. Ketcham, Dell St. Julien, K. W. Lay, and J. E. Tkaczyk

New Facilities for Fundamental Studies of Laser-Cenerated Plasmas and for Laser-Ablation Film Growth-D. H. Lowndes, D. B. Geohegan, R. Feenstra, I. W. McCamy, und S. Zhu.

Combined Spectroscopic and Ion Probe Characterization of the Laser Ablation Transport Process-D. B. Geohegan.

First Direct Measurements of Ground-State Neutral and lon Transport During Laser Ablation of YBCO Using Transient Optical Absorption Spectroscopy-D. B. Geohegan

Development of Optical Absorption, Emission, and Ion Probe Methods and Facilities to Characterize Laser Ablation Plumes-D. B. Ceohegan.

The Effect of Deposition Rate on the Growth of Epitaxial Ce on CaAs(100)-D. Eres, D. H. Lowndes, I. Z. Tischler, I. W. Sharp, T. E. Haynes, and M. F. Chisholm.

Gas-Phase Characterization of Digermane Molecular Jets Used in Thin-Film CrowthD. Eres and I. W. Sharp 
Mechanism of Ge Thin-Film Growth from Digermane Molerular Jets-D. Eres and

J. N. Sharp.

Apparatus for Investigation of Thin-Film Growth by Utilizing Supersonic Molecular

Beams-D. Eres and J. W. Sharp.

120

\section{Structure of Solids and Surfaces}

\section{SURFACE PHYSICS}

Growth Kinetics in the Phase Tra:isition from $A g(111)^{\prime}$ to $(\sqrt{3} \times \sqrt{3}) R 30^{\circ}$ Structures for Ag/Si(111)-J.-K. Zuo a.d J. F. Wendelken.

Scaling of the $(\sqrt{3} \times \sqrt{3})$ R30 Domain Size Distribution with Coverage for Ag/Si(111)-I.-K. Zuo and I. F. Wendelken.

A Low-Energy Li Ion Scattering Study of the Surface Structure of Stepped NiAl(111)-

S. H. Overbury, D. R. Mullins, and I. F. Wendelker

Confirmation of an Exception to the "General Rule" of Surface Relaxations-I. R. Noonan

and H. L. Davis

Structure and Phases of the Au(001) Surface: X-Ray Scattering Measurements-

S. G. J. Mochrie, D. M. Zehner, B. M. Ocko, and Doon Gibbs.

Phase Buhavior of the Au(i11) Surface: Discommensurations and Kinks-K. G. Huang,

Doon C.bbs, D. M. Zehner, A. R. Sandy, and S. G. I. Mochrie.

Enhanced Surface Anharmonicity Observed in Vibrations on Cu(110)-A. P. Baddorf and E. W. Plummer.

Radiation-Enhanced Dissociation of Oxygen on a Copper Surface-A. P. Baddorf and

I. F. Wendelken.

Chemisorption of Nitrogen on Cu(110): Coverage and Site Detemination-A. P. Baddorf and D. M. Zehner.

Charge-Distribution Changes Accompanying the Formation and Changes in the

Composition of $\mathrm{HfC}_{\mathrm{x}}$ and $\mathrm{TaC}_{\mathrm{x}}-\mathrm{C}$. R. Gruzalski and D. M. Zehner

Work-Function Changes Accumpanying Changes in Composition of (100) Surfaces of $\mathrm{HCC}_{x}$ and $\mathrm{TaC}_{\mathrm{z}}-\mathrm{C}$. R. Gruzalski, S.C. Lui, and D. M. Zehner. 


\section{ELECTRON MICROSCOPY}

Hign-Resolution Incoherent Imaging of Crystals-S. J. Pennycook and D. E. Jesson

Incoherent Imaging of Thin Specimens Using Coherently Scattered Electrons-D. E. Iesson and S. J. Penmycook

Angular Mapping of Electron Channeling Phenomena Using Scanning Transmission Electron Microscopy-D.E. Jesson and S. J. Pennycook

Z-Contrast Imaging of High-Angle $\mathrm{YBa}_{2} \mathrm{Cu}_{3} \mathrm{O}_{7-6}$ Grain Boundaries-M. F. Chisholm and S. I. Pennycook.

Solid-Phase-Epitaxial Recrystallization of Ion-Implanted $\mathrm{YBa}_{2} \mathrm{Cu}_{3} \mathrm{O}_{7-\mathrm{x}}$ Thin Films-

S. I. Pennycook, R. Feenstra, M. F. Chisholm, J. D. Budai, D. P. Norton, and

D. H. Lowndes

Growth Mechanisms and Interdiffusion of $\mathrm{YBa}_{2} \mathrm{Cu}_{3} \mathrm{O}_{7-x} / \mathrm{PrBa}_{2} \mathrm{Cu}_{3} \mathrm{O}_{7-x}$ Superlattices-

S. I. Pennycook, M. F. Chisholm, D. E. Jesson, H. R. Kerchner, D. P. Norton, and

D. $H$. Lowndes

Structural Origin of Reduced $J_{c}$ in $\mathrm{YBa}_{2} \mathrm{Cu}_{3} \mathrm{O}_{7-8}-\mathrm{M}$. F. Chisholm and S. I. Pennycook.

Direct Imaging of Interfacial Ordering in Ultrathin $\left(\mathrm{Si}_{m} \mathrm{Ge}_{n}\right)_{p}$ Superlattices Using

Z-Contrast Scanning Transmission Electron Microscopy-D. E. Jesson,

S. J. Pernycook, and J. M. Baribeau.

A Novel Ge Atom Pump Mechanism Operating During Si Molecular Beam Epitaxy Growth on Ge-D. E. Jesson, S. J. Pennycook, and J. M. Baribeau

Strained Ge/Si Heterostructures-M. F. Chisholm and S. I. Pennycook.

Misfit Accommodation in Epitaxial Ge Films on Si-M. F. Chisholm, S. J. Pennycook, and $O$. W. Holland

Direct Imaging of Interface Structure and Chemistry in $\mathrm{GaAs} / \mathrm{In}_{0.5} \mathrm{Ga}_{0.5} \mathrm{P}$ Quantum

Wells-S. J. Pennycook, M. F. Chisholm, D. E. lesson, and G. Y. Robinson..

Interfacial Defects in Epitaxial $\mathrm{CoSi}_{2}$ rilms on $\mathrm{Si}(100)-S$. I. Pennycook, D. E. lesson,

S. M. Yalisove, and R. T. Tung.

Ion Beam Synthesis of Buried Erbium Silicide-I. L. Park, A. Colanski, S. I. Pennycook,

R. Feenstra, M. D. Galloway H. E. Harmon, and C. W. White

\section{X-RAY DIFFRACTION}

Resonant Monochromators for Submicrovolt Energy Resolution X-Ray Diffraction-

J. Z. Tischler, B. C. Larson, and G. E. Ice. 
Time Spectrum of Resonant Nuckear Bragg Scattering ficm a Mosaic Crystal-

Time-Domain Spectrum of Resonantly Filtered X Rays from a Nuctear Bragg

Monochromator-I. Z. Tischler, B. C. Larson, and P. Zschack.

X-Ray Study of Epitaxial $\mathrm{YBa}_{2} \mathrm{Cu}_{3} \mathrm{O}_{x} / \mathrm{PrBa}_{2} \mathrm{Cu}_{3} \mathrm{O}_{2}$ Superdattices-I. D. Budai,

D. H. Lowndes, and D. P. Norton.

Preferred Alignment of Twin Boundaries in $\mathrm{YBa}_{2} \mathrm{Cu}_{3} \mathrm{O}_{\mathbf{r}}$ Thin Films and Superlattices on $\mathrm{SrTiO}_{3}$-I. D. Budai, M. F. Chisholm, R. Feenstra, D. H. Lowndes,

D. P. Norton, L. A. Boatner, and D. K. Christen.

Direct Determination of Precipitate Strain and Sizes Using High-Order X-Ray

Diffuse Scattering-B. C. Larson, M. D. Calloway, and P. Zschack.

The Effect of Microgravity Disturbances on the Growth of Indium Crystals-

J. D. Budai, R. M. Nicklow, D. A. Thomas, and B. I. Dunbar .

A Structural Investigation of Several Mixed Lanthanide Orthophosphates-

D. F. Mullica, E. L. Sappenfield, and L. A. Boatner

PUBLICATIONS AND PAPERS.

SEMINARS

SCIENTIFIC ACTIVITIES, AWARDS, AND HONORS.

PERSONNEL CHANGES. 


\section{Acronyms}

AES - auger electron spectroscopy

AFS - Adianced Photon Source

ANS - Advanced Neutron Suunce

BCA - binary collision approximation

CBED - convergent beam electron diffraction

CHESS - Comell High-Energy

Synchrotron Source

CPA - constant-phase angle

ECR - electron cydotron resonance

EELS - electron energy-loss spectroscopy

EPR - electron paramagnet resonance

FWHM - full width at half maximum

FZ - float-zone

HFIR- High Flux Isotope Reactor

HIXSE - heavy-ion-induced x-ray satellite emission

HPLC - high-performance liquid chromatography

HREM - high-resolution clectron microscopy

HREELS - high-resolution electron energy loss spectroscopy

HTSc - high-temperature superconductors

IANS - intermediate angle neutron scattering

IBD - ion beam deposition

IPNS - Intense Pulsed Neutron Source

LDA - local density approximation

LEED - low-energy electron diffraction

MBE - molecular beam epitaxy

MIB - mutual inductance bridge

MOCVD - molecular orbital chemical vapor deposition
NSLS - National Synchrotron Light Source

PEP-PEF - Folyfethylene-propylene)poly(ethylethylene)

PIXE - particle-induced $x$-ray emission

PLA - pulsed-laser ablation

QMS - quadrupolp mass spectrometer

RBS - Rutherford backscattering spectroscopy

RHEED - reflection high-energy electron diffraction

RR - resistance ratio

SANS - small-angle neutron scattering

SAXS - small angle $x$-ray scattering

SEM - scanning electron microscopy

SF - stacking faults

SSL - strong segregation limit

SPEC - solid-phase-epitaxial growth

STEM - scanning transmission electron microscopy

TCR - thermochemical reduction

TDS - thermal desorption spectroscopy

TEM - transmission electron microscopy

TOF - time-of-flight

TRR - time-resolved reflectivity

UHV - ultrahigh vacuum

VSM - vibrating sample magnetometer

WAXD - wide-angle $x$-ray diffraction

WSL - weak-segregation limit

XDCD - x-ray double-crystal diffraction

XPS - $x$-ray photoemission spectroscopy

YSZ - yttria-stabilized zirconia 


\section{Introduction}

This report covers research progress in the Soli', State Division from April 1, 1989, to September 30, 1990. During this period, division research programs were significantly enhanced by the restart of the High-Flux lsotope Reactor (HFIR) and by new initiatives in processing and characterization of materials.

HFIK restart has been accompanied by a major effort to reestablish and expand the neutron scattering user program and to sake full research advantage of the new instruments and instrumentation upgrades which were installed during the sluutdown. Preparations for the Advanced Neutron Source (ANS) are accelerating at ORNL, and the Neutron Scattering Program is playing a crucial role in the scientific direction, instrumentation, and research community coorciination for this projact.

New capabilities in thin-film processing have been added to the substantial existing resources in surface modification and ion implantation. New programs at the state of the art have been developed in laser ablation fabrication of superconducting superlattices, formation of thin-film composites for microbattery applications, and advanced plasma etching for SEMATECH. These programs are complemented by traditional strengths in surfa_e physics and microstructural characterization.

Progress in the development and application of Z-contrast scanning transmission electron microscopy for the atomic-scale characterization of materials has continued. The technique was put on a firm theoretical basis, new and unforeseen interface structures were discovered in semiconductor and superconductor systems, and preparations for the development and ourchase of a new instrument with unprecedented high resolution were completed.

The broad divisional effort in high-T $T_{c}$ superconductivity has continued with substantial progress in theory, process development, materials characterization, and transport investigations. Applied research in support of the ORNL High-T ${ }_{c}$ Superconductivity Pilot Center included cooperative projects with more than ten high-technology industries and provided an important focus and outlet for the fundamental work.

While maintaining a broad research prugram ranging from the theory of "sand piles" to new experimental descriptions of the amorphous state, the division continued 
a tra dition of service and leadership in the sirientific community. During this reporting period, 15 division staff members chaired or co-chaired national conferences and workshops, 6 served as nationai officers or executive committee members for professional societies, 9 served on editorial boards for technical journals, and 8 edited or co-edited books and proceedings. 


\section{Theoretical Solid State Physics}

A comprehensive review of current research activities of the Solid State Theory Program is presented in this chapter. The work covers a broad range of reseanch which exitends from "sand piles" to particles with fractional siatistics. This diversity results from coilaborations with the broad spectrum of the division's experimental programs and inherent interests of the staff, a combination which is crucial to maintaining a strong and productive theoretical effort.

A significant fraction of the research described in thes chapter is associated with highly correlated systems and includes work on high-temperature superconductivity, electrons in very high magnetic fields, and itinerant electron magnetism in meials. The lattice dynamics of $\mathrm{La}_{2} \mathrm{CuO}_{4}$ has been studied, and phonon spectra have been predicted. The superconducting properties of $\mathrm{YBa}_{2} \mathrm{Cu}_{3} \mathrm{O}_{7}$ and $\mathrm{PrBa}_{2} \mathrm{Cu}_{3} \mathrm{O}$ layered films have been analyzed both in terms of a Kosterlitz-Thouless transition and a spin-polaron model. A theory based on interlayer pairing of Cooper pairs has been used to describe selfconsistently the superconducting properties of flanes and chains in $\mathbf{Y 1 2 3}$ materials. Work on anyons has raised new questions as to the applicability of this concept to high- $T_{c}$ systems.

In other theoretical work, the spin-wave spectra for hcp cobalt have been predicted to be remarkably isotropic with energies reaching about $300 \mathrm{meV}$. Results from fits to the phonon spectra of insulators based on an indirect interaction model have confirmed speculation that three-body forces are larger for ions with filled $d$-shells. The theory of low-energy electron diffraction has been used to determine the surface structure and composition of the substitutionally random binary alloy Mo85Re15. An analysis of collision cascades in $\mathrm{Cu}$ has predicted less damage when collisions between particles are properly time ordered instead of being velocity ordered. In one of the new areas of research, results from a study of "sand piles" have raised doubts about the universal scaling of their static and dynamic properties and have established a connection between $1 / f$ fluctuations and diffusion through self-generated complex patterns. 


\section{SUPERCONDUCTIVITY}

\section{INTRALAYER VERSUS INTERLAYER PAIRING IN THE COPPER OXIDE SUPERCUNDUCTORS 1}

\section{R. A. Klemm ${ }^{2}$ and S. H. Liu}

Crystals of high- $T_{c}$ copper oxide superconductors are highly stratified, and it is known that they have ni,gh normal-state conauctivity in directions parallel to the planes and low conductivity in the perpendicular d.reclions. There is increasing experimental evidence that these materials also have highly snisotropic superconducting energy gaps. Existing theories of superconductivity in la:ered systems, which assume that the Croper pairs always form in the same layer, predict either isotropic or only minutely anisotropic $\boldsymbol{g}$ P functions. Since the gap function is of fundamental importance to the understanding of superconducting properties, it is imperative that the theory be modified so that the anisotropic gap is a central feature. We investigated in detail possible mechanisms for gap anisotropy and discovered that the only one capable of producing the observed gap anisotropy is interlayer pairing (i.c., the two electrons that form the Cooper pair actually reside in different $\mathrm{CuO}_{2}$ planes). This new type of pairing introduces a new complication (i.e., that the Pauli principle allows the Cooper pair to be in singlet or triplet states). As a result the order parameter is in general a fourvector, 'with one component for singlet pairing and three for triplet pairing. If the system has two or more layers in a unit cell, both types of paining produce highly anisotropic gaps. The competition between intralayer and interlayer pairing and the possible coexistence of intralayer and interlayer order parameters have been studied in detail. The resulting theory, now much more complex in its formulation, may be a more realistic starting point for investigating the many intriguing electromagnetic properties of these new superconductors.

1. Summary of paper: Physica $C$ (in press).

2. Present address: Argonne National Laboratory, Argonne, Ill.

\section{SELF-INDUCED EFFECTIVE GAUGE FIELDS IN THE COPPER-OXYGEN PLANE OF HIGH-T, PEROVSKTTES: A LATTICE AND A CONTINUUM FORMULATION'}

\section{Merk Resolt and G. Vignale ${ }^{2}$}

One of the exciting proposals for the mechanism of high-T $T_{c}$ superconductivity is the creation of "particles" with fractional statistics called anyons. The existence of these anyons depends crucially on the twodimensional aspect of the $\mathrm{Cu}-\mathrm{O}$ planes and the existence of a particular gauge field whose origin is the spontaneously broken symmetry of the ground state; the so-called chiral spin liquid. It has been assumed that such a chiral ground state can exist in the high- $T_{c}$ materials; three available experiments give conflicting results. Following such an assumption, the scenario of anyon superconductivity is very plausible. The fractional statistics places the 
carriers between fermions and bosons leading to a real space-like condensation, not unlike the Bose condensation of $\mathrm{He}^{4}$, which could require no pairing mechanism. Morc precisely, the fractional statistics can be mapped to another gauge field, called the Chern Simon field, which shares similarities with a spontaneously created uniform magnetic field leading to many of the properties shared by the quantum Hall cffect.

An exact formulation of these self-induced effective gauge fields was derived, ioth in the Hubbard model and in the true continuum description of the Cu-O planes of the high- $T_{c}$ perovskites. The solution for the ground state was bypassed in favor of the chirality order parameter as the intrinsic variable. The constraint of gauge symmetries was found to narrow greatly the admissible forms for these gauge fields. It was found that these fields can produce broken-symmetry chiral ground states on a battice, but the intrinsic frustration in the continuum form of the gauge field seems to rule out such a state in the real $\mathrm{Cu}-\mathrm{O}$ plane.

1. Summary of paper: Phys. Rev. Lett. 65, 1498 (1990).

2. University of Missouri, Columbia, Mo.

\section{ON THE ORDERING OF CHIRALTTY FOR MANY PLANES OF ANYONS'}

\section{A. G. Rojo' and C. S. Canright ${ }^{2}$}

Identical anyons are particles that give an arbitrary complex phase $\mathrm{e}^{\mathrm{j} \theta}$ upon interchange which is neither +1 (Bose) nor - 1 (Fermi), and due to simple topological reasons, they can live only in two dimensions. ${ }^{3}$ The high-temperature superconductors are based on copper-oxide planes, which are in a useful approximation, two dimensional. It has been argued that particles doped over the insulating phase of these compounds become dressed quasiparticles that obey fractional statistics. One essential feature of the anyon models is the violatif, the time reversal symmetry $T$; and in predicting observable quantities in the bulk system, it is of crucial importance to have a picture of the ordering of the signs of the spontamous symmetry breaking (statistical angles) in different layers. We snow that if one includes a twobody Coulomb interartion $V$ between anyons in different planes, the antiferromagnetic configuration of statistical angles in different planes is favored.

The ground-state energy for many planes of anyons $(p=2-4$, where $p$ is the number of coupled planes) has been calculated. All planes were identical (small lattices with free boundary conditions and $\boldsymbol{N}$ particles per plane) except for the sign of the statistics angle $\theta$. Ordering schemes for $\operatorname{sgn}(\theta)$ included strictly alternating order (AFM-e.g., +-+ for $p=3$ ), uniform order (FM: +++ ), and intermediate schemes for $p>2$. The quantity $\Delta E$. $E(F M)$ - $E(A F M)$ was calculated; thus $\Delta E>0$ implies that alternating order is favored energetically. It was found that $\Delta E>0$ for a wide variety of cases: for either sign of $V$, for the entire range of $\theta$, and for either sign of an in- 
plane near-neighbor coupling $U$. It follows, threfere, that the bulk system does not break time-reversal symmetry. A possible experimental test of our picture will be the dichroism for transmission in which it is predicted that the rotation angle should be independent of the sample thickness.

1. Summary of paper to be published.

2. Cuest scientist from the ORNL/UT Distinguished Scientist Program.

3. G. S. Canright and S. M. Girvin, Science 247, 1197 (1990).

\section{FIRST-PRINCIPLES HARTREE-FOCK CLUSTER AND BAND CAICULATIONS FOR La $\mathrm{CuO}_{4}^{1}$}

\section{P. Saalfrank, 2 R. F. Wood, I. Ladik, M. A. Abdel-Reouf, 2 and C.-M. Liegener?}

By use of an ab initio Hartree-Fock mxthod that avoids the 'ocal density approximation (LDA), $\mathrm{La}_{2} \mathrm{CuO}_{4}$ in undoped and doped forms is studied by cluster calculations that take the La atoms explicitly into account. In these computations the important effects of the crystal field are studied in detail. Density-of-states curves are computed and compared with experiment and other calculations. The charge and spin distributions and the extent of covalent bonding in $\mathrm{La}_{2} \mathrm{CuO}_{4}$ are examined. The $\mathrm{CuO}_{2}$ planes in the high- $T_{c}$ materials have sometimes been described as "islands of covalency" in otherwise ionic material. We find that, before doping, these materials are highly ionic throughout. Covalency becomes more important if one $\mathrm{La}$ atom in $\mathrm{La}_{2} \mathrm{CuO}_{4}$ is replaced by one of the alkaline-earth elements (Ba, Sr, Ca, or Mg). This "doping" produces holes in the $\mathrm{CuO}_{2}$ planes that are mainly oxygen-like and are of $\sigma$ symmetry. The alkaline-earth atoms are fully ionized, thus suggesting that theoretical models based on the polarizability of these substituents may no: be valid.

Hartree-Fock energy bands for a single $\mathrm{CuO}_{2}$ plane modeling tetragonal $\mathrm{La}_{2} \mathrm{CuO}_{4}$ were computed. The effects of (1) interplane chargetransfer and (2) Madelung potentials arising from the surrounding ions are taken into acceunt. The states around the Fermi level are found to be strongly dominated by in-plane $\mathrm{O} 2 \mathrm{p}-\mathrm{Cu} 3 d$ hybridization. Doping effects are studied by varying (1) the number of electrons associated with the $\mathrm{CuO}_{2}$ plane and (2) the threedimensional Madelung potential as functions of the doping value. It was found that in the "low-doping limit" rigid band models for high- $T_{c}$ ceramics are valid.

1. Summary of papers: Phys. Rev. B 41, 8824 (1990); Physical Reoiew B (in press).

2. Friedrich-Alexander-University, Erlangen-Nümberg, Germany.

\section{AP SPIN-POLARON MODEL OF HIGH-T SUPERCONDUCTIVTTY'}

\section{R. F. Wood, Mark Mostoller, J. F. Cooke, and M. A. Abdel-Reouf}

Development of the $d^{9}$ spin-polaron model of high- $T_{c}$ superconductivity has continued during the period covered by this report. Using stoichiometric $\mathrm{La}_{2} \mathrm{CuO}_{4}$ as the prototypical 
material, the most important features of our is spin-polaron model can be summarized as follows. $\mathrm{La}_{2} \mathrm{CuO}_{4}$ is a strongly correhted syster with long-range antiferromagnetic (AF) fluctilations of the $\mathrm{Cu}$ spins in the $\mathrm{CuO}_{2}$ planes. The material is a somewhat complicated MottHubband (M-H) insuiator with the $\mathrm{Cu} 3 \mathrm{~d}^{\mathrm{P}} \mathrm{con}$ figuration playing a predominant role. Doping with $\mathrm{Sr}$ introduces holes into the $\mathrm{CuO}_{2}$ planes and gives rise to the observed hole-type conductivity. Holes in the $\mathbf{O} 2 \mathbf{0}$ orbitals induce spin deriations on the $\mathrm{Cu}$ sublattice to form the spin polarons. The mechanism by which this happens is a slight modification of that discussed by Mott in anotiner context. It depends on the lowering of the kinetic energy of a carrier by the local transition from an AF to a ferromagnetic or random alignment of $\mathrm{Cu}$ spins. Pairing comes about because the localized spin deviations associated with two polarons can repair or "heal" one another by partially reestablishing local AF ordering.

The results of parameterized AF band calculations show how a strong electron-electron correlation, modeled by an on-site Hubbard term, opens a gap in the band structure obtained by more conventional calculations. The relative ordering in energv of $\mathrm{O} 2 p \sigma$ and $2 p \pi$ bands and the delocalization energy involved in spinpolaron formation are obtained from t:ese calculations. The gap $(\Delta)$ equation is studied, and approximate expressions for $T_{c}$ and $\Delta$ suitable for numerical work are derived. Numerical solutions of these expressions show that the model can fit the observed experimental results in $\mathrm{La}_{2-y} \mathrm{St}_{y} \mathrm{CuO}_{4}$ and $\mathrm{Y}_{1} \mathrm{Ba}_{2} \mathrm{Cu}_{3} \mathrm{O}_{7-y}$ for $T_{c}$ as a function of hole concentration. $A d^{8}$ spin polaron model proposed by Kamimura et al. ${ }^{3}$ has been contrasted to the $d^{9}$ spin-polaron model described here. The experimental evidence strongly argues against any significant role for the $\mathrm{Cu} d^{8}$ ion in these materials, whereas the configuration is almost invariably observed. Consequently, if a spin-polaron mechanism is applicable to high- $T_{c}$ superconductivity, it will likely be along the lines of the approach we have developed.

1. Summary of papers: Physica C 165, 97 (1990); Solid State Commun. 74, 371 (1990); to be published.

2. Friedrich-Alexander-University, Erlangen-Nümberg, Germany.

3. H. Kamimura, S. Matruno, and R. Saito, Solid Stale Commun. 67, 363 (1988).

\section{SPIN POLARONS, HOLE FILING, AND HHGH-T SUPERCONDUCTTVITY IN $\mathrm{YBa}_{2} \mathrm{Cu}_{3} \mathrm{O}_{7} \mathrm{AND} \mathrm{PrBa}_{2} \mathrm{Cu}_{3} \mathrm{O}_{7}$ ALLOYS AND LAYERED FILMS'}

\section{R. F. Wood}

Several groups have reported on the superconducting properties of alloys of $\mathrm{YBa}_{2} \mathrm{Cu}_{3} \mathrm{O}_{7}$ (YBCO) and $\mathrm{PrBa}_{2} \mathrm{Cu}_{3} \mathrm{O}_{7}$ (PBCO), and more recently superconductivity in layered structures of YBCO and PBCO has been extensively studied. In the $\mathrm{Y}_{1-x} \mathrm{Pr}_{8} \mathrm{Ba}_{2} \mathrm{Cu}_{3} \mathrm{O}_{7}$ alloys, $T_{c}$ shows a monatonic decrease with $x$ from $\sim 91 \mathrm{~K}$. at $x=0.0$ to $0 \mathrm{~K}$ at $x=0.60$. A complex dependence of $T_{c}$ on the structure of the $\mathrm{YBCO} / \mathrm{PBCO}$ supercells making up the layered films is found. The results are frequently discussed in terms of pair 
breaking by the Pr ions and/or filling of holes in the $\mathrm{CuO}_{2}$ planes. Here it is demonstrated that the $T_{c}$ data on both the alloys and the layered structures can be fit with precision and self-consistency using results from a spinpolaron model, 2 together with the hole-filling concept.

In this cakculation it is assumed that when YBCO; PBCO c-axis layered films are formed, the electrons from the Pr redistribute to minimize the total energy. The energy $\varepsilon$ of a supercell with $N_{Y}$ YBCO and $N_{P}$ PBCO layers is minimized with respect to the cell charges and subjet to the constraint of charge neutrality on the entire supercell. This assumes that the charge distribution in a unit cell is much less important than the magnitude in determining the energy. In this way. the problem of calculating $\varepsilon$ is decoupled from that of determining the distribution of the charge within a cell. Figure 1.1 shows the results for a calculation in which $N_{P}=16$ while $N_{Y}$ is varied from 1 to 8; the experimental data are from Lou'ndes and coworkers. ${ }^{2}$ Comparable fits to the data are obtained when $N_{Y}$ is held fixed and $N_{P}$ is varied. The work reported here strongly suggests that superconducting pairing between unit cells is not necessary for achieving the high values of $T_{c}$ observed in the new superconductors.

1. Summary of paper to be published.

2. R. F. Wood et al., " $d^{9}$ Spin-Folaron Model of High- $T_{c}$ Superconductivity." this report.

3. D. H. Lowndes, D. I'. Norlon, and J. D). Budai, Phys. Rei. Iell. 65, I(K) (19\%)).

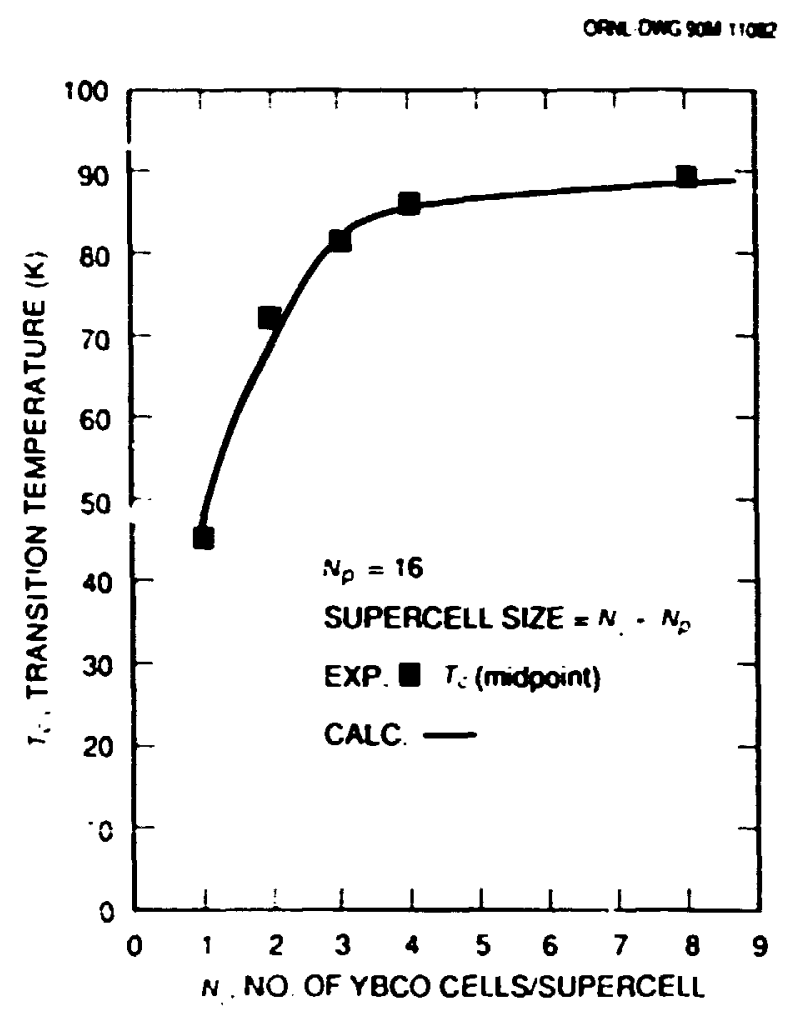

Fig. 1.1. Mid-point transition temperatures for layered structures with $N_{p}$ held at 16 while $\Lambda_{Y}$ is varied.

\section{KOSTERLITZ-THOULESS TRANSITION AND CHARGE REDISTRIBUTION IN THE SUPERCONDUCTIVITY OF YBCO/PBCO SUPERLATTICES'}

\section{Mark Rasolt, Taner Edis, ${ }^{2}$ and Zlatko Tesanovic ${ }^{2,3}$}

In recent experiments, YBCO/PBCO superlattices of varying layer thicknesses of both constituents were studied in order to probe effects of interlayer coupling by examining how the intervening insulating Pr layers would act in lowering the $T_{c}$. These experiments provide some interesting possibilitics for studying the crossiver belween (wo- and three-dimensional 
critical fluctuations in the superconducting order parameter.

A preliminary theoretical study of Ref. 4 was completed. The experimentally measured critical temperatures were successfully correlated with the measured resistive transition width by combining the effects of both the Kosterlitz-Thouless (KT) suppression of $T_{c}$ and the charge transfer from the YBCO layers. The signature of a KT transition is a linear dependence of $\log [R(7)]$, where $R(T)$ is the resistivity just above $T_{c}$, on the function $\tau^{-1 / 2}=(T$ $\left.T_{c} / T_{c}\right)^{-1 / 2}$. This is cleaily demonstrated in Fig. 1.2(a) for 4 different thicknesses of YBCO, corresponding to 1, 2, 3, and 8 YBCO layers, with 16 intervening PBCO layers. Theoretical predictions of the depression in $T_{c}$, based on these results are shown in Fig. 1.2(b), and are found to be in excellent agreement with the experimenial values."

1. Summary of paper to be published.

2. Johns Hopkins University, Baltimore. Md.

3. Los Alamus Natic al Laboratory, Baltimore, Md.

4. D. H. Lowndes, D. P. Norton, and J. D. Budai, Phys. Reo. Lett. 65, 1160 (1990).

\section{DIELECTRIC FUNCTION OF LAYERED MATERIALS'}

\section{D. Mahan ${ }^{2}$}

The first complete dielectric function for the interaction of electrons and ions in a layered electron gas has been derived. The dielectric function is the most important quantity for determining many of the propertics of these
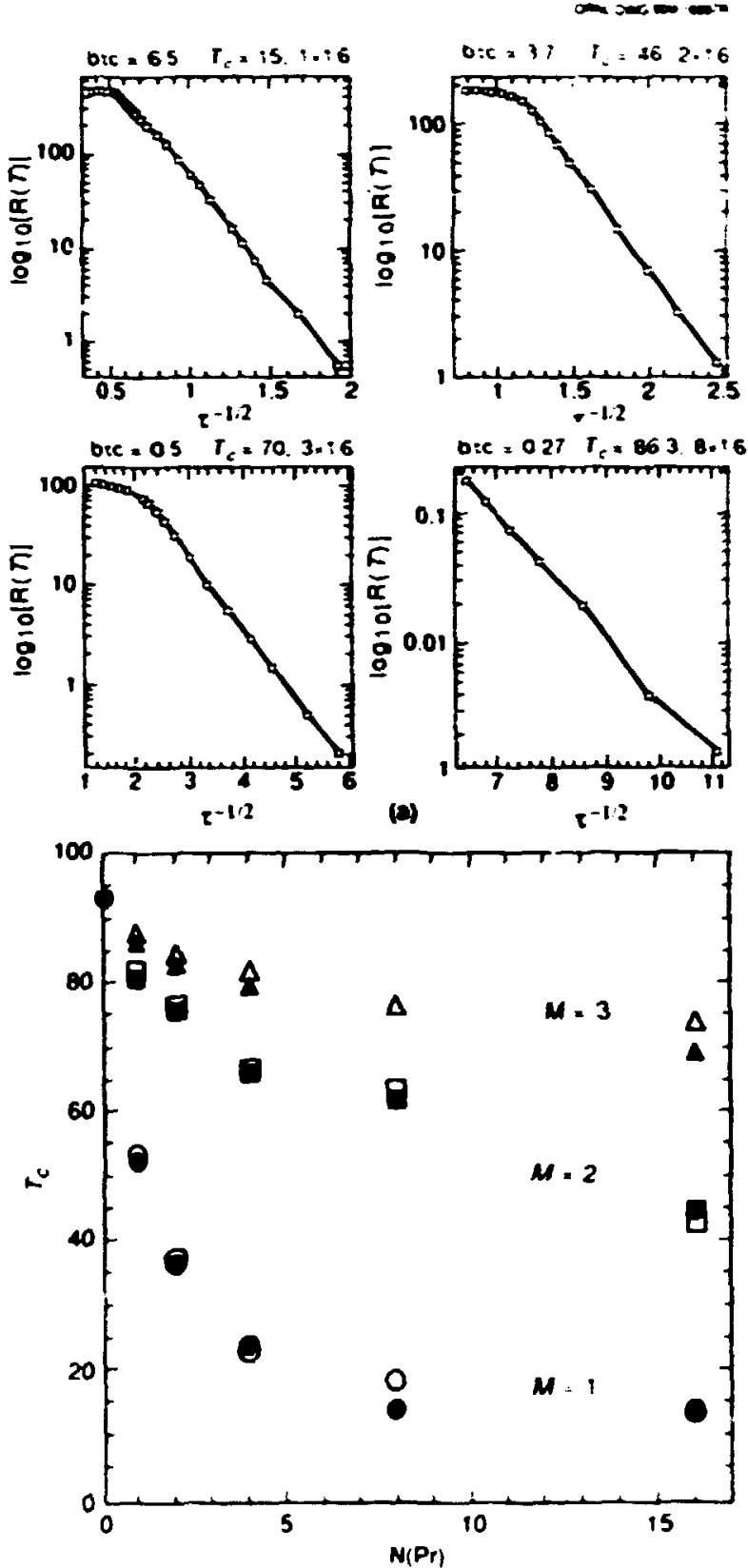

(b)

Fig. 1.2. (a) The linear region of $\log _{10}(R(T))$ vs $\tau^{-1 / 2}$ representing the $K T$ resistive transition region. Four samples are displayed with $M=1,2,3$, and 8 layers of $Y B C O$ and a fixed and very wide $(N=16)$ intervening layers of PBCO. (b) Comparison of the measured $T_{i}$ s, solid markers |from the position of the linear KT region, Fig. 1.2(a)), and therretical predicted values, npen markers (from KT depression of $T_{c}$ and charge transfer, soc t(ext) for $M=1,2,3$, and a range of luyer Ihickne'seses. 
systems. The general theory will be spplied to two classes of solids. One group of solids are the high-temperature superconductors. The electrical conductivity is confined to the planes of copper oxide. Our squations can be used to model the electron-phonon interaction in these systems. Another class of solids which can be described by this theory is multiple quantum wells of semiconductors. The conducting electrons are also in layers, and these systems are also well described by the present theory.

A model which consisted of twodimensional layers, which are stacked parallet separated by a distance $c$, was considered. Each layer can cenduct electrons, but there is no conduction between layers. The ions are in the layers and can vibrate either perpendicular or parallel to the plane of the layers. Pievious workers ${ }^{3}$ had derived the diclectric response of the electrons in the layered systems. Our derivation was the first to include the vibrations of the ions as well. The vibrations of the ions which are perpendicular to the plane are only weakly screened by clectrons. In contrast, the ion vibrations parallel to the planar surface are strongly screened by the el.xtrons in the layers.

1. Summary of paper: Phys. Rev. B 42, 4351 (1990).

2. ORNI./UT Distinguished Scientist.

3. S. Das Sarma and I. I. Quinn, Phys. Rin' B 25, 76033 (1982).

\section{LATTICE DYNAMICS OF HIGH-T SUPERCONDUCTORS'}

\section{Mark Mostoller}

The measured phonon frequencies in stoichiometric single crystals of $\mathrm{La}_{2} \mathrm{CuO}_{4}$ have been critically examined and fitted with a simple shell model. Reasonably good agreement with the experimental results was obtained with a model with variable ionic charges and all ions polarizable. The calculations reproduce the soft mode at $X=[1 / 2,1 / 2,0]$ that drives the tetragonal-to-orthorhombic structural phase transition, and the calculated soft-mode displacement pattern compares fairly well with the observed displacements. The largest short-range forces are those for Cu-in-plane $O$ and La-axial $O$ interactions, while the $0-O$ forces appear to be smaller by almost an order of magnitude.

Using the best fit to the data, calculations were done for the anisotropic mean-square vibrational amplitudes and equivalent Debye temperatures as functions of the temperature. These were compared with values determined by Rietveld refinement of neutron powder diffraction data taken on the High-Intensity Powder Diffractometer at the Manuel Lujan, Jr., Los Alamos Neutron Scattering Center (LANSCE). None of the measured thermal parameters displayed any pronounced anomalies near $T_{c}$. Agreement between the calculated and experimental Debye $\theta$ 's was generally fond, except that the calculations gave values for the in-plane oxygens that were somewhat 
high and also yielded negligible anisotropy for the Cu vibrations. Calculations of the anisotropic, T-dependent thermal kinetic energy of $\mathrm{Cu}$ and the other ions in $\mathrm{La}_{2} \mathrm{CuO}_{4}$ were also done to investigate an anomalous softening of the in-plane $\mathrm{Cu}$ vibrations at $T_{c}$ observed in the compound $\mathrm{Bi}_{2} \mathrm{Sr}_{2} \mathrm{CaCu}_{2} \mathrm{O}_{8}\left(T_{c} \sim 83 \mathrm{~K}\right) .^{2}$

1. Summary of papers: Phys. Reo. B 41, 6488 (1990); Physica $C$ (in press).

2. H. A. Mook et al., "Observation of Phonon Softening at the Superconducting Transition in $\mathrm{Bi}_{2} \mathrm{Sr}_{2} \mathrm{CaCu}_{2} \mathrm{O}_{8}$," this report; Physical Reoiew Letters (in press).

\section{EFFECT OF ZEEMAN SPLITTING AND IMPURITIES ON SUPERCONDUCTIVITY IN THE QUANTUM LIMTT OF STRONG MAGNETIC FIELDS ${ }^{1}$}

\section{Rasolf and Z. Tesanovit²}

In a classic paper Abrikusov ${ }^{3}$ showed that in type-Il superconductors the Meissn:r effect is incomplete and part of the external magnetic ficld ilux penetrates into the superconductor, 1e: silting in a lattice of quantized vortex lines. This theory and its more microscopic extensions lead to a critical temperature which decreases monotonically to zero as a function of the external field $H_{c_{2}} . T_{c}$, the critical temperature, is identically zero when $H_{c_{2}}=\phi_{0} / 2 \pi \xi_{0}^{2}$, where $\phi_{0}$ is the elementary flux and $\xi_{0}$ is the coherence length at zero temperature. This well-known result has been shown to be incorrect. The Abrikosov-Gorkov theory is the consequence of a commonly employed semiclassical approximation in which the Landau level structure is neglected. Detailed analytical and exact numerical calculations with full Landau level structures reveal a nonmor stonic $T_{d}(H)$ as the flux lattice crosses over to the quantum limit. In particular, for external fields larger than $H_{c_{2}}(0)$, superconductivity is enhanced as the field is increased. 4,5

Detailed analytical and numerical caículations on the effect of Zeeman splitting and impurities on this new superconductivity in the high-quantum limit $H>H_{Q L}$ have been completed and are shown in Fig. 1.3. It has been found that the state is stable for Zeeman splittings and impurities found in real systems (like Ge or GaAs) over a wide range of the magnetic field in the quantum limit.

1. Summary of paper to be published.

2. Harvard University, Cambridge, Mass.

3. A. A. Abrikosov, Zh. Eksp. Teor. Fiz. 32, 1442 (1957).

4. M. Rasolt, Phys. Rev. Lett. 58, 1482 (1987).

5. Z. Tesanovic, M. Rasolt, and L. Xing, Phys. Rev. Lett. 63, 2425 (14989). 


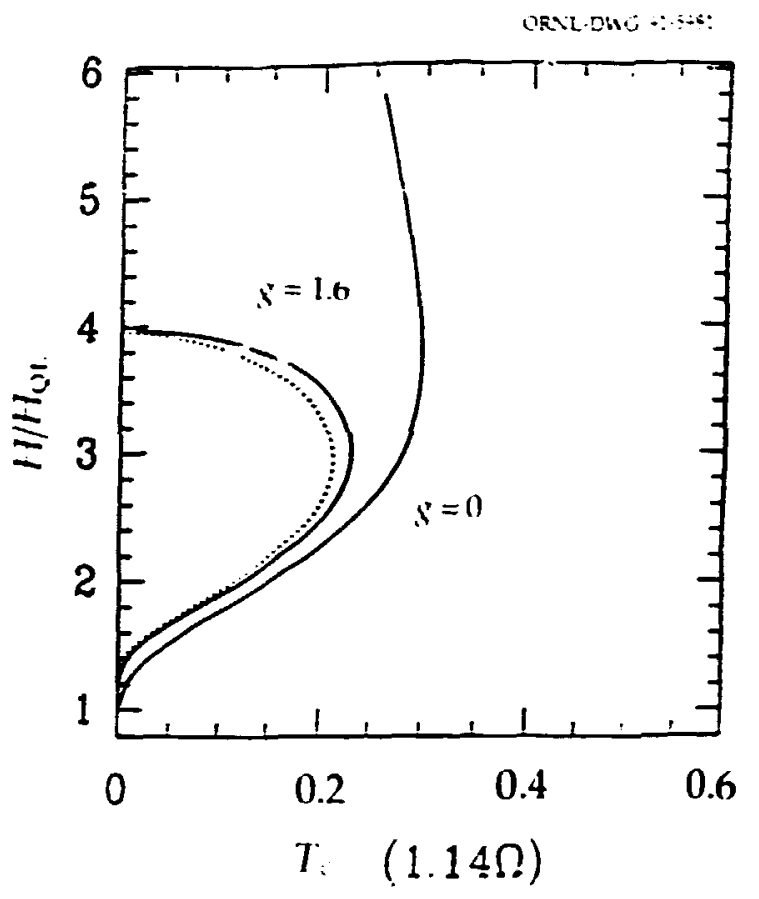

Fig. 1.3. The critical temperatures $T_{c}$ $(g=0)$ (i.e., no Zeeman splitting) and $T_{c}(g)$ (i.c., with Zeeman splitting) vs the magnetic field. The dashed line is $T_{c}(g)$ for a nonuniform state with disorder $1 / 2 \tau E_{3 D F}=10^{-3}$, which can be readily achieved in, e.g., Ge or GaAs. $\mathrm{HQL}$ is the magnetic field necessary to place all the electrons in the lowest Landau level; for lowcarrier-density semimetals or semiconductors (like Ge or GaAs) $\mathrm{HQL}_{\mathrm{Q}}$ is of the order of $20 \mathrm{~T}$ and, therefore, readily accessible experimentally.

\section{ELECTRONIC PROPERTIES AND LATTICE DYNAMICS}

\section{ITINERANT-ELECTRON SUSCEPTIBILITY CALCULATIONS FOR IRON AND COBALT'}

\author{
J.F. Cooke, I. A. Blackman, 2 \\ and K. N. Trohidou ${ }^{2}$
}

Several years ago we reported the results of calculations of the frequency and wave-vector dependent susceptibility for ferromagnetic iron based on itinerant-electron theory. Spin-wave excitations were found to extend up to about 600 $\mathrm{meV}$ in energy and were particularly sharp at about halfway to the Brillouin zone boundary in the $(100)$ direction. The general behavior of these predictions have now been confirmed bv experiment. An extensive series of calculations indicates that the susceptibility for iron is very sensitive to the band structure and exchange matrix elements (EME), especially along the (110) direction. These calculations were based on a relatively simple form of the EME in terms of parameters which could be determined from magnetic moment and form factor data. The theory has now been generalized to include the most general form of the EME which is consistent with symmetry and includes first-neighbor overlap of the electronic wave functions. The results depend on over 650 terms which are independent of band and wave-vector indices and can, therefore, be treated as parameters. Symmetry arguments can and have been used to relate all of these terms to 11 unique priameters. One interesting aspect of this generalization is that in the limit of very narrow bands, the susceptibility reduces to the form one obtains from local moment theory. Numerical calculations have revealed a broad range of spin-wave behavior, from single branch to a wide variety of multiple branch dispersion curves which vary dramatica'ly with wavevector direction. The analysis of results obtained from neutron scattering experiments currently under way in terms of our therretical 
approach will provide sensitive tests of itinerant electron theory.

Calculations of the susceptibility for hcp cobalt have also been initiated. The theory for cobalt is more complicated than for iron because of the lattice structure, with two atoms/cell, and the nature of the point group which must be explicitly built into computer programs. Because there are two atoms/cell, our previous experience with iron and nickel leads us to expect even more complex spin-wave behavior. Preliminary results, however, yield only two branches: the expected acoustic and optic branches. Furthermore, the acoustic branch is remarkably isotropic with energies reaching about $300 \mathrm{meV}$. Preliminary experimental data appear to be in broad agreement with these predictions.

1. Summary of paper to be published.

2. University of Reading, Reading, United Kingdom.

\section{THE SCREENED ANDERSON MODEL ${ }^{1}$}

\section{H. O. Frota ${ }^{2}$ and G. D. Mahan ${ }^{3}$}

The question of the electronic transitions in intermediate valence compounds is reexamined. The 4 f orbital was treated as an s orbital, and it was assumed that the electrons in this orbital did not inferact with the $4 f$ electrons in the neighbor sites. The qualitative behavior of the clectronic transition in intermediatc valence compounds is analyzed in terms of the electron transition in the one-impurity Anderson model.
The calculation was made using the Wilson renormalization technique. The Hamiltonian is given by

$$
\begin{aligned}
& H=H_{c}+H_{f}+H_{V}+H_{G} \\
& H_{c}=\sum_{k} \varepsilon_{k} c_{k \mu \mu}^{\dagger} c_{\mu \mu} \\
& H_{f}=\varepsilon c_{f \mu}^{\dagger} \varepsilon_{f \mu}+u_{f f}^{\dagger} c_{f f}^{\dagger} f_{f l}^{\dagger} c_{f l} \\
& H V=V \sum_{k}\left(c_{L_{\mu}}^{\dagger} c_{\mu^{2}}+c_{\mu \mu}^{\dagger} c_{\mu_{\mu}} c_{L_{\mu}}\right) \\
& H G=G\left(c_{H^{\prime}}^{\dagger} c_{H^{\prime}}\right)+\eta \sum_{k k^{\prime}} c_{k v k^{\prime} v^{\prime}}^{\dagger}
\end{aligned}
$$

where repeated spin indices are assumed to be summed over. The parameter $\eta$ denotes the valence on the local site, $\eta=0, \pm 1$. The operator $c_{k \mu}^{\dagger}\left(c_{f^{2}}^{\dagger}\right)$ creates an electron with spin $\mu(v)$ and energy $\varepsilon_{k}\left(\varepsilon_{p}\right)$ in the conduction band (localized orbital). The term $H_{c}$ represents the conduction band Hamiltonian; $H_{f}$ is the Hamiltonian of the impurity ion, where $U$ is the Coulomb repulsion between electrons in the impurity orbital; $H_{v}$ is the hybridization between the impurity orbital and the conduction band via the interaction $V$; and $H_{C}$ represents the screening of the impurity orbital via the Coulomb interaction $G$. The screening is separated in two parts: the part that depends on the occupation number of the localized orbital $n f=c_{\mu \mu_{\mu}}^{\dagger}$, guen by $G c_{\mu_{\mu} c_{\mu \mu}} \Sigma_{k k^{\prime}} c_{k v}^{\dagger} \varepsilon_{k^{\prime} v^{\prime}}$ and the part that does not depend on $n$, given by $\pm C \Sigma_{k t} c_{k v}^{\dagger} c_{k^{\prime} v^{*}}$

For $\eta=0,-1$ and $G$ comparable to $U$, it was found that the screening interaction induces in the model an effective $U$ that is negative. This 
leads to a direct transition $f^{0} \rightarrow f^{2}$, without having the configuration $f^{1}$ as an intermediate stage. For $\eta=+1$ the effective $U$ is always positive, with the transition $f^{0} \rightarrow f^{2}$ happening in two stages $\rho \rightarrow f \rightarrow f$.

1. Summary of paper to be published.

2. Cuest scientist from the ORNL/UT Distinguished Scientist Program.

3. ORNL/UT Distinguished Scientist.

\section{SHAPE OF THE KONDO RESONANCEI}

\section{H. O. Frota ${ }^{2}$}

Electron scattering from a local impurity spin has its density of states (DOS) altered by spin-flip scattering. This phenomenon causes a peak in the local DOS which is called the "Kondo resonance." Earlier a simple formula for the shape of this resonance using the law of Doniach and Sunjic was deduced. ${ }^{3}$ The formula was in agreement with our numerical solution of the Kondo problem using the method of renormalization group. The Kondo resonance has the spectral shape

$$
D(\varepsilon)=\frac{2}{\pi \Gamma_{1}} R\left[\frac{i \Gamma_{k}}{\varepsilon+i \Gamma_{k}}\right]^{\frac{1}{2}}
$$

The width $\Gamma_{k}$ is proportional to the Kondo temperature, while the width $\Gamma_{A}$ is given by the Friedel sum rule. The validity of this formula vas recently confirmed by Silva et al. 4 using quantum Monte Carlo calculations.

The above formula for the DOS at the Kondo resonance was used to calculate a number of thermodynamic properties of the Kondo effect. These include the low-field magnetic susceptibilitly $\chi(T)$, the electrical resistivity $\rho(T)$, and the heat capacity $C(T)$. All are known to be anomalous because of the Kondo effect. Our calculations gave the known results in every case. Thus, the macroscopic measurements of the Kondo effect can be described by using our DOS function for the Kondo resonance.

1. Summary of paper to be published.

2. Guest scientist from the ORNL/UT Distinguished Scientist Program.

3. H. O. Frota and L. N. Oliveira, Phys. Reo. B 33, 7871 (1986).

4. R. N. Silver et al., Phys. Rev. Left. 65, 496 (1990).

\section{QUANTUM FLUCTUATIONS AND CHARGING EFFECTS IN SMALL TUNNEL JUNCTIONS ${ }^{I}$}

\section{Karsten Flensberg ${ }^{2}$ and $M$. Jonson ${ }^{2}$}

Novel and interesting effects have been predicted and observed in tunnel junctions with low capacitance $c$, so that the charging energy voltage $\mathrm{e}^{2}$ /e from one electron is larger than the energy scale of thermal fluctuation. In particular, the Coulomb blockade has been observed in recent experiments on single-tunnel junctions. ${ }^{3}$ The measured blockade is, however, much reduced compared to what one expects from scmiclassical theory. It is clear that in addition to thermal fuctuations, quanlum flucluations have to be included in order for a theory to be able to describe the experiments in any 
detail. Two different physical processes may give important contributions to the quantum fluctuations: (1) change transfer across the junction and (2) charge fluctuations along the leads connecting the junction to the external circuit. Both effects reduce the effective Coulomb barrier.

A microscopic theory for the finite-bias conductance of a tunnel junction that contains the effects of quantum fluctuations caused both by couping to transmission line modes and by charge trarsfer across the junction is presented. Using an "harmonic approximation" for the charge transfer nuctuations, it was found that the two different kinds of charge fuctuations enter the theory on an essentially equal footing. The harmonic approximation is much like the spin-wave approximation in the classical $X Y$ model, both neglect the periodicity in the angular variables. In the $X Y$ model this corresponds to the neglect of vortices; while in the present context, it corresponds to neglect of the discreteness of the charge transfer in the tunnel events.

It was found that the impedance of the environments and the resistance of the junction add in parallel to produce an effective coupling constant for the coupling to the fluctuating degrees of freedom. In a sense this is a natural generalization of the results in Refs. [3] which are valid for infinite junction resistance and treats only charge fluctuations along the leads. On the other hand there is a fundamental difference between discharge along the leads, which is continuous, and discharge back through the junction, which is necessarily dis- crete. Although our theory, where we use an harmonic approximation which neglects this difference, qualitatively accounts for the variation of the size of the Coulomb blockade with junction resistance found in the recent experiments, we speculate that it is necessary to go beyond this approximation to get a fully satisfactory agreement with experiments in the limit of vanishing bias.

1. Summary of paper to be published.

2. Guest scientist from the ORNL/UT Distinguished Scientist Program.

3. Delsing et al., Phys. Rev. Lett. 63, 1180 (1989); Cleland et al., ibid. 64,1565 (1990).

\section{CHARGING EFFCTS IN TUNNEL JUNCTIONS: A FOUR-LEVEL STUDY'}

\section{Karsten Flensberg ${ }^{2}$ and H. O. Frota 2}

For small tunnel junctions it may be possible to observe the charging energy associated with the tunneling of the individual electrons which then blocks the transfer of electrons. This cffect is referred to as the Coulomb effect. The semiclassical theory which predicted this effect is based on the assumption that quantum charge fluctuations are unimportant (i.e., the theory ignores the fluctuations caused by charge transfer). The effect of the fluctuations is to reduce the blockade. Except in the case of extremely high-resistance junctions (compared to the quantum resistance), charge transfer fluctuatior. nust be included in the description. This has previously been studied using a path integral technique and an expansion in the 
hybridization between the two sides; an approximation which is, strictly speaking, only valid for high-resistance junctions.

The purpose of this work is to study the effect of fluctuations and to go beyond the limitation of small coupling. A modet Hamiltonian which contains the essential physics: the competition between the potential energy and fluctuations in the charge difference is used. This model is studied via a transformation which, following Wilson, ${ }^{3}$ allows us to treat the hybridization between the two sides and the charging energy exactly. The two conduction bands are transformed to the so-called "hopping" Hamiltonian which leads to a basis where the two sides couple through the Coulomb term and lowest energy level on cach side. An iterative solution where each iteration includes one more site of each of the two hopping Hamiltonians is now possible.

In the present work, a simplified version of this Hamiltonian is studied (i.e., we keep only the first term that hybridizes directly the two bands and which involves the charging energy and the junction coupling strength). The resulting four-level system is solved exactly, and the eigenvalues and the mean-square charge difference are calculated. The first levels of the hopping Hamiltonian can be thought of as the one electron level closest to the interface, and building more levels on top of this corresponds to taking levels further away from the junction into account. This motivales our truncation to four one-electron levels which enables us to study (in an exactly solvable model) the effect of and competition between the sunncling term and the Coulomb term. In accordance with the physical picture, it was found that for weak tunnel coupling the Coulomb interaction strongly suppress any charge difference; whereas in the strongly coupled limit the kinetic enet 6 ; dominates, and the charging energy is less important.

1. Summary of paper to be published.

2. Guest scientist from the ORNL/UT Distinguished Scientist Program.

3. K. G. Wilson, Reo. Mod. Phys. 47, 773 (1975).

\section{INDIRECT IONIC INTERACTION'}

\section{G. D. Mahan" and M. Mostoller}

Several years ago we proposed ${ }^{3}$ a model of three-body forces between ions in insulators. A central ion is polarized by a displacement of one of its neighbors. This polarization causes a force on all of the other neighbors of the central ion, which serves as a medium for causing indirect interactions between its neighbors. Of course, each ion can serve as a central ion, and the effect is particularly large for ions with filled $d$-shells.

A model of indirect ionic interactions was used to explain the lattice dynamics of four insulators: $\mathrm{KCl}, \mathrm{KBr}, \mathrm{RbCl}$, and $\mathrm{AgBr}$. Experimental values for the parameters of this thenry were obtained by fitting the theoretical model to the phonon spectra measured by neu. Iron scattering. Very goxod fits were obtained. 
Experimental parameters for the $\mathrm{Ag}_{\mathbf{B}}{ }^{+}$ion in AgBr were much larger than for the other ions. This confirmed our expectation that the three body forces are much larger for ions whose valence electrons come from a filled $d$-shell.

1. Summary of paper: Phys. Reo. B 41, 10824 (1990).

2. ORNL/UT Distinguished Scientist.

3. G. D. Mahan and M. Mostoller, Phys. Reo. Lett. 57,357 (1986); Phys. Reo. B 34, 5726 (1986)

\section{PHONON DISPERSION AND KOHN ANOMALIES IN Cuasallays'}

Henry Chou, 2 S. M. Shapiro, 2

S. C. Moss, ${ }^{3}$ and Mark Mostoller

The phonon dispersion curves of $\mathrm{Cu}_{0.84} \mathrm{~A}_{0.16}$ were measured along the [500], 1550], [550], and [051] directions by inelastic neutron scattering at the High-Flux Breeder Reactor at Brookhaven National Laboratory. The measured spectra could be fitted very well with a fifth nearest neighbor (5NN) Born-von Kármán model. Near the zone boundaries, the phonon frequencies are lower than those in pure $\mathrm{Cu}$ by as much as $15 \%$, which may be attributed to mode repulsion from high-frequency localized vibrations of the lighter mass $\mathrm{Al}$ defects in the Cu host lattice.4

The Fermi surface of $\mathrm{Cu}$ and of $\mathrm{Cu}$-rich $\mathrm{Cu}_{1-x} \mathrm{Al}_{x}$ alloys has a fat portion perpendicular to the $<110\rangle$ direction in reciprocal space, and this can lead to Kohn anomalies in the dispersion curves at the appropriate $q=2 k_{F}$ phonon wave vectors. Kohn anomalies have, in fact, been detected in the spectra of pure $\mathrm{Cu}$ by careful study of the slope of the measured dispersion curves compared to the results of a Bomvon Kámin analysis. 5 We have carried out a similar study for the $\mathrm{Cu}_{0.84} \mathrm{~A}_{\mathbf{0}} \mathrm{.16}_{6}$ alloy and find no persuasive evidence of Kohn anomalies Ore plausible reason for the absence of the anomalies is the smearing-out of the Fermi surface by disorder in the alloy, as indicated by electronic struciure calculations for this system. 6

1. Summary of paper: Phys. Reo. B 42, 500 (1990).

2. Brookhaven National Laboratory, Upton, New York.

3. University of Houston, Houston, Texas.

4. T. Kaplan and M. Mostoller, Phys. Reo. B 9, 353 (1974).

5. G. Nilsson and S. Rolandson, Phys. Reo. B 9, 3278 (1974).

6. F. Pinski, private communication.

\section{THERMAL BOUNDARY RESISTANCE'}

\section{S. Pettersson ${ }^{2}$ and C. D. Mahan ${ }^{3}$}

When heat flows at the boundary between two solids, it is usually assumed that the temperature is continuous. However, it is known that there is a small temperature step $\Delta T$ at the boundary. It is given in terms of the thermal boundary resistance $R$ and the heat flux as $\Delta T=R \dot{Q}$.

The first theory of the thermal boundary resistance $R$ at the interface between two dif- 
ferent solids has been derived. The theory can also be applied to the boundary between two different grains of the same solid when the grains have different crystal orientations. The boundary resistance is found by a calculation of the reflection and transmission of sound waves at the interface. A major new result in this work is that a single sound wave, which is incident at the interface, can cause many waves to be reflected and transmitted. The multitude of waves comes from the matching of the ion motions at the interface. These many waves carry away much energy and significantly increase the thermal boundary resistance. Interfaces which have the same acoustic mismatch at low temperatures can have very different thermal boundary resistances at high temperatures. (1990).

1. Summary of paper: Phys. Reo. B 42, 7386

2. Guest scientist from the ORNL/UT Distinguished Scientist Program. Present address: University of Umes, Umed, Sweden.

3. ORNL/UT Distinguished Scientist.

4. E. T. Schwartz and R. O. Pohl, Rev. Mod. Phys. 61, 605 (1989).

\section{SURFACES, SAND PILES, AND ION SCATTERING}

\section{LAYER-BY-LAYER SEGREGATION AND DEEP MULTILAYER RELAXATION IN THE RANDOM ALLOY SURFACE Mo85Re15(100)!}

\section{H. L. Davis, D M. Zehner, B. Dötsch,2 D. v. Gemünden, ${ }^{3}$ and K. Müller ${ }^{3}$}

The layer-by-layer constituent segregation in the (100) surface of the substitutionally ran- dom binary alloy Mos5 $\mathrm{Re}_{15}$ has been determined by LEED. After verification that the surface exhibits a $(1 \times 1)$ diffraction pattem, I-V profile data were collected with the incident clectron beam normal to the surface and the sample maintained at a temperature of $100 \mathrm{~K}$. The data base consisted of eight inequivalent I-V profiles which exterded over the energy range $50-600 \mathrm{eV}$. Analysis of the data base differed from conventional LEED procedures in that a layer-by-layer version of the average $t$-matrix approximation was used to mimic the clectron scattering from the randomly occupied atomic sites.

Calculated and experimental I-V profiles were compared by use of the $R_{2} \boldsymbol{R}$-factor, and the best comparison had a total $R_{2}$ value of 0.0259. The final results indicate that the Mo concentrations in the first five atomic layers of the surface are $97,67,79,78$, and $87 \%$, respectively, which differ from the bulk concentration of $85 \%$. In addition, the results indicate that the first eight interlayer spacings are relaxed from the bulk value $(=1.567 \AA$ ) by -10.6 , $+5.1,-2.7,+3.0,-1.8,+2.6,-1.8$, and $+1.2 \%$, respectively. It is interesting that the signs of these relaxations have an oscillatory character when proceeding into the surface region. Although loss of sensitivity prevented obtaining information about spacings deeper than the eighth, it is unusual and exciting that explicit nonzero relaxations were obtained for the first cight spacings. Mcaningful information about the decper relaxations was obtained because the experimental profiles contained considerable struclure up to $6(1) \mathrm{eV}$ where high-encergy 
electrons have a longer mean-free path than low-energy electrons. I-V profiles for two beams fron the surface are illustrated in Fig. 1.4.

1. Summary of paper to be published.

2. Consultant from Universitat ErlangenNümberg, Erlangen, Germany.

3. Universität Erlangen-Nümberg. Erlangen, Germany.

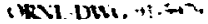
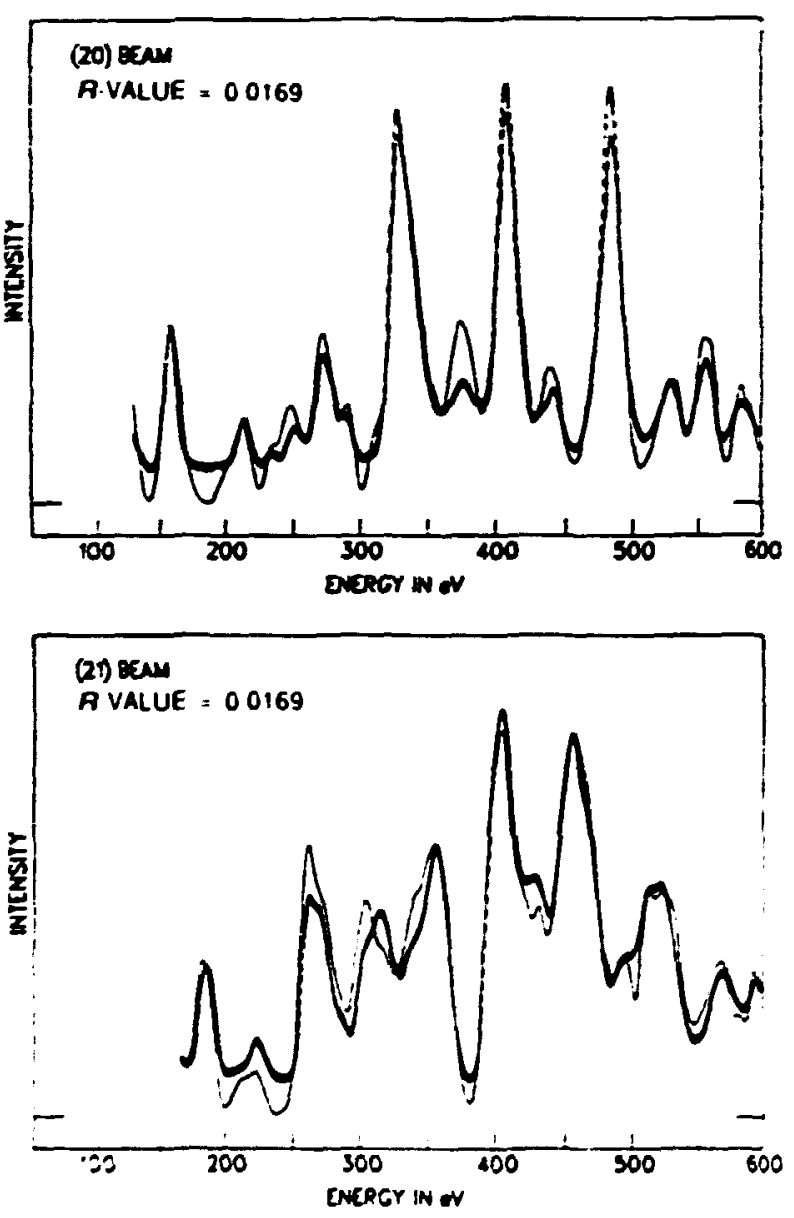

Fig. 1.4. Experimental (broken and/or heavy curve) and calculated (light curve) I.V profiles for the (20) and (21) beams from Mog5 $_{8 e_{15}}(100)$.
MULTLAYER RELXATION OF THE

Ru(000) SURFACE AND CTUNGES

INDUCED BY MONOLAYER COVERAGE OF

Cur STRUCTURE OF A SIMPLE METALUC INTERFACE!

\section{H. L. Drovis, J. E. Houston, ${ }^{2}$ and D. G. O'Neip}

The multilayer relaxation of the clean Ru(0001) surface and the relaxation of a Ru(0001) substrate covered by 1 ML of Cu have both been determined by LEED, I-V profile analysis. For both systems, the I-V profile data were collected with a video acquisition system-the incident electron beam normal to the surface and the respective sample maintained at a temperature of $90 \mathrm{~K}$. The data base for each system consisted of profiles, 6-beam averaged, for three lowest order diffracted beams (i.e., the (10) with energy range 70-40 eV and the (11) and (20) with energy range 310-570 eV). Each of the experimental profiles contained as intense peak structure in its higher energy range as its lower energy range; thus, since electrons have a longer mean-free path at higher energies than at lower energies, a search was made in each of the LEED analyses for the possible existence of reasonably deep relaxations.

Results for the clean Ru(0001) surface indicate that the first six interlayer spacings are rclaxed from the truncated bulk values $(=2.137$ A) by, respectively, $-2.1,-0.1,+0.5,-0.1,-0.6$, and $-0.3 \%$. The $R_{2}$ value was 0.0136 , which is the smallest $R$-factor ever reported for a LEED analysis. For the system of 1-ML $\mathrm{Cu}$ on $\mathrm{Ru}(0001)$, results indicate that the $\mathrm{Cu}$ atoms are 
in a normal registry position, and the $\mathrm{Cu}$ layer is $2.123 \AA$ above the first layer of the $R u$ substrate. The $R_{2}$ value for the analysis was 0.0160 , and the first six interlayer spacings of the Ru substrate are relaxed by, respectively, $2.8,0.0,+0.5,+0.5,-0.7$, and $-0.6 \%$. It is to be noted that creation of the $\mathrm{Cu}-\mathrm{Ru}$ interface induces a further contraction of $0.7 \%(=0.015 \AA)$ in the first interlayer spacing of the Ru substrate. I-V profiles fo: the (11) beams of the two surface systems are illustrated in Fig. 1.5.

1. Summary of papers to be published.

2. Sandia National Laboratories, Albuquerque, N. Mex.

3. Present address: Sandia National Laboratories, Albuquerque, N. Mex., Corporate Research-3M, St. Paul, Minn.

\section{GEOMETRY AND DYNAMICS OF DETERMINISTIC SAND PILES ${ }^{1}$}

\section{S. H. Liu, T. Kaplan, and L. J. Gray'}

A wide variety of natural objects are fractals, which means that they look the same under different length scales. Similarly, many dynamical systems exhibit scale invariance in the time domain. The frequency spectrum of a scale invariant time sequence rises in the lowfrequenry end and is commonly called the 1/f spectrum. Recently, there have been many studies on whether spatial and temporal scale invariance may evolve from a common physical origin. A nonlincar cellular automaton model called a "sand pile," proposed by Bak, Tang, and Wisenfeld, ${ }^{3}$ seems to be both simple and germane. It is susceptible to detailed study,
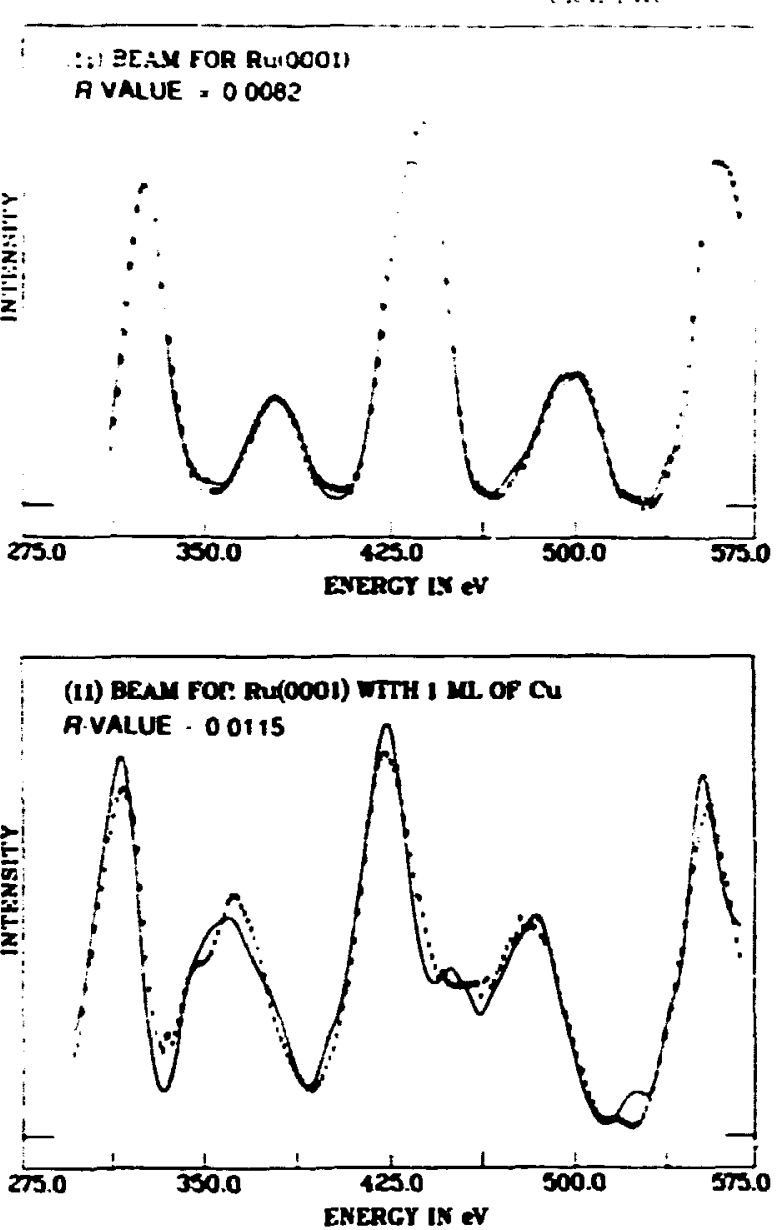

Fig. 1.5. The (11) I-V profiles for Ru(0001) and $1 \mathrm{ML}$ of $\mathrm{Cu}$ on Ru(0001). The broken curves are experimental, while the solid curves are calculated.

and the results may shed light on a class of very complex problems in nature.

In existing literature, the problem is commonly studied under random initial conditions. The necessity of such a starting point was questior ed, and a series of studies of the time evolution of sand piles under totally deterministic initial conditions were made. Drspite the lack of randomness, the relaxation process exhibits I/] behavior under a variety of circumstances. 
The fully relaxed states often have compiex patterns, some of which are self-similar. The temporal scaling is found to be intimately linked to the growth of the spatial pattern. This demonstrates for the first time that 1/f fluctuations can be the result of diffusion through self-generated complex patterns.

1. Summary of paper: Phys. Rev. A 42 , 3207 (1990).

2. Engineering Physics and Mathematics Division, ORNL.

3. P. Bak, $C$ Tang and $k$ Wiesenfeld, Phys. Reo. Lett. 59, 381 (1987); Phys. Rev. A 38, 364 (1988).

\section{CRITCAL EXPONENTS IN SAND PILE'}

TheodGre Kaplan, S. H. Liu, and L. J. Gray'

Many systems in nature show scaling in space and/or time. Mountain ranges, coast lines, and cloud formations show fractal symmetry (spatial scaling), while earthquakes, current flow in a resistor, and sun-spot activity show 1/f-like power spectra (te.nporal scaling). Bak, Tang, and Wiesenfeld (BTW) have suggested a possible connection between these two types of scaling phenomena. 3 They have studied a nonlinear dynamical system which mimics a sand pile, in that a pile of sand with a slope higher than a critical value tends to collapse. They showed that under very gencral conditions the distributions of slopes evolves naturally into a stste with spatial scaling, which they call a "sclf-organized ciitical state." When the critical state is perturbed, an avalanche occurs, and the size distribution of the avalanches obeys spatial scaling while the distribution of relaxation times obeys temporal scaling.

Motivated by some findings in our study of the BTW sand pile under deterministic initial conditions, a detailed stud'y of the BTW sand pile for three different two-dimensional lattices-square, triangular, and honeycomb-was initiated. Two distinct regions for each distribution which showed power-law behavior were found. Power-law behavior indicates scaling. The first region, which occurred at smallet life times and avalanche sizes, displayed a sensitivity to lattice structure. The sccond region showed little dependence on the lattice structure. As the coordination number increased, the exponents in the first region approached those in the second. Less than 35\% of the avalanches reached the boundary in the first region, while between $35 \%$ and $85 \%$ reached the boundary in the second region. Despite the larger boundary interaction, it is the second region which one would generally associate with conventional critical phenomena, because it shows the expected insensitivity to battice structure.

1. Summary of paper to be published.

2. Engineering Physics and Mathematics Division, ORNL.

3. P. Bak, C. Tang, and K. Wiesenfeld, Phys. Reo. Lett. 59, 381 (1987); Phys. Rev. 138, 364 (1988).

4. S. H. Liu, T. Kaplan, and L. J. Cray, Phys. Rev. A 42, 3207 (1990). 


\section{THE TEMPORAL DEVELOPMENT OF COLLISION CASCADES IN THE BINARY-COLUSION APPROXIMATIONI}

\section{Mark T. Robinson}

A method developed ${ }^{2}$ to evaluate explicitly the time in the binary collision approximation (BCA) was applied using the BCA code MARLOWE ${ }^{3}$ to calculate the slowing-down time of energetic recoils in $\mathrm{Cu}$ crystals. In the present work, an algorithm was developed for ordering the collisions in a BCA cascade properly in time, rather than ordering them in velocity as has zenerally been done before. Cascades initiated by primary atoms of kinetic energy $\leqq 10 \mathrm{keV}$ were calculated in $\mathrm{Cu}$ and Au. In addition, cascade nonlinearities were approximated by allowing stopped cascade atoms to become tangets in later collisions.

If stopped cascade atoms cannot become targets, the only interferences between particle traj-ctories in MARLOWE involve target sites: sinc e none emits more than one atom, projectiles mi.y compete for particular sites, and the collision order may be significant. Computations showed that the differences between velocilyordered (VO) and time-ordered (TO) cascades in $\mathrm{Cu}$ were very small: up to $5 \mathrm{keV}$, the numbers of Frenkel pairs in VO cascades were not statistically different from those in TO cascades; at $10 \mathrm{keV}$, the results differed by about $3 \mathrm{O}$. The differences in Au were greater: about $4 \sigma$ at 5 $\mathrm{keV}$ and 90 at $10 \mathrm{keV}$. The VO cascades show more defects because low-energy atoms are not followed until the fast-particle trajectorics are nearly complete. Thus, lowenergy particles encounter more vicant sites than in TO calculations, move slightly farther, and have a greater likelihood of becoming Frenkel pairs. The trajectories of slow particles are followed from the start of TO calculations and are more likely to move in undamaged crystal. It appears to be a general result that less damage will be calculated in TO cascades than in VO ones.

The differences between $\mathrm{TO}$ and VO cascades are more significant when stopped cascade atoms can become targets in later collisions and be "redisplaced." These extra collisions dissipate kinetic energy without producing additional defects; fewer Frenkel pairs are produced when redisplacements are allowed than when they are not. More redisplacements occur in TO cascades, a further cause of the differences between the two ordering schemes. Redisplacement events mainly evolve encounters of slowly moving atoms with stopped ones.

The model with redisplacements could not be used at initial kinetic energies above about $10 \mathrm{keV}$ because of excessive computing time. Recent work has improved the searching algorithm in MARLOWE to accelerate these calculations, and new calculations are in progress at energies up to $100 \mathrm{keV}$, where nonlincar cascade effects are expected to be more important, especially in heavy elements.

1. Summary of paper: Nucl. Instr. and Methods in Phys. Res. Sect. B 48, 408 (1990).

2. M. T. Robinson, Phys. R(1). B 40, 10717 (1989).

3. M. T. Robinson and I. M. Torrens, Phys. Rev. B 9, 5 SOD8 (1974). 


\section{POSSIBLE RESONANCE IN POSTTRON UTHIUM SCATTERING'}

M. A. Abded-Reouf ${ }^{2}$ and R. F. Wood

The possible appearance of resonances in the partial cross sections of the inelastic collisions of positrons with lithium atouns at energies below $5 \mathrm{eV}$ is investigated. It is assumed that only elastic and rearrangement channels are open, while excitation channels are closed. A coupled static formalism, in which the polarization potentials of the lithium and positronium are switched on, is employed. The basis set of Clementi and Roetti is used for describing the target model. In order to cakculate the reactance and transition matrices required for the evaluation of the partial and total cross sections of the first and second channels, an iterative Green's-function partialwave expansion technique within the framework of the restricted coupled static approximation (RCSA) was employed. Comparison between the resulting total cross sections and those cbtained by other authors is presented.

Our results indicate the existence of s-wave resonance states around 0.5 and $4.5 \mathrm{eV}$ and a pwave resonance at $15 \mathrm{eV}$. Thus, they suggest the possible formation of $e^{+}-\mathrm{Li}$ (or $\mathrm{Ps}-\mathrm{Li}^{+}$) bound states at these energies. However, since the appearance of false resonances is the main defect of most mathematical approaches (including the RCSA and the close-coupling approximation) applied to scattering problems, thi need for further investigation of the resonance problem in $e^{+}-L^{-}$scattering using other techniques is emphasized. It is commonly agreed that the confirmation of resonance at a certain energy by various mathematical approaches supports the existence of a true resonance at that energy.

1. Summary of paper: Phys. Rev. A 42, 3117 (1990).

2. Friedrich-Alexander-University, Erlangen-Nümberg, Germany. 


\section{Neutron Scattering}

It is a pleasure to report that the High-Flux Isotope Reactor (HFIR) is back in operation after a shutdown which lasted for more than three years. After a period of low-power operation which began on January 29, 1990, the new fullpower level of $85 \mathrm{MW}$ was reached on May 16, 1990. The operating record since then has gradually improved, reaching an availability of $61 \%$ during the fourth quarter of 1990.

There are presently seven instruments in operation, including two new ones-a high-resolution powder diffractometer and an Ames-ORNL tripleaxis unit. Three older triple-axis spectrometers have been improved with new analyzer and detector shields, absolute encoders, and motor-driven goniometers and diaphragms. The triple-axis spectrometer at HB-2 has a new multiple-crystal, variable-focus monochromator assembly. A new data acquisition and analysis system is in operation at the 30 -m small-angle instrument.

The U.S.-Japan Cooperative Program on Neutron Scattering is in full operacion, but the general user program has not yet been formally restarted. Nevertheless, there have been a number of users on an informal basis. The total number of outside users since the restart of the HFIR is 40 , including participants in the U.S.-Japan program.

The long-range future is dominated by the Advanced Neutron Source (ANS). This project continues to show excellent progress and has passed all revirsws. The current schedule calls for start of construction in FY 1994 with completion expected in FY 1999. Two important shorter range proposals r.wait funding decisions. One is a $\$ 12 M$ upgrade of neutron instrumentation at the HFIR, including five new instruments and a small guide hall. The other one is a national neutron scattering scholarship plan for graduate students to do their thesis work a! any of the national laboratories with neutron scattering programs.

Current research highlights include a variety of experiments on high- $T_{c}$ superconductors: magnetic excitations, phonon softening, structures, and fluxoid-lattice observations. As usual, the small-angle instrument has been in heavy use on a variety of polymer and micelle studies. Of particular importance is the work on nhase transitions in block copolymers. 


\section{SMALL-ANGLE SCATTERING}

\section{NEUTRON SMALLANGLE SCATTERING MEASUREMENT OF THE FLUXOID LATTICE IN $\mathrm{B}_{20.6} \mathrm{~K}_{0.4} \mathrm{BiO}_{3}$}

\author{
H. A. Mook, G. D. Wignall, \\ M. L. Norton, ${ }^{1}$ and H-Yi Tang'
}

$\mathrm{Ba0.6} \mathrm{K}_{0.4} \mathrm{BiO}_{3}$ is a high-temperature superconducting material with a transition temperature of about $32 \mathrm{~K}$. Measurements of the fluxoid lattice were undertaken on the HFIR smallangle scattering spectrometer using a simple of five small crystals giving a total sample volume of about $0.25 \mathrm{~cm}^{3}$. The fluxoid lattice was established by cooling the sample in a 0.3-T field placed along the neutron beam. The neutrons diffracted from the fluxoid lattice were counted by using an area detector at a distance of $18 \mathrm{~m}$ from the sample. The diffraction pattem from the fluxoid lattice consisted of spots on the area detector at the momentum transfer expected for a triangular lattice with a 0.3-T applied field. The fact that the pattern consisted of discrete spots showed that the fluxoid lattice had crystalline order and was not a polycrystal or a glass. The temperature dependence of the scattering from the fluxoid lattice was also established and is shown in Fig. 2.1.

The highest temperature at which the fluxoid lattice could be observed was $24 \mathrm{~K}$. However, even at this temperature, the diffraction spots remained sharp, showing the

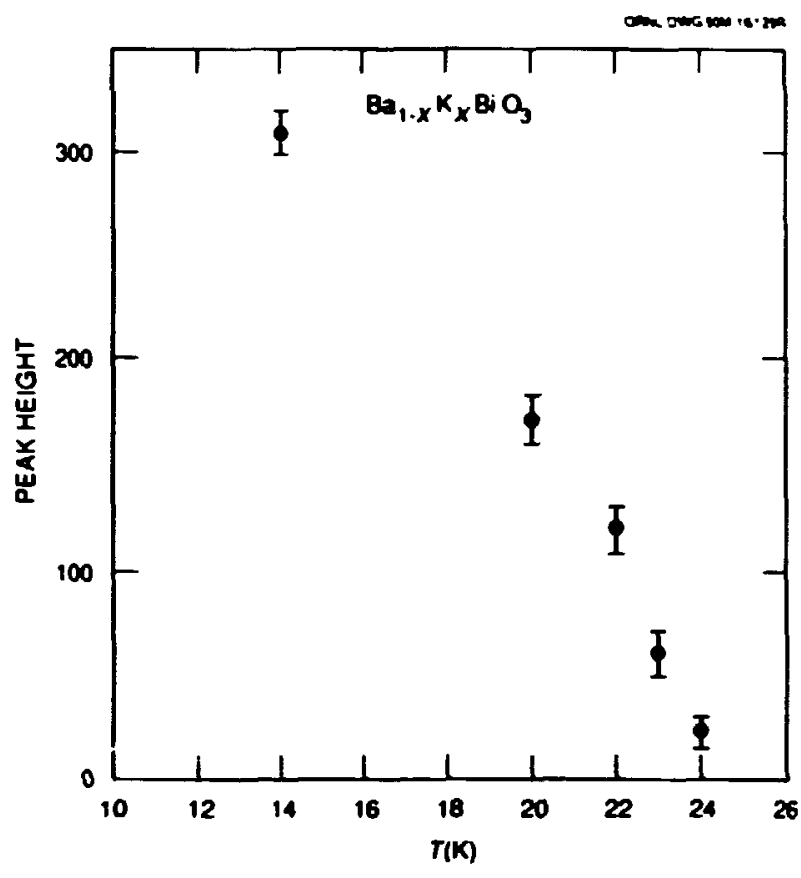

Fig. 2.1. Temperature dependence of the scattering from the fluxoid lattice.

fluxoid lattice was crystalline and had not undergone melting. It is not clear what the exact crystal structure of the fluxoid lattice is, although it seems to be similar to that of $\mathrm{Nb}$ when the field is applied along the (100) direction, as it was in this case. More measurements are planned to clarify this point. Measurements of the scattering from the fluxoid lattice were made as the sample was rotated in the beam. It was found that the scattering was very sensitive to rotation of the sample, and thus, the fluxoids are very straight along the direction of the applied ficld.

1. University of Georgia, Athens, Ga. 
THEOKY OF CORRELATIONS IN A BINARY FLUID MIXTURE OF SPHERICAL PARTICLES WITH EMBEDDED ORIENTED DIPOLES'

John B. Hayter and Roger Pynn

Fluids of colloidal-sized particles with embedded dipoles form an important class of materials whose properties may be controlled by the application of an external electric or magnetic field (examples are electrorheological fluids and magnetic fluids). Many systems of interest may be modeled by suspensions of spherical particles with embedded point dipoles, which are usually oriented under enduse conditions (external field applied). Calculating the correlations in these fluids provides a novel inveried Ising problem, in which the spin orientations are fixad, and the problem is to compute their positions in the equilibrium fluid. Since the interparticle potential is central plus dipolar only, such model systems are relatively tractable theoretically, and the correlation functions were calculated analyticaily to dipolar order for the onccomponent case some time ago. ${ }^{3}$

Subsequent experiments and simulations have shown that this order of approximation is satisfactory only in the case of modest dipolar coupling and that for many practical systems, spatial correlations have non-negligible quadrupolar character. Further, there is now substantial interest in the structures which form in mixtures of these fluids, in which one component is used to modulate the structure of the other. Unfortunately, the theoretical method of solution for the one-component case, which relies on reducing the Ornstein-Zernike equation for the fluid to a set of equivalent scaled equations, whose solution is known for many systems, does not work in the case of mixtures, since the scaling factor becomes a singular matrix in the mixture. A different theoretical approach has now been developed and solved to quadnipole onder in the correlarions for the binary-mixture case. The solution overcomes the deficiency of the dipolar solution at high coupling.

1. Summary of papet to be published.

2. Los Alamos National Laboratory, Los Alamos, N. Mex.

3. J. B. Hayter and R. Pynn, Phys. Reo. Lett. 49, 1103 (1982).

\section{DIFFERENTULL DEUTERATION EFFECTS IN BLOCK COPOL MMERS AS OBSERVED BY SMALLANGLE NEUTRON SCATTERING (SANS) ${ }^{1}$}

C. D. Wignall, J. H. Rosedale, ${ }^{2}$ and F. S. Bates 2

Block copolymers are self-associating amphiphilic molecules which have applications in a wide range of industrial products and in fundamental s!udies of condensed-state thermodynamics. SANS is a unique tool for investigating these systems because of the scattering contrast which may be obtained by isotopically labeling (staining) the parts of the molecule of interest. Experiments on model poly (ethylenc-propylenc) - poly(ethylethylene) (PEP-PEE) copolymers have been undertaken on samples synthesized at the University of Minnesota, and a surprising result is the 
demonstration that strong SANS contrast can be obtained by treating the protonated precursor (polyisoprene-polybutadiene) molecules with deuterium gas. The $D^{2}$ atoms have been shown to locate preferentially on the PEP units, thus giving rise to a SANS signal an order of magnitude higher than would be expected for the case of uniform deuteration. Figure $\mathbf{2 . 2}$ shows the differential scattering cross section $d \Sigma / d \Omega(Q)$ vs $Q$ for one such model block copolymer. For this sample the PEP and PEE components are phase separated into a lamellar structure, and the strength of the signal is determined by the scattering contrast between the domains. The strong SANS signal allowed the rescarch to commence during the first wecks after the HFIR start up, tespite the fact that the reactor was operating at very low power. The preferential deuteration allows

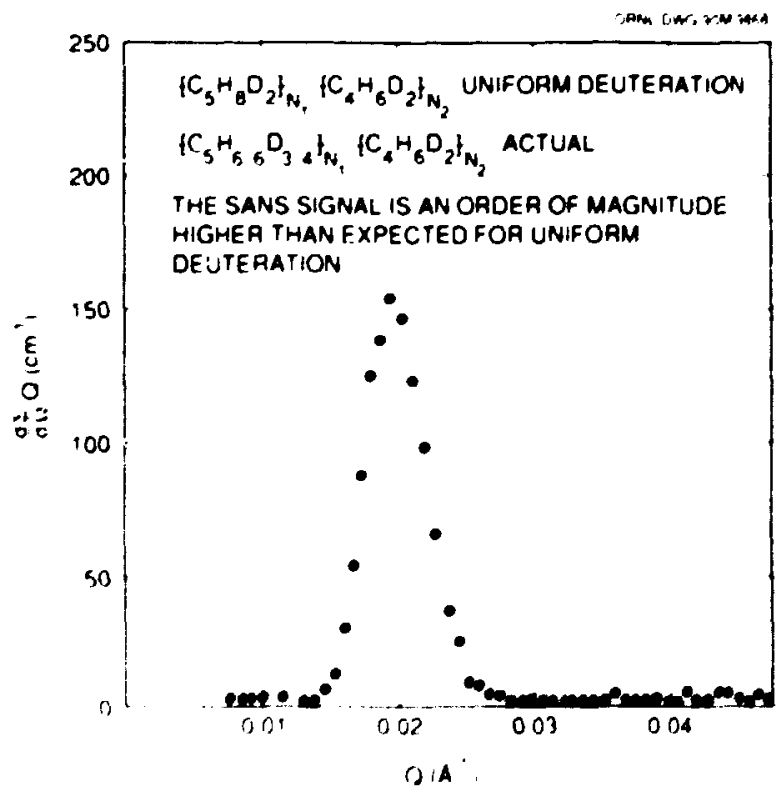

Fig. 2.2. $d \Sigma / d \Omega(Q)$ vs $Q$ for PEP-PFE. block copolymer. contrast to be obtained by treating existing well-characterized molecules. Previously, contrast would have to have been obtained by performing a separate polymerization using expensive deuterated monomer. The new procedure eliminates not only this step but also the need for separate characterization of the labeled molecules. It also removes the possibility of introducing differences between the labeled and unlabeled molecules and ensures that both have exactly the same scattering characteristics (molecular weight, polydispersity, etc.). SANS data are being used to probe the domain sizes, order-disorder transitions, and symmetry as a function of temperature, composition, and molecular weight. ${ }^{3}$

1. Summary of paper to be published.

2. University of Minnesota, Minneapolis, Minn.

3. K. Almdahl et al., "Gaussian-toStretched-Coil Transition in Block Copolymer Melts," this report.

\section{GAUSSIAN-TO-STRETCHED-COIL TRANSITION IN BLOCK COPOL MMER MELTS ${ }^{1}$}

\section{K. Almdahl, ${ }^{2}$ F. S. Bates, ${ }^{2}$ G. H. Fredrickson, ${ }^{3}$ J. H. Rosedale, ${ }^{2}$ and G. D. Wignall}

Block copolymers have commanded both theoretical and experimental attention for over two decades. Central to block copolymer theory and practice is the issue of order and disorder. A variety of phases exist below the orderdisorder transition (ODT), and phase segregation is controlled by threc parameters-... the 
segment-segment interaction parameter, composition, and overall degree of polymerization (N). All current theories dealing with copolymer-phase behaviur make the assumption that in the vicinity of this transition the interactions are sufficiently weak that the individual copolymers remain essentially Gaussian and the microdomain period scales as $N^{1 / 2}$. This is referred to as the weaksegregation limit (WSL) assumption. The second limiting regime of phase behavior is referred to as the strong segregation limit (SSL) where narrow interfaces separate welldeveloped, nearly pure $A$ and $B$ microdomains. This morphology leads to stretched-coil configurations and ordered-phase periods that scale as $N^{2 / 3}$. Although there exists extensive literature dealing with block copolymer melts, we are unaware of any experimental evidence of a Gaussian-to-stretched-coil transition. We have, therefore, undertaken small-angle neutron scattering experiments on a series of nearly monodisperse poly(ethylene-propylene). poly(ethylethylene) (PEP-PEE) diblock copolymers containing $55 \%$ by volume PEP ranging in size from $N=125-1890$. Neutron scattering contrast was obtained by preferentially deuterating one of the blocks. The characteristic lamellae period $D$ for symmetric block copolymers is inversely proportional to the small-angle scattering peak position $Q^{*}$ so that $Q^{*} \sim N^{\delta}$, where $\delta=1 / 2$ and $2 / 3$ for the WSL and SSL theories, respectively. The results are summarized in Fig. 2.3, and two scaling regions are clearly evident. For the three lowest molecular-weight samples $\delta=0.49 \pm 0 .(12$, essen-

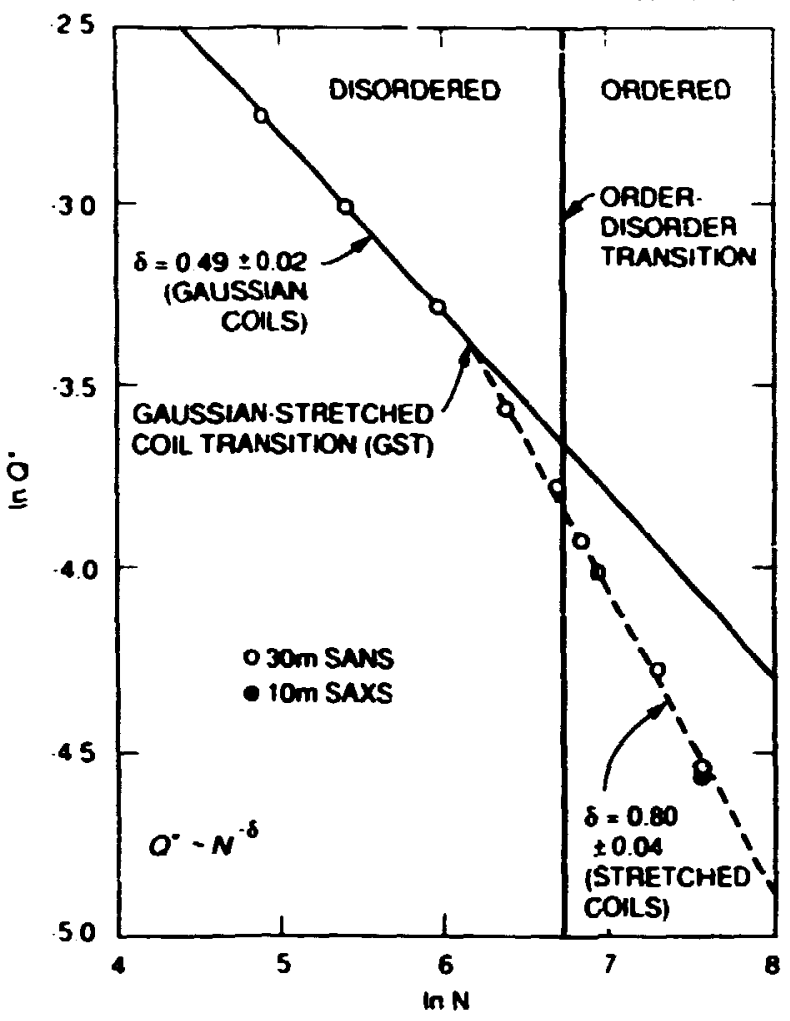

Fig. 2.3. Gaussian-stretched coil transition for PEP-PEE block copolymers as a function of polymerization index, $N$.

tially in exact agreement with Gaussian-coil statistics. The six highest molecular-weight samples follow a distinctly different scaling behavior, with $\delta=0.80 \pm 0.04$. Here the polymer coils are clearly in a stretched configuration. The Gaussian-to-stretched-coil transition is identified as the intersection of these two scaling regimes. To our knowledge, this represcnts the first experimental evidence for such a transition in block copolymer melts. Departure from Gaussian-coil statistics precedes the order-disorder transition and extends well into the ordered state. These results are not accounted for by current theory. 
1. Summary of paper. Phys. Rev. Lett. 65, 1112 (1990).

2. University of Minnesot3, Minneapolis, Minn.

3. University of California, Santa Barbara, Calif.

\section{SMALL-ANGLE NEUTRON SCATTERING CHARACTERIZATION OF POLYPHENYLENE SULFIDE'}

C. B. Caflisch, D. R. Fagerburg, ${ }^{2}$ C. Sass, ${ }^{2}$ and G. D. Wignall

Polyphenylene sulfide (PPS) has been used for over a decade in lightweight, hightemperature materials applications. Because of its high heat-deflection temperature and outstanding resistance to solvents, it may be used in a variety of applications and environments which would degrade and damage normal polymeric materials. Commercial PPS suffers from : rittleness owing to insufficient molecular weight and halide salt contamination, both of which tend to limit the material utility in either structural or electronic applicatinns. In order to overcome these difficultics, Eastman Chemical Co. has developed a novel melt synthesis o! PPS which can produce very high molecular weights and has no salt contaminant. A characteristic of this new method is that a fraction of disulfide linkages is created in the polymer chain. Characterization of this polymer in solution has resulted in molecular weights (chain lengths) which are very low compared to values expected on the basis of the melt viscosity. One explanation of this phenomenon is that the polymer chain breaks at the disulfide linkages in solution, and the molecular weight represents the chain length between disulfide linkages. In the solid state, it is believed that the disulfide linkages reform, and the m!t riscosity is characteristic of a high molecular-weight polymer. Conventional methods for molecular-weight determination (e.g., light scattering) are conducted in solution, but the unique small-angle neutron scattering (SANS) scattering contrast available from deuterium labeling methods allows the determination of this parameter in the bulk (solid) polymer. For mixtures of deuterated PPS with commercial PPS (which docs not contain end groups reactive to disulfide linkages), the observed SANS molecular weight is in agreement with that expected from melt-viscosity measurements thus confirming the above hypothesis. Further measurements in the solid state and in solution are planned at a variety of concentrations of deuteriumlabeled polymer. If these measurements further confirm the above hypothesis, the new synthesis route to PPS could provide a polymer which has improved mechanical and electrical properties while retaining the outstanding high-temperature stability of this material.

1. Summary of paper to be published.

2. Eastman Chemical Company, Kingsport, Tenn. 


\section{SMALL-ANGLE NEUTRON SCATTERING FROM BIMODAL POLYMER MELTS'}

\section{Tangari, ${ }^{2} R$. Ullman, 2}

\section{J. S. King, and G. D. Wignall}

It is well established ${ }^{3}$ that in the bulk polymer the radius of gyration $\left(R_{g}\right)$ of a molecule is the same as in an ideal solvent and scales as $N^{1 / 2}$ where $N$ is the number of units on the chain. Conversely, in a good solvent the polymer coil is expanded and $R_{8}$ scales as $N^{3 / 5}$. In general, the monomer is a good solvent so that the polymer chain is again expanded when dissolved in its own monomer (e.g., polystyrene swollen in styrene). If the solvent is a low molecular-weight polymer, the solvent power is intermediate, and successive increases in molecular weight of the solvent correspond to lesser degrees of molecular swelling and reduced second virial coefficients $\left(A_{2}\right)$. There has been only one systematic study of this effect, 4 and here new results on this phenomenon are provided for further consideration. The polymer chosen for these experiments is polystyrene in the deuterated form (MW = $2.3 \times 10^{5}$ ), and the solvents are protonated polymer (PSH) with molecular wrights in the range 580-19,800.

The measured values of $R_{g}$ and $A_{2}$ are shown in Table 2.1. Also shown are values of $A_{2}$ calculated by means of a power-law approximation based on the assumption that the enthalpy of mixing of a polymer with a solvent, which is itself a polymer of the same species, should be close to zero. The decrease in $A_{2}$ with increasing molar volume of the solvent in these systems is far less than expected.
Table 2.1. Experimental and calculated second virial coefficients $D$-polystyrene in H-polystyrene solvents.

\begin{tabular}{|c|c|c|}
\hline Solvent & $R_{g}(\boldsymbol{A})$ & $\begin{array}{r}A_{2} \times 10^{s} \\
(\exp )\end{array}$ \\
\hline
\end{tabular}

Benzenes

4.68

PSH 580

150.0

3.14

3.1

PSH 3600

145.5

2.16

1.9

PSH 9100

145.5

1.78

1.8

PSH 19,800

Taking the position that a solution of a polymer in a polymer of the same species is primarily driven by the entropy of mixing, one of two conclusions can be reached. Either two polymers of the same chemical species but different molecular weights mix much less efficiently than anticipated, or the errors in a mean-ficld approach to polymer thermodynamics are more serious than is currently believed. These conclusions point to the need for further experiments on the influence of the size of the solvent molecule on the thermodynamics of polymer solutions and call for the possibility that ideas on the mixing of polymers which are widely held may, in fact, be in error.

1. Summary of paper: Macromolecules (in press).

2. University of Michigan, Ann Arbor, Mich.

3. G. D. Wignall, p. 645 in Concise Encyclopedia of Polymer Science, ed. by J. I. Kroschwitz, Wiley-Interscience, New York, 1989.

4. R. G. Kirste and B. R. Lehnen, Macromol. Chem. 177, 1137 (1976).

5. M. Fukuda et al., I. Polymer Sci. Polymer Phys. 12, 871 (1974). 


\section{ESTIMATION OF INCOHERENT \\ BACKGROUNDS IN SMALL-ANGLE \\ NEUTRON SCATTERING (SANS) STUDIES OF POLYMER'}

\section{G. D. Wignall, W. S. Dubner, ${ }^{2}$ and I. M. Schultz $z^{2}$}

Blends of deuterated and hydrogenous polymers have been used for well over a decade in conjunction with SANS to provide information on polymer structure, configuration, and compatibility. How'ever, the subtraction of the flat background due principally to the $H^{1}$ incoherent cross section remains an unsolved problem, which becomes increasingly important in the intermediate-angle neutron scattering (IANS) range $\left(0.1<0<0.7 A^{-1}\right)$, where the incoherent background can actually exceed the coherent signal. In principle, it is possible to estimate the background by producing random copolymers of the same overall isotopic ratio as the partially labeled blend, though in practice this is rarely done because of the difficulties of synthesis. In the vast majority of SANS experiments, the background is measured for pure $H$ and D-polymer blanks, and some fraction of this background is subtracted from the scattering of the partially labeled system using various extrapolation techniques.

Based on work by McAlea et al., ${ }^{3}$ an exact solution of this problem is now possible. Due to the transesterification reaction, partially labeled blends of deuterated and hydrogenous poly(ethylene terepthalate) (PET) may be converted to random copolymers by bricf annealing in the melt. The coherent and incoherent back- grounds from a series of PET random copolymers have been measured as a function of the percent deuteration, and the results have been compared with calculations used in previous extrapolation techniques used in IANS studies of polymers. Scaling via the measured sample transmission works well for predominantly protonated mixtures but fails when the fraction of deuterated polymer exceeds $\phi_{D} \sim 0.6$. Scaling via the component of the sample transmission attributable to the incoherent scattering from $H$ nuclei works well over the whole concentration range. This method involves calculations of the sample transmission via the bound atom cross section, and this is a good approximation at the wavelength employed $(\lambda \sim 4.75 \AA)$, where the hydrogen incoherent cross section is close to the bound atom cross section $\left(\sigma_{H} \sim 80 x\right.$ $10^{-24} \mathrm{~cm}^{2}$ ). However, $\sigma_{\| l}$ is a strong function of wavelength, and the method cannot be used where the measured and calculated Iransmissions diverge. A recently developed theory describing the scattering from random copolymers was used to analyze the residual coherent cross section of the copolymers and led to a statistical segment length $b=14.3 \pm 1 \AA$, which is in good agreement with literature values for I'ET.

1. Summary of paper: Physical Review Letfers (in press).

2. University of Delaware, Newark, Del.

3. K. P. McAlea et al., Polymer 27, 1581 (1986). 
TIME-RESOLVED X-RAY STUDIES ON PREMELTING DURING ANNEALING OF GEATION CRYSTALIZED ULTRAFIGH MOLECULAR WEGHT POLYETHYLENE'

M. H. Cho, T. Kyus, J. S. Lin,
K. Saijo, ${ }^{3}$ and T. Hashimoto

A semicrystalline polymer consists of alternate layers of amorphous and crystalline material with a well-defined periodicity. The phenomenon of premelting in these materials is generally inferred from a continuous decrease in crystalline volume during annealing below the equilibrium melting temperature $\left(T_{m}\right)$. Smallangle $x$-ray scattering (SAXS) measurements have revealed an increase in the amorphouscrystalline period with increasing temperature or isothermal annealing. There is no agreedupon opinicn on the mecinanism underlying the premelting process. A classical interpretation of premelting is based on lamellar thickening in which solid state diffusion of intercrystalline chains takes place. However, there are at least three other mechanisms that have been postulated: (1) selective melting of small lamellae within the lamellar stacks, (2) alternating melting and recrystallization, and (3) surface melting associated with an entropy change in the amorphous regions resulting in an increase in thickness of the interlamellar amorphous region.

In a previous paper, the phenomena of melting and crystallization of gelationcrystallized, ultrahigh molecular-weight polycthylene using SAXS and wide-angle $x$-ray scattering (WAXD) has been investigated. It was observed that the morphology of the gelation-crystallized polyethylene is similar to stacked lamellae of single-crystal mats and postulated that the increase in long period may be due to an increase in the periodic distance of lamellar structure associated with the partial melting of certain lamellae.

In this study our effort was continued to elucidate the phenomenon of annealing based on time-resolved SAXS and WAXD. Isothermal annealing studies were undertaken by conducting temperature-jump experiments from ambient to elevated temperatures around the onset of $T_{m}$. Sheets of gelatin-crystallized polyethylene $\left(M W \sim 6 \times 10^{6}\right)$ were used and stacked tightly for $x$-ray studies.

The time-resolved WAXD results clcarly reveal that the apparent crystallinity decreases during isothermal annealing below $T_{m}$, suggesting a premelting transition without recrystallization. Recrystallization was not detected within our experimental time scalc, and this could be due to slow molecular diffusion because of the extremely high viscosity of our samples. It is reasonable to conclude from the WAXD results that premelting is the dominant mechanism during annealing below $T_{m}$. The increase in the SAXS long period may be due to the melting of successive lamellae. This is exactly the reverse of crystallization, which can be explained in terms of a lamellar insertion model in which new lamellac form in interlamellar spaces. It should be pointed out that wur time-resolved SAXS and WAXD observations do not preclude the possible 
lamellar thickening which can occur concurrently with successive lamellar metting.

1. Summary of paper to be published.

2. University of Akron, Akron, Ohio.

3. Kyoto University, Kyoto, Japan.

4. T. Kyu, Macromolecules 22, 2238 (1989).

\section{STRUCTURAL DETERMINATION OF MICROSTRUCTURES IN MODEL BILE'}

\section{E. W. Kaler, 2 M. Long, ${ }^{2}$ \\ A. Martino, ${ }^{2}$ and G. D. Wignall}

Vesicles are water-filled spherical shells formed from a bilayer of surfactant molecules, and they are typically less than $1000 \AA$ in diameter. Micelles are closed, usually roughly spherical aggregates of surfactants and are the usual morphology found in dilute surfactant solutions. Small-angle neutron scattering (SANS) experiments have been undertaken to demonstrate micelle-to-vesicle transitions as a function of composition in the aqueous system lecithin/cholesterol/bile salt, which serves as an excellent model of bile. Promotion of the solubility of cholesterol in bile is crucial for the prevention of gallstone disease, and these physical chemical characterizations of model systems have direct physiolsgical relevance. When a concentrated micellar solution of bile salt and lecithin is rapidly diluted, vesicles form. 3,4 At intermediate dilutions, before the appearance of vesicles, the particle size increases, peaks, and then decreases to unilamellar vesicle dimensions. The shapes of the intermediate structures are not known. We used SANS to provide information on the shape of the particles along the dilution path and to confirm the presence of vesicles. Figure 2.4 shows one such dilution series, and similar transitions are observed in three-component mixtures containing cholesterol. At highdilution factors, the characteristic maxima and minima of the vesicle form factor are obscrved. The fitted outer radius of the vesicle agrees well with light-scattering measurements. The scattering of the concentrated solutions is more difficult to analyze. The scattering curves are featureless, but attempts to fit them with the scattering of cylinders, ellipsoids, and disks are under way. The role of interparticle interactions in determining the arrangement of micelles and thus the scattered intensity is

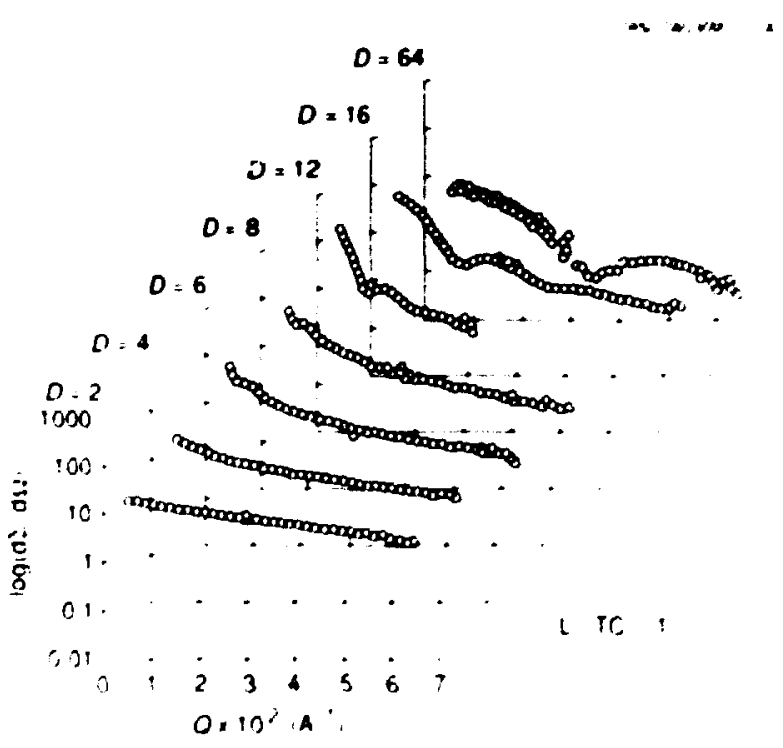

Fig. 2.4. Scattered intensity $/(Q)(1 / \mathrm{cm})$ for an initial lipid concentration of $5 \mathrm{~g} / \mathrm{dl}$ of $1: 1$ lecithin/taurocholatc as a function of dilution ratio $D$. The spectra evolve from those characteristic of micelles $(D<6)$ to those charactiris. tic of vesicles $(D>16)$. 
important, but the nature and range of the interactions are not known. Models of intermicellar interaction potentials are being developed, and spectra are being fit considering only simple excluded volume effects with a hardsphere fluid structure factor.

1. Summary of paper to be published.

2. University of Delaware, Newark, Del.

3. R. P. Hidm, P. Thiyagarajan, and H. Alkan, J. Appl. Crystallogr. 21, 858 (1988).

4. P. Schurtenberger, N. Mazer, and W. Kanzig, J. Phys. Chem. 89, 1042 (1985).

MONOVALENT COUNTER-ION DISTRIBUTIONS AROUND FERSISTENCE LENGTH DNA FRAGMIENTS IN SOLUTIONI

\section{S. L. Chang, S. H. Chen, R. L. Rill, ${ }^{3}$ and J. S. Lin}

In a DNA molecule, the phosphate groups $\left(\mathrm{PO}_{4}\right)^{-}$are uniformly distributed along the helical backbone with 3.4- $A$ separation in the helix-axis direction, and at neutral $\mathrm{pH}$, the counter ions (such as $\mathrm{Na}^{+}$) are fully dissociated. The DNA is, in fact, a highly charged polyelectrolyte in solution. It is well known that the thermodynamic and transport properties of a polyelectrolyte solution are highly dependent on the counter-ion distribution around the molecule. The sequence and environmentdependent local variations in secondary DNA structures are likely to play essential roles in the specific interactions of DNA with protcins and other ligands, and a detailed knowledge of the structures and structural transitions of DNA in solution is essential for inderstanding gene expression and regulation at the molecular level.

It has previcusly been demonstrated that it is possible to detect the degree of countertion condensation along the surface of highly charged cylindrical micelles in solutions by using $\mathrm{Cs}^{+}$(54 electrons) as higher contrast counter ions.4 In the case of DNA, the nucleotides have a high electron density which may be comparable to the heavy-metal ions around the macro-ion surface. Therefore, the heavy monovalent counter-ion $\mathrm{n}^{+}$was selected to substitute for the $\mathrm{Na}^{+}$ions in our counter-ion distribution study.

Small-angle $x$-ray scattering (SAXS) measurements were made on solution samples of DNA fragments and varying concentrations of counter ions. DNA fragments with a narrow length distribution about a most probable length of 146 base pairs $(500 A)$ were prepared from calf thymus chromatin digested with micrococal nuclease. The deproleinized $\mathrm{Na}^{+}$ DNA was dialyzed exhaustively to exchange over $90 \%$ of the $\mathrm{Na}^{+}$ions with $\mathrm{M}^{+}$. The absolute scattering intensity distributions were compared with numerical solutions of the nonlinear Poisson-Boltzmann equation in a cell modei. 5.6

Quantitative agreement between caliulations and experiments was obtained when conIributions to the intensity from both the DNA and the diffuse ion cloud are included. The agreement is slightly improved if it is assumed that $10 \%$ of the counter ions are within the cylindrical DNA core; namely, in the major and minne grooves of the double helices. 
To our knowledge, this study clucidates for the first time the contribution of the diffuse ion distributions to the SAXS intensity from. persisrence kngth DNA fragments in solution.

1. Summary of paper. I. Phys. Chem. 94, 8025 (1990).

2. Massachusetts Institute of Technology, Cambridge, Mass. Fla.

3. Florida State University, Tallahassee,

4. C. F. Wu et al., Phys. Rec. Lett. 61, 645 (1988).

5. A. Katchalaky, Pure and Appl. Chem. 26,320 (1971).

6. C. Cametti and A. DiBiasio, Ber. Bunsen-Ces. Phys. Chem. 92, 1089 (1988).

\section{NEUTRON DIFFRACTION}

\section{PRESSURE EFFECTS ON THE MARTENSIIIC TRANSFORMATION IN METAUUC UTHIUM'}

\section{H. G. Smith, R. Berliner, ${ }^{2}$ J. D. Jorgensen, 3 $M$. Nielsen, and I. Trivisonnos}

This neutron diffraction study investigated the effects of modest hydrostatic pressures on the bec-9R transformation temperature in a polycrystalline sample of ${ }^{7} \mathrm{Li}$ and a subsequent 9R-fcc-bec transition on warming (at the Intense Pulsed Neutron Source, Argonne National Laboratory). A brief study of the 9R-fcc-bec transition in a single crystal of natural lithium was also made (at Riso, Denmark). The initial powder diffraction scans were made at room temperature, at atmospheric pressure, and at 2.9. 4.5, and 6.5 kbar under hydrostatic conditions. There was no evidence of any transforma. tion even though the volume change at $6.5 \mathrm{kbar}$ was twice as large as the volume change from room temperature to $75 \mathrm{~K}$ at atmospheric pressure (the average temperature of previous transformations at zero pressure). The fact that the bec structure did not transform by volume changes alone suggests that theoretical total cnergy calculations should include the effects of vibrational entropy in the search for the most stable structure, even at low temperatures. The sample was then cooled in stages down to $109 \mathrm{~K}$, keeping the pressure as nearly constant as possible at 6.5 kbar. Upon further cooling to $90 \mathrm{~K}$, a partial transformation to the $9 R$ structure had taken place. This indicates $T_{c}$ increased about 15 or $20 \mathrm{~K}$ above the atmospheric pressure $T_{c}$, or roughly $3 \mathrm{~K} /$ khar of applied pressure. (This ratio is similar to that obtained in one roomtemperature $x$-ray diffraction study of $\mathrm{Li}$ in a diamond anvil apparatus at a pressure of 70 kbar, in which a change to the fec structure was reported.)

Upon warming in stages from $70-140 \mathrm{~K}$ at maximum pressure, the $9 R$ structure disappeared. A small amount (-20,t) of the fec phase appeared ano then disappesred on further warming to $175 \mathrm{~K}$, leaving only the bec phase. The existence of the fce phase was investigated further with a single crystal at atrrospheric pressurc on a triple-axis spectrometer at Rise National Laboratory. This was accomplished by monitoring the intensity at the positions of the (104) and (10)? $9 R$ reflections while slowly warming the somple after the $9 R$ iransformation. The intensity of the 
(103) reflection is zero for the $9 R$ structure, but not for the fec structure indexed on the 9R lattice [with proper foc index of (111)]. The intensity of the strong (104) $9 \mathrm{R}$ reflection began to decrease at about $90 \mathrm{~K}$, while the (103) $9 R$ reflection increased, clearly indicating growth of the foc phase at the expernse of the 9R phase. but not necessarily on a volume-for-volume basis.

The results of these experiments are at variance with another $x$-ray study in which a bec-to-fec transition occurred at 2.94 kbar at room temperature. These data were obtained by photographic methods, but the pressure techniques used are uncertain; however, it is likely that i.snhydrostatic methods were used, in contrast to the hydrostatic conditions in the He gas pressure system used in the neutron studies. The large discrepancy in transformation pressure between the two $x$-ray studies is not resolved, but may be due to the methods of applying pressure. Clearly, much work needs to be done to fill in the gap between the low- and high-pressure regions.

1. Summary of paper: Phys. Rev. B 41, 1231 (i990).

2. University of Missouri, Columbia, Mo.

3. Argonne National Laboratory, Argonnc, III.

4. Riso National Laboratory, Roskilde, Denmark.

5. John Carroll University, Cleveland, Ohio.

\section{PRESSURE EFFECTS ON THE MARTENSITC TRANSFORMATION TN METALUC SODTMI}

H. G. Smith, R. Berliner,?

$J$. D. Jorgensen, ${ }^{3}$ and I. Trivisonnot

Neutron scattering experiments on polycrystalline and single crystals of $\mathrm{Na}$ have shown that the metal undergoes a partial martensitic transformation from the bec lattice to a combination of the $9 \mathrm{R}$ lattice and a trigonal or hexagonal lattice below $35 \mathrm{~K}$, somewhat similar to that of lithium, which transforms to a 9R lattice plus an foc hattice below about $75 \mathrm{~K}$. Recent neutron scattering experiments on $\mathrm{L}$ under hydrostatic pressure at the Intense Pulsed Neutron Source showed a pronounced increase in the transition temperature from 75 to about $90 \mathrm{~K}$ at a pressure of $6.5 \mathrm{kbar}$. Similar experiments on $\mathrm{Na}$, however, show a decrease in the phase-transitiot temperature with pressure, actually suppressing the $9 \mathrm{R}$ transition at $1 \mathrm{kbar}$ to $15 \mathrm{~K}$ (Fig. 2.5). The amount transformed is also reduced considerably with increasing pressure.

Several theoretical groups have shown that, in general, close-packed structures (fcc, hcp, 9R, etc.) are more stable than bcc. They often differ in the type of close-packed lattice that is most stable, but the energy differences are extremely small (only a few $K$ in the case of $\mathrm{Na}$ and are undoubtedly dependent on the approximations made in the various approaches. Experimental observation of the coexistence of several phases, including the bec 


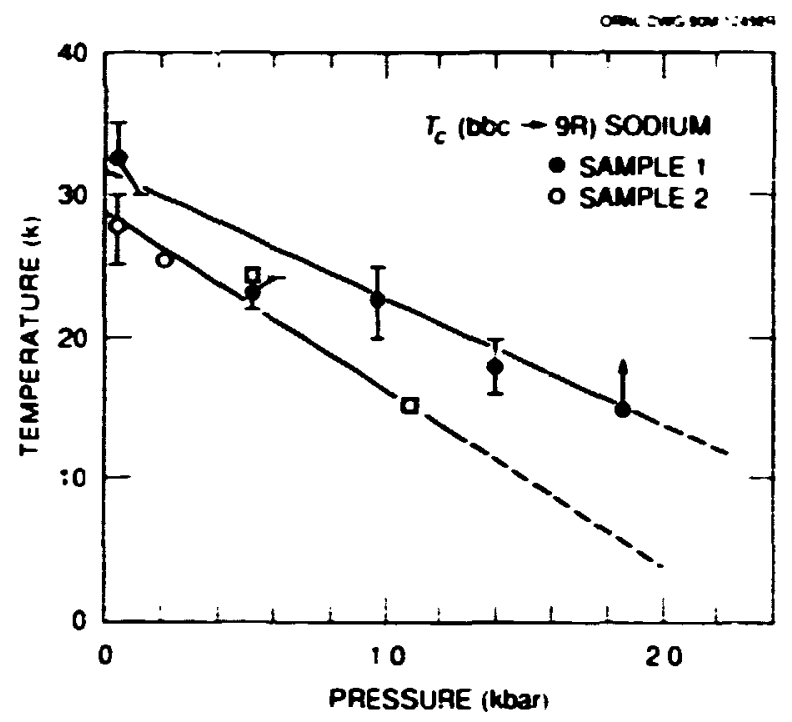

Fig. 2.5. Phase boundaries for bce and $9 R$ structures for the two sample preparations. (Sample 2 was cold worked by an extrusion process)

phase, indicates that the differences in total energies should be small. The results presented here, however, are consistent with severai theories which predict that $\mathrm{Na}$ will transform back from a close-packed lattice (hcp) to bec at a pressure of about $10 \mathrm{kbar}$, which is considered in agreement with the extrapolated 2-3 kbar observed in the neutron scattering and acoustic emission measurements.

It is not possible from these experiments to determine readily the effects of pressure on the stacking-fault densities or to what extent thr presence of the "hexagonal" phase has been affected. These questions will best be answerrd by single-irystal experiments under pressure at low temperatures.
1. Sumniary of paper: Physical Reciew B (in press).

2. University of Missouri, Columbia, Mo. III.

3. Argonne National Laboratory, Argonne,

4. John Carroll University, Cleveland, Ohio.

\section{ATOMIC MAGNETIC MOMENT SPATIAL CORRELATIONS IN Fe-13.5:, $\mathrm{V}$}

\section{J. W. Cable, B. Gillon,' I. Mirebeau,'}

G. Parette, and Y. Nakar?

Recently, censiderable theoretical effort has been directed toward a general understonding of the thermally induced concentration and magnet. : moment fluctuations in alloys and the relationship of these fluctuations to alloy phase stability. The atom-atom, atommoment, and moment-moment spatial correlations that are determined from neutron diffuse scattering measurements can be directly compared to those based on electronic structure calculations and thereby provide stringent tests for the theoretical approaches as well as theoretical assistance in the interpretation of experimental results. Such a comparison was made recently for the atom-atom correlations in an Fe-13.5\% V alloy. The first-principles calculation $^{3}$ not only was successful in reproducing our experimental observations ${ }^{4}$ but also provided a connection between the q-dependent structure in the diffuse scattering and the underlying elecIronic structure of the alloy. Johnson, Staunton, and Pinski ${ }^{5}$ a!so calculated the atom-moment correlations and suggested that a polarized- 
neutron determination of these spatial correlations would provide a valuable test of their theoretical approach. We decided to pursue this problem using the polarized-neutron diffractometer HC-1 located at the ORPHEE reactor at the Laboratoire Léon Brillouin, CENSaclay, France. The diffuse-scattering measurements were made for the same Fe-13.5\%V alloy, and the observed atom-moment correlations $M(Q)$ are compared with the calculated correlations in Fig. 2.6. These are in absolute units of Bohr magnetons per atom and correspond to the magnetic response of the system to a concentration fluctuation. Clearly, the calculated shape is essentially correct in all three high-symmetry directions, while the magnitude of the q-dependent moment response is not adequately reproduced.

1. Laboratoire Léon Brillouin, CENSaclay, France.

2. Osaka University, Osaka, Japan.

3. J. B. Staunton, D. D. Johnson, and F. J. Pinski, Phys. Rev. Lett. 65, 1259 (1990).

4. J. W. Cable, H. R. Child, and Y. Nakai, Physica B 156/157, 50 (1990).

5. J. B. Staunton, D. D. Johnson, and F. J. Pinski, Phys. Rev. Letl. 65, 1259 (1990).

\section{HIGH-RESOLUTION POWDER DIFFRACTOMETER}

\section{A. Fernandez-Baca and H. A. Mook}

Construction of a powder-diffractometer option for the HB4 spectrometer' has been completed, and this instrument is now in operation. The instrument has a $\mathrm{Ce}(115)$ monochromator
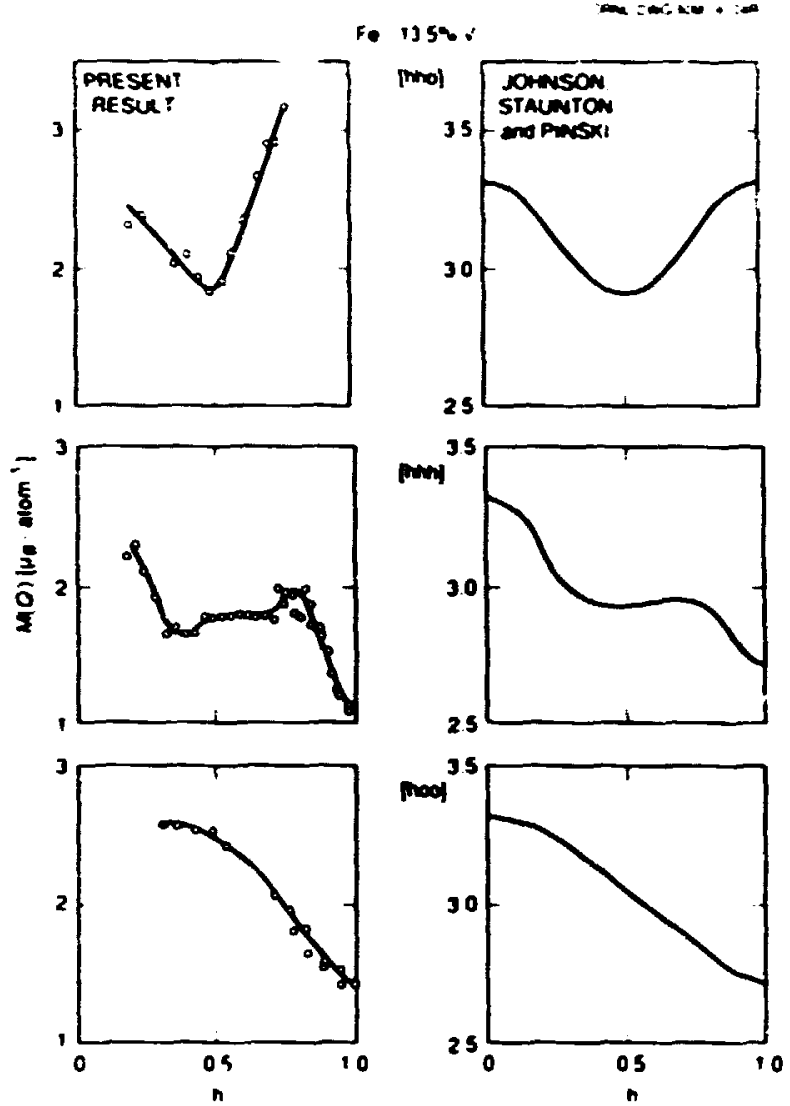

Fig. 2.6. Comparison of the observed (left; and calculated (right) atom-moment corrclations for Fe-13.5\%V.

which, when used at a takeoff angle $2 \theta_{M}=20^{\circ}$, selects a neutron incident beam of $1.4 A$. Sollerslit collimators of $12^{\prime}$ and $20^{\circ}$ are placed before and after this monochromator crystal. A bank of 32 equally spaced (2.70 apart) ${ }^{3}$ He detectors, each with a $6^{\prime}$ mylar-foil collimator, can be step-scanned in order to obtain powderdiffraction patterns in the scattering angle range $11-135^{\circ}$. At $1.4 \AA$, this angular range corresponds to $d$-spacings between $0.76-7.3 \AA$. The resolution of this instrument (Fig. 2.7) makes it very convenient for us to perform the refinement of structures by the Rietveld technique. This resolution is sufficient to refine 


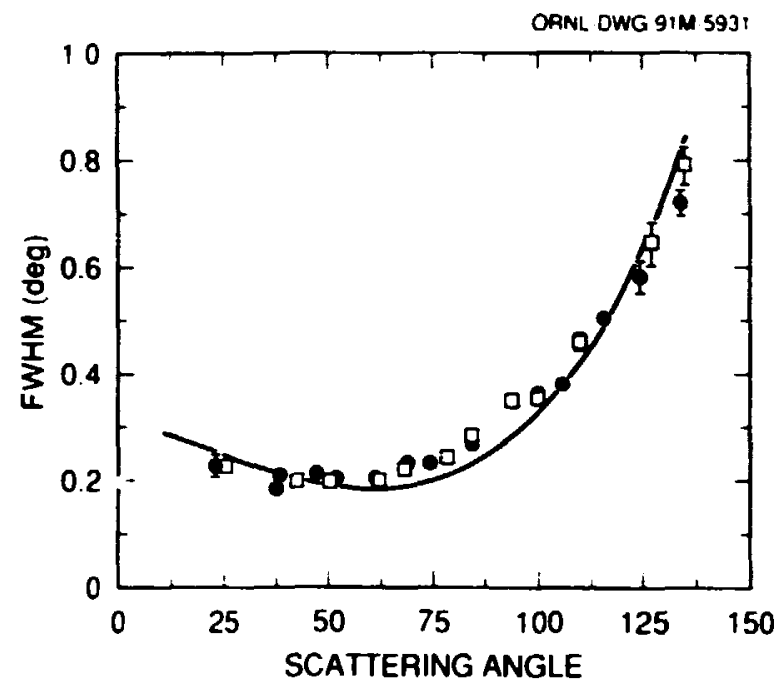

Fig. 2.7. Resolution curve of the new HB4 powder diffractometer. The data points correspond to actual FWHM from $\mathrm{Al}_{2} \mathrm{O}_{3}$ (solid circles) and Si (operi squares) powders; the solid line is the calculated resolution.

rather complex structures, while it is not very sensitive to peak-broadening effects resulting from particle size, strain, etc. Other areas that will benefit from this instrument include the study of magnetic structures and phase transitions. The data acquisition of this instrument is controlled by a MicroVAX-II computer which is also used for the data analysis.

1. R. M. Nicklow and J. W. Cable, Solid Stale Division Progress Report for the Period Ending Seplember 30, 1987, ORNL6453, p. 70.

\section{RIETVELD ANALYSIS OF \\ $\mathrm{Bi}_{2} \mathrm{Sr}_{2-x} \mathrm{Ia}_{x} \mathrm{CuO}_{6+y}$ FROM NEUTRON POWDER DIFFRACTION DATA ${ }^{1}$}

\section{B. C. Chakot'makos, J. A. Fernandez-Baca, B. C. Sales, and H. A. Mook}

With increasing La content, the title compound changes from semiconducting to superconducting and then back to semiconducting. For the superconducting compositions, $T_{c}$ has a parabolic dependence on the La content, with the maximum $T_{c}$ between $x=0.4\left(T_{c}=23 \mathrm{~K}\right)$ and $x=0.6\left(T_{c}=17 \mathrm{~K}\right)$ depending upon the oxygen content. The lowes' uxygen content is associated with the highest $T_{c}$. The crystal structure is isotypic with $\mathrm{Bi}_{2} \mathrm{Sr}_{2-x} \mathrm{CuO}_{6+y}$ which is known approximately on the basis of single-crystal $x$-ray diffraction data. The extent of Bi-Sr-La cation disorder and the nature of the exchangeable oxygen have not been determined by crystal structure analysis. A pronounced superlattice modulation complicates accurate structure determinations. The substitution of rare carth for $\mathrm{Sr}$ not only raises $T_{c}$ but also enables more nearly single-phase samples to be obtained (over the range $x=0.4-1.0$ for $\mathrm{La}$ ). Powder data have been collected on the newly constructed HB4 high-resolution powder diffractometer at the HFIR. Rietveld analysis is being used to determine the detailed roomtemperature structure, the variation of structural parameters with temperature, and the correlations of bond valence sums with $T_{c}$.

Preliminary refinements of the data have employed a variety of subcell models (i.e., the 
superstructure is ignored). The quality of the refinements as measured by the agreement indices are poor (e.g., $R_{w p}=0.20$ for the best case). For most of the structural parameters, meaningful values can be refined only with an improved structural model. So far, the estimated cell dimensions and $\mathrm{Cu}-\mathrm{O}$ bond lengths are relatively stable in adjusting other model parameters. Therefore, it is concluded that these are reliable estimates and that the systematic variations of the apical and in-plane CuO distances are reasonable. Moreover, the bond strength sum to the $\mathrm{Cu}$ site from the inplane oxygens correlates with $T_{c}$. Future efforts will explore: (1) combined refinement of both neutron and $x$-ray data sets, (2) incorporation of the superlattice modulation into the Rietveld analysis, and (3) sample preparation to suppress the superlattice modulation by $\mathrm{Pb}$ doping.

1. Summary of paper to be published.

\section{SEAKCH FOR STRAIN EFFECTS IN THE SPINODAL DECOMPOSITION OF Mn-Cu ALLOYS}

Steve Spooner, 1. A. Fernandez-Baca, and Y. Morii

Manganese-rich alloys of coppermanganese upon decomposition generate a finescale microstructure consisting of manganeserich and manganesc-poor regions on a common crystallographic lattice. A small compositiondependent lattice strain is expected which influences the side-band peak intensities appearing at the nuclear Bragg peaks [including the $(000)$ position]. Side-band intensities, including those at small angle, can be related to the coherency strains in the alloy. Preliminary measurements performed at the new HB4 high-resolution powder diffractometer on a null-lattice alloy (32.6 at.\% $\mathrm{Cu}$ ) decomposed by annaling at $450^{\circ} \mathrm{C}$ show a weak intensity asymmetry consistent with the above lattice strains. These high-resolution measurements are an improvement over earlier observations of decomposition with the wideangle neutron diffractometer spectrometer, because it is now possible to define the shape of side-band intensity in detail. New measurements on a larger sample of this null-lattice alloy (in preparation) will allow us to obtain more quantitative results.

1. Guest scientist from Japan Atomic Energy Rescarch Institute, Tokai, Japan.

\section{COHERENT NEUTRON SCATTERING LENGTH OF ${ }^{4} \mathrm{Ca}$}

R. M. Moon, 1. A. Fernandez-Baca, and S. Raman ${ }^{1}$

In a previous study, ${ }^{2}$ the coherent neutron scattering lengths of all the $\mathrm{Ca}$ isotopes except ${ }^{46} \mathrm{Ca}$ were measured. The supply of this rare 
(0.003\% abundant) isotope was too small to make an adequate sample for neutron diffraction measurements. By combining all the various lots of $\mathrm{CaCO}_{3}$ enriched in ${ }^{46} \mathrm{Ca}$, a sample of about $0.5 \mathrm{~g}$ with an isotopic enrichment of $32 \%$ has now been obtained. The major isotopic impurity is ${ }^{\circ 0} \mathrm{Ca}(59 \%)$.

Powder-diffraction data on both the HB4 and HB3 spectrometers have been obtained. Analysis of these data gives an average $\mathrm{Ca}$ scattering length of $4.05 \pm 0.06 \mathrm{fm}$ for this sample. Using the known isotopic distribution and our earlier measurements of the other isotopes, it is deduced that the scattering length for ${ }^{40} \mathrm{Ca}$ is $3.55 \pm 0.21 \mathrm{fm}$.

1. Physics Division, ORNL.

2. S. Raman et al., Phys. Reo. C 39, 1297 (1989).

\section{NEUTRON DIFFRACTION STUDY \\ OF RESIDUAL STRESSES IN A \\ MULTIPLE-PASS WELD IN A THICK STAINLESS STEEL PLATE}

T. M. Holden, ' I. H. Root,' loyce Coldstone,?

M. A. M. Bourke, S. A. David, 3 and Steve Spooner

Steady-state and pulsed neutron source methods of residual stress analysis have been applied to study a multiple-pass weld in a 1-in. thick 302 stainless steel plate. The neutron measurements were performed at the National Reactor Universal facility at Chalk River Nuclear Laboratories and at the Los Alamos
Neutron Scattering Center at Los Alamos National Laboratory. The variation of residual stresses through the plate thickness and perpendicular to the weld line within the plate were mapped out with the steady-state neutron source method which uses the shift in the position of the (111) and (200) Bragg peaks. The data from the pulsed source neutron experiments do not provide the same spatial detail as in the case of the steady-state source experiment. However, because the entire diffraction pattern is collected simultaneously in pulsed neutron sources, these data contain details such as texture, peak broadening, presence of second phases, and peak shifts which provide struclural information not available in the singlepeak shift method. The two types of measurements are therefore complementary. The steady-state results on residual stress are consistent with earlier results on sinjle-pass welds where a peak in the longitudinal stress along a path perpendicular to the weld line is found within the heat-affected zone and is at a level close to the yield point stress of the matcrial. Through-thickness variation is small except within the weld metal where effects duc to multiple weld passes are important.

1. Chalk River Nuclear Laboratorics, Chalk River, Ontario, Canada,

2. Los Alamns National Laborratory, l.os Alamos, N. Mex.

3. Metals and Ceramics Division, ORNI.. 


\section{INELASTIC NEUTRON SCATTERING}

\section{MAGNETIC FLUCTUATIONS DN}

$\mathrm{La}_{1-9} \mathrm{X}_{\mathbf{y}} \mathrm{CuO}_{4}: X=\mathrm{Ba}$ or S

\section{H. A. Mook, G. Aeppli, I S. M. Hayden, ${ }^{2}$ D. Ryiz, ${ }^{3}$ and Z. Fist4}

Work on the 2-1-4 compounds has been continued in an attempt to understand the nature of the spin fluctuations at various doping concentrations $y$ of either Ba or Sr. We now have a good picture of the magnetic excitations for the parent compound where $y=0$. This material appears to be an excellent example of a twodimensional Heisenberg antiferromagnet in which classical spin-wave theory can account for the behavior of the magnetic excitations without the need for quantum corrections. Temperature-dependent effects can be explained in terms of a spin-spin correlation length which grows as the temperature is lowered and diverges at the ordering temperature.

As $y$ is increased, the ordering temperature decreases, and at $\boldsymbol{y}=\mathbf{0 . 0 5}$ for Ba, only spin-glass ordering with the Edwards-Anderson order parameter increasing sharply below $5 \mathrm{~K}$ is found. It was also found that the spin-spin correlation length is constant below $250 \mathrm{~K}$ so that the temperature dependence of the spin dynamics is determined only by changes in the electronic susceptibility. A useful quantity to consider is the momentum integral of the dynamic susceptibility $X(\omega)$, which is well described by the function $A \tan ^{-1}(\omega / \Gamma)$ at all temperatures.
$X(\omega)$ thus crosses smoothly from the linear form $A \omega / \Gamma$ to the constant form $A \pi / 2$ at high $\omega$. This is the type of behavior one expects from a marginal Fermi liquid. This behavior also explains the results found in the $\mathrm{Cu}$ nuclear spin-lattice relaxation experiments where $T_{1}{ }^{-1}$ is found to be temperature independent for the 2-1-4 materials.

As the doping is increased to $y=0.14$ for $\mathrm{Sr}$, the material becomes superconducting at $33 \mathrm{~K}$. The spin fluctuations are now not found at the antiferromagnetic Bragg point, but rather peak on an incommensurate position. This position appears to lie on a square in the reciprocal lattice corresponding to the a-c plane, $b$ being the long axis. The size of the square is sensitive to the doping concentration $y$. At the present time, the nature of the magnetic excitations for the superconductor are not completely understood, and additional experiments are now in progress.

1. AT\&T Bell Laboratories, Murray Hill, N.J.

2. Institut Laue Langevin, Grenoble, France.

3. Sandoz Optoelectronics, Switzerland.

4. Los Alamos National Laboratory, Los Alamos, N. Mex.

\section{OBSERVATION OF PHONON SOFTENING AT THE SUPERCONDUCTING TRANSITION IN Bi $\mathrm{Sr}_{2} \mathrm{CaCu}_{2} \mathrm{O}_{6}{ }^{1}$}
H. A. Mook, M. Mostollet, I. A. Haroey, N. W. Hill, ${ }^{3}$ B. C. Chakoumakos, and B. C. Sales

Neutron resonant absorption spectroscopy has been used to determine the average kinetic 
encrgy $<E>$ of the $\mathrm{Cu}$ aloms for the directions along and perpendicular to the $c$ axis for the high- $\mathrm{T}_{6}$ superconductor $\mathrm{Bi}_{2} \mathrm{~S}_{2} \mathrm{CaCu}_{2} \mathrm{O}_{8}$. The measurements were made at the Oak Ridge Electron Linear Accelerator and consist of highresolution studies of the shape of the neutron absorption resonances of the $\mathrm{Cu}$ atoms in the superconductor. Most of our results were based on data taken for the $230 \mathrm{eV}$ resonance in ${ }^{63} \mathrm{Cu}$, although other resonances were studied as well. $\langle E\rangle$ is given by an integral of the phonon density of states weighted by the phonon energy so the measurement is most sensitive to the higher energy phonons.

The sample was made by applying high pressure to quantities of small, single-crystal plates of the superconductor, resulting in a sample with one direction well aligned along the $c$ axis. The superconducting transition temperature was determined to be about $83 \mathrm{~K}$. Measurements were made at a series of temperatures, with the sample $c$ axis both along and perpendicular to the neutron beam. For the case where $c$ was along the beam, c-axis motions were determined, and $\langle E\rangle$ showed a rather normal temperature dependence. $\langle E\rangle$ was about equal to the temperature at elevated temperatures and was given by the zero-point motion at low temperatures. However, $\langle E\rangle$ for the directions perpendicular to the $c$ axis showed an unusual behavior. In this case, $\langle E\rangle$ is determined by motions within the $\mathrm{Cu} \cdot \mathrm{O}$ planes, and a noticeable decrease in $\langle E\rangle$ was found at the superconducting transition as shown in Fig. 2.8. The dashed line is from a

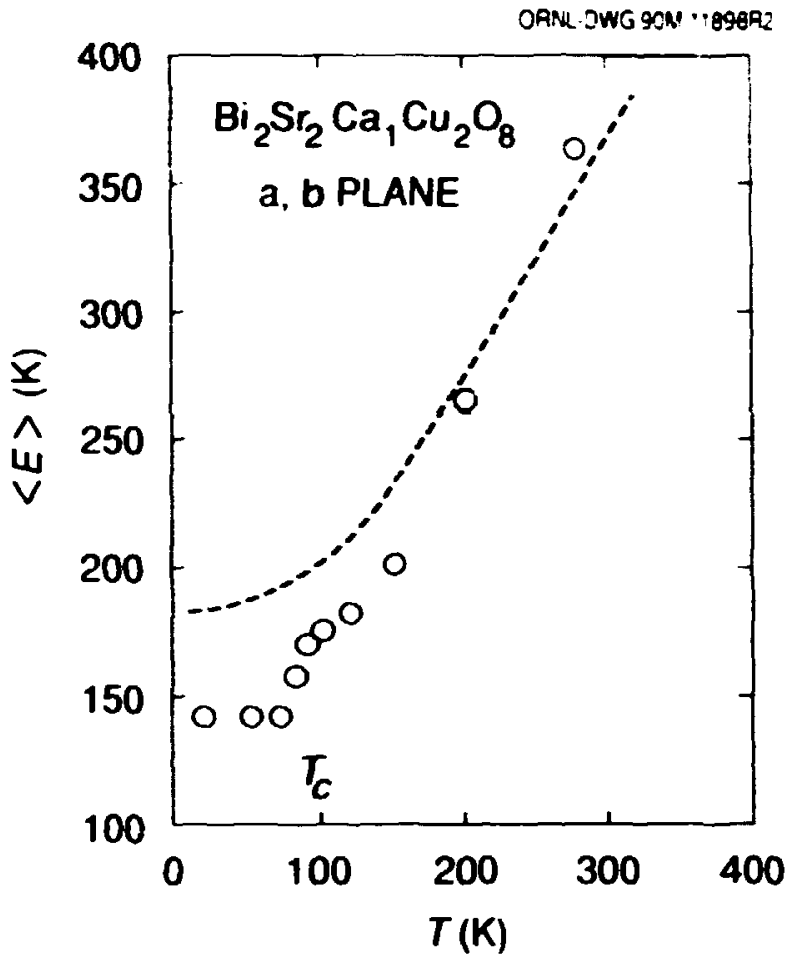

Fig. 2.8. $<E>$ for the $a, b$ plane as a function of temperature.

calculation for $\mathrm{La}_{2} \mathrm{CuO}_{4}$ which shows the normally expected behavior. This is the first observation of phonon softening at the superconducting transition in a high- $T_{c}$ material and demonstrates that certain of the phonons are coupled closely to the superconductivity.

1. Summary of paper: Phys. Rev. Lelf. 65, 2712 (1990).

2. Engineering Physics and Mathemalics Division, ORNL.

3. Instrumentation and Controls Division, ORNL. 


\section{MAGNETIC EXCTTATIONS IN THULUMI}

\section{J. A. Fernandez-Baca, R. M. Nicklow, Z. Tun, ${ }^{2}$ and I. I. Rhyne ${ }^{3}$}

A polarized neutron inelastic scattering experiment with full polarization analysis has been performed in order to confirm our recent report' that the excitation spectrum of $\mathrm{Tm}$, in its low-temperature ferrimagnetic-antiphasedomain phase, consists of one magnon mode with limited dispersion (8.3-9.6 meV) and lower energy excitations originating from magnetovibrational scattering from the TA phonon. The experiment was performed at the NRU reactor at Chalk River Nuclear Laboratories. A polarizing magnetic field was applied at the sample position along its $c$ axis, and the measurements were performed at the constant wave vector $x=(1,1,0)$. In this geometry the spin-flip (SF) scattering consists of onehalf of the transverse magnetic cross section, while the nonspin-flip (NSF) scattering contains the longitudinal and nonmagnetic processes. 5 The results of the measurements are shown in Fig. 2.9, where the open and closed circles denote the observed NSF and SF intensities, respectively. It is clear that the two excitations shown in this figure are of different characters. The lower energy excitation ( $E=3.3 \mathrm{meV}$ ) is NSF, while the highest energy excitation ( $E=8.4 \mathrm{meV}$ ) is SF. It is therefore concluded that this experiment confirms our previous report that the higher energy excitation is a magnon (transverse excitation), while the lower energy excitation is

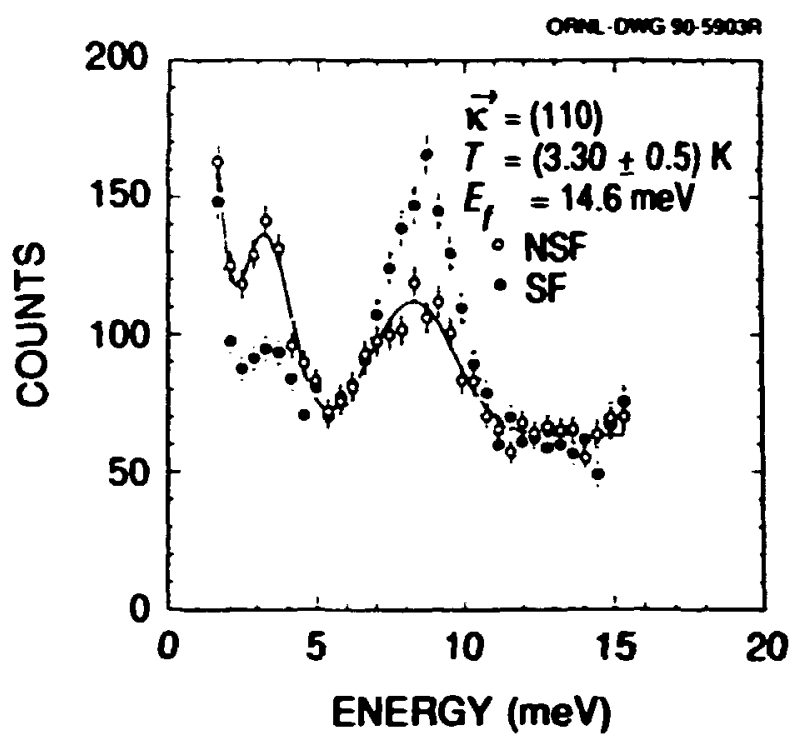

Fig. 2.9. Thulium constant- $x \operatorname{scan}$ at $k=$ $(1,1,0)$ showing the neutron polarization dependence of the scattering. The open circles correspond to NSF scattering, while the full cirdes correspond to SF scattering. The lower energy excitation is NSF, while the higher encrgy excitation is SF.

magnetovibrational scattering (longitudinal excitation).

1. Summary of paper: Physical Review B (in press).

2. Chalk River Nuclear Laboratories, Chalk River, Ontario, Canada.

3. National Institute of Standards and Technology, Gaithersburg, Md.

4. J. A. Fernandez-Baca, R. M. Nicklow, and J. J. Rhyne, J. Appl. Phys. 67, 5283 (1990).

5. R. M. Moon, T. Riste, and W. C. Kochler, Phys. Reo. B 181, 920 (1969).

\section{MAGNETIC CRITCAL SCATTERING FROM THULUM}

M. Hagen, ${ }^{1}$ H. R. Child,

J. A. Fernandez-Bace, and J. L. Zarestky ${ }^{2}$

The magnetic critical phenomena associated with the 57-K paramagnetic-to- 
aniifertumagñelic phase transition in tep thulium metal has been examined. At temperatures below $T_{N}=57 \mathrm{~K}$ but above $32 \mathrm{~K}$, thulium has a magnetic structure in which the magnetic moments are collinear with the hexagonal $c$ axis and have a sinusoidal variation in magnitude along the $c$ axis. $^{3}$ The modulation wave vector corresponding to this variation is $(0,0, \tau)$ with $\tau=0.285$. As a consequence, this phase transition is expected to belong to a universality class governed by the parameters $d=3$ and $n=2$, where is the spatial dimensionality and $n$ is the number of independent order parameters. Although thulium is uniaxial and there is only one spin degree of freedom, $n$ takes a value of 2 because the star of the modulation wave vector has two components. This universality class corresponds to that associated with the $d=3 X-Y$ model, which has the critical exponents $\beta=0.35, v=0.67$, and $\gamma=1.32 .4$ This transition in thulium is isomorphic with that in erbium at $85 \mathrm{~K}$. In a recent measurement, du Plessis et al.5 have reported an exponent $\beta=0.39$ for this transition in erbium. This value is significantly larget than the $d=3 X-Y$ moded value.

Measurements on a single crystal of thulium of dimensions $18 \times 10 \times 2.5 \mathrm{~mm}$ which is, to our knowledge, the best-quality crystal available in the roold tare been made. This is the same crystal that was used by Fernandez-Baca et al. 6 in their spin-wave measurements. For temperatures below $T_{N}$, the Bragg peak intensity at $(1,1, \bar{\tau})$ was measured as a function of temperature to extract a value for the $\beta$ exponent, while for temperatures $T>T_{N}$, the lineshape and intensity of the critical scattering around $(1,1, \bar{\tau})$ were measured as a function of temperature 20 obtain values for the exponents $v$ and $\mathrm{r}$.

Preliminary analysis of the Bragg intensity has resulted in an exponent value of $\beta=0.36$. However, the potentially large effects of extinction need to be considered carefully before this value can be finally accepted. Analysis of the critical scattering line shape above $T_{N}$ is still in progress.

1. University of Keele, Keele, Staffordshire, United Kingdom.

2. Ames Laboratory, Ames, lowa.

3. W. C. Koehler et al., Phys. Reo. 126, 1672 (1962).

4. Magnetic Critical Scallering by M. F. Collins, Oxford University Press (1989).

5. P. de V. du Plessis et al., J. Phys. France C8, 35.3 (1988).

6. j. A. Fernandez-Baca and R. M. Nicklow, J. Appl. Phys. 67, 5283 (1989). 


\section{SYNTHESIS AND PHYSICAL PROPERTIES OF MATERIALS}

Significant advances in the related fields of solid state physics and materials science are often predicated on the synthesis and subsequent physical characterization of new and novel materials. The research effort in the Solid State Division has a strong tradition of emphasizing materials synthesis activities on a broad scale ranging from metals and alloys to insulating materials, superconductors, glasses, and solid electrolytes. Materials synthesis and characterization activities are carefully coordinated with other research activities in the division and are designed to address either outstanding basic scientific questions or important energy-related technical issues.

This chapter is divided into three sections with the first section devoted to descriptions of the major portion of the division's research effort in the relatively new field of high-temperature oxide superconductors. Additional specialized high- $T_{c}$ research activities are also described in other chapters in this report. The Solid State Division's superconductivity research program encompasses investigations of single-crystal, bulk polycrystalline, and thinfilm materials as well as superlattice structures. The research effort includes transport and magnetic characterization studies in addition to investigations of flux pinning and critical currents in the yttrium-, bismuth-, and thalliumbased superconducting oxides.

The second section describes the division's efforts in the emerging field of the synthesis and characterization of thin-film ceramics and macromolecular thin-film composites. This research effort addresses both basic scientific issues and technologically important problems associated with the development of a new type of thin-film microbattery for integrated circuits and other applications. Additionally, this work has the potential for developing a new class of advanced materials that would combine the characteristics of both organic and inorganic substances.

The third section describes investigations in the areas of optical, magnetic, and physical characterization of materials that are synthesized primarily within the division. This work includes new investigations of the glass and amorphous states of matter through the application of high performance liquid chromatography, novel approaches to the formation and characterization of textured nanophase ceramics, and a pioneering approach to the study of solidification phenomena and microstructural formation in welds. 


\section{SUPERCONDUCTORS}

ORIENTATION-DEPENDENT CRTICAL CURRENTS IN YBa2 $\mathrm{Cu}_{3} \mathrm{O}_{7-x}$ EPITAXIAL THIN FILMS: EVIDENCE FOR INIRINSIC FLUX PINNING ${ }^{1}$

D. K. Christen, C. E. Klabunde, R. Feenstra, D. H. Lowndes, D. P. Norton, I. D. Budai, H. R. Kerchner, J. R. Thompson, 2 S. Zhu, ${ }^{3}$ and A. D. Marwick4

The basal-plane transport critical current density $I_{c}$ of high- $T_{c}$ oxide epitaxial thin films depends sensitively on the orientation of the crystal with respect to the applied magnetic field $H$. In particular, $l_{c}$ is sharply peaked and greatly enhanced when $H$ is precisely paralled to the copper-oxygen planes. Experiments on a series of epitaxial single-layer $\mathrm{YBa}_{2} \mathrm{Cu}_{3} \mathrm{O}_{7-x}$ and superconductor-insulator multilayer thin films of $\mathrm{YBa}_{2} \mathrm{Cu}_{3} \mathrm{O}_{7-x} / \mathrm{PrBa}_{2} \mathrm{Cu}_{3} \mathrm{O}_{7-x}$ provide clear evidence that the enhancement is a bulk rather than a surface or thin-sample phenomenon. Measurements of the field, temperature, and orientation dependence indicate that the effect exceeds that expected from the material anisotropy alone and point to a highly anisotropic-pinning microstructure. These results have been compared with a model of "intrinsic flux pinning" by the layered crystal structure.

The large anisotropy in $I_{c}$ is illustrated in Fig. 3.1, which shows the normalized critical current density $I_{c}(\mathrm{H}) / I_{c}(0)$ at $77 \mathrm{~K}$ for eight different epitaxial c-oriented films. For Hlab and in fields $108 \mathrm{~T}$, the data can be represented as a

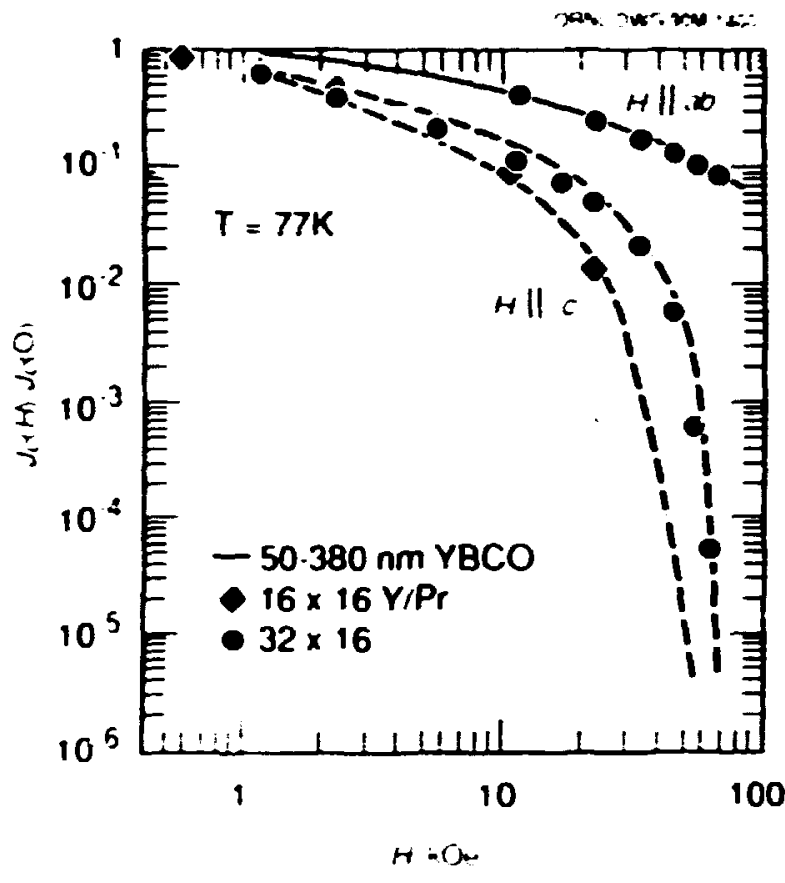

Fig. 3.1. The normalized critical current densities $I_{c}(H) / I_{c}(0)$ at $77 \mathrm{~K}$ for a series of single-layer epitaxial films with thicknesses indicated and for two multilayer superlattices. The data are shown for the two high-symmetry field orientations, $H \mid c$ and $H \mid a b$. In all cases, the zero field values were in the range $2-5 \times 10^{6} \mathrm{~A} / \mathrm{cm}^{2}$.

nearly universal curve, indicative of a common, strong flux-pinning mechanism for this orienlation. Proposed intrinsic flux-pinning mechanisms predict weak field dependences and large values of $J_{c}$ that are consistent with these obscrvations.

In contrast with $H \mid c$, the observed $I_{c}$ decreased abruptly for fields greater than $2-T$ and displayed orders-of-magnitude variation from sampic to sample, as indicated in Fig. 3.1 by the shaded region. The rapid decay of $I_{e}$ has been associated with flux lattice melting, 
thermally activated flux motion, or a vortex glass transition. These large, sampledependent variations in $l_{c}$ for $H / c$ are not directly correlated with the sample thickness. and are probably related to differences in the flux-pinning defect structure which results during the film growth process.

1. Summary of papers: Physica C 162-164, 653 (1989) and Proceedings of the Fourth Annual Conference on Superconductivity and Applications, Buffalo, N.Y. (in press).

2. Adjunct research and development participant from The University of Tennessee, Knoxville, Tenn.

3. Graduate student from The University of Tennessee, Knoxville, Tenn.

3. IBM Thomas J. Watson Research Center, Yorktown Heights, N.Y.

\section{DEFECT INDEPENDENT FLUX CREEP IN AS-GROWN AND PROTON-IRRADIATED SINGLE-CRYSTAL YBa2 $\mathrm{Cu}_{3} \mathrm{O}_{7}^{\prime}$}

J. R. Thompson, ${ }^{2}$ D. K. Christen, Yangren Sun, ${ }^{3}$

L. Civale, A. D. Merwick, M. W. McElfresh,"

T. K. Worthington, A. P. Malozemoff, and F. H. Holtzbergs

Flux pinning has been studied in single crys tals of the high- $T_{c}$ superconductor $\mathrm{YBa}_{2} \mathrm{Cu}_{3} \mathrm{O}_{7}$, both as-grown and irradiated with 3-MeV protons. The critical current density $I_{c}$, initially relatively low, increased substantially after irradiation with $10^{16}$ protons $/ \mathrm{cm}^{2}$ and reached $0.37 \mathrm{MA} / \mathrm{cm}^{2}$ at $75 \mathrm{~K}$ in a $1-\mathrm{T}$ magnetic fieldnearly a factor of 100 increase. The $I_{c}$ varied approximately exponentially with temperature $T$ for $T<T_{c} / 2$.

Flux-creep studies consisted of measurements of the magnelization $M(H, T, \ell)$ vs time $t$, for fixed $T$ and fietd $H=1 \mathrm{~T}$ parallet to the c-axis. Conventional flux creep theory provides the model relation $M(t)=$ $M_{0}\left(l-\left(k_{B} T / U_{e f f}\right) \ln \left(t / t_{0}\right)\right)$. We found $M / M_{0} \propto$ $\ln (t)$ and, thereby, obtained values for the effective vortex pinning energy $U_{\text {\&f }}$ Figure 3.2 shows $\left.d M / M_{\sigma} d \mid \ln (t)\right]=k_{B} T / U_{q f}$ as a function of $T$. The effective pinning potential was changed very little by proton irradiation, while $l_{c}$ increased substantially. This suggests an increase in density of pinning centers, all of similar strength.

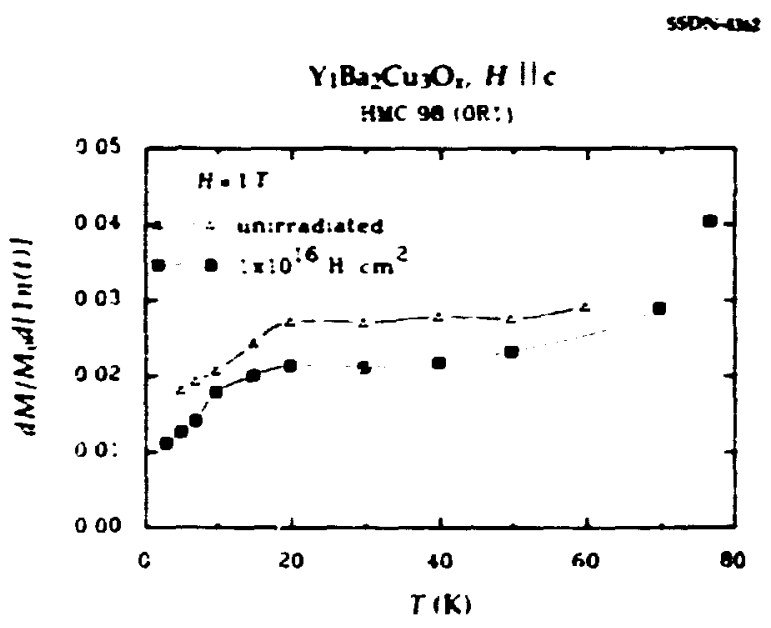

Fig. 3.2. Normalized flux creep rate vs temperature $T$ for single-crystal $\mathrm{Y}_{1} \mathrm{Ba}_{2} \mathrm{Cu}_{3} \mathrm{O}_{x}$ as-grown and proton-irradiated.

These results corroborate $1 \mathrm{MHz}$ ac studies of the irreversibility line, which showed that the boundary between reversible and irreversible behavior was not affected by proton irradiation.

1. Summary of paper: Phys. Rev. Iell. 65, 1164 (1990)). 
2. Adjunct research and development participant from The University of Tennesser, Knoxvilk, Tenn.

3. Graduate student from The University of Tennesser, Knoxville, Tenn.

4. IBM Thumas J. Watson Rescarch Center, Yorktown Heights, N.Y.

\section{MAGNETIC FLUX PINNING IN $\mathrm{YB}_{2} \mathrm{Cu}_{3} \mathrm{O}_{-2}$ FILMS}

\section{H. R. Ketchnet, R. Feenstra. I. O. Thomson,?}

I. R. Thompson, ${ }^{3}$ D. K. Christen,

\section{S. T. Sekula, and L. A. Boatner}

The magnetic hysteresis was studied for $\mathrm{YBa}_{2} \mathrm{Cu}_{3} \mathrm{O}_{7-x}$ films of different epitaxial orientations on single-crystal $\mathrm{SrTiO}_{3}$ and $\mathrm{KTaO}_{3}$ substrates. Deposition by coevaporation of $Y$, $\mathrm{BaF}_{2}$, and $\mathrm{Cu}$ was followed by annealing in flowing, wet oxygen at $800-850^{\circ} \mathrm{C}$. Magnetic hysteresis was measured by using a vibrating sample magnetometer (VSM) and a mutual inductance bridge (MIB).

The VSM provided a continuous reading of a film's magnetic moment, while the applied magnetic field $B$ was slowly swept from $B=0$ to $8 \mathrm{~T}$ and back to zero. The critical current density $l_{c}\left(\mathrm{~A} / \mathrm{cm}^{2}\right)$ was then deduced from the difference $\Delta m$ between the magnetic moments $\left(6-\mathrm{cm}^{3}\right)$, while $B$ was increasing and decreasing, using $l_{c}=15 \Delta \mathrm{m} / \pi a^{3} t$. Here $a$ is the substrate radius, and $t$ is the measured film thicknass, both in $\mathrm{cm}$.

The MIB yields tire ac magnetic moment $m_{a c}$ of the film induced by an audio-frequency, applied ac field $B_{\text {ac. }}$. parallel to a much larger applied dc field $B$. When $B_{\alpha C}$ is large enough, $l_{c}$ nows uniformly throughout the film, and $m_{a c}$ is saturated at the value $\Delta m$.
Measurements of $\Delta m$ made by the two techniques were compared for a variety of films. The values of $J_{c}$ deduced from $\Delta m$ were also compared with four-terminal transport-current results on similar films. Figure 3.3 shows a typical set of data. The MIB technique systemati-

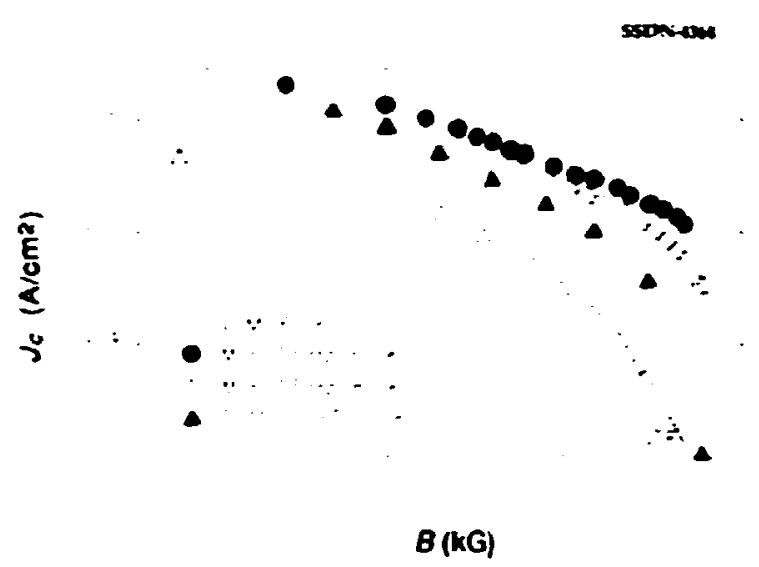

Fig. 3.3. Critical current density /c v s applied magnetic field $B$.

cally gave higher values due to faster magnetic field changes and the resulting larger electric ficlds. Films grown epitaxially on (100)-oriented $\mathrm{SrTiO}_{3}$ substrates apparently had the highest fc's due to their single-crystal qualities.

1. Summary of paper: p. 903 in High-Temperature Superconductors: Fundamental Properties and Novel Materials Proceising, ed. by David K. Christen, Jagdish Narayan, and Lynn F. Schneemeyer, Matcrials Rescarch Society, Pittsburgh, Pa., 1990.

2. Consultant from The University of Tennessee, Knoxville, Tenn.

3. Adjunct research and development participant from The University of Tennesser, Knoxville, Tenn.

4. Deceased. 


\section{DYNAMIC MAGNETC HYSTERESIS \\ OF YBaz $\mathrm{Cu}_{3} \mathrm{O}_{7-x}$ THIN FILMS'}

\section{H. R. Kerchner, J. O. Thomson,? \\ R. Feenstra, and D. K. Christen}

Magnetic hysteresis is commonly used as a means of measuring current-carrying capacity of superconducting materials. The deduced value of the critical current density $I_{c}$ depends on the nature of measurement and is sensitive to the electric field present. The $I_{c}$ deduced fron: a dc-magnetic moment, measured by a vibrating sample magnetometer when the applied field is slowly swept, is systematically smaller than the value deduced from audio-frequency ac magnetometry. In addition, values can be separately deduced from the two phases of a lock-in amplifier when the applied ac field has sufficient amplitude to change the $J_{c}$ direction throughout the sample within one-quarter cycle. To the extent that the electric-field dependence can be approximated by the simple, linear flux-flow conductivity relation, $J(E)=$ $I_{c}+\sigma_{f} E$, the observed moment change $\Delta m$ associated with the change in current direction is

$$
\begin{aligned}
& \Delta m_{i}=\pi a^{3} t l_{c} / 15 \\
& \Delta m_{r}=\pi a^{3} t J_{c} / 15+\pi^{2} a^{4} t h_{0} \sigma_{f} / 10,
\end{aligned}
$$

where the subscripts $i$ and $r$ refer to the inductive and resistive phases of the applied ac field of amplitude $\boldsymbol{h}_{0}$.

The theoretical arguments given above were used to interpret data of a high-quality thin film deposited by coevaporation of $Y$, $\mathrm{BaF}_{2}$ and $\mathrm{Cu}$, followed by annealing in flowing. wet oxygen at $800-850^{\circ} \mathrm{C}$. Shown in Fig. 3.4 is a collection of interpreted data taken at different frequencies. Although no discrepancy in the deduced critical current density is visible, the apparent flux-flow conductivities differ substantially. The observations strongly support the thermally activated flux-flow picture, but they also indicate a need for extensive, complex theoretical analysis in order to obtain satisfying, quantitative agreements.

1. Summary of paper to be published.

2. Consultant from The University of Tennessee, Knoxville, Tenn.

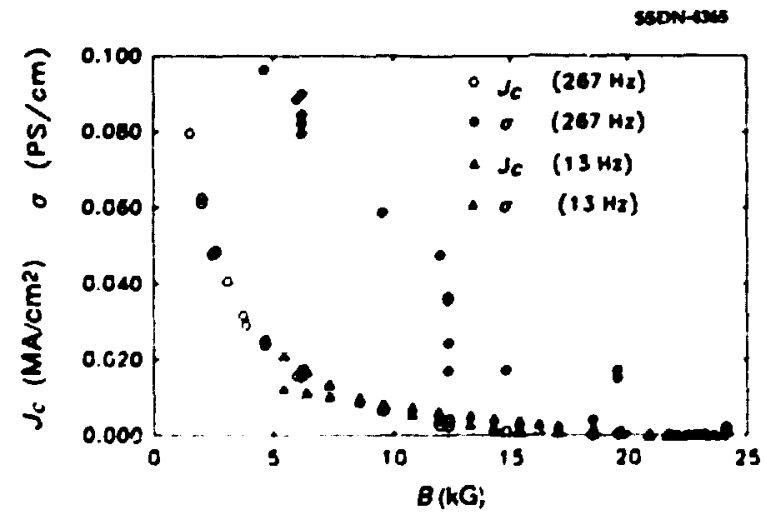

Fig. 3.4. Pot of the critical current density $l_{c}$ and the flux-flow conductivity of of a $\mathrm{YBa}_{2} \mathrm{Cu}_{3} \mathrm{O}_{7-x}$ film as deduced at the indicated frequencies. 


\section{SUPERCONDUCTIVITY IN NONSYMMETRIC EPITAXAL $\mathrm{YBa}_{2} \mathrm{Cu}_{3} \mathrm{O}_{7-2} \mathrm{PrB}_{2} \mathrm{Cu}_{3} \mathrm{O}_{7-x}$ SUPERLATTICES: THE SUPERCONDUCTING BEHAVIOR OF $\mathrm{C} n-0$ BILAYERS ${ }^{\prime}$}

Douglas H. Lowndes, David P. Norton, und J. D. Budai

The layered structure of high-temperature superconductors (HTSc), particularly the existence of closely spaced pairs of $\mathrm{Cu}-\mathrm{O}$ planes, is widely considered to be responsible for both their remarkable superconducting properties and their (as yet unidentified) superconducting pairing mechanism. However, until recently it was unresolved whether interactions are necessary between the $\mathrm{Cu}-\mathrm{O}$ bilayers in adjacent unit cells for superconductivity to occur or how $T_{C}$ would vary with the number of interacting unit cells in a YBCO layer.

To address these questions, the pulsedlaser-ablation method has been used to grow epitaxial, nonsymmetric $M \times N$ superlattices in which $\mathrm{YBa}_{2} \mathrm{Cu}_{3} \mathrm{O}_{7-x}(\mathrm{YBCO})$ layers with $\mathrm{M}=1$, $2,3,4$, or $8 c$-axis unit cell thicknesses are separated by insulating $\mathrm{PrBa}_{2} \mathrm{Cu}_{3} \mathrm{O}_{7-x}$ (PBCO) layers $N$ unit cells thick $(N=1-\sim 16) .^{1}$ By varying $N$ and $M$, the dependence of $T_{c}$ has been systematically mapped out on YBCO layer thickness and interlayer separation in a set of nearly lattice-matched periodic epitaxial structures that contain up 1060 layers 130 periods) of alternating composition.

Figure 3.5 demonstrates that increasing the thickness $d_{p}$, of the P'BCO layers results in an initial decrease of the zero-resistance Iransjtion temperature $T_{\text {eo }}$ followed by saturation of

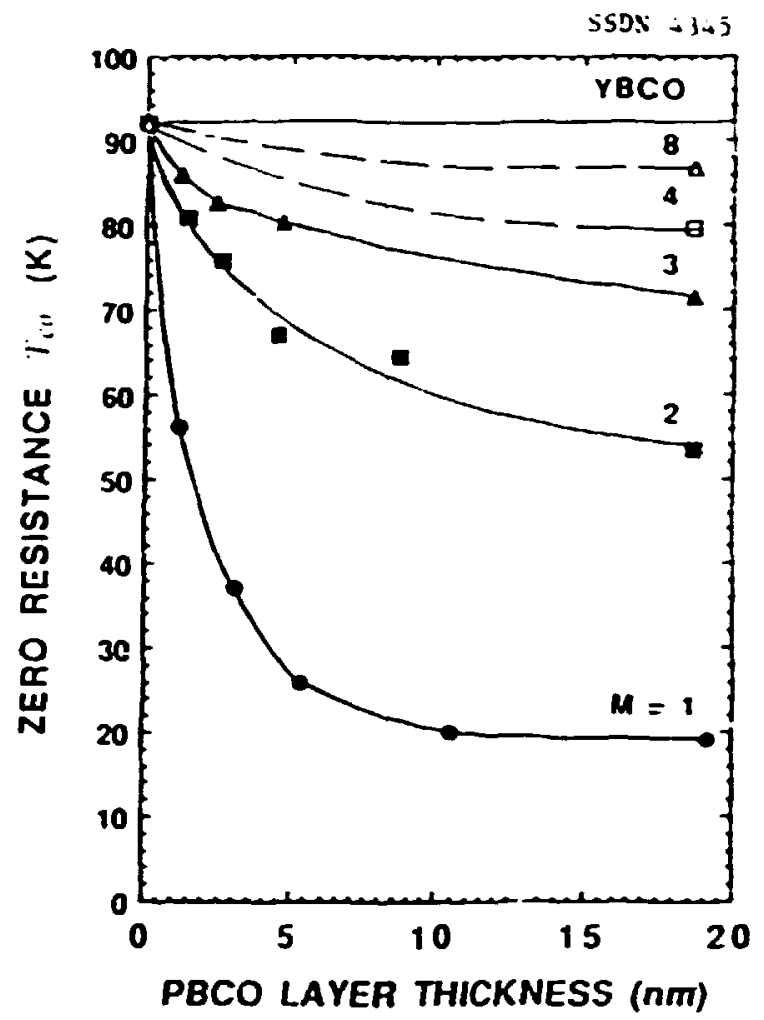

Fig. 3.5. Zero-resistance superconducting transition temperature $T_{\text {co }}(K)$ vs PBCO layer thickness fer nonsymmetric YBCO/PBCO superlattices containing 1- $(O), 2-(B), 3-(\Delta)$, 4- (Q), and 8- ( $\Delta)$ cell-thick YBCO layers and for pure YBCO (solid line).

$T_{\text {co at a nonzero value for large PBCO layer }}$ thicknesses, for all of the $1 \times N, 2 \times N$, and $3 \times N$ superlattices. Single-cell-thick YBCO layers, in particular, are superconducting in a PBCO matrix. It is noted that the $T_{c o}$ values for $M \times N$ superlattices are very different f:om those for alloys having the same average composition. For large $d_{p_{r}}, \tau_{\text {co }}$ saturates for all of the superlattices, while $T_{c o}$ for Pr-rich alloys goes to zero.

Figure 3.5 shows that the superlattice $i_{\text {co }}$ depends strongly on the number of adjacent 
YBCO-containing unit cells in a layer, suggesting either that coupling is required between YBCO cells in ordet to increase their $T_{\infty}$ or that YBCO cells immediately adjacent to PBCO have their $T_{\text {co }}$ depressed by PBCO. Recent model calculations suggest that the latter effect dominates and that the depression of $T_{\infty}$ for isolated, very thin YBCO layers is due to hole filling in the superconducting layers by electrons transferred from the PBCO layers, ${ }^{3}$ modified by effects of reduced dimensionality.4

1. Summary of paper: Phys. Reo. Lett.65, 1160 (1990).

2. ORNL Eugene P. Wigner Fellow.

3. R. F. Wood, Physical Review Letters (submitted for publication).

4. M. Rasolt, Physical Review Letters (submitted for publication).

\section{SUPERCONDUCTING TRANSITIONS OF $\mathrm{YBa}_{2} \mathrm{Cu}_{3} \mathrm{O}_{7-2} / \mathrm{PrBa}_{2} \mathrm{Cu}_{3} \mathrm{O}_{7-y}$ SUPERLATTICE THIN FILMS ${ }^{1}$}

\section{H. R. Kerchner, J. O. Thomson, ${ }^{2}$ \\ I.R. Thompson, ${ }^{3}$ I. Ossandon, \\ D. Norton, and D. H. Lowndes}

Epitaxial thin films were fabricated by the pulsed-laser deposition method with superlattice layers of $\mathrm{YBa}_{2} \mathrm{Cu}_{3} \mathrm{O}_{7-x}$ and $\mathrm{PrBa}_{2} \mathrm{Cu}_{3} \mathrm{O}_{7-y} .^{5}$ Superconductive properties were investigated by magnetic measurements. Although the electrical resistances of such films showed broad superconducting transitions, the magnetic transitions were quite sharp, very near the temperatures where four-terminal resistances reached zero. The magnetic observations indicate that the films are homoge- neous and that macroscopically wide layers of $\mathrm{YBa}_{2} \mathrm{Cu}_{3} \mathrm{O}_{7-x}$ transform into the superconductive state uniformly at temperatures determined by the concentrations of holes. The broad resistive transitions may be due to microscopic fluctuations that are not visible magnetically.

When the applied magnetic fields are sufficiently large, the magnetic transitions also broaden. Within such broad transitions, the films clearly are in the critical state-not the ideal field-exclusion state. Two examples of such broad magnetic transitions are shown in Fig. 3.6. The critical current density $I_{c}$ of the $2 \times 1$ sample (i.e., two lattice cells of $\mathrm{YBa}_{2} \mathrm{Cu}_{3} \mathrm{O}_{7-x}$ separared by one cell of $\left.\operatorname{PrBa}_{2} \mathrm{C}_{3} \mathrm{O}_{7-y}\right)$ approached the magnitude of pure $\mathrm{YBa}_{2} \mathrm{Cu}_{3} \mathrm{O}_{7-3}$ films. In contrast, even the earth's field eliminated shielding current

\section{ZERO-FIELD HYSTERESIS}

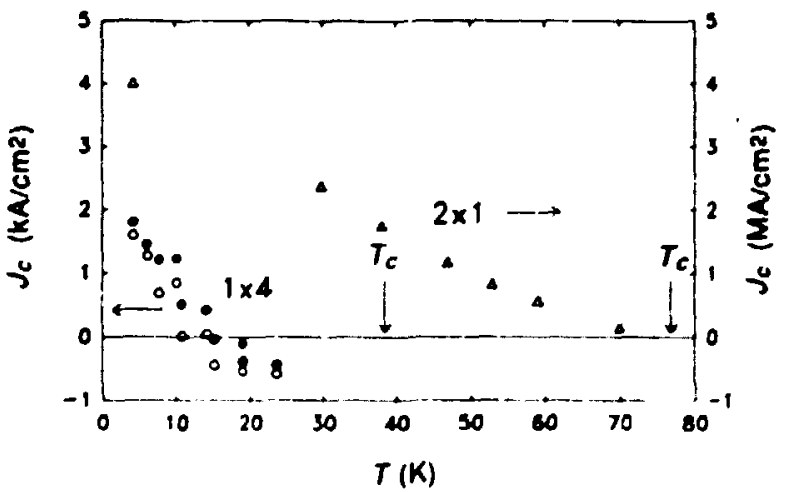

Fig. 3.6. Critical current densities for two films deduced from ac magnetometry results in broad magnetic transitions plotted as functions of temperature. The ac applied ficld was $40 \mathrm{C}$ for the stronger film, a sequence of two lattice cells of $\mathrm{YBa}_{2} \mathrm{Cu}_{3} \mathrm{O}_{7 \cdot x}$ scparated by one cell of $\mathrm{PrBa}_{2} \mathrm{Cu}_{3} \mathrm{O}_{7-y}$. The field was $1 \mathrm{G}$ for the $1 \times 4$ film. 
within the $1 \times 4$ film. The apparent $/ c$ was reduced sharply as the superconductive layers were made thin, below three cells thick. The reason for this sharp reduction of current-carrying capacity may well be due to intrinsic barriers to current flow, or it may be associated with the decoupling of very thin superconductive layers.

1. Summary of paper to be published.

2. Consultant from The University of Tennessee, Knoxville, Tenn.

3. Adjunct research and development participant from The University of Tennessec, Knoxville, Tenn.

4. Graduate student from The University of Tennessce, Knoxville, Tenn.

5. Technique is described in Phys. Rev. Lett. 65, 1160 (1990).

\section{STABILITY OF $\mathrm{YBa}_{2} \mathrm{Cu}_{3} \mathrm{O}_{7-x} / \mathrm{PrBa}_{2} \mathrm{Cu}_{3} \mathrm{O}_{7-x}$ SUPERLATTICES: EFFECT OF ELEVATED TEMPERATURE, LOW.PRESSURE OXYGEN ANNEALING}

\section{H. Lowndes, D. P. Norton,} R. Feenstra, and I. D. Budai

The stability of $1 \times N$ and $2 \times N$ $Y B C O / P B C U$ superlattices was investigated by subjecting them to postannealing in lowpressure oxygen for periods of 50-185 $\mathrm{min}$ at temperatures up to $840^{\circ} \mathrm{C}$, more than $150^{\circ} \mathrm{C}$ higher than their original laser ablation growth temperature. In general, it was found that the only significant effect of low-pressure oxygen annealing at $\mathrm{T} \leq 800^{\circ} \mathrm{C}$ was to reduce slightly $(\sim 20 \%)$ the normal-state resistance, consistent with graill growth and/or an improvement in the continuity of the very thin
YBCO layers in these specimens. The zeroresistance transition temperature $T_{\text {co }}$ often increased by $-1-2 \mathrm{~K}$ after an initial postanneal, mainly as a result of removing the lowtemperature "tail" of the superconducting transition. Figure 3.7 shows results of a series of six postanneals of a $2 \times 8$ superlattice with varying temperatures, durations, and oxygen pressures
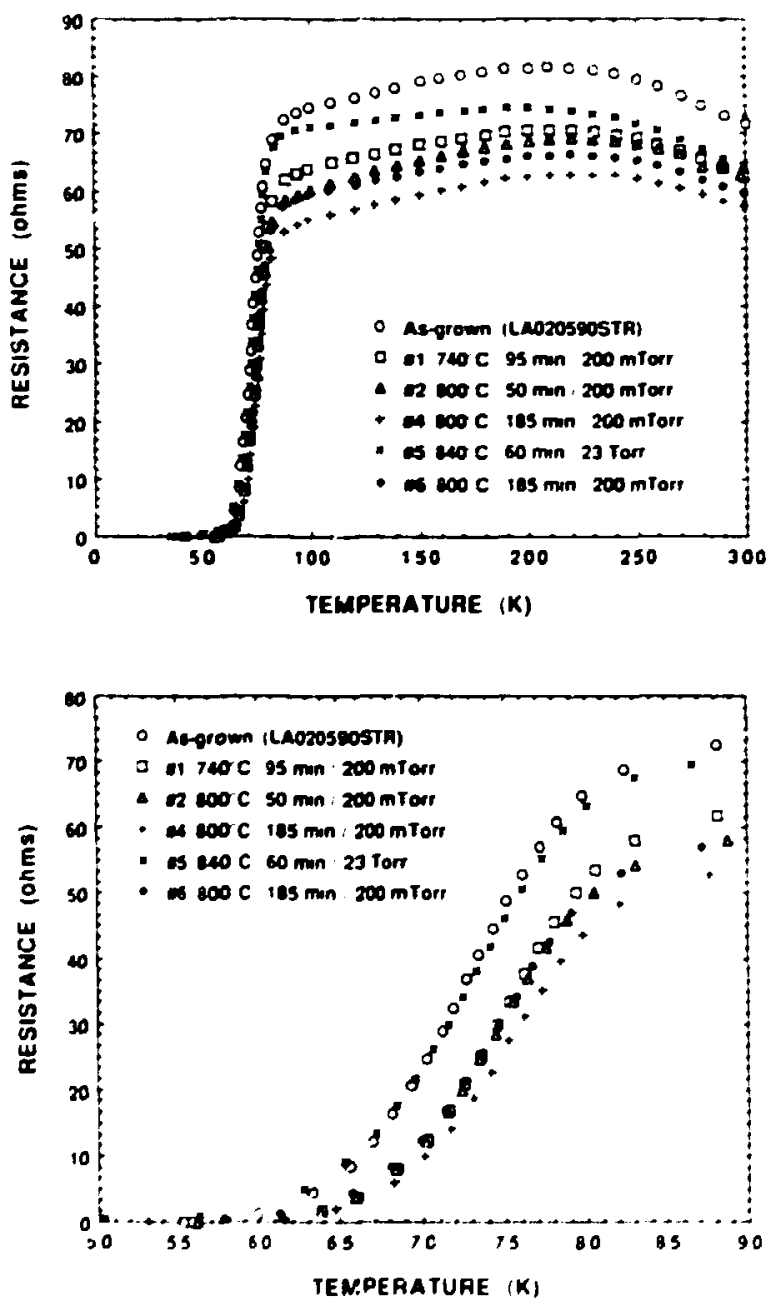

Fig. 3.7. Resistance vs temperature for a 30-period $2(\mathrm{YBCO}) \times 8(\mathrm{PBCO})$ superlattice, both as-grown and after low-pressure oxygen annealing at temperatures above the original growth temperature $\left.(\sim 670)^{\circ} \mathrm{C}\right)$. 
$p\left(\mathrm{O}_{2}\right)$. The first and second anneals at 740 and $800^{\circ} \mathrm{C}$ decreased the normal-state resistance below the as-grown value, while a third anneal at $800^{\circ} \mathrm{C}$ (not shown) produced essentially no change. After a fourth (much longer duration) anneal at $800^{\circ} \mathrm{C}$ resulted in another $R(T)$ decrease, the annealing temperature was increased to $840^{\circ} \mathrm{C}$, and the oxygen pressure was increased -100 -fold to 23 Torr for the fifth anneal. $R(T)$ then increased. Significantly though, when the low-p $\left(\mathrm{O}_{2}\right)$ conditions for anneal number four were used in the sixth anneal, $R(T)$ returned to nearly the same low value.

These results demonstrate that YBCO/PBCO superlattices are highly stable, and they strongly suggest that any diffusion during $-300^{\circ} \mathrm{C}$ annealing at low-oxygen pressure occurs along the $a-b$ planes, with very little diffusion along the $c$-axis direction of compositional modulation (i.e., the chemical superlattice modulation is preserved).

1. ORNL Eugene P. Wigner Fellow.

\section{ELECTRICAL TRAIJSPORT DISSIPATION EFFECTS IN EPITAXIAL $\mathrm{YBa}_{2} \mathrm{Cu}_{3} \mathrm{O}_{7-1} \mathrm{l}$}

D. K. Christen, C. E. Klabunde, R. Feenstra, D. H. Lowndes, D. Norton. H. R. Kerchner, I. R. Thompson, ${ }^{2}$ S. T. Sekula, ${ }^{3}$ J. D. Budai, L. A. Boatner, I. Narayan, and R. Singh 5

Superconductive electrical-transport propertics were investigated on a series of $\mathrm{YBa}_{2} \mathrm{Cu}_{3} \mathrm{O}_{7-x}$ thin films deposited onto single-crystal substrates by thermal coevaporation and laser ablation. For fully epitaxial, c-oriented films on (001) substrate surfaces, the magnetic field-dependent critical current density $I_{c}$ showed large enhancements for the film aligned with $\boldsymbol{H}$ parallel to the copper-oxygen planes and a precipitous decay of $J_{c}$ with increasing field for H/k. The dissipative resistivity at high temperatures and fields was thermally activated and could be described by

$$
p=\frac{E_{0}}{J_{\infty}} \exp \left[-\frac{U_{0}}{k T}\right]
$$

Here, $E_{o}$ and $I_{c o}$ are related to details of the flux-pinning system, and the simple, physical interpretation of $J_{c o}$ is that of the critical ct:srent density in the absence of thermally activated processes. The deduced flux-pinning energy barriers $U_{0}$ were observed to obey $U_{0}=$ $u_{\infty}\left(1-T / T_{c}\right)^{1.8} / H$.

Parameters determined from measurements of this dissipative bchavior at high ficlds can be used as a self-consistent test of the ther.nally activaied flux-creep model in the nondissipative regime at lower fields. From the flux-creep relationships, the "creeplimited" critical current is given by

$I_{c}=I_{\infty} \frac{k T}{U_{o}} \sinh ^{-1}\left[\frac{\left(E_{c} / E_{o}\right) \exp \left(U_{o} / k T\right)}{I_{c o}\left(k T / U_{o}\right)}\right]$

where $E_{c}$ is the electric field criterion for defining $/ c$. These results are shown in Fig. 3.8, where the solid curves, generated from Eq. (2), are compared to the data taken at several tem- 


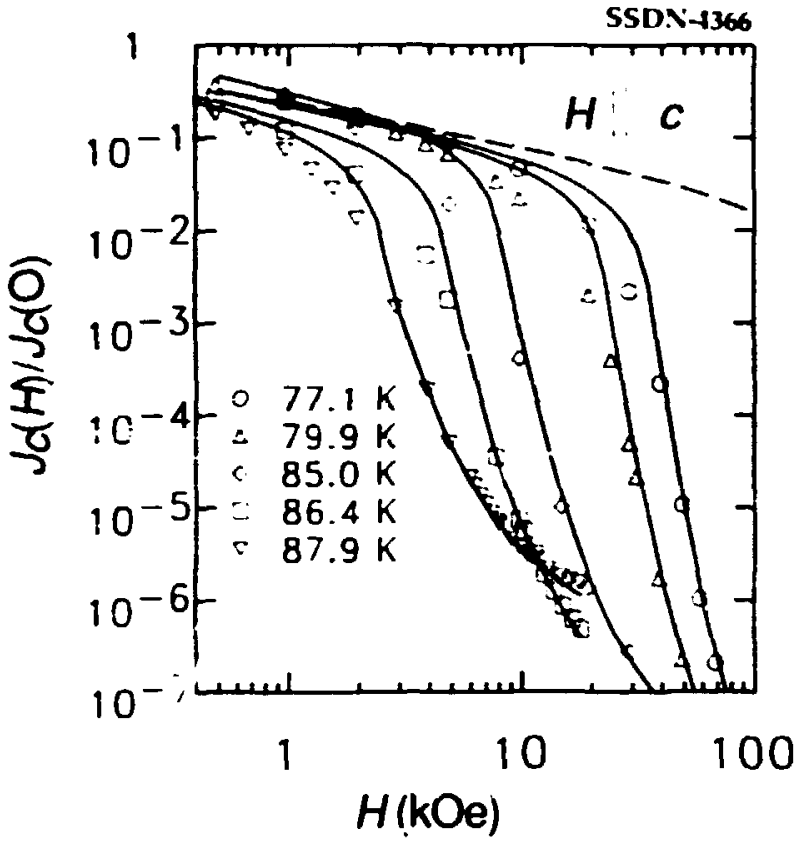

Fig. 3.8. The observed $l_{c}(B, T)$ for a c-oriented film on $\mathrm{LaAlO}_{3}$, compared to the predictions of Eqs. (1) and (2), shown as solid curves. Results are normalized to the experimental $I_{c}(0)$ at each temperature. The dashed curve is $I_{\infty}$ at $77 \mathrm{~K}$.

peratures where thermally activated processes are important. Overall, the agreement is gond-considering the crude assumptions and the fact that we have not included realistic effects such as a distribution in $U_{0}$ values or the role of flux lattice elasticity in the model for Io.

1. Summary of paper: Mat. Res. Soc. Sym. Proc. 169, 883 (1990).

2. Adjunct research and development participant from The University of Tennessee, Knoxville, Tenn.

3. Deceased.

4. Consultant from North Carolina Statc University, Raleigh, N.C. Present address:

National Science Foundation, Washington, D.C.

5. North Carolina State University, Ralcigh, N.C.

\section{EFFECT OF OXYGEN PRESSURE ON THE SYNTHESIS OF $\mathrm{YBa}_{2} \mathrm{Cu}_{3} \mathrm{O}_{7-x}$ THIN FILMS BY POSTDEPOSITION ANNEALING'}
R. Feenstra, T. B. Lindemer,
I.D. Budai, and M. D. Galloway

The effect of oxygen pressure on the synthesis of epitaxial $\mathrm{YBa}_{2} \mathrm{Cu}_{3} \mathrm{O}_{7-x}$ films on (100) $\mathrm{SrTiO}_{3}$ by postannealing of coevaporated $Y$, $\mathrm{BaF}_{2}$, and $\mathrm{Cu}$ amorphous precursor films was studied at oxygen partial pressures $\mathrm{PO}_{2}$. between 1.0 and $8.0 \times 10^{-5}$ atm and temperatures between 650 and $890^{\circ} \mathrm{C}$. The results show evidence of a strong dependency of growth properties on the oxygen pressure with enhanced c-oriented epitaxy, denser and more platelike surface morphologies, improved crystalline quality, and lower metallic resistivities at lower $\mathrm{pO}_{2}$ values.

By varying both oxygen pressure and temperature, films having comparable structural features wete produced at various $\left(T-\mathrm{PO}_{2}\right)$ combinations. These systematics are illustrated in the $\mathrm{PO}_{2}-1 / \mathrm{T}$ diagram of Fig. 3.9, where annealing conditions explored in this study have been plotted and connected to those leading to similar structural properties by straight lines. The epitaxial orientations corresponding to these lines progress from exclusively $c \perp$ films at the highest temperatures, to predominantly a 1 films at the lowest temperatures, but with film orientation and morphology constant over the range of $\mathrm{T}, \mathrm{PO}_{2}$ values on a given line. This remarkable correlation implies that the enhanced growth properties at lower oxygen pressures (and oxygen stoichiometry in 


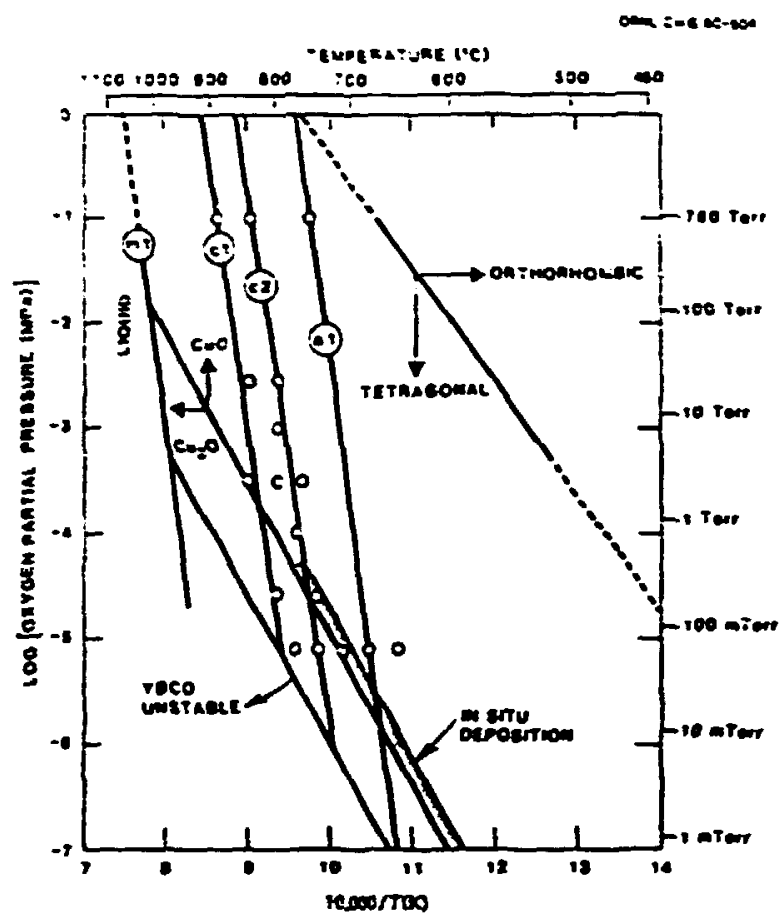

Fig. 3.9. Oxygen partial pressure vs temperature diagram showing annealing conditions explored in this study in relation to $\mathrm{YBa}_{2} \mathrm{Cu}_{3} \mathrm{O}_{7-x}$, stability line, melting line $\mathrm{m}$ !, tetragonal-to-orthorhombic transition line, and $\mathrm{CuO}-\mathrm{Cu}_{2} \mathrm{O}_{2}$ equilibrium/Hammond-Bormann line indicating conditions for optimal in situ film growth. ${ }^{3}$ Annealing conditions yiclding films of comparable epitaxial and structural properties are connected by straight lines denoted by $c 1, c 2$, and $a 1$, respectively. Predominantly $c$-oriented films were obtained betwcen $c 1$ and $c 2$ and mixed $c$ - and $a$-oriented films between $c 2$ and $a 1$.

$\mathrm{YBa}_{2} \mathrm{Cu}_{3} \mathrm{O}_{7-x}$ ) may compensate for lower annealing temperatures.

As a result of this compensation for pressures $\mathrm{PO}_{2}<0.5$ Torr, one may employ annealing temperatures which extend well into the range of subst ate temperatures $\left(700-750^{\circ} \mathrm{C}\right)$ currently employed for the in situ growth of high. quality $c \perp \mathrm{YBa}_{2} \mathrm{Cu}_{3} \mathrm{O}_{7-8}$ films. A practical implication of this result is that one may resort to the simpler deposition methods used for growing precursor films (with $\mathrm{BaF}_{2}$ ) rather than the more difficult to control in situ growth methods while still keeping the maximal processing temperature reasonably low.

1. Summary of paper to be published.

2. Chemical Technology Division, ORNL.

3. R. H. Hammond and R. Bormann, Physica C 162-164, 703 (1989).

\section{STATIC AND DYNAMIC MAGNETIZATION STUDIES OF $\mathrm{YBa}_{2} \mathrm{Cu}_{3} \mathrm{O}_{7}$ THIN FILMS'}

\section{S. T. Sekula, 2 R. Feenstra, J. R. Thompson, 3 D. K. Christen, H. R. Kerchner, L. A. Boatner, and J. Budai}

Magnetization measurements were carried out on a series of $\mathrm{YBa}_{2} \mathrm{Cu}_{3} \mathrm{O}_{7}$ thin films, using dc and ac magnetometry methods. The films were prepared by codeposition and postannealing techniques on (100) and (110) $\mathrm{SrTiO}_{3}$ and (100) $\mathrm{KTaO}_{3}$ substrates, using different annealing protocols to vary the epitaxy of the resulting films. Th.e irreversible magnetization, obtained with $H \perp$ to the film plane, and deduced magnetic critical current density $l_{c}$ were very sensitive to the type of epitaxial growth. As expected, Ic was largest for films with the $c$-axis $\perp$ to the film plane, intermediate for the case of mixed $c$-and $a$-axes, and relatively small for triaxially oriented films on (110) $\mathrm{SrTiO}_{3}$. Flux ereep effects were always evident as significant time dependences in the magnetization $M$. Cood agreement was found between 
the ac and swept ficld dc measurements of $I_{c}$. while "quasi-static" de measurements gave lower $/ c$ values due to flux creep. In studies of the time dependence of $M$, the standard proportionality $M \propto \ln (t)$ was observed, and this was used to estimate the effective flux-pinning potential in selected films.

1. Summary of paper: p. 385 in Adoances in Cryogenic Engineering: Materials, Vol. 36A, Plenum Publishing Company, New York, N.Y., 1990.

2. Deceased.

3. Adjunct research and development participant from The University of Tennessec, Knoxville, Tenn.

\section{EFFECT OF GRAIN BOUNDARIES ON CRITICAL CURRENT DENSTTY IN $\mathrm{YBa}_{2} \mathrm{Cu}_{3} \mathrm{O}_{7-x}$ FILMS ON POLYCRYSTALLINE ZIRCONIA ${ }^{\mathfrak{l}}$}

David P. Norton, 2 Douglas H. Lowndes, J. D. Budai, D. K. Christen, E. C. Jones, ${ }^{3}$ K. W. Lay, and I. E. Tkaczyk

In situ pulsed-laser ablation growth of $\mathrm{YBa}_{2} \mathrm{Cu}_{3} \mathrm{O}_{7 \cdot x}$ (YBCO) thin films on wellpolished but randomly oriented polycrystalline yttria-stabilized zirconia (poly-YSZ) substrates has bern demonstrated. The large anisotropy in growth kinetics for YBCO results in c-axis-perpendicular $(c 1)$ films having an $x$-ray diffraction rocking curve width of only $1^{\circ}$. but with randomly oriented in-plane alignment (i.e., no prefersed orientation of the $a$ and $b$ axes). Consequently, large-angle grain boundaries are present in these YBCO films.

For growth on poly-YSZ, it was found that substrate temperature and, secondarily, surface finish are quite important for obtaining a high critical current density Je, probably because of nonnegligible film/substrate interactions accompanied by diffusion along grain boundarics. Under optimum growth conditions (Fig. 3.10), $J_{c}(77 \mathrm{~K}, H=0)=11 \mathrm{kA} / \mathrm{cm}^{2}$ and $l_{c}(4.2 \mathrm{~K}, \mathrm{H}=0)=122 \mathrm{kA} / \mathrm{cm}^{2}$ were obtained. These $I_{c}$ values are among the highest reported for YBCO films on polycrystalline substrates. More importantly it was shown that these Ic values approach the "intrinsic" upper limit for YBCO films that contain large-angle grain boundaries. This conclusion is based on a comparison of our results with recent experiments by Dimos et al., 5 in which YBCO films were grown on bicrystal specimens containing pairs of grains with known misorientations. From this latter work there appears to be a limit for

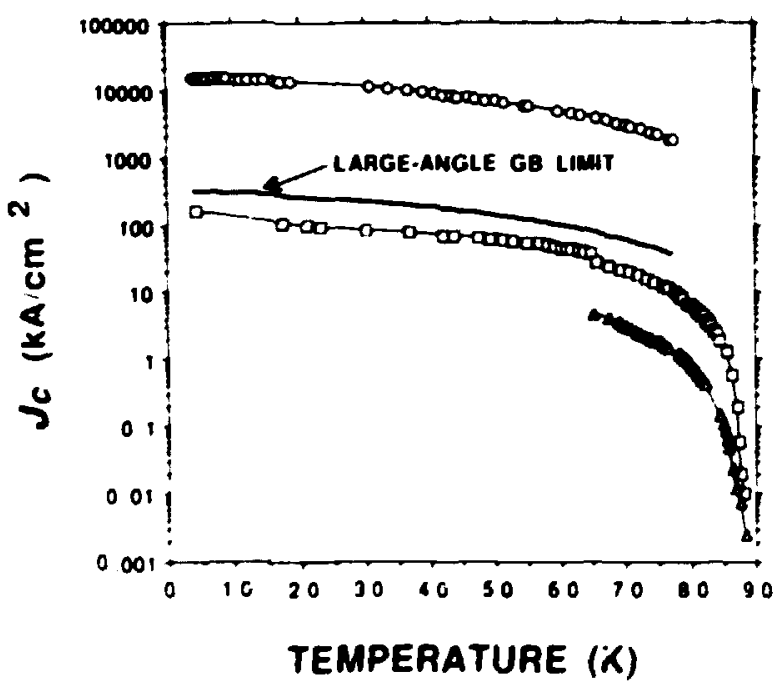

Fig. 3.10. Critical current density vs temperature for $Y-123$ thin films grown on (10)) YSZ fol, as well as on polycrystalline YSZ at $680)\left(\mathrm{J} \mid\right.$ and $7300^{\circ} \mathrm{C}|\Delta|$. Also shown is the intrinsic upper limit for $I_{c}(T)$, hased on transport measurements across large-angle grain boundaries. 
$J_{c}(77 \mathrm{~K}, H=0)$ of $-40-80 \mathrm{kA} / \mathrm{cm}^{2}$ in films that contain large-angle grain boundaries. The temperature dependence of this limiting /c for a polycrystalline film containing large-angle grain boundaries was estimated by simply reducing $I_{c}(T)$ for the corresponding epitaxial film (on a single-crystal substrate) by the ratio of $J_{C}(77 \mathrm{~K}, \mathrm{H}=\mathrm{O})$ for the epitaxial film to the Dimos value as is also shown in Fig. 3.10. This yields empirical $I_{c}(T)$ values for films on polyYSZ that approach the highest values observed by Dimos et al. for bicrystal specimens and implies that in-plane alignment of grains will be required in order to obtain really significant improvements of $I_{c}$ when using polycrystalline substrates.

1. Summary of paper: Appl. Phys. Lett. 57, 1164 (1990).

2. ORNL Eugene P. Wigner Fellow:

3. Graduate student from The University of Tennessee, Knoxville, Tenn.

4. Ceneral Electric Corporate Rescarch and Development Center, Schenectady, N. Y.

5. D. Dimos et al., Phys. Rev. Lett. 61, 1653 (1988).

HOLE FILLING AND HOLE CREATION IN THE SUPERCONDUCTING COMPOUNDS $\mathrm{Bi}_{2} \mathrm{Sr}_{2-x} \mathrm{RE}_{x} \mathrm{CuO}_{6+y} \mathrm{RE}=\mathrm{La}, \mathrm{Pr}, \mathrm{Nd}, \mathrm{AND} \mathrm{Sm^{3 }}$

\section{B. C. Sales and B. C. Chakoumakos}

The superconducting properties, hole content, and latlice constants of several rareearth-doped compounds $\mathrm{Bi}_{2} \mathrm{Sr}_{2-x} \mathrm{RE}_{x} \mathrm{CuO}_{6+y}$ have been investigated as a function of rarecarth doping and oxygen content. The maximum superconducting transition temperature $T_{c}$ obtained for each of the rare-earth dopants was $23 \mathrm{~K}$ (La), $25 \mathrm{~K}$ (Pr), $21 \mathrm{~K}$ (Nd), and $12 \mathrm{~K}$ $(\mathrm{Sm})$. The $T_{c}$ 's of these compounds exhibited a parabolic dependence on the $\mathrm{L} a$ and $\mathrm{Pr}$ concentration $(0<x<1)$, with the maximum $T_{c}$ occurring for $x=0.4$ in samples with low oxygen content (Fig- 3.11). For La- and Pr-doped samples treated in pure oxygen, the position of the maximum shifted to $x=0.6$ and 0.5 , respectively, with a concurrent reduction in the maximum value for $T_{c}(17 \mathrm{~K}$ for $\mathrm{L} a$ and $18 \mathrm{~K}$ for $\mathrm{Pr}$ ). Absolute values of the oxygen content and the changes of the oxygen content with heat treatments were determined using thermogravimetric analysis.

The qualitative features of the dependence of $T_{c}$ on $x$ and $y$ can be understood by assuming an excess of holes in the $\mathrm{Cu}-\mathrm{O}$ planes of the undoped compound which are filled by the

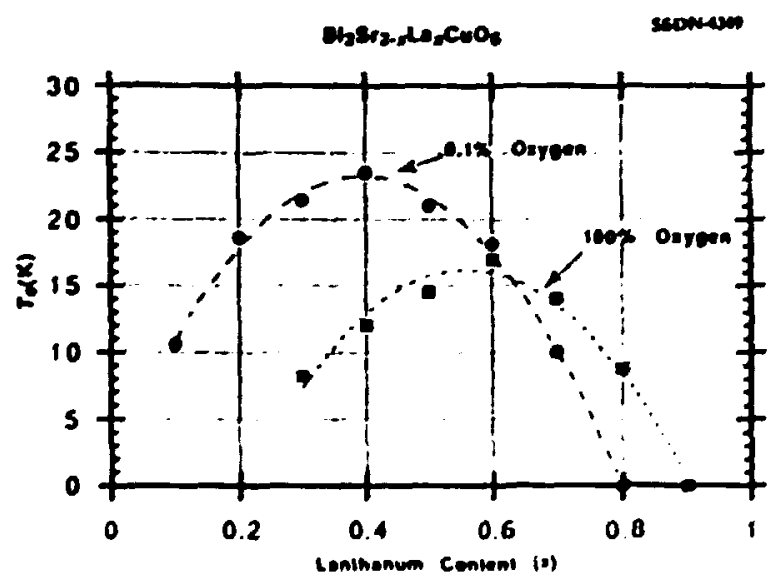

Fig. 3.11. Superconducting transition temperature (10\% magnetic) vs La content for $\mathrm{Bi}_{2} \mathrm{Sr}_{2-x} \mathrm{La}_{x} \mathrm{CuO}_{6+y}$ samples treated al $7500^{\circ} \mathrm{C}$ in either $0.1 \%$ or $100 \%$ oxygen. 
extra electrons contributed with rare-carth doping or increased with the addition of oxygen. For La-doped samples treated in pure oxygen, there was a smooth variation of $T_{c}$ with holc content with the maximum $T_{c}(18 \mathrm{~K})$ occurring at a hole content of $0.2 \pm 0.02$. For Ladoped camples treated in $0.1 \%$ oxygen, the maximum $T_{c}$ occurred at the same hole content (0.2), but the value of $T_{c}$ was significantly higher ( $23 \mathrm{~K})$. These data illustrate that, even within one alloy system, composition can affect $T_{c}$ as well as hole content. Finally, lattice constant and oxygen content data from the $\mathrm{Pr}$ doped materials suggest that Pr substituted onto the Sr site has a formal valence of approximately 3.3 .

1. Summary of paper: Physical Review B (in press).

\section{TEMPERATURE-DEPENDENT MAGNETIC PENETRATION DEPTH $\lambda(T)$ IN HIGH- TEMPERATURE SUPERCONDUCTORS ${ }^{1}$}

I. R. Thompson, 2 D. K. Christen, Yangren Sun, 3 S. T. Sekule, J. G. Ossandon, ${ }^{3}$ H. R. Kerchner, J. Brynestad, 5 B. C. Chatoumakos, B. C. Sales, I. Budai. and E. Sonder

Studies of the temperature-dependent magnetic-penetration depth $\lambda(T)$ have been performed on several high- $T_{c}$ compounds including the Bi-(Pb)-Sr-Ca-Cu oxide containing two or three adjacent $\mathrm{Cu}-\mathrm{O}$ layers and $\mathrm{Tl}_{2} \mathrm{Ca}_{2} \mathrm{Ba}_{2} \mathrm{Cu}_{3} \mathrm{O}_{10}$. Materials included highly textured samples of Bi-(Pb)-2223 and Bi-2212, single-crystal Bi-2212, and grain-aligned T1-2223. Characterization methods included $x$ ray diffraction, electrical resistivity, vibrating sample, and SQUID magnetometry. Values for the resistive midpoint of the transition temperature $T_{c, y}$ magnetic midpoint $T_{c, m,}$ and other results are summarized in Table 3.1.

Table 3.1. Experionental Properties of Migh-T, Cuprate Superconductors

\begin{tabular}{|c|c|c|c|c|c|}
\hline Compound & $\begin{array}{l}\text { Prepara- } \\
\text { tion T, } \\
\text { almosphere }\end{array}$ & $\begin{array}{c}\lambda_{2}(\mu \mathrm{m}) \\
\text { (cxccpt as } \\
\text { noled) }\end{array}$ & $T_{c, \lambda}(K)$ & $\begin{array}{c}T_{c y} \\
(50 \% \\
\text { resistance) }\end{array}$ & $\begin{array}{l}T_{(\Omega, d}(K) \\
\text { magnetic } \\
\text { midjpoint }\end{array}$ \\
\hline $\begin{array}{l}\mathrm{Bi}_{1}+\mathrm{Ib}_{0} \mathrm{Sr}_{2} \\
\mathrm{Ca}_{2} \mathrm{Cu}_{3} \mathrm{O}_{10}\end{array}$ & $\ldots$ & 0.22 & 105.9 & 108 & 105.5 \\
\hline $\begin{array}{l}\mathrm{Bi}_{2} \mathrm{Se}_{1}{ }_{5} \mathrm{Ca}_{1}{ }_{17} \\
\mathrm{Cu}_{2} \mathrm{O} .\end{array}$ & $\begin{array}{l}\text { PArC. } \\
1 \times \mathrm{O}_{2}\end{array}$ & 0.30 & 80.7 & 80.7 & $\cdots$ \\
\hline $\begin{array}{l}\text { Bi.2212 } \\
\text { (llicelit AC) }\end{array}$ & Astre, air & 0.28 & 72.7 & 77.5 & 71.8 \\
\hline $\begin{array}{l}\text { B1.2212 } \\
\text { (Illexche AC) }\end{array}$ & $\begin{array}{l}\text { MrT C. } \\
2 \%, \mathrm{C}_{2}\end{array}$ & 0.30 & 80.3 & 81.8 & 78.9 \\
\hline $\begin{array}{l}\text { Is. } .2212 \\
\text { single crysl.ol }\end{array}$ & $\cdots$ & $\begin{array}{l}\lambda_{2}=0.27 \\
\lambda_{r}=3.7\end{array}$ & 73.8 & $\cdots$ & $\cdots$ \\
\hline $\mathrm{Tl} \cdot(., \mathrm{B} \cdot \mathrm{B}, \mathrm{C} \cdot \mathrm{u} \cdot \mathrm{O})$ & $\begin{array}{l}\text { st.tpicd: } \\
10 x)^{2}+\mathrm{C}_{2}\end{array}$ & $\begin{array}{l}\lambda_{3}=0.17 \\
\lambda_{c}=0.48\end{array}$ & 122.8 & $\cdots$ & 121.2 \\
\hline
\end{tabular}


Measurement of the magnetic-penetration depth $\lambda(T)$ was based on a London-limit formalism for the magnetization $M$ in the equilibrium mixed state of a uniaxial, type-ll superconductor, which provides $M=\ln (H)$, with $d M / d[\ln (H)]=\phi_{0} /\left(32 \pi^{2} \lambda_{i} \lambda_{j}\right)$. Here $\lambda_{j}$ is the anisotropic magnetic-penetration depth corresponding to supercurrent flow in the $j$-th directic 1 and $H$ is the magnetizing field, $H_{c l} \ll H$ $\ll H_{C}$. Near $T_{c}$ Ginzburg-Landau theory provides that $(1 / 2)^{2} \propto\left(T_{c} T\right)$. This linear variation with $T$ was observed experimentally and used to obtain accurate values of $T_{c, \lambda}$, appropriate to the penetration depth study. Results for $\lambda(T)$ were compared with theoretical forms, including weak couplir: $\hat{b}$ Bardeen-Cooper-Schrieffer calculations in the clean and dirty limits, the empirical two-iluid model, and a strong-coupling calculation. In every case, the weak-coupling, clean-limit theory provided the best description of the experimental results. Extrapolation of the best theoretical form to $T=0$ yielded the values for $\lambda(0)$ in Table 3.1.

In summary, comparison of the experimental evidence with theory indicates that these high-T, $T_{c i-}$, and Tl-based materials are weak-coupling siperconductors-a result consistent with the earlier arguments of Little. 6

1. Summary of papers: Phys. Rev. 41, 7293 (1990); Physica B 165/166, 1453 (1990).

2. Adjunct rescarch and development participant from The University of Tennessec, rusuxville, Tenn.

3. Graduate student from The University of Tennessec, Knoxville, Tenn.
4. Deceased.

5. Chemistry Division, ORNL.

6. W. A. Little, Science 242, 1390 (1988).

\section{SOLID ELECTROLYTES} AND CERAMIC FILMS

\section{ELECTRICAL PROPERTIES OF $\mathrm{Li}_{2} \mathrm{O}_{-} \mathrm{SiO}_{2} \mathrm{P}_{2} \mathrm{O}_{5}$ AMORPHOUS UTHIUM ELCTROL YTE THIN FILMS'}

\section{J. B. Bates, N. J. Dudney, and C. F. Luck}

Amorphous electrolyte films which are suitable for application in rechargeable lithium microbatteries should have a lithium ion conductivity of $10 \mathrm{nS} / \mathrm{cm}$ or higher and a negligible electronic conductivity. Amorphous $\mathrm{Li}_{2} \mathrm{O}-\mathrm{SiO}_{2}-\mathrm{P}_{2} \mathrm{O}_{5}$ films measuring about $1.5-\mathrm{cm} \times$ $2-\mathrm{cm} \times 1+\mu \mathrm{m}$ thick were deposited by if magnetron sputtering ${ }^{2,3}$ over a $0.15-\mathrm{cm}$ wide gold strip electrode on a polycrystalline alumina substrate. A top gold strip electrode was sputtered over the electrolyte film to form a sandwich structure with an overlap area of 0.023 $\mathrm{cm}^{2}$ between the top and bottom contacts. Impedance measurements were made on the films at temperatures from 25 to $200^{\circ} \mathrm{C}$ using 10 to 100-mv ac signals at frequencies from $0.1 \mathrm{~Hz}$ to $10 \mathrm{MHz}$. The dc resistance of the films was determined from analysis of the ac impedance.

Examples of the conductivity plotted as $\log (\sigma T)$ vs $1 / T$ are given in Fig. 3.12. The solid lines through the data points are graphs of $\sigma T=c_{\rho} \exp (-E / k T)$ where the pre-exponential factor $\sigma_{0}$ and the activation energy $E$ were determined from least-squares fits to the data. 
Conk DWG 20-17400

\section{TEMPERATURE $\left({ }^{\circ} \mathrm{C}\right)$}

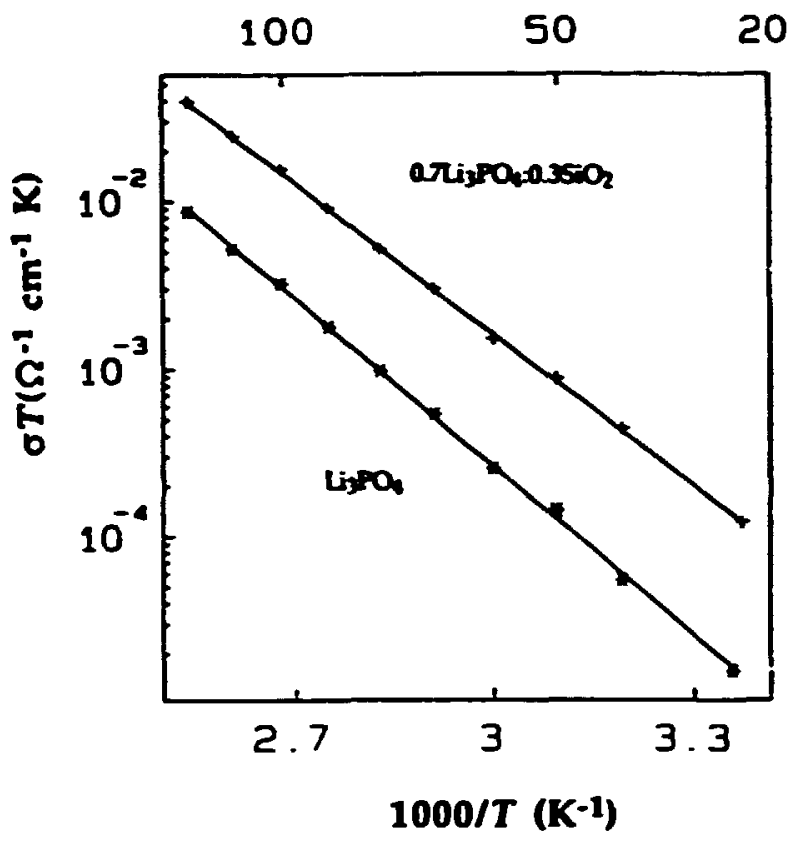

Fig. 3.12. Graphs of $\log (\sigma T)$ vs $1 / T$ for films with the approximate compositions (a) $\mathrm{Li}_{3} \mathrm{PO}_{4}$ and (b) $0.7 \mathrm{Li}_{3} \mathrm{PO}_{4}: 0.3 \mathrm{SiO}_{2}$.

In general, the highest conductivities and lowest activation energies were observed in films with the highest $\mathrm{Li}_{2} \mathrm{O}$ content. However, the conductivity is mainly affected by changes in the $\mathrm{L}^{+}$ion mobility (presumably due to changes in the glass structure) rather than the number of charge carricts. An example is shown by the results in Fig. 3.12. These data illustrate the important but unexplained "mixed fomer" effect that is generally observed in bulk amorphous electrolytes. For the film that had approximately the net composition $0.7 \mathrm{Li}_{3} \mathrm{PO}_{4}: 0.3 \mathrm{SiO}_{2}, \sigma=400 \mathrm{nS} / \mathrm{cm}$ at $25^{\circ} \mathrm{C}$ and $E=0.62 \mathrm{cV}$ compared to $\sigma=70 \mathrm{nS} / \mathrm{cm}$ and $E=0.68 \mathrm{cV}$ for the film with the approximate composition $\mathrm{Li}_{3} \mathrm{FO}_{4}$. This increase in conductivity of the amorphous lithium orthophosphate was achieved by adding a second glass former $\mathrm{SiO}_{2}$, but no additional $\mathrm{LO}_{2}$. The added $\mathrm{SiO}_{2}$ evidently modifies the glass structure resulting in an increase in the $\mathrm{L}^{+}$ion mobility.

1. Summary of paper to be published.

2. J. B. Bates, el al., Fabrication, Composition, and Structure of $\mathrm{Li}_{2} \mathrm{OSiO}_{2}: \mathrm{P}_{2} \mathrm{O}_{5}$ Thin Films," this report.

3. N. J. Dudney, J. B. Bates, and J. D. Robertson, "Sputtering of $\mathrm{Li}_{4} \mathrm{SiO}_{4}, \mathrm{Li}_{3} \mathrm{PO}_{4}$, and Li2 O," this report.

\section{SEGRECATION OF LTHIUM DURING RF MAGNETRON SPUTIERING OF LTTIUM ORTHOSILCATE}

\section{N. I. Dudney. J. B. Bates and I. D. Robertson'}

Amorphous electrolyte thin films containing $\mathrm{Li}_{2} \mathrm{O}, \mathrm{SiO}_{2}$ and $\mathrm{P}_{2} \mathrm{O}_{3}$ have been prepared by If magnetron sputtering. ${ }^{2,3}$ One of the frequently used target materials, $\mathrm{Li}_{4} \mathrm{SiO}_{4}$ does not sputter stoichiometrically in this system, and much of the lithium segregates outside of the sputtered area. A number of experiments have been performed that are aimed at impeding the segregation or elucidating the mechanism responsible for this phenomenon.

The planar magnetron source contains two concentric magnets of opposite polarity bencath the sputter target. This confines the plasma in a ring at the target surface. The lithium segregation is observed as a large accumulation of material at the center and rim of the target away from the spultered ring. The segregated 
material has been identified as $\mathrm{Li}_{2} \mathrm{O}$ by $x$-ray diffraction. When sputtered in pure argon the segregated $\mathrm{Li}_{2} \mathrm{O}$ material is black, whereas when spultered in the presence of oxygen, it is generally white.

The $\mathrm{Li}_{2} \mathrm{O}$ segregation at the surface has been observed for every $\mathrm{Li}_{4} \mathrm{SiO}_{4}$ target that has been prepared. Targets have been prepared from a varicty of starting powders with a range of sizes and densities. In addition, $\mathrm{Li}_{4} \mathrm{SiO}_{4}$ has been sputtered under a variety of if powers, gas pressures, sputter gas compositions, and magnetic fields. Changes in the sputter gas imive had the largest influence on the degree of lithium segregation, but none of these variations eliminated the tendency for decomposition of the target. The addition of $\mathrm{O}_{2}$ to the .ert sputter gas appears to promote the lithium segregation. Film compositions of 0.6 $\mathrm{Li}_{2} \mathrm{O} \cdot \mathrm{SiO}_{2}$ were obtained in pure argon, while the 3/2 argon/oxygen mixture gave films of $<0.4$ $\mathrm{Li}_{2} \mathrm{O} \cdot \mathrm{SiO}_{2}$. Sputtering of $\mathrm{Li}_{4} \mathrm{SiO}_{4}$ in neon rather than argon has resulted in the smallest lithium loss due to segregation. Films prepared by sputtering in neon have compositions of 1.3 $\mathrm{Li}_{2} \mathrm{O} \cdot \mathrm{SiO}_{2}$.

The location of the segregated $\mathrm{Li}_{2} \mathrm{O}$ on the surface of sputter targets composed of sectors of $\mathrm{Li}_{4} \mathrm{SiO}_{4}$ and $\mathrm{Li}_{3} \mathrm{PO}_{4}$ suggests that the lithium is transported along or very near the surface of the target rather than in the gas phase. The direction of the magnetic ficld has been observed to reverse the pattern of segregated $\mathrm{Li}_{2} \mathrm{O}$ on the target surface suggesting that lithium ions are moving out of the plasma ring; however, attempts to identify $\mathrm{Li}^{+}$in the emission spectra have been unsuccessful. Further investigation is needed to identify the transport mechanism and the reason that lithium segregates during sputtering of $\mathrm{Li}_{4} \mathrm{SiO}_{4}$ (and also $\mathrm{Li}_{2} \mathrm{SiO}_{3}$ and $\mathrm{Li}_{2} \mathrm{O}$ ) but does not segregate during the sputtering of $\mathrm{Li}_{3} \mathrm{PO}_{4}{ }^{3}$

1. University of Kentucky, Lexington, Ky.

2. J. B. Bates et al., "Fabrication, Composition, and Structure of $\mathrm{Li}_{2} \mathrm{OS}_{5} \mathrm{~S}_{2}: \mathrm{P}_{2} \mathrm{O}_{5}$ Thin Films," this report.

3. N. J. Dudney, J. B. Bates, and J. D. Robertson, Sputtering of $\mathrm{Li}_{4} \mathrm{SiO}_{4} \mathrm{Li}_{3} \mathrm{PO}_{4}$ and $\mathrm{Li}_{2} \mathrm{O}$," this report.

\section{FABRICATION, COMPOSTMON, AND STRUCTURE OF Li2 $\mathrm{OSIO}_{2}: \mathrm{P}_{2} \mathrm{O}_{5}$ THIN FILMS ${ }^{1}$}

\section{J. B. Bates, N. J. Dudney, I. D. Robertson, B. C. Sales, R. A. Zuhr, A. L. Wachs, and C.F. Luck}

The deposition of thin-film lithium clectrolytes with reproducible properties is an important step in the development of rechargeable microbatteries suitable for sircuit integration. Amorphous films are best suited for this application because of their isotropic conductivity and superior mechanical properties. Lithium electrolyte thin films $1-1.5-\mu \mathrm{m}$ thick in the ternary system $\mathrm{Li}_{2} \mathrm{O}: \mathrm{SiO}_{2}: \mathrm{P}_{2} \mathrm{O}_{5}$ were prepared by single- and dual-source if magnetron sputtering of $(1-x) \mathrm{Li}_{4} \mathrm{SiO}_{4}: x \mathrm{Li}_{3} \mathrm{PO}_{4}, \mathrm{Li}_{2} \mathrm{O}$, and $\mathrm{SiO}_{2}$ targets in $20 \mathrm{mTorr}$ of $3 / 2 \mathrm{Ar} / \mathrm{C}_{2}$. The $\mathrm{Li} / \mathrm{P}$ and $\mathrm{Li} / \mathrm{Si}$ ratios in the films were determined by protoninduced gamma ray emission, whilc the $P$ and 
Si contents were determined by energydispersive $x$-ray fuorescence and by Rutherford backscattering spectrometry. Representative compositions of the films are plotted on the ternary diagram shown in Fig. 3.13. The Si/P ratios in the films were close to the Si/P ratios in the $(1-x) \mathrm{Li}_{4} \mathrm{SiO}_{4}: \mathrm{xLi}_{3} \mathrm{PO}_{4}$ targets in the case of single-source depositions or to the ratio of the deposition rates in the case of the dualsource depositions. However, the $\mathrm{Li}_{2} \mathrm{O}$ content varied dramatically depending on the nature of the targets, deposition method (single or dual source) and on the sputtering gas composition.

The shaded region in Fig. 3.13 is the approximate composition domain in which the

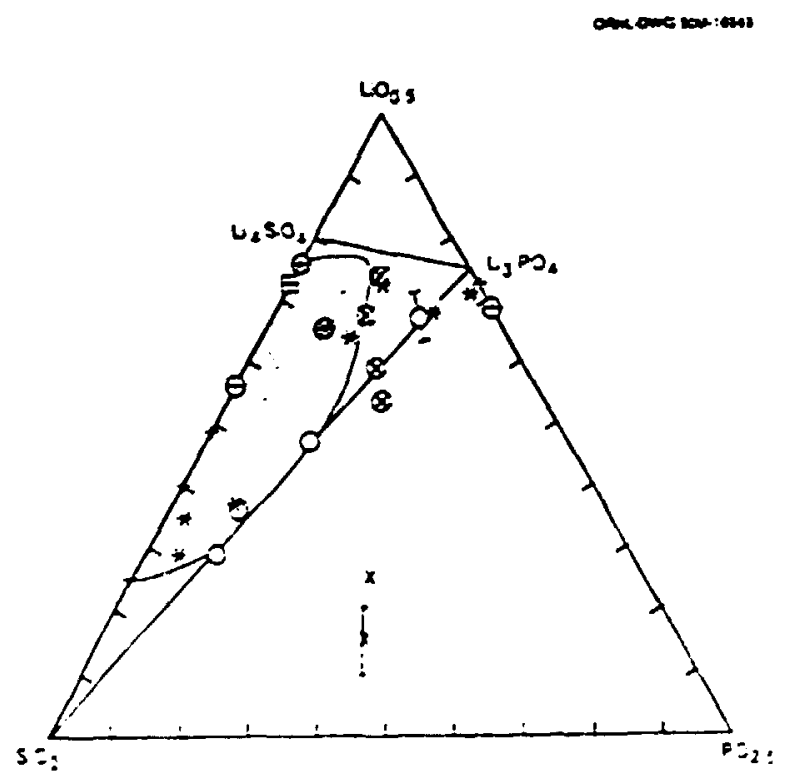

Fig. 3.13. The composition of $\mathrm{Li}_{2} \mathrm{O}: \mathrm{SiO}_{2}$ $\mathrm{P}_{2} \mathrm{O}_{3}$ clectrolyte thin films, +: single $\mathrm{Liz}_{3} \mathrm{PO}_{4}$ or $\mathrm{Li}_{6} \mathrm{SiO}_{6}$ targets $(\rightarrow$ in Ar only; $y$ in Ne only; $\left(\mathrm{Li}_{4} \mathrm{SiO}_{4}+\mathrm{Li}_{2} \mathrm{O}\right.$ ); $\mathrm{x}$ : single (1-x) $\mathrm{Li}_{4} \mathrm{SiO}_{4} x \mathrm{Li}_{3} \mathrm{PO}_{4}$ largets (x sinf(cred; holpressed; $y$ additional l.i2 $O$ from second source); *: dual-scource $l_{4} i_{4} S i O_{4}+1 . i_{3} \mathrm{PO}_{4}(0$ Ar only); $O$ : dual-source $\mathrm{l}_{13} \mathrm{PO}_{4}$ and $\mathrm{SiO}_{2}$. Uncertainly in l.iOn; content indicated by error bars. presence of two phases is indicated by optical and electrical measurements. By contrast, in bulk $\mathrm{Li}_{2} \mathrm{O}-\mathrm{Si}_{2} \mathrm{O}$ glasses, phase separation is not observed when the fraction of $\mathrm{LOO}_{0.5}$ is higher than 0.5. The ionic conductivities of the films are as much as two orders of magnitude lower than the conductivities of bulk glasses of the same composition. These differences between the properties of the thin-film and bulkamorphous electrolytes are probably due to the process by which the films are formed (i.c., by recombination at the substrate of atomic and small molecular species, Li, LiO, P, PO, Si, SiO, ... ), which are the principal components of the sputtered nux. 3

1. Summary of paper to be published.

2. University of Kentucky, Lexington, Ky.

3. A. L. Wachs. J. B. Bales, N. J. Dudncy, and C. F. Luck, "Masma Diagnostic Studies of the Influence of Process Variables upon the Alomic and Molecular Species Ejected from (1-x) $\mathrm{Li}_{4} \mathrm{SiO}_{4}: \mathrm{xLi}_{3} \mathrm{PO}_{4}$ Targets During RF Magnetron Sputtering," this report.

\section{SPUTTERING OF $\mathrm{Li}_{4} \mathrm{SiO}_{\downarrow} \mathrm{Li}_{3} \mathrm{PO}_{\downarrow}$ and $\mathrm{Li}_{2} \mathrm{O}^{1}$}

\section{N. I. Dudncy, I. B. Bates, and I. D. Roberlson ${ }^{2}$}

Various targets of lithium orthosilicate, lithium orthophosphatc, and lithium oxide have been used in the preparation of amorphous lithium electrolyte films by if magnetron sputtering. These targets have been sputtered at various if powers, gas pressures, and in pure argon as well as argon plus oxygen gas mixtures. The composition of the targets was found to have a pronounced effect on the lithium content of the films. The film compositions were 
deternined by a combination of results from energy-dispersive $x$-ray fluorescence, protoninduced gamma emission, and atomic emission spectroscopies.

Films prepared by sputtering pure $\mathrm{Li}_{3} \mathrm{PO}_{4}$ targets are found to have compositions near that of the target, with $\mathrm{Li} / \mathrm{i}^{\prime}$ ratios 2.5-2.9. Films sputtered from $\mathrm{Li}_{4} \mathrm{SiO}_{4}$ targets, however, are extremely lithium deficient. The largest $\mathrm{Li} / \mathrm{Si}$ ratio obtained by sputtering in argon is 1.3. The loss of lithium is due to segregation of the lithium at the surface of the $\mathrm{Li}_{4} \mathrm{SiO}_{4}$ target. ${ }^{3}$

As for the pure $\mathrm{Li}_{4} \mathrm{SiO}_{4}$ targets, there is considerable loss of lithium due to segregation for mixed $\mathrm{Li}_{3} \mathrm{PO}_{4}+\mathrm{Li}_{4} \mathrm{SiO}_{4}$ targets. Targets with approximately equal mole fractions of $\mathrm{Li}_{3} \mathrm{PO}_{4}$ and $\mathrm{Li}_{4} \mathrm{SiO}_{4},(1: 1)$, have been prepared by both sintering and hot pressing the mixed powders. Relative densities were approximately 75\% of theoretical density for the sintered targets and $96 \%$ for the hot-pressed ones. Films grown using (1:1) targets made by sintering contained relatively little lithium with $\mathrm{Li} /(\mathrm{Si}+\mathrm{P})<0.5$. Films grown using $(1: 1)$ hotpressed targets generally had a higher lithium concentration (approximately $\mathrm{Li} /(\mathrm{Si}+\mathrm{P})=1.5$ ], but this lithium content is still well below that of the larget. The increased lithium concentration from the hot-pressed targets is not simply related to the target densily, as a dense hotpressed target made from $\mathrm{Li}_{3} \mathrm{PO}_{4}+\mathrm{Li}_{4} \mathrm{SiO}_{4}$ (1:1), which had been prereacted by calcining at $800^{\circ} \mathrm{C}$, gave films with $\mathrm{l} . \mathrm{i} /(\mathrm{Si}+\mathrm{P})=0.4$.

Films were also grown using $t_{\text {wo }}$ independently controlled sputlering sources operated simultaneously. Typically the sputter sources were pure sintered targets of $\mathrm{Li}_{3} \mathrm{PO}_{4}$ and $\mathrm{Li}_{4} \mathrm{SiO}_{4}$. Such dual-source films frequently have higher lithium contents than those obtained from single mixed targets. For example, a dual-source film with a $\mathrm{Si} / \mathrm{P}$ ratio of approximately 1 has a ratio $\mathrm{Li} /(\mathrm{Si}+\mathrm{P})=2.45$. To increase the lithium content in the films further, dual-source depositions were made using a $\mathrm{Li}_{2} \mathrm{O}$ target as one of the sputier sources to compensate for the lithium loss in the silicatecontaining target. In this way, $\mathrm{Li} /(\mathrm{Si}+\mathrm{P})$ ratios as high as 3.4 have been obtained. By using multiple sputter sources, electrolyte films can be deposited with reproducible and controlled compositions.

1. Summary of paper to be published.

2. University of Kentucky, Lexington, $\mathrm{Ky}$.

3. N. J. Dudney, J. B. Bates, and J. D. Robertson, Segregation of Lithium During RF Magnctron Sputtering of Lithium Orthosilicate," this report.

\section{PLASMA DIAGNOSTIC STUDIES OF THE INFLUENCE OF PROCESS VARIABLES ON THE ATOMIC AND MOLECULAR SPECIES EJECTED FROM $(1-x) \mathrm{Li}_{4} \mathrm{SiO}_{4}-\mathrm{LL}_{3} \mathrm{PO}_{6}$ TARGETS DURING RF MAGNETRON SPUTTERDNG ${ }^{1}$}
A. L. Wachs, J. B. Bates, N. J. Dudney, and C. F. L.eck

As a first step toward understanding the mechanisms of $(1-x) \mathrm{Li}_{2} \mathrm{SiO}_{4}: x \mathrm{Li}_{3} \mathrm{PO}_{4}$ target sputtering and thin-film growth, the relative concentrations of neutral species in the $r f$ plasma have been measured, in real time. rhese experiments were performed with a 
vacuum chamber similar to the systems in which the actual films for microbattery applications are grown. This system includes an optical-fiber arrangement for optical spectroscopy of the the $\mathrm{rf}$ plasma and a quadrupole mass spectrometer (QMS).

The targets were sputtered in high-purity Ar- $\mathrm{O}_{2}$ working-gas mixtures at total pressures and flow rates used in our other thin-film deposition systems. The chemicai species identified by the QMS during sputtering runs are shown in Table 3.2. Amorphous electrolyte films grown by the rf sputtering of $(1-x) \mathrm{Li}_{3} \mathrm{PO}_{4}: \mathrm{xLi}_{4} \mathrm{SiO}_{4}$ targets are formed by the chemical recombination of atomic and small molecular species at the substrate.

It was found that a high-lithium content in the sputtered films is critical for their use as electrolytes in thin-film batteries. With an optical fiber arrangement substrate, it was possible to measure the emission from excited lithium and argon atoms in the plasma during film growth. With the same targets and sputtering conditions used in grewing the clectrolyte thin films, a positive correlation between the Li(670.8 nm):Ar(675.2 nm) optical emission intensity ratios and the $\mathrm{Li}$ mass peak intensities measured by the QMS was consistently found. Hence, achieving and maintaining a high Li:Ar emission intensity ratio during the sputter deposition is one criterion for the growth of high-lithium content films.

1. Summary of paper: loumal of Vacuum Science and Technology $A$ (in press).

Table 3.2. Singly ionized species observed for selected sputter target and working gas compositions as determined from peak assignments from a quadrupole mass spectrometer. Species whose molecular weights are the same as residual gas species in the chamber are enclosed in square brackets. The mass-to-charge ratio (amu/e) of the peak assignments are shown in parentheses where applicable.

Working Cas Composition

\begin{tabular}{|c|c|c|}
\hline Target & Ar Only & $3: 2 \mathrm{Ar}: \mathrm{O}_{2}$ \\
\hline \multirow[t]{3}{*}{$\mathrm{Li}_{4} \mathrm{SiO}_{4}$} & $\operatorname{Li}(7),|S i|(28), O(16)$ & $\mathrm{Li},|\mathrm{Si}|, \mathrm{O}_{2}, \mathrm{O}, \mathrm{LiO}$ \\
\hline & $\mathrm{LiO}(23), \mathrm{Li}_{2} \mathrm{O}(30)$ & $\mathrm{L}_{2} \mathrm{O},(\mathrm{SiO}, \mathrm{LiSiO}(51)$, \\
\hline & {$[\mathrm{SiO}](44)$} & $\mathrm{LiSiO} \mathrm{O}_{2}(67)$ \\
\hline $\mathrm{Li}_{3} \mathrm{PO}_{4}$ & l.i,l.i. $\mathrm{O}, \mathrm{P}(31), \mathrm{PO}(47)$ & $\mathrm{I} . \mathrm{i}, \mathrm{L}, \mathrm{i}_{2} \mathrm{O}, \mathrm{P}, \mathrm{PO}$ \\
\hline \multirow[t]{2}{*}{$L_{i} i_{4} \mathrm{SiO}_{4}: 1 . i_{3} \mathrm{PO}_{4}$} & li, $|\mathrm{Si}|, \mathrm{O}, \mathrm{O}_{2}, \mathrm{l}, \mathrm{i}_{2} \mathrm{O}$ & I.i,ISil,O, $\mathrm{O}_{2}, \mathrm{~L}_{2} \mathrm{O}$, \\
\hline & $P, P O$ & $P, I O)$ \\
\hline
\end{tabular}




\section{MICROBATTERY RESEARCH}

G. R. Gruzalski, J. B. Bates,

N. I. Dudney, and C. F. Luck

A battery is an electrochemical cell consisting of an anode (electron source), a cathode (electrun sink), and an electrolyte, which allows ionic conduction but prevents electronic conduction. Because solid state thin-film microbatteries have favorable properties for potential application as miniature selfcontained secondary power sources for electronic devices on integrated circuits and because voltage and energy-density requirements can be fulfilled best by Li-based systems, we have begun a study of thin-film cells consisting of $\mathrm{Li}$ anodes, $\mathrm{V}_{2} \mathrm{O}_{5}$ cathodes, and $\mathrm{Li}$-ion conducting amorphous electrolytes of composition $x \mathrm{Li}_{4} \mathrm{SiO}_{4}: y \mathrm{Li}_{3} \mathrm{PO}_{4}: 2 \mathrm{P}_{2} \mathrm{O}_{5}$. These electrolytes were chosen because of their high ionic conductivity and promising mechanical properties, and $\mathrm{V}_{2} \mathrm{O}_{5}$ was chosen because of the relatively high free energy associated with the cathode reaction:

$$
x \mathrm{Li}+\mathrm{V}_{2} \mathrm{O}_{5} \rightarrow \mathrm{Li}_{x} \mathrm{~V}_{2} \mathrm{O}_{5}
$$

which is expected to give rise to an open-circuit voltage (OCV) near $3.6 \mathrm{~V}$.

The materials problems involved in making useful cells are challenging. Current research is aimed at determining (1) the stability of the clectrolyte in contact with elemental Li, (2) the best composition of the electrolyte, (3) the optimal growth conditions for depositing $\mathrm{V}_{2} \mathrm{O}_{5}$, (4) suitable raterials for anode and cathode current collectois, and (5) acceptable cell configurations consistent with potential packaging and device applications.

A functional cell has been fabricated using the following procedures. The cathode current collector was deposited onto a glass substrate by dc magnetron sputtering of $V$ in $10^{-2}$ Torr Ar; the amorphous cathode, having a composition close to that of $\mathrm{V}_{2} \mathrm{O}_{5}$, was deposited by reactive dc magnetron sputtering or $V$ in $2 \times 10^{-2}$ Torr $A r$ containing about $14 \% \mathrm{O}_{2}$. The amorphous electrolyte was deposited by if magnetron sputtering of $\mathrm{Li}_{3} \mathrm{PO}_{4}$ in $2 \times 10^{-2}$ Torr Ar containing about $40 \% \mathrm{O}_{2}$, and the anode was deposited by evaporating elemental $\mathrm{Li}$ in vacuum $\left(-5 \times 10^{-7} \mathrm{Torr}\right)$. The anode current collector (and its protective cuvering) was deposited by electron-beam evaporation of elemental $V$. The total thickness of the cell was $-3.7 \mu \mathrm{m}$. Several charge and discharge tests have been performed on this cell with encouraging results. The OCV of $-1.4 \mathrm{~V}$ was lower than expected, but the cell could be discharged at current densities as high as $10 \mu \mathrm{A} / \mathrm{cm}^{2}$.

\section{ENHANCED IONIC CONDUCTION IN SILVER HALIDE-ALUMINA COMPOSITES'}

\section{N. I. Dudney}

The addition of fine alumina particles or fibers to $\mathrm{AgCl}, \mathrm{AgBr}$, and $\mathrm{Agl}$ can give marked increases in the low-temperature silver-ion conductivities. Most of the particle-containing composites have been prepared with 10 to 40 vol. \% submicron powders, but even a 1 to 3 vol. \% of such particles can give a factor of 2 
or 3 increase in the room-temperature conductivity.

The reasons for the observed enhanced conductivity are still not completely clear. Many researchers have attributed the enhanced con ductivity to the formation of a space-charge layer at the interface of the silver halide with the alumina particles. Calculations show that a space-charge layer may greatly enhance the conductivity along an interface of $\mathrm{AgCl}$, but it is unlikely to have a significant effect for Agl, which has a much smaller Debye length.

Experiments demonstrate that the alumina may have important effects on the microstructure and phase stability of the silver halide. Specifically, for $\mathrm{AgCl}$, the grain boundaries stabilized by the alumina phase contribute to the high-conductivity network in the composite. For AgI, the metastable gamma phase is stabilized by the dispersed alumina and is responsible for the enhanced conductivity of the composites.

1. Summary of paper: J. Imag. Sci. 34, 104 (1990).

\section{INTERFACE AND BULK RELAXATION IN SOLID IONIC CONDUCTORS ${ }^{I}$}

\section{B. Bates, J. C. Wang, ${ }^{2}$ and Y.T. Chu ${ }^{3}$}

The frequency-dependent response of solid ionic conductors to a small amplitude ac voltage applied through de blocking metal contacts is due to polarization from two sources: (1) charge accumulation at the metal-electrolyte interfucces and (2) displacement of bound charges and defects within the electrolyte. These two polarization sources can be identified, for example, in the impedance spectrum of a thin sample of an ionic conductor with gold elec. trodes sputter-dsposited on opposite faces. The observed impedance measured at frequencies $f$ from $0.1 \mathrm{~Hz}$ to $10 \mathrm{MHz}$ can be represented accurately by the function

$Z=A(j \omega)^{-n}+\left(\frac{1}{R}+j \omega C(\omega)\right)^{-1}$

where $j=\sqrt{-1}, A$ and $n$ are constants, $0<\pi<1$, $\omega=2 \pi f, R$ is the resistance of the sample, and $C(\omega)$ is its frequency-dependent capacitance.

The first term in Eq. (1) has the so-called constant phase angle (CPA) form and represents the impedance of the two electrode-electrolyte interfaces. The value of the exponent $n$, which is typically about 0.9 , depends on the detailed microstructure of the electrode-electrolyte interface. The second term represents the bulk response of the electrolyte. With the ratio of the electrode area to the sample thickness given by $L, R=1 / \sigma L$, where $\sigma$ is the ionic conductivity of the electrolyte, and $C(\omega)=$ $L \kappa_{0} \varepsilon(\omega)$, with $\kappa_{0}$ as the vacuum permittivity and $\varepsilon(\omega)=\varepsilon^{\prime}(\omega)-j \varepsilon^{\prime \prime}(\omega)$ as the permittivity of the solid. A characteristic of all solid electrolytes is that above the frequency of the dielectric loss peak (the frequency at which $\varepsilon^{\prime \prime}((0)$ is a maximum), $\varepsilon((0))$ reduces to the CPA form,

$$
\varepsilon(\omega)-\varepsilon_{\infty}=\left(\varepsilon_{0}-\varepsilon_{m}\right)(j(\omega))^{-m},
$$

whire $\varepsilon_{0}$ and $\varepsilon_{m}$ are the static and highfrequency diclectric constants, respectively. 
Recently, i: was shown that a permittivity $\varepsilon(\omega)$ that is in good agreement with experiments can be obtained from a model based on a temperature-dependent uistribution of activation energies of ions $i$ opping in double potential wells.

1. Summary of paper to be published.

2. Present address: Energy Division, JRNL.

3. Present address: Schaffinet Technologies, Inc., Knoxville, Tenn.

4. J. C. Wang and J. B. Bates, submitted to foumal of Chemiral Physics.

\section{SPUTTER DEPOSITION OF $\mathrm{CaF}_{2}$}

\section{N.I. Dudney}

Attempts have been made to prepare thin films containing $\mathrm{CaF}_{2}$ by if magnetron sputtering, but frequently etching rather than film growth has been observed on substrates positioned directly above the target. Similar results have been reported for other materials, and these effects are generally attributed to the acceleration of sputtered anions away from targets with a negative self bias. ${ }^{1}$ Since the difference in the ionization potential for $\mathrm{Ca}$ and the electron affinity of $F$ is relatively small, a similar mechanism leading to highsnergy fluorine bombardment of the substrate seems likely.

Comparison of the sputter-rate distribution above a $\mathrm{CaF}_{2}$ target under a variety of sputter gases leads to a surprising result. For $20 \mathrm{~m}$ Torr of $\mathrm{Ar}$ or $\mathrm{Kr}$, the sputter rate monitored with a quartz oscillator indicated a negative deposi- tion rate at 5-6 cm directly above the target. A small positive rate was observed if the quartz monitur was rotated away from a position directly above the target. Both the etching and deposition rates were larger for the Ar gas than for $\mathrm{Kr}$ at the same target power and gas pressure. When Ne was used as the sputter gas, however, a positive deposition rate was observed at all positions above the target, with the maximum rate observed directly above the target. The self bias of the target was as large or even larger than that observed for the Ar and $K_{r}$ sputtering. This result is unexpected bascd on the theory of negative-ion-induced etching published in the recent literature.

1. J. J. Cuomo et al., I. Vac. Sci. and Technol. A 15, 281 (1978).

\section{OPTICAL, MAGNETIC, AND PHYSICAL CHARACTERIZATION OF MATERIALS}

CHARACTERIZATION OF TEXTURED CERAMICS BY ELECTRON PARAMAGNETC RESONANCE (EPR) SPECTROSCOPY: FORMATION AND PROPERTIES OF TEXTURED MBO'

\section{I.-L. Boldú O., 2 L. A. Boatner, and $M . M$. Abraham}

A method for the formation of macroscopic quantities of faceted, nailophase $\mathrm{MgO}$ precursor powders has been developed in which it is possible to exercise some control over the particulate size distribution. By employing powders of 
this type, it was possible to form textured $\mathrm{MgO}$ ceramics by means of cold-pressing and sintering. The theoretical basis for the application of EPR techniques to texture analysis, as developed previously, ${ }^{3}$ was used to establish the nature of the preferred orientations in these sintered $\mathrm{MgO}$ ceramics. Both EPR and $x$-ray analyses showed that preferred orientations of the (111) axes of the $\mathrm{MgO}_{\mathrm{g}} \mathrm{micros}$ tructural grains occur along or near the direction of forc: used to compact the green body. EPR results also show that this texture develops during the sintering process and that no preferted orientation effects are present in the unsintered body formed by cold-pressing the faceted, nanophase $\mathrm{MgO}$ powder. Effects of parametric variations such as sintering temperature, compaction force, and faceted particle size on the degree of preferred crientation in the sintered $\mathrm{MgO}$ ceramics were investigated using EPR techniques. The results show that the amount of texturing increases with increasing compaction force or temperature up to a limiting value and, as illustrated in Fig. 3.14, that it also increases with decreasing faceted particle size.

1. Summary of paper: I. Am. Cerain. Sox. 73, 2345 (1990).

2. Universidad Nacional Autónoma de México, D.F., México.

3. L. A. Boatner, J.-L. Boldú O., and M. M. Anraham, J. Am. Ceram. Soc. 73, 2333 (199()).

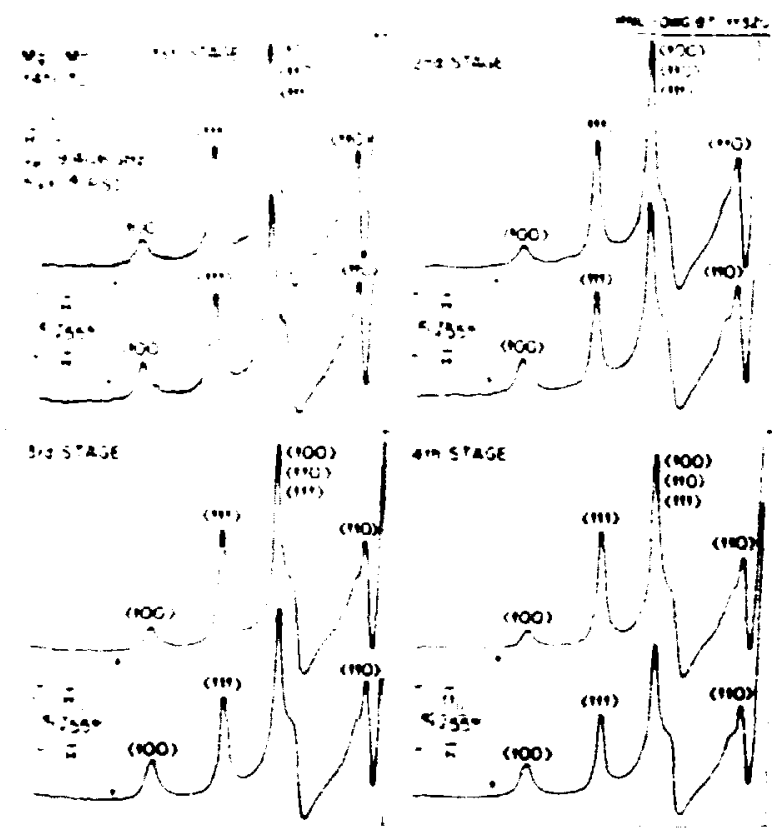

Fig. 3.14. First-derivative EPR spectra of the low-field fine-structure lines of $\mathrm{Mn}^{2+}$ in $\mathrm{MgO}$ are shown for ceramics formed using nanophase powders collected in stages $1-4$ of the apparatus shown in the figure. These results illustrate the effects of varying precursor particle size distribution on the degree of texturing in ceramics sintered at $1450^{\circ} \mathrm{C}$ for $24 \mathrm{~h}$. A comparison of the relative intensities of the (111) and (100) peaks for the two applied mag. netic field orientations in the case of the ceramic from stage 1 powder shows a relatively low degrec of texturing in coinparison with that evidenced by the results for the ceramic formed using fourth-stage powder.

\section{STRUCTURAL RELAXATION DYNAMICS OF PHOSPHATE GLASSES: THE EFFECTS OF NETWORK TOPOLOGY ON THE GLASS TRANSITION'}

B. C. Sales

Scanning calorimetry measurements were made on a series of lead $f(y) \mathrm{PbO}+(1-y) \mathrm{P}_{2} \mathrm{O}_{5}$, 
$0.3<y<0.55]$ and lead-iron phosphate glasses $\left.(1-x) \quad \mathrm{Pb}_{(} \mathrm{PO}_{3}\right)_{2}+(x) \mathrm{Fe}_{2} \mathrm{O}_{3}$. $0<x<0.25$ ] over a temperature range encompassing the glass transition. The apparent activation energy for structural relaxation $\Delta H$, determined for each composition from plots of the inverse of the glass-transition temperature $T_{g}$ vs the $\log$ of the cooling rate, was found to increase with the metal-to-phosphorus ratio. The value of $\Delta H$ increased from $44 \mathrm{kcal} / \mathrm{mole}$ for $\mathrm{P}_{2} \mathrm{O}_{5}$ to $350 \mathrm{kcal} / \mathrm{mole}$ for a lead-iron phosphate glass with $x=0.25$ (Fig. 3.15). The calorimetry data were analyzed using the Adam-Cibbs-Scherer model of the glass transition. This analysis suggests that these glasses undergo a transition from the "strong" to the "fragile" glass regime as the metal-tophosphorus ratio is increased. It is also shown

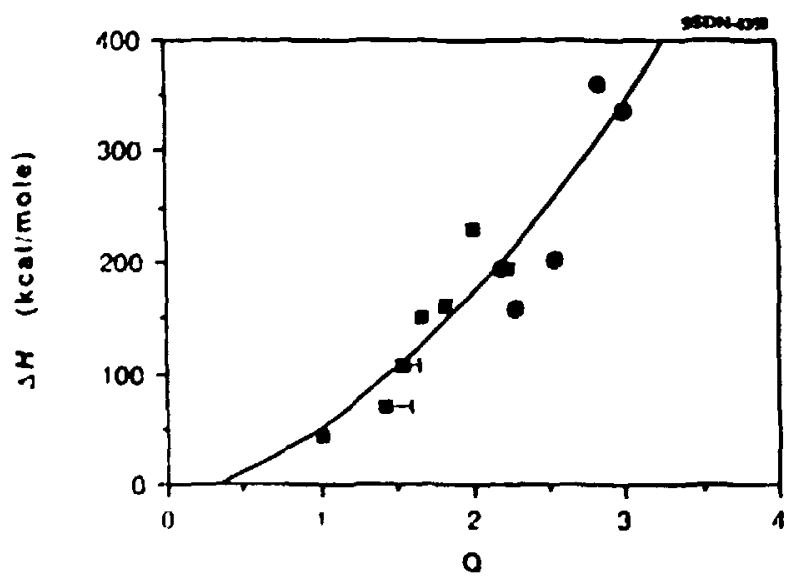

Fig. 3.15. The variation of the apparent activation energy for structural relaxation $\Delta H$ with $Q$ for lcad phosphate (D) and lcad-iron phosphate glasses (O). The parameter $Q$ is equal to the number of nonbridging oxygen atoms per $\mathrm{PO}_{4}$ tetrahedron and was determined from the composition of each glass. The horizontal errors bars for two of the lead phosphate glasses refer to the uncertainty in the amount of structural water present. that this transition may be related to the phase transition predicted by $\mathrm{He}$ and Thorpe ${ }^{2}$ for glasses with underconstrnined networks. From scanning calorimetry data on $\mathrm{P}_{2} \mathrm{O}_{5}, \mathrm{~B}_{2} \mathrm{O}_{3}$. and a lead-iron phosphate glass $(x=0.15)$, the temperature dependence of the shear viscosity for each material above $T_{8}$ was calculated and compared to published viscosity data.

1. Summary of paper: I. Non-Cryst. Solids 119,136 (1990).

2. H. He and M. F. Thorpe, Phys. Reo. Letl. 5A, 2107 (1985).

\section{Q-DECAY-INDUCED CONDENSATION OF PHOSPHATE ANIONS IN A MINERAL'}

\section{B. C. Chakoumakos, B. C. Sales, and L. A. Boatner}

Griphite is a U-Th-bearing phosphate mineral with the unusual property that structural a!terations due to the radioactive decay of uranium and thorium are preserved over geologic time periods. Differential-scanning calorimetry results show that the recrystallization temperature required for the annealing of displacive damage in griphite is relatively high, and therefore such damage can accumulate unchecked with time. Accordingly, griphite that contains $U$ or Th occurs in the amorphous or metamict state unlike other U-Th-bearing phosphate minerals (e.g., monazite) that are found in the crystalline state even after large displacive radiation doses. In its crystalline form, griphite has a structure in which isolated phosphate anions 
are bonded to adjacent metal cations. In this work, however, linked phosphate groups (i.e., chains of $\mathrm{PO}_{4}$ tetrahedra) have been detected in metamict, amorphous $U$-Th-bearing griphite by means of a chromatographic technique. The occurrence of such linked phosphate units in mineralogical systems is unusual since the P-O-P bond is relatively easy to hydrolyze. The radiation damage process in griphite condenses isolated phosphate groups into chains composed of two, three, and four phosphate units. To balance this reaction, free-metal oxide is produced that is not associated with the phosphate anions. Some of the free-metal oxide has reacted with absorbed water to form hydroxide, which may provide additional stability to the structural arrangement of the radiation-damaged state.

1. Summary of paper: Am. Mineral. 75, 1449 (1990).

\section{LOW-FREQUENCY RELAXATION MODES AND STRUCTUKAL DISORDER IN KTa $\mathrm{a}_{1-x} \mathrm{Nb}_{2} \mathrm{O}_{3}{ }^{1}$}

\section{P. Sokoloff, ${ }^{2}$ L. L. Chase, ${ }^{3}$ and L. A. Boalner}

Previous investigations of phase transitions in the perovskite-structure, mixed-crystal system potassium tantalate-niobate $\left(\mathrm{KTa}_{1-x} \mathrm{Nb}_{x} \mathrm{O}_{3}\right.$, or $\mathrm{KTN}$ ) have been carricd out with the goal of accounting for the mechanisms responsible for the observed structurai alterations. In the KTN solid-solution system, a multicritical point accurs at a niobium concentration corresponding to $x \equiv 0.05$. Above this $\mathrm{Nb}$ concentration, KTN undergoes a cubictetragonal-orthorhombic-rhombohedral sequence of phase transitions where the various transition temperaturc $s$ increase with increasing niobium concentration. A number of carlier investigations have produced results suggesting that the phase transitions in KTN are not simply displacive, and there are structural, diclectric, bulk, and microscopic indications that the phase transitions in KTN have some intrinsic order-disorder character.

In the present work, a light-scattering study of the low-frequency excitations in single crystals of cubic and tetragonal $\mathrm{KTa}_{1-x} \mathrm{Nb}_{x} \mathrm{O}_{3}$ ( $x=0.26$ and 0.28 ) has been carried out by employing a technique that uses an iodine filter to remove elastically scattered light. Low-frequency Raman and Fabry-Pérot components related to structural disorder in the mixed-crystal, tantalate-niobate system were observed. The spectral shape, symmetry properties, and thermal behavior of these components are consistent with an eight-site order-disorder model of the sequence of structural phase transitions observed in ferrodistortive perovskites.

1. Summary of paper: Phys. Rev. B 41, 2398 (1990)).

2. University of Arizona, Tucson, Ariz.

3. Lawrence Livermore National Laboratorics, Livermore, Calif. 
INFRARED STUDY OF OXYGEN VACANCIES IN KTaO,

\section{S. JandI, 2 M. Banoille, 2 P. Dufour,?} S. Coulombe, ${ }^{2}$ and L. A. Boutner

$\mathrm{KTaO}_{3}$ (i.e., potassium tantalate) is a quantum paraelectric or incipient ferroelectric that does not undergo a ferroelectric phase transition like $\mathrm{BaTiO}_{3}$ or $\mathrm{KNbO}_{3}$. Nevertheless, the $\mathrm{KTaO}_{3}$ dielectric constant does increase rapidly with decreasing temperature while its lowest infrared active mode softens. Accordingly, $\mathrm{KTaO}_{3}$ is a modet system in which to study soft-mode behavior and phonon-phonon interactions. ${ }^{3}$

One of the prominent impurities found in $\mathrm{KTaO}_{3}$ is hydrogen, as evidenced by the $\mathrm{OH}$ stretching vibration mode in the near-infrared at about $3500 \mathrm{~cm}^{-1}$. D. Houde et al." have claimed that the splitting of the $\mathrm{OH}$ mode observed at low temperakives is related to fuctuations of the ferroelectric soft mode. A. Jovanovic et al., 5 however, have attributed the $\mathrm{OH}$ mode side-bands to interactions with impurities and defect centers.

In the present work, the effects of oxygen vacancies on the ferroelectric soft mode and the $\mathrm{OH}$ stretching vibration in $\mathrm{KTaO}_{3}$ have been investigated by comparing the infrared spectra of high-purity and reduced high-purity samples. The temperature dependence of the reflectance in the range $30-1500 \mathrm{~cm}^{-1}$, the transmission in the vicinity of $3500 \mathrm{~cm}^{-1}$, and the Raman spectrum at $15 \mathrm{~K}$ of high-purity $\mathrm{KTaO}_{3}$ have been compared with the same propertics of reduced $\mathrm{KTaO}_{3}$ with nxygen vacancies. Phonons at critical points in the Brillouin zone were observed while disruption of the Ta-O-Ta chains in the reduced material generated (at all temperatures) an overdamping of the ferroelectric soft mode. The ferroelectric soft mode is strongly overdamped due to the induced disorder in the Ta-O-Ta chains, and consequently, the $\mathrm{OH}$ strctching-mode frequencies are affected pointing to the existence of an interaction between the $\mathrm{OH}$ radicals and the ferroelectric fluctuations in pure $\mathrm{KTaO}_{3}$.

1. Summary of paper: Physical Revicw B (in press).

2. Université de Sherbrooke, Québec, Canada.

3. R. L. Prater, L. L. Chase, and

L.A. Boatner, Phys. Rev. B 23, 221 (1981).

4. D. Houde et al., Fermelectrics 77, 55 (1988).

5. A. Jovanovic et al., Ferroelectrics 107,85 (1990).

\section{LONGITUDINAL ELASTIC BEHAVIOR IN THE MIXED FERROELECTRIC KTN ${ }^{1}$}

\section{M. Wang, ${ }^{2}$ I. Toulouse, ${ }^{2}$ and L. A. Boatner}

Because of the essential role played by anharmonic elastic forces and strain in ferroelectric transitions, elastic measurements can provide direct evidence of the various stages through which ferroelectrics pass when approaching a phase transition. In particular, the elastic properties of pure cubic perovskite ferroelectrics exhibit significant anomalies both above and below the phasc transition. The present work was carricd out with the goals of obtaining a comprehensive picture of 
the longitudinal lastic anomalies in KTN $\left(K \mathrm{Ta}_{1-\mathrm{x}} \mathrm{Nb}_{\mathbf{x}} \mathrm{O}_{3}\right)$ as a function of the nisbium concentration and of identifying the nature and origin of such elastic anomalies in mixed ferroclectrics.

In the case of mixed ferroclectrics like KTN, the transition was found to occur in two distinct stages-clearly revealed by ultrasonic measurements of the Jongitudinal $c_{i l}$ elastic constant. In the first stage, increasing anharmonicity (enhanced by the presence of niobium ions) results in a softening of $c_{11}$ with a $\left(T-T_{c}\right)^{-\mu}$ dependence. For high niobium concentrations, the exponent value is about 0.5 and can be explained by the interaction of the sound waves with small fluctuations of the polarization. At low concentrations, $\mu$ increases to 2 . In a second stage, $c_{I I}$ deviates from the above divergence indicating a change in the elastic bchavior. This change can be explained by the appearance of ferroclectric clusters, and it is accompanied by a steep rise in the ultrasonic attentiation.

1. Summary of paper: Ferroelectrics (in press).

2. Lehigh Universily, Bethlehem, Pa.

\section{FLUORESCENCE AND FERROELECTRIC MICROREGIONS IN KTaO ${ }_{3}{ }^{\mathfrak{I}}$}

P. Grenier, ${ }^{2}$ G. Bernier, 2 S. Jandl, B. Salce, ${ }^{3}$ and L. A. Boatner

Optical properties of impurities in ferroelectric and related materials are of great interest since they probe the lesal crystalline environment. In the case of mixed tantalates, Salce ef al. 4 have proposed that residual $\mathrm{Nb}$ or $\mathrm{Na}$ impurities should be at the origin of the ferroelectric regions. In the present work, we have investigated the fluorescence in the near-infrared region of pure $\mathrm{KTaO}_{3}$, $\mathrm{KTa}_{0.982} \mathrm{Nb}_{0.018} \mathrm{O}_{3}$, and doped $\mathrm{KTaO}_{3}$ samples. The luminescent impurity introduces an important charge and mass defect in the lattice and has the properties of a transition-meta! ion. It is also located in a ferroclectric microdomain and is universally present in either pure or doped $\mathrm{KTaO}_{3}$ samples, independent of the crystal growing procedure. In the neighborhood of $14,570 \mathrm{~cm}^{-1}, 11$ peaks are observed at $16 \mathrm{~K}$ that are associated with the fluorescence of $\mathrm{a} \mathrm{Ta}^{3+}$ ion nearby an oxygen vacancy. In the ferroelectric $\mathrm{KTa}_{0.982} \mathrm{Nb}_{0.018} \mathrm{O}_{3}$ crystal, the fluorescence is not affected below $T_{c}=25 \mathrm{~K}$ indicating that the $\mathrm{Ta}^{3+}$ inn in the $\mathrm{KTaO}_{3}$ materials is located in a ferroclectric microdomain.

This study identifies one of the possible origins of the symmetry-breaking defects responsible for microscopic ferroclectric regions that extend over a few unit cells in pure $\mathrm{KTaO}_{3}$.

1. Summary of paper: 1. Phys., Condens. Malter 1. 2515 (1989).

2. Universite de Sherbrooke, Quebec, Canada.

3. C.F.N.G., Grenoble, France.

4. B. Salce, A. M. De Coer, and

L. A. Boatner, I. Phys. 42, C6-424 (1981). 


\section{STUDY OF PROTONS IN ACCEPTOR-DOPED $\mathrm{KT} \mathrm{O}_{3}$ CRYSTALS BY IR AND EPR TECHNIQUES'}

\section{S. Q. Fu, ${ }^{2}$ W.-K. Lee, ${ }^{2}$ A. S. Nowick,}

L. A. Boatner, and M.M. Abraham

Although there have been many detailed studies of the defect chemistry of perovskite-structure oxides such as $\mathrm{BaTiO}_{3}$ and $\mathrm{SrTiO}_{3}$, it is only relatively recently that attention has been paid to the role of protons in materials of this type. A number of authors have shown that the presence of acceptor dopants makes it possible to introduce protons readily by treatment in water vapor. 3,4 It has been shown by means of infrared (IR) absorption measurements that an interstitial proton combines with an adjacent $\mathrm{O}^{2-}$ ion to form an $\mathrm{OH}^{-}$ center, thus the IR absorption due to $\mathrm{OH}^{-}$provides the most direct evidence for the presence of protons in the lattice. Of particular interest is the possibility of obtaining protonic conductors from such materials.

In order to understand the manner of incorporation of protons into perovskite-structure oxides, $\mathrm{KTaO}_{3}$ crystals containing acceptor dopants $\mathrm{Co}^{2+}, \mathrm{Mn}^{2+}, \mathrm{Cu}^{2+}$, and $\mathrm{Fe}^{3+}$ were investigated following thermal treatments in various partial pressures of $\mathrm{O}_{2}$ and $\mathrm{H}_{2} \mathrm{O}$. IR measurements were used to monitor the proton content through the $\mathrm{OH}^{-}$absorption bands, while quantitative EPR measurements were used to determine the changes in the local environment of the dopant ion. It was shown that the introduction of protons during high-temperature treatments in $\mathrm{H}_{2} \mathrm{O}$ is accompanied by the replacement of the oxygen-ion vacancies $\left(V_{0}\right)$ that initially compensated the acceptor dopants by $\mathrm{O}^{2-}$ ions. In the case of $\mathrm{M}^{2+}$ dopants, the dopant-vacancy-association free energy is sufficiently strong to retain the $M-V_{0}$ pairs even during high-temperature $\left(900^{\circ} \mathrm{C}\right)$ treatments; thus, only $\langle 100\rangle$ axial EPR spectra due to such pairs are observed. For $\mathrm{Fe}^{3+}$ dopants, however, virtually all of the $V_{0}$ can be replaced by $\mathrm{O}^{2}$, leaving the $\mathrm{Fe}^{3+}$ ion in a cubic environment. The results also show evidence for valence changes of the Co, Mn, and Cu dopants when treatments change from oxidizing to reducing atmospheres.

1. Summary of paper: I. Solid State Chen. 83, 221 (1989).

2. Henry Knumb School of Mines, Columbia Universily, New York, N.Y.

3. H. Eagstrom, J. B. Bates, and

L. A. Boatner, J. Chem. Phys. 73, 1073 (1980).

4. W.-K. Lee, A.S. Nowick, and

L. A. Boatner, Adv. Ceram. 23, 387 (1987).

\section{DETERMINATION OF THE OPTICAL FUNCTIONS OF TRANSPARENT GLASSES USING SPECTROSCOPIC ELIPSOMETRY}

$$
\text { C. E. Jellison, Jr., and B. C. Sales }
$$

In order to examine the suitability of the new two-channel spectroscopic ellipsometer for the study of insulators, measurements were performed on four different glasses. Figure $\mathbf{3 . 1 6}$ shows the data for one of these glasses (In-Pb-PO 4 ), expressed in terms of the effective refractive index $\langle n\rangle$ and extinction coefficient $<k>$. Since this glass is transparent in the visible, $k=0$; the uncorrected result that $<k>>0$ implics that there is a thin microrough over- 


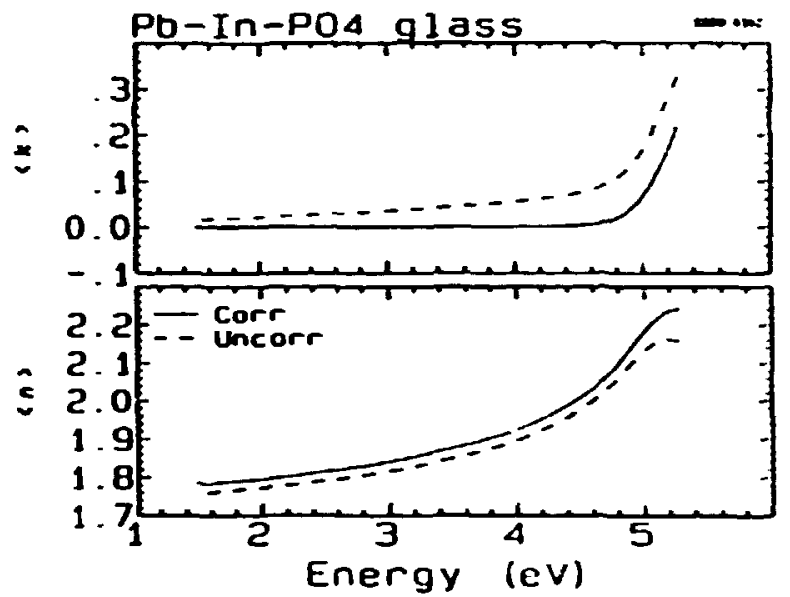

Fig. 3.16. The effective refractive index (bottom) and extinction coefficient (top) for In-Pb-PO 4 glass plotted vs photon energy. The solid lines represent the corrected data, using a rough interfacial layer, and the dashed lines represent the uncor:seicis values obtained directly from the ellipsometry data.

layer on the sample surface. If an interfacial region $-2.3 \mathrm{~nm}$ thick is now assumed and modeled using a Bruggeman effective medium consisting of $50 \%$ voids and $50 \%$ underlying matcrial, new calculated values of $n$ and $k$ can be obtained and are shown in Fig. 3.16. Since the predicted interface region is very thin and the expected result of $k \sim 0$ is obtained over an

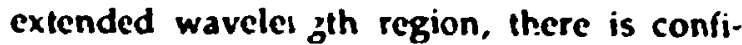
dence that this approximation is valid. Using this interface correction, the raw experimental data can be modified over the entire spectrum, yielding more accurate optical functions in all regions, including photon energies above the optical band gap.

Using this correction technique, the errors in the refractive index and extinction coefficient are \pm 0.002 . Clearly, these results are not nearly as accuratc as minimum deviation methods, but there are several advantages to this technique: (1) measurements on $\boldsymbol{n}$ and $k$ can be made for photon energies greater than the band gap of the material whare interface layers that arise from surface roughness effects are taken into account; (2) the spectroscopic measurements can be performed and analyzed in $-1 \mathrm{~h}$; (3) only one surface has to be carefuliy polished; even in this case, the effects of surface roughness resulting from the polishing can be taken into account; and (4) this technique provides a measurement of the thickness of a surface-roughness layer with dimensions less than the wavelength of light, providing information that is nut otherwise available.

1. Summary of paper to be published.

\section{USE OF THE BIASED ESTIMATOR RN THE INTERPRETATION OF SPECTROSCOPIC ELUPSOMETRY DATA'}

\section{E. Jellison, Ir.}

In order to interpret spectroscopic ellipsometric data properly, well-established numerical analysis procedures must be followed; unfortunately, the spectroscopic ellipsometry community has not been following these procedures. Ellipsometric data are often expressed by

$$
\rho(\lambda, \phi)=r_{p} / r_{3}=\tan \psi(\lambda, \phi) e^{i \Delta(\lambda, \phi)}
$$

where $r_{p}$ and $r_{s}$ are the complex Fresnel reflection ratios for light polarized parallel and 
perpendicular to the plane of incidence, respectively. The ratio $\rho$, as well as conventional ellipsometric angles $\boldsymbol{\psi}$ and $\Delta$, depends explicitly on the wavelength $\lambda$ and the angle of incidence $\$$.

In performing a fitting procedure, the data in the form cf Eq. 1 (or possibly some other. equivalent form) are compared with a calculated spectrum using some figure of merit. According to established numerical amalysis procedure, the proper figure of merit is the biased estimator, given by

$$
\begin{aligned}
& x^{2}=[1 /(n-m-1)] \\
& \times \sum_{i=1}^{n}\left[\left|p_{\exp }\left(\lambda_{i}\right)-p_{\text {calc }}\left(\lambda_{i} z\right)\right| 2 /\left|\delta \rho\left(\lambda_{i}\right)\right| 2\right] .
\end{aligned}
$$

The index $i$, which is the number of wavelength points in the spectrum, goes from 1 to $\pi$, and the vector $z$ (with $m$ components) represents the parameters in the calculation model, such as film thickness, constituent fractions, or Sellmeier coefficients. The fitting procedure minimizes $\chi^{2}$, using a numerical analysis method such as that due to LevenbergMarquardt.

The standard way in which spectroscopic ellipsometry data are fit uses the unbiased estimator, where $\delta \rho\left(\lambda_{i}\right)=1$. This procedure has three major flaws: (1) very accurate parts of the spectrum are weighted the same as inaccurate parts, distorting the final fit; (2) different representations of the ellipsometric data (such as $\psi$ and $\Delta$ ) will result in a different fit and figure of merit; and (3) the unbiased estimator has no statistical significance.

The biased estimator is obtained by including the errors in Eq. 2, whereby the three flaws mentioned above are corrected: (1) the fit is now spectrally weighted; (2) the fit results (including the figure of merit) are independent of representation, if the error is propagated into the new representation; and (3) the biased estimator is a meaningful quantity. As a rule of thumb, the fit is good if $\chi^{2}-1$. If $\chi^{2} * 1$, then the fit deviates significantly from the experimental data, while if $\chi^{2} \times 1$ for too many samples and situations, the error may have been overestimated.

1. Summary of paper to be published.

\section{ACCURATE CALIBRATION OF A PHOTO- ELASTIC MODULATOR IN POLARIZATION MODULATION ELIPSOMETRY ' \\ G. E. Jellison, Ir., and F. A. Modine}

A photoelastic modulator is a device that uses the photoelastic effect to impart a timedependent phase retardation to a light beam; photoelastic modulators have been used for many years in spectroscopic ellipsometry experiments. However, in order to perform very accurate polarization modulation ellipsometry experiments, it is essential that the modulator be properly calibrated.

Techniques have been developed to determine (1) the drive voltage (which determines the amplitude of the modulation) as a function 
of wavelength, (2) the modulator static strain as a function of wavelength, and (3) the azimuthal-angular orientation of the photoelastic modulator with respect to other optical elements in the system and to the sample itself.

The modulator-drice voltage $V_{m}$ is determined by setting the ellipsometer in the straight-through configuration (no sample) and orienting the modulator azimuthal angle $\theta_{m}$ at $\pm 45^{\circ}$ with respect to the analyzer-azimuthal angle $\theta_{e}$. The proper value of $V_{m}$ is obtained when the dc light intensity is just one-half that when the modulator is turned off. The staticstrain $\delta$ is obtained in the same configuration by measuring the ratio of the fundamental intensity ( $50 \mathrm{kHz}$ in our rase) to the dc light intensity. If the values of $V_{m}$ and $\delta$ are properly determined, ther, $V_{m} \delta=$ constant.

Proper orientation of the angles $\theta_{m}$ and $\theta_{a}$ is obtained in the ellipsometer configuration (light reflecting off a sample), where $\theta_{m}-\theta_{a} \sim 0^{5}$. The angle $\theta_{a}$ is adjusted such that the fundamental intensity goes to zero, and $\theta_{m}$ is adjusted such that second-harmonic intensity goes to zero. Since the zero position of these angles can easily be set to $\pm 0.02^{\circ}$, the accuracy of any other angle setting is preduminantly a function of the accuracy of the rotators used to set the azimuthal angles.

1. Summary of a paper: SPIE 116E, 231 (1989).

\section{TEMPERATURE DEPENDENCE OF SINGLY AND DOUBLY IONIZED ACCEPTOR-HOLE RECOMBNATION IN GERMANIUM ${ }^{l}$}

\section{S. Darken ${ }^{2}$ and G.E. 'ellison, Ir.}

Recombination of mobile carriers with attractive centers is important to understanding a variety of processes in semiconductors. In paiticular, nuclear radiation detectors fabricated from high-purity Ge and operated as depleted diodes at $7 \mathbf{K}$ must have effective carrier-drift lengths $>10 \mathrm{~m}$ without recombination or trapping. In this work, the temperature dependence of hole recombination at several acceptor centers in high-purity $G e$ is reported.

The recombination kinetics of several centers $\mathrm{Zn}^{-}, \mathrm{Cu}^{-}, \mathrm{B}^{-}, \mathrm{CuH}_{x}^{-} \mathrm{CuH}_{2}, \mathrm{Zn}^{-}, \mathrm{Cu}^{=}$, and $\left.\mathrm{CuH}_{\mathrm{x}}{ }^{\bar{*}}\right)$ in high-purity Ge were measured as a function of temperature from 8 to $160 \mathrm{~K}$ by transient capacitance techniques and were found to be significantly faster than expected from cascade theory (Fig. 3.17). The cascade theory also gives the wrong temperature dependence and the wrong dependence on the charge $z$ of the scattering center. Therefore, the data are generally fit by the expression $N_{v} / 4 p \tau_{c} \equiv k T / h$ ( $p$ and $\tau_{c}$ are, respectively, the free-hole concentration in the sample and the experimental mean-caplure time for a center). $N_{0}$ is the cffective density of states in the valence band, and $h$ is Planck's constant. $N_{v} / 4 p \tau_{\text {r }}$ is the pre factor in the expression for emission e from the ground state obtained via detailed balance

$$
e=\frac{1}{\tau_{e}}=\frac{N_{v}}{4 p \tau_{c}} \exp \frac{-\Delta G}{k T} .
$$




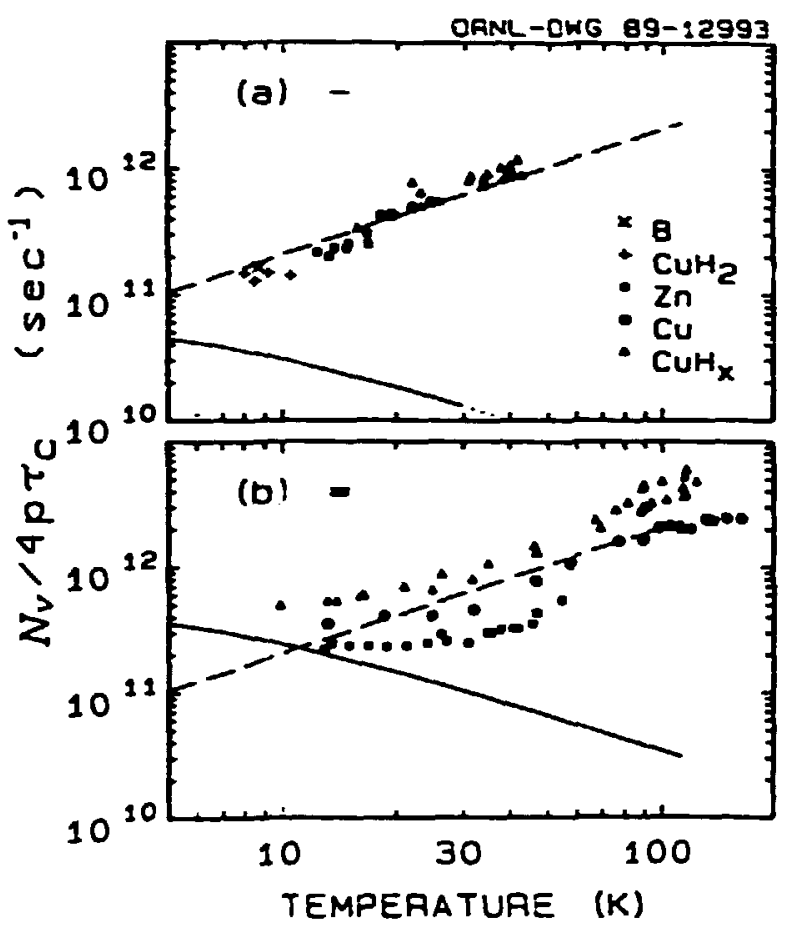

Fig. 3.17. Recombination data for acceptors in germanium (a) $z=1$ and (b) $z=2$. Data for a ceiter are typically from two or more samples. The solid lines represent the expected value from the cascade theory, while the dashed line represents $k T / h$.

In this expression, $\Delta G$ is the Gibbs-free energy for ionization less the entropy term for the change in electronic degeneracy. Thus, $N_{u} / 4 p \tau_{c}$ may be roughly considered an "attempttoescape" frequency. The results of this work infer that this attempt-to-escape frequency is that of thermal vibrations.

1. Summary of paper: Appl. Phys. Lelt. 55, i424 (1989).

2. Tenrelec, Oak Ridge, Tenn.
EXAMINATION OF THIN SiO 2 FILMS ON Si USING SPECTROSCOPIC POLARRATION MODULATION ELIPSOMETRY!

G. E. Jallison, Ir.

In order to characterize properly the sensitivity of the new two-channel polarization modulation ellipsometer, a series of $\mathrm{SiO}_{2}$ films were grown on Si and spectroscopic ellipsometry measuremk nis were made. This new ellipsometer is particularly effective for very thin films of $\mathrm{SiO}_{2}$ on $\mathrm{Si}$, since it can measure the ellipsometric parameter $\Delta$ very accurately when $\Delta$ is near $0^{\circ}$ or $180^{\circ}$. Particular care was taken to reduce errors to a minimum and to incorporate the experimental errors into the fitting procedure using a biased estimator as a figure of merit.

The spectroscopic ellipsometry data were fit to a four-medium structure model, consisting of air, $\mathrm{SiO}_{2}$, an interface layer, and crystalline Si. The refractive index $\boldsymbol{n}$ of $\mathrm{SH}_{2}$ was approximated using a single-term Sellmeier expression $\mid n^{2}-1=A \lambda^{2} /\left(\lambda^{2}-\lambda_{0}{ }^{2}\right)$, where $\lambda$ is the wavelength of light, and $A$ and $\lambda_{0}$ are the fitting parameters], while the optical functions of the interface layer were calculated using an effective medium approximation, assuming 50\% $\mathrm{Si} / 50 \% \mathrm{SiO}_{2}$.

The fits to the experimental data show that the Sellmeier coefficient $A$ (or equivalently, the refractive index) increases with decreasing $\mathrm{SiO}_{2}$ film thickness (Fig. 3.18). Even for the thickest samples, the $A$ coefficient is significantly larger than $A$ for fused quartz, 


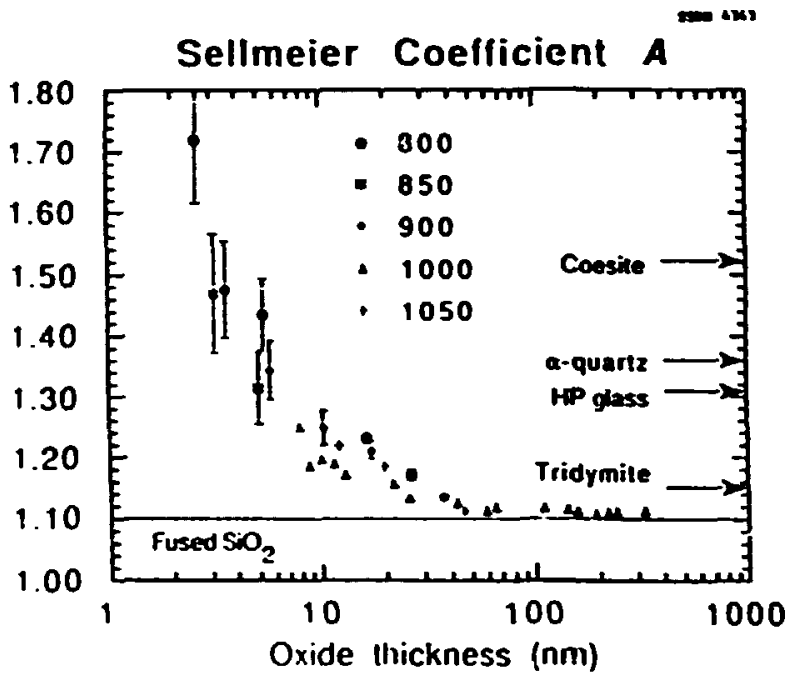

Fig. 3.18. Sellmeier coefficient $A$ plotted vs the $\log$ of the thickness of the $\mathrm{SiO}_{2}$ layer. The values of $A$ for various high-index polymorphs of crystalline $\mathrm{SiO}_{2}$ are shown to the right by the arrows. The straight line at $A=$ 1.099 corresponds to the $A$ for fused silica. The data sets are labeled by their growth temperatures.

showing that thin-film $\mathrm{SiO}_{2}$ is denser than fused quartz. For the very thinnest films, the $A$ parameter becnmes as large as the $A$ parameter for the denser forms of crystalline $\mathrm{SiO}_{2}$, such as coesite.

For the thicker films, the fits indicate that there is an interface region between the $\mathrm{SiO}_{2}$ and the $\mathrm{Si}$ substrate. The thickness of this region increases with increasing film thickness, and the nature of the interface layer is consistent with a microroughness layer.

1. Summary of paper to be published.

\section{TRAPPED-HOLE CENTERS IN ALKALINE-EARTH OXIDES'}

\section{Y. Chen and M.M. Abraham}

Wide-band-gap materials, such as refractory oxides and alkali halides, are commonly used as hosts for tunable lasers. The active component in these materials can either be an impurity dopant, such as $\mathrm{Ti}: \mathrm{Al}_{\mathbf{2}} \mathrm{O}_{\mathbf{3}}$, or a vacancy-impurity complex, such as the $F_{A}$ center in the alkali halides. A serious impediment to laser action in a given system, however, is solarization (i.e., the tendency of a crystal to color upon either optical excitation or exposure to ionizing radiation such as e'ectrons or $X$ rays). This coloration is due to absorption that may be detrimental to laser action. Such absorption in the oxides is frequently caused by the trapping of holes by cation vacancies that are charge compensated by aliovalent impurities (Fig. 3.19).

The production mechanism of the trappedhole center by ionizing radiaticn is obviously quite different from that of its antimorph, the anion vacancy or $F$-type center. Whereas $F$ type centers are produced by clastic collisions, the trapped-hole centers are produced by the capture of holes by existing vacancies. Aliovalent impurities are always present and may be either cations or anions. Impurities such as $\mathrm{H}, \mathrm{F}, \mathrm{Al}, \mathrm{Si}$, and $\mathrm{Fe}$ are ubiquitous in the starting powders used in the crystal growth, and trapped-hole centers are caused by existing cation vacancies which are formed via valence compensation of these aliovalent impurities. Even after a long gamma irradiation of the alkaline-carth oxides, there may be a substan- 


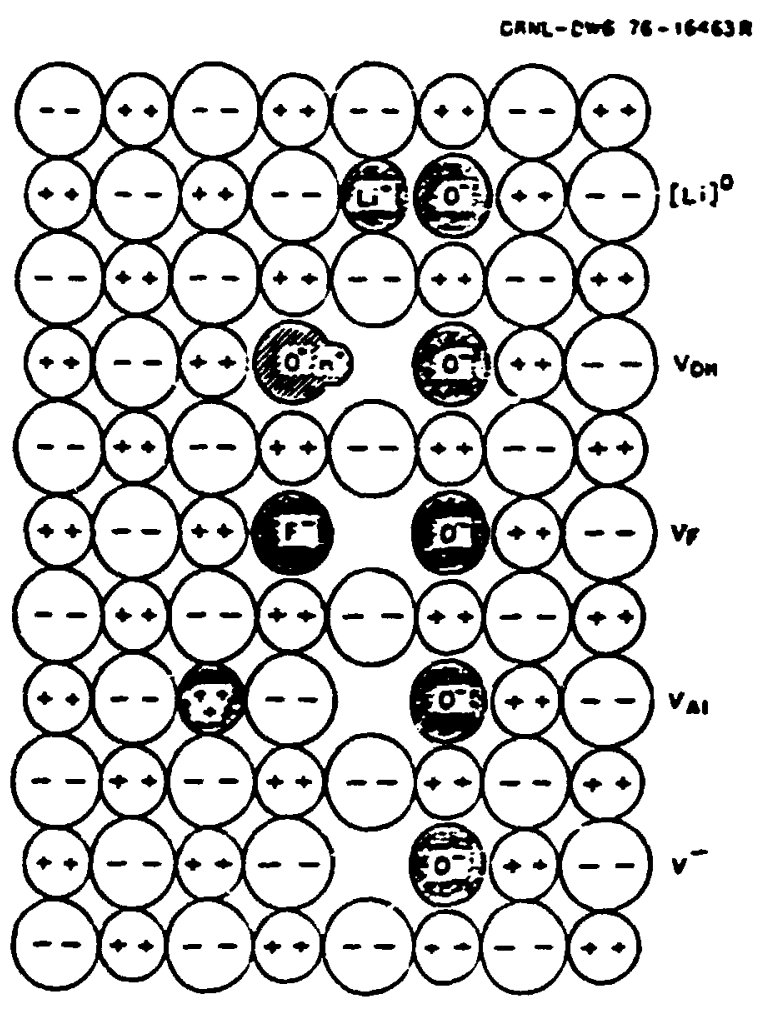

Fig. 3.19. Schematic depiction of five trapped-hole defects for $\mathrm{MgO}$ in order of increasing distance of the aliovalent impurity from the trapped-holc: [Li] ${ }^{\circ}, V_{O H}, V_{F}, V_{A l}$, and $\mathrm{V}^{-}$centers. The $\mathrm{O}^{-}$ion represents a hole lucalized on an $\mathrm{O}^{2-}$ ion site.

tial number of positive-ion vacancies that have not trapped holes. The concentration of trapped-hole centers is determined not only by the concentration of positive-ion vacancies, perturbed or otherwise, but also by the availability of impurities that can trap electrons during ionizing radiztion. Irradiation with fission neutrons tends to suppress trapped-hole centers, in general. There are two competing mechanisms: suppression of trapped hole centers due in lattice damage by the massive neu. trons and enhancement of trapped hole centers due to Compton electrons caused by gamma rays attendant to the neutron irradiation. The existence of trapped-hole centers appears to be quite common in oxide crystals, simply by virtuc of the existence of aliovalent impuritics and car :. as much as $10^{18} \mathrm{~cm}^{-3}$ in materials such as $\mathrm{MgO}, \mathrm{CaO}, \mathrm{SrO}$, and lanthanum magnesium aluminate.

1. Summary of paper: I. Phys. Chem. Solids 51,747 (1990).

\section{PRUSPECTS FOR WAVELENGTH TUNABLE LASERS BASED ON VACANCY DEFECTS IN ALKALINE-EARTH OXIDES'}

\section{Y. Chen, V. M. Orera, ${ }^{2}$ and R. Gonzales 3}

Research on color centers in the alkali halides has proven fruitful in that the recent development of tunable lasers was a direct outgrowth of such studies. It is expected that research on the alkaline-earth oxides will also yield similar results in this area. Vacancies suct as the $t^{*}$ center (an anion vacancy with one electror which is positively charged with respect to the lattice) constitute a four-level system and generally provide a higher gain than transition-metal or rare-carth ions. As in the alkali halides, the two methods for producing anion vacancies in the oxides are either by irradiation with energetic particles or by thermochemical reduction (TCR). There are, however, aiso problems fur TCR-specifically, crystal darkening and long-lived phosphorescence. In the first casc, transmission clectron 
microscopy studies in $\mathrm{MgO}$ have revealed the presence of Fe and $\mathrm{Fe}-\mathrm{Cr}_{\mathrm{r}}$ precipitates that cause Mie scattering. The second problem associated with TCR is F-center phosphorescence. We have identified the cause of this phosphorescence as hydride $(\mathrm{H})$ ions. The problems of hydride ions and Mie scattering from second phases in TCR crystals can be resolved. The most serious problem encountered in attempting to use anion vacancies as a laser-active color center, however, is of an intrinsic nature: photoconversion. It is independent of the method of defect production, whather by. TCR or neutron irradiation. Therefore, to avoid this problem, it is necessary to find a way to localize the electronic wave functions at the defect. One possibility is to introduce a perturbing defect.

1. Summary paper to be published.

2. Guest scientist from University of Zaragoza, Zaragoza, Spain.

3. Guest scientist from University of Madrid Complutense, Madrid, Spain.

\section{EFFECT OF SUBSTITUTIONAI HYDRIDE IONS ON THE CHARGE STATES OF OXYGEN VACANCIES IN THERMOCHEMICALY REDUCED CaO AND MgO'}

Y. Chen, V.M. Orera, 2 R. Gonzalez, 3 R.T. Williams, G. P. Williams, G. H. Rosenblatt, and C. I. Pogatshnik

Continuous-wave laser action based on the $F^{*}$ center and the $F$ center in an electronirradiated $\mathrm{CaO}$ crystal has been reported by others. 6,7 However, the reproducibility of these results has not been achieved. Themf, e, the present investigation was undertaken to examine the optical properties of these defects further in order to shed new light on this discrepancy. In the alkaline-earth oxides, anion vacancies are often produced by thermochemical reduction (TCR) or bombardment with energetic particles. In the present work, the optical properties of crystals from which protons had been removed either before or during TCR have been examined.

Different methods were used to produce thermochemically reduced $\mathrm{CaO}$ and $\mathrm{MgO}$ crystals with different hydride concentrations and, as a result, strongly contrasting optical characteristics. The excited states of the $F$ center in both $\mathrm{MgO}$ and $\mathrm{CaO}$ are sufficiently close to the conduction band $(-0.01$ and $=0.1 \mathrm{eV}$, respectively) that photoconversion is inevitable, as evidenced by the trapping of electrons by hydride ions. Therefore, the $F$ center is not a viable candidate for tunable laser;. The situation for the $\mathrm{F}^{+}$center is less obvious. The magnitude of the absorption coefficient of the trapped-hole $V$-type centers formed is, however, of the order of a few $\mathrm{cm}^{-1}$ and, in our estimation, is more than enough to negate any gain derivable from the $\mathrm{F}^{+}$centers. Additionally, in $\mathrm{CaO}$ the $F$ absorption band overlaps the $F^{+}$ emission band. As a result, exciting the $F^{+}$ center is tantamount to exciting the $F$ center simultaneously. Finally, recent time-resolved studies of luminescence spectra from $F$ and $F^{*}$ centers in $\mathrm{MgO}$ and $\mathrm{CaO}$ suggest that the $F^{+}$ first-excited state lies close to the conductionband edge. ${ }^{8}$ Because of the instability of the 
excited states of both $F$ and $F^{*}$ centers against ionization (by electron or hole release), it is concluded that tunable lasers based on the intrinsic $F$ or the $F^{+}$centers in $\mathrm{C}=\mathrm{O}$ and $\mathrm{MgO}$ ase not possible.

1. Summary of paper: Phys. Reo. 3 42, 1410 (1990).

2. Guest scientist from Us iversity of Zaragoza, Zaragoza, Spain.

3. Guest scientist from University of Madrid Complutense, Madrid, Spain.

4. Wake Forest University, WinstonSalem, N.C.

5. Southem Illinois University, Edwardsville, II.

6. B. Henderson, Opt. Lett. 6, 437 (1981).

7. B. Henderson, p. 383 in Proceedings of the International Conference on Lasers, ed. by Cart B. Collins, STS, McLean, Va, 1981.

8. G. H. Rosenblatt et al., Phys. Reo. B 39, 10309 (1989).

\section{ANALYSIS OF SOLDIFICATION MICROSTRUCTURES IN FE-NI-C STNGLE-CRYSTAL WELDSI}
M. Rappaz,, S. A. David, 3
J. M. Vitek, ${ }^{3}$ and L. A. Boatnes

The development of the fusion-zone grain structure in weld metal is a complex phenomenon in which it is difficult to identify the extent to which growth crystallography and the direction of the thermal gradient influence the grain growth selection process and the associated microstructural growth behavior in a polycrystalline weld metal. However, by making welds along specific crystallographic directions on a well-characterized single crystal, it is possible to relate the combined influence of the growth crystallography and the heat flow direction to the derelopment of weld-metal microstructures.

A geometric analysis technique for the evaluation of the microstructures in autogenous single-crystal electron beam welds has been developed, and a general procedure for predicting the scidification microstructure of single-crystal welds with any arbitrary orientation established. It was shown that a nonsymmetric cell structure is expected in transverse micrographs for most welding geometries. The development of steady-state conditions in the weld pool was also examined in terms of the weld-pool size, its shape (as revealed by the dendritic growth patterm), and the size of the dendritic cells. It was found that a steady state is established within a few millimeters of the beginning of the weld. A general analysis of the three-dimensional (3-D) weld-pool shape based on the dendritic structure, as revealed in two-dimensional (2-D) transverse micrographs, was also developed. It was shown that, in combination with information on the preferred growth direction as a function of the solidification front orientation, the entire dendritic growth pattern in single-crystal welds can be predicted. Finally, the theoretical analysis of the dendrite tip radius was extended from binary systems to include the case of ternary systems. The theoretical dendrite trunk spacing in a ternary $\mathrm{Fe}-\mathrm{Ni}-\mathrm{Cr}$ alloy was calculated from the dendrite tip radius, and in Fig. 3.20, computed variations as a function of tip velocity are compared with the 


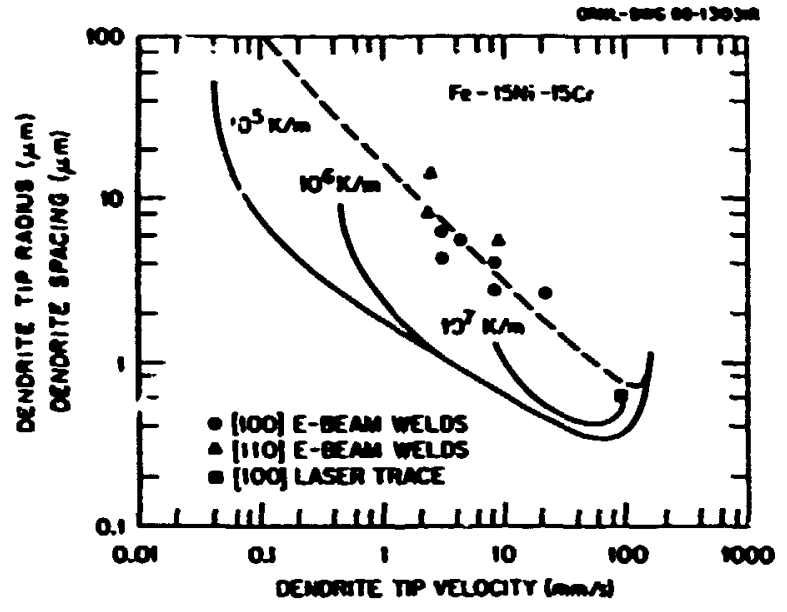

Fig. 3.20. A plot of dendrite tip radius vs dendrite tip velocity for an $\mathrm{Fe}-15 \mathrm{Ni}-15 \mathrm{Cr}$ alloy with three different assumed temperature gradients. The dashed curve correspords to the dendrite trunk spacing calculated using the computed dendrite tip radii shown in this figure. The data points (circles and triangles) correspond to previously measured dendrite trunk spacings in electron beam welds ([100) and [110] welds, respectively). The square point is the trunk spacing measured for the $[001]$ dendrites in a laser beam run.

experimental values for several weld conditions.

1. Summary of paper: Metall. Trans. A 21, 1767 (1990).

2. Ecole Polytechnique Fédérale de Lausanne, Lausanne, Switzerland.

3. Metals \& Ceramics Division, ORNL.

\section{MICROSTRUCTURE OF STANLESS STEEL SINGLE-CRYSTAL ELECTRON BEAM WELDS ${ }^{1}$}

\section{S. A. Darid, ${ }^{2}$ I. M. Vitek, \\ M. Rappaz, ${ }^{3}$ and L. A. Boaliner}

The development of the fusion-zone grain structure in a weld is prim rily controlled by the base-metal grain structure and the urelding conditions. The base metal acts as a substrate upon which growth of the solid phase occurs epitaxially, and both crystallographic effects and welding conditions (e.g., welding speed and other process parameters, such as heat and thic flow) can influence the development of microstructures in the fusion zone. Techniques for the microstructural analysis of welds, that include effects of both the growth crystallography and weld-pool shape, have been applied to several cases involving the single-crystal electron beam welding of an Fe-15Ni-15Cr alloy.

This evaluation of weld microstructures and associated dendritic growth patterns is based on a three-dimensional geometrical analysis. It includes examinations of the effects observed in overlapping, multipass-autogenous welds and bult welds of two single crystals with different orientations, as well as effects due to variations in the welding speed. The weld-pool shapes were found to change significantly with increasing welding speed. Additionally, all of the electron beam welds showed evidence of a plateau region in the center of the weld pool. The pool shapes, howcver, were found to be independent of the crystallographic orientation. Therefore, it is possible to extend the pool-shape results to crystals welded in any orientation and even to polycrystals.

The overlapping multipass welds showed remarkable reproducibility from pass-to-pass, as shown in Fig. 3.21, and duplicated the struc- 

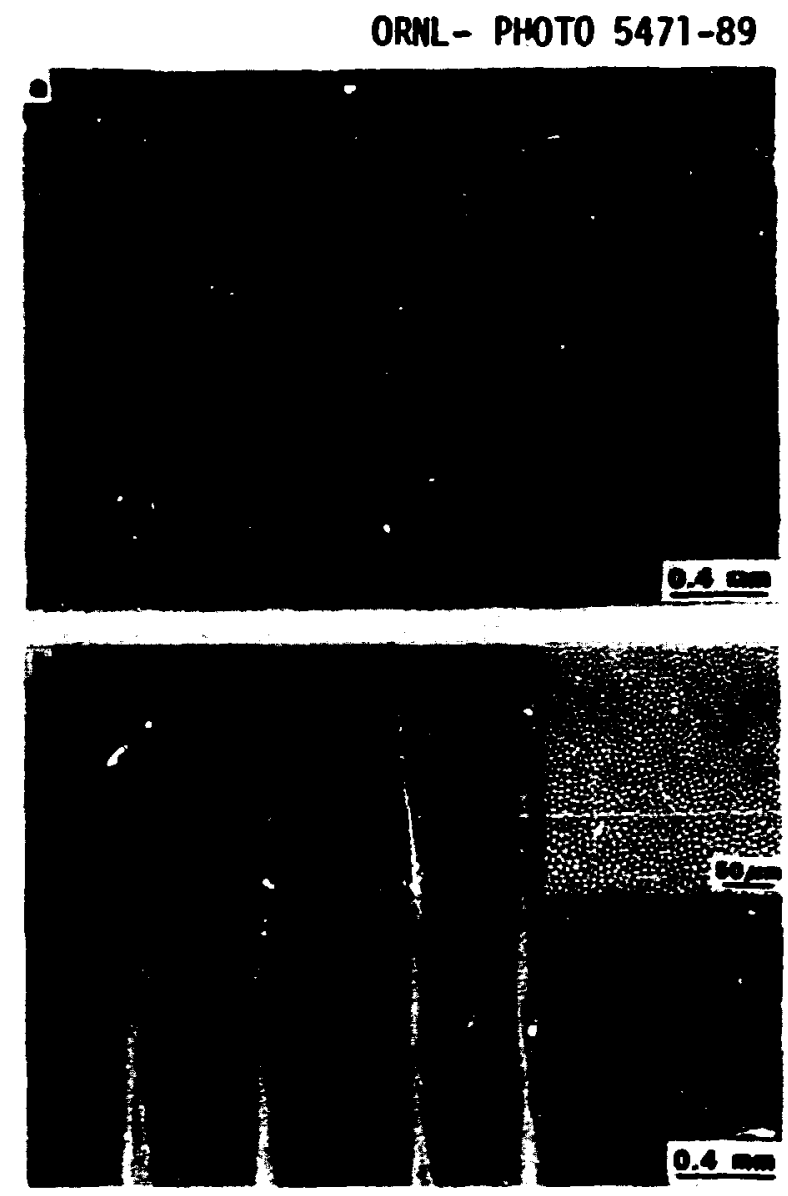

Fig. 3.21. Transverse sections of multipass overlapping welds made on a single crystal of Fe-15Ni-15Cr at $4.2 \mathrm{~mm} / \mathrm{s}$ along a [100] direction and on an (001) surface. The magnified insert in (b) shows the "honeycomb" dendritic arrangement.

tural pattems found in single-pass welds. The similarity in dendritic patterns within each pass indicates that the weld-pool shapes were identical in all the passes. The microstructure of butt welds of two single crystals with different relative orientations showed a remarkable relationship to that associated with cach individual crystallographic orientation, and the microstructure was, in effect, simply a com- posite of two single-pass microstructures. The results show that modeling of weld-pool shapes can be directly carried out by comparing the predirted dendritic growth patterns based on the modeled shapes with the actual experimentally observed dendrite growth patterns.

1. Summary of paper: Metall. Truns. A 21, 1753 (1990).

2. Metals and Ceramics Division, ORNL.

3. Ecole Polytechnique Fédérale de Lausanne, Lausanne, Switzerland.

\section{ANOMALOUSLY SMAL YSSA OSCILATOR STRENGTHS AND \&f-4 ELECTRONIC RAMAN SCATTERING CROSS SECTIONS FOR Ceit IN CRYSTALS OF LnPOA}

\author{
G. M. Williams, 2 N. Edelstein, 2 \\ L. A. Boatner, and M.M. Abraham
}

Transparent crystals containing trivalent rare-earth ions form a unique and interesting class of optical materials, and accordingly, a great deal of effort has been directed toward establishing a quantitative description of the intensities of optical processes in these crystals. Recently, Judd ${ }^{3}$ has derived a simple expression for the sum of oscillator strengths for transitions of the type $4 f^{N} \rightarrow 4 f^{N-1} 5 d$. Using this expression, oscillator strength sums were computed for $\mathrm{f} \rightarrow d$ transitions in $\mathrm{Ce}^{3+}, \mathrm{Tb}^{3+}$, and $\mathrm{Bk}^{3+}$ and compared to the observed values for these ions in aqueous solutions. It was found that the calculated values exceeded the obscrved values by factors ranging from 2 to greater than 10 . This result is relevant to the intra-4 N one- and two-photon transition inten- 
sities because the expressions describing these intensities contain matrix elements of the electric-dipole operator between states of the $4 f^{N}$ and $4 f^{N-1} 5 d$ configurations. Thus, if the measured $4 f^{N}$ and $4 f^{N-1} 5 d$ oscillator strengths are smaller than theoretically expected, this implies that the intensities of the intra- $4 f^{N}$ one- and twr-photon transitions also should be smaller than expected. This idea can be readily tested by comparing the observed absolute cross sections to those cakculated from theory.

In the present work, the oscillator strengths for the $4 f \rightarrow 5 d^{\prime}$ transitions of $\mathrm{Ce}^{3+}$ in LuPO, were measured from absorption spectra and compared to calculated values. The measured oscillator strengths were found to be between 2.5 and $\mathbf{2 0}$ times smaller than the corresponding theoretical values. In addition, absolute cross sections for electronic Paman scattering between the levels of the $4 f^{4}$ configuration of $\mathrm{Ce}^{3+}$ in LuPO, were measured and found to be significantly smaller than those expected from theory. Both of these discrepancies may be explained by a reduction in the radial integral, $\langle 4 f|r| 5 d \mid\rangle$, for $\mathrm{Ce}^{3+}$ in the solid state. Absorption data obtained from the literature for the $4 f \rightarrow 5 d^{1}$ transitions of $\mathrm{Ce}^{3+}$ in a number of host crystals were used to establish a correlation betwcen the cerium-ion-ligand distance and the reduction in the $\langle 4 f f|5 d|\rangle$ integral.

1. Summary of paper: Phys. Rev. B 40,4143 (1989).

2. Lawrence Berkelcy Latoratory, Berkeley, Calif.

3. B. R. Judd, Inorg. Chim. Acla 139, 341 (1987).

\section{EXCTATION PROFILES OF RESONANCE ELECTRONIC RAMAN SCATTERING IN ERPO, CRYSTALS'}

\section{G. M. Williams, 2 P. C. Becker, 2 N. Edelstein,2 L. A. Boatner, and M. M. Abraham}

Resonance enhancements of the intensities of electronic Raman scattering transitions between crystal-field levels of the ground $\mathbf{I}_{15 / 2}$ multiplet of $\mathrm{Er}^{3+}\left(4 \mathrm{f}^{11}\right)$ in erbium-doped phwsphate crystals have been reported previously. ${ }^{3}$ The previous results were unusual in that the intensity enhancements were anomalously large (on the order of 10-100). New results have now been obtained by varying the frequency of the exciting light. The intensities of electronic Raman scattering transitions between the ground and excited crystal-field states of the ground $\mathrm{I}_{15 / 2}$ multiplet of $\mathrm{Er}^{3+}$ in crystals of ErrO, have been measured as a function of excitation frequency in the region of an intermediate-state resonance between the $I_{15 / 2}$ ground state and a crystal-field state of the ${ }^{4} F_{7 / 2}$ multiplet. It was shown that for excitation frequencies near the intermediate-state resonance, the observed spectra are the result of electronic Raman scattering and are not due to absorption followed by fluorescence. Enhancements of the intensities of the electronic Raman scattering on the order of 100 were found. A striking feature of the data is the observed asymmetry in the excitation profile about the resonance-a feature that is attributed to interference between nonresonant and resonant scattering amplitudes.

A general understanding of resonances of this type is uscful in analyzing the resonant 
enhancement of other inherently weak multiphonon processes such as two-photon absorption and four-wave mixing.

1. Summary of paper. Phys. Reo. B 10,1288 (1989).

2. Lawrence Berkeley Laboratory, Berkeley, Calif.

3. P. C. Becker et al., Opt. Lett. 11, 282 (1986).

\section{INIENSTIES OF ELECTRONIC RAMAN} SCATTERING BETWEEN CRYSTALFIELD LEVELS OF $\mathrm{C}^{3+}$ IN LUPO: NONRESONANT AND NEAR-RESONANT EXCTTATION!

\section{G. M. Williams, ${ }^{2}$ P. C. Becker,}

J. G. Conway, ${ }^{2}$ N. Edelstein, 2

L. A. Boatner, and M.M. Abraham

$\mathrm{Ce}^{3+}$ has a unique electronic structure that makes it an interesting case for electronic Raman scattering studies. Two important features of $\mathrm{Ce}^{3+}$ are: (1) $\mathrm{Ce}^{3+}$ has only one optically active if electron. This lends simplicily to all of the calculations and reduces the numbet of physical interactions that may contribute to the electronic Raman srattering process, and (2) $\mathrm{Ce}^{3+}$, relative to the other trivalent rareearth ions, has a first-excited configuration (5d) that is rather low in energy. For $\mathrm{Ce}^{3+}$ in LuPO, the states of the $5 d^{l}$ configuration span the range from 30,000 to $50,000 \mathrm{~cm}^{-1}$. This low energy of the first-excited configuration makes $\mathrm{Ce}^{3+}$ an sspecially stringent test of the clc'sure approximation used in the standard electronic Raman scattering theory.
In this work an electronic Raman investigation of $\mathrm{Ce}^{3+}$ in $\mathrm{LuPO}_{4}$ has been carried out. The results were used to locate and determine the symmetries of all the states associated with the if configuration of $\mathrm{Ce}^{3+}$. The energies of the $5 d^{\prime}$ configuration have been determined from optical-absorption measurements.

The relative intensities of the electronic Raman scaltering between individual crystalfield states of the $4 f^{1}$ configuration of $\mathrm{Ce}^{3+}$ in LuPO, hame been compared te those calculated by using the standard second-order theory and also by explicitly evaluating the sum over the virtual intermediate states using the crystalfield wave functions and observed energies of the $5 d^{1}$ configuration. The results show that the explicit calculation predicts the observed relative intensities much more accurately than the standard theory. In addition, a change in the incident laser energy from the argon-ion green line $(514.5 \mathrm{~nm})$ to that of the frequencytripled output of a $\mathrm{Nd}^{3+}$ YYA $(355 \mathrm{~nm})$ laser results in enhancements of the electronic Raman scattering intensities by factors on the order of 100. These enhancements are accurately predicted by the explicit calculation.

1. Summary of paper: Phys. Reo. B 40,4132 (1989).

2. Lawrence Berkeley Laboratory, Berkeley, Calif. 
A PHOTOVOLTAIC-FOWERED MICROTRANSMIITER USED FOR MATING-FABIT STUDIES OF AFRICANIZED BEES

R. D. Westbrook,', G. E. Jellison, Ir., P. H. Flewing, D. D. Faller, ${ }^{2}$ G. T. Alley, ${ }^{2}$

K. G. Faller, ${ }^{2}$ and J. M. Rochelle 2

A photovoltaic-powered infrared microminiature transmitter and tracking system is being developed at ORNL for the purpose of contimuously monitoring the movements of the Africanized bee. The first prototype transmitter (measuring $4 \times 6 \mathrm{~mm}$ and weighing $65 \mathrm{mg}$ ) is powered by an array of nine series-connected $1-\mathrm{mm}^{2}$ solar cells, which are used to charge a $1-\mu F$ capacitor. The capacitor discharges through a GaAlAs (830-nm) laser diode, emitting -5ys light pulses from 50 to 300 times per second. The prototype receiving unit consists of an 8* Schmitt-Cassegrain telescope, a narrow. bandwidth interference filter, and a Si-photodiode detector. Field tests of the system have shown that a signal-to-noise ratio of $7 \mathrm{can}$ be obtained with the transmitter placed -1700 ft from the receiver on an overcast day.

The nine $1-\mathrm{mm}^{2}$ pholovoltaic cells were made from 0.2-0.3 $\Omega-\mathrm{cm}$ p-type silicon, using glow-discharge implantation for function for- mation, followed by a thermal-heat treatment io anneal the damage and to grow a $-15 \mathrm{~nm}$ of $\mathrm{SiO}_{2}$ overlayer for front surface passivation. No antireflection coating was used for these cells. Measurements of the photovoltaic properties of the $1-\mathrm{mm}^{2}$ cells showed that $I_{\text {sc }}=$ 23-24 mA $/ \mathrm{cm}^{2}, V_{o c}=0.595-0.605 \mathrm{~V}$, and efficiencies ranged from 10.3\% to 11.7\%. Shunt resistances generally were large $(>10,000 \Omega)$, while series resistances were moderate (1.92.4 Q) due to the wire bonding used to comnect the cells to the pads for measurement.

An I-V curve was determined for the 9-series-connected cells, illuminated with AM 1.0 simulated sunlight. A fill factor of $-71 \%$ was obtained, in spite of the large series resistance arising from the wire bond interconnects. The open-circuit voltage (538 V) was far greater than the $4.5 \mathrm{~V}$ needed to charge the capacitor to the firing voltage of the transmitter, while the short-circuit current (206 $\mu A$ ) was roughly four times that needed by the transmitter. The overall efficiency of the photovoltaic array was $8.7 \%$.

1. Consultant representing self.

2. Instrumentation and Controls Division, ORNL. 


\section{Ion Beam and Laser Processing}

Basic research and materials development using ion beams, molecular beams, and lasers are emphasized in this chapter. This research is performed at the Surface Modifiration and Characterization Research Center and the pulsed-laser ablation and molecular jet-growth facilities.

Ion beam processing research has focused on ion beam deposition of $\mathbf{A l}$ films, effects of implantation on corrosion of $\mathrm{Al}$ alloys, production of optical waveguides by stoichiometric implantation, differing amorphous states of glass following ion implantation, and intermixing across the boundary of $\mathrm{MgF}_{2}$ films on LiF substrates. Substantial progress has been made in characterizing and understanding electron cyclotron resonance microwave plasma sources and related thin-film processing applications.

Damage formation and morphology, annealing, crystallization, and precipitation in both elemental and compound semiconductors continue to be extensively investigated using ion beam analysis techniques. New results on dose-rate effects in ion-irradiated $\mathrm{Ge}$ and $\mathrm{GaAs}$ indicate fundamental differences in the cascade relaxation in these two materials. High-dose ion implantation has been used for the first time to form buried $\mathrm{ErSi}_{2}$ layers. The ability to use ion beams to change the local epitaxial orientation of thin-film high- $r_{c}$ superconductors has been demonstrated.

Within the Semiconductor Physics, Thin Films, and Photovoltaic Materials Program, advances in pulsed-laser ablation deposition have resulted in highly uniform deposition of high- $T_{c}$ YBCO at temperatures of $600-750^{\circ} \mathrm{C}$, and well-textured YBCO has been grown on thin polyc:ystalline yttria-stabilized zirconia. This research has also led to the development of high- $T_{c}$ superconducting superlattices which are described in Chapter 3 of this report. First measurements of the transport kinetics of "ground state" particles in the plume of laser-ablated YBCO have been made through the use of optical absorption measurements rather than the usual optical emission measurements, which monitor only "excited state" particles. Mol- ular jet growth of Ge on GaAs, using digermane, has shown that fas.er growth improves both the electrical and mechanical properties of the Ce films. 


\section{ION BEAM PROCESSING}

\section{ORIENTED ALUMTNUM FILMS ON SILICON BY DIRECT ION BEAM DEPOSITIONI}

\section{R. A. Zuhr and T. E. Haymes}

The Al-on-Si system is especially interesting not only because $A l$ is presently the conductor of choice for microelectronics fabrication but also because the system exhibits unusual interface properties. It has been demonstrated that oriented crystalline Al films can be grown on Si(111) and Si(100) surfaces at room temperature, even though there is a large mismatch in the size of the respective lattices (25\%). In this work, the quality of Al films formed on Si(111) substrates by ion beam deposition (IBD) has bcen investigated.

Scanning electron micrographs of three A(111) films grown by loD on ion beam cleaned Si(111) are shown in Fig-4.1. The films were grown with an ion energy of $120 \mathrm{eV}$ at cemperatures of 30 (bottom), 150 (center), and $300^{\circ} \mathrm{C}$ (top). The effects of temperature are clear; the morphology of the surface roughens with increasing temperature, forming at $300^{\circ} \mathrm{C}$ a collection of micron-size islands separated by a network of canyons. For the sample deposited at $150^{\circ} \mathrm{C}$, a relatively smooth surface with a nctwork of depressions is observed. For roomcemperature deposition, the surface is smooth and featureless to the limit of resolution of the scanning electron microscope. This can be understood in terms of the interface propertics of Al on $\mathrm{Si}$. Both the interface encrgy and the mobil-
ORNL-PHOTO 7290-90
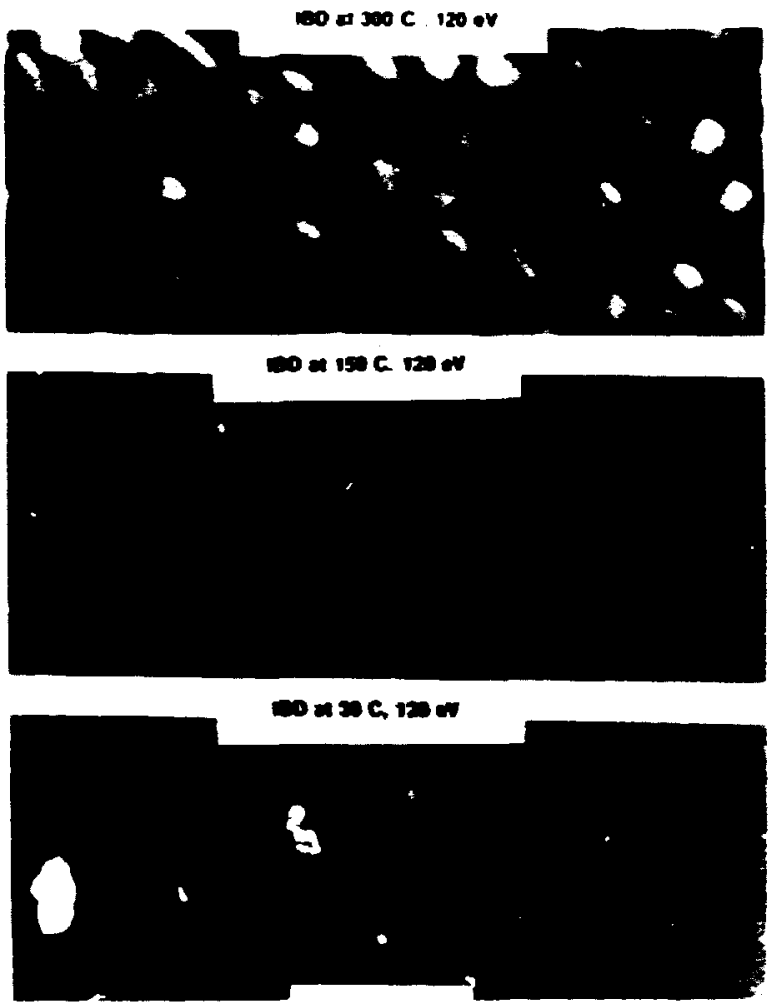

Fig. 4.1. Scamning electron micrographs of oriented Al films formed on Si(111) by IBD at $120 \mathrm{eV}$ illustrate the changes in surface morphology that occur as a function of ut mpcrature.

ity of AI/Si are high. This results in conditions that are energetically favorable for the formution of Al clusters. At temperatures above ambient, surface diffusion increases and threedimensional island growth predominates, resulting in the coarsely textured surface observed for the sample formed at $300^{\circ} \mathrm{C}$.

The best A films formed on Si(111) by IBD have been deposited at $30 \mathrm{eV}$ and show a channeling minimum yield of $28 \%$, which is comparable to the best minimum yields achieved on this surface by other technologies (22\%). Therefore, it is clear that good quality epi- 
taxial $\mathrm{A}$ films can be formed on Si by IBD and that the quality of such films is a function of both incident energy and substrate temperature.

1. Summary of paper to be published.

\section{ION-ANDUCED DAMAGEFORMATION BY FRENTI PAIR SEPARATIONI}

O. W. Hollond and C. W. White

The effects of spatial separation of Frenkel pair defects on ion-induced damage growth is discused. For room-lemperature implantation, it was observed that damage growth during high-energy, self-ion irradiation of Si varies markedly over the path of the ion-increasing monotonically with dose near the end of range (EOR), while saturating at a very low level in the region ahead. These different behaviors apo attributed to the effects of a local imbalance in the getreration rates of the complementary defects (i.e., interstitial and vacancies) in the EOR region. This imbalance is created, in part, by momentum transfer between =ר ion and interstitial during fonnation of a Frenkel-pair defect which separates ts: pair into regions of excess interstitials and vacancies.

In an attempt to isolate the effects of this separation, heavy ions were used to separate the Frenkel pair more efficiently. TRJM computer simulation of excess defects produced by a $100-\mathrm{keV}, \mathrm{As}^{+}$ion implantation at a fluence of $10^{16} \mathrm{~cm}^{-2}$ is shown in Fig. 4.2(a). (Negative values of the distribution denote regions of excess vacancies.) Excess peak concentrations
SSD -4323
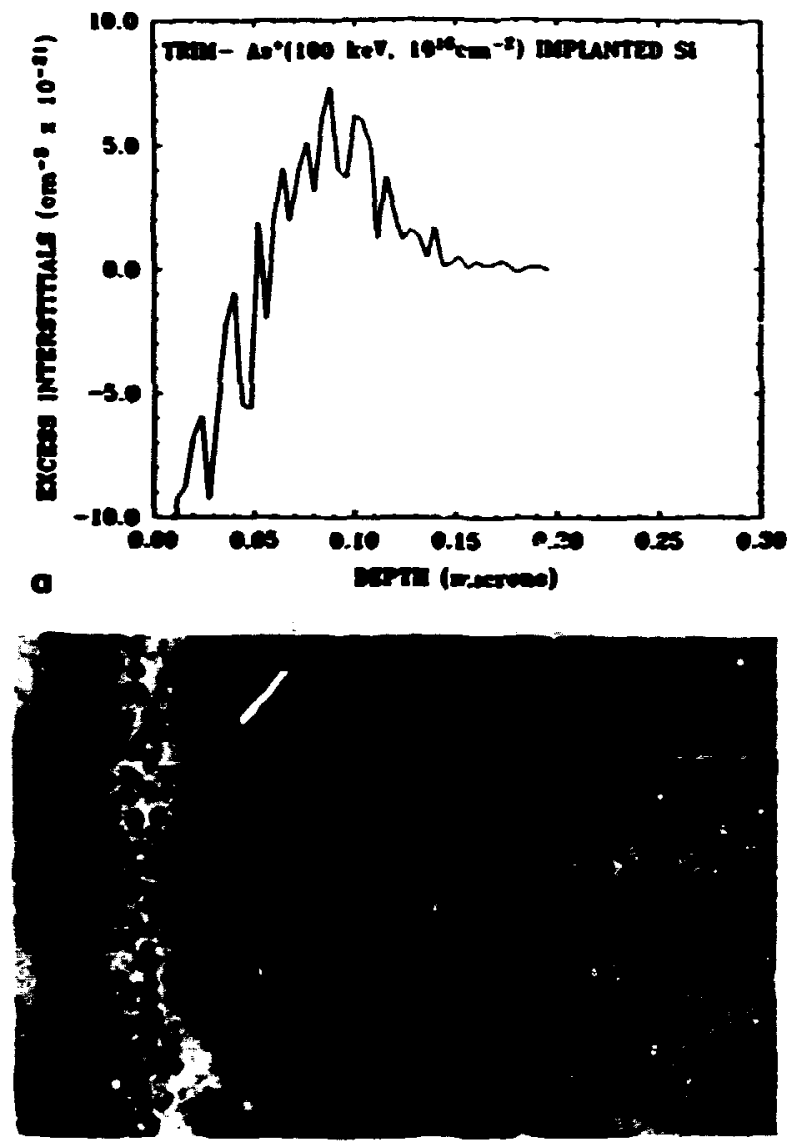

Fig. 42. Comparison of (a) TRIM simulation of excess interstitials in Si and (b) a crosssectional TEM micrograph of a Si(100) crystal implanted with 100-keV $\mathrm{As}^{+}$ions at a fluence of $10^{16} \mathrm{~cm}^{-2}$. The depth scale of the micrograph is identi-1 to the scale of the TRIM distribution.

for vacancies and interstitials are seen to be $-10^{22}$ and $5 \times 10^{21} \mathrm{~cm}^{-3}$, respectively. A crosesectional, transmission electron microscopy (TEM) micrograph of a Si(100) crystal implanted at the simulation conditions is shown in Fig. 4.2(b) wit. a matching depth scale to allow direct comparison to the TRIM results in Fig. 4.2(a). To prevent amorphizatiun by beam heating of the sample, a high- 
flux beam, $185 \mu \mathrm{A} / \mathrm{cm}^{2}$, was used during irradiation. The sample is seen to be crystalline with a damage morphology consisting of a $45-\mathrm{nm}$ region at the surface containing a high density of voids, followed by a band of interstitialtype dislocation tangles extending to $-0.2 \mu \mathrm{m}$. A comparison with the TRIM simulated distribution shows that it accurately replicates this damage morphology. The vacancy distribution (extending $50 \mathrm{~nm}$ from the surface) correspond: almost exactly with the rgion in the crystal containing voids, while the distribution of excess interstitials closely matches the band of interstitial dislocations. This correlation provides strong evidence to establish Frenkel-pair separation as an important mechanism contributing to ion-induced damage accumulation in Si.

1. Summary of paper to be published.

\section{MECHANISMS OF LOW-TEMPERATURE CRYSTALLIZATION IN Si ${ }^{1}$}

O. W. Holland, C. W. White, M. K. El-Ghor, ${ }^{2}$ and J.D. Budai

Ion irradiation of Si at cryogenic temperatures produces an interesting damage morpholOEy which consists in part of isolated regions of amorphous ( $\alpha) \mathrm{Si}$ embedded in the crystalline lattice. A transmission electron microscopy (TEM) micrograph is shown in Fig. 4.3(a) of a Si(100) crystal implanted with $1.25 \cdot \mathrm{MeV} \mathrm{Si}{ }^{+}$ self-ions at a fuence of $3 \times 10^{14} \mathrm{~cm}^{-2}$ and a temperature of $80 \mathrm{~K}$. A distinct buried layer is located beyond $-1.0 \mu \mathrm{m}$, which is predomi-
SSD-4228
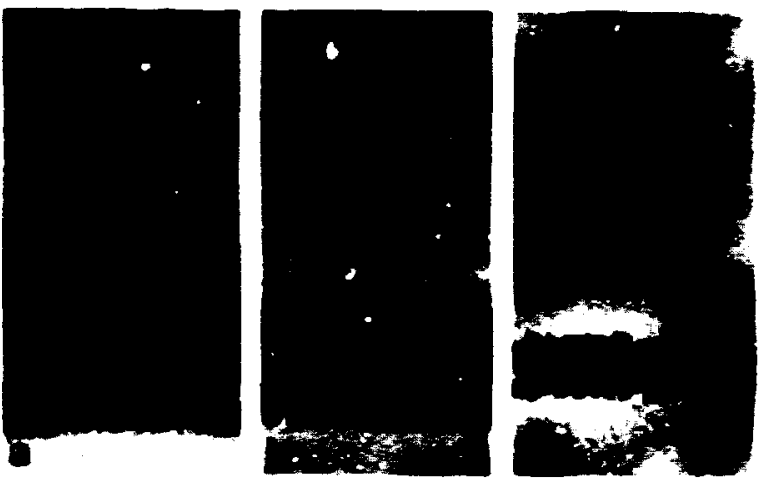

Fig. 43. Cross-sectional TEM from (1.25 MeV, $3 \times 10^{14} \mathrm{~cm}^{-2}$ ) self-ion-implanted Si(100) after (a) implantation, (b) 573 $\mathrm{K} / 30 \mathrm{~min}$ annealing, and (c) $673 \mathrm{~K} / 30 \mathrm{~min}$ annealing.

nantly amorphous, although selected-area microdiffraction from the layer indicated the presence of crystalline (c) Si. Damage ahead of this layer was found to consist of islands of $\alpha-S i$ and small extended defects. The $\alpha$-islands were examined by high-resolution TEM and found to be $-3 \mathrm{~nm}$ in diameter. The density of these islands increases away from the surface, but their diameter remains fairly constant to near the boundary of the buried layet. Thermal cycling at $573 \mathrm{~K}$ for $30 \mathrm{~min}$ left little or no visible damage ahead of the buried layer and substantially annealed the heavily damaged, front interfacial region of the buried layer, as seen in the micrograph of Fig. 4.3(b). Cycling at $673 \mathrm{~K}$ for $30 \mathrm{~min}$, as shown in Fig. $4.3(\mathrm{c})$, produced further annealing in the interfacial regions of the buried layer. Damage remaining after each of the thermal cycles is stable during subsequent cycles at the same temperature. Also, both annealing stages involve crystallization of isolated $\alpha \cdot S i$ islands, which is con- 
sidered to be somewhat anomalous since macroscopic growth of continuous a-layers occurs at anuch higher temperatures (>773 $\mathrm{K}$ ).

Different mechanisms were proposed to account for annealing in each of the stages. Dissociation of small defect complexes formed during implantation are thought to produce a transient condition during 573-K cycling, which assists thermally activated crystallization in lowering the transition temperature. This transient is not expected to persist to higher teanperatures, so it cannot account for the 673-K stage. Rather, a lange strain was observed in the lattice surrounding the isolated a-regions, which is thought to arise because of a volumetric mismatch in the reaction region during the a-c transition. The kinetics of solid-phase crystallization of a-Si are known to follow a simple Arrhenius relation with a pressuredependent activation energy. ${ }^{3}$ The stress in the lattice surrounding an $\alpha$-island was calculated and shown to be sufficient to account for crystallization during the 673-K stage.

1. Summary of paper: i. App. Phys.68, 2081 (1990).

2. Oak Ridge Associaled Universitics postgraduate research participant. Present address: Texas Instruments, Dallas, Tex.

3. G. Q. Lu et al., Mat. Res. Soc. Sym. Proc. 100, 135 (1988).

PRECIPTTATION IN SILICON DMPLANTED BY OXYGEN AND CARBON PLUS OXYGEN'

Y. L. Chen, ${ }^{2}$ R. W. Carpenter, 2 and T. P. Sjoreen

High-resolution electron microscopy (HREM) observations of precipitate morphol-
Ogy in (CO1) float-zone (FZ) Si implanted with $O$ to a concentration of $2.3 \times 10^{20} \mathrm{~cm}^{-3}$ at the projected range $\left(R_{\text {p }}\right)$ of $3.0 \mathrm{pm}$ and with $O$ plus $C$ each having concentrations of $1.2 \times 10^{20} \mathrm{~cm}^{-3}$ at $R_{\text {p }}$ are reported. After implantation, the specimens were postannealed at $923 \mathrm{~K}$ for $120 \mathrm{~h}$ to precipitate the excess $\mathbf{O}$ or $\mathrm{C}$. Wafers were examined in (011) cross section using HREM and electron energy-loss spectroscopy (EELS). The defect arrays created in the wafers by implantation plus aging treatment were planar bands of precipitates and dislocations located at a depth approximately equal to $\boldsymbol{R}_{\boldsymbol{r}}$ However, there was also an extended band with a lower precipitate density about $0.3-\mu m$ thick below the main band in the O-implanted specimen, which was absent in the samples implanted with C. Some of the morphological forms found in the O-implanted specimen by HREM observations at (011) zone axes are stacking faults (SF) on Si(111) planes, plate-like defects (PLD) on Si(100) planes, asymmetrically truncated octahedra (TO) bsunded by Si(100) facets, (113) defects, teeth-like defects (TLD) on Si(100), dot-like defects (DLD), and a ribbon-like defect (RLD) on a Si(100) plane associated with a (113) defect. These morphological forms have also been observed in nonimplanted Czochralski (CZ) Si, thermally aged at the same or higher temperatures. ${ }^{3}$ Furthermore, 0 has been detected in TO, PLD, RLD, and DLD.4-6 In the present study, the most important difference between the two implants was in carbon suppression of RLD, which was only found in the O-implanted wafer and in thermally aged $\mathrm{CZ} S \mathrm{Si}$ with a low $\mathrm{C}$ to $\mathrm{O}$ 
concentration. No $O$ was detected by EELS on primary (113) defects which were the dominant defects in our specimens.

The results show that the defects found in ion-implanted $\mathrm{FZ} \mathrm{Si}$ are similar to those in thermully aged CZ Si and that $\mathrm{C}$ is one of the major impurities which can influence $O$ precipitation, especially by suppression of RLD. The dominant primary (113) defects in innimpianted $\mathrm{Si}$ appear to be the imporr. nnt nucleation sites for impurities surh as $\mathrm{C}$ and $\mathrm{O}$.

1. Research sponsored in part by the Naticnal Science Foundation and the DuPont Committee on Educational Aid.

2. Arizona State University, Tempe, Ariz.

3. R. W. Carpenter et al., Mat. Res. Soc. Sym. Proc. 14, 195 (1983).

4. R. W. Carpenter et al., Mat. Res. Soc. Sym. Proc. 59, 309 (1986).

5. Y. L. Chen et al., Proc. Ann. EMSA Mecting 27, 222 (1989).

6. R. W. Carpenter, Inst. Phys. Conf. Ser. 100,543 (1989).

\section{OPTICAL ABSORPTION OF CU-AND Bi-IR.PLANTED SILICA ${ }^{1}$}

\section{R. H. Magruder, 2 R. A. Weeks, 2 and R.A. Zuhir}

The optical absorption spectrum of $\mathrm{Cu}$ implanted fused silica has been measured. It depends strongly on total dose, dose rate, and substrate temperature during implantation. At doses $23.0 \times 10^{16}$ jons $/ \mathrm{cm}^{2}$, the optical absorption between 2 and $5 \mathrm{eV}$ is attributed to the formation of copper colloids of varying diaiileters. A peak at $2.2 \mathrm{eV}$ is observed to grow in intensity with total dose in a noriinear fash- ion. This particular peak is attributed to prolate copper spheroids with an aspect ratio of -2. Absorption at energies $>5 \mathrm{eV}$ is attributed to defects caused by the implantation process.

For samples with a total dose of $6.0 \times$ $10^{16}$ ions $/ \mathrm{cm}^{2}$, the optical absorption from 2 to $5 \mathrm{eV}$ is observed tn increase with dose rate as scen in Fig. 4.4. The dose-rate dependence is believed to be due to local heating. The local heating results in greater diffusivity of $\mathrm{Cu}$ ions allowing for the growth of the colloids.

The optical absorption is also a function of substrate temperature during implantation. $\mathrm{Cu}$ was implanted for a total dose of $6.0 \times$ $10^{16}$ ions $/ \mathrm{cm}^{2}$ at three substrate temperatures, 100,293 , and $693 \mathrm{~K}$. The optical absorption increases throughout the range examinnd $(1.8-6 \mathrm{eV})$ with increasing substrate temperatures. These increases are due to increased diffusivity of $\mathrm{Cu}$ with substrate temperatures resulting in growth of the metallic colloids.

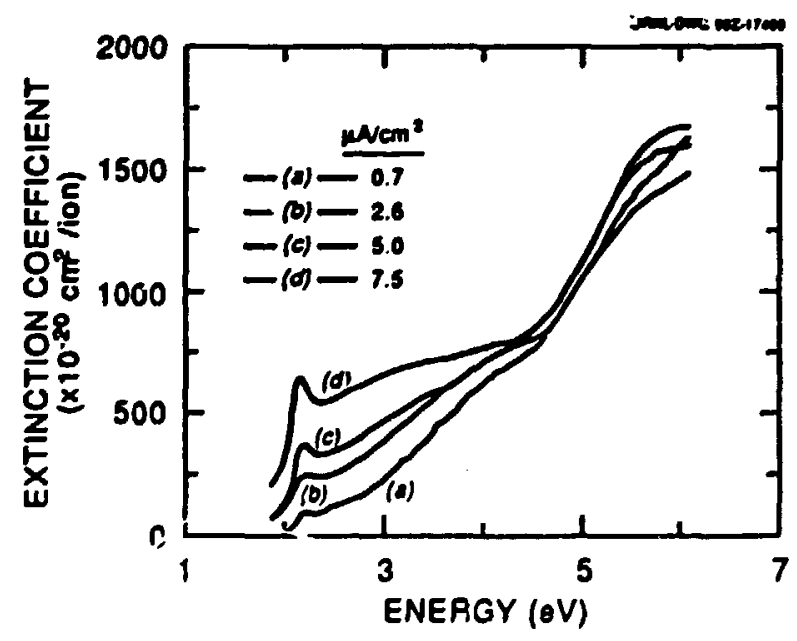

Fig. 4.4. Extinction cuefficient as a function of energy for sample with a total dose of $6.0 \times 10^{16}$ ions $/ \mathrm{cm}^{2}$ for dose rates of (a) 0.7 , (b) 2.6 , (c) 5.0 , and (d) $7.5 \mu \mathrm{A} / \mathrm{cm}^{2}$. 
Optical absorption of samples implanted with $\mathrm{Bi}$ to a total dose of $6.0 \times 10^{16} \mathrm{jons} / \mathrm{cm}$ for dose rates from 7 to $20 \mu \mathrm{A}$ was also measured. The spectra display increasing absorption from 2 to $6 \mathrm{eV}$ and are dominated by a broad shoulder at $5 \mathrm{eV}$ that is attributed to $\mathrm{Bi}^{3+}$ ions. Absorption of the Bi-implanted samples does not display a dependence on dose rate.

Laser bleaching experiments were performed on these Bi-implanted samples. After 100 pulses of 5-eV irradiation from a KrF excimer laser $(190 \mathrm{~mJ} /$ pulse), the spectra display an -75\% drop in absorption. Subsequent laser bleaching, up to 300 pulses, produces small additional drops in absorption. The absorption remains constant with subsequent irradiation for up to 1000 pulses. This change in optical absorption is attributed to electronic interactions caused by the laser irradiation that alters the chemical state of the $\mathrm{Bi}$ ions.

1. Summary of papers to be published. Tenn.

2. Vanderbilt University, Nashville,

\section{NONLINEAR OPTICAL MEASUREMENTS} OF ION-IMPLANTED SILICA ${ }^{1}$

\section{K. Becker, ${ }^{2}$ L. Yang, ${ }^{2}$ R. F. Hoglund, 2 R. H. Magruder, 2 R. A. Weeks, ${ }^{2}$ and R.A. Zuhr}

Using a Z-scan technique which utilizes the self-focusing property of the third-order nonlinearity, the nonlinear index of refraction has been measured for Ti- and Cu-implanted glasses. The absolute value of $\pi_{2}$ can be calcu- lated from the difference of the normalized peak and valley transmission ratios of the spectra. The shape of the spectra can be used to indicate whether $\boldsymbol{n}_{\mathbf{2}}$ is positive or negative in sign. Measurements were made with a CWmode-locked, frequency-doubled Nd:YAG laser.

Ti-implanted samples show a threshold for nonlinear response between $1 \times 10^{16}$ and $6 \times 10^{16} \mathrm{ions} / \mathrm{cm}^{2}$. The value of $n_{2}$ calculated for the Ti samples implanted with doses of $6 \times 10^{16} \mathrm{ions} / \mathrm{cm}^{2}$ is on the onder of $10^{-8} \mathrm{~m}^{2} \mathrm{w}^{-1}$, and the shape of the spectro suggests a positive $n_{2}$. Electron paramagnetic resonance spectroscopy and optical data show that a large fraction of the $\mathrm{Ti}_{\mathrm{i}}$ is in the $\mathrm{T}^{3+}$ and $\mathrm{T}^{4+}$ states. The ratio of the concentrations of the oxidation states depends on total dose. The mechanism for the nonlinear response in these $\mathrm{Ti}$ implanted samples may be due to the ionic polarization of the $\mathrm{Ti}$ ion.

The nonlinear indices of refraction of samples implanted with $6 \times 10^{16} \mathrm{Cu}$ ions $/ \mathrm{cm}^{2}$ at different dose rates were measured. As can be seen in Fig. 4.5, the maximum far-field intensity, which is related to $m_{2}$, depends on the dose rate. Absorption differences are attributed to changes in the formation of metallic colloids due to dose rate. Also seen in Fig. 4.5 are the spectra for an unimplanted sample marked pure silica.

The nonlinear response may arise from electronic excitation of either the metallic colloids or highly polarizable ions. Based on linear absorption data, it is suggested that metallic colloids are formed in these glasses. Hence, the 
manes

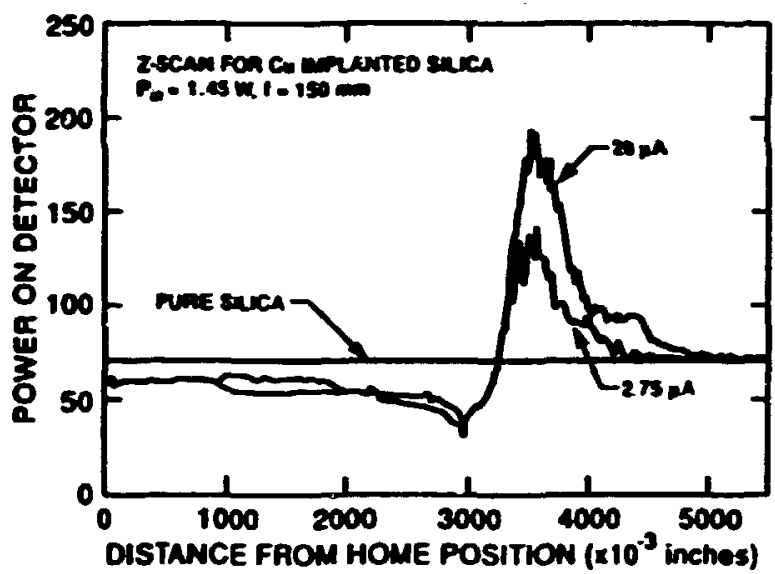

Fig-45. Far-field intensity measured using Z-scan technique for $\mathrm{Cu}_{\mathrm{SiO}}$ sample implanted at 20 and 2.75 uA to a total dose of $6 \times 10^{16} \mathrm{ions} / \mathrm{cm}^{2}$.

nonlinear response may result from excitation of surface plasmon resonances of copper particles. The values calculated for $n_{2}$ are also on the order of $10^{-8} \mathrm{~m}^{2} \mathrm{~W}^{-1}$. As seen in Fig. 4.5 , the signal initially shows a decreasing intensity on approaching the focal spot, indicating a positive value for the nonlinear index of refraction.

1. Summary of paper to be published.

2. Vanderbilt University, Nashville, Tenn.

\section{ION MMPLANTATION FOR CORROSION INHIBIION OF ALUMINUM ALLOYS DN SALINE MEDU'}

J. M. Williams, A. Conzales, 2 J. Quintara, 3 I-S. Lee, R. A. Buchanan,' F. C. Burns,' R. I. Culbertson, ${ }^{5}$ M. Leoy, and I. R. Tregliob

Helicopters often operate in marine atmospheres. Moreover, shipping, storing, and repairing of suct aircraft may involve exposure to marine environments. There is a high incidence of failure of components made of aluminum alloys due to salt-pitting corrosion. The present experiments have test-d the effects of ion implantation of N, Si, Ti, or Cr on salt-pitting corrosion of a $2014 \mathrm{~A}$ alloy. This alloy is used for construction of UH-1 pillow blocks, a component that is classified as critical for fight safety. Comparison studies were also drme on $1100 \mathrm{Al}$.

The engineering test used was the salt fog test (ASTM Designation B117-73). The test consists of exposure of sample surfaces to an atmosphere of atomized salt fog. Other investigative techniques included electroctremical studies and Rutherford backscattering spectrometry.

Among the implanted constituents named, Cr produced the best results, and the effect can be regarded as striking. Whereas a salt-fog exposure of $168 \mathrm{~h}$ produced an oxide thickness of $30 \mathrm{~km}$ over most of the area of unimplanted 2014 Al, there was an oxide thickness of only about $0.05 \mu \mathrm{m}$ on the $\mathrm{Cr}$-implanted areas. The oxide was uniform, and no evidence of pitting or localized attack was found for the $\mathrm{Cr}_{\text {- }}$ implanted alloy.

The other constituents produced favorable results to varying degrees for one alloy or the other in one test or the other (salt fog or electrochemical). There were some correlations between favorable performance on electrochemical studies and favorable performance in salt-fog studies, but overall it was judged that 
electrochemical results were not highly predictive indicators of petformance in the engineering test.

Ion implantation of $\mathrm{Cr}$ to doses of about $3 \times 10^{17} / \mathrm{cm}^{2}$ at energies $100-150 \mathrm{keV}$ would appear to be quite effective as protection for 2014 Al against salt-pitting corrosion. Practical utilization of the process could occur $\operatorname{son}$

1. Summary of paper. Nuclear Instruments and Methods Physics Resenrch Section B (in press).

2. AVSCOM-DERSO, Corpus Christi, Tex.

3. Corpus Christi Army Depot, Corpus Christi, Tex.

4. The University of Tennessee, Knoxville, Tenn.

5. U.S. Amy Materials Technology Laboratory, Watertown, Mass.

6. ISM Technologies, Inc. San Diego, Calif.

7. ASTM Designation B117-73 (Reapproved 1979), Standard Method of Salt Spray (Fog)

Testing "Metal Corrosion, Erosion and Wear," p. 32 in 1984 Annual Book of ASTM Standards, Vol. 03/02, in ASTM, Philadelphia, Pa., 1984.

\section{OPTCAL WA VEGUIDE FABRICATION BY STOICHIOMETRIC MMPLANTATION OF $\mathrm{TI}$ AND O INTO LNBO ${ }_{3}{ }^{\mathrm{I}}$}

\section{B. Poker and W. Xia}

The introduction of $\mathrm{Ti}$ into $\mathrm{LiNbO}_{3}$ can be used to modify the index of refraction near the surface, forming a region capable of sustaining a propagating mode of light transmission. Ion implantation of $\mathrm{Ti}$ has been shown to exhibit several advantages over thermal diffusion; namely, shallower waveguides, a higher concentration of $\mathrm{Ti}$, steeper concentration gradi- ents, and better lateral control of the Ti concentration. However, the implantation process produces damage at room lemperature sufficient to drive the $\mathrm{LiNb}_{3}$ amoiphous. Ion implantation at high temperatures would offer advantages over low-temperature implantation, since the formation of the amorphous phase might be avoided, eliminating the need for sclid-phaseepitaxial regrowth. Howe rer, when $\mathrm{LiNbO}_{3}$ is implanted at high tenuperatures with $T$ il alone and suosequently annealed in oxygen, segregation of $\mathrm{Li}$ and $\mathrm{Ti}$ occurs at the surface. The purpose of this study was to determine whether stoichiometric implantation at high temperatures could be used to produce optical waveguides.

$X$-cut substrates of $\mathrm{LiNbO}_{3}$ were implanted at $500^{\circ} \mathrm{C}$ with $\mathrm{Ti}$ and $\mathrm{O}$ to doses of 2.5 and $7.5 \times$ $10^{17}$ ions/ $\mathrm{cm}^{2}$, respectively. The high substrate temperature during implantation ensures dynamic recrystallization, preserving the crystallinity of the $\mathrm{LiNbO}_{3}$. The stability of the stoichiometric implants is enhanced sufficiently that annealing at $1000^{\circ} \mathrm{C}$ proceeds with no surface degradation of the substrate. Annealing under identical conditions without the $O$ implant usually results in phase separation of an oxide at the surface, even when annealing is performed immediately following implantation. Samples implanted with $\mathrm{Ti}$ and $O$ to preserve the stoichiometric metal:oxygen ratio of the substrate can be stored at room temperature for several months without phase separation. I'lanar optical waveguides have been produced by stoichiometric implantation 
followed by annealing in water-saturated oxygen for $1 \mathrm{~h}$ at temperati: es of 900 and $1000 \mathrm{C}$. The sample annealed at $900^{\circ} \mathrm{C}$ supported a single lossy mode at $\lambda=0.6 \mu \mathrm{m}$, while the 1000C sample supported two propagating nodes and one lossy mode.

1. Summary of paper: Mat. Res. Sac. Sym. Proc. 157, 531 (1989).

2. University of California-San Diego, La Jolla, Calif.

\section{STRUCTURAL DIFTERENCES BETWEEN THE GLASS STATE AND ION BEAM AMORPTIZED STATES OF IEAD MYOFHOSPHATE'}

\author{
B. C. Sales, J. O. Ramey, \\ J. C. McCallum, ${ }^{2}$ and L. A. Boatner
}

Amorphous lead pyrophosphates $\left(\mathrm{Pb}_{2} \mathrm{P}_{2} \mathrm{O}_{7}\right)$ were prepared by three techniques: thermally quenching the molten phosphate to produce a glass, by implanting $\mathrm{O}^{2+}$ or $\mathrm{Pb}^{3+}$ ions into lead pyrophosphate single crystals, or by implanting $\mathrm{O}^{2+}$ ions into lead pyrophosphate glass. The structures of the amorphous phases produced by these methods were determined using the technique of high-performance liquid chromatography (HPLC) which measures the distribution of phosphate anions (i.e., the num- ber and length of $\mathrm{PO}_{4}$ tetrahedral chains) present in each type of amorphous material.

Ion channeling measurements of $\mathrm{Pb}$ implanted (540-keV) lead pyrophosphate crystals showed that the amorphous layse produced via ior bombandment (1015 $\mathrm{Pb}$ ions/( $\left.\mathrm{cm}^{2}\right)$ was about $200 \mathrm{~nm}$ thick. For all implant doses. the structure of the damaged layer produced by ion bosibandment was found to be significantly different than the structure of lead pyrophosphate glass prepared via thermal quenching (Fig 4.6). The structure of the amorphous phase produced by ion implantation was, however, esontially independent of the implanted species since the implantation of high doses of either lead or oxygen produced virtually the same distribution of phosphate anions. The final amorphous phase formed via ion implantation was determined to be structurally different depending on whether the implanted lead pyrophosphate surface was initially a crystal or a gass.

1. Summary of paper: Joumal of NonCrystalline Solids (in press).

2. Oak Ridge Associated Universities postgraduate research participant; present address: University of Westem Ontario, London, Ontario, Canada. 


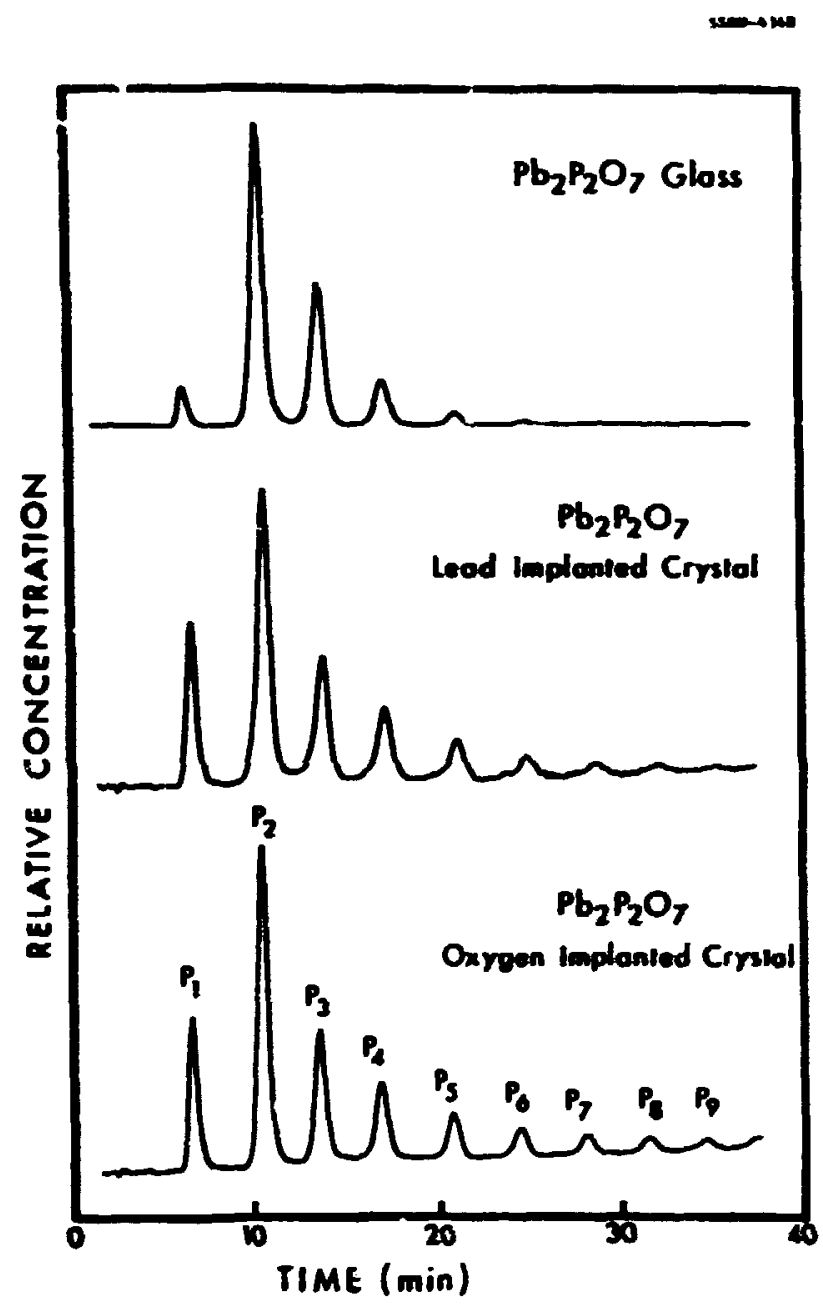

Fig. 4.6. A comparison of the HPLC chromatograms from amorphous lead pyrophosphates produced by: (1) quenching a melt to form a conventional phosphate glass (top), (2) ion bombardment of the crystal surface with $10^{15} \mathrm{Pt}^{3+}$ ions $/ \mathrm{cm}^{2}$ at $540 \mathrm{keV} / \mathrm{ion}$ (middle), and (3) ion bombardment of the crystal with $10^{17}$ $\mathrm{O}^{2+}$ ions $/ \mathrm{cm}^{2}$ at $55 \mathrm{keV} /$ ion (bottom). The labels $\mathbf{P}_{1}, \mathbf{P}_{2}, \mathbf{P}_{3} \ldots$ denote chains of phosphate tetrahedra that are, respectively, 1, 2, $3 \ldots$ tetrahedra in length. A comparison of the ratios of the areas under each peak (representing the total amount of phosphorus contained in chains of that length) for the three chromatograms, shows that the structure of the pyrophosphate glass is significantly different from that of the ion beam amorphized layers whose structures are the same within the limiis of accuracy of the analysis.

\section{IOIN TRRADIATION EFFECTS FOR MEF FIMS ON UF SUISTRATES'}

\author{
G. C. Farlow and L. A. Bantner
}

Although ion beam mixing results are relatively common for metal-semiconductor and metal-insulator systems, there are very few reported investigations of the fundamental properties of ion beam mixing between dielectric bilayers. From the practical point of view. previous work on ion beam mixing of metal films has shown that enhanced adhesion on be achieved due to alteration of the electronic bonding at the film-substrate interface, and the observation of similar effects for dielectric bilayers would have important implications for improving the quality of dielectric thin-film antireflection coatings on a wide variety of optical materials. In the present case, the effects of ion beam mixing of dielectric thin films on dielectric substrates have beer. investigated using a model system consisting of single-crystal LiF substrates canted with $53 \mathrm{~nm}$ of $\mathrm{MgF}_{2}$. The $\mathrm{MgF}_{2}$ films were deposited on cleaved (100) Lif surfaces using e beam evaporation. These samples were subsequently irradiated with $126 x$ e at $210 \mathrm{keV}$ to doses of $0.5,1.0$, and $1.5 \times 10^{16} \mathrm{ions} / \mathrm{cm}^{2}$. The sample nomal was about $7^{\circ}$ away from the $X e$ beam direction, and the ion beam irradiations were performed at room temperature. The combined results of RBS and SEM observations indicate that Xe irradiation produces a significant degree of intermixing across the film-substrate bounsary in the case of MgFi films on LiF substrates and suggest that 
ion beam mixing techriques may have applica. tions in the optical coating area.

1. Summary of paper. Nud. Instrum. and Methols in Ptys. Ras. Sect. B 46, 422 (1990).

2. Wright State University, Dayton, Ohio.

\section{ELCTION CYCIOTION RESONANCE MTCOWAVE RLAMA SOURCE STUDIESI}

\section{S. M. Gortatkin, L. A. Berry, ${ }^{2}$ and I. B. Roberto}

Inert ges clectron cyclotron resonance (ECR) microwave plasmas operated in a parameter space useful for thin-film deposition and etching have been studied in a mirror field ECR microwave f's ma source. Langmuir probes, visual observation, and monitoring of downstream microwave penetration were used to study Ar gas behavior. Distinct operating modes were observed and comelated to transitions in the plasma density from below to above the plasma resonance. Aks, significant neutral depletion was found to exist due to the the high innization efficiency of the source. Such mutral depletion was found to cause an illusion of bistability, which leads to inconsisient source operation if the pressure in the mirror rojon is used as the referense for downstream presaure control.

The pressure dependence of the plasene density was found to be different when meacured in the minor region and downstrem of the source. Downstrean, the plasma density peaked at $-4 \mathrm{mTor}$ and decreased with further pressure increases (Fig. 4.7). In the source, the plasma density continued to increase at presoures near 25 mTorr. Previously, it has been proposed that

Orin Dugeay istos
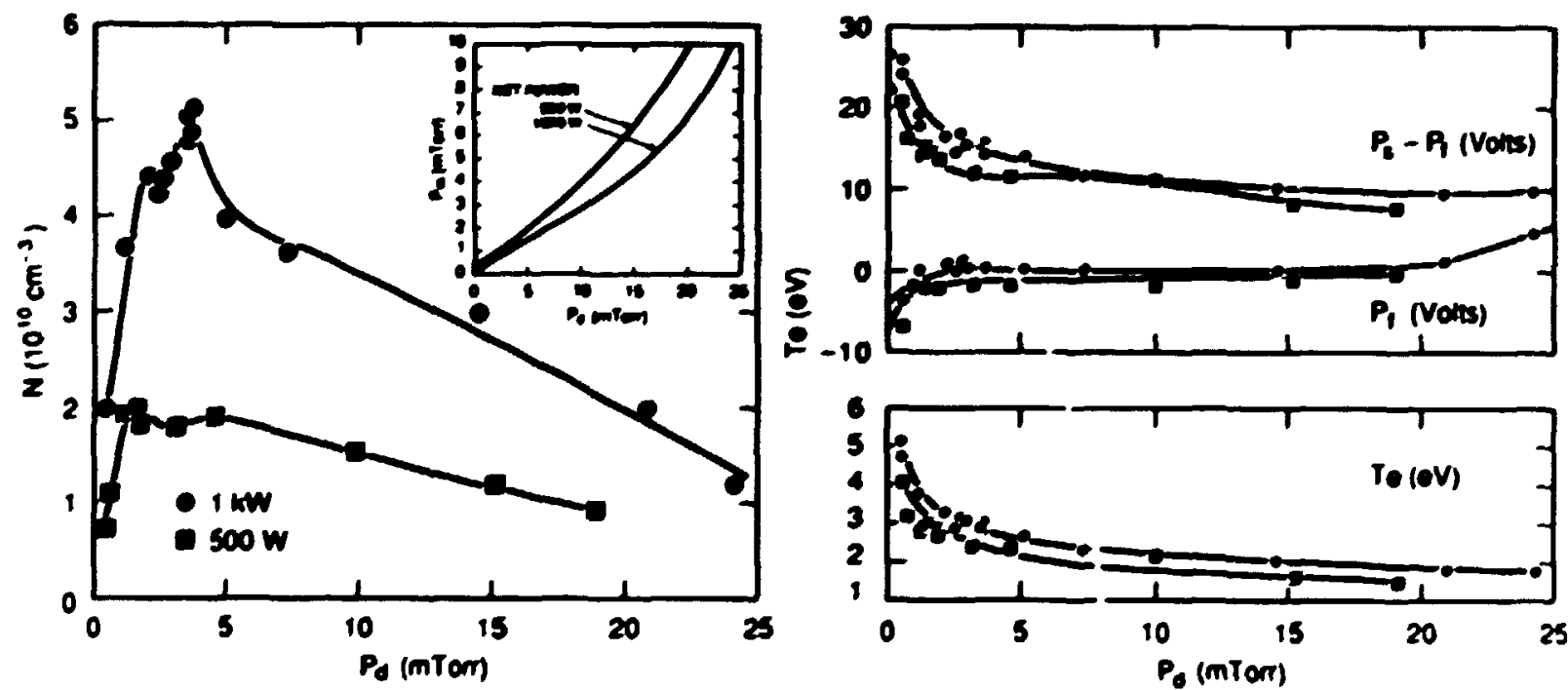

Fig. 4.7. Plasma density $n_{i}$ electron temperature $T_{e}$ floating poten ial $P_{f}$ and $\left(P_{s}-P_{f}\right)$, where $P_{s}=$ plasm potential, as a function of downstream presoure for net power $=50 \mathrm{C}$ and $1000 \mathrm{~W}$ and flow $=5 \mathrm{sccm}$ as measured by the downstream Langmuir probe. The inset shows the retation between the mirror prescure $P_{m}$ and downstream pressure $P_{d}$ at $5 \mathrm{scm}$ (with downstream pressu re control). 
decreases in the plasma density with increasing pressure seen downstream are due to the decreased electron temperature at higher pressures. These measurements suggest that the plesma density is dominated by production in the source, and the electron temperature decrease is offset by the increased neutral gas pressure.

Contamination by sputtering was also found to cocur due to the presence of high ( $30 \mathrm{~V}$ ) potentials in the source, which implies that care must be taken in selecting source materials and operating conditions if high-purity thinfilm processing is desired.

1. Summary of paper: I. Vec. Sci. and Technol. A 8, 2893 (1990).

2. Fusion Energy Division, ORNL.

\section{ELECTHON CYCLOTRON RESONANCE MICROWAVE PLASMA-ETCH TOOL REVELOPMENT'}

L. A. Berry, 2 S. M. Gorbatkin, G. H. Henkel, ${ }^{2}$ and I. B. Roberto

Electron cyclotron resonance (ECR) microwave plasmas are being increasingly used for etching and deposition research. They can be operated over a wide pressure range (0.1-10 mTorr), offer electrodeless operation, and yield high-current densities $\left(10\right.$ s of $\left.\mathrm{mA} / \mathrm{cm}^{2}\right)$ at low ion energies (a few 10 s of eV).

For semiconductor etching, ion energy controi plays a key role in process development. For example, in a typical complementary metal-on-silicon device, a $-0.5-\mu m$ layer of polysilicon (which will ultimately forsit the gate electrode) is deposited on top of a much thinner layer of $\mathrm{SiO}_{2}$ (the gate oxide). Since the gate is formed by etching the polysilicon, an attractive etch process must simultaneoushy maximize the removal rate of silic on while minimizing both the etching and damage to the underlying oxide. RF bias can be used as an aid in ion energy control independent of other operating parameters, such as presure or ion density; however, even moderate bias dc voltages (40 V) can result in unacceptably high-etch rales of the underlying oxide. 3

Since ECR microwave plasma systems are also serious contenders for a variety of microelectronic and optical device production processes, the next step in ECR source evolution is to optimize ECR reactor configurations for specific processes. In the current joint program between ORNL and SEMATECH (a consortium of U.S. semiconductor equipment manufacturers), members of the Solid State and Fusion Energy divisions are working together to op.. mize and evaluate various ECR configurations for use in semiconductor etching.

After SEMATECH selects the best technolOgy, it will be transferred to a U.S. tool manufacturer who will incorporate the technology into a production tool.

1. Research sponsored by SEMATECH Corporation (Work For Others Contract No. ERD-89-876).

2. Fusion Energy Division, ORNL.

3. L. A. Berty and S. M. Corbatkin, Nuc'ear Instruments and Methods Physics Resenrch Section B (submitted for publication). 


\section{ION IMPLANTATION AND ANALYSIS}

\section{DOSE-RATE ETECTS ON DAMAGE ACCUMULATION IN ION-DMLANTED GAIIIUM ARSENIDEI}

\section{T. E. Hames and O. W. Holland}

lon channeling measurements of the damage produced in GaAs by ion implantation of 100-keV ${ }^{30}$ Sit at room temperature (RT) have shown that the damage increases dramatically with increasing dose rate for current densities J between 0.05 and $12 \mu \mathrm{A} / \mathrm{cm}^{2}$. The dose-rate dependence of the damage is shown as the filled circles in Fig-48. These data show that the damage $\mathbf{N}_{\boldsymbol{d}}$ obeys a single power law over nearly two orders of magnitude of ion current density (i.e. $N_{d} \sim m$, where the exponent $m$ is 0.37). Under our implant conditions, this damage was more sensitive to the average $I$, rather than to the instantaneous $J$, indicating that the lifetime of mobile precursor defects is greater than $10^{-4} \mathrm{~s}$. The threshold fluence for this effect $\$ 21 \times 10^{14} / \mathrm{cm}^{2}$ appears to be correlated with the onset of doseratedependent electrical activation reported recently by Moore et al. 2

The size of the dose-rate effect in GaAs was found to be dependent on the ion mass and implantation temperature, becoming weaker with increasing ion mass and virtually disappearing at liquid nitrogen temperature (LNT). This behavior is qualitatively consis tent with a model in which the damage forma. tion at RT is dominated by homogencous nucleatton. For purposes of comparison, 100-k.V

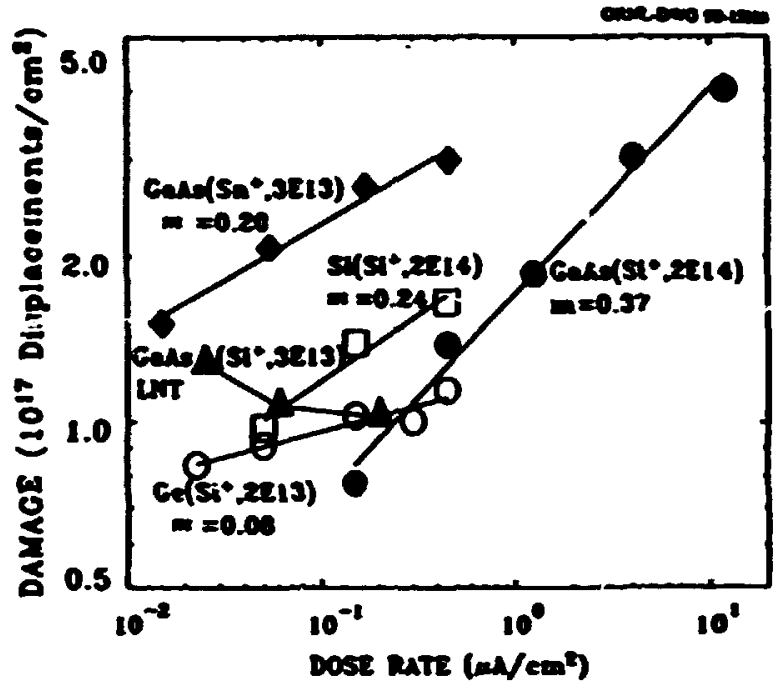

Fig. 48. The dose-rate dependence of the total damag: produced in as-implanted substrates is shown for the indicated ion and substrate combinations (all at RT except as indicated by "LNT"). Labels indicate the substrate and, in parentheses, thr :on species and fluence in $\mathrm{cm}^{-2}$. The exponent of of the best-fit power law is given for each set of data.

${ }^{30 \mathrm{Si}^{+}}$ions were . Iso implanted into $\mathrm{Si}$ and $\mathrm{Ce}$ substrates at RT. These materials exhibit much weaker dose rate effects than CaAs, with exponents $m=0.24$ and 0.08 for Si and Ge, respectively. Hence, the dose-rate effect in CaAs is comparatively strong and is expected to have a significant effect on damage and annealing studies in GaAs. Furthermore, in light of the physical similarities (atomic number, mass, and density) between Ge and GaAs, the large difference in the dose-rate effect inaicates a fundamental difference in the cascade relaxation in these two materials.

1. Summary of papers: Applied Physics Letters (in press): to be published.

2. F. G. Monre et al., Appl. Phys. Lett. 57, 911 (1990). 


\section{COMPARATIVESTUDY OF DAMAGE ACCUMULATION IN ION-MMLANTED GAIIUM ARSENIDE AND GERMANUUM}

\section{T. E. Haymes, O. W. Holland, and S. I. Penarycoot}

The collision kinematics and cascade densities in GaAs and Ge are essentially identical due to the similarity of their average atomic number, mass, and density. Therefore, a comparison of the damage accumulation during ion implantation in these materials should be useful for understanding the compound semiconductor. Although the damage accumulation in GaAs and Ge is very different at room lempereture (RT), a study of the temperature dependence has indeed uncovered a remarkable correspondence, as illustrated in Fig. 4.9. The damage levels in GaAs at an arbitrary temperature are similar to those in $\mathrm{Ge}$ for the some implants performed at a temperature approximately $110^{\circ} \mathrm{C}$ higher. Furthermore, in both materials, the as-implanted damage decreases rapidly toward zero at a well-defined transition temperature.

Such a temperature dependence of the damage can be described by a model which assumes that the bulk of the damage arises from heterogeneous nucleation in the cascade core and that the volume of this core is red'xced by the temperature-dependent out diffusion of mobile primary defects. Differences between GaAs and Ge are thus attributed to the different mobilities of simple defects in the two materials. The solid curves in Fig. 4.9 represent the best fit to this simple model for cach mate-

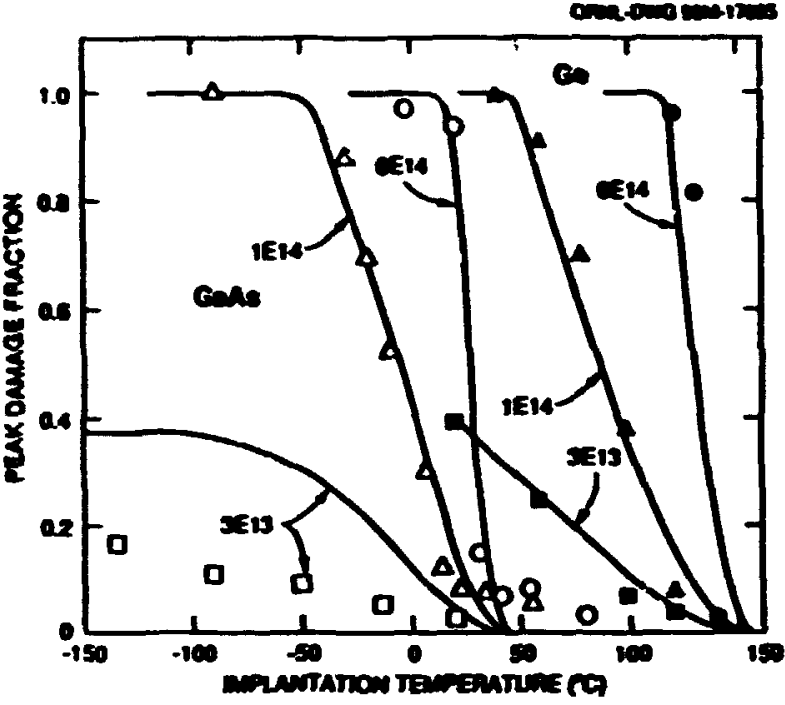

Fig. 4.9. Symbols indicate values of the peak damage (measured by ion channeling) in GaAs and Ge as a function of the substrate iemperature during implantation for implants of sasit at various indicated fluences. The solid lines are model calculations.

rial. On the basis of the model and our previous observations of dose-rate effects in GaAs near the transition temperature, a strong dose-rate effect on damage accumulation in Ce at $120^{\circ} \mathrm{C}$ was predicted and observed. Additional evidence that damage development in GaAs parallels that in Ce at $T+110^{\circ} \mathrm{C}$ was obtained by a microscopic comparison of the microstructural development in Ce and CaAs at equivalent doses and effective temperatures. Since the transition temperature for $\mathrm{GaAs}$ is between 20 and $30^{\circ} \mathrm{C}$, the damage is very sensitive to temperature near RT, and strict temperature control is necessary during RT implantation of this material.

1. Summary of paper to be published. 
EURIED ER SILICIDE FORMATION BY ION MMLANTATION AND ANNEALNG:

A. Golansti, ${ }^{2}$ C. W. White, M. D. Gallowery. W. H. Christie, ${ }^{3}$ H. E. Hanmon, I. L. Part, and S. J. Pennycoot

High-dose ion implantation is being used to Ebricate high-quality buried conducting hyes (silicides) in silicon. ${ }^{5}$ In this work, we have used high-dose Er implantation followed by thermal annealing to form a buried ErSiz layer in (111)-oriented silicon. This is the first time that the formation of buried $\mathrm{ErSi}_{2}$ by ion implantation has been reported.

Figure 4.10 shows the Er part of Rutherford backscattering (RBS)-ion channeling spectra from a (111) Si crystal implanted by Er (170 keV, $\left.8 \times 10^{16} / \mathrm{cm}^{2}\right)$ at $450^{\circ} \mathrm{C}$ and subsequently annealed at $800^{\circ} \mathrm{C}$ for $1 \mathrm{~h}$. Random and aligned spectra are shown in the as-implanted state and after annealing. Implantation at temperatures greater than $400^{\circ} \mathrm{C}$ gives rise to the formation of coherent precipitates in the Si matrix during implantation. During subsequent thermal annealing at $800^{\circ} \mathrm{C}$, the Er distribution narrows due to Ostwald ripening, and preferential alignment of precipitates occurs to form a buried layer of single-crystal ErSi 2 in silicon.

At a dose of $8 \times 10^{16} / \mathrm{cm}^{2}$, a discontinuous layer is formed during subsequent annealing. for a dose $>1.1 \times 10^{17} / \mathrm{cm}^{2}$ (at an energy of 170 keV), a continuous layer can be formed. We have also observed that nearly continuous layers can be formed by scquential implantation steps as reported elsewhere. 6

1. Summary of paper to be published.

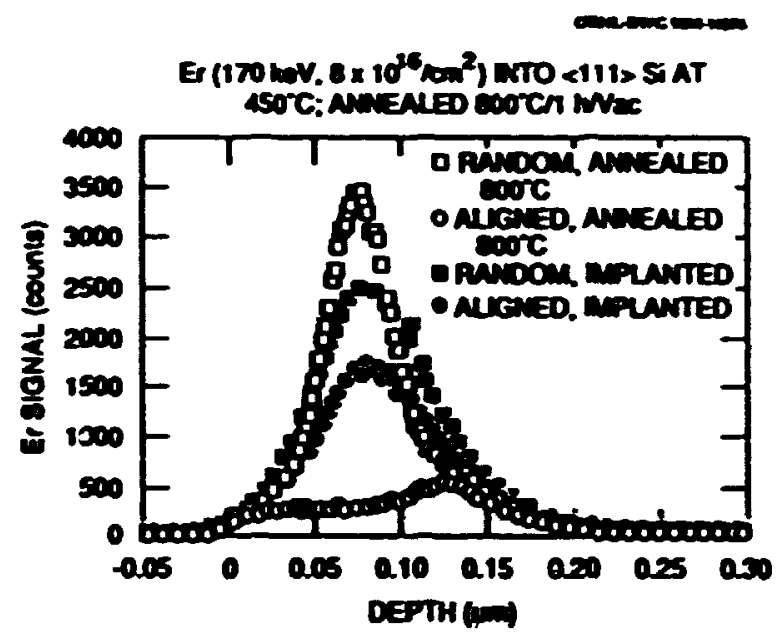

Fig-4.10. Silicide formation by ion implantation and annealing.

2. Consultant from CNET-RPT, Meylan, France.

3. Analytical Chemistry Division, ORNL

4. Postgraduate reseanch participant from North Carolina State University, Raleigh, N.C.

5. A. E. White et al., Appl. Phys. Lett. 50, 95 (1980).

6. J. L Park et al., Ton Beam Synthesis of Buried Erbium Silicide," this report.

\section{PLANAR CHANNEIING FOR DOPANT STTE LOCATION IN DUALIMIRANTED GAUUUM PHOSTHDE'2}
N. R. Parith, ${ }^{3}$ C. T. Keo, 3 D. R. Lee, 3

I. Muse, ${ }^{3}$ M. L. Swonson, ${ }^{3}$ T. E. Haymes,

R. Venketasubramanian, and M. Timmonst

The use of dual implantation of host atoms along with amphoteric dopants can be beneficial for effective doping of compound semiconductors. In this approach, the stoichiometric imbalance caused by preimplanted host atoms of one type is believed to produce vacancies on the complementary sublattice which may then be occupied preferentially by the dopant atoms 
implanted afterward. A new ion channeling analysis method has been applied to determine the butice boction of Sn atous following deal implantation in GaP. This method takes advantage of asymmetry in the backscattering yield oscillations of ions channeled abong (111) planes in zinctlerde crystals. For instance, in GaP, with the analysis beam tilked slightly off of planar alignment, the yields of ions backscattered from $G a$ and from $P$ oscillate with depth in a complementary way. The correlation of these oscillations with similar oscillations in the yield beckscattered from an implanted dopant such as Sn can then be used to determine the preferred lattice site of the dopant.

In this study, Sn was implanted into GaP at $400 \mathrm{C} \mathrm{to}$ a fluence of $1 \times 10^{15} / \mathrm{cm}^{2}$, either alone or following implantation of an equal fluesce of either $G$ or P. Implant energies were chosen to match the ranges of all three species. Channeling analyses have shown that the Sn atoms had a slight preference ( $-10 \%$ excess) for occupation of the $G$ sublattice in all cases, irrespective of the preimplanted species. However, Hall effect measurements on these samples showed that the carrier concentration was an order of magnitude greater than the implanted dopant concentration, indicating that the conduction was controlled by defect complexes rather than by activation of the dopant. Therefore, the sublattice occupation of the Sn could not have had a significant effect on the doping. Additional studies are planned to (1) identify the defects responsible for the unexpectedly large carriet concentration and (2) determine the implant conditions for which dual implantation has a significant effect on the carrier concentration. The corretation of this activation with sublattice occupation of the dopant will then be re-examined.

1. Summary of paper Materials Resenrch Society Symposium Procedings (in press).

2. Supported in part by the SURA Summer Cooperative Research Program at ORNL.

3. University of North Carolina, Chapel Hill, N.C.

4. Research Triangle Institute, Research Triangle Park, N.C.

\section{ANNEALING OF BURIED AMORPHOUS LAYERS IN MEVION-IMILANTED Ge}

\section{T. P. Sjoreen, S. J. Pennycood: and $O$. W. Holland}

Buried amorphous layers in Ge were produced by $2.5-\mathrm{MeV}$ self-icn implantation at doses between 0.5 and $1.8 \times 10^{14} \mathrm{Ce}^{+} / \mathrm{cm}^{2}$ and temperatures of 80 or $300 \mathrm{~K}$. Al both temperatures, a dose of $1.0 \times 10^{34} / \mathrm{cm}^{2}$ produced a buried amorphous layer about $1.3-\mu m$-thick that extended nearly all the way to the surface. Annealing at $626 \mathrm{~K}$ resulted in crystallization of this buried layer by solid-phase-epitaxial growth (SPEC) at both the layer interfaces. Ruthertord backscattering analysis showed that in the 300-K-irradiated sample, the regrowth rates at the two interfaces were about equal and very nearly the value reported for SPEC of amorphous layers formed at the surface in Ge. However, for the 80-K irradiation, 
the upper interface regrew at a rate which was slower by about 15\%. This is similar to results in self-implanted $\mathrm{Si}$, where the regrowth rate of the upper interface was observed to be about $30 \%$ less than the bottom interface. 1,2 This difference has been attributed to the vacancy concentration. ${ }^{2}$ The difference between regrowth rales in $\mathrm{Ge}$ and $\mathrm{Si}$ is presently being analyzed in terms of this model.

Transmission electron microscopy analysis of the sample implanted at $300 \mathrm{~K}$ and annealed at $626 \mathrm{~K}$ for $80 \mathrm{~min}$ revealed that the buried layer was completely recrystallized with the residual damage consisting primarily of dislocation loops and hairpin dislocations. The loops are found in bands centered at $0.15,0.80$, and 1.35 um below the surface. Comparison of the annealed morphology with that for the asimplanted case ${ }^{3}$ shows that the bands of disbocations at 0.15 and $1.35 \mu \mathrm{m}$ are formed in the interfacial regiuns of the buried amorphous layer during implantation. Hairpin dislocations are observed 10 originate at the dislocation bands at 0.15 and $1.35 \mu \mathrm{m}$, thread through the interfacial growth region, and terminate at $0.80 \mu \mathrm{m}$, where a central dislocation band is formed due to closure requirements for the hairpins. This damage morphology is similar to that observed in Si.l However, the density of loops and hairpins is much grealer in Ce and is thought to be due to a very diffuse nature of the amorphous-crystalline interface after irradiation. (1990).
2. E. F. Krimmel et al., Phys. Status Solidi a 66, 565 (1981).

3. T. P. Sjoreen et al., Mat. Res. Soc. Sym.

Proc. 128, 593 (1989).

\section{ION BEAM INDUCED EPTTAXAL CRYSTALIZATION OF HEAVILY DOPED Si'}

\section{S. P. Withrow, O. W. Holland, and S. J. Pennycook}

Ion implantation into Si at high fluences and low temperatures results in an amorphous layer at the surface that extends through the implanted ion distribution. Solid-phaseepitaxial regrowth (SPEG) of this amorphous layer onto the single-crystal substrate can be achieved if the crystalline-amorphous interface is irradiaied with high-energy ions. The activation energy for SPEG using ion beams is $0.3 \mathrm{eV}$, which is considerably lower than that for thermal annealing, $2.7 \mathrm{eV}$. Hence, ion beam induced epitaxial crystallization of amorphous layers and phenomena, such as dopant redistribution, solute trapping supersaturated alloying etc., associated with SPEG, can be studied at temperatures lower than the $450^{\circ} \mathrm{C}$ that is required for thermal annealing.

Silicon (100) samples have been implanted with pither $G$ or overlapping distributions of Ga plus As to produce peak dopant concentrations of up to 15 at. \%. Regrowth of the silicon lattice was induced by bombardment of the implanted samples with ${ }^{28} \mathrm{Si}$ at 375 or $750 \mathrm{keV}$ with the sample held at $300-400^{\circ} \mathrm{C}$. Some samples were thermally annealed for comparison. Damage to the Si lattice and the distribution and substitutionality of the implanted 
species were measured using Rutherford backscattering (RBS)/ion chanioling. Lattice morphology was studied using transmission electron microscopy.

For the Ga-implanted samples only, defectfire growth, without $G$ precipitates, proceeds until the onset of an amorphous-topolycrystalline transition at the regrowing interface. This transition occurs when the concentration of $\mathrm{Ca}$ transported at the interface reaches a critical concentration of approximately $7 \times 10^{20} / \mathrm{cm}^{3}$ as the Si regrows. The amount of $\mathrm{Ga}$ trapped in the regrown $\mathrm{Si}$ is somewhat greater than that reported for thermal annealing and is temperature dependent.

For dual-implanted Si, the entire anrorphous layer is regrown epitaxially, and the $\mathrm{Ga}$ and As go highly substitutional, even for the highest initial dopant concentrations used. This is in contrast to regrowth of Ga-implanted sumples only. The presence of the As impurity offers competition to precipitation by paining with the Ga. No macroscopic transport of either dopant is observed in RBS spectra. It can be noted that thermal annealing of samples with peak concentrations of $\mathrm{Ga}$ and $A$ s above approximately 2.5 at. \% does not produce epitaxial regrowth.

1. Summary of papers: Appl. Surf. Sci.43, 191 (1989); Materiols Reseipch Society Symposium Proceedings (in press)

\section{TRAPTING OF D IN SIC AND DAMAGE DUE TO MMPANTATION'}

\author{
R. Siggele, 2 S. P. Withrow, \\ 1. Rolk, ${ }^{2}$ and B.M. U. Scherzer 2
}

The effects of particle bombardment, as well as inventory phenomena, have been studied for D implantation in SiC, a candidate material for the first wall of magnetic confinement devices. Singlecrystal SiC samples were implanted at normal incidence with $24-\mathrm{keV} \mathrm{D}_{2}{ }^{+}$ at both room temperature (RT) and $77 \mathrm{~K}$. Trapping of $\mathbf{D}$ was measured by detecting protons from the nuclear reaction $D\left({ }^{3} H e, H\right) a$. For low fluences, mearly all incident $D$ is retained at both temperatures. Saturation effects begin for RT implantation at $-8 \times 10^{17} \mathrm{D} / \mathrm{cm}^{2}$ and for half that dose at $770 \mathrm{~K}$. At saturation, approximately $1.4 \times 10^{18} \mathrm{D} / \mathrm{cm}^{2}$ and $0.8-1.0 \times$ $10^{18} \mathrm{D} / \mathrm{cm}^{2}$ are retained for $\mathrm{RT}$ and $770-\mathrm{K}$ impiantations, respectively. The maximum amount trapped at RT is approximately 0.5-D atom per host atom.

Damage to the S.C crystal lattice and location of the implanted ions were monitored using Rutherford backscattering/ion channeling. For $R T$ implantation, the peak in the distribution of damage to the Si sublattice occurs at a depth corresponding to the maximum in energy deposition, as calculated using the TRIM code. In contrast, for the 770-K implantation this peak shifts closer to the surface or to near the peak in the theoretical retained $D$ profile, suggesting that under these high-temperature conditions the damage to the Si lattice is stabilized by retained $D$. Experiments correlating the distri- 
bution of detected $\mathbf{D}$ with the angular position of the sample's channeling axes showed a very weak preference for the $D$ to be substitutional.

Isochronal annealing of the samples for $10 \mathrm{~min}$ at temperatures up to $1073 \mathrm{~K}$ revealed that retained lattice damage could be reduced if the lattice were not amorphous. All the implanted $D$ is retained up to $1073 \mathrm{~K}$, but by $1273 \mathrm{~K}$ most of the $D$ is lost from the implanted layer.

1. Summary of paper: Proceedings of the Ninth International Conference on ParticleSolid Interactions, Boumemouth, United Kingdom, 1990 (in press).

2. Max-Planck-Institut für Plasmaphysik, Carching Germany.

\section{LOCALIZED CONVERSION OF EPITAXIAL $\mathrm{YBa}_{2} \mathrm{Cu}_{3} \mathrm{O}_{7-x}$ FROM $c \perp$ TO a $\perp$ BYION IMPLANTATION AND LOW OXYGEN PRESSURE ANNEALING ${ }^{1}$}

\section{R. Feenstra, S. J. Pennycook, D. P. Norton, D. H. Lowndes, I. D. Budai, and M.D. Galloway}

Due $k$ s the highly anisotropic nature of the high-Tc supe; nducting cuprates, the properties of their films depend strongly on epitaxial orientation. For example, with epitaxial $\mathrm{YBa}_{2} \mathrm{Cu}_{3} \mathrm{O}_{7-x}(\mathrm{YBCO})$ films, high currentcarrying capability is achieved if the c-axis is oriented normal to the substrate. On the other hand, the larger coherence length along the $(a, b)$ directions of YBCO may make a 1 films more suitable for controlled fabrication of planar Josephson junctions. By selecting growth conditions, one can grow films which are either entirely $c \perp$ or $a \perp$ or mixed in some uncontrolled way. In this study, ion implantation followed by thermal processing was used to change the local epitaxial orientation of the films.

Epitaxial YBCO films and bilayers composed of 200-nm YBCO on 100-nm PrBa $\mathrm{Cu}_{3} \mathrm{O}_{-2}$ (PBCO) were grown on (100) $\mathrm{SrTiO}_{3}$ substrates by in situ laser ablation at $680^{\circ} \mathrm{C}$ in a $0.2-$ Torr oxygen background. Prior to implantation, the c-axes of YBCO and PBCO layers were normal to the substrate. Using a shadow mask, the films were locally implanted with oxygen ions $\left(10^{16} \mathrm{O}^{+} / \mathrm{cm}^{2}\right)$. Annealing was performed at $700-800^{\circ} \mathrm{C}$ in atmospheric argon/oxygen mixtures with $\mathrm{PO}_{2}=0.2$ Torr and resulted in predominantly al regrowth of the amorphized YBCO layer. Implantation energies in the range 68-110 keV were used for the bilayers to fine tune the depths of amorphized and damaged layers relative to the YBCO/PBCO interface.

Temperature dependences of the sheet risistance after each of the individual processin'g steps for one of the bilayer structures are illustrated in Fig.4.11. After implantation, electrical conduction takes place mainly through the semiconducting PBCO layer below the implanted YBCO layer. After regrowth, superconductivity is restored in the YBCO layer and the resistance curve is typical for $a \perp$ YBCO films, as confirmed by $x$-ray diffraction 


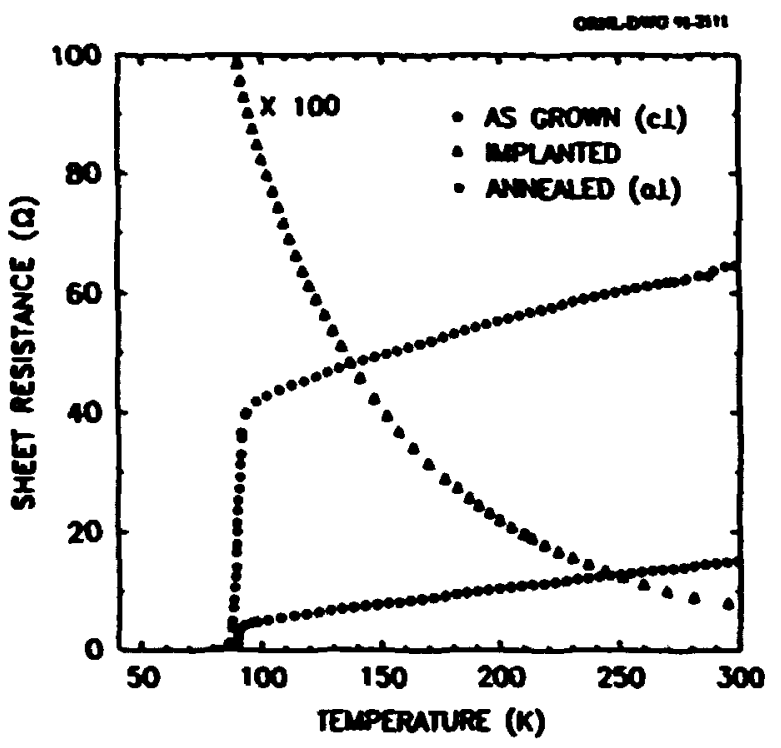

Fig. 4.11. Temperature dependence of the sheet resistance of a 200-nm $\mathrm{YBa}_{2} \mathrm{Cu}_{3} \mathrm{O}_{7-x}$ on 100-nm PrBa2 $\mathrm{Cu}_{3} \mathrm{O}_{7-x}$ bilayer on (100) $\mathrm{SrTiO}_{3}$ before and after oxygen implantation $(80 \mathrm{kV}$, $10^{16} \mathrm{O}^{+} / \mathrm{cm}^{2}$ ) and after regrowth at $760^{\circ} \mathrm{C}$ and $\mathrm{PO}_{2}=0.2$ Torr. After regrowth, superconductivity is restored with $T_{c}(\mathrm{R}=0) \equiv 86 \mathrm{~K}$.

and electron microscopy. An issential feature of this procedure is that the masked-off, unimplanted $c \perp$ YBCO layer remains intact after the low-pO $\mathrm{O}_{2}$ annealing process.

1. Summary of paper to be published.

\section{EFFECT OF $\mathrm{H}_{2} \mathrm{O}$ VAPOR ON THE EPITAXIAL RECRYSTALLIZATION OF ION BEAM AMORPHIZED STTIO,'}

\section{J. Rankin, J. C. McCallum, ${ }^{2}$ and L. A. Boatner}

Although it has been established that $\mathrm{SrTiO}_{3}$ can be made amorphous with a relatively low dose of implanted ions and that the amorphous layer can be made to regrow epitax- ially upon heating, many details of the effect of the annealing environment on the regrowth process have not been previously investigated. White et al. ${ }^{3}$ have monitored the epitaxial growth kinetics in air of amurphous layers on singas crystals of as-grown $\mathrm{SrTiO}_{3}$ throigh Rutherford backscattering (RBS) spectroscopy and channeling (RBS-C) measurements. They observed two distinct regrowth regimes- the first stage of regrowth, referred to as the "induction period," and the final, longer stage, referred to as the period of "fast growth." Additionally, the activation energy for the fast growth period under these conditions was found to be $0.77 \mathrm{eV}$. Thry were not able to measure the activation energy of the induction period; however, because of the limits inherent in the use of conventional fumace anneals in the production of small amounts of regrowth. Short regrowth distances are necessary to obtain amorphous-layer thicknesses as a function of time during the initial stages of regrowth.

In this work the effects of water vapor on the solid state epitaxial regrowth of ion beam amorphized, near-surface layers on singlecrystal $\mathrm{SrTiO}_{3}$ have been investigated using RBS and time-resolved reflectivity (TRR). The effects of alteration of the H/OH content of the crystal prior to ion implantation and amor. phization have been studied. The measured activation energy for the regrowth of amrorphous layers was $1.2 \pm 0.1 \mathrm{eV}$ over a range of ambient water vapor concentrations. However, when the concentration of water in the environment is increased, the activation energy for 
the regrowth process appears to decrease to $-0.95 \mathrm{eV}$. Additionally, when annealed in atmospheres containing $\mathrm{H}_{2} \mathrm{O}$ vapor, $\mathrm{SrTiO}_{3}$ exhibits the two distinct stages of regrowth originally reported by White et al. ${ }^{3}$ The two-stage regrowth process can be explained by the diffusion of $\mathrm{H} / \mathrm{OH}$ from the annealing atmosphere through the amorphous layer to the regrowing amorphous/crystalline interface.

1. Summary of paper to be published.

2. Onk Ridge Associated Universities postgraduate research participant. Present address: University of Westem Ontario, London, Ontario, Canada.

3. C.W. White et al., Nucl. Instrum. and Methods in Phys. Res. Sect. B 32, 11 (1988).

\section{HIGH-RESOLUTION X-RAY STUDY OF MMPLNTED SULFUR AS A PROBE OF COMMPLANTED OXYGEN IN AN OXIDE SUBSTRATE'}

\section{T. M. Rossed, 2 R. S. Peterson, ${ }^{3}$ C. R. Vane, J. P. Young, ${ }^{2}$ and R.A. Zuhr}

One of the most difficult chemical measurement problems is the characterization of a small change in the concentration profile of a major species in a complex sample. If the small change in concentration is limited to a narrow spatial band below the surface of the sample, such as in an ion-implanted layer, new or alternative approaches must be developed. One such alternative approach is to find a chemical difference induced by the localized change in concentration. If a chemical variation can be discerned, the technique must also be able to piobe the narrow band without destroying that chemical information. Heavy-ion-induced $x$-ray satellite emission (HIXSE) spectroscopy. because it nondestructively probes the nearsurface region (5-1000 nm) and yields chemical information, is ideally suited for this task. HIXSE combines the advantages of particleinduced $x$-ray emission (PDE) (i.e., the ability to detect an element in almost any matrix) with the chemical information obtainable from electron spectroscopy methods.

A series of high-resolution sulfur $K_{\alpha} L$ $x$-ray satellite spectra have been produced by 24-MeV Si ion irradiation of quartz glass previously implanted with 70-keV sulfur ions at $2.5 \mu \mathrm{A} / \mathrm{cm}^{2}$ to doses ranging from 2 to $8 \times 10^{16} / \mathrm{cm}^{2}$ and quartz glass coimplanted with 70-keV sulfur and 35-keV oxygen ions to equal depths and doses. A comparison of the HIXSE spectra from the sulfur- and oxygen-implanted samples with the sulfur only implanted targets reveals a clear shift in the satellite intensity distribution from higher order to lower order satellites. This is consistent with oxygen increasing the valence electron density of the local sulfur environment. An increase in the valence electron density will increase the L-vacancy refilling which will be observed as a lower yield of the higher order (e.g., $\mathrm{KL}^{5}$; satellite lines. These results not only demonstrate the sensitivity of HIXSE to small changes in the subsurface local chemical environment, but they also suggest that with an appropriate choice of an implanted probe ion, high-resolution HIXSE measurements may be capable of detecting not only atomic oxygen in an oxide matrix but also almost any impurity 
ion in any matrix, including matrices that normally mask the impurity.

1. Summary of paper to be published.

2. Analytical Chemistry Division, ORNL.

3. University of the South, Sewance, Tenn

3. Physics Division, ORNL.

\section{HON MDING OF SEMCONDUCTOR SURDRATTICES'}

\author{
W. Xia,2 S. A. Peppert, ${ }^{2}$ C. Cozzolino,? \\ J. Zhang, ${ }^{2}$ C. W. Tu, ${ }^{2}$ P. K. L Y $u_{0}^{2}$ \\ S. S. Lau, ${ }^{2}$ and D. B. Poker
}

Compositional disordering of III-V compound superiattice structures has received considerable attention recently due to its potential application for photonic devices. The conventional method to induce compositional disorder is to implant a moderate dose of impurity ions $\left(-10^{15} / \mathrm{cm}^{2}\right)$ into the structure at room temperature, followed by a high-temperature annealing step (designated IIHT). Ion irradiation at room temperature alone does not cause any significant intermixing of layers. The subsequent high-temperature annealing step, however, tends to restrict device processing fexibility. Ion mixing is capable of enhancing compositional disordering of layers at a rate that increases exponentially with the ion irradiation temperature. Hence, as a processing tech- nique to planarize devices, ion mixing appears to be an attractive technology. In this work. the compositional disordering in the AlGaAs/GaAs and the InGaAs/InP systems during ion mixing was investigated. It was found that the ion mixing behavior of these two systems shows a thermally activaled regime as well as an athermal regime, similar to that observed for metal-metal and metalsemiconductor systems. Ion mixing was observed to induce compositional disordering at significantly lower temperatures than that for the IHT process. In the AGaAs/GaAs system, disordering due to ion mixing was observed at $400^{\circ} \mathrm{C}$ [Fig $4.12(\mathrm{~b})$ ]. At $650^{\circ} \mathrm{C}$, both ion mixing [Fig-4.12(c)] and IHT [Fig-4.12(d)] produced disondering, but the ion-mixed samples showed a much higher crystalline quality, as evidenced by the decrease in amplitude of the satellite peaks and the flatness of the baseline.

1. Summary of paper: Nuclear Instruments and Melhods in Physics Research Section B (in press).

2. University of Califomia-San Diego, La Jolla, Calif. 
ORNL-DWG 91.2111

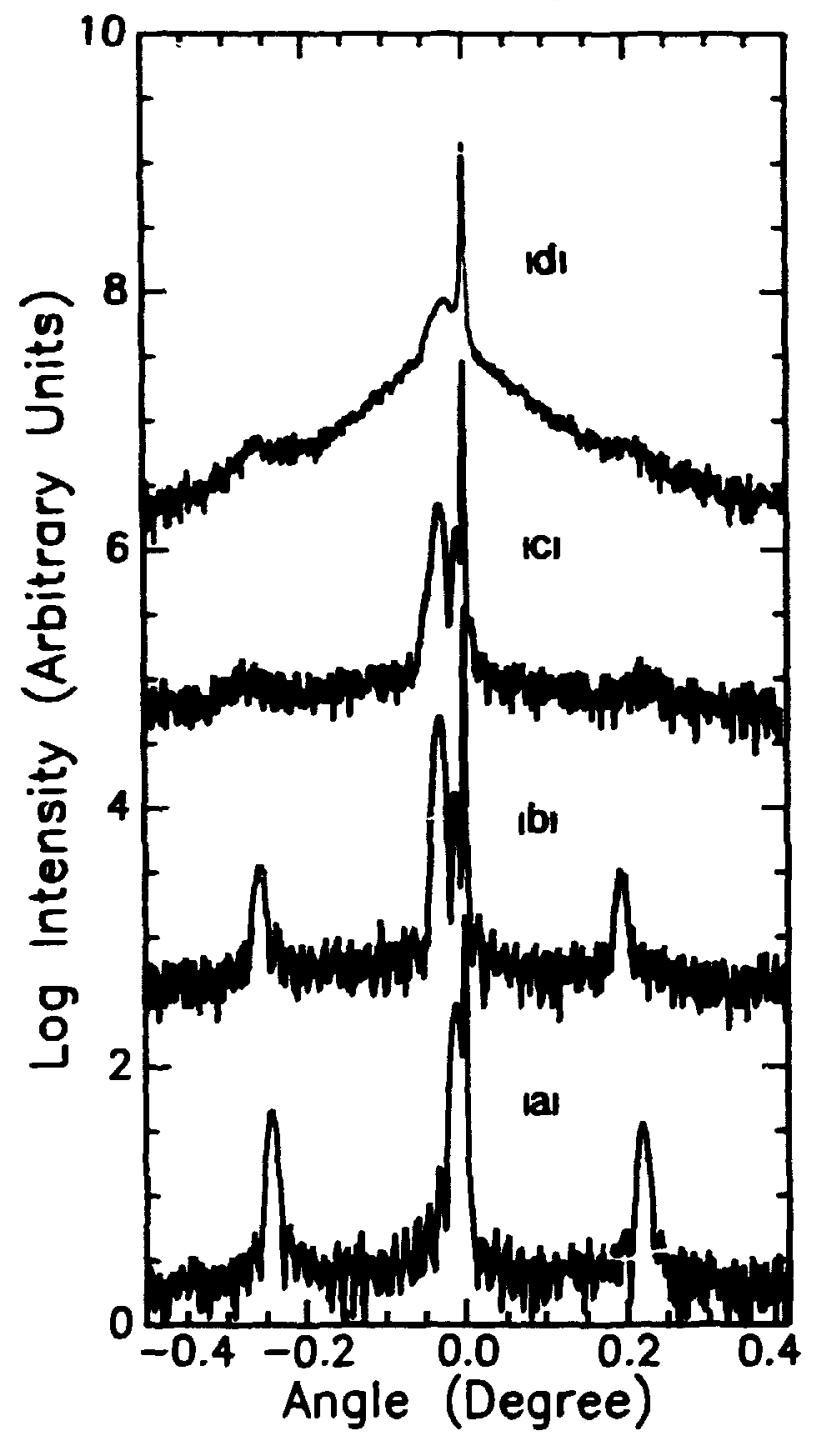

Fig. 4.12. $X$-ray rocking curves $(400$ reflec(ion) for a $A l_{0.5}$ Ga0.5As(100A)/GaAs(100A) sample with 16 periods: (a) as-grown; (b) after ion mixing at $400^{\circ} \mathrm{C}$ with $1 \times 10^{15} \mathrm{Ar}^{+} / \mathrm{cm}^{2}$, with an energy of $190 \mathrm{keV}$; (c) after ion mixing at $650^{\circ} \mathrm{C}$; and (d) after ion implantation at room temperature, followed by annealing at $650{ }^{\circ} \mathrm{C}$ for 20 minutes (IIHT).

\section{LASER AND MOLECULAR BEAM PROCESSING OF THIN FILMS}

\section{IN STIU GROWTH OF HIGH-QUALTY

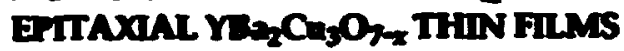 \\ DY PULSED-LASER ABLATIONI}

\author{
D. H. Lowndes, D. P. Nortom, 2 I. W. McCany, 3 \\ R. Feenstine, I. D. Budri. \\ D. K. Christen, and D. B. Paker
}

Pulsed-laser ablation (PLA) has the advantages that smooth, stoichiometric highcemperature superconducting films can be grown in situ at temperatures of only $600-7500^{\circ} \mathrm{C}$ and that this can be accomplished in a few minutes for films $100-200-\mathrm{nm}$ thick $(0.1-0 \mathrm{Am} / \mathrm{p}$ ilse). We have recently prepared high-quality $\mathrm{YBa}_{2} \mathrm{Cu}_{3} \mathrm{O}_{7-2}(\mathrm{YBCO})$ films using a pulsed $\mathrm{KrF}$ (248-nm) excimet laset beam (1.1), -38-ns FWHM pulse duration) that is passed through an aperture and brought to a horizontal line focus $\left(-2.5-3.0 \mathrm{~J} / \mathrm{cm}^{2}\right)$ on a 2.5-cm-diam polycrystalline YBCO target and scanned over the target perpendicular to the line-focus direction in onder to form an extended region of more uniform film deposition. This combination results in films that are completely uniform in composition over areas $-8 \mathrm{~cm}^{2}$, maintains a smooth (nondendritic) target surface even after many laser pulses, and results in thickness variations 
of only -15-25\% over $2.5 \mathrm{~cm}$ in both the horizontal and vertical directions for argetsubstrate separations $-7 \mathrm{~cm}$.

Our best films were grown at $T_{\text {abb }}-730^{\circ} \mathrm{C}$. 1.1-Hz laser-pulse rate, and $p\left(O_{2}\right)-200$ mTor for single-crystal substrates such as $\mathrm{SrTiO}_{3}$ $\mathrm{KTaO}_{3}, \mathrm{LaCaO}_{3}, \mathrm{LaAlO}_{3}$, and cubic $\mathrm{ZrO}_{2}$ These films have an extrapolated normal-state resistivity, $p(T=0 \mathrm{~K})$, that is zero or a few percent negative (in units of the roomtemperature resistivity) and a resistivity p(100K) - $85 \mu \Omega-\mathrm{cm}$ on $\mathrm{SrTiO}_{3}$ (Table 4.1). Films grown at bower $T_{\text {sob }}$ have higher resistivity and $\rho(0 K)>0$. For MgO, the best results were obtained for a bower $T_{\text {sub }} \sim 66^{\circ} \mathrm{C}$, apparently because of interdiffusion and/or civemical reaction between YBCO and $\mathrm{MgO}$ at $730^{\circ} \mathrm{C}$.

X-ray diffraction studies show that PLA films remain in the $c \perp$ orientation regardless of film thickness; consequently the sheet conducance of the films increases lincarly with film thickness (unlike some coevaporated and postannealed film processes). Athough films normally were cooled in $-100-600$ Tort oxygen at $10^{\circ} \mathrm{C} /$ min to minimize stress due to differences is Film and substrate thermal expansion coefficients, excellent YBCO films could be produced even when "quenched" at $1^{\circ} \mathrm{C} / \mathrm{s}$, provided that they were grown on a thermally wellmatched substrate such as SrTIO,

1. Summary of paper: Mat. Res. Soc. Sym. Proc. 169, 431 (1990).

2. ORNL Eugene P. Wigner Fellow.

3. Oak Ridge Associated Universities Professional Internship Program graduate student from The University of Tennessee, Knoxville, Tenn.

Table 4.1. Chrracteristics of the highest $\mathrm{T}_{c} \mathrm{YBa}_{2} \mathrm{Cu}_{3} \mathrm{O}_{7}$, films on selected substrates.

\begin{tabular}{lcccccc}
\hline Substrate & $\begin{array}{c}\text { Thickness } \\
(\mathrm{sm})\end{array}$ & $T_{\text {sab }}^{(\mathrm{a})}$ & $T_{c}^{(\mathrm{b})}$ & $\Delta T_{c}^{(\mathrm{c})}$ & $\rho(100 \mathrm{~K})^{(\mathrm{d})}$ & $R R^{(\mathrm{e})}$ \\
\hline $\mathrm{SrTO}_{3}$ & 502 & 730 & 92.5 & 0.65 & 86 & -0.03 \\
$\mathrm{KTaO}_{3}$ & 774 & 730 & 92.1 & 0.9 & 215 & 0.00 \\
$\mathrm{LaCaO}_{3}$ & 333 & 730 & 91.7 & 1.5 & 105 & -0.01 \\
$\mathrm{LaAlO}_{3}$ & 47 & 730 & 90.3 & 1.8 & 124 & 0.09 \\
$\mathrm{ZrO}_{2}$ (cubic) & -275 & 730 & 90.3 & 2.2 & $\sim 97$ & 0.04 \\
$\mathrm{M}_{8} \mathrm{O}$ & -175 & 665 & 88.2 & 1.6 & $\sim 101$ & -0.01 \\
\hline
\end{tabular}

(a) Film-growth temperature ( $\left.{ }^{\circ} \mathrm{C}\right)$; (b) zero-resistance $T_{C}$; (c) 10-90\% transition width; (d) resistivity in $\mu \Omega-\mathrm{cm}$ at $100 \mathrm{~K}$; and (e) resistance ratio $(R R)=R(0 \mathrm{~K}) / R(300 \mathrm{~K})$. 
Y-BA-CU-0 THIN FILMS GROWN ON RIGID AND FLEXIELE FOLYCRYSTALUNE MTRIA-STABILRED ZIRCONA BY PULSED-LASER AOLATION'

Devid P. Norton, Dougles H. Lowndes, J. W. McComy, J.D. Budai, D. K. Christen, E.C. Jones, Thomes D. Ketchan, 5 Dell St. Inlien, ${ }^{5}$ K. W. Lyy, ${ }^{6}$ and J. E. Mreczyt6

Polycrystalline yttria-stabilized zirconia (YSZ) substrates appear potentially useful for growth of $\mathrm{YBa}_{2} \mathrm{Cu}_{3} \mathrm{O}_{7-2}(\mathrm{MBCO})$ supperconducting films. The sizes and shapes of the substrates needed for specific applications are not constrained, as with single-crystal substrates, and flexible, thin-polycrystalline YSZ substrates can be obtained. The in situ growth of highly oriented $\mathrm{YBa}_{2} \mathrm{Cu}_{3} \mathrm{O}_{7-x}$ thin films (200$500 \mathrm{~nm}$ in thickness) by pulsed KrF (248-nm) laser ablation on both rigid and nexible randomly oriented poly-YSZ substrates was recently obtained. C-axis-perpendicular YBCO films were obtained with mosaic spreads of only $1^{-}$on these randomly oriented polycrystalline substrates. Superconducting thin films were obtained with $T_{c o} \sim 89 \mathrm{~K}$ on well-polished poly-YSZ substrates. For films deposited on the flexible substrates, $T_{c o}$ was not degraded by repeated bending of the nexible substrate/film composite over a $2.25-\mathrm{cm}$ radius arc, although the normal-state resistivity increased slightly suggesting the creation of microcracks. The resistance vs temperature characteristics for YBCO thin films on flexible polycrystalline, rigid polycrystalline, and (100) single-crystal YSZ substrates are shown in Fig. 4.13. The zero-resistance superconducting Iransition temperatures for films deposited on

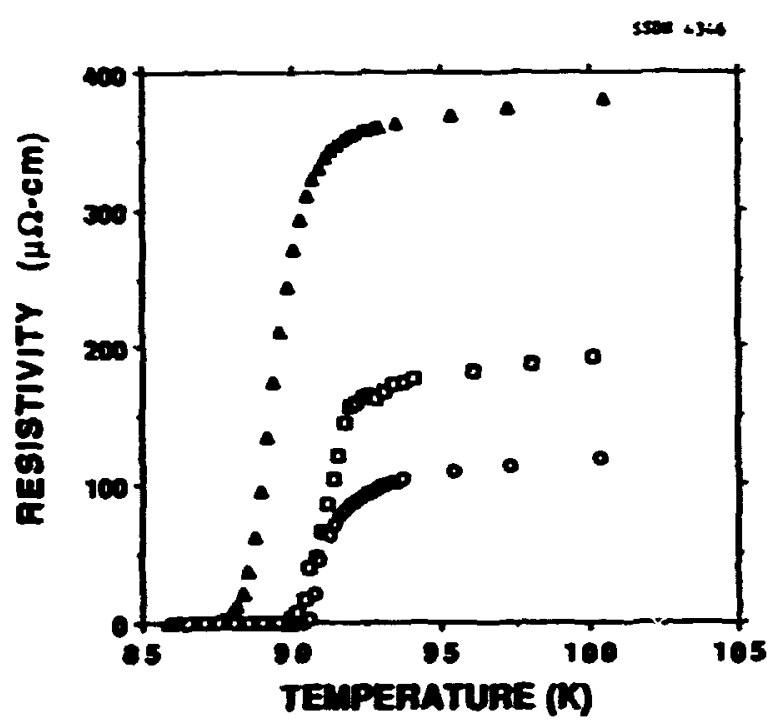

Fig. 4.13. Resistivity vs temperature for YBa2 $\mathrm{Cu}_{3} \mathrm{O}_{7-x}$ thin films grown on (0) (100) single-crystal, (O) cold-pressed and sintered polycrystalline (General Electric), and (A) flexible polycrystalline (Coming) YSZ substrates.

polyerystalline YSZ substrates are seen to be only slightly loner than for those grown on the single-crystal substrate. However, for the films deposited on both types of polycrystalline substrates, an extrapolation of the normal-state resistance curve $100 \mathrm{~K}$ gives a nonzero resistance intercept. This has been found to be the case for $c$-axis perpendicular YBCO thin films that do not exhibit an in-plane epitaxial relationship. Finally, it was found that the critical current density, $I_{c} \sim 4000 \mathrm{~A} / \mathrm{cm}^{2}$, was not affected by flexing the film/substrate composite three times, but did gradually decrease with repeated flcxing.

1. Summary of paper: J. Appl. Phys. 68, 223 (1990).

2. ORNL Eugene P. Wigner Fellow. 
3. Oak Ridge Associated Universities Professional Internship Program graduate siudent from The University of Tennessee, Knoxville, Tenn.

4. Graduate student from the University of Tennesce, Knoxville, Tenn.

5. Coming Incorporaled, Coming N.Y.

6. Ceneral Electric Corporate Research and Development, Schenectady, N.Y.

\section{NEW FACIITIES FOR FUNDAMIENTAL STUDIES OF LASER-GENIEATED TLAMNS AND FO: LAST-ADATLON FIM GIOWTH}

D. H. Lowndes, D. B. Geohroun, R. Fenstion, J. W. MCCony, and S. Zux ${ }^{2}$

During FY 1990, the design and purchase of three new laser ablation facilities for growth of highly uniform, larger area hightemperature superconductor (HTSc) films for growth of wide-band-gap II-VI semiconductor films and for spectroscopic studies were completed.

The HTSc film-growth facility consists of a laser ablation deposition chamber, vacuum system, pressure measurement and control systems, laser beam handling components, and control electronics. This system will relieve present limitations on the experimental time available for film growth and will provide several new film-growth capabilities, including (1) a larger area of uniform-thickness film growth, (2) in situ deposition of both superconducting and dielectric buffer/protective layers without breaking vacuum, (3) in situ deposition rate monitoring for accurate film thickness measurements, (4) an ion gun port for in situ substrate cleaning and for studies of ion-assisted film growth, and (5) indirect substrate heating for studies of HTSc film growth on continuously fed substrates. When coupled to a new highpower excimer laser, this system is expected to provide epitaxial HTSc growth rales $>100 A$ $\mathrm{cm}^{2} / \mathrm{s}$

The II-VI semiconductor film-growth system incorporates similar capabilities. Pulsedlaser ablation of wide-band-gap II-VI films from solid targets is attractive because it is expected most of the material can be transported from target to substrate as molecules (not atoms or dusters) and because the process is inherently free of carbon or hydrogen contamination (unlike metalorganic chemical vapor deposition, for example). Doping to control the carrier type and concentration in II-VI films will be accomplished either by ablating into an ambient low-pressure vapor or gas that rontains the dopant element or by alternating between doped and undoped targets. Epitaxial compositional superlattice structures also will be grown by alternating targets.

Spectroscopic studies are carried out in a functional-deposition chamber that has been modified for optical access to the ablation plume from three-orthogonal axes. Film growth feasibility surveys can be carried out for a wide variety of materials. Optical absorption and gated-optical emission measurements can be carried out, as well as ion probe measurements of the plasma plume during its -10 us propagation time. New experimental capabilities include: (1) studies of the spatial evolution of the plume over the entire plume propagation region; (2) doppler velocity-profiles of 
plume fluorescence; (3) studies of background gas-pressure effects, which include scattering. slowing and conversion of species in the plume; (4) studies of the spatial variation of the ion density in the plasma plume, using ion probes; and (5) two laser-crossed-beam experiments in which the plume is created and then probed or modified by a second laser pulse.

1. Oak Ridge Associated Universities Professional Intemship Program graduate student from The University of Tennessee, Knoxville, Tenn.

2. Graduate student from The University of Tennessee, Knoxville, Tenn.

\section{COMBNED STECTROSCORC AND ION PROBE CHARACTEREATION OF THE LASER ABLATION TRANSFOKT PROCESS '}

\section{B. Geokegen}

Spatial and temporal measurements were made of the absorption, emission, and ion probe response in the ablation "plume" of material formed following pulsed $248-\mathrm{nm}$ irradiation of YBCO targets. Laser-energy densities ranging from near threshold up to the film-growth regime $\left(-1 / \mathrm{cm}^{2}\right)$ were used in order to understand the formation, composition, acceleration, and propagation of plumes utilized for film growth. The background gas pressure in the chamber also was varied.

As shown in Figs. 4.14(a) and 4.14(b) for Ba+ and Ba, time-of-flight absorbance-velocity profiles in vacuum indicate the formation and acceleration of a plasma front with ions leading neutrals on the edge of the expanding plume. Estimates of densitics for $\mathrm{Ba}$ and $\mathrm{Ba}^{+}$
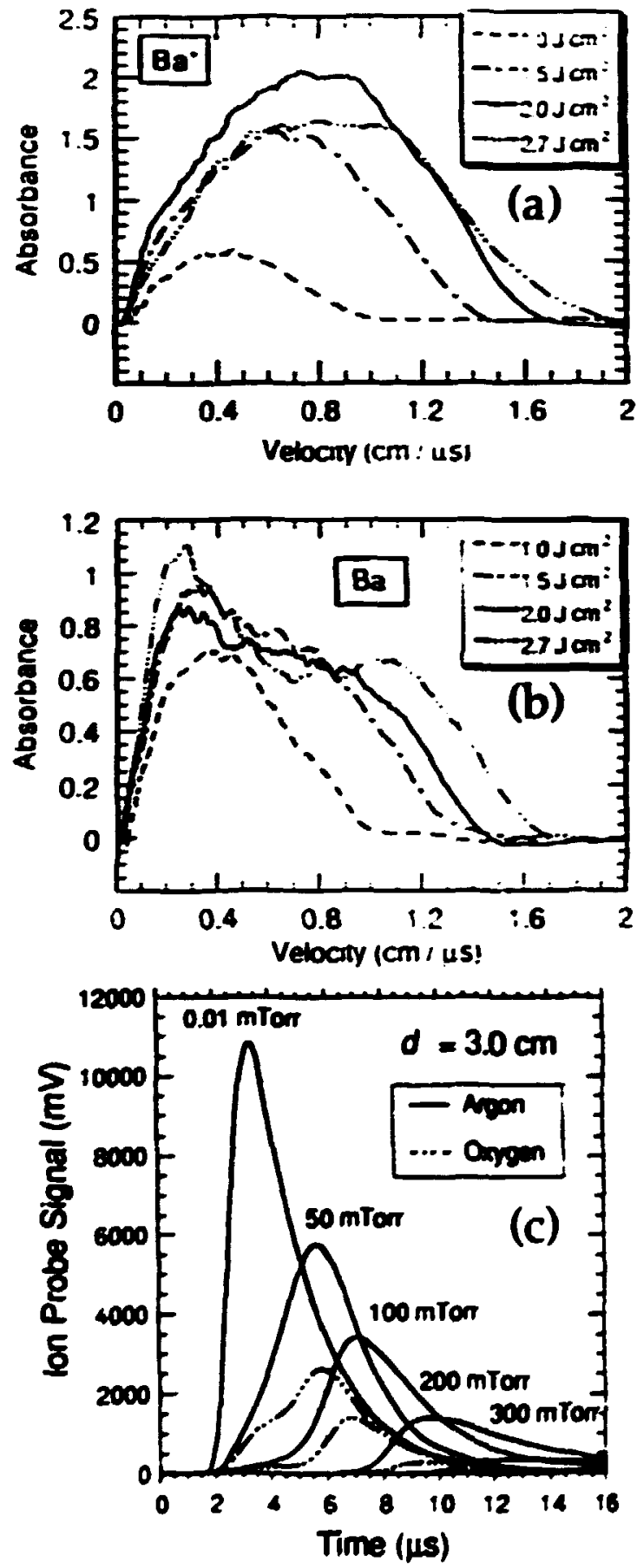

Fig. 4.14. (a) and (b) Measured absorbances vs time-of-flight velocity for ground state $B a$ $(553.5 \mathrm{~nm})$ and $\mathrm{Ba} *(445.4 \mathrm{~nm})$ at $d=1 \mathrm{~cm}$ from a $\mathrm{Y}_{1} \mathrm{Ba}_{2} \mathrm{Cu}_{3} \mathrm{O}_{2}$ pellet at $10^{-5}$ Torr following $\mathrm{KrF}$ irradiation at four energy densities. (c) Comparison of ion current waveforms $3.0 \mathrm{~cm}$ from Krf-irradiated $\mathrm{Y}_{1} \mathrm{Ba}_{2} \mathrm{Cu}_{3} \mathrm{O}_{7}$ in 0.01 and $50,100,200$, and $300 \mathrm{mTon}$ background pressures of argon and oxygen. 
indicale that at the higher laser-energy densiies, the Bat ions outnumber Ba neutrals by 5 b 1. Plasma kinetic procesves such as three body recombination appear chiefly responsible for the fluorescence (not shown), which follows the leading edge of the ion palse and "feeds" the neutral ground steve popolation, resulting in the apparent two-component Ba profile of Fig-4.14.

The increase in plume velocity and ion density with boerenegy dencity was also weosured with ion probes. The number of ions in the ploure increased nonlinearly ove two regimes. Below $0.32 / \mathrm{cm}^{-2}$, the dblation threshold, the cotal charge varies shaply as $\mathrm{P}$, where $I$ is the laser intensity. Above $0.7 / \mathrm{cm}^{-2}$, the charge increases more gradually as $I^{1.6}$. These energy density ranges correlate with the formation of the bright hser plasina, indicated by a rapid increase in fluorescence of all species, and indjcate the probable interaction of the laser light with the ejected, high-density plasma vapor. Figure 4.14(c) shows the effect of collisions on the laser-plasma propagation, at a fixed distance, in background argon and oxygen. The integrated charge displays an exponential attenuation with pressure and distance, while the plasma front is slowed exponentially in time and linearty with distance. This results in a limiled range for useful film deposition and in a wide variety of possible incident kinetic energies.

1. Summary of paper: p. 211 in Laser Ablation for Materials Symthesis, ed. by David C. Paine and John C. Bravman, Materials Research Society, Pittsburgh, Pa., 1990.

\section{FIRST DRECT MEASUREMITIS OF GROUNDSTATENIJIRAL AND ION TRANSTOXT DURING LASTR ARATION OF YCO USANG TMANSIENT ORTCAL ALSONTON STECTMOSCOMY'}

\section{B. Ceakegan}

Transieal optical absorption spectroscopy was utilized for the first tine to study the trensport of ground-state Y, Ba, Cu, Yt, and Bo+ following excimer-laser ablation of MaCO under film-growth conditions. Although the mpiority of aloms and singly cherged ions cour prising the high-relocity $\left(0-10^{6} \mathrm{~cm} \mathrm{~s}^{-1}\right)$ palse of expanding plasma are in the grownd stake, optical enisoion spectroscopy of exciled staves has been the vechnique typically employed for characterization of the plume transport, until this work. Time-of-flight (TOF) velocity distributions of the ronemitting nevtrals and cons determined by the absorption technique are broadened and shifted to bower velocities rebtive to the velocity distributions inferred from excited-state fuorescence in the plume (Fig- 4.15). By revealing the temporal history of the ground-state species, the kinetic processes producing the excited state populations were uncovered.

Strong abcorption on the resonance lines of $\mathrm{Y}, \mathrm{Ba}, \mathrm{Cu}, \mathrm{Y}^{+}$, and $\mathrm{Ba}^{+}$was observed with a relatively simple experimental arrangement. An Xe flashlomp provided -500-ns pulses of brnadband continuum emission, which was focused to provide a $-0.5-\mathrm{mm}$ beam through the plume. Due to spectral broadening in the laser plasma, a 1-m spectrometer provided sufficient spectral resolution to resolve the absorption. 


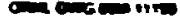
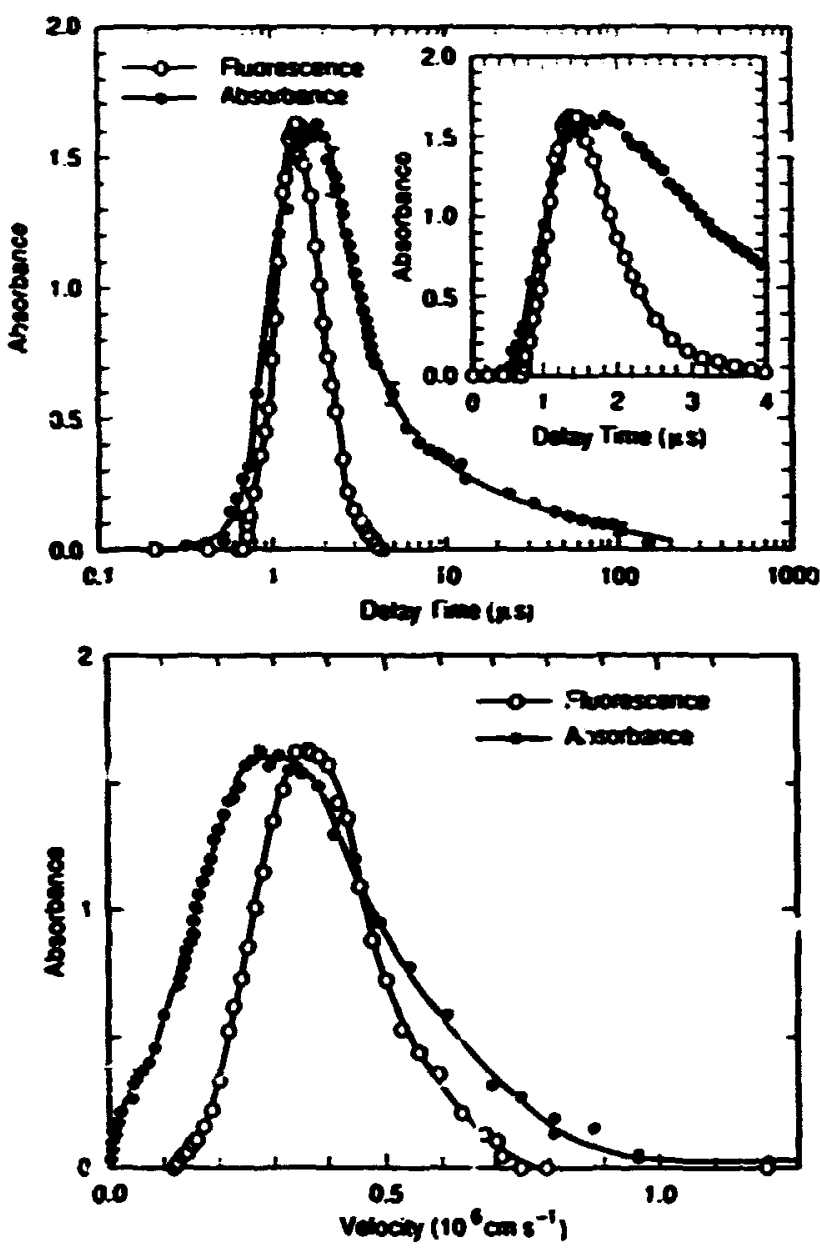

Fig. 4.15. (Top) Temporal history for atomic $\mathrm{Ba}, 5 \mathrm{~mm}$ from the inradiated pellet, as munitored by Ba* emission and ground-state Ba absorption using the $6 p^{1} P_{1}-60^{2} S_{0}$ transition at 5535 A $A$. The peak of the fluorescence signal was normalized to the peak of the measured absorbance, $\ln \left(I_{a} / \mathrm{h}\right.$. The arrival of the $1-\mathrm{J} \mathrm{cm}^{-2}$ Arf laser pulse at the pellet is defined as $t=0$. (Bottom) The resulting TOF velocity distributions show a pronounced low-velocity component not revealed by the plume fluorescence.

By simply tuning the spectrometer, the absorbance and lineshape of the resonance transitions of each of the species could be quickly studied at specific spatial locations and time delays after arrival of the laser pulse at the target.
1. Summary of paper. Appl. Phys. Lett. 55. 2345 (1990).

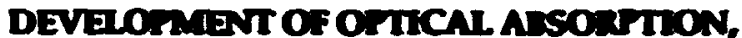 EMISSION, AND EON FOOTEMIETLODS AND FACIITIES TO CHARACTEAVA LASE ARATON TUUMTES}
D. B. Geoheran

Three complementary plasma diagnostic techniques have been developed for in situ diagnostics of the polsed-Haser ablation plume during thin-film growth These nonintrusive techniques operate in high-density, wellscreened laser plasinas that are typically produced in beckground gases where collisions are important. These techniques address the need for implementable in situ diagnostic techniques to measure the flux of species, kinetic energy of components, fractional ionization, and plasma temperatures in real time during film growth. The experimental apparatus developed for the diagnostics is shown in Fig. 4.16. Each diagnostic is described in detail in the following:

(1) Optical emission spectroscopy is used to study the formation and decay of excited species in the plume. A high-resolution spectrometer $(0.04 A)$ is used to measure the temporal evolution of excited species with $-50-\mu m$ spatial resolution. High-resolution scans of the emission reveal Stark-broadened line shapes that can be used to estimate boal-electron temperature and dencities.

(2) Optical absorption spectroscopy inver tigates the (nonemitting) ground state populations of atoms, ions, and molecules. Absorption 


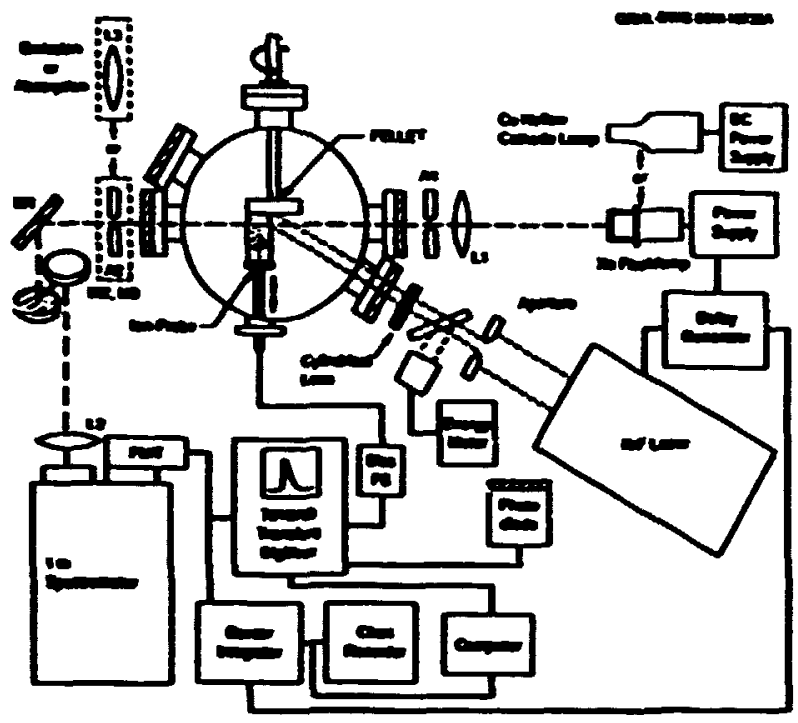

Fis 4.16. Schematic diagram of the experimental apparalus used for diagnostics of the laser ablation transport process. Optical absorption and emisoion are used to characterize spatially and temporally the populations of ground-state and excited species in the plume. Ion probes are used for measurements of the velocity and flux of the expanding plasma front.

of broadband emission from a pulsed, Xe flashlamp is observed with the spectrometer, and absorption of narrowbandwidth emiscion from a dc hollow cathode lamp can also be performed with a simple photomultiplier using an interference filter. Typical absorption line shapes are Stark-broadened in the laser plasma to 0.3-1.5 A.

(3) Ion probes are employed to measure the bulk ion and electron densities (with low species selectivity), to m-asure the total flux, and to make time-of-flight measurements of the velocities of the charged species. A series of ion probes was developed to investigate the local velocities and charge densities in the laser-produced plasma; the small dimensions and pulsed mature $(\tau-10 \mu s)$ of the plasma required small (area $-0.02 \mathrm{~cm}^{2}$ ) and fast ( $\tau<3$ ns) probes for the necessary spatial and temporal resolution.

1. Summary of paper. p. 153 in Superconductioily and Applications, ed. by H.S. Kwok et al., Plenum Publishing Company, N.Y., 1990.

\section{THE EFFCT OF D JOSMION RATE ON THE GROWTH OF ETTAXILI Ge ON Gasetion!'}

D. Eres, D. H. Lowndes, I. Z. Tischler, I. W. Shapp' T. E. Haymas, and M. F. Chisholm

A molecular jet-growth technique utilizing high-pressure expansions of digermane molecules seeded in helium was used for Ge thin-film growth. The capability of this growth technique to provide a wide range of average growth rates was utilized to investigate the effect of growth kinetics on thin-film quality. Microstructural and electrical properties of epitaxial Ge films grown on GaAs(100) at average growth rates from $10 \mathrm{~mm} / \mathrm{min}$ to over $1100 \mathrm{~nm} / \mathrm{min}$ were investigated.

Transmission electron microscopy (TEM), Rutherford backscattering spectroscopy (RBS), and $x$-ray double-crystal diffraction (XDCD) revealed high-quality epitaxial Ge films (regardless of growth rates) that were free of extended defects and misfit dislocations. However, films grown slowly $(10 \mathrm{~nm} / \mathrm{min})$ had rough surfaces characteristic of film growth by 
island formation. At high-growth rates (1100 $\mathrm{mm} / \mathrm{min}$ ) a dramatic reduction of islanding was observed. The density of islands at intermediate growth rates was found 10 be in inverse relationship with the growth rates.

Another consequence of rapid growth is a marked improvernent in electrical propenties. Ce films grown on GaAs (100) are either n-type or p-type as a result of autodoping from the substrate. The switch in carrier type from n-type to p-type is a consequence of compensation, and the temperature at which it occurs is a function of growth rates. For Ge films grown rapidly at $1100 \mathrm{~nm} / \mathrm{min}$, this switch occurs above $600 \mathrm{C}$. while molecular beam epitaxy (MBE) groun Ge films tum p-i,jpe around $40 \mathrm{CC}^{3}$ Molecular jetgrown films at slow-growth rates (-MBE rates) exhibit the same carrier type and carrier concentration as the MBE-grown material. Molecular jet-grown Ge films are less compensated and consequently have highes mobilities than the MBE-grown material. These results are interpreted in terms of an interplay between thermodynamics and kinetics. At high-growth rates there is less time to reach equilibrium. Consequently, rapid growth provides a means to eliminate the deleterious effects of equilibrium processes such as surface roughening. autodoping, and impurity incorporation on thin-film materials properties.

1. Summary of paper: 1. Appl. Phys.67, 1361 (1990).

2. Graduate student from the University of Tennessee, Knoxville, Tenn.

3. M. Kawanaka et al., I. Cryst. Growth 95, 421 (1989).

\section{GAS-MUASE CHARACTERIZATION OF \\ DIGERMANE MOLECULAR JETS USED \\ IN THIN-FILM GROWTH?}

$$
\text { D. Eres and I. W. Shap? }
$$

Gas-phase characterization of supersonic molecular jets containing film-growth source molecules is an important step toward understanding and using molecular jets to control thin-film-growth processes. Measurements of velocity distributions, radial intensity distributions, and the centerline intersity in the jet all provide information that can help to elucidate growth proceses and mechanisms through direct comparisons with film thickness distributions and growth rates.

Measurements of radial intensity distributions in continuous molecular jets were carried out using a mass spectrometer in a differentially pumped two-chamber system. The radial distributions of both helium carrier gas atoms and digermane molecules were measured at several distances within the $10-30-\mathrm{mm}$ range. An $l_{0} \cos ^{m} \theta$ function gave a good fit for both helium (with $m=15$ ) and digermane (with $m=22$ ) intensity distributions.

Thin-film growth rates from digermane molecular jets show a linear dependence on digermane intensity. The thickness distribu. tions of films grown on Si(100) substrates show excellent agreement with the gas-phase distribution of digermane molecules, indicating that film growth occurs from molecules supplied directly by the jet and not as a result of growth from the low-pressure, steady-state background gas. 
Supersonic expansions provide tunable transhtional energy sources by seeding heavy film-growth molecules in various carrier gases. Preliminary data obtained by time-of-flight measurements of pulsed molecular jets show that digermane molecules seeded in helium carrier gas at $5 \%$ concentration travel with a velocity about 4 times higher (and kinetic energy 16 times higher) than their corresponding room-temperature thermal velocity. Investigations are presently under way to determine whether and how these neutral molecules with superthermal (1-2 eV) energies can enhance thin-film growth.

1 Summary of paper to be published.

2. Graduate student from The University of Tennessee, Knoxville, Tenn.

\section{MECHANISM OF Ge THINHFHM GROWTH FROM DIGERMANE MOLECULAR JETS'}

\section{Eres and J. W. Shanp}

The use of molecular jets facilitates inves tigation of thin-film growth processes free from the background interference caused by the mase and heat-transport effects typical of flowinggas-reactor environments used in chemical vapor deposition. Contributions to film growth from homogeneous gas-phase reaction pathways also can be neg!ected, because all the unreacted source molecules and reaction products are pumped away immediately following collision with the substrate surface. As a result, it was possible to construct a model for epitaxial Ge growth on GaAs(100) from digermane $\left(\mathrm{H}_{3} \mathrm{Ge}-\mathrm{CeH}_{3}\right)$ molecules using experimental results obtained with a digermane supersonic-molecular jet.

The growth-rate dependence on digermane arrival rates shows two distinctly different growth regimes. The earty stage of film growth (up to -2-nm thickness) is known as the incubation period, because it is characterized by slow growth rates. In this regime, the growth rate increases exponentially with the inbensity of digermane molecules. After the incubation period, growth rates are much higher and increase linearly with the intensity of digermane molecules. No plateaus or saturation regions are observed throughout the entire intensity range.

In a substrate temperature rangi from $\mathbf{4 0 0}$ to $700 \mathrm{C}$, the Ge growth rates exhibit a nonlinear dependence on reciprocal substrate temperature. The growth curve starts out with a negative slope (growth rate increasing with temperature), followed by a short plateau, and then changes to a positive slope (decreasing growth rate at high temperatures). The position of the plateau shifts toward higher substrate temperatures with increasing digermane arrival rate.

These observations were used to construct a phenomenological model of Ge film growth based on sequential elimination of molecular hydrogen from a hydrogenated growth surface. The hydrogenated growth surface is produced during the incubation period through chemisorption of digermane molecular frag- 
inents, $\mathrm{H}_{3} \mathrm{Ce}-\mathrm{GeH}$.. Film growth occurs on this surface by incorporation of digermane molecular fragments, $\mathrm{H}_{3} \mathrm{Ge}-\mathrm{GeH}$; at growth sites created by molecular hydrogen elimination. In the model, the occurrence of the plateau region followed by a decrease of growth rate at high substrate temperatures is caused by a loss of growth sites through rapid Ge-Ge surface dimer formation. Film growth on such a surface is slow because the surface dimer has to be reopened before film growth can occur.

1. Summary of paper to be published.

2. Graduate student from The University of Tennessee, Knoxville, Tenn.

\section{APPARATUS FOR INVESTIGATION OF THIN-FILM GROWTH BY UTILIZING SUPERSONIC MOLECULAR BEAMS}

\section{Eres and J. W. Sharp 1}

Supersonic molecular beams provide unique capabilities to control independently some experimental parameters that cannot be controlled using other film-growth methods. The translational (kinetic) energy of neutral filmgrowth molecules can be tuned within a 0.05-2 $\mathrm{eV}$ range by seeding the molecules in various carrier gases in the supersonic expansion. Furthermore, the ability of supersonic expansions to induce cluster formation also can be exploited to investigate the role of neutral molecular clusters in thin-film growth.

An ultrahigh vacuum molecular beam apparatus is being constructed for investigation at a monolayer level of fundamental processes in thin-film growth of semiconductor materials. This apparatus consists of a subsystem for supersonic molecular beam generation and characterization and a second subsystem for surface analysis and characterization. It is equipped with a load-lock chamber to avoid atmospheric exposure during sample transfer.

The surface-analysic subsystem consists of a 100-mm radius hemispherical analyzer, an electron gun for Auger electron spectroscopy, and an $x$-ray source for $x$-ray photoelectron spectroscopy measurements. It is used for chemical analysis of the substrate surface prior to film growth and for composition-depth profiling during or after thin-film growth experiments. A reflection high-energy electron diffraction (RHEED) system is used for surface orientation and in situ monitoring of surface structure and film growth.

Supersonic molecular beams of source gases for semiconductor film growth are generated in a differentially pumped system of chambers. The beam source is a high-pressure supersonic expansion that is skimmed and collimated before it is introduced into the film-growth chamber. The system is designed to handle both pulsed and continuous beams.

The apparatus will be used to investigate fundamental aspects of heteroepitaxial growth of $\mathrm{Si}$ and $\mathrm{Ce}$ as well as Si-Ge alloy thin films. The ultimate goal is growth of ultrathin $\mathrm{Si}_{n} \mathrm{Ge}_{m}(n, m=1-3)$ strained-layer superlattices. It is believed that growth from low-energy, high-fluence beams of hydrogenated source molecules can produce chemically sharp inter- 
faces that are free of $\mathrm{Ge}$-Si interdiffusion observed in films grown from elemental soürces by molecular beam epitaxy.

1. Graduate student from The University of Tennessee, Knoxville, Tenn. 


\section{Structure of Solids and Surfaces}

This chapter focuses on the structural properties of solids and surfaces. A variety of techniques including surface spectroscopies, electron microscopy, electron diffraction, and both conventional and synchrotron $x$-ray diffraction are utilized.

The Surface Physics Program has continued to emphasize the atomic-scale structure and dynamics of surfaces and molecules on surfaces. The spot profile analysis technique has been used to study the growth kinetics of $\mathrm{Ag}$ on Si(111) and to provide the first experimental evidence for two-dimensional scaling of the domain-size distribution with coverage. Synchrotron radiation has been employed to examine the phase behavior and structure of the Au(001) and (111) surfaces between $300 \mathrm{~K}$ and the triple point. Enhanced surface anharmonicity has been observed by measuring the temperature dependence of vibrations on $\mathrm{Cu}(110)$. Radiation-enhanced oxidation and ordered chemisorption of nitrogen on $\mathrm{Cu}(110)$ have been studied.

In the Electron Microscopy of Materials Program, a mathematical inderstanding of the incoherent imaging properties of Z-contrast scanning eleciron microscopy has been achieved, and the enormous power of this new micrc scopy technique has been applied to a wide range of atomic-resolution studies. Results include direct compositional information on hightemierature superconductor superlattices, information on high-angle grain boundary structures in high-temperature superconductors, the observation of unexpected ordering in $\left(\mathrm{Si}_{m} \mathrm{Ge}_{n}\right) p$ superlattices, and compositional characterization of quantum well structures.

Research in the Structural Properties of Materials-X-Ray Diffraction Program has led to an order of magnitude intensity increase in submicrovolt resolution $x$-ray beams using mosaic ${ }^{57} \mathrm{Fe}_{2} \mathrm{O}_{3}$ resonant monochromators in a time-resolved mode, and time-domain resonant filter techniques have been developed that provide a direct means for separating elastic and inelastic scattering for submicrovolt scattering spectrescopy. X-ray diffraction has been used to characterize the composition of superconductor superlattices, and studies of thin-film superconductors have indicated methods of preferentially aligning twin-boundaries using miscut substrates. 


\section{SURFACE PHYSICS}

\section{GROWIH RINETICS IN THE PHASE TRANSITION FROM Ag(111) TO $(\sqrt{3} \times \sqrt{3}) R 30^{\circ}$ STRUCTURES FOR A\&/Sili11)}

\section{I.-K. Znol and I. F. Wendelken}

Recent studies of growth kinetics of disorder-order phase transitions, in which systems are quenched from a disondered phase to an ordered phase, have revealed many interesting phenomena. ${ }^{2,3}$ One of the striking features is that the growth law has a universal form, $\bar{R}(t) \propto t^{n}$, where $t$ is the elapsed time after the quench and $\bar{R}(t)$ is the average ordered domain size.

To understand domain growth in an orderorder phase transition, we have chosen Ag/Si(111) as a model system. For a submonolayer of $A_{g}$ deposited on a Si(111)7x7 surface below $-190^{\circ} \mathrm{C}$, epitaxial Ag(111) islands form which transform irreversibly to a $(\sqrt{3} \times \sqrt{3}) R 30^{\circ}$ (or simply $\sqrt{3}$ ) structure when the temperature is increased above $190^{\circ} \mathrm{C}$. Experimentally, Ag was evaporated on a clean Si(111)7x7 surface near room temperature. Then, the sample was held at a temperature above $190^{\circ} \mathrm{C}$ and the angular profile of a $\sqrt{3}$ superlattice low-energy electron diffraction (LEED) beam, which reflects the $\sqrt{3}$ domain size distribution, was measured as a function of time.

Since the full width at half maximum (FWHM) of the angular profile is inversely proportional to the average linear domain size li.e., FWHM $\propto 1 / \bar{R}(t)$ ], a power growth law would imply the following behavior for the
FWHM

$$
w^{2}(t)-w^{2}(0) \propto t^{2 n}
$$

where $w=1 / F W H M \propto \bar{R}$ and $w(0)$ is the initial value just after the quench. As seen in Fig. 5.1, a power-growth law can be established for the initial growth with the data for different temperatures all giving $2 n=0.68 \pm 0.03$ which is consistent with $n=1 / 3$, a characteristic of Lifshitz-Slyozov kinetics governed by a diffusion mechanism. 24 Also, we have observed a continuous decay of the peak intensity with time for a Ag(111) LEED beam. According to these observations we

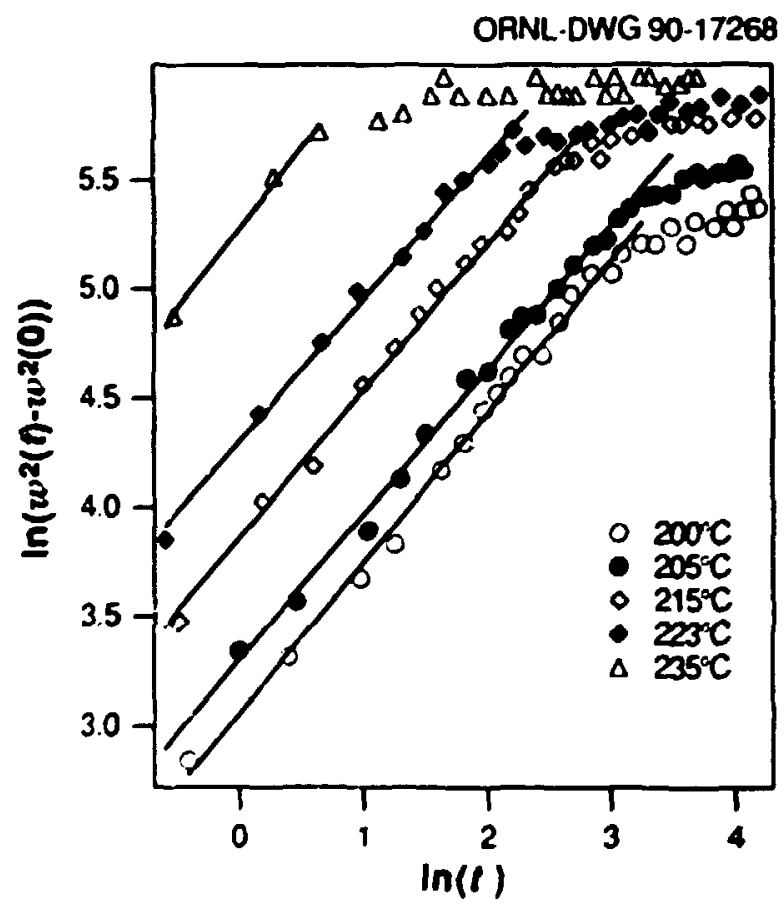

Fig. 5.1. The In-ln plot of the inversesquared FWHM of a $\sqrt{3}$ superlattice LEED beam vs time $t$ in minutes at different quench lemperatures for a $\mathrm{Ag}$ coverage of $-1 \mathrm{ML}$, where $w(t)=1 /$ FWHM(f). 
suggest a growth model in which Ag atoms are gradually dissolved from the Ag(111) ishands co form surrounding $\sqrt{3}$ domains, and simultaneously Ostwald ripening occurs among the $\sqrt{3}$ domains. 24

1. Oak Ridge Associated Universities postgraduate research participant.

2. O. G. Mouritsen, P. J. Shah, and J.V. Anderson, Phys. Reo. B 42, 4506 (1990); D. A. Huse, Physs. Reo. B 34, 7845 (1986).

3. M. C. Tringides, Phys. Reo. Lett. 65, 1372 (1990); J.-K. Zwo, G.-C. Wang, and T.-M. Lu, Phys. Reo. Lett. Co, 1053 (1988).

4. I. M. Lirshitz and V. V. Slyozov, J. Chem. Phys. Solids 15, 35 (1961).

\section{SCALING OF THE $(\sqrt{3} \times \sqrt{3})$ R30 DOMAN STEE DISTRIDUTION WIIH COVERAGE FOR AP/SI(111)!}

\section{I.-K. Zuo and J. F. Wendelken}

Recent theoretical and computer simulation studies of thin-film growth kinetics in various systems have shown that the domain size distribution and its moments are scale invariant." This implies that the domain size distribution $P(R, \theta)$, the probability of finding a domain with a diameter of $R$ lattice spacings at a coverage $\theta$, can be written as

$$
P(R, \theta)=\frac{1}{\bar{R}} P^{\prime}(x),
$$

where $x=R / \bar{R}$ and $P^{\prime}(x)$ is a scaling function independent of coverage. In this study we have obtained the first experimental evidence for 2-D scaling of the domain size distribution with coverage on $\mathrm{Ag} / \mathrm{Si}(111)-(\sqrt{3} \times \sqrt{3}) \mathrm{R} 3 i^{\circ}$ using a newly installed high-angular resolution low-energy electron diffractometer (LEED), which allows us to detect an ordered domain growth up to $>1000 A$.

The deposition of $\mathrm{Ag}$ on a fat Si(111) surface was performed in a UHV chamber with base pressure in the $10^{-11}$ Torr range. For a submonolayer of Ag grown on a clean Si(111)7x] surface above $\sim 190^{\circ} \mathrm{C}$, a $\sqrt{3}$ superlattice forms. Figure 5.2(a) shows LEED angular profiles narrowing with increasing coverage, indicating the growth of the $\sqrt{3}$ domains. In order to determine the domain size distribution at different coverages, we have used a simple model to fit the angular profiles. Assuming a random distribution of the $\sqrt{3}$ domain boundaries, the intensity of any superlattice beam is the sum of intensities diffracted from individual domains weighted by the sizedistribution function $P(R, \theta)$. In Fig. 5.2(b) a considerable increase of the mean-domain size and distribution width with coverage clearly indicates that the domain growth is governed by domain coalescence at $T=450^{\circ} \mathrm{C}$. Because coalescence simply rescales length but leaves the basic morphology of domains unchanged, one expects the existence of scaling in the growth process. According to Eq. (1), we replot $P^{\prime}(x)=\bar{R} P(R, \theta)$ vs $x$ in the inset of Fig. 5.2(b). Remarkably, although the $P(R, \theta)$ broadens with coverage, the $P^{\prime}(x)$, after being rescaled by their own mean sizes, superpose on each other independent of coverage.

1. Summary of paper: Applied Surface Science (in press). 
2. Oak Ridge Associated Universities postgraduate research participant.

3. F. Family and P. Meakin, Phys. Reo. Lett. 61, 228 (1988) and references therein; J. D. Gunton, p. 239 in Kinetics of Interfoce Renctions, ed. by M. Grunze and H. J. Kreuzer, SpringerVerlag. Berlin, 1987.

4. T.-M. Lu et al., Surf. Sci. 122, 519 (1982).

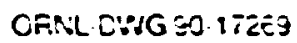
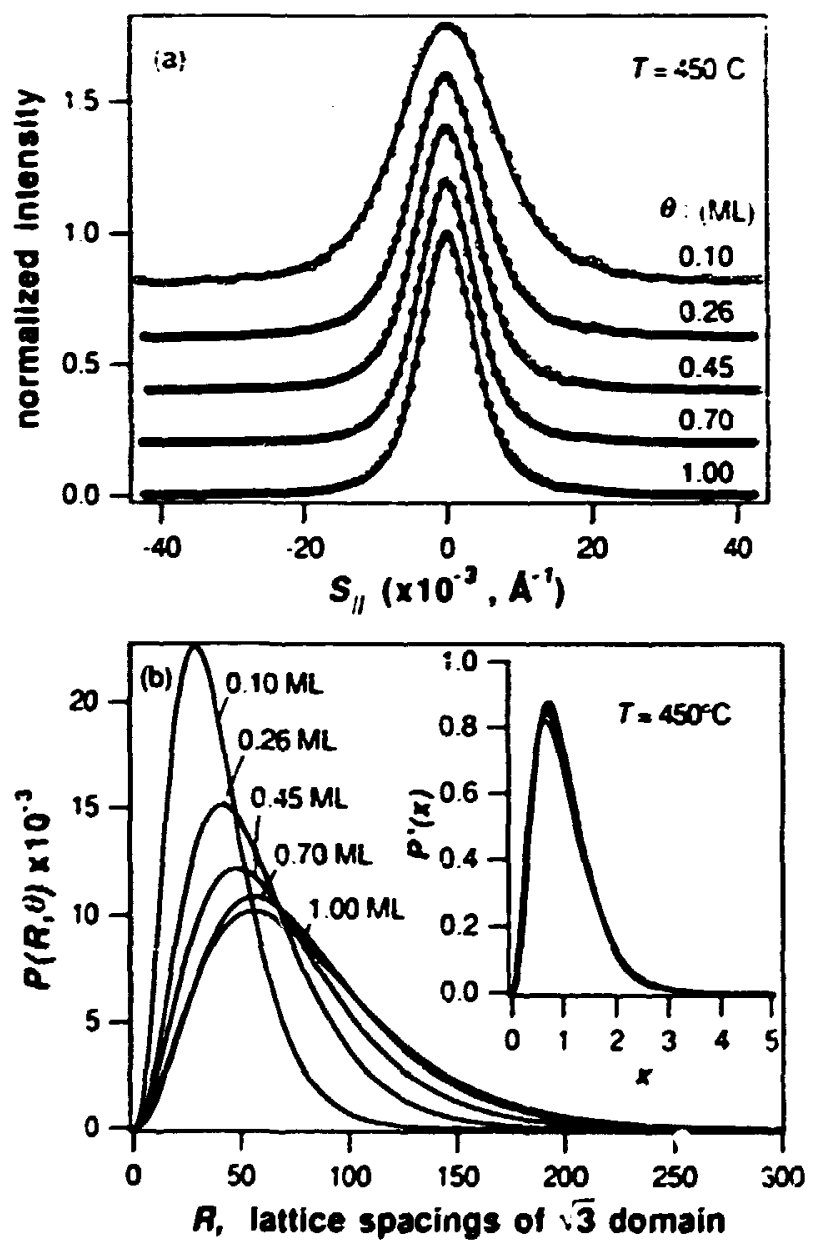

Fig. 5.2. (a) Angular profiles of a $\sqrt{3}$ superlattice beam at different coverages for $T=450^{\circ} \mathrm{C}$. The solid curves represent the best fits of the incoherent scattering model using the gamma distribution in $P(R, \theta)$ and convoluted with the instrument response function. (b) Evolution of domain-size distribution. with coverage obtained in (a). The inset is a $p ! n t$ of the corresponding scaling functions $P^{\prime}(x)$ vs $x$.

\section{A LOW-ENERGY Li+ ION SCATTERING STUDY OF THE SURFACE STRUCTURE OF STEPPED NIAI(111)'}

\author{
S. H. Ooerbury, 2 D. R. Mullins, 2 \\ and I. F. Wendelken
}

In NiAl, (111) planes composed only of Al alternate with planes composed only of $\mathrm{Ni}$. Despite an expectation that this would lead to a preferential surface termination, previous LEED intensity ${ }^{3}$ and low-energy ion scatteringt studies showed that the NiAk(11) surface was composed of a nearly equal mixture of $\mathrm{Ni}$ terminated and Al-terminated domains. The LEED intensity study also determined a contraction of the first interlayer spacing which is different for Ni-terminated vs Al-terminated domains. A subsequent angle-resolved LEED study 5 of a miscut, highly stepped NiAl(111) surface indicated a preference for double-step heights, suggesting the possibility of a preferential termination for this surface.

Using the same stepped crystal as in the angle-resolved LEED study, scattering of $\mathrm{Li}^{+}$ ions was examined as a function of incidence angle and energy. Direct shadowing of second layer by first layer atoms occurs for incident angles below a critical angle or shadowing edge S21 when the scattering plane is in the [211] azimuth. Figure 5.3 shows the energy distributions of the scattered ions obtaincd for incidence angles just below and above the $\mathbf{2 1}$ shadowing edge. From this, it is observed that the ratio of Ni to $A l$ is essentially the same in the first and second layers. Since the intensity of both the $\mathrm{Ni}$ and $\mathrm{Al}$ peaks is nearly doubled when scat- 


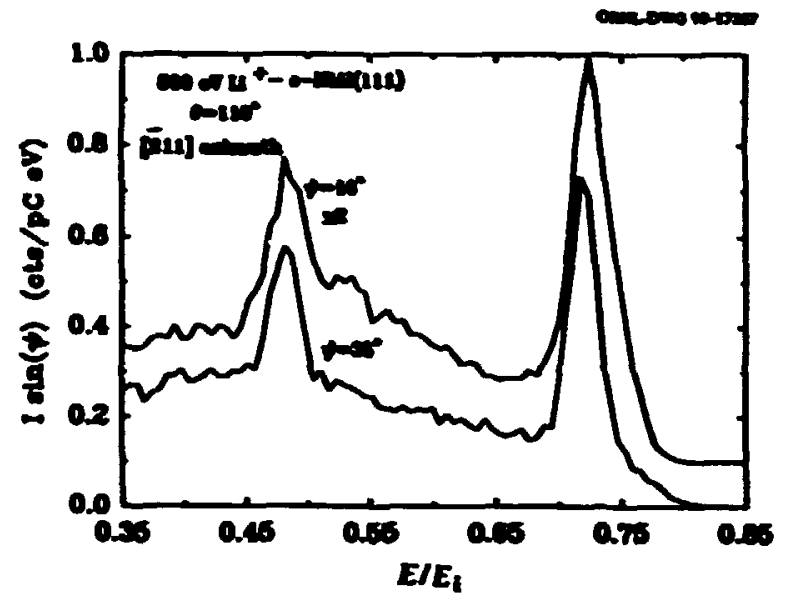

Fig. 5.3. Energy distributions obtained in the [211] aximuth are shown for incidence angles just below (top curve) and above (bottom curve) the S21 edge. The Al peak is at the bower energy. The top curve is offset for clarity.

tering from the second layer occurs, it is apparent that an equal number of each type of atom occurs in each layer. A comparison of the $\mathrm{Ni} / \mathrm{Al}$ peak ratio for scattering from the first layer alone to a $\mathrm{NiAl(110)}$ standard indicates an equal mixture of $\mathrm{Ni}$ and $\mathrm{Al}$ in the firt layer. Hence, it must be concluded that even for this stepped crystal, there is no preferential termination, despite the angle-resolved LEED observation which indicated a predominance of double-step heights. The first layer spacing was also measured, and the results are consistent with the earlier LEED intensity study.

1. Summary of paper: Surf. Sci. 236, 122 (1990).

2. Chemistry Division, ORNL.

3. J. R. Noonan and H. L. Davis, Phys. Reo. Lett. 59, 1714 (1987).

4. H. Nichus, Nucl. Instrum. and Methods Phys. Res. Sect. B 33, 876 (1988).

5. J. F. Wendelken, Solid State Division Progress Report for Period Ending March 30. 1989, ORNL-6571 (August 1989), p. 140.

\section{CONFIRMATION OF AN EXCETMON TO THE "GENERAL RUUE- OF SURFACE RELAXATIONS'}

\section{R. Noonan and H. L. Devis}

A new analysis of low-energy electron diffraction (LEED) data obthined from Al(111) has been performed in order to resolve a controversy about the relaxation of its first interlayer spacing. Although a "general rule" that the first interlayer spacing of a monatomic metal surfece should be contracted from the value for bulk spacings had evolved from considerable experimental and theoretical research, previous LEED analyses for AJ(111) had indicated that its first interlayer spacing was slightly expanded.2 Recent theoretical model simula. tions predict that the first interlayer spacing of Al(111) should be contracted, 3 while an earlier theory predicted an expansion. In an atternpt to resolve this controversy, a new data base for the Al(111) surface was analyzed at ORNL. The data base was measured for a sample at $160 \mathrm{~K}$ to reduce thermal effects. Experimental I-V spectra that extended over the energy range $50-380 \mathrm{eV}$ were collected for seven distinct LEED beams. Information about the relaxation of the Al(111) surface was then cxtracted by comparison with results from extensive LEED dynamical calculations which considered variation of the first two interlayer spacings and the possibility that thermal vibrations were layer dependent. Comparison of the experimental I-V spectra with various sets of calculated spectra has determined that the first interlayer spacing for Al(111) is expanded by $1.7 \%$ and the second spacing is expanded by 
0.7\%. This comparison for the best theoretical model is illustrated in Fig. 5.4. The agreement between calculated and experimental spectra is unprecedented. The experimental evidence is now compelling and clear. The first spacing of Al(111) is expanded. Any general theoretical model simulation for surface relaxations that does not predict this result for Al(111) must be improved first before it can be extended to treat reliably other problems such as grain boundaries, epitaxy, or any other form of interface.
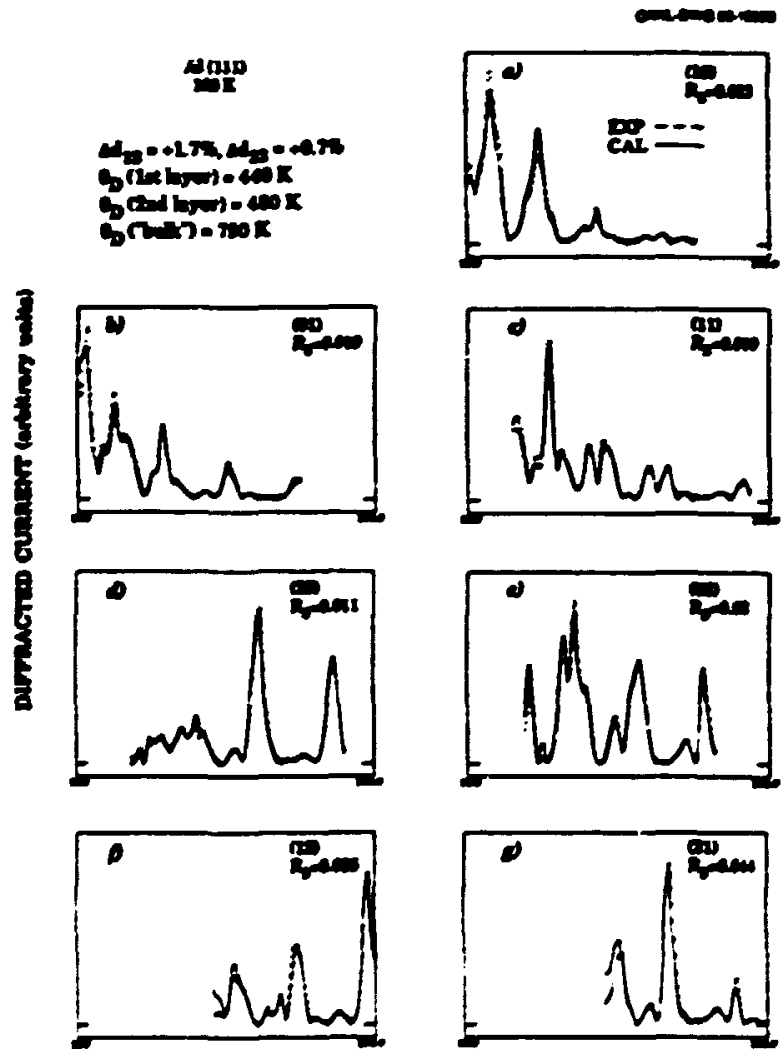

\section{Exection enact ion}

Fig. 5.4. Comparison of experimental (EXP) and calculated (CAL) I-V spectra for Al(111) at a temperature of $160 \mathrm{~K}$.
1. Summary of paper: I. Vac. Sci. and Technol. A 8, 2671 (1990).

2. D. W. Jepsen, P. M. Marcus, and F. Jona, Phys. Reo B 6, 3684 (1972); F. Jona, D. Sodericker, and P. M. Marcus, I. Phys. C 13, L155 (1980); H. B. Nielsen and D. L Adams, I. Phys. C 15, 615 (1982).

3. J. R. Smith and A. Banerjea, Phys. Reo. $B$ 37, 10411 (1988); T. Ning, Q. Yu, and Y. Ye, Surf. Sci. 206, L857 (1988).

4. R. N. Bamett, U. Landman, and C. L. Cleveland, Phys. Rev. B 28, 1685 (1983).

\section{STRUCTURE AND PHASES OF THE An(001) SURFACE $X$-RAY SCATTERING MEASUREMIENTS'}

\section{S. G. I. Mochrie,2 D. M. Zehner, B. M. Ocko, ${ }^{3}$ and Doon Gibbs 3}

$X$-ray diffraction, using synchrotron radiation, has been used to study the phase behavior and structure of the Au(001) surface between 300 $K$ and the triple point. The diffraction pattern observed in the high-temperature phase between 1170 and $1337 \mathrm{~K}$ is shown in Fig. 5.5(a). Solid squares are indexed in bulk-cubic reciprocal-lattice units $(H, K, L)$ and represent the reflectivity along directions normal to the surface joining all the bulk Bragg reflections. At elcvated temperatures, the in-plane diffraction pattern has the (1x1) symmetry of the bulk, consistent with an unreconstructed or disordered surface layer. More importantly, the $Q_{\mathbf{z}}$-dependence of the reflectivity is consistent with the existence of a thin disordered film (1-2 layers thick) and not with an ideally terminated crystal.

Below $1170 \mathrm{~K}$, there is a reversible transition to an incommensurate, two-dimensional 
OENL-DWG 91-5786

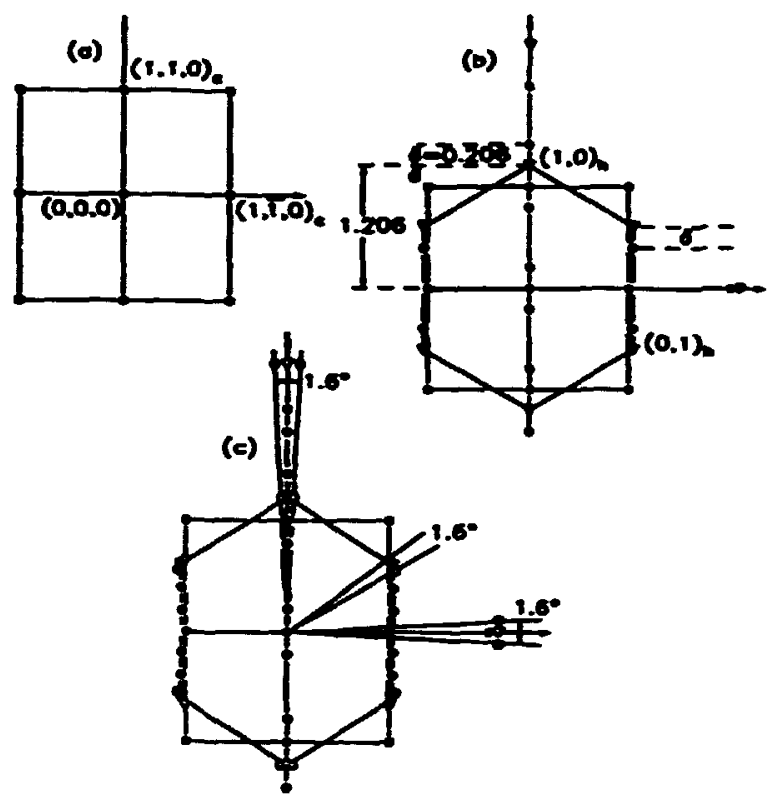

Fig. 5.5. In-plane diffraction pattern $(H$, K, 0.07) for (001) surface of Au: (a) disordered phase, (b) distorted-hexagonal phase, and (c) rotated, distorted-hexagonal phase. Each point represents a rod of scattering extending norma: to the surface. Bulk [110]c and [110]c directions are indicated. Peaks corresponding to domains rotated by $90^{\circ}$ were observed but are not shown.

structure of hexagonal symmetry. Hexagonal reciprocal-lattice vectors $(1,0) \mathrm{h}$ and $(0,1) \mathrm{h}$ are indicated by open triangles in Fig. 5.5(b). An important feature of this structure is an incommensurate comugation along the [110] direction, which gives rise to satellites about each hexagonal position. These satellites arise from modulations of the surface layer by the underlying square lattice and vice versa. Although also incommensurate in the $[1 \overline{1} 0 \mid$ direction, there are no corresponding satellite peaks.
This structure is called the distortedhexagonal phase.

Below 970 K, additional rods appear around each of the hexagonal rods, as shown by the open circles in Fig. 5.5(c). These occur at a fixed angle equal to $\pm 0.81^{\circ}$ and reveal the existence of rotated domains (in coexistence with the aligned domains). In addition, higher harmonics are visible along the [110] direction [solid cincles, Fig-55(c)]. The transition to the rotated, distorted-hexagonal phase is reversible. In this phase, the relative population of aligned and rotated hexagonal domains depends sensitively on temperature and on sample history, clearly displaying hysteresis.

1. Summary of paper. Phys. Reo. Lett. 64, 2925 (1990).

2. Massachusetts Institute of Technology, Cambridge, Mass.

3. Brookhaven National Laboratory, Upton, N.Y.

\section{PHASE BEHA VIOR OF THE Am(111) SURFACE: DISCOMMIENSURATIONS AND KINKS' 1}

\section{K. G. Huang, ${ }^{2}$ Doon Gibbs, ${ }^{2}$ D. M. Zehner, A. R. Sandy, ${ }^{3}$ and S. G. J. Mochrie ${ }^{3}$}

The Au(111) surface structure between 300 and $1250 \mathrm{~K}$ has been studied using $x$-ray diffraction and synchrotron radiation. For $T<865 \mathrm{~K}$, the surface exhibits a $(p \times \sqrt{3})$ reconstruction with $p=23$, which itself is composed of discommensurations, separating $A B C$ stacking regions from ABA stacking regions. This structure wot ld give rise to the diffaction pattern 
shown in Figs 5.6(a) and 5.6(b). Here open circles represent lateral periodicitics of the bulk crystal (truncation rods), and the solid symbols surrounding each trunction rod arise from the reconstruction (surface rods). However, the $x$-ray diffraction pattern cannot be explained solely on the basis of the $(p \times \sqrt{3})$ reconstruction. Instead, its interpretation leads to a structural model involving an equilibrium density of kinks scparating domains whose discommensuration orientations differ by $120^{\circ}$, as shown in Fig. 5.6(e). These kinks are thernselves ordered and produce a structure in which two of the three possible rotationally equivalent domains of the $(p \times \sqrt{3})$ reconstruction altemate across the surface. This is called the chevron phase and gives rise to the diffraction pattern shown in Fig-5.6(c). Al $865 \mathrm{~K}$, the reconstruction begins to lose large-range order. The first-order phase transition to a disordered phase is complete by $880 \mathrm{~K}$. In this phase, the reconstruction exhibits hexagonal symmetry, and the surface layer is isotropically compressed relative to pylk (111) planes. This results in the diffraction pattern shown in Fig. 5.6(d) and is called the discommensuration-fluid phase. The area per atom in this phase is strikingly similar to that for the hexagonally reconstructed Au(001), which suggests the possibility that a hexagonal overlayer of Au on a Au substrate has a natural lattice constant.

1. Summary of paper. Phys. Rev. Letl. 65, 3313 (1990).

2. Brookhaven National Laburatory, Upton, N.Y.

3. Massachusetts Institute of Technology, Cambridge, Mass.

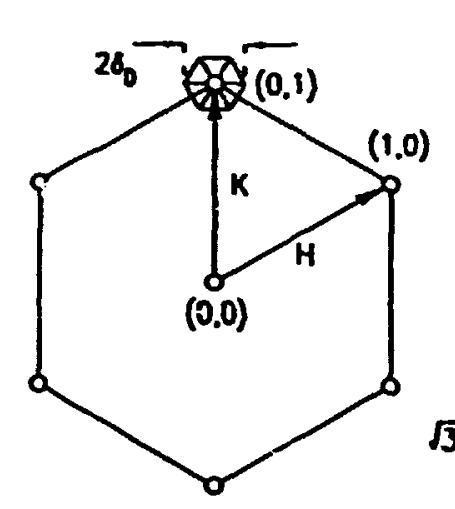

(o)

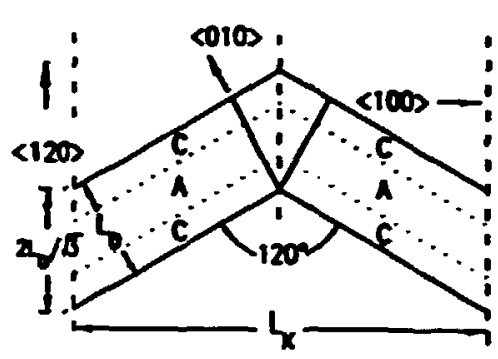

(e) $T<865 \mathrm{~K}$
ORNL-DWG 91-5m7

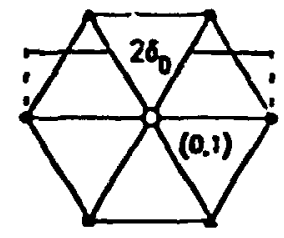

(b)

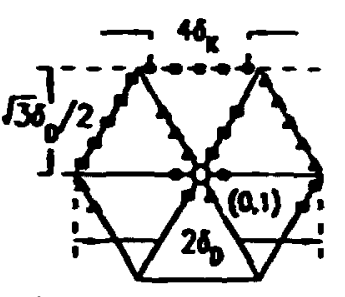

(c) $T<805 \mathrm{~K}$

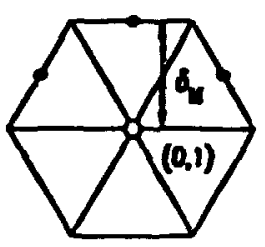

(d) $T>800 \mathrm{~K}$
Fig. 5.6. (2-d) Diffraction pattern of a reconstructed Au(111) surface. Open circles are truncation rods; solid symbols are surface rods. (a) Reciprocal space as defined by the hexagonal coordinate system. The small hexagon about the $(0,1)$ rod is reproduced in detail in (b)-(d). (b) Uniaxially compressed surface, (c) chevron surface, (d) discommensurationfluid phase. (e) Chevron unit cells shown in hexagonal coordinates.

\section{ENHAPSCED SURFACE ANHARMONICITY OBSERVED IN VIBRATIONS ON Cu(110)!}

\section{A. P. Baddorf and E. W. Plummer?}

The anharmonicity of vibrations in solids is well recognized and is required to account for many macroscopic propertics. At a solid surface, the crystal symmetry is broken, and the anharmonicity is expected to be greater than in the bulk. If true, surface anharmonicity is important in phasc transitions. diffraction 
intensities, and thin-film growth at surfaces. A wide variety of surfece-sensitive techniques indirectly sugzest the Cu(110) surface has an enhanced anharmonicity. 3

This study determines the surface anharmonicity on $\mathrm{Cu}(110)$ by examining the lemperture dependence of a surface phonon at the zone center using high-resolution electron energy loss spectroscopy (HREELS). The atomic motion of this phonon is perpendicular to the surface. The temperature dependence can be seen in Fig. 5.7. Between 21 and $766 K$, the phonon

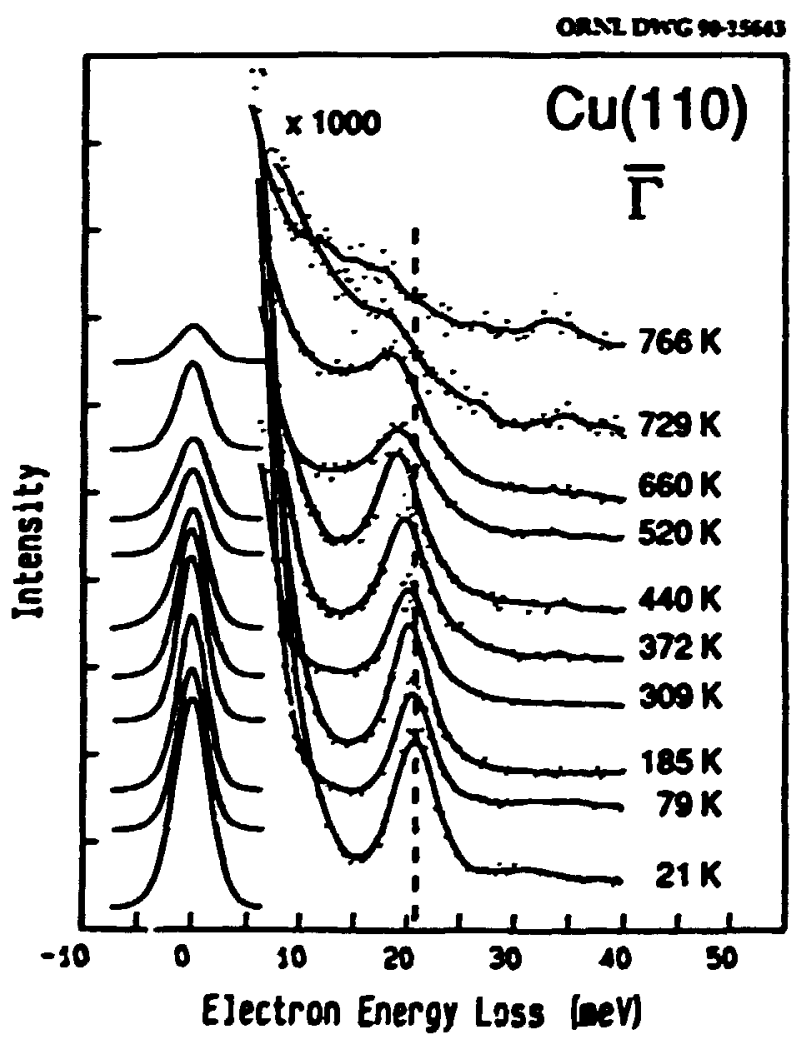

Fig. 5.7. HREELS spectra of clean Cu(110) at crystal temperatures between 21 and $766 \mathrm{~K}$. Spectra were obtained with an incident beam energy of $2.5 \mathrm{eV}$ and with incident and exit angle both $60^{\circ}$ from normal. The solid lines are smooth curves drawn through the data. energy decrenses by $3.2 \mathrm{meV}$ and the intrinsic width increases by $8.6 \mathrm{meV}$. The energy decreases at a rate 4.1-4.8 times greater than a comparable bulk phonon. A simple model is used to show that the changes in phonon energy and width can be explained as thermal occupetion of vibrational levels which have different excitation energies doe to the anharmonicity of the potential These results show that the anharmonicity of the Cu(110) surface is enhanced 4-5 times over that of the bull, with significant implications for descriptions of energy trancer and vibrational amplitudes at this surface.

1. Summary of paper: Physical Revieu Letters (in press).

2. University of Pennsylvania, Philadelphia, Pa.

3. P. Zeppenfeld et al., Phys. Reo. Lett. 62, 63 (1989).

\section{RADIATION-ENHANCED DISSOCIATION OF OXYGEN ON A COPPER SURFACE'}

\section{A. P. Baddorf and J. F. Wendelken}

Several studies have reported enhanced oxidation of transition-metal surfaces during irradiation by light. 2 Despite possible technological importance, this process has not been completely described. It has been suggested that the light promotes oxidation by dissociating a chemisorbed molecular $\mathrm{O}_{2}$ precursor on the metal surface.

We have studied the surface species on $\mathrm{Cu}(110)$ after exposure to $\mathrm{O}_{2}$ with and without 
$x$-ray and $e^{-}$radiation. The crystal was mainbined at $19 \mathrm{~K}$ to slow down chemical reactions and allow spectroscopic observations of the reaction intermediates. High-resolution electron energy loss spectroscopy (HREELS) was used to identify oxygen species by their vibrational signatures. Spectra before and after $\mathrm{Mg}_{\mathbf{8}}$ Ka $x$-ray irradiation (1253.6 eV) are shown in Fig. 5.8. In the lower curve only condensed $\mathrm{O}_{2}$ is observed, identified by the same 192-meV O-O stretch seen for gas-phase $\mathrm{O}_{2}$ - After irradiation, the vibrational spectrum shows vibrations

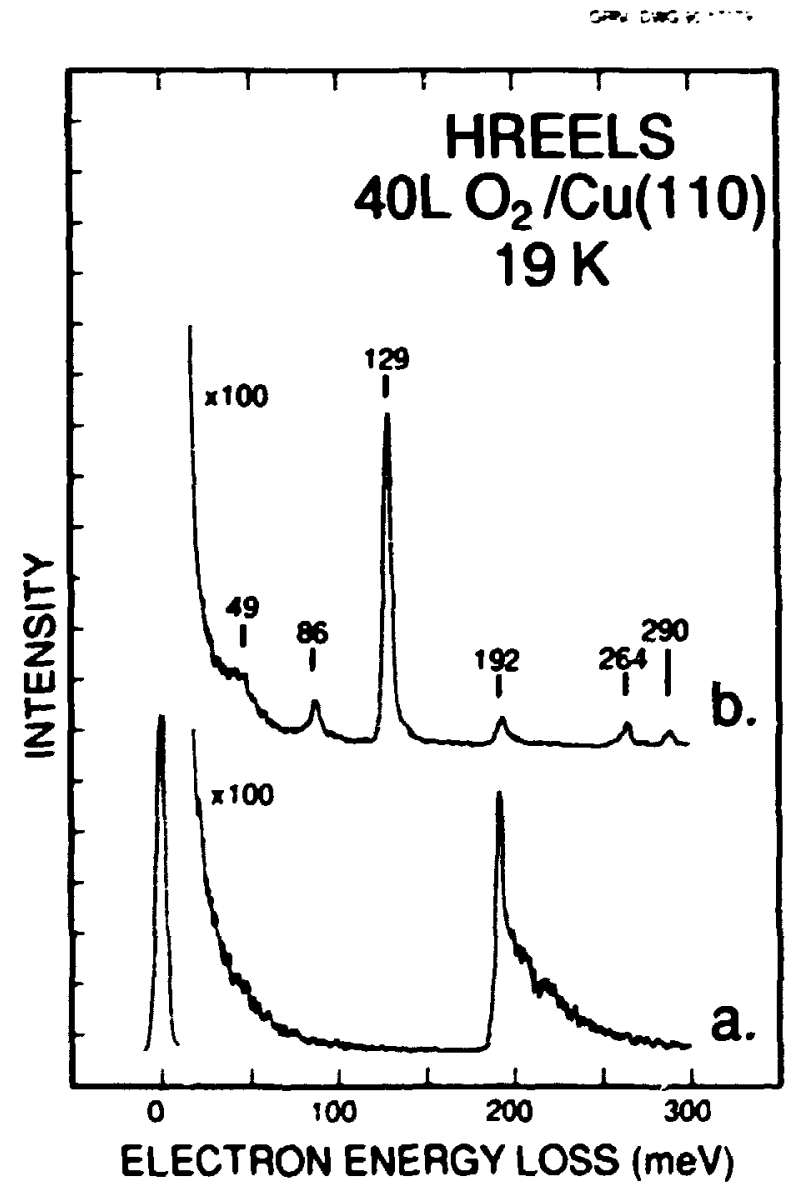

Fig. 5.8. Vibrational spectra of $40 \mathrm{~L} \mathrm{O}$ exposed at $19 \mathrm{~K}$; (a) as exposed and (b) after $135 \mathrm{~min}$ of $x$-ray exposure. of adsorbed O (49 meV), absorbed O (86 meV), and chemisorbed $\mathrm{O}_{2}$ (129 meV), identified in a previous study. 3 A similar spectrum is produced after $e^{-}$beam bombardment with beam energies between 60 and $600 \mathrm{eV}$. When warmed to $300 \mathrm{~K}$, the irradiated samples contain 2-3 times more oxide than unirradiated samples. This study reveals that radiation not only dissociates chemisorbed $\mathrm{O}_{2}$, as previously predicted, but more importantly converts gas-phase $\mathrm{O}_{2}$ into chemisorbed $\mathrm{O}_{2}$. This latter mechanism appears to be the rate-limiting step in the oxidation of the outermost layers of copper.

1. Summary of paper to be published.

2. A. Mesarwi and A. Ignatiev, J. Vac. Sci. and Technol. A 7, 1754 (1989); L. Hanley, $X$. Guo, and J. T. Yates, Js., I. Chem. Phys. 91, 7220 (1989).

3. A. P. Baddorf and J. F. Wendelken, to be published.

\section{CHIEMSORPIION OF NITROGEN ON Cu(110): COVERAGE AND STIE DETERMINATION'}

\section{A. P. Baddorf and D. M. Zehner}

Only two stable wel! ordered structures have been observed for chemisorbed nitrogen on copper surfaces. On $\mathrm{Cu}(110)^{2}$ and $\mathrm{Cu}(210)^{3}$ this structure produces a $(2 \times 3)$ LEED pattern. This structure is not yet understood, and studies have shown disagreement even on the coverage of $N$ involved. 3,4

In this work, the $\mathrm{N} / \mathrm{Cu}(110)$ system was studied with v-ray photoemission spectroscopy (XPS), auger electron spectroscopy (AES), 
thermal desorption spectroscopy (TDS), and low-energy electron diffraction (LEED). Gasphase nitrogen was activated and accelerated to $200 \mathrm{eV}$ to promote interaction with the surface. Coverages were oblained from XPS N1s areas and calibrated using $a p(2 \times 1) 0$ structure. Exposures at $300 \mathrm{~K}$ produced a surface which was not well ordered, with nitrogen coverages saturated near $1 \mathrm{ML}$ after exposure at $300 \mathrm{~K}$ (Fig. 5.9). The width of the N1s core in XPS indicated that only one site is occupied for coverages below $2 / 3 \mathrm{ML}$, but additional site(s) were occupied at higher coverages. Annealing the crystal to $650 \mathrm{~K}$ (Fig. 5.9) produced a stable (2×3) structure consisting of $0.63 \pm 0.03 \mathrm{ML}$ of nitrogen in a single site. The lineshape of the nitrogen auger signal was independent of coverage or surface structure, so little structural information was obtained. TDS reveals that annealing the crystal to $800 \mathrm{~K}$ removes all nitrogen from the surface by a combination of

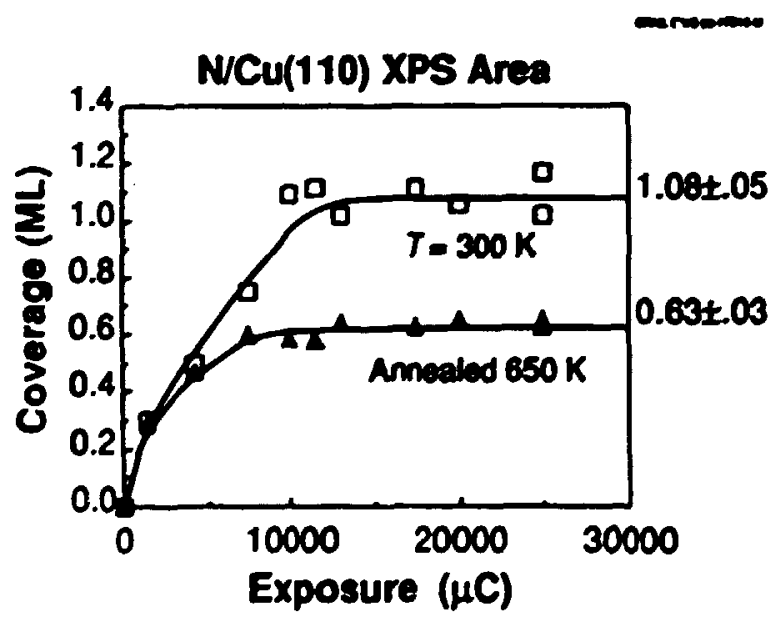

Fig. 5.9. Nitrogen coverage as a function of exposure at $300 \mathrm{~K}$ and after annealing to $650 \mathrm{~K}$. desorption and absorption into the bulk. The results obtained from these spectroscopies severely limit the choice of models for the (2×3) structure.

1. Summary of paper. Surf. Sci. 238, 255 (1990).

2. D. Heskett, A. P. Baddorf, and E. W. Plummer, Surf. Sci. 195, 94 (1988).

3. R. E Kirby, C S. McKee, and L. V. Renny, Surf. Sci. 97, 457 (1980).

4. E. Roman and R. Riwan, Surf. Sci. 118, 682 (198?).

\section{CHARGE-DISTREUTION CHANGES ACCOMPANMNG THE FORMATION AND CHANGES IN THE COMPOSTION OF HFC $C_{x}$ AND TaC ${ }_{x}$}

\section{G. R. Gruzalski and D.M. Zehner}

Core-level binding energies (BEs) and valence-band structures, determined with $x$-ray photoelectron spectroscopy, and C KVV Auger electron spectra were obtained for $\operatorname{TaC}_{x}(0.5 \leq x$ $\leq 1.0)$ and $H F C_{x}(0.6 \leq x \leq 1.0)$, and the $4 f$ BEs of $\mathrm{Ta}$ and $\mathrm{Hf}$ in the elemental metals were measured. The data were explained in terms of changes in the charge distributions occurring with changes in $x$ or upon the formation of $H_{i} C_{x}$ and $\mathrm{TaC}_{\mathbf{x}}$. In particular, the following conclusions were drawn: (1) Upon formation of the carbides, the electron charge increases in the vicinity of carbon ions and decreases in the vicinity of metal ions. Over the range of $x$ investigated, more charge resides in the vicinity of carbon ions in $\mathrm{TaC}_{x}$ than in $\mathrm{HfC}_{x}$; it is likely that $\mathrm{TaC}_{x}$ is more ionic than $\mathrm{HfC}_{x}$. (2) For both $\mathrm{TaC}_{x}$ and $\mathrm{HfC}_{\mathrm{x}}$, as $\mathrm{x}$ decreases, the electron 
charge increases in the vicinity of metal ions and decreases in the vicinity of carbon ions. An analysis of the C KVV Auger electron spectra is consistent with this interpretation in that the analysis suggests a reduction in C-2p electron occupancy per carbon atom with decreasing $x$. (3) The increase in electron charge in the vicinity of metal ions that accompanies a decrease in $x$ is larget in $\mathrm{TaC}_{x}$ than in $\mathrm{HfC}_{\mathbf{x}}$, whereas the corresponding depletion of charge from carbon ions is approximately equal in the two sets of materials. (4) In $\mathrm{HFC}_{x}$ the charge redistribution accompanying changes in $x$ causes relatively little redistribution of $\mathrm{Hf}$ states below $E_{F}(x)$-the valence band near $E_{F}$ behaves like a rigid band-and $E_{F}(x)$ moves higher in the bands with decreasing $x$. Presumably, there is a significant redistribution of $\mathrm{Hf}$ states just above $E_{F}(x)$. (5) In $\mathrm{TaC}_{x}$ this charge redistribution causes a significant redistribution of Ta states below $E_{F}(x)$, with more states being created than destroyed below the original fermi level, and $E_{F}(x)$ moves lower in the bands with decreasing $x$.

It is the difference in valence-band behavior described in (4) and (5) and not some significant difference in charge distributions that is primarily respunsible for the spectroscopic data of $\mathrm{HFC}_{x}$ differing from those of $\mathrm{TaC}_{x}$.

1. Summary of paper: Priys. Rev. B 42, 2768 (1990).

\section{WORK-FUNCTION CHANGES \\ ACCOMPANMNG CHANGES IN \\ COMPOSITION OF (100) SURFACES \\ OF HIC : AND TaC $C_{x}$}

\author{
G. R. Gruzalshi, S.-C. Lui, \\ and D. M. Zehner
}

In a recently published study ${ }^{3}$ of $\mathrm{HFC}_{x}$ $(0.6 \leq x \leq 1.0)$ and $\mathrm{TaC}_{x}(0.5 \leq x \leq 1.0)$, it was found that as the carbon content $x$ decreased, the Fermi level moved higher in the bands for $\mathrm{HfC}_{\mathrm{x}}$ and lower in the bands for $\mathrm{TaC}_{\mathrm{x}}$. It was felt that this behavior could be detected by work-function measurements, and such mezsurements were performed on four (100) surfaces having near-surface compositions estimated as $\mathrm{TaC}_{1.0}, \mathrm{TaC}_{0.5}, \mathrm{HFC}_{1.0}$ and $\mathrm{HFC}_{0.6}$ These measurements were performed at the National Synchrotron Light Source at Brookhaven National Laboratory. The results are summarized in Table 5.1.

Table 5.1. Work functions $\Phi$ in $\mathrm{eV}$ for (100) surfaces of $\mathrm{T}_{\mathrm{x}} \mathrm{C}_{\mathrm{x}}$ and $\mathrm{HF} \mathrm{C}_{\boldsymbol{x}}$ Work functions were determined with an estimated accuracy of $\pm 0.05 \mathrm{eV}$.

\begin{tabular}{lll}
\hline Surface & $\Phi$ \\
\hline $\mathrm{TaC}_{1.0}$ & 4.38 \\
$\mathrm{TaC}_{0.5}$ & 4.73 & \\
$\mathrm{HfC}_{1.0}$ & 4.63 & \\
$\mathrm{HfC}_{0.6}$ & 3.87 & \\
\hline
\end{tabular}


The work function $\Phi$ can be written as a sunı of two terms, $E_{1}$ and $E_{2}$, where $E_{1}$ is the energy difference between the Fermi level and a reference potential termed the crystal zero and $E_{2}$ is the energy difference between the crystal zero and the vacuum zero. It therefore can be written as $\Delta \Phi=\Delta E_{1}+\Delta E_{2}$, where the quantities refer to values of $\Phi, E_{1}$, and $E_{2}$ for surfaces of $\mathrm{TaC}_{x}$ or $\mathrm{HfC}_{x}$ having the lower carbon content minus those having the higher carbon content.

If the crystal zero is defined as that energy level which uiffers from the vacuum level by only a surface-barrier potential, then $E_{2}$ is identical to the surface-barrier potential. If one then supposes that the bulk valence bands are tied to the crystal zero, the variation of $E_{1}$ with $x$ is given by the Fermi-level behavior described in Ref. 3. Arguments for the following relationships can then be made:

$$
\begin{aligned}
& \Delta \Phi^{H f C}<0, \\
& \left|\Delta \Phi^{T A C}\right|<\left|\Delta \Phi^{H K C}\right|
\end{aligned}
$$

and

$\Delta \Phi^{T \sim C}-\Delta \Phi^{H K C}=0.9 e V+\Delta E_{2}^{T a C}-\Delta E_{2}^{H F C}$.

Given the qualitative nature of the ideas applied, the agreement between Eqs. (1) and the experimental results given in Table 5.1 is quite good. This good agreement is interpreted as supporting these ideas and, in particular, for the difference in work-function bchavior exhibited by $\mathrm{TaC}_{x}$ and $\mathrm{HfC}_{x}$, as being due primarily to a bulk effect (i.e., to the difference in Fermi-level behavior described in Ref. 3).

1. Summary of paper. Surf. Sci. 239, LS17 (1990).

2. Oak Ridge Associated Universities postgradiate research participant. Present address: Allied Signal, Inc., Morristown, N.J.

3. G. R. Gruzalski and D. M. Zehner, Phys. Reo. B 12, 2 \%68 (1990).

\section{ELECTRON MICROSCOPY}

\section{HIGH-RESOLUTION INCOHERENT MAGING OF CRYSTAIS ${ }^{1}$}

\section{S. I. Pennycook and D. E. Jesson?}

For the last $\mathbf{4 0}$ years, the imaging of materials by high-resolution transmission electron microscopy has been based entirely on coherent interference techniques. ${ }^{3}$ Resolution and contrast are intrinsically linked in an image, which depending on sample thickness and objective lens defocus, can reverse contrast and show complicated interference effects particularly at defects and interfaces. This makes image interpretation possible only through computer simulation of model structures. Unanticipated effects are easily missed by this procedure. A fundamentally new approach to electron microscopy is now possible using a high-angle annular detector on a scanning transmission electron microscope (STEM). This provides atomic-resolution images showing strong compositional sensitivity together with 
incoherent characteristics. There are no contrast reversals and no interference effects at defects and interfaces, therefore resulting in a unique image for each object. Furthermore, the optimum imaging conditions are the same for all materiais so that unanticipated structures will be immediately apparent.

These characteristics arise since the highangle detector is sensitive only to the electron intensity very close to the nucleii. The compositional scnsitivity arises through the nearly unscrecned scattering cross section, which is proportional to $Z^{2}$, where $Z$ is the atomic number, so that we refer to this technique as Z-contrast imaging. The incoherent characteristics arise because with a coherent incident probe, the electron intensity close to the atom sites is almost entirely due to s-type Bloch states tightly bound to the atomic columns. These states peak close to the atom sites and are excited in phase by all plane waves comprising the incident STEM probe. This coherent addition does not occur for more weakly bound states, such as p-type states, which also tend to have a high amplitude away from the atomic columns. Since only s-states are involved, the complex interference effects scen in conventional images are removed. Because s-statcs are insensitive to the positions and compositions of ncighboring columns (in marked contrast to p-states for example), images from complex unit cells, interfaces, and superlattices show equally simple characteristics. The image reduces to a simple convolution between the incident probe intensity profile and the integrated s-state intensity at the atomic sites, weighted by the scattering cross section. This convolution is relatively easy to vis'salize, resulting in an unambiguous, intuitivcly interpretable atomic resolution image of a materials structure and composition, even on a scale below the resolution limit as shown in Fig. 5.10.

SSDN-4173b
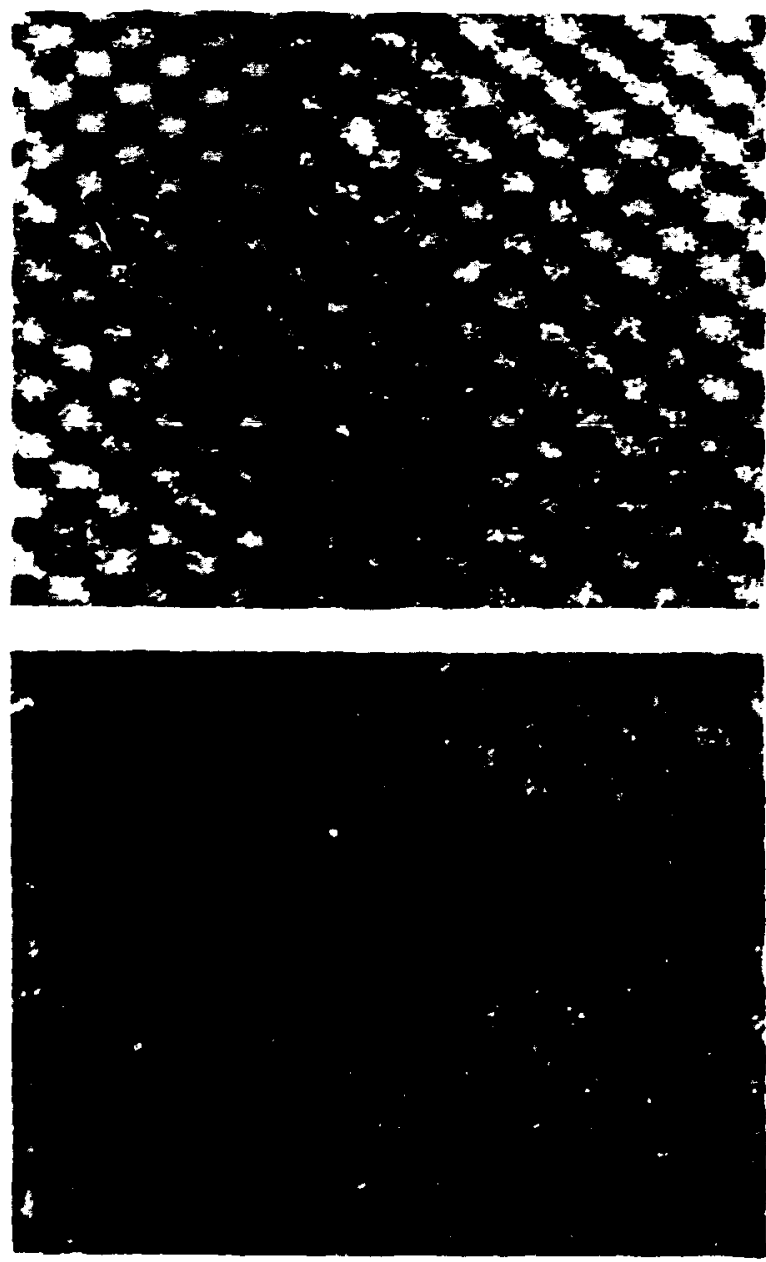

Fig. 5.10. $Z$ contrast images demonstrating intuitively interpretable images on a scale well below our 2.2-A resolution limit. (a) $S(110)$ in which the 1.4- $A$ separation of the closely spaced pairs of strings, though not resolved, clearly results in the expected elongation of the image along $(100\rangle$. (b) $\ln P(110)$ in which scattering from the In strings dominates, giving an array of circular image features. Simulated images are inset. 
A 300-kV STEM currently on order will provide these benefits in an image having an unprecedented $1.4-\AA$ resolution together with a simply interpretable information content to well below the 1-A level, providing an exciting opportunity for fundamental advances in all areas of materials research.

1. Summary of paper. Phys. Reo. Lett.64, 938 (1990).

2. Oak Ridge Associated Universities postgraduate research participant.

3. O. Scherzer, J. Appl. Phys. 20,20 (1949).

4. S. J. Pennycook and L. A. Boatner, Nature 336,565 (1988).

\section{INCOHERENT IMAGNG OF THIN SPECIMIENS USING COHIERENTIY SCATTERED ELCTRONS'}

\section{E. Jesson ${ }^{2}$ and S.J. Pennycook}

Contrary to long-standing ideas $3 A$ we have shown that images obtained in the scanning transmission electron microscope (STEM) with a high-angle annular detector can be interpreted in terms of the theory for incoherent imaging provided that incoherently generated thermaldiffuse scattering is collected.5 Here, these arguments are extended to the important case of coherent scattering, which can dominate in the case of very thin crystals or small clusters of atoms. Essentially, as long as the inner radius of the annular dark-field detector is much greater than the scale of interference effects between atomic columns in the detector plane, the detected intensity will approach the result for columns scattering incoherently. Thus, con- trary to popular belief, transverse incoherent imaging of a phase object is indeed possible using an annular detector with a large inner angle. We note, however, that the detector cannot eliminate interference effects between atoms within a particular column so that the thickness dependence increases initially as the number of atoms squared and rapidly becomes more complicated due to Fresnel effects (Fig. 5.11). Being aware of such limitations in advance means that intuitive imaging of single heavy atoms, atom clusters, and microcrystals

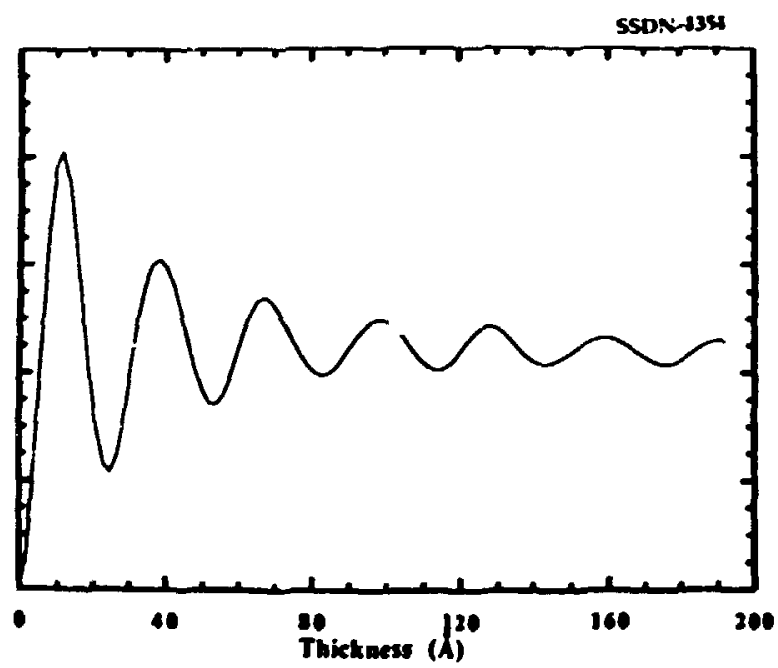

Fig. 5.11. Plot of intensity against thickness for Sil110) with a $2.2-\AA$ electron probe located at the center of a dumbbell. The annular detector covers the range 50-150 mrad.

using STEM (without requiring complex image simulation) is a real possibility.

1. Summary of paper to be published.

2. Oak Ridge Associated Universities postgraduate research participant. 
3. G. Ade, Optik 49, 113 (1977).

4. J. M. Cowley, Ultramicroscopy 2,3 (1976).

5. S. J. Pennycook and D. E. Jesson, Phys. Reo. Lett. 64, 938 (1990).

\section{ANGULAR MAPPING OF EIECTRON CHANNELING PHENOMENA USING SCANNING TRANSMISSION ELECTRON MICROSCOPYl}

\section{E. Iessont and S. I. Penurycook}

The utilization of electron channeling effects to obtain localized crystallographic information is now well established. ${ }^{3}$ Such experiments exploit the orientational dependence of the thickness integrated electron intensity $W^{*}\left(\underline{R}_{i}\right)$ which for highly localized interactions at the atom sites $R_{i}$ profoundly influences the relevant scattering cross section.

Using a large inner angle annular detector on a scanning transmission electron microscope (STEM), we have developed a new method to map $\nabla^{*}\left(\underline{R}_{i}\right)$ as a function of incident-beam orientation. We refer to such maps as largeangle channeling patterns, and a relevant result for Sil110) is shown in Fig-5.12(a). The patterns clearly contain detailed structural infor. mation and represent a unique probe of the electron wave function close to the atom sites. Although at first sight complex, the features in the patterns can usually be interpreted in terms of just two excited Bloch states. The thickness variation of the patterns, therefore, directly reveals the transition from dependent to independent Bloch-wave thickness regimes, and enormous potential exists to obtain other structural information directly by inspection. The
SSDN-4191
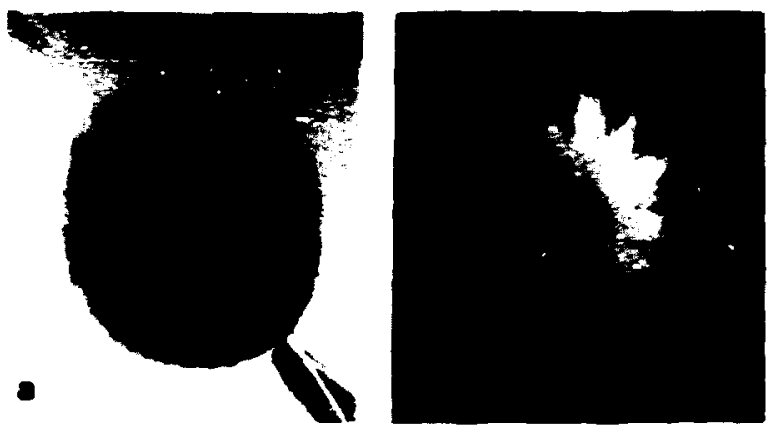

Fig. 5.12. (a) Large-angle channeling pattern and (b) convergent beam electron diffraction pattem obtained simultaneously from Si(110) in the STEM.

technique is strongly allied to Z-contrast imaging methods since it enables the accurate orientation of thin specimen regions. Furthermore, the bright lines crossing the pattern in Fig. 5.12 directly map the strength of three-dimensional diffraction effects. Since no bright lines are present in the center of the pattem, incoherently generated thermal diffuse scattering must dominate the signal within the angular range of the electron probe used for Z-contrast imaging.

It is worth pointing out that by choosing a suitable collector aperture, a convergent beam electron diffraction (CBED) pattern is simulaneously obtained at the bright-field detector [Fig-5.12(b)]. Although readily attainable, this simple method for obtaining CBED patterns in the STEM with good angular resolution does not appear to have been reported previously.

1. Summary of paper to be published. 
2. Oak Ridge Associated Universities postgraduate research participant.

3. S. J. Pennycook and J. Narayan, Phys. Rev. Lett. 54, 1513 (1985).

\section{ZCONIRAST IMAGNG OF HIGH-ANGLE YBaz $\mathrm{Cu}_{3} \mathrm{O}_{78} \mathrm{GRAN}$ BOUNDARIES}

\section{F. Chisholm and S.J. Penmyecok}

Special high-angle misorientations, where a coincidence site lattice is produced for two adjacent grains, have been observed ${ }^{2}$ in $\mathrm{YBa}_{2} \mathrm{Cu}_{3} \mathrm{O}_{7}$ \& Recently published results ${ }^{3}$ indicate that there appear to be exceptions to the conclusion of Dimos et als that all grain boundaries with misorientations greater than $10^{\circ}$ are weak links. We have begun a study to investigate the structure of these special boundaries to determine their specific atomic arrangements. Z-contrast imaging with its incoherent characteristics allows intuitive interpretations of these special structures associated with highangle (001) tilt boundaries. All special boundaries studied contain both symmetric and asymmetric sections; the asymmetric portions normally involve (100) faceting of one of the adjacent grains. Figure 5.13 is an image from a symmetric section of a near $\Sigma 25$ boundary, where the grains share a common (430) plane. A simple geometric construction predicts the essential features observed in this boundary. The presence of the periodic bridges of relatively undisturbed material across the boundary is highly suggestive that there may be special high-angle boundaries with struclures suitable for superconducting device applica-

tions. The determination of these previously unknown boundary structures has been made a relatively simple procedure by $Z$-contrast imaging. These atomic arrangements are the first step in quantifying the relaxation associated with the grain boundary and in correlating structure with superconducting properties.

1. Summary of paper to be published.

2. D. A. Smith, M. F. Chisholm, and J. Clabes, Appl. Phys. Lett. 53, 2344 (1988).

3. S. E. Babcock et al., Nature 347, 167 (1990).

4. D. Dimos, P. Chaudhari, and J. Mannhart, Phys. Rev. B 41, 4038 (1990). 


\section{SOLID-PHASE-EPTTAXAL RECRYSTALUZATION OF ION- MIILANIED YBa2 $\mathrm{Cu}_{3} \mathrm{O}_{7-x}$ THIN FILMS} S. J. Pennycook, R. Feenstra, M. F. Chisholm,
I. D. Budei, D. P. Norton, and D. H. Lowndes

There are two major problems for the ion implantation doping of high-temperature superconductors compared to the standard procedures used for elemental semiconductors. The first is the uncertainiy in the thermodynamic stability of the phase at the low temperatures at which recrystallization is desired, and the second is the complex kinetics, involved in ordering a large unit cell. Growth of $\mathrm{YBa}_{2} \mathrm{Cu}_{3} \mathrm{O}_{7-x}$ along the $c$ axis involves ondering on a 12- $\AA$ scale ahead of the interface, a far larger distance than the $4 \AA$ required for growth in the $a-b$ plane, so that a large anisotropy is anticipated. Evidence for this comes from the growth of single crystals and also from the deposition of superlattices where growth along the $c$ axis occurs primarily through the migration of ledges in the $a-b$ plane. ${ }^{2}$ Similar behavior is found to occur at an amorphous/crystal interface during the annealing of oxygen-implanted films. Grains or films with their $a$-axis normal to the substrate recrystallize by epitaxial growth; the slower growth of c-axis normal films allows nucleation of polycrystalline $\mathrm{YBa}_{2} \mathrm{Cu}_{3} \mathrm{O}_{7-x}$ at the surface or even decomposition to occur. Such behavior is typical for processing in oxygen at atmospheric pressures.

Recently, however, we have begun to process implanted films at subatmospheric oxygen pressures. A substantially reduced oxygen par- tial pressure is advantageous ${ }^{3}$ both for thermodynamic stability of the desired phase and for enhanced diffusion kinetics due to increased oxygen vacancy concentrations. Implanted $c$ axis normal films may be recrystallized in 0.2 Torr oxygen at only $740^{\circ} \mathrm{C}$ (Fig. 5.14). Under these conditions, nucleation at the amorphous/crystal interface is greatly enhanced. The amosphous material becomes

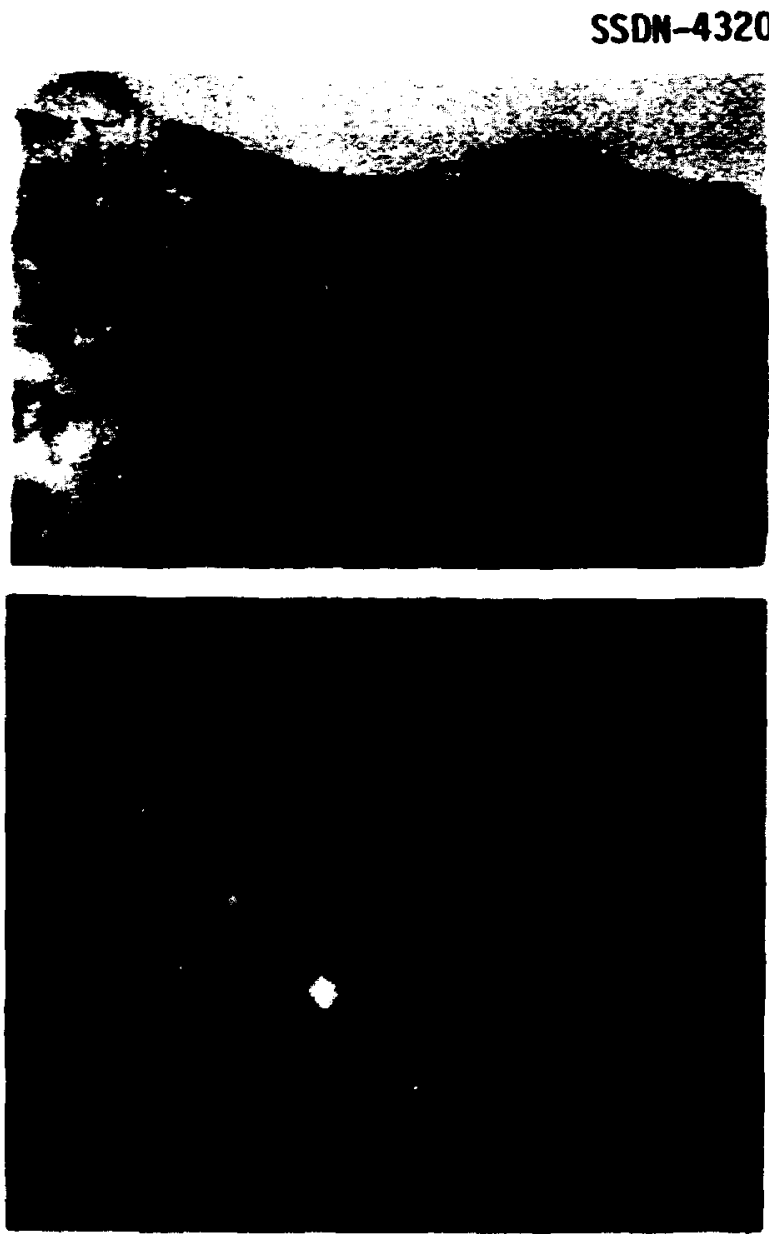

Fig. 5.14. (a) Crose-section micrograph of a laser-deposited $\mathrm{YBa}_{2} \mathrm{Cu}_{3} \mathrm{O}_{7-x}$ thin film tollowing implantaticn with $\mathrm{O}^{+}\left(80 \mathrm{kcV}, 1 \times 10^{16}\right.$ $\left.\mathrm{cm}^{-2}\right)$ and annealing $\left(0.2-\right.$ Torr oxygen, $740^{\circ} \mathrm{C}, 10$ min) showing small domains with $c$ lattice fringes normal and parallel to the substrate; (b) corresponding diffraction pattern. 
increasingty filled with thin sheets of crystallized $\mathrm{YBa}_{2} \mathrm{Cu}_{3} \mathrm{O}_{7-2}$ resulting eventually in a dense intergrowth of small domains in all three epitaxial orientations. The ability to implant and recrystallize these superconducting materials offers exciting new possibilities for materials modification and device applications.

1. Sumanary of paper to be publisted.

2. S. J. Pennycook et al, to be poblished. See also S. J. Pernnycook et al., Direct Imaging of Interface Structure and Chemistry in CaAs/InosGons Qunatum Wels, this report.

3. R. Feenstra et al, Jound of Applied Physics (in press).

\section{GROWHH MECHANLSMS AND} INTERDIFFUSTON OF $\mathrm{Ma}_{2} \mathrm{Cu}_{3} \mathrm{O}_{7-2} \mathrm{PrBa}_{2} \mathrm{Cu}_{3} \mathrm{O}_{7-x}$ SUTERLATICES

\section{S. J. Penmycook, M.F. Chishalm, D.E. Jesson," H. R. Kerchner, D. P. Norton, and D. H. Lowndes}

In view of the fact that the alloy $\mathrm{Y}_{3} \mathrm{Pr}_{1-y} \mathrm{Ba}_{2} \mathrm{Cu}_{3} \mathrm{O}_{7-2}$ shows a rapid reduction of $T_{c}$ with increasing $\operatorname{Pr}$ content, ${ }^{3}$ the extent to which interdiffusion contributes to the interesting transport properties of $\mathrm{YBa}_{2} \mathrm{Cu}_{3} \mathrm{O}_{7-2} / \mathrm{PrBa}_{2} \mathrm{Cu}_{3} \mathrm{O}_{7-x}$ superlattices is a question of key importance. There is apparently no technique capable of answering this question apart from $Z$-contrast scanning transmission electron microscopy (STEM). Conventional phase contrast imaging, for example, has been unable to distinguish $Y$ from Pr. Figure 5.15(a) shows a Z-contrast image from part of such a superlattice deposited by laser ablation, showing perfect crystallinity
SSDN-4255
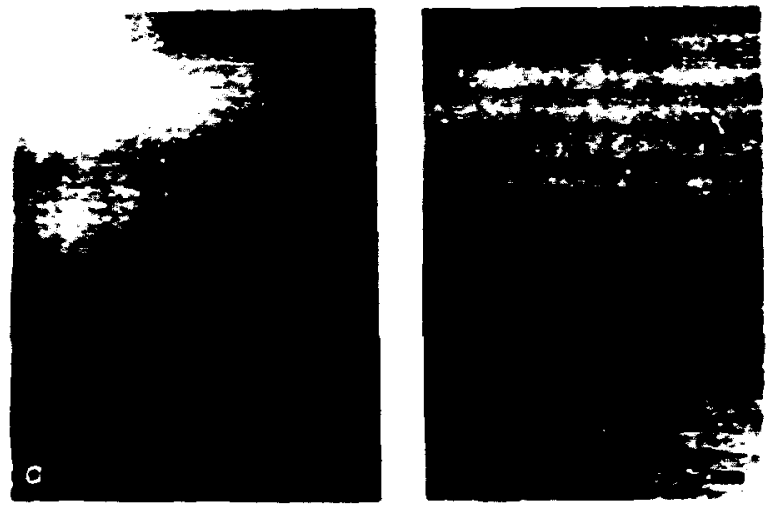

Fig-5.15. Part of a $\mathrm{YBa}_{2} \mathrm{Cu}_{3} \mathrm{O}_{7-2}$ PrBa2 $\mathrm{Cu}_{3} \mathrm{O}_{7-x}$ superlattice showing compositionally abrupt but stepped interfaces; (a) generl view showing wavy compositional modulation and (b) higher magnification showing interface step.

although the compositional modulation is somewhat wavy. Each unit cell in the $i$ direction is separated by dark horizontal lines representing the CuO layers. Within each cell, the three metal layers are individually resolved, with the $Y$ layer imaging darket and the Pr layer brighter than the two adjacent Ba planes. It is quite obvious from the image that interdiffusion is insignificant. Even at an interfacial step [Fig. 5.15(b)] the compositional abrupiness within the $a-b$ plane is of the order of a singha-lattice spacing $(-4 A)$. Since diffusion in this plane is expected to be considerably higher than along the $c$ axis, this is further confirmation that no significant interdiffusion has occurred.

The observation of abrupt interfacial steps also provides great insight into the growth mechanism. It can only be explained if grow'th 
proceeds by a simple terrace sweeping mechanism where the height of the ledge is the full c-axis unit cell itseif.

The effect of the compositional waviness is to force the current to cross from cell to cell. It might be expected that this effect would be most severe for the single-unit-cell superbattices in which there is only a very small lateral overlap at interfacial steps (which would be extremely sensitive to the exact amount of $\mathrm{YBa}_{2} \mathrm{Cu}_{3} \mathrm{O}_{7-x}$ deposition). Magnetic hysteresis measurements do, in fact, indicate a dramatic reduction of critical current per unit cell for a single cell (1x4) superlattice, with an estimated $10 \%$ overlap, compared to a $(2 \times 1)$ superlattice.

1. Summary of paper to be published.

2. Oak Ridge Associated Universities postgraduate research participant.

3. J. L. Peng et al., Phys. Rev. B 40, 4517 (1989).

\section{STRUCTURAL ORIGIN OF REDUCED Ic $\mathrm{N} \mathrm{YB}_{2} \mathrm{Cu}_{3} \mathrm{O}_{7} \mathrm{l}$}

\section{F. Chisholm and S. J. Pennycook}

Z-contrast scanning transmission electron microscopy imaging demonstrates that chemical segregation need not occur at grain boundaries in $\mathrm{YBa}_{2} \mathrm{Cu}_{3} \mathrm{O}_{7-8}$ superconductors. ${ }^{2}$ The rapid reduction of the grain boun lary critical current density with increasing grain misorientation is therefore thought to arise through an intrinsic structural effect. In order to model the observed behavior, it is proposed that the order parameter for superconductivity is only depressed within a strained region associated with the grain boundary dislocations. Figure 5.16(a) shows a linear plot of the data of Dimos et al., 3 which clearly reveals the linear behavior expected in the small-angle regime. From a least-squares fit of the slope between $\theta=0$ and $10^{\circ}$, the dimension of this disturbed region in the boundary plane is $2.9|\bar{b}|$, where $b$ is the dislocation Burgers' vector. A physical rationalization for this large disturbed region is obtained by examining the strain field associated with an array of grain boundary dislocations.

Figure 5.16(b) is a plot of the roughly elliptical area around the dislocations where one of the strain components is calculated to be $\geq 1 \%$. It can be seen that there is a "conducting plate" of relatively undistorted crystal between dislocations. For misorientation angles less than $10^{\circ}$, the equations for the lateral extent of $1 \%$ strain predict a linear dependence on misorientation angle, providing an excellent explanation of the available experimental data. The grain boundary dislocations effectively only reduce the area of the grain houndary plane. This model predicts the entire boundary loses the required structural order for superconductivity for misorientations beyond $10^{\circ}$ and becomes a weak link very sensitive to small applied magnetic fields, as is indeed observed experimentally. 4

1. Summary of paper to be published. 
SSDN-4358
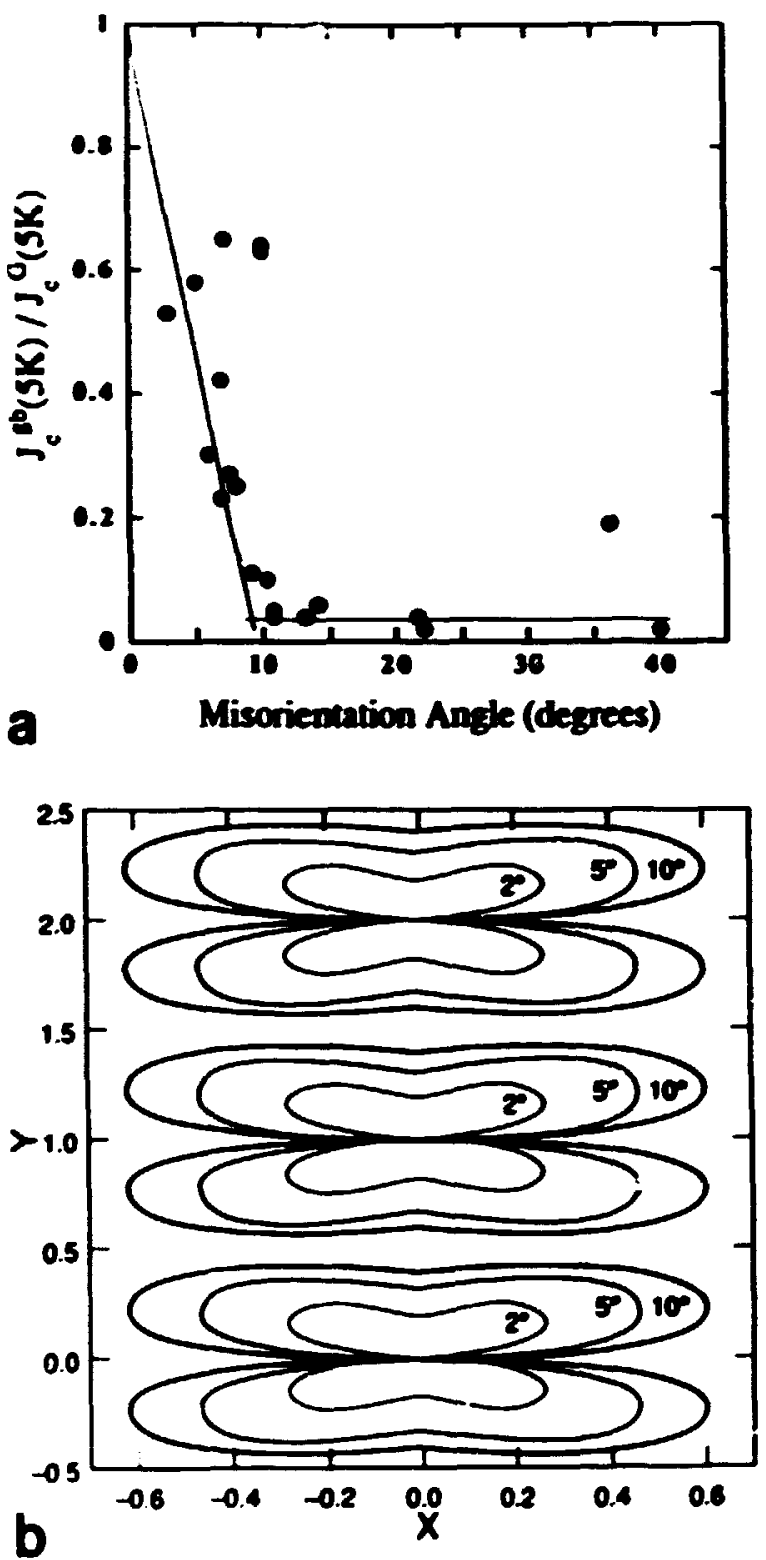

Fig. 5.16. (a) Normalized critical current density as a function of misorientation from Ref. 2, plotted on a linear scale showing a rapid drop in critical current for micorientations up to $10^{\circ}$ and then little additional drop for larger misorientations. (b) Calculated strain ficld for an infinite array of edge dislocations in a 2,5, and $10^{\circ}$ tilt boundary showing the extent to which $\varepsilon_{x x}$, the largest strain component exceeds 1\%, plotted using reduced distances $Y=y / D$ and $X=x / D$, where $D$ is the dislocation spacing.
2. M. F. Chisholm and S. J. Pennycook, Proc. of Electron Micros. Soc. Amer. 47, 198 (1989).

3. D. Dimos, F. Chaudhari, and J. Mannhart, Phys. Rev. B 41, 1038 (1990).

4. J. Mannhart et al., Phys. Reo. Lett. 61, 2476 (1988).

\section{DTRET MAGNG OF NTIEFACIAL OADERNG IN ULTRATIIN (ST)Cen) SURELATICES USTNG ZCONII AST SCANINIG TIANSMISSION EIECTRONMTCIOSCOYY}

\section{E. Jesom, 2 S. J. Pevurgcook, and J.M. Baribean?}

There is currently great interest in the growth of ultorathin (SimGe, superlattices due to their predicted direct band gap, which, if realized, would bypass the difficull and costly problem of the growth of III/V materials and their integration into Si technology. The first direct images of interfacial ordering in the Si-Ge system have been obtained. Figure 5.17 shows a Z-contrast image of a (Si, Cegh4 superlattice grown by molecular beam epitaxy (MBE) at $350^{\circ} \mathrm{C}$ directly onto a Ce substrate. The (001) growth direction is toward the top of the image. Remarkably, three distinct types of ordering can be observed to occur within the Si laycrs. A strong $(2 \times n)$ interfacial periodicity is evident in the top Si layer, directional (111) ordering in the central Si layer, and cross-like structures in the bottom Si layer.

These phases differ from all previously proposed ordered structures. 5 An asymmetric interfacial abruptness is also observed; that is, the Si on Ce interface is less abrupt than the Ce on Si interface. Utilizing the intuitive imaging 
SSDN-4276

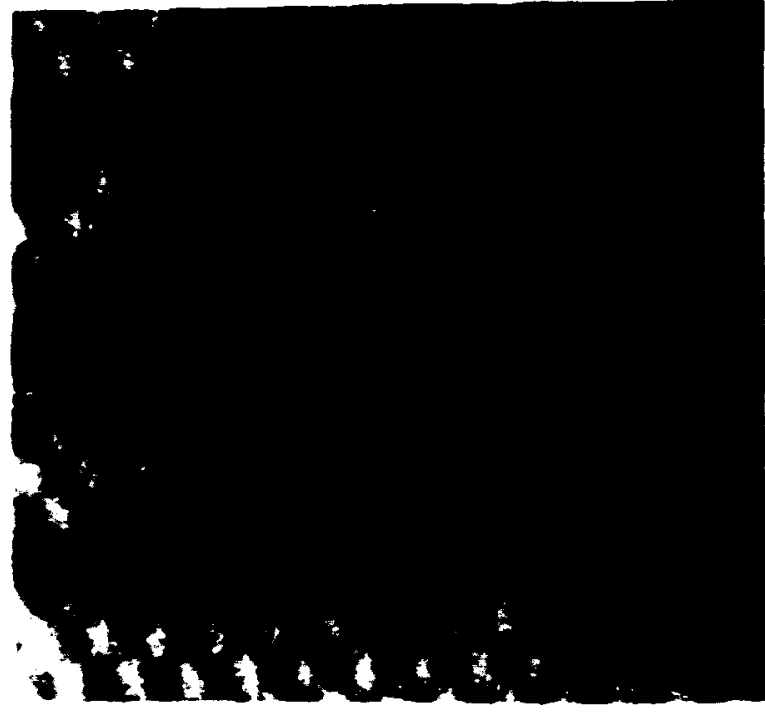

Fig. 5.17. Z-contrast image of a (Sir $\mathrm{Ceg}_{24}$ superlattice revealing unintentional lateral ordering (arrowed). Each bright spot in the image represents a single-atomic dumbbell. The bright horizontal bands are the deposited Ge layers, and the darker regions are the deposited Si layers which contain the ordering.

Capabilities of Z-contrast scanning transmission electron microscopy, it is possible to examine the interface structures over a wide range of specimen thickness. Only clear projections of the ordered configurations in the very thinnest regions are obtained, so an ordered domain size of $\leq 5 \mathrm{~nm}$ is deduced. Our observations clearly provide dramatic insight into the atomistic processes occurring during epitaxial growth and are highly relevant to the band structure calculations pertaining to the design of a direct band gap optoelectronic material.

1. Summary of paper: Physical Review Letters (in press).

2. Oak Ridge Associated Universities postgraduate research participant.
3. National Research Council of Canada, Otawa, Canada.

4. S. Froyen, D. M. Wood, and A. Zunger, Phys. Reo. B 36, 4547 (1987).

5. A. Ourmazd and J. C. Bean, Phys. Reo. Lett. 55, 765 (1985).

\section{A NOVEL GE ATOM PUMP MECHUNISM OFIRATING DURING SI MOLECULAR BEAM ETTAXY GROWTH ON Gel}

\author{
D. E. Jesson,2 S. J. Penunycook, \\ and I.M. Baribenu
}

From the insights provided by $Z$-contrast imaging, we have been able in deduce a noval Ge atom pump mechanism which cocurs during molecular beam epitaxy (MBE) growth of Si on Ge. The model explains all the features observed experimentally, in addition to the whllknown but little understood effect of "Ce drag." which complicates Si-Ge MBE growth." Our model is illustrated in Fig-5.18, which schematically represents ti:e growth of a Si dimet row on a $\mathrm{Ce}(2 \times 1)$ reconstructed surface along (110). The growth is forced through two distinct step configurations, nonrebonded [Fig. 5.18(b)] and rebonded [Fig. 5.18(c)] edges, type $S_{B}$ steps in the notation of Chadi.5 Using bond-energy arguments, it can be established that the nonrebonded edge [Fig. 5.18(r)] remains stable during growth, but the rebonded edge [Fig. 5.18(c)] is unstable compared to an interchange of a surface $\mathrm{Si}$ atom with a relatively weakly bound Ge atom at the step edge. This replaces a surface $S i$ dangling bond with an energetically favorable Ce dangling bond, saving in total $0.45 \mathrm{eV} /$ exchange. Thus, the rebonded edge step configuration acts as a 

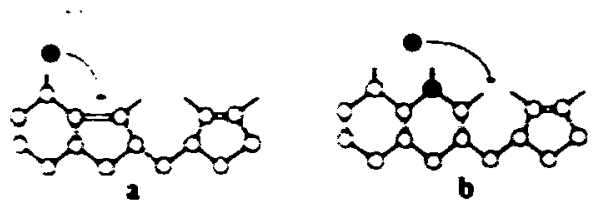

[001]
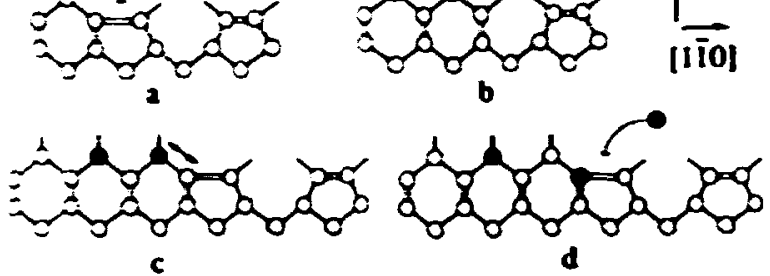

d

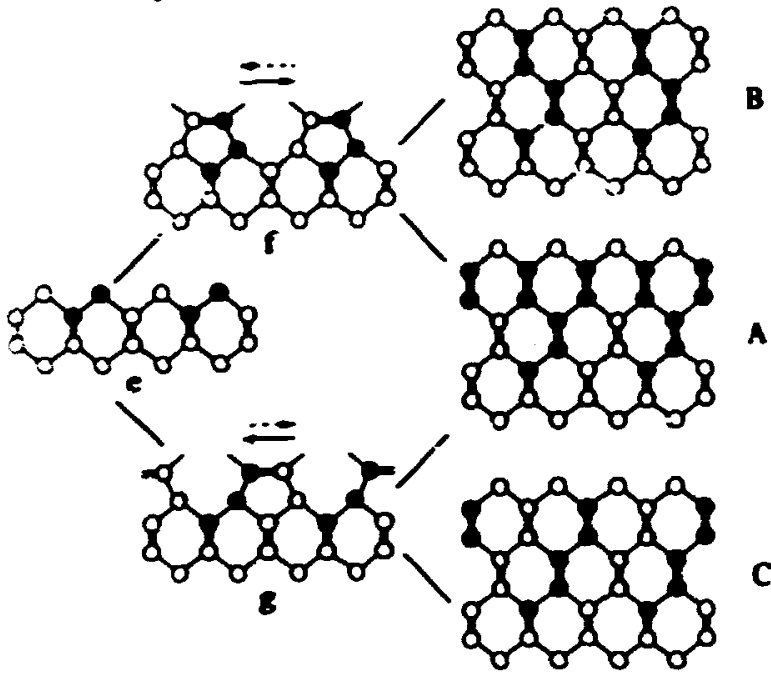

Fig- 5.18. Growth kinetics model for Ge segregation and oxdering resulting from Si deposition on a (2x1) reconstructed Ge surface. Open circles represent Ge volumes, solid circles $\mathrm{Si}$ columns, and shaded circles alloy columns. The exchange arrowed in (c) replaces the Si surface dangling bond with a Ce dangling bond (d).

chemically driven atom pump for Ge segregation.

Since the growth is forced alternately through stable and unstable step configurations, the asymmetric pumping mechanism configures the dimer string into alternating Si and SiGe alloy columns as the growth proceeds along [1i0] (Fig 5.18(e)]. Carrying the model through predicts all the structures observed experimentally [Figs. 5.18(a)-5.18(c)]. Each structure is also associated with a compositional modu- btion along the growth direction. Defining an adatom/substrate exchange parameter $a$, the asymmetric interfacial abruptness can be explained and the kemperature dependence of the pumping mectarism, which has important implications for the growth of extremely abrupt interfaces, predicted.

1. Sumnary of paper: Physical Review Letiers (in press).

2. Onk Ridge Aseccialted Universities postgraduate research participant.

3. National Research Council of Canada, Ottawa, Canada.

4. S. S. Iyer et al, Appl. Phys. Lett. 54, 219 (1909).

5. D. J. Chadi, Phys. Reo. Lett. 59, 1691 (1987).

\section{STRAINED CESI HETEROSTRUCTURES'}

\section{F. Chisholim and S.I. Penmycook}

A recently developed notion that strain can play a beneficial role in modifying optical and electronic properties of heterostructures has led to a number of advances in heterojunction physics and device technology.2 The simplest application of this concept is the heterojunction bipolar transistor (HBT). Using $\mathrm{Si}_{1-x} \mathrm{Ge}_{x}$ in the base region of the transistor offers several potential advantages. These include higher emitter injection efficiency, lower base resistance, shorter base transit time, and superior low-temperature operation due to both the intrinsic properties of Se as well as the additional strain effect on the band gap. ${ }^{2}$ Although the best performance should be obtained with a 
nearly pure Ge base region, it has not been possible to grow a continuous disbeation-free film of Ge on Si using conventional deposition techniques, inctuding molecular beam epitaxy. It has been found that after the deposition of the initial three monolayers, Ge begins to grow as islands on Si and with the equivalent of six monolayers of Ce, dislocations begin to form ${ }^{3}$

In the present work, we have applied the novel growth technique of implantation and oxidation to obtain a film of Sio.18Geose ch Si over 40 monolayers thick, which is coherent, chemically abrupt, and planar (Fig. 5.19). Our

SSDN-4196

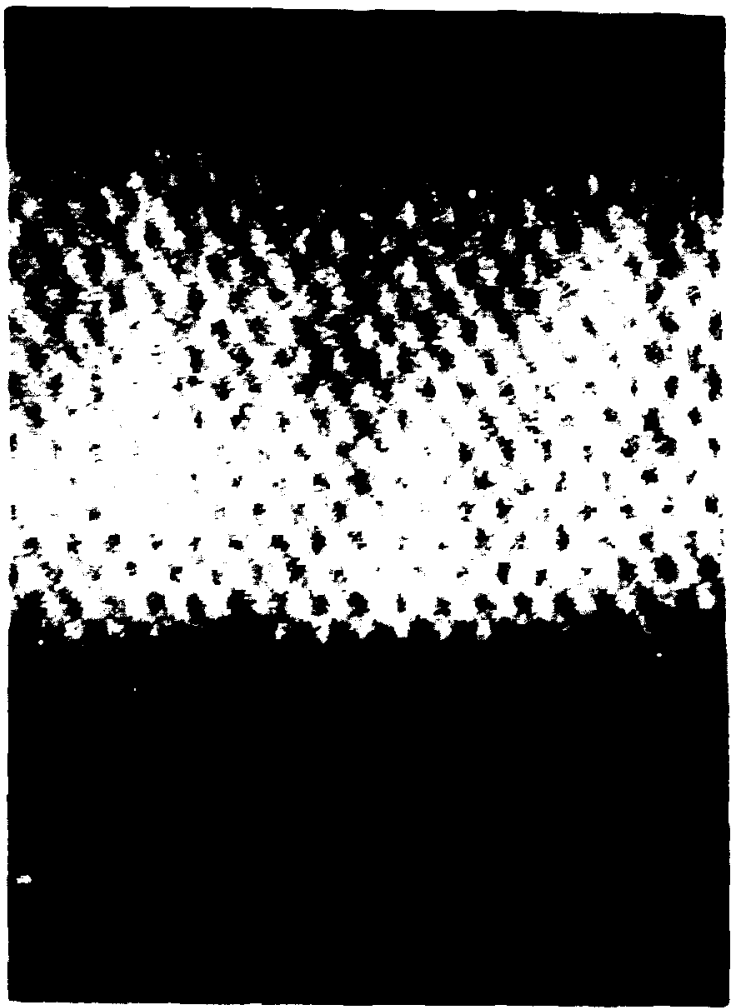

Fig.5.19. A $Z$-contrast image of a disbcation-free 5-nm-thick Ge film on (001) $\mathrm{Si}$ produced by stcam oxidation of a $\mathrm{Ge}\left(2 \times 10^{16}\right.$ ions $/ \mathrm{cm}^{2}$ )-implanted wafer. results indicate that we can take advantage of the slow rate at which distocations nucleate at the film/oxide interface to produce a continuous, highly strained and distocation-free layer. High-performance SiadCeo.12-base HBTs have been fabricated with a $45 \%$ improvement in intrinsic transistor speed compared with Si transistors. 5 Even greater improvements with a Sio.18Geo..2 base grown by implantation and oxidation can now be pursued.

1. Summary of paper to be published.

2. See, for example, T. P. Pearsall, Thin Solid Filins 184, 451 (1990).

3. J. Bevk et al., Appl. Phys. Lett. 49, 286 (1986).

4. D. Fathy et al., Appl. Phys. Lett. 51, 337 (1987).

5. J. H. Comfort et al., Proceadings of the Topical Symposium on Silicon-Based Heterostructures, Toronto, Canada (1990).

\section{MISFT ACCOMIMODATION IN EPTTAXIAL Ge FILMS ON Si'}

\author{
M. F. Chisholm. S. J. Pennycoot, \\ and O.W. Holland
}

High-resolution Z-contrast imaging is well suited for determinations of the structure of interfaces and dislocation cores. ${ }^{2}$ This technique has been used in this study to investigate film formation and misfit accommodation in epitaxial Ge films grown on Si. The epitaxial Ge films were produced by steam oxidation of Ce-implanted (001) $\mathrm{Si}^{3}$ The implanted $\mathrm{Ce}$ is rejected by the growing $\mathrm{SiO}_{2}$ layer and does not have sufficient mobility to diffuse into the $\mathrm{Si}$. A range of $\mathrm{Ge}$ film thicknesses, formed by vary- 
ing the Ge implant dose, can be produced to study the various stages of misfit accommodation in these epitaxial films. With this growth process the Ge films are constrained to grow layer-by-layer instead of the normally observed island mode.

It is observed that the $m^{-1}$ fit distocations are nucleated at a free surface as Shockley partials, glide to the interface, and then combine to form a perfect dislocation. The $30^{\circ}$ partial dislocation nucleates first and glides to the interface producing a stacking fault [Fig-5.20(a)]. Subsequently, a $90^{\circ}$ partial nucleates at the free surface and follows the $30^{\circ}$ Fartial, annihilating the stacking fault. This mechanism, which has been proposed earlier, 4,5 is for the first time conclusively demonstrated by this investigation. In supercritical thick films (>7 $\mathrm{nm})$ a fairly regular array of
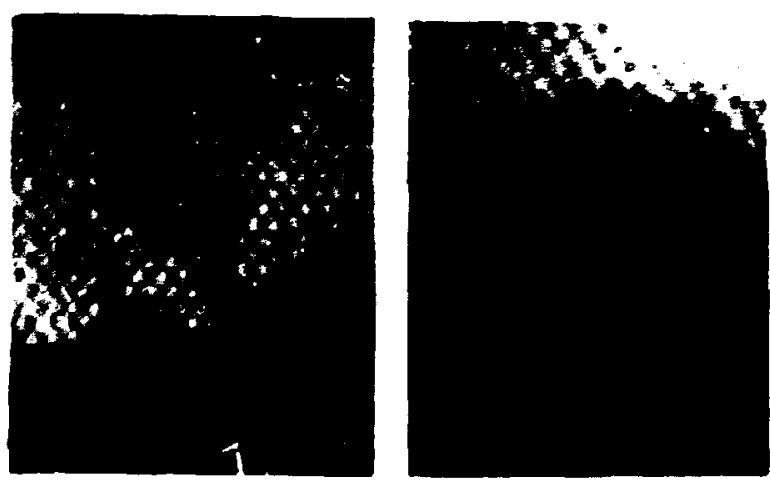

Fig. 5.20. 2-contrast images of Ce films on (001) Si viewed in cross section: (a) Image showing the initial stages of misfit accommodation. A $30^{\circ}$ Shockley partial dislocation (arrowed) has glided to the $\mathrm{Gc} / \mathrm{Si}$ interface creating a stacking fault in the Ge film. (b) Image of the Ce/Si interface showing that the edge dislocation core (arrowed) lies in the Ge film. interfacial dislocations spaced every $-17 \mathrm{~nm}$ is developed. It is observed that the dislocation cores are entirely in the Ge layer, which is the elastically softer material (Fig- 5.20(b)). This is an important observation and is one that could not be made easily without $Z$-contrast imaging.

1. Summary of puper. Mat. Res. Soc. Sym. Proc. 159, 447 (1990).

2. S. J. Pennycook, S. D. Benger, and R. J. Culbertson, J. Micrusc. 14, 229 (1986).

3. D. Fathy, O. W. Holland, and C. W. White, Appl. Phys. Lett. 51, 1337 (1987).

4. G. Abstreiter et al., J. Cryst. Growth 95, 431 (1989).

5. W. A. Jesser and J. W. Matthews, Philos. Mag. 17, 461 (1968).

\section{DIRECT MMGING OF NIERFACE STRUCTURE AND CHEMISTRY IN GaAelino.sGans? QUANTUM WELLS}
S. J. Pennycook, M.F. Chisholm,
D. E. Jesson, and G. Y. Robinson' ${ }^{2}$

The most sensitive technique used to date for the characterization of quantum-well interface quality has been photoluminescence spectroscopy. The best wells show narrow luminescence peaks with quantized energy differences corresponding to monolayer variations in the width of the wells, from which it is inferred that the quantum-well interfaces are compositionally abrupt and smooth over distances which are large compared to the diameter $(-100 \AA)$ of the two-dimensional exciton. ${ }^{3}$ Using the highly localized compositional sensitivity of $Z$-contrast imaging, these 
assumptions for the thinner quantum wells have been directly confirmed. Figure 5.21(a) shows part of a quantum well, nominally $5.7-\AA$ thick, clearly indicating abrupt interfaces and also the presence of an ir.terface step. The CaAs well appears brighter in the image and has elongated features corresponding to individual CaAs dumbbells, whereas in the barrier layers of $\ln _{0.5} \mathrm{Ca}_{0.5} \mathrm{P}$, the dumbbells appear as more circular features located over the Ino.5Ga.5 column of the dumbbell. Since cach dumbbell contributes to the image independently of its neighbors, it is possible to distinguish GaAs, GaP, Im ${ }_{5} \mathrm{Ga}_{0.5}$ As and $\operatorname{In}_{0.5} \mathrm{Ga}_{0.5} \mathrm{P}$ dumbbells from their shape and contrast characteristics to give compositional information on a scale well below that which can be achieved by conventional high-resolution imaging.

SSDN-4351
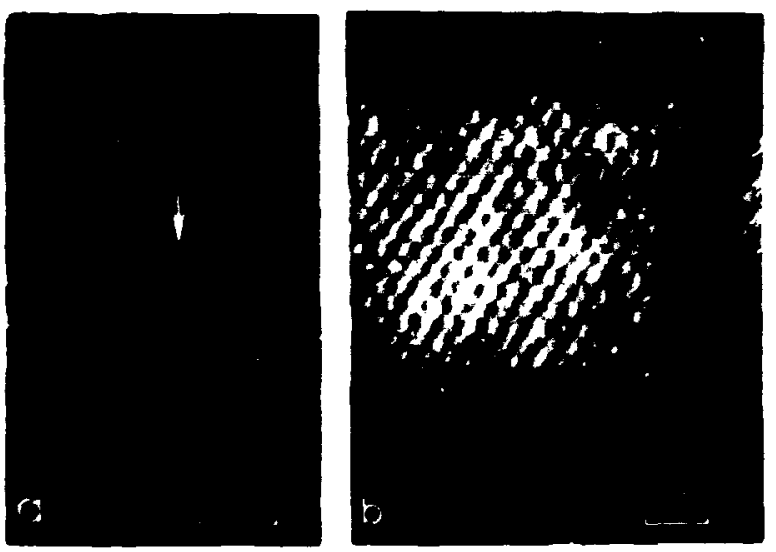

Fig. 5.21. Z-contrast imaging of GaAs quantum wells in $\ln _{0.5} \mathrm{G} \mathrm{ad}_{0.5} \mathrm{P}$ showing: (a) abrupt interfaces with monolayer interfacial steps (arrowed) and (b) interfacial roughening occurring during growth of a $52-\AA$ well.
Figure 5.21(b) shows a thicker quantum well in which the lower interface is stepped as with the thin well, but the upper interface is significantly roughened. The exciton will, therefore, experience a more diffuse interface, which may be the reason that multiple photoluminescence peaks are not observed from thicker wells.

\section{Oak Ridge Associated Universities} postgraduate research participant.

2. Colorado State University, Fort Collins, Colo.

3. M. J. Hafich et al., Appl. Phys. Lett. 54, 2686 (1989).

\section{INTERFACIAL DEFECTS IN EPTTAXIAL $\mathrm{COSi}_{2}$ FILMS ON Si(100)'}

\author{
S. J. Pennycook, D. E. Jesson,2 \\ S. M. Yalisooe, ${ }^{3}$ and R. T. Tung
}

For the last decade, there has been extensive study of epitaxial interfaces between silicon and the two silicides $\mathrm{NiSi}_{2}$ and $\mathrm{CoSi}_{2}$ in an attempt to correlate the measured Schottkybarrier height to the atomic structure as measured by other techniques. However, the assumption that such interfaces are atomically smooth and abrupt so that the electrical measurements are characteristic of the average boundary structure needs furth 2 examination in light of our recent $Z$-contrast observations. A high density of interfacial defects has been observed in $\mathrm{CoSi}_{2} / \mathrm{Si}(100)$ films grown by a template method, in the form of monolayer deep protrusions of the silicide into the $\mathrm{Si}$. These protrusions are one, two, or three atomic 
spacings wide and are most clear in the thinnest regions of the sample and must, therefore, only be 1-2 $\mathrm{nm}$ in length along the beam direction. Since they are not periodic on a large scale, they have not been observed in previous diffraction or high-resolution imaging studies. 5 The protrusions most likeły originate from surface defects present on the original cleaned surface layer. The film was grown by the template method in which 2-3 monolayers of Co metal are deposited at room temperature onto the cleaned substrate and annealed briefly at $300^{\circ} \mathrm{C}$ to form a thin template, followed by stoichiometric coevaporation of $\mathrm{CoSi}_{2}$ to form the desired final film thickness.

Figures 5.22(a)-5.22(c) indicate a likely mechanism for the formation of such protrusions starting from a vacancy channel, a defect seen even on clean $S i$ surfaces and stabilized markedly by the presance of a metal such as $\mathrm{Ni}$. The monolayer stryps at each side of these protrusions represent small segments of $[111]$ interfaces and may bo contributing to or even controllirig the observed Schottky-barrier height.

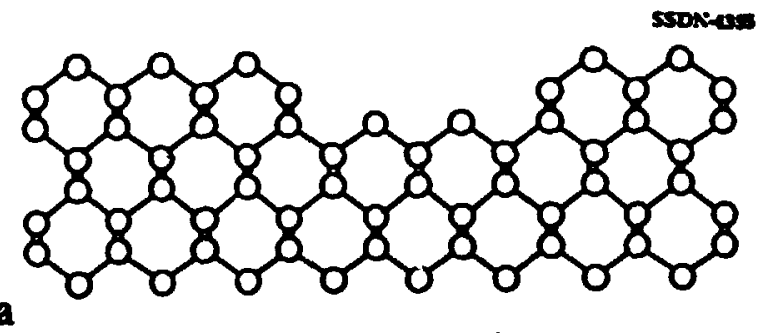

a
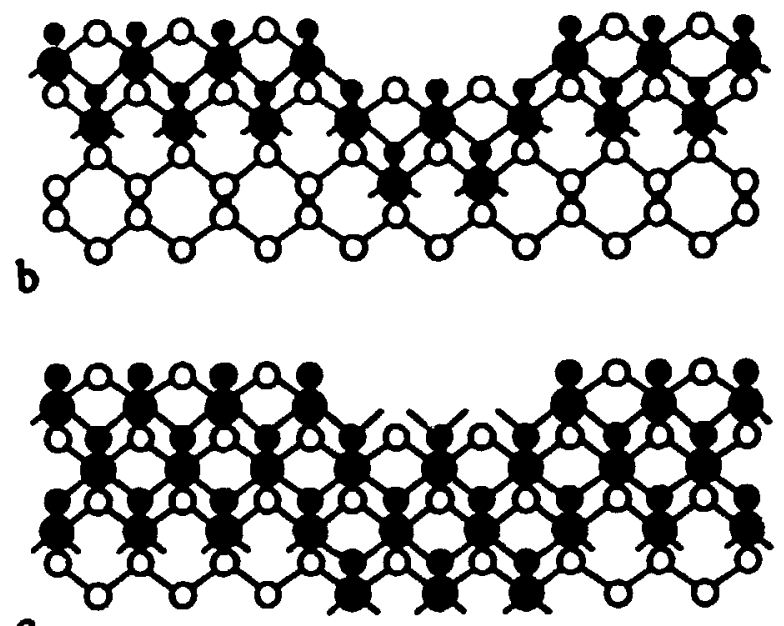

c

Fig. 5.22. Mechanism for the formation of interfacial defects from a vacancy channel on the original Si surface (a) which is preserved during growth of the template layer either two (b) or three (c) monolayers thick. Open circles represent Si atoms in their initial position, while small solid circles represent Si displaced by reaction with $\mathrm{Co}_{0}$, represented by large solid circles.

ION BEAM SYNTHESIS OF BURIED EREIUM SILICTDE'

\section{J. L. Park, ${ }^{2}$ A. Golanski, ${ }^{3}$ S. I. Pennycook, R. Feenstra, M.D. Galloway, H. E. Harmon, and C. W. White}

Rare-earth silicides are of particular interest since they give the lowest known Schottky-barrier heights on n-type $\mathrm{Si}$. In the 
present work, we demonstrate for the first time the formation of buried single-crystalline $\mathrm{ErSi}_{2}$ via ion implantation and annealing and its subsequent mesotaxial growth through a second implantation and annealing step.

High-dose, $8 \times 10^{16} / \mathrm{cm}^{2}$ of $170-k e V ~ \mathrm{Er}^{+}$ions were implanted into (111)Si resulting in the formation of precipitates of various sizes correlated to the implanted Er distribution. Figure 5.23(a) shows that $\mathrm{ErSi}_{2}$ precipitates (showing Moiré patterns) are preferentially oriented along the $<111\rangle$ axes. After annealing at $800^{\circ} \mathrm{C}$ in vacuum following the implant, the growth and coalescence of $\mathrm{ErSi}_{2}$ precipitates lead to a continuous buried $\mathrm{ErSi}_{2}$ layer [Fig. 5.23(b)]. The samples were then reimplanted with $8 \times 10^{16} / \mathrm{cm}^{2}$ of $170-\mathrm{keV} \mathrm{Er}$ at implantation temperatures of $250<T_{i}<520^{\circ} \mathrm{C}$ and reannealed at $800^{\circ} \mathrm{C}$. During the second implantation process the buried disilicide layer grows mesotaxially [Fig. 5.23(c)]. The mechanism of the growth was shown to be related to the ion-beam-induced, defectmediated diffusion of Er atoms. Transmission electron microscopy (TF.M) diffraction patterns confirm the $\mathrm{ErSi}_{2}$ phase and show the orientation relationship to be (0.01) $\mathrm{ESSi}_{2} / /(111) \mathrm{Si}$ and (10ī0)ErSi $i_{2} / /(\overline{4} 22) S i$. No evidence of vacancy ordering was observet, indicating that the implantation process produces a dense stoichiometric ErSi, ${ }_{2}$ phase superior to that formed by deposition and annealing."

1. Summary of paper to be published.

2. Postgraduate research participant from North Carolina State University, Ralcigh, N.C.
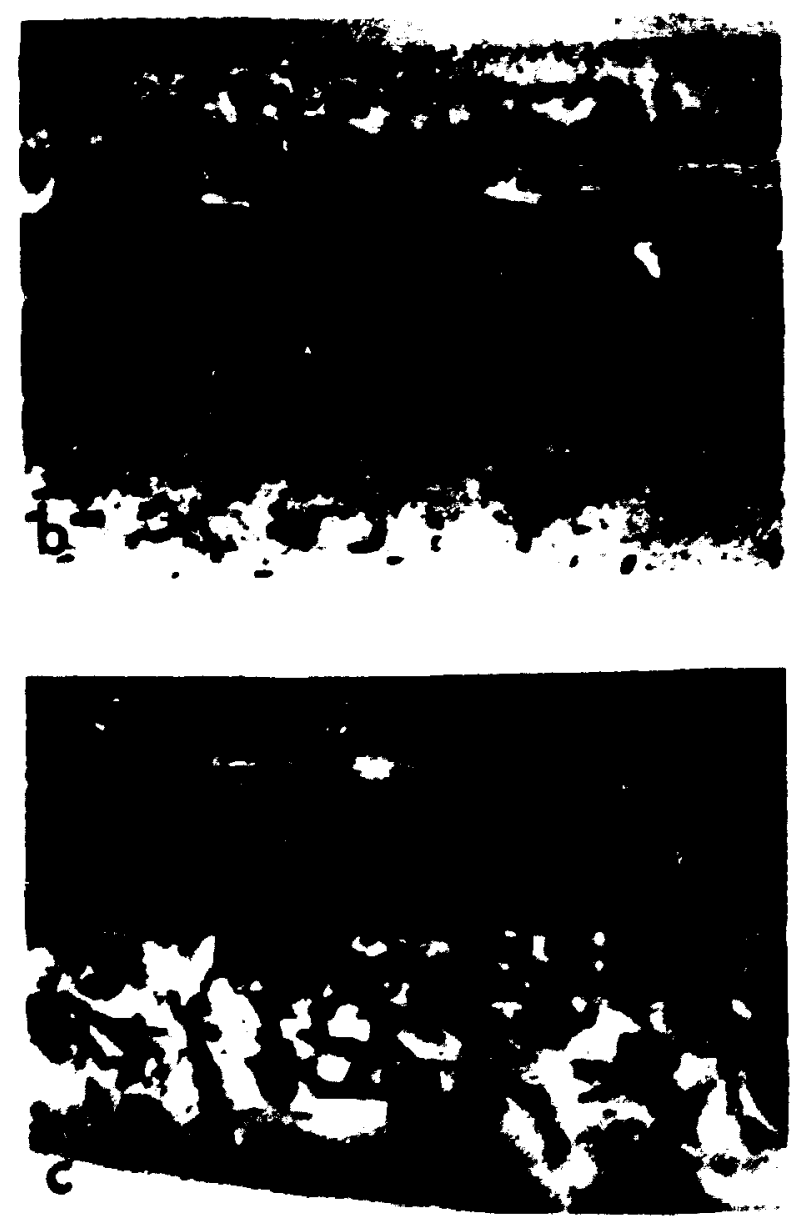

Fig. 5.23. Cross-section TEM micrographs from Er $\left(170 \mathrm{keV}, 8 \times 10^{16} / \mathrm{cm}^{2}, 520^{\circ} \mathrm{C}\right) \mathrm{im}$ planted Si(111); (a) as-implanted, (b) following $800^{\circ} \mathrm{C}$ annealing, and (c) following sequential implantation and annealing. 
3. Consultant from Centre National d'Etudes des Telecommunications, Meylan, France.

4. F. Amaud d'Avitaya et al., Appl. Phys. Lett. 54, 2198 (1989).

\section{X-RAY DIFFRACTION}

\section{RESONANT MONOCHROMATORS FOR SUBMICKOVOLT ENERGY RESOLUTION X-RAY DIFPACTION}

\section{Z. Tischler, B. C. Larson, and G. E. Ice'}

The development of techniques for $x$-ray scattering with submicrovolt energy resolution is dependent on the development of efficient Mössbauer resonant monochromators to select photons in an $-10^{-6} \mathrm{eV}$ bandwidth from the continuous synchrotron $x$-ray spectrum. Although widespread application of resonant scattering to condensed matter problerns is not expected to become feasible until the increased power and brightness of the Advanced Photon Source (APS) are available, it is important to optimize techniques now so that they can be used when the APS becomes operational. In this work, investigations of epitaxial ${ }^{5 /} \mathrm{Fe}_{2} \mathrm{O}_{3}$ on natural $\mathrm{Fe}_{2} \mathrm{O}_{3}$ substrates as Mössbauer monochromators have been made at the BNL National Synchrotron Light Source (NSLS), and it was found that significant increases in resonant photon yield can be achieved th angh the use of low-resolution premonochromator elements rather than the ultrahigh resolution premonochromators used by other investigators.

Measurements were performed on ${ }^{57} \mathrm{Fe}_{2} \mathrm{O}_{3}$ crystals with $\sim 15$ arc second mosaic widths using silicon (333) and (111) channel-cut premonochromators (in the dispersive mode) on the sagittally focused $X-14$ beam line at NSLS. These premonochromators have significantly lower angular and energy resolutions than the silicon (1064) premonochromator used by other investigators. 2 Lower angular resolution was chosen to ensure that the sagittally focused $X$ rays would be transmitted by the premonochromator and because they are more closely matched to the mosaic width of our ${ }^{57} \mathrm{Fe}_{2} \mathrm{O}_{3}$ crystal. Resonant photon beams of 6 counts/s were obtained using the (333) channelcut premonochromator, and a 22 counts/s resonant beam was obtained in the (111) case. The (111) results represent more than an order of magnitude increase over the -1.5 counts/s reported using a high-resolution premonochromator, ${ }^{2}$ indicating the advantage that can be gained through simultaneous use of sagittal focusing and low-resolution premonochromators. As might be expected though, the higher intensity resonant beams are obtained at the expense of a larger nonresonant noise accompanying the signal. The signal/noise ratios were found to be $-6 / 1$ and $-1 / 1$, for the (333) and (111) premonochromators, respectively, whercas ratios $\sim 100 / 1$ have been reporied for the ultrahigh resulution case.

1. Metals and Ceramics Division, ORNL.

2. C. Faigeret et al., Phys. Reo. Lell. 58, 2699 (1987). 


\section{TIME SPECTRUM OF RESONANT NUCLEAR BRACG SCATTERING FROM A MOSAIC CRYSTAL}

\section{J. Z. Tíchler and B. C. Larson}

It has been shown elsewhere that it is possible to obtain significant increases in the number of Mōssbauer resonant photons from pure nuclear Bragg monochromators by lowering the angular resolution of the premonochromator elements. In this report, measurements of the time spectrum of resonant nuclear Bragg scattering from mnsaic ${ }^{57} \mathrm{Fe}_{2} \mathrm{O}_{3}$ are described. The measurements demonstrate that additional increases in resonant scattering can be obtained by eliminating the premonochromator elements and using time resolution to separate (prompt) nonresonant $X$ rays from the resonant signal.

Through the use of an ${ }^{57} \mathrm{Fe}_{2} \mathrm{O}_{3}$ crystal with a 15 arc second mosaic width (many times the intrinsic diffraction width), the undesirably strong sensitivity of the resonant time spectrum to small angular variations in the monochromator ${ }^{2}$ angle has been removed. The resulting time-resolved spectrum measured on the A-2 beam line at the Cornell High-Energy Synchrotron Source (CHESS) is shown in Fig. 5.24. These data correspond to - 60 resonant photons/s using a plastic scintillator detector and represent about a factor of 5 improvement in count rate over that attained using a premonochromator element. There is a large prompt ('e., zero delay) background, and the time spectrum oscillates ${ }^{2}$ as a result of interference beating of the Zeeman split reflectivity

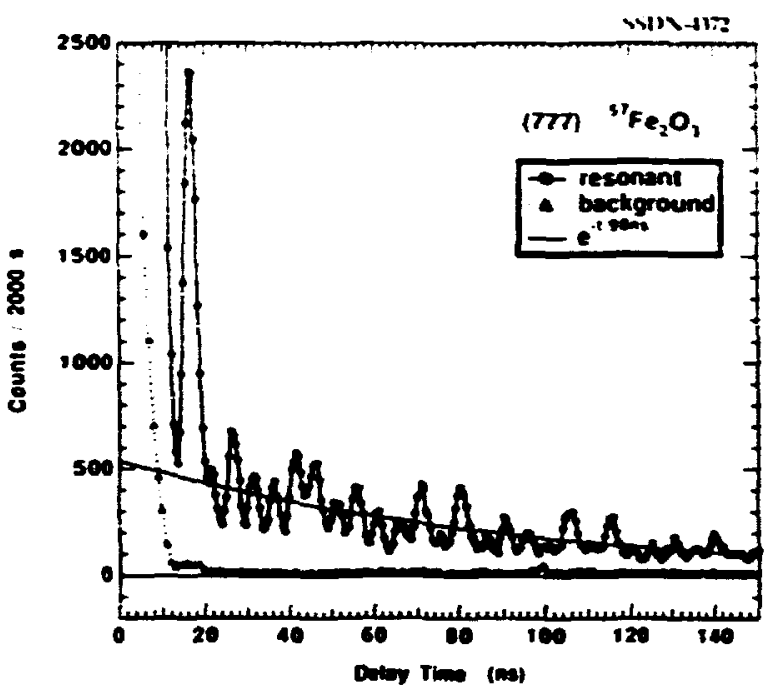

Fig. 5.24. Time spectrum of the (777) superlattice reflection and background scattering measured under off-resonance conditions. The small spike in the background at $100 \mathrm{~ns}$ is an artifact from the storage ring.

spectrum of antiferromagnetic ${ }^{57} \mathrm{Fe}_{2} \mathrm{O}_{3}$; however, we note that after $-20 \mathrm{~ns}$ the spectrum falls off with a time constant near the 98-ns lifetime of an isolated ${ }^{57} \mathrm{Fe}$ Mössbaucr state. This last observation indicates that a significant component of the resonant scattering has a band width dose to that of ordinary Mossbauer absorbers and suggests the possibility of using conventional Mōssbauer techniques in timedomain resonant scattering applications.

1. I. Z. Tischler, B. C. Larson, and G. E. Ice, "Resonant Monochromators for Submicrovolt Energy Resolution X-Ray Diffraction," this report.

2. E. Gerdau et al., Phys. Rev. Lett. 57, 1141 (1986). 
TIME-DOMAIN SPECTRUM OF RESONANTLY FILTERED X RAYS FROM A NUCLEAR BRAGG MONOCHROMATOR

\section{J. Z. Tischler, B. C. Larson, and P. Zschack'}

It has been shown ${ }^{2}$ that significant increases in the number of Mössbauer resonant photons can be obtained from a nuclear Bragg monochromator using time-resolved techniques. In this report, it is shown that the time structure of a resonant beam is dramatically affected by a simple powdered ${ }^{57} \mathrm{Fe}_{2} \mathrm{O}_{3}$ filter, and an application of the effect in submicrovolt $x$-ray spectroscopy is discussed.

The use of a Mósbauer resonant beam in a scattering experiment requires a means of analyzing the scattered beam to determine changes in its energy spectrum. The simplest type of experiment, for instance, requires separation of the elastic scattering (due to static structural variations) from $X$ rays scattered inelastically (by dynamical phenomena), where inelastic means an energy shift $\geq 10^{-7} \mathrm{eV}$. In the present work, experiments performed at the BNL National Synchrotron Light Source (NSLS) have shown that a powdered filter should provide a convenient means for making such measurements.

Figure 5.25 shows the measured time spectra of $X$ rays reflected from the 777 reflection of a mosaic ${ }^{57} \mathrm{Fe}_{2} \mathrm{O}_{3}$ crystal with and without a powdered ${ }^{57} \mathrm{Fe}_{2} \mathrm{O}_{3}$ absorber. The data in Fig. 5.25 were normalized to have the same intensity at time $t=0$ to account for the effects of nonresonant absorption in the filter. Note that the shapes of the Iwo curves are quite different; the relative difference between filtered

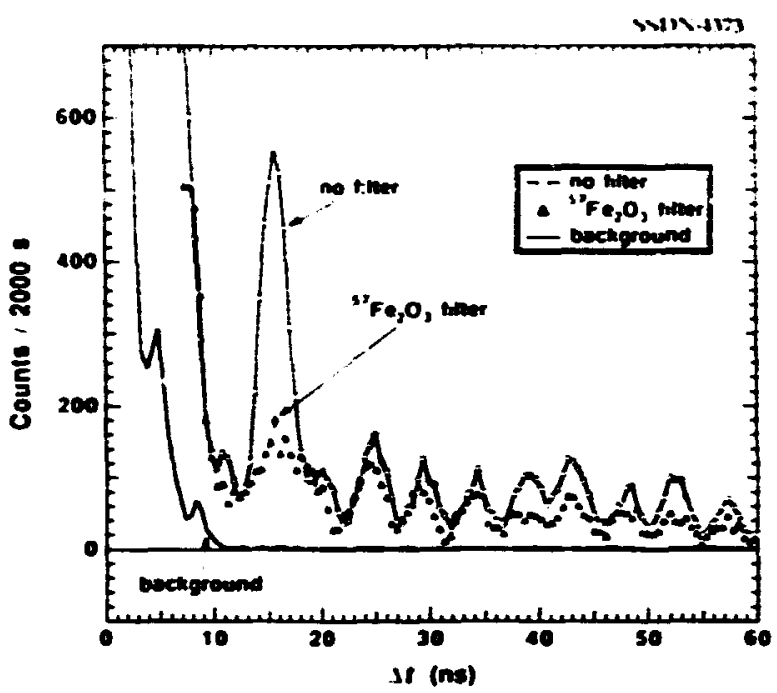

Fig. 5.25. Time spectra of the (777) superlattice reflection in $\mathrm{Fe}_{2} \mathrm{O}_{3}$ for resonant Mossbauer photons with and without an ${ }^{57} \mathrm{Fe}_{2} \mathrm{O}_{3}$ filter. The background was measured off-resonance.

and unfiltered peaks varies from peak to peak (in particular, note the peaks at 15 and $24 \mathrm{~ns}$ ). Since the final time spectrum in a resonant fraction measurement must be a linear combination of the two curves in Fig. 5.25, this simple filter should provide a convenient analyzer for measuring the resonant fraction in a beam scattered from a sample under investigation.

1. Metals and Ceramics Division, ORNL.

2. J. 2. Tischler and B. C. Larson, TimeSpectrum of Resonant Nuclear Bragg Scattering from a Mosaic Crystal," this report.

\section{X-RAY STUDY OF EPTTAXIAL} $\mathrm{YBa}_{2} \mathrm{Cu}_{3} \mathrm{O}_{2} / \mathrm{PrBa}_{2} \mathrm{Cu}_{3} \mathrm{O}_{2}$ SUPERLATTICES

\section{J. D. Budai, D. H. Lowndes, and D. P. Norton}

$X$-ray diffraction measurements have been used to investigate the epitaxy and microstructure of $\mathrm{YBa}_{2} \mathrm{Cu}_{3} \mathrm{O}_{x} / \mathrm{PrBa}_{2} \mathrm{Cu}_{3} \mathrm{O}_{x}$ 
(YBCO/PrBCO) superlattices grown on $\mathrm{SrTiO}_{3}$ i001) substrates using an in situ pulsed-laser ablation process. ${ }^{1,2}$ Structural information obtained from $x$-ray scattering plays an important role in the interpretation of electrical measurements from these superconductor/ insulator multilayer samples. $X$-ray scans through diffraction peaks indicated that the films are aligned almost completely in the $c \perp$ orientation with a small ( $\leq 0.4^{\circ}$ FWHM) mosaic spread and that the in-plane epitaxy is such that the <110 directions of the film and substrate are aligned.

Figure 5.26 shows $\theta-2 \theta$ scans for three different YBCO/PrBCO superlattices in which the YBCO layers are approximately one-unitcell thick. In addition to the large (truncated) Bragg peaks due to the underlying $(\mathrm{Y}, \mathrm{Pr}) \mathrm{Ba}_{2} \mathrm{Cu}_{3} \mathrm{O}_{2}$ crystal structure, satellite peaks associated with the Y/Pr compositional modulation are also evident, and their positions provide a measurement of the modulation wavelength $\Lambda$. The presence of a satellite for the $\Lambda=2.3 \mathrm{~nm}(1 Y \times 1 \mathrm{Pr})$ superlattice confirms directly that interdiffusion must be limited to less than a single c-axis cell during the in situ growth process. X-ray measurements obtained after heating a sample to $800^{\circ} \mathrm{C}$ revealed that the satellites were enhanced rather than diminished, indicating that the compositional modulation is remarkably stable to thermal annealing.

Information concerning the extent of structural and compositional order has been obtained as well. The widths of the main (002)

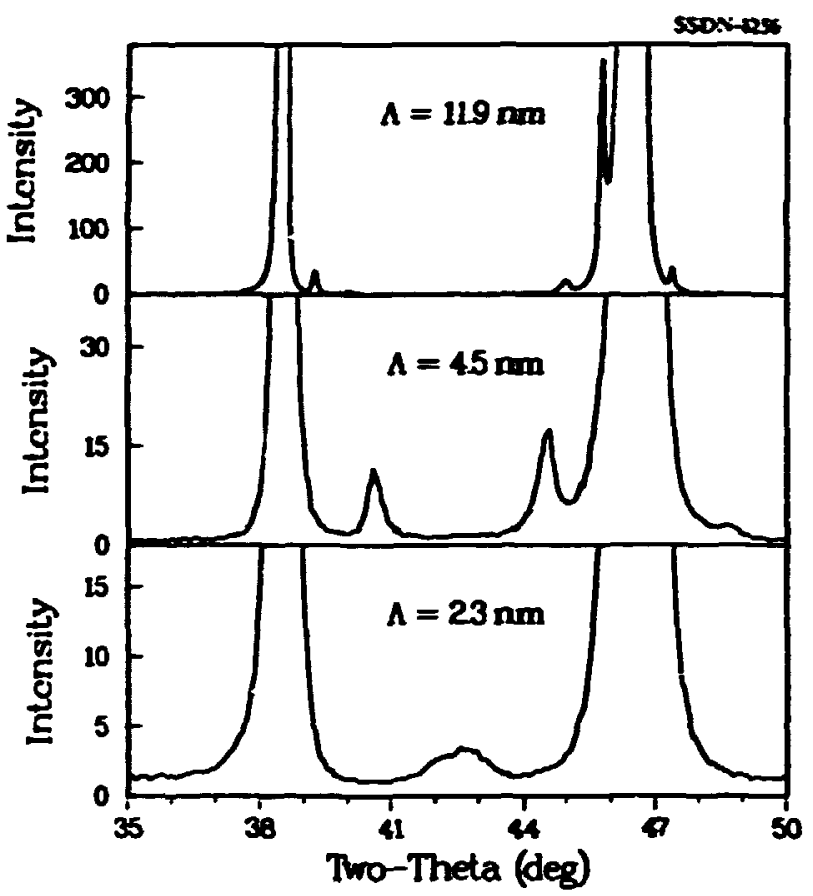

Fig. 5.26. $\theta-2 \theta$ x-ray diffraction scans along the (002) axis from YBCO/PrBCO supcrlattices with modulation wavelengths $\boldsymbol{A}$ as noted.

peaks are found to increase only slightly with order and yield $(\mathrm{Y}, \mathrm{Pr}) \mathrm{Ba}_{2} \mathrm{Cu}_{3} \mathrm{O}_{x}$ structural domain sizes on the order of the film thickness. In contrast, the widths of the superlattice reflections increase more rapidly with order and yield coherence lengths several timcs smaller. Thus, although the superlattices are fairly well ordered, the periodicity of the chemical modulation is not perfect throughout the film.

1. D. H. Lowndes, D. P. Norton, and J. D. Budai, Phys. Reo. Lett. 65, 1060 (1990).

2. D. H. Lowndes et al., Materials Research Sociely Symposium Proceedings (in press) 
FERERDD AUGNMIDNT OF TWIN COUNDARIES IN YB+2 ${ }_{3} \mathrm{O}_{2}$ THIN FIIMS AND SUTELATTCES ON SrTto ${ }^{\prime}$

J. D. Buda, M.F. Chisholu, R. Feanstre, D. H. Lowndes, D. P. Norton,?

L. A. Boatner, and D. K. Christen.

The detailed epituxial and morphological properties of $\mathrm{YBa}_{2} \mathrm{Cu}_{3} \mathrm{O}_{2}$ thin films and $\mathrm{YBa}_{2} \mathrm{Cu}_{3} \mathrm{O}_{2} / \mathrm{PrBa}_{2} \mathrm{Cu}_{3} \mathrm{O}_{2}$ superlattice films grown on (OCL) $\mathrm{SrTO}_{3}$ substraties have teen investigated using $x$-ray diffraction and transmission electron microscopy. The films are found to have their caxis aligned with the substrate [OOL] axis and consist of four symmetry-equivalent orthorhombic domains for which the low-order reciprocal lattice peaks are shown schematically in Fig. 5.27.

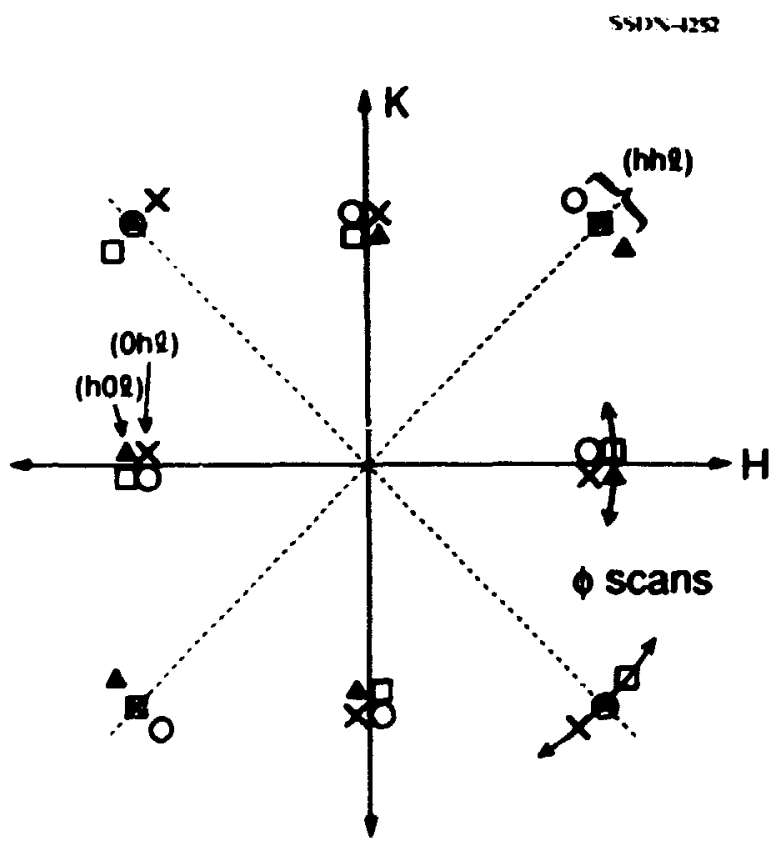

Fig. 5.27. Schematic drawing of a YBCO thin-film reciprocal lattice plane perpendicular to the $(002)$ axis and its epitaxial relation to the $\mathrm{SrTiO}_{3}$ substrate. The solid and dashed lines represent the in-plane substrate $\langle\mathrm{HOO}\rangle$ and $\angle \mathrm{HH} 0\rangle$ directions, respectively.
These four domains can be considered as one Iwin-related pair (squares and $x$ 's) aligned with the cubic substrate [HHO] direction and another twin-related pair (circles and trian-

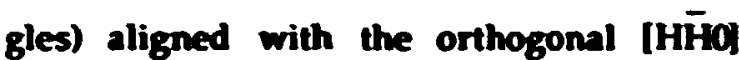
direction. Twin boundaries and other defects are known to play an important role in controlling the fuxx pinning and current-carrying copebilities of hight- $T_{c}$ superconductors.

By measuring the $x$-ray intersities associated with different domains, it has been found that one twin orientation is often highly favored relative to the other; this results in films containing a high density of twin boundories preferentially aligned in a single direction. This in-plane symmetry breaking has becn correlated with small $\left(0.2-3^{\circ}\right)$ miscuts of the substrate away from the exact (001) surface. and in all samples in which a domain bias was observed, the preferred twin planes are aligned perpendicular to a tilt of the substrate surface normal toward an in-plane [110] direction. These observations can be understood on a microscopic level by considering the influence of miscut-induced surface steps on the nucleation of twin boundaries during the tetragonal-toorthorhombic phase transition that occurs during film processing. It is expected that high- $T_{c}$ superconductor films containing oriented boundaries will prove particularly useful in studying the influence of twins on physical propertics, and measurements of critical current densities both perpendicular and parallel to the twin boundarics in such films have been initiated.

1. Summary of paper to be published.

2. ORNL Eugene P. Wigner Fellow. 


\section{DIRECT DETERMINATION OF PRECIPTTATE STRAIN AND STZES USING HIGH-ORDER $X$-RAY DIFFUSE SCATTERING}

B. C. Larson, M. D. Galloway, and P. Zschack'

$X$-ray diffuse scattering near Bragg reflections provides a sensitive, nondestructive tool for the investigation of clustered lattice defects (e.g., coherent precipitates and dislocation loops', and it is anticipated that this technique can bu applied to detailed investigations of the structure and thermal evolution of irradiation or ion implantation-induced displacement cascades in materials.

High-order diffuse-scattering measurements on coherent precipitates in (aged) $\mathrm{Cu} 1 \%$ Co crystals have been combined with numerically calculated diffuse scattering cross-scetion calculations to determine coherency strains and the sizes of the precipitates. Scattering measurements were made near the $(800),(1000)$, and (1200) Bragg reflections using synchrotron $X$ rays at the ORNL beam line at the National Synchrotron Light Source, and calculations were made using anisotropic elasticity. Figure 5.28 shows diffuse scattering measured near the $(800)$ reciprocal lattice point in which direct Bragg scattering from the precipitates is present in the form of a completely resolved subsidiary peak. The separation of the "precipitate" scattering peak from the "host lattice" Bragg peak provides a direct measure of the lattice parameter of the precipitates relative to the host lattice. In addition, the diffraction width of the precipitate scat-

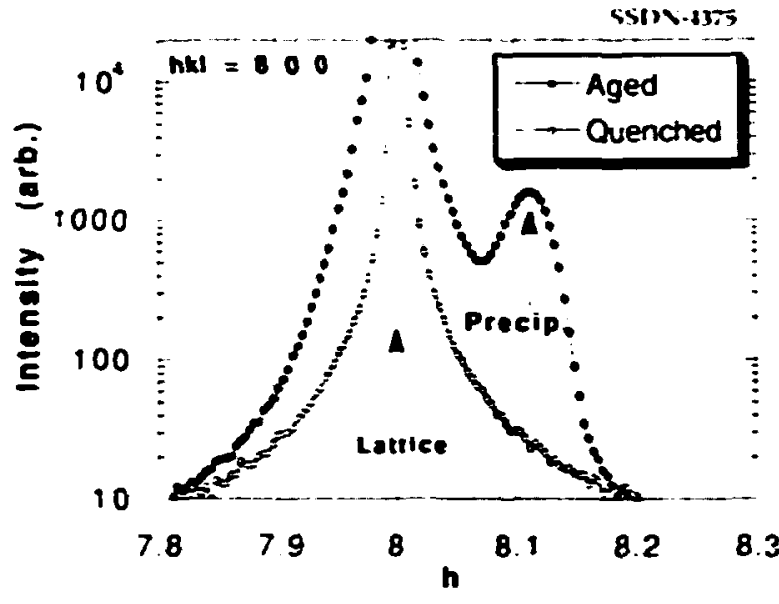

Fig. 5.28. Diffuse scattering measured in the radial direction near the $(800)$ Bragg reflection on aged (containing precipitates) and quenched (no precipitates) Cu $1 \% \mathrm{Co}$.

tering peak provides a direct measure of the characteristic size of the precipitates when analyzed using the numerical colculations.

The strain within the cobalt precipitates (relative to the host lattice) was found to be $-1.4 \%$, in good agreement with the $-1.42 \%$ strain inferred indirectly from previous loworder diffuse scattering measurements. A value of $-1.41 \%$ was calculated using elasticity and the $-1.8 \%$ unconstrained lattice parameter difference between fec cobalt and copper. The characteristic radius of the precipitates was determined to be $68 A$; however, the use of the precipitate scattering alone does not provide information on the distribution of sizcs.

1 Metals and Ceramics Division, ORNL. 


\section{THE DFFCT OF MCROGRAVIX DISTURBANCES ON THE GROWTH OF INDIUM CRYSTALS}

\section{J. D. Budai, R. M. Nictlow,}

\section{A. Thomes,' and B. I. Duaborl}

$X$-ray and neutron scattering measurements have been used to characterize the domain structure at various positions along a large (18con-longe 1-con-diam) indium cystal which was grown from a seed during the NASA Microgravity Disturbances Experiment (MDE) on the spence shuttle Columbia in January 1990 Although microgravity generally enhances size and perfaction during crystal growth, the quality of space-grown crystals may be affected by the numerous disturbances such as engine/thruster firings and crew treadmill exercise activity which occur during manned spacecraft operations. The primary purpose of the MDE is to investigate the effect of such disturbances on the microstructure of indium crystals which are grown from a polycrystalline rod by a float zone technique.

Figure 5.29 shows a composite of neutron scattering rocking curves measured at six different positions along an indium rod that was grown during a time period which contained treadmill runs. These measurements show that the crystal orientation of the sced has propagated along the rod during the float-zone process in spite of the macroscopic vibrations of the molten zone which were visible in videotapes. However, it is also clear that the seed itself was not a perfect crystal and that the mosaic spread diverges slowly as growth proceeds.

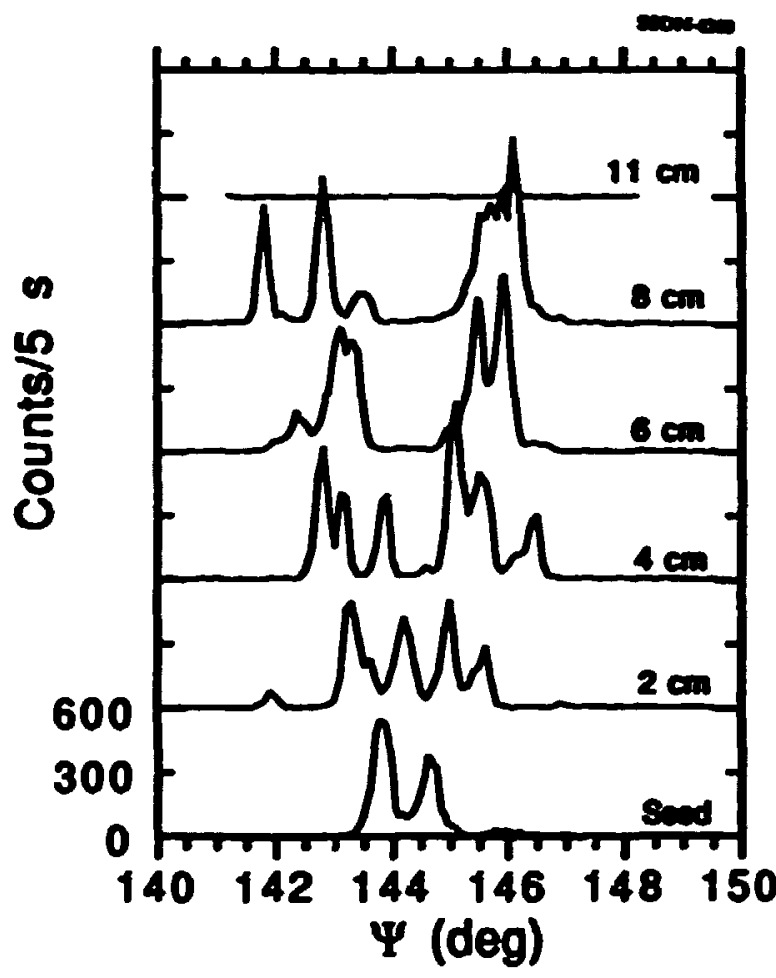

Fig 5.29. Neutron scattering rocking curves through the (112) reflection from a float-zone indium crystal at various distances from the seed crystal.

The decreased intensity at $11 \mathrm{~cm}$ indicates the original polycrystalline material associated with the furthest extent of the zone melting. No clear correlation of increased mosaic spread with disturbances on the space shuttle were obcerved. Examination of samples grown during other disturbance time periods is planned.

1. NASA Johnson Space Center, Houston, Tex. 


\section{A STRUCTURAL INVESTIGATION OF SEVIBAL MDCED \\ LANTHANIDE ORTHOPHOSPHATES'}

\section{F. Mullica, 2 E. L. Sappenfield,? and L. A. Bontuer}

Pure lanthanide orthophosphates exist in two different structural types. The first half of the lanthanide series $(\mathbf{L n}=L, \ldots, \mathrm{Gd})$ crystallizes in the monoclinic monazite-type structure while the higher atomic-number rare earths (RE $=T b, \ldots, L u, S c$, and $Y$ exist in the xenotine or zircon-type structure. In contrast to our earlier structural refinement work on pure orthophosphates, the present investigation is concerned with the structural properties of mixed orthophosphates with relatively high concentrations of different heavy-metal cations. In particular, the single-crystal structures of $\mathrm{Cd}_{0.5} \mathrm{Er}_{0.5} \mathrm{PO}_{4}, \mathrm{Gd}_{0.5} \mathrm{Y}_{0.5} \mathrm{PO}_{4}$, $\mathrm{Cd}_{0.5} \mathrm{Yb}_{0.5} \mathrm{PO}_{4}$ and $\mathrm{Cd}_{0.75} \mathrm{Yb}_{0.25} \mathrm{PO}_{4}$ have been determined by means of single-crystal $x$-ray diffraction techniques. These mixed phosphate compounds have the zircon-type structure and are, therefore, isostructural with the pure orthophosphates of the higher lanthanides (i.e., Tb, Dy, ..., Lu). The subject mixed orthophosphates crystallize in space group $14_{1} /$ and $\left(D^{19}\right.$ No. 141). Anisotropic refinements of the structures using a full-matrix least-squares program yielded final reliability factors in the range 0.040-0.053. The metal atoms in each compound are eight-coordinated to oxygen atoms, with two distinct $\mathrm{M}-\mathrm{O}$ bond distances. The important bond distance $P-O_{0 v}$. was found to be $1.533(6) \AA$. The coordination geometry about the central metal atom is bisphenoidal $\left(D_{2 d}\right)$ and is represented by two interpenetrating tetrahedra. In each mixed compound, the phosphate group is found to be a distorted tetrahedron.

1. Summary of paper: Inorg. Chim. Acte 174, 155 (1990).

2. Bayior University, Waco, Tex. 


\section{Publications and Papers}

\section{(April 1, 1989-September 30, 1990)}

\section{JOURNAL ARTICLES}

M. A. Abdel-Roouf and R. F. Wood, Possible Resonance in Positron-Lithium Scattering," Phys. Reo. A 42, 3117 (1990).

G. Aeppli, S. M. Hayden, H. A. Mook, Z. Fisk, S.-W. Cheong, D. Rytz, J. P. Remeika, G. P. Espinosa, and A. S. Cooper, Magnetic Dynamics of $\mathrm{La}_{2} \mathrm{CuO}_{4}$ and $\mathrm{La}_{2-x} \mathrm{Ba}_{x} \mathrm{CuO}_{4}{ }^{*}$ Phys. Reo. Lett. 62, 2052 (1989).

Carmen Almasan, T. Datta, R. D. Edge, E. R. Jones, J. W. Cable, and H. M. Ledbetter, LowTemperature Phase and Magnetic Interactions in FCC Fe-Cr-Ni Alloys," I. Magn. and Magn. Mater. 80, 329 (1999).

Kristoffer Almdal, Jeffrey H. Rosedale, Frank S. Bates, George D. Wignall, and Glenn H. Fredrickson, "Gaussian-to-Stretched-Coil Transition in Block Copolymet Melts," Phys. Reo. Lett. 55,1112 (1990).

C. Ballesteros, L. S. Cain, S. J. Pennycook, R. Conzalez, and Y. Chen, "Optical and Analytical Transmission Electron Microscopy Characterization of Thermochemically Reduced $\mathbf{M g A l}_{2} \mathrm{O}_{4}$ Spinel," Philos. Mag A. 59, 907 (1989).

John H. Barrett, "Methods of Channeling Simulation," Nud. Instrum. and Methods Phys. Res. Sect. B 4, 367 (1990!.

R. A. Bartynski, D. Heskett, K. Garrison, G. M. Watson, D. M. Zehner, W. N. Mei, S. Y. Tong, and X. Pan, "Photoelectron Diffraction Determination of the Geometry of a Clean Metal Surface: Ta(100)," Phys. Reo. B 40, 5340 (1989).

R. A. Bartynski, D. Heskett, K. Garrison, G. Watson, D. M. Zehner, W. N. Mei, S. Y. Tong, and $X$. Pan, "The First Interlayer Spacing of Ta(100) Determined by Photoelectron Diffraction," J. Vac. Sci. and Technol. A 7, 1931 (1989).

J. R. Beene, F. E. Bertrand, M. L. Halbert, R. L. Auble, D. C. Hensley, D. J. Horen, R. L. Robinson, R. O. Sayer, and T. P. Sioreen, "Heavy-Ion Excitation and Photon Decay of Giant Resonances in ${ }^{200} \mathrm{~Pb}$, , Phys. Reo. C 39, 1307 (1989).

R. Berliner, O. Fajen, H. G. Smith, and R. L. Hitterman, "Neutron Powder-Diffraction Studies of Lithium, Sodium, and Potassium Metal," Phys. Rev. B 40, 12086 (1989). 
R. S. Bhattacharya, A. K. Rai, and J. M. Williams, Titanium and Carbon Coimplantation into Sintered $Q$-SiC and Hot-Pressed $\mathrm{Si}_{3} \mathrm{~N}_{4}$ " I. Appl. Phys. 65, 1885 (1989).

N. Biunno, I. Narayan, A. R. Srivatsa, and O. W. Holland, "Laser Deposition of Epitaxial Titanium Nitride Films on (100) MgO," Appl. Phys. Lett. 55, 405 (1989).

L A. Boatner and S. A. David, Discovering Weld Microstructures," Microscope Technology and News 1, 4 (1989).

Lymn A. Boatner, Jose Luis Boldú O., and Marvin M. Abraham, "Characterization of Textured Ceramics by Electron Paramagnetic Resonance Spectroscopy: I. Concepts and Theory," I. Am. Cerrave. Soc. 73, 2333 (1990).

J. L Boldú O, L A. Boatner, and M. M. Abraham, Electron Paramagnetic Resonance Investigations of Divalent Cobalt in Single Crystals of Perovskite (CaTrO 3 ), J. Chem. Phys. 91, 5117 (1989).

Jose Luis Boldú O., Lynn A. Boatner, and Marvin M. Abraham, "Characterization of Textured Ceramics by Electron Paramagnetic Resonance Spectroscopy. II. Formation and Properties ol Textured MgO," J. Am. Cermu. Sac. 73, 2345 (1990).

Hugh R. Brown and George D. Wignall, "A SANS Study of the Dimensions of Polystyrene Formed by Freeze Drying from Dilute Solution," Mecromol. 23, 683 (1990).

Ch. Buchal, P. R Ashley, and D. K. Thomas, Ti-Implanted Optical Waveguides in LiNbO3," Mater. Sci. and Eng. A 109, 189 (1989).

R. A. Buchanan, In-Seop Lee, and J. M. Williams, Surface Modification of Biomaterials Through Noble Metal lon Implantation," J. Biomed. Mater. Res. 24, 309 (1990).

J. D. Budai, R. Feenstra, and L A. Boatner, "X-Ray Study of In-Plane Epitaxy of $\mathrm{VBa}_{2} \mathrm{Cu}_{3} \mathrm{O}_{x}$ Thin Films," Phys. Reo. B 39, 12355 (1989).

J. R. Buschert, J. Z. Tischler, D. M. Mills, Q. Zhao, and R. Colella, Time-Resolved X-Ray Diffraction Study of Laser Annealing in Silicon at Grazing Incidence," I. Appl. Phys. 66, 3523 (1989).

J. W. Cable and R. M. Nicklow, "Spin Dynamics of Cd at High Temperatures," Phys. Reo. B 39, 11732 (1989).

J. W. Cable and R. M. Nicklow, "Spin Dynamics of Gd in an Applied Magnetic Field," J. Phys., Condens. Matter 1, 7425 (1989).

B. C. Chakoumakos, P. S. Ebey, B. C. Sales, and Edward Sonder, "Characterization and Superconducting Properties of Phases in the Bi-Sr-Cu-O System," I. Mater. Res. 4, 747 (1989).

Szu-Li Chang Sow-Hsin Chen, Randolph R. Rill, and J. S. Lin, "Measurements of Monovalent and Divalent C "nterion Distributions Around Persistence Length DNA Fragments in Solution," J. Phys. Chem. 94, 8025 (1990).

Y. K. Chang, W. Uelhoff, A. Fattha, and C. Hanke, "Correlation of Crystal Perfection and Growth Parameters in Czochralski-Grown Cu3Au Single Crystals," I. Cryst. Growth 85, 396 (1987). 
T. Chattopadyay, P. J. Brown, B. C. Sales, L. A. Boatner, H. A. Mook, and H. Maletta, SingleCrystal Neutron Diffraction Investigation of the Magnetic Ordering of the High-Temperature Superconductor ErBayO)-8" Phys. Red. B 40, 2624 (1989).

C. Y. Chen, J. L. Park, L. S. Cain, G. J. Pogatshnik, M. R. Kokta, M. M. Abraham, and Y. Chen, Tonizing Radiation Effects in Lanthanum Magnesium Aluminate Crystals," Phys. Reo. B 40, 8522 (1989).

Y. Chen and M. M. Abraham, "Trapped-Hole Centers in Alkaline-Earth Oxides," I. Phys. Chem. Solids 51, 747 (1990).

Y. Chen, V. M. Orera, K. Conzalez, R. T. Williams, G. P. Williams, G. H. Rosenblatt, and G. J. Pogatshnik, Effect of Substitutional Hydride lons on the Charge States of Oxygen Vacancies in Thermochemically Reduced C2O and MgO," Phys. Reo. B 12, 1410 (1990).

Hersy Chou, S. M. Shapiro, S. C. Moss, and Mark Mostoller, Phonon Dispersion and Kohn Anomalies in CuouAlo.16" Phys. Reo. B 42, 500 (1990).

D. K. Christen, C. E Klabunde, J. R. Thompson, H. R. Kenchner, S. T. Set aly, R. Feenstra, and J. D. Budai, Transport Critical Currents in Epitaxial $\mathrm{Y}_{1} \mathrm{Ba}_{2} \mathrm{Cu}_{3} \mathrm{O}_{7-x}$ " Physica C 162-164, 653 (1989).

D. K. Christen, C. E. Klabunde, R. Feenstra, D. H. Lowndes, D. Norton, J. D. Budai, H. R. Kerchner, J. R. Thompson, L. A. Boatner, J. Narayan, and R. Singh, Large, Orientation-Dependent Enhancements of Critical Currents in $\mathrm{Y}_{1} \mathrm{Ba}_{2} \mathrm{Cu}_{3} \mathrm{O}_{7-x}$ Epitaxial Thin Films: Evidence for Intrinsic Flux Pinning?" Physica B 165/166, 1415 (1990).

L Givale, A. D. Marwick, M. W. McElfresh, T. K. Worthington, A. P. Malozemoff, F. H. Holtzberg J. R. Thompson, and M. A. Kirk, "Defect Independence of the Irreversibility Line in ProtonIrradiated Y-Ba-Cu-O Crystals," Phys. Reo. Lett. 65, 1164 (1990).

F. Claro and G. D. Mahan, "Transient Heat Transport in Solids," J. Appl. Phys. 66, 4213 (1989).

R. R. Coltman, Jr., C. E Klabunde, H. R. Kerchner, S. A. Richardson, and L. R Greenwood, The Low-Temperature Neutron Irradiation Facility at Oak Ridge National Laboratory," I. Nucl. Mater. 166, 379 (1989).

i. S. Darken and G. E. Jellison, Jr., Temperature Dependence of Acceptor-Hole Recombination in Cermanium," Appl. Phys. Lett. 55, 1424 (1989).

L.. S. Darken, G. E. Jellison, Jr., and Pat Sangsingkeow, "Hole Capture at Acceptors in p-Type Cermanium," J. Electron. Mater. 19, 105 (1990).

S. A. David, J. M. Vitek, M. Rappaz, and L. A. Boatner, Microstructure of Stainless Steel SingleCrystal Electron Beam Welds," Metall. Trans. A 21, 1753 (1990).

N. J. Dudney and J. B. Bates, "Ion Exchange Reaction of Silver and Sodium $\beta^{\prime \prime}$-Alumina in Molten Mercury Salts," Solid State lonics 34, 53 (1989).

N. J. Dudney, "Enhanced lonic Conduction in Silver Halide-Alumina Composites," I. Imaging Sci. 34, 104 (1990). 
M. K. El-Ghor, O. W. Holland, C. W. White, and S. J. Pennycook, "Structural Characterization of Damage in Si(100) Produced by MeV Sit-lon Implantation and Annealirg," I. Mater. RE. 5, 352 (1990).

M. K. El-Ghor, S. J. Pennycook, F. Namavar, and N. H. Karam, "Formation of Low Dislocation Density Silicon-on-Insulator by a Single Implantation and Annealing," Appl. Phys. Lett. 57, 156 (1990).

Djula Eres, Douglas H. Lowndes, and Jon Z. Tischler, Rapid Heteroepitaxial Growth of Ge Films on (100) GaAs by Pulsed Supersonic Free Jet Chemical Beam Epitaxy," Appl. Phys. Lett. 55. 1008 (1989).

Diula Eres, Douglas H. Lowndes, J. Z. Tischler, J. W. Sharp, D. B. Geohegan, and S. J. Pennycook, Heteroepitaxial Growth of Ge Films on (100) GaAs by Pyrolysis of Digermane," Appl. Phys. Lett. 55, 858 (1989).

Djula Eres, D. H. Lowndes, J. Z. Tischler, J. W. Sharp, T. E. Haynes, and M. F. Chisholm, The Effect of Deposition Rate on the Growth of Epitaxial Ce on CaAs(100)," J. Appl. Phys. 67, 1361 (1990).

N. Fanjat, G. Lucazeau, J. Bates, and A. J. Dianoux, "Dynamics of $\mathrm{Na}^{+}$in a Single Crystal of Nap" $\mathrm{Al}_{2} \mathrm{O}_{3}$," Physica B 156/157, 342 (1989).

G. C. Farlow and L. A. Boatner, Ton Irradiation Effects for MgF 2 Films on Lif Substrates," Nucl. Instrum. and Methods Phys. Res. Sect. B 16, 422 (1990).

G. C. Farlow, P. S. SkJad, C. W. White, and C. J. McHargue, Microstructural Development in the Near-Surface Region During Thermal Annealing of $\mathrm{Al}_{2} \mathrm{O}_{3}$ Implanted with Cationic Impurities," I. Mater. Res. 5, 1502 (1990).

R. Feenstra, J. D. Budai, M. D. Galloway, and L. A. Boatner, "Comparison Between $\mathrm{LaCaO}_{3}$, $\mathrm{LaAlO}_{3}, \mathrm{KTaO}_{3}$, and $\mathrm{SrTiO}_{3}$ Substrates for the Epitaxial Giowth of $\mathrm{YBa}_{2} \mathrm{Cu}_{3} \mathrm{O}_{7-x}$ Thin Films by a Baf 2 Process'," Physica C 162-164, 655 (1989).

J. A. Fernandez-Baca, 'Neutron Scattering Studies of Amorphous Invar Alloys," Physica B 161, 211 (1989).

J. A. Fernandez-Baca, J. J. Rhyne, G. E. Fish, M. Hennion, and B. Hennion, "Spin Dynamics of Amorphous Fego-x $\mathrm{Ni}_{x} \mathrm{Zr}_{10}$, I. Appl. Phys. 67, 5223 (1990;.

J. A. Fernandez-Baca, R. M. Nicklow, and J. J. Rhyne, Magnetic Excitations in Thulium Metal," J. Appl. Phys. 67, 5283 (1990).

R. S. Fishman, "Correlation of Phase Fluctuations in Granular Superconductors," Phys. Rev. B 40, 11014 (1989).

R. S. Fishman, "Expansion in 1/2 for the Transiuon Temperature of Granular Superconductors," F'hys. Reo. Lett. 63, 89 (1989).

R. S. Fishman and S. H. Liu, "Expansion in $1 / 2$ for the Transition Temperature and Specific Heat of Ferromagnets," Phys. Rev. B 40, 11028 (1989). 
R. S. Fishman and G. D. Mahan, "Binding of Charged Particles in Lattice Defects," Phys. Rev. B 40, 11493 (1989).

E. M. Forgan, D. McPaul, F. A. Mook, P. A. Timmins, H. Keller, S. Sutton, and J. S. Abell, "Observation by Neutron Small-Angle Diffraction of the Magnetic Flux Lattice in SingleCrystal $\mathrm{YBa}_{2} \mathrm{Cu}_{3} \mathrm{O}_{7-8}$." Nature 343, 735 (1990).

S. Q. Fu, W.-K. Lee, A. S. Nowick, L. A. Boatner, and M. M. Abraham, Study of Protons in Acceptor-Doped $\mathrm{KTaO}_{3}$ Crystals by IR and EPR Techniques," I. Solid State Chem. 83, 221 (1990).

S. Funahashi, Y. Morii, S. Katano, M. lizumi, H. R. Child, R. M. Nicklow, and R. M. Moon, "Single-Crystal Measurements with Wide-Angle Neutron Diffractometer," Physica B 156/157. 577 (1989).

B. D. Gaulin, "Kinetics of Spinodal Decomposition in One Dimension," Phys. Rev. B 38, 7184 (1988).

B. D. Gaulin, E. D. Hallman, and E. C. Svensson, "Phonons in $\mathrm{Cu}_{3} \mathrm{Au}$ near the Order-Disorder Transition," Phys. Rev. Lett. 64, 289 (1990).

D. J. W. Geldart and Mark Rasolt, "Effective Interactions, Structure Factors, and Nonlocal Correlations in Many-Electron Systems," Int. J. Quantum Chem. Quantum Chem. Symp. 23, 631 \{1989).

D. B. Geohegan and D. N. Mashburn, "Characterization of Ground Siate Neutral and lon Transport During Laser Atlation of $\mathrm{Y}_{1} \mathrm{Ba}_{2} \mathrm{Cu}_{3} \mathrm{O}_{7-x}$ Using Transient Optical Absorption Spectroscopy," Appl. Phys. Lett. 55, 2345 (1989).

Doon Gibbs, B. M. Ocko, D. M. Zehner, and S. G. J. Mochrie, "Structure and Phases of the Au(001) Surface i: In-Plane Structure," Phys. Reo. B 12, 7330 (1990).

S. M. Girvin, L. I. Glazman, M. Jonson, D. R. Penn, and M. D. Stiles, "Quantum Fluctuations and the Single-Junction Coulomb Blockade," Phys. Reo. Lett. 64, 3183 (1990).

Mukund j. Codbole, Anthony I. Pedraza, Douglas H. Lowndes, and Edward A. Kenik, "LaserInduced Interface Reactions of Copper Thin Films on Sapphire Substrates," I. Mater. Res. 4, 1202 (1989).

R. Conzalez, R. Hantenzadeh, C. Y. Chen, L. F. Halliburton, and Y. Chen, "Deuterium Diffusion and Magnetic Resonance Investigations in $\mathrm{LiTaO}_{3}$ Single Crystals," Phys. Rev. B 39, 1302 (1989).

R. Gonzalez, C. Ballesteros, Y. Chen, and M. M. Abraham, "Diffusion of Tritons, Deuterons, and Protons in LiNbO 3 Crystals," Phys. Reo. B 39, 11085 (1989).

S. M. Corbatkin, L. A. Berry, and J. B. Roberto, "Behavior of Ar Plasmas Formed in a Mirror Field ECR Ion Source," J. Vac. Sci. and Technol. A 8, 2893 , 1990).

P. Grenier, G. Bemier, S. Jandl, B. Salce, and L. A. Boatner, "Fluorescence and Ferroclect:ic Mictoregions in $\mathrm{KTaO}_{3}$," J. Phys., Condens. Matter 1, 2515 (1989). 
G. R. Gruzalski, D. M. Zehner, J. R. Noonan, H. L. Davis, R. A. DiDio, and K. Müller, “(1×1) Rippled Relaxation of (100) Transition-Metal Carbide Surfaces," I. Vac. Sci. and Technol. A 7. 2054 (1989).

G. R. Gruzalski ar 1 D. M. Zehner, "Charge-Distribution Changes Accompanying the Formation and Changes in the Composition of $\mathrm{HFC}_{\mathrm{z}}$ and $\mathrm{TaC}_{\mathrm{z}}$ " Phys. Reo. B 12, 2768 (1990).

H. Haghighi, J. H. Kaiser, S. Rayner, R. N. West, M. J. Fluss, R. H. Howell, P. E. A. Turchi, A. L. Wachs, Y.C. Jean, and Z. Z. Wang "A Positron Study of the Electronic Structure of $\mathrm{YBa}_{2} \mathrm{Cu}_{3} \mathrm{O}_{7-8}$ " I. Phys., Condens. Matter 2, 1911 (1990).

C. C. Havener, K. J. Reed, K. J. Snowdon, D. M. Zehner, and F. W. Meyer, "Interaction of kiultiply Charged lons with Solid Surfaces," Radiat. Eff. Defects Solids 109, 99 (1909).

C. C. Havener, K. J. Reed, K. J. Snowdon, N. Stohiterfoht, D. M. Zehner, and F. W. Meyer, "Evidence for Production of Inner-Strell Vacancies in Slow Multicharged F lons Interacting with a Cu Surface," Surf. Sci. 216, 1357 (1969).

T. E. Haynes, R. A. Zuhr, S. J. Pennycook, B. C. Larson, and B. R. Appleton, Meteroepilaxy of 76e Films on GaAs by Direct Deposition from a Low-Energy lon Beam," J. Vac. Sci. end Technol. A 7, 1372 (1989).

T. E. Hayres, R. A. Zuhr, S. J. Pennycook, and B. R. Appleton, "Heteroepitaxy of GaAs on Si and Ce Using Alternating, Low-Enengy lon Beams," Appl. Phys. Lett. SA, 1439 (1989).

J. B. Hayter, "Interparticle Interactions and Polarization Effects in Colloids," I. Appl. Crystallogr. 21, 737 (1988).

John B. Hayter, Roger Pynn, Stuart Charles, Ame T. Skjeltorp, Jill Trewhella, Gerald Stubbs, ard Peter Timmons, "Ordered Macromolecular Structures in Ferrofluid Mixtures," Phys. Reo. Lett. 62, 1667 (1989).

J. B. Hayter, "Applications of the Advanced Neutron Source," Trans. Am. Nucl. Soc. 61, 379 (1990).

O. W. Holland and J. Narayan, "Model for Boron Diffusion in Si at High Concentrations," Nucl. Instrum. and Methods Phys. Res. Sect. B 40141, 537 (1989).

O. W. Holland, 5. J. Pennycook, and G. L. Albert, "New Model for Damage Accumulation in Si During Self-lon Imadiation," Appl. Phys. Lett. 55, 2503 (1989).

O. W. Holland, J. D. Budai, and C. W. White, "Uniaxial Lattice Expansion of Self-Ion-Implanted Si," Appl. Phys. Lett. 57, 243 (199)).

O. W. Holland, C. W. White, M. K. El-Ghor, and J. D. Budai, "MeV, Self-Ion Implantation in Si at Liquid Nitrogen Temperature: A Study of Damage Morphology and lis Anomalous Annealing Behavior," I. Appl. Phys. 68, 2081 (1990).

R. H. Howell, H. B. Radousky, A. L. Wachs, M. J. Fluss, P. E. A. Turchi, Y. C. Jeari, C. S. Sundar, C. W. Chu, J. L. Teng, T. J. Folkerts, R. N. Shelton, and D. G. Hinks, "Systematics in Positron Annihilation Lifetime Analysis of High-T, Superconducting Transitions," Physica C 162-164, 1377 (1989). 
W. F. Huang, Z. J. Xu, S. H. Liu, and M. K. Wu, "Evidence for Charge-Density Wave or Spin-

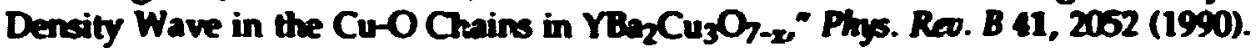

Alan J. Hund, Dale W. Schaefer, Douglas M. Smith, Steven B. Ross, Alain Le Mehaute, and Stephen Spoone, "Study of Surface Areas of Fractally Rough Particles Studied by Scattering." Phys. Reo. B 39, 9742 (1909).

A. P. Jauhw and M. Jonson, Tunneting Through a Time-Modulated Barrier-Relation to Tunneling Times," I. Phys., Condens. Mattor 1, 9020 (1909).

Y. C. Jean, H. Nakanichi, M. J. Fuss, A. L. Wachs, P. E. A. Turchi, R. H. Howell, Z. Z. Wang, R. L Meng, P. H. Hor, Z J. Huang, and C. W. Chu, "A Comparison of the Temperature Dependence of Electron-Positron Momentum Density Characteristics in $\mathrm{T}_{22} \mathrm{Ca}_{2} \mathrm{Ba}_{2} \mathrm{Cu}_{3} \mathrm{O}_{103+8} \mathrm{YBa}_{2} \mathrm{Cu}_{3} \mathrm{O}_{7}$, and $\mathrm{L}_{1}$ esta $_{15} \mathrm{CuO}_{4}$ " I. Phys., Condens. Alatter 1, 2909 (1989).

Y. C. Jean, C. S. Sundar, A. Eharathi, J. Kyle, H. Nakanishi, P. K. Tseng, P. H. Hor, R. L. Meng, Z. J. Huane C. W. Chu, Z.Z Wane P. E. A. Turchi, R. H. Howell, A. L Wachs, and M. J. Fluse, Zocal Charge-Density Change and Superconductivity: A Position Study," Phys. Rev. Lett. 64, 1593 (1990).

G. E. Jellisom, Jr., and F. A. Modine, "A Simple Implementation of a Power Supply for Constant Pholotube Current in Light Modulation Spectroscopy," Reo. Sci. Instrum. C0, 3345 (1989).

G. E. Jellison, Ir., and F. A. Modine, Two-Channel Polarization Modulation Ellipsometer," Appl. Opt. 29, 959 (1990).

R. G. Jordan, D. M. Zehner, N. M. Harrison, P. J. Durham, and W. M. Temmerman, "Án XPS Investigation of the Electronic Structure in AgZn, Z. Phys. B 75, 291 (1989).

M. H. Kang S.C. Lui, E. J. Mele, E. W. Plummer, and D. M. Zehner, "Alomic and Electronic Structure of the NiAl (111) Surface," Phys. Reo. B 41, 4920 (1990).

Eugene J. Kelly, C. E. Vallet, and C. W. White, "Application of Ion Implantation/RBS to the Study of Electrocatalysis-Comparison of Chlorine Evolution at Ir-Implanted and Ru-Implanted Titanium Electrodes," I. Electrochem. Soc. 137, 2482 (1990).

H. R. Kerchner, R. Feenstra, J. O. Thomson, J. R. Thompson, Y. C. Kim, D. K. Christen, and S. T. Sekulo, "Magnetic Hysteresis and AC Response of $\mathrm{YBa}_{2} \mathrm{Cu}_{3} \mathrm{O}_{2}$ Films," Physica C 162-164, 1557 (1989).

J. Kwo, M. Hong, D. B. McWhan, Y. Yafet, R. M. Fleming, F. J. Disalvo, J. V. Waszczak, C. F. Majkrzak, D. Gibbs, A. I. Coldı.un, P. Boni, J. Bolu, H. Grimm, C. L. Chien, and J. W. Cable, "Magnetic Superlattices," I. Phys. (France) 49, C8-1651 (1988).

Thein Kyu, Kenichi Fujita, Myung H. Cho, Takeshi Kikutani, and J. S. Lin, "Melting and Crystallization of Gelation-Crystallized Ultrahigh Molecular Weight Polyethylene," Macromol. 22, 2238 (1989).

Janos J. Ladik, Peter Saalfrank, and Richard F. Wood, "Theoretical Considerations of High-T Superconductivity," Int. I. Quantum Chem. Quantum Chem. Symp. 23, 621 (1989). 
P. Lamparter, S. Steeb, D. M. Kroeger, and S. Spooner, Neutron and X-Ray Small-Angle Scattering with Fe-Based Metallic Glasses," Mater. Sci. and Eng. 97, 227 (1988).

Youngehul Lee, Roger S. Porter, and J. S. Lin, "On the Double-Melting Behavior of Poly(Ether Ether Ketone)," Mecromol. 22, 1756 (1989).

S. H. Liu, "Spin-Fuctuation Resonance in Mixed-Valence Materials," Phys. Reo. B 39, 73 ?' (1989).

S. H. Liu, "Critical Temperature of Pseudo One- and Two-Dimensional Magnetic Systerns," I. Magn. and Magn. Mater. 82, 294 (1989).

S. H. Liu, Reply to 'Comment on Magnetic Interaction Between Rare-Earth Moments in HighTemperature Superconductors RBa2 $\mathrm{Cu}_{3} \mathrm{O}_{7-2}$;" Phys. Reo. B 40, 7316 (1989).

S. H. Liu, T. Kaplan, and L. J. Gray, "Geometry and Dynamics of Deterministic Sand Piles," Phys. Reo. A 22, 3207 (1990).

Douglas H. Lowndes, David P. Norton, and J. D. Budai, Superconductivity in Nonsymmetric Epitaxial $\mathrm{YBa}_{2} \mathrm{Cu}_{3} \mathrm{O}_{7-x} / \mathrm{PrBa}_{2} \mathrm{Cu}_{3} \mathrm{O}_{7-x}$ Superlattices: The Superconducting Behavior of $\mathrm{Cu}-\mathrm{O}$ Bilayers," Phys. Reo. Lett. 65, 1160 (1990).

G. Q. Lu, E. Nygren, M. J. Aziz, D. Tumbull, and C. W. White, "Interferometric Measurement of the Pressure-Enhanced Crystallization Rate of Amurphous Si," Appl. Phys. Lett. 54, 2583 (1989).

G. Q. Lu, E. Nygren, M. J. Aziz, D. Tumbull, and C. W. White, "Pressure-Enhanced Solid Phase Epitaxy of Germanium," Appl. Phys. Lett. 56, 137 (1990).

D. Lubben, T. Motooka, J. E. Greene, and J. F. Wendelken, High-Resolution Electron Energy Loss Spectroscopy Study of the UV-Laser Photodissociation of Adsorbed $\mathrm{Al}_{2}\left(\mathrm{Ch}_{3}\right)_{6}$ on $\mathrm{Si}(100)_{2 \times 1}$ and Si(111)7×1 Surfaces," Phys. Reo. B 39, 5245 (1989).

S.C. Lui, M. H. Kang, E. J. Mele, E. W. Plummer, and D. M. Zehner, "Surface States on NiAl(110)," Phys. Reo. B 39, 13149 (1989).

S.-C. Lui, J. W. Davenport, E. W. Plummer, D. M. Zehner, and G. W. Ferando, "Electronic Structure of NiAl," Phys. Reo. B 42, 1582 (1990).

J. W. Lynn, W.-H. Li, H. A. Mook, B. C. Sales, and Z. Fisk, "Antiferromagnetic Order of the Cu in $\mathrm{RBa}_{2} \mathrm{Cu}_{3} \mathrm{O}_{6+x}$ "J. Phys. Colloq. (France) C8, 2153 (1988).

R. H. Magruder III, S. H. Morgan, R. A. Weeks, and R A. Zuhr, "Effects of Ion Implantation on Intermediate Range Order: IR Spectra of Silica," J. Non-Cryst. Solids 120, 241 (1990).

R. H. Magruder III, J. M. Jackson, D. L. Kinser, R. A. Weeks, and E. Sonder, "Thermal Analysis of the Radiation-Induced Conductivity in $\mathrm{GeO}_{2}$ Glasses," Radiat. Eff. Defects Solids 112, 69 (1990).

G. D. Mahan and B. E. Sernelius, "Electron-Electron Interactions and the Bandwidth of Metals," Phy=. Rev. Lett. 62, 2718 (1989).

G. U. Mahan and Zhongcheng Wang, "Kushino-Taylor Coefficient in Electrical Resistivity," Phys. $k_{2}=B 39,4926$ (1989). 
G. D. Mahan and Francisco H. Claro, Nonlocal Thermal Conductivity," High Temp.-High Pressure 21, 1 (1989).

Cerald D. Mahan, "Static Coulomb Model for High-Temperature Superconductivity," Phys. Reo. B 10,2200 (1989).

Gerald D. Mahan, "Anisotropic Energy Gaps in Copper Oxide Superconductors," Phys. Reo. B 40, 11317 (1989).

G. D. Mahan and Mark Mostoller, "Lattice Dynamics with Indirect lonic Interactions," Phys. Rep. B 41, 10824 (1990).

G. D. Mahan, Lattice Dynamics of lonic Crystals," Phys. Reo. B 11, 10808 (1990).

G. D. Mahan, Dielectric Function of Layered Materials," Phys. Reo. B 42, 4351 (1990).

T. E. Mason, B. D. Gaulin, and M. F. Collins, Neutron Scattering Measurements of Critical Exponents in CsMnBry, A $Z_{2} \times S_{1}$ Antiferromagnet," Phys. Reo. B 39, 586 (1989).

J. C. McCallum, C. W. White, and L A. Boatner, The Annealing Behavior of lon-Implanted Single Crystals of the Type $\mathrm{YBa}_{2} \mathrm{Cu}_{3} \mathrm{O}_{x}, "$ Nucl. Instrum. and Methods Phys. Res. Sect. B 40/41, 608 (1989).

J. C. McCallum, J. Rankin, C. W. White, and L. A. Boatner, Time-Resolved Reflectivity Measurements in Pb-Implanted SrTiOz," Nucl. Instrum. and Methods Phys. Res. Sect. B 46, 98 (1990).

J. C. McCallum, C. W. White, P. S. SkJad, and C. J. McHargue, "Annealing Environment Effects in Solid-Phase-Epitaxial Regrowth of Fe-Implanted $\mathrm{Al}_{2} \mathrm{O}_{3}, "$ Nucl. Instrum. and Methods Phys. Ras. Sect. B 46, 137 (1990).

C. J. Mchargue, P. S. Sklad, J. C. McCallum, C. W. White, A. Perez, and G. Marest, The Structure of $\mathrm{Al}_{2} \mathrm{O}_{3}$ Implanted with Iron at $77 \mathrm{~K}, "$ Nucl. Instrum. and Methods Phys. Res. Sect. B 46, 144 (1990).

C. I. Mchargue, P. S. Sklad, J. C. McCallum, C. W. White, A. Perez, E. Abonneau, and G. Marest, The Residual Charge State of Tin Implanted into Sapphire," Nucl. Instrum. and Methods Phys. Res. Sect. B 46, 74 (1990).

C. J. McHargue, P. S. SkJad, and C. W. White, "The Structure of lon-Implanted Ceramics," Nucl. Instrum. and Methods Phys. Res. Sect. B 46, 79 (1990).

C. J. McHargue, D. L. Joslin, and J. M. Williams, "The Hardness and Elastic Modulus of ChromiumImplanted Silicon Carbide," Nucl. Instrum. and Methods Phys. Res. Sect. B 46, 185 (1990).

C. J. Mchargue, M. E. OHern, C. W. White, and M. B. Lewis, "Ion Implantation in CeramicsResidual Stress and Properties," Mater. Sci. and Eng. A 115, 361 (1989).

D. F. McMorrow, R. A. Cowley, R. M. Nicklow, P. W. Mitchell, A. D. Taylor, and M. Mostoller, "A Neutron Scattering Study of the Impulse Approximation in Single-Crystal Beryllium," J. Phys., Condens. Matter 2, 1045 (1990). 
S. G. J. Mochrie, D. M. Zehner, B. M. Ocko, and Doon Gibbs, "Structure and Phases of the Au(001) Surface: X-Ray Scattering Measurements," Phys. Reo. Lett. 64, 2925 (1990).

F. A. Modine, R. B. Wheeker, Youngiee Shim, and James F. Cordaro, "Origin of the Pulse Response Characteristics of Zinc Oxide Varistors," J. Appl. Phys. 66, 5608 (1989).

F. A. Modine, M. D. Foegelle, C. B. Finch, and C. Y. Allison, Electrical Properties of TransitionMetal Carbides of Group I :- Phys. Reo. B 40, 9558 (1989).

F. A. Modine and R. W. Wheeler, "Pulse Response Characteristics of ZnO Varistors," I. Appl. Phys. 67,6560 (1990).

F. A. Modine, R. W. Major, S.-I. Choi, L. B. Bergman, and M: N. Silver, Polarization Currents in Varistors," I. Appl. Phys. 68, 339 (1990).

H. A. Mook, J. A. Harvey, and N. W. Hill, Measurement of $\mathrm{T}_{\text {eff for }} \mathrm{Cu}$ in $\mathrm{YBa}_{2} \mathrm{Cu}_{3} \mathrm{O}_{7}$ by Neutron Resonance Absorption," Phys. Reo. B 41, 764 (1990).

R. M. Mloon, "Status Report: Neutrons at Oak Ridge," Neutron News 1, 22 (1990).

F. G. Moore, H. B. Dietrich, E. A. Dobisz, and O. W. Holland, Silicon Implantation into GaAs: Observations of Dose Rate Dependent Electrical Activation and Damage," Appl. Phys. Lett. 57, 911 (1990).

Mark Mostoller, R. M. Nicklow, D. M. Zehner, S.-C. Lui, J. M. Mundenar, and E. W. Plummer, "Bulk and Surface Vibrational Modes in NiAl," Phys. Reo. B 40, 2856 (1989).

Mark Mostoller, Jiquang Zhang, A. M. Rao, and P. C. Eklund, "Lattice Vibrations in La $\mathrm{CuO}_{4}$ " Phys. Rev. B 41, 6488 (1990).

Michael F. Müller, John D. Ferry, and J. S. Lin, "Small-Angle X-Ray Scattering Studies of Fibrin Film: Comparisons of Fine and Coarse Films Prepared with Thrombin and Ancrod," Biopulymers 28, 1011 (1989).

D. F. Mullica, E. L. Sappenfield, G. A. Wilson, and L. A. Boatner, "The Crystal Structure of $\mathrm{Ce}_{0.9} \mathrm{U}_{0.1} \mathrm{PO}_{4}$," Lanthanide and Actinide Res. 3, 51 (1989).

D. F. Mullica, E. L. Sappenfield, and L. A. Boatner, "A Structural Investigation of Several Mixed Lanthanide Orthophosphates," Inorg. Chim. Acta 174, 155 (1990).

D. M. Newns, P. Pattnaik, M. Rasolt, and D. A. Papaconstantopolos, "Realistic Anderson Lattice Model of the 214 Material Solved in Mean Field," Physica C 153-155, 1287 (1988).

J. R. Noonan and H. L. Davis, "Confirmation of an Exception to the 'Ceneral Rule' of Surface Relaxations," I. Vac. Sci. and Technol. A 8, 2671 (1990).

David P. Norton, Douglas H. Lowndes, J. D. Budai, D. K. Christen, E. C. Jones, J. W. McCamy, Thomas D. Ketcham, Dell St. Julien, K. W. Lay, and J. E. Tkaczyk, "Y-Ba-Cu-O Thin Films Grown on Rigid and Flexible Polycrystalline Yttria-Stabilized Zirconia by Pulsed-Laser Ablation," I. Appl. Phys. 68, 223 (1990). 
David ' Norton, Douglas H. Lowndes, J. D. Budai, D. K. Christen, E. C. Jones, K. W. Lay, and J. E. Thaczyk, "High Critical Current Densities in $\mathrm{YBa}_{2} \mathrm{Cu}_{3} \mathrm{O}_{7-x}$ Films on Polycrystalline Zirconia," Appl. Phys. Lett. 57, 1164 (1990).

M. E. OHern, C. J. McHargue, C. W. White, and G. C. Farlow, The Effect of Chromium Implantation on the Hardness, Elastic Modulus, and Residual Stress of $\mathrm{Al}_{2} \mathrm{O}_{3}$, Nucl. Instrum. and Methods Phys. Res. Sect. B 46, 171 (1990).

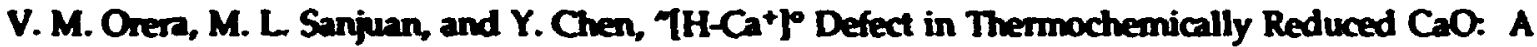
Static and Dynamic EPR Study," Phys. Reo. B 42, 7604 (1990).

S. H. Overbury, D. R. Mullins, and I. F. Wendelken, "Surface Structure of Stepped NiAl(111) by Low-Energy Li+ Ion Scattering" Surf. Sai. 236, 122 (1990).

R. Pareja, R. M. de la Cruz, M. A. Pedrosa, R. Gonzalez, and Y. Chen, Positronium Hydride in Hydrogen-Laden Thermochemically Reduced MgO Single Crystals," Phys. Reo. B 41, 6220 (1990).

S. J. Pennycook, "Z-Contrast STEM for Materials Science," Ulthmicroscopy 30, 58 (1989).

S. J. Pennycook, Tigh-Resolution Imaging with Large-Angle Elastically Scattered Electrons," EMSA Bull. 19:1, 67 (1989).

S. J. Pennycook, Reply to 'Comment on High-Resolution Imaging with Large-Angle Elastically Scattered Electrons'" EMSA Bull. 19:2, 15 (1989).

S. J. Pennyccok and D. E. Jesson, "High-Resolution Incoherent Imaging of Crystals," Phys. Reo. Lett. 64, 938 (1990).

5. J. Pennycook, "Toward a One-Angstrom Electron Microscope," ORNL Review 23, 54 (1990).

Sune Pettersson, "Theoretical Considerations on the Hot-Wire Method Applied to Solids Under Pressure," Reo. Sa. Instrum. 61, 1308 (1990).

Sune Pettersson and K. R. Subbaswamy, "Volume Dependence of Ion Polarizabilities in AlkaliHalide Crystals," Phys. Reo. B 42, 5883 (1990).

Sune Pettersson and C. D. Mahan, "Theory of the Thermal Boundary Resistance Between Dissimilar Lattices," Phys. Rev. B 12, 7386 (1990).

S. Raman, S. Kahane, R. M. Moon, J. A. Fernandez-Baca, J. L. Zarestky, j. E. Lynn, and J. W. Richandson, Jr., "Thermal-Neutron Scattering Lengths and Capture by Even Calcium Isotopes," Pliys. Rev. C 39, 1297 (1989).

M. Rappaz, S. A. David, J. M. Vitek, and L. A. Boatner, "Analysis of Solidification Microstructures in Fe-Ni-Cr Single-Crystal Welds," Metall. Trans. A 21, 1767 (1990).

Mark Rasolt and F. Perrot, "Collective Enhancement of Three-Body Recombinations in Highly lonized Classical Plasmas," Phys. Reo. Lett. 62, 2273 (1989).

M. Rasolt and F. Perrot, "Erratum on 'Collective Enhancement of Three-Body Recombinations in Highly lonized Classical Masnus'," F nys. Rev. Lett. 63, 2317 (1989). 
Mark Rasolt and D. J. W. Geldart, "Comment on 'Quantum-Mechanical Interpretation of the Exchange-Correlation Potential of Kohn-Sham Density-Functional Theory":- Phys. Reo. Lett. 65,276 (1990).

Mark Rasolt and Giovanni Vignale, "Self-Induced Effective Gauge Fields in the Copper-Oxygen Plane of High- $T_{c}$ Perovskites: A Lattice ard a Continuum Formulation," Phys. Reo. Lett. 65, 1498 (1990).

N. M. Ravindra, T. Fink, W. Savin, T. P. Sjoreen, R. L. Pfeffer, L. G. Yerke, R. T. Lareau, J. G. Gualtieri, R. Lux, and C. Wren, "Oxygen-lon-Implanted Cermanium-Structural Properties," Nucl. Instrum. and Methods Phys. Res. Sect. B 46, 409 (1990).

R. T. Richard, R. L. Chaplin, R. R. Coltman, Jr., H. R. Kerchner, and C. E. Klabunde, "Stored Energy Recovery of Irradiated Copper," Rediat. Eff. Defects Solids 112, 161 (1990).

C. T. Rieck, K. Scharnberg, and R. A. Kemm, "Reentrant Superconductivity Due to Landau Level Quantization?" Physica C 170, 195 (1990).

J. B. Roberto, "Ion Implantation Technology in the National Laboratories: Four Decades of Technology Transfer," Nucl. Instrum. and Methods Phys. Res. Sect. B 40/41, 510 (1989).

Mark T. Robinson, "Slowing-Down Time of Energetic Atoms in Solids," Phys. Reo. B 40, 10717 (1989).

Mark T. Robinson, The Temporal Development of Collision Cascades in the Binary Collision Approximation," Nucl. Instrum. and Methods Phys. Res. Sect. B 48, 408 (1990).

G. H. Rosenblatt, M. W. Rowe, G. P. Williams, Jr., R. T. Williams, and Y. Chen, "Luminescence of F and $F^{*}$ Centers in Magnesium Oxide," Phys. Rev. B 39, 10309 (1989).

T. M. Rosseel, C. R. Vane, J. P. Young, R. A. Zuhr, and R. S. Peterson, "High-Resolution HIXSE Studies of the Chemical Environment of Sulphur Implanted in Quartz Glass," Nucl. Instrum. and Methods Phys. Res. Sect. B 43, 14 (1989).

P. Saalfrank, M. A. Abdel-Raouf, J. Ladik, and R. F. Wood, "Electronic Structure of Undoped and Doped $\mathrm{La}_{2} \mathrm{CuO}_{4}$ : A Hartree-Fock Cluster Study," Phys. Reo. B 41, 8824 (1990).

B. C. Sales, "Structural Relaxation Dynamics of Phosphate Classes: The Effects of Network Topology on the Glass Transition," I. Non-Cryst. Solids 119, 136 (1990).

Dale W. Schaefer, L. Jian, C.-C. Sun, D. McCarthy, C.-Y. Jiang, Y.-P. Ning, J. E. Mark, and Steve Spooner, The Structure of Silica-Polysiloxane Composites and Its Effects on Their Elastomeric Properties," Polym. Preprints 30, 102 (1989).

P. W. Schmidt, A. Hohr, H. Neumann, H. Kaiser, D. Avnir, and J. E. Lin, "Small-Angle X-Ray Scattering Study of the Fractal Morphology of Porus Silicas," J. Chem. Phys. 90, 5016 (1989).

B. E. Sernelius, "Free-Carrier Absorption from Fibonacci Sequences of Delta-Doped Layers in Silicon," Phys. Rev. B 40, 6218 (1989).

R. K. Singh, O. W. Holland, and J. Narayan, "Theoretical Model for Deposition of Superconducting Thin Films Using Pulsed-Laser Evaporation Technique," J. Appl. Phys. 68, 233 (1990). 
P. S. Stzlad, J. C McCallum, C. J. McHargue, and C. W. White, The Amorphous-to-Gamma Transformation in Ion-Implanted $\mathrm{Al}_{2} \mathrm{O}_{3}$," Nuch. Instrum. and Methods Phys. Res. Sect. B 46, 102 (1990).

H. G. Smith, R. Bertiner, J. D. Jorgensen, M. Nielsen, and J. Trivisonno, Pressure Effects on the Martensitic Transformation in Metallic Lithium," Phys. Reo. B 41, 1231 (1990).

J. P. Sokoloff, L L C Chase, and L A. Boatner, Low-Frequency Relaxation Modes and Structural Disorder in KTN," Phys. Ra. B 41, 2398 (1990).

E. Sonder, L. A. Boatner, B. C. Chakoumakos, and B. C. Sales, The Effect of Gamma Irradiation on the Superconducting Properties of $\mathrm{CdB}_{2} \mathrm{Cu}_{3} \mathrm{O}_{7-2}$ " Mater. Lett. 8, 171 (1989).

Edward Sonder, B. C. Chakoumakos, and B. C. Sales, "Effects of Oxygen and Strontium Vacancies on the Saperconductivity of Single Crystals of $\mathrm{Bi}_{2} \mathrm{Sr}_{2-x} \mathrm{CuO}_{6 y}$ " Phys. Reo. B 40, 6872 (1989).

A. R. Srivatsa, S. Sharan, O. W. Holland, and J. Narayan, Nature of Interfaces and Oxidation Processes in Get-Implanted Si," I. Appl. Phys. 65, 4028 (1989).

M. A. Subramanian, R. D. Shannon, B. H. T. Chai, M. M. Abraham, and M. C. Wintersgill, Dielectrir Constants of BeO, MgO, and CaO Using the Two-Terminal Method," Phys. Chem. Minerals 16, 7\$1 (1989).

Zatko Tesanoric, Mark Rasolt, and Lei Xing "Quantum Limit of a Flux Lattice: Superconductivity and Magnetic Field in a New Relationship," Phys. Reo. Lett. 63, 2425 (1989).

J. R. Thompson, J. Brynestad, D. M. Kroeger, Y. C. Kim, S. T. Sekula, D. K. Christen, and E. D. Specht, "Superconductivity, Intergrain and Intragrain Critical Current Densities of $\mathrm{T}_{2} \mathrm{Ca}_{2} \mathrm{Ba}_{2} \mathrm{Cu}_{3} \mathrm{O}_{10+8}$ and $\mathrm{T}_{2} \mathrm{CaBa}_{2} \mathrm{Cu}_{2} \mathrm{O}_{3+8}$ Materials," Phys. Reo. B 39, 6652 (1989).

J. R. Thompson, D. K. Christen, H. A. Deeds, Y. C. Kim, J. Brynestad, S. T. Sekula, and I. Budai, Weak Coupling and Anisotropy in the Magnetic Penetration Depth of the High-Temperature Superconductor $\mathrm{T}_{2} \mathrm{Ca}_{2} \mathrm{Ba}_{2} \mathrm{Cu}_{3} \mathrm{O}_{10+2}$ "Phys. Rev. B 41, 7293 (1990).

J. R. Thompson, D. K. Christen, Yang Ren Sun, B. C. Chakoumakos, B. C. Sales, H. R. Kerchner, J. C. Ossandon, and E. Sonder, 'Evidence of Weak Pair Coupling in the Penetration Depth of BiBased High-T, Superconductors," Physica B 165/166, 1453 (1990).

R. Triolo, E. Caponetti, F. Boschetti, and S. Spooner, "Small-Angle Scattering from a Commercial Al-Li Alloy," Philos. Mag. 60, 401 (1989).

C. C. Tsai, L. A. Berry, S. M. Corbatkin, H. H. Haselton, J. B. Roberto, and W. L. Stirling, Putential Applications of an Electron Cyclotron Resonance Multicusp Plasma Source," I. Vac. Sci. and Technol. A 8, 2900 (1990).

P. E. A. Turchi, A. L. Wachs, K. H. Wetzler, J. H. Kaiser, R. N. West, Y. C. Jean, R. H. Howell, and M. J. Fluss, "Electron Momentum Density Studies in High-T, Materials by Positron Annihilation Spectroscopy: Theory and Experiment," I. Phys., Condens. Matter 2, 1635 (1990).

C. E. Vallet, D. E. Heatherly, and C. W. White, "In Situ Photoacoustic Spectroscopy of MixedOxide Electrocatalysts," I. Electrochem. Soc. 137, 579 (1990). 
L J. V. van IIzendoorn, G. F. A. van de Walle, A. A. van Gorkum, A. M. L. Theunissen, R. A. van den Heuvel, and J.H. Barrett, "Diffusion and Strain Relaxation in $\mathrm{Si} / \mathrm{Si}_{1-x} \mathrm{Ge}_{\mathbf{x}} / \mathrm{Si}$ Structures Studied with Rutherford Backscattering Spectrometry," Nuct. Instrum. and Methods Phys. Res. Sect. B 50, 127 (1990).

A. L. Wachs, P. E. A. Turchi, R. H. Howell, Y. C. Jean, M. J. Fluss, R. N. West, J. H. Kaiser, S. Rayner, H. Haghighi, K. L. Merkle, A. Revcolevshci, and Z. Z. Wang "Electron-Positron Momentum Distribution Measurements of High- $T_{c}$ Superconductors and Related Systems," Physica C 162-164, 1375 (1989).

J. C. Wang, "Comments on a Fractal Model for Blocking Interfaces'," Electrochimica Acta 34, 787 (1989).

J. C. Wang "Construction of CPA Admittance with Distributions of Series RC-Pairs," Solid State Ionics 39, 227 (1990).

J. C. Wang, "Model for Lithium Intercalation into $\mathrm{TS}_{2}$," Solid State lonics 40141,548 (1990).

G. Whichard, H. Hosono, R. A. Weeks, R. A. Zuhr, and R. H. Magruder III, "Electron Paramagnetic Resonance Spectroscopy of Titarium-Ion-Implanted Silica," I. Appl. Phys. 67, 7526 (1990).

C. W. White, C. J. Mchurgue, P. S. Sklad, L. A. Boatner, and G. C. Farlow, "Ion Implantation and Annealing of Crystalline Oxides," Mater. Sci. Rep. 4, 11 (1989).

C. W. White, J. C. MCCallum, and L A. Boatncr, "Ion Implantation and Thermal Annealing of High- $T_{c}$ Single $\mathrm{Crystals}_{\text {of }} \mathrm{YBa}_{2} \mathrm{Cu}_{3} \mathrm{O}_{2}, "$ Mater. Sci. and Eng. A 109, 311 (1989).

G. D. Wignall, "Neutron Scattering Studies of the Crystallization of Polymers," Polym. Reprints 30,268 (1989).

G. D. Wignall, V. Ramakrishnan, M. Linne, A. Klein, L. Sperling, M. Wai, R. Celman, M. Fattica, R. Hoerl, L. Fisher, S. Melpolder, and J. O Reilly, "The Morphology of Emulsion-Polymerized Latex Particles," Mol. Cryst. Liq. Cryst. 180A, 25 (1990).

G. D. Wignall, N. R. Farrar, and S. Morris, "Characterization of Carbon-Filled Polymers by SmallAngle Scattering Techniques," I. Mater. Sci. 25, 69 (1990).

G. D. Wignall, J. S. Lin, and S. Spooner, "Reduction of Parasitic Scattering in Small-Angle X-Ray Scattering by a Three-Pinhole Collimating System," J. Appl. Crystallogr. 23, 241 (1990).

G. M. Williams, P. C. Becker, N. Edelstein, L. A. Boatner, and M. M. Abraham, "Excitation Profiles of Resonance Electronic Raman Scattering in ErPO4 Crystals," Phys. Reo. B 40, 1288 (1989).

G. M. Williams, P. C. Becker, J. G. Conway, N. Edelstein, L. A. Boatner, and M. M. Abraham, "Intensities of Elecironic Raman Scattering Between Crystal-Field Levels of $\mathrm{Ce}^{3+}$ in $\mathrm{LuPO}_{4}$ : Nonresonant and Nizat Resonant Excitation," Phys. Rev. B 40, 4132 (1989).

G. M. Williams, N. Ede'stein, L. A. Boatner, and M. M. Abraham, "Anoma'ously Sinall 4f-5d Oscillator Strengths ar.d 4f-4f Electronic Raman Scattering Cross Sections for $\mathrm{Ce}^{3+}$ in Crystals of LuPO," Phys. Rev. B 40, 4143 (1989). 
R. K. Williams, J. B. Bates, R. S. Graves, D. L. McElroy, and F. J. Weaver, "Comparison of Thermal Conductivity Data for Partially Stabilized Zirconia with Values Derived from Thermal Diffusivity Results," J. Thermophys. 9, 587 (1988).

S. P. Withrow, O. W. Holland, and S. J. Pennycook, Ton Beam Annealing of Ga-Implanted Si," Appl. Surf. Sci. 13, 191 (1989).

S. P. Withrow, K. L. More, R. A. Zuhr, and T. E. Haynes, Mon Beam Deposition of B-SiC Layers onto a-SiC Substrates," Vacuum 39, 1065 (1989).

K. Wittmaack and D. P. Poker, "Interface Broadening in Sputter Depth Profiling Through Alternating Layers of Isotopically Purified Silicon," Nucl. Instrum. and Methods Phys. Res. Sect. B 47, 224 (1990).

R. F. Wood, Mark Mostoller, and John F. Cooke, "A Spin Polaron Model of High-T, Superconduntivity," Physica C 165, 97 (1990).

R. F. Wood and M. Abdel-Raouf, "Comparison of Spin Polaron Models of High-T, Superconductivity," Solid State Commun 74, 371 (1990).

W. Xia, C. A. Hewett, M. Fernandes, S. S. Lau, and D. B. Poker, Moving Species During Ion Mixing in GerSi $1-x$ Metal Systems," I. Appl. Phys. 67, 1814 (1990).

P. Young, R. S. Stein, T. Kyu, and J. S. Lin, "Static Small-Angle X-Ray Scattering Study of Polymet Deformation," Polym. Sci-Polym. Phys. 28, 1791 (1990).

\section{BOOKS AND PROCEEDINGS}

J. B. Bates, N. J. Dudney, Y. T. Chu, and P. Mazumdar, "Properties of Electrolyte and Electrode Films Prepared by RF and DC Magnetron Sputtering, p. 143 in Solid State lonics, ed. by Gholamabbas Nazri, Robert A. Huggins, Duward F. Shriver, and P. T. Wu, Materials Research Society, Pittsburgh, Pennsylvania, 1989.

J. B. Bates, N. J. Dudney, C. F. Luck, and J. L. Katt, "Deposition of Electrolyte and Cathode Thin Films by Magnetron Sputtering," p. 35 in Ceramic Transactions, Ceramic Thin and Thick Films, Vol. 11, ed. by Basavaraj V. Hiremath, The American Ceramic Society, Colurnbus, Ohio, 1990.

L. A. Berry, S. M. Corbatkin, J. B. Roberto, C. C. Tsai, W. Holber, and J. Yeh, "Characteristics of the ORNL ECR Multiple Plasma Source," p. 156 in SRC Topical Research Conference on Plasma Etching, ed. by J. Richard Burke, Semiconductor Research Corporation, Research Triangle Park, North Carolina, 1989.

L. A. Boatner and C. W. White, "The Application of Rutherford Backscattering and Channeling Techniques to the Characterization of Ceramics," p. 112 in Ceramic Transactions, Advanced Characterization Techniques for Ceramics, ed. by G. McVay, W. Young, and G. Pike, The American Ceramic Society, Columbus, Ohio, 1989. 
R. A. Buchanan, In-Seop Lee, and J. M. Williams, "Iridium Ion Implantation of Surgical Titanium Alloy: Corrosion Inhibition and Charge Injection Effects," p. 53 in Ion Implantation and Plasme Assisted Processes for Industrial Applications, ed. by Robert F. Hochman, Hillary SolnickLeg8, and Keith O. Legg, ASM International, Metals Park, Ohio, 1988.

B. C. Chakoumakos, J. D. Budai, B. C. Sales, and Edward Sonder, "Variation of Superlattice Structure of the $\mathrm{Bi}_{2} \mathrm{Sr}_{2-x} \mathrm{CuO}_{6-y}$ Superconductor with Composition and Thermal History," p. 329 in High-Temperature Superconductors: Relationships Between Properties, Structure, and Solid State Chemistry, ed. by J. B. Torrance, K. Kitazawa, J. M. Tarascon, J. R. Jorgensen, and M. Thompson, Materials Research Society, Pittsburgh, Pennsylvania, 1989.

Bryan C. Chakoumakos, Brian C. Sales, and EJward Sonder, "Physical Properties of $\mathrm{Bi}_{2} \mathrm{Sr}_{2} \mathrm{CuO}_{6}$ the Semiconducting Phase, Structurally Distinct from the $N=1$ Bi-Cuprate Superconductor," p. 103 in High-Temperature Superconductors: Fundemental Properties and Novel Materials Processing, ed. by David K. Christen, Jagdish . Jarayan, a1.1 Lynn F. Schneemeyer, Materials Research Society, Pittsburgh, Pennsylvania, 195.

Y. Chen, R. Conzalez, and V. M. Orera, "Hydride lons and Tunable Lasers Based on Anion Vacancies in Refractory Oxide Crystals," p. 91 in Hydrogen Absorbing Materials/Catalytic Materials, ed. by Y. Mornoka, S. Ono, Y. Sasaki, and S. Suda, Materials Research Society, Pittsburgh, Pennsylvania, 1989.

Y. Chen, R. Gonzalez, V. M. Orera, and G. J. Pogatshnik, "Optical Properties of Oxygen Vacancies and Hydride lons in MgO and CaO Crystals," p. 96 in Disordered Systems and New Materials, ed. by M. Borisson, N. Kirov, and A. Vanick, World Scientific, Teaneck, New Jersey, 1989.

Y. L. Chen, R. W. Carpenter, and T. P. Sjoreen, "Precipitation in Silicon Implanted by Oxygen and Carbon Plus Oxygen," p. 640 in Electron Microscopy 1990, Proceedings of the XIIth International Congress for Electron Microscopy, Vol. 4, ed. by L. D. Peachey and D. B. Williams, San Francisco Press, San Francisco, Califomia, 1990.

M. F. Chisholm and S. J. Pennycook, "High-Resolution Z-Contrast Imaging of $\mathrm{YBa}_{2} \mathrm{Cu}_{3} \mathrm{O}_{7-8} \mathrm{Grain}$ Boundaries," P. 198 in Proceedings of the 47th Annual Meeting of the Electron Microscopy Society of America, ed. by G. W. Bailey, San Francisco Press, San Francisco, California, 1989.

M. F. Chisholm, S. J. Pennycook, and D. E. Jesson, "Atomic Structure and Chemistry of SiCe Interfaces Determined by Z-Contrast STEM," p. 447 in Atomic Scale Structure of Interfaces, ed. by R. D. Bringans, R. M. Feenstra, and J. M. Gibson, Materials Research Socicty, Pittsburgh, Pennsylvania, 1990.

M. F. Chisholm and S. J. Pennycook, "Misfit Accommodation in Epitaxial Ge Films on Si," p. 340 in Electron Microscopy 1990. Proceedings of the XIIth International Congress for Electron Microscopy, Vol. 4, ed. by L. D. Peachey and D. B. Williams, San Francisco Press, San Francisco, California, 1990.

Henry Chou, S. M. Shapiro, S. C. Moss, and Mark Mostoller, "l'honon Dispersion and Kohn Anomalies in the Alloy $\mathrm{Cu}_{0.84} \mathrm{Al}_{0.16, "}$, p. 237 in Neutron Scattering for Materials Science, ed. by S. M. Shapiro, S. C. Moss, and J. D. Jorgensen, Materials Rescarch Socicty, Pittsburgh, Pennsylvania, 1990. 
D. K. Christen, C. E. Klabunde, R. Feenstra, D. H. Lowndes, D. Norton, H R. Kercinner, J. R. Thompson, S. T. Sekula, J. D. Budai, L. A. Boatner, J. Narayan, and i.. Singh, "Electrical Transport Dissipation Effects in Epitaxial $\mathrm{Y}_{1} \mathrm{Ba}_{2} \mathrm{Cu}_{3} \mathrm{O}_{7-x}$ Thin Fi'ms," p. 883 in HighTemperature Superconductors: Fundamental Properties and Nood Meterials Processing, ed. by David K. Christen, Jagdish Narayan, and Lynn F. Schneemeyer, Materials Research Society, Pittsburgh, Pennsylvania, 1990.

Francisco Claro and R. Fuchs, Multipolar Excitations in Small Metallic Spheres," p. 601 in Physics and Chemistry of Small Clusters, ed. by P. Jena, B. K. Rao, and S. N. Khanna, Plenum Publishing Company, New York, 1987.

F. C. Difilippo, R. M. Moon, W. G. Gambill, R. T. Primm III, and C. D. West, “An Intense Steady State Neutron Source-The CNR Reactor," p. 792 in Proceedings of the Meeting on Adonences in Reactor Physics and Safely, Vol. 2, F. Feimer, Compiler, (NUREG/CP-0080), U.S. Nuclear Regulatory Commission (August, 1986).

M. K. El-Ghor, S. J. Pennycook, and R. A. Zuhr, "Shallow Junction Formation in As-Implanted Si by Low-Temperature Rapid Thermal Annealing," p. 19 in Ion Beam Processing of Adoanced Electronic Materials, ed. by N. W. Cheung, A. D. Marwick, and J. B. Roberto, Materials Research Society, Pittsburgh, Pennsylvania, 1989.

Djula Eres, D. H. Lowndes, J. Z Tischler, J. W. Sharp, D. B. Geohegan, and S. J. Pennycook, Prrolytic and Laser Phowlytic Growth of Crystalline and Amorphous Germanium Films from Digermane $\left(\mathrm{Ge}_{2} \mathrm{H}_{6}\right)$," p. $\mathbf{5 1 7}$ in Chemical Perspectioes of Microelectronic Materials, ed. by Mihal E. Gross, Joseph Jasinski, and John T. Yates, Jr., Materials Research Society, Pittshurgh, Pennsylvania, 1989.

Djula Eres, J. W. Sharp, and Dorglas H. Lowndes, "Heteroepitaxial Growth of Germanium Films by Supersonic Free Jet Chemical Beam Epitaxy," p. 359 in Loyered Structures-Heteroepitaxy, Superlattices, Strain, and Metastability, ed. by I. J. Showalter, F. H. Pollack, B. W. Dodson, and J. E. Cunningham, Materials Ressarch Society, Pittsburgh, Pennsylvania, 1990.

R. Feenstra, J. D. Budai, D. K. Christen, M. F. Chisholm, L. A. Boatner, M. D. Galloway, and D. B. Poker, "Microstructure and Critical Currents of $\mathrm{YBa}_{2} \mathrm{Cu}_{3} \mathrm{O}_{7-2}$ Thin Films on $\mathrm{SrTiO}_{3}$ and a New Substrate: $\mathrm{KTaO}_{3}, "$ p. 327 in Science and Technology of Thin-Film Superconduclors, ed. by Robert D. McConnell and Stuart A. Wolf, Plenum Publishing Company, New York, 1989.

L. Ganapathi, Ashok Kumar, J. Narayan, and O. W. Holland, "Superconducting and Semiconducting Thin Films of La123 and Y123 and Their Superlattices," p. 561 in HighTemperature Superconductors: Fundamental Properties and Novel Materials Processing, ed. by David K. Christen, Jagdish Narayan, and Lynn F. Schneemeyer, Materials Research Society, Pittsburgh, Pennsylvania, 1990.

D. B. Geohegan and D. N. Mashbum, "Studies of Neutral and Ion Transport During Laser Ablation of 1:2:3 Superconductors by Optical Absorption Spectroscopy," p. 153 in Superconductivily and Applications, ed. by Hoi S. Kwok, Yi-Han Kao, and David T. Shaw, Plenum Press, New York, 1990. 
David B. Geohegan and Douglas N. Mashburn, "Characterization of Ground State Neutral and lon Transport During Laser Ablation of 1:2:3 Superconductors," p. 501 in High-Temperature Superconductors: Fundamental Properties and Nooel Materials Processing, ed. by David K. Christen. Jagdish Narayan, and Lynn F. Schneemeyer, Materials Research Society, Pittsburgh, Pennsylvania, 1990.

D. B. Geohegan and Dougias N. Mashburn, -Spectroscopic and Ion Probe Characterization of the Transport Process Following Laser Ablation of $\mathrm{YBa}_{2} \mathrm{Cu}_{3} \mathrm{O}_{x, "}, \mathrm{p} .211$ in Laser Ablation for Materials Synthesis, ed. by David C. Paine and John C. Bravman, Materials Research Society, Pittsburgh, Pennsylvania, 1990.

T. E. Haynes, R. A. Zuhr, and S. J. Pennycook, "Heteroepitaxy of GaAs on Si and Ge by Low-Energy Ion Beam Deposition Using Alternating Beams," p. 311 in Adoances in Materials, Processing and Devices in III-V Compound Semiconductors, ed. by Devendra K. Sadana, Lester Eastman, and Russell Dupuis. Materials Research Society, Pittsburgh, Pennsylvania, 1989.

T. E. Haynes, R. A. Zuhr, S. J. Pennycook, and B. C. Larson, "Ion Beam Depusition of Epitaxial Germanium and Gallium Arsenide Layers," p. 363 in Procedings of the Twedfth Symposium on Ion Sources and Ion-Assisted Technology, ed. by Toshinori Takagi, Kyoto University, Kyoto, Japan, 1989.

J. B. Hayter and H. A. Mook, "Design of High-Reflectivity Supermirror Structures," p. 23 in ThinFilm Nenitron Optical Devices: Mirrors, Supermirrors, Multilayer Monochromators, Polarizers, and Beam Guides, ed. by C. F. Majkrzak, Society of Photo-Optical Instrumentation Engineers, Bellingham, Washington, 1988.

J. B. Hayter, "SANS from Polymers and Colloids," p. 345 in Neutron Scattering Adoances and Applications, ed. by M.M. Elcome and T.J. Hicks, Trans-Tech Publications, Geneva, Switzerland, 1988.

J. B. Hayter, "Concentrated Dispersions," p. 827 in Foundations of Colloid Science, ed. by R. J. Hunter, University Press, Oxford, 1989.

John B. Hayter, "The Advariced Neutron Source," p. 30 in Neutron Scattering for Materials Science, ed. by S. M. Shapiro, S. C. Moss, and J. D. Jorgensen, Materials Research Society, Pittsburgh, Pennsylvania, 1990.

O. W. Holland, M. K. El-Ghor, and C. W. White, "Damage Growth in Si During Self-lon Imadiation: A Study of Ion Effects Over an Extended Energy Range," p. 169 in lon Beam Processing of Adoanced Electronic Materials, ed. by N.W. Cheung, A.D. Marwick, and J. B. Roberto, Materials Research Society, Pittsburgh, Pennsylvania, 1989.

G. E. Jellison, Jr., and F. A. Modine, "Accurate Calibration of a Photo-Elastic Modulator in Polarization Modulation Ellipsometry," p. 231 in Polarization Considerations for Optical Systems II, Vol. 1166, Society of Photo-Optical Instrumentation Engineers, Bellingham, Washington, 1989.

D. E. Jesson, S. J. Pennycook, and M. F. Chisholm, "Simulation and Quantification of HighResolution Z-Contrast Imaging of Semiconductor Interfaces," p. 439 in Atomic Scale Structure of Interfacis, ed. by R. D. Bringans, R. M. Feenstra, and J. M. Gibson, Materials Research Society, Pittsb :.rgh, Pennsylvania, 1990. 
D. E. Jesson and S. J. Pennycook, "Atomic Imaging of Crystals Using Large-Angle E ectron Scattering in STEM," p. 74 in Electron Microscopy 1990, Procedings of the XIlth Intermational Congress for Electron Microscopy, Vol. 1, ed. by L. D. Peachey and D. B. Williams, San Francisco Press, San Francisco, California, 1990.

D. E. Jesson, S. J. Pennycook, and J.-M. Baribeau, "Column-by-Column Compositional Mapping at Semiconductor Interfaces by Z-Contrast STEM," p. 223 in High-Resolution Electron Microscopy of Defects in Materials, ed. by Robert Sinclair, David J. Smith, and Ulrich Dahmen, Materials Research Society, Pittsburgh, Pennsylvania, 1990

H. R. Kerchner, R. Feenstra, J. O. Thomson, J. R. Thompson, D. K. Christen, S. T. Sekula, and L. A. Boatner, "Magnetic Flux Pinning in $\mathrm{YBa}_{2} \mathrm{Cu}_{3} \mathrm{O}_{7}$ Films," p. 903 in High-Temperature Superconductors: Fundamental Properties and Novel Materials Processing, ed. by David K. Christen, Jagdish Narayan, and L.ynn F. Schneemeyer, Materials Research Society, Pittsburgh, Pennsylvania, 1990.

C. B. Lee, R. Prasad, A. K. Singh, S. Sharan, R. K. Singh, J. Narayan, O. W. Holland, and L. A. Boatner, In Situ Processing of Epitaxial and Textured High-T $\mathrm{T}_{c}$ Superconducting $\mathrm{HoBa}_{2} \mathrm{Cu}_{3} \mathrm{O}_{7-x}$ Thin Films by Pulsed-Laser Evaporation Technique," p. 459 in High-Temperature Superconductors: Fundamental Properties and Novel Malerials Processing, ed. by David K. Christen, Jagdish Narayan, and Lynn F. Schneemeyer, Materials Research Society, Pittsburgh, Pennsylvania, 1990.

Lionel M. Levinson, Herbert R. Philipp, and E. Sonder, Interface Effects in Zinc Oxide Varistors," p. 665 in Ceramic Microstructures 86, Materials Science Research, Vol. 21, ed. by Joseph A. Pask and Anthony G. Evans, Plenum Publishing Company, New York, 1987.

Lionel M. Levinson, H. R. Philipp, and G. D. Mahan. "Evidence for Parallel Conduction Paths in ZnO Varistors," p. 145 in Adoances in Varistor Tecinology, Ceramic Transactions, Vol. 3, ed. by Lionel M. Levinson, The American Ceramic Society, Westerville, Ohio, 1989.

S. H. Liu, "Theory of Strongly Fluctuating Superconductivity," p. 455 in Superconductivity and Applications, ed. by Hoi S. Kwok, Yi-Han Kao, and David T. Shaw, Plenum Publishing Company, New York, 1990.

D. H. Lowndes, D. Eres, D. B. Geohegan, J. Z. Tischler, D. N. Mashburn, and S. J. Pennycook, "Excimer Laser-Controlled Photochemical Deposition of Thin Films and Artificially Structured Materials," p. 382 in Addances in Laser Science IV, ed. by James L. Gole, Donald F. Heller, Marshall Lapp, and William C. Stwalley, American Institute of Physics, New York, 1989.

Douglas H. Lowndes, David P. Norton, J. W. McCamy, R. Feenstra, J. D. Budai, D. K. Christen, E. Jones, and D. B. Poker, "In Situ Growth of High-Quality Epitaxial $\mathrm{YBa}_{2} \mathrm{Cu}_{3} \mathrm{O}_{-2}$ Thin Films at Moderate Temperatures by Pulsed-Laser Ablation," p. 431 in High-Temperature Superconductors: Fundamental Properties and Novel Materials Processing, ed. by David K. Christen, Jagdish Narayan, and Lynn F. Schneemeyer, Materials Research Society, Pittsburgh, Pennsylvania, 1990. 
Douglas H. Lovmdes, David P. Norton, J. D. Budai, S. J. Pennycook, D. K. Christen, B. C. Sales, and R. Feenstra, "Superconductivity in Nonsymmetric Epitaxial $\mathrm{YBa}_{2} \mathrm{Cu}_{3} \mathrm{O}_{7-\delta} / \mathrm{PrBa}_{2} \mathrm{Cu}_{3} \mathrm{O}_{7-\delta}$ Superlatices Grown by Pulsed-Laser Ablation," p. 153 in Leser Ablation for Materials Syminesis, ed. by David C. Paine and John C. Bravman, Materials Research Society, Pittsburgh, Pennsylvania, 1990.

D. Lubben, T. Motooka, J. F. Wendelken, and J. E. Greene, "Laser-Induced Photodissociation of $\mathrm{Al}_{2}\left(\mathrm{CH}_{3}\right)_{6}$ : Cas-Phase and Adsorbed Layer Dissociation Mechanisms for Al Film Growth," p. 198 in Proceedings of the NATO Workshop on Mechonisms of Reactions of Organometallic Compounds with Surfaces, ASI Series D, Plenum Publishing Company, New York, 1989.

H. Maeta, B. C. Larson, T. P Sjonen, D. K. Thorr s, O. S. Oen, and J. D. Lewis, "X-Ray Diffuse Scattering Study of Vacancy and Interstitial Loops in Ion-Irradiated Copper," p. 81 in Characterization of the Structure and Chemistry of Defects in Materials, ed. by Bennett C. Larson, Manfred Rühle, and David N. Seidman, Materials Research Society, Pittsburgh, Pennsylvania, 1989.

R. H. Magruder, D. L. Kinser, R. A. Weeks, and R. A. Zuhr, Mnteraction Between Implanted Ions and Intrinsic Defects in Silica," p. 519 in Beam Solid Interactions: Physical Phenomena, ed. by James A. Knapp, Peter Borgesen, and Raymond A. Zuhr, Materials Research Society, Pittsburgh, Pennsylvainia, 1990.

G. D. Mahan and K. R. Subbaswamy, Local Density Theory of Polarizability, Plenum Publishing Company, New York, 1990.

J. W. McCamy, David P. Norton, Douglas H. Lowndes, L. A. Boatner, D. K. Christen, R. Feenstra, and E. Sonder, "In Situ Growth of Epitaxial Superconducting $\mathrm{YBa}_{2} \mathrm{Cu}_{3} \mathrm{O}_{7-x}$ Films on Insulating, Serniconducting, and Ferroelectric $\mathrm{K}\left(\mathrm{Ta}, \mathrm{Nb}_{3} \mathrm{O}_{3}\right.$ by Pulsed-Laser Ablation," p. 469 in HighTemperature Superconductors: Fundamental Properties and Nooel Materials Processing, ed. by David K. Christen, Jagdish Narayan, and Lynn F. Schneemeyer, Materials Research Society, Pittsburgh, Pennsylvania, 1990.

C. J. Mchargue, C. W. White, P. S. Sklad, M. E. O'Hern, D. L. Joslin, and G. C. Farlow, "StructureMechanjcal Propcrty Relationships in Ion-Implanted Ceramics, p. 561 in Procedings of the 2nd International Seminar on Surface Engineering with High Energy Beams, CEMUL, Lisbon, Portugal, 1989.

C. J. McHargue, P. S. Sklad, P. Angelini, C. W. White, and J. C. McCallum, "Structure of Amorphous $\mathrm{Al}_{2} \mathrm{O}_{3}$ Produced by 'on Implantation," p. 505 in Beam-Solid Interactions: Physical Phenomena, ed. by James A. Knapp, Peter Borgesen, and Raymond A. Zuhr, Maierials Research Society, Pittsburgh, Pennsylvania, 1990.

C. J. McHargue, P. S. Sklad, J. C. McCallum, C. W. White, A. Perez, and G. Marest, "The Effects of Annealing on the Structure and Charge States of Iron Implanted into $\mathrm{Al}_{2} \mathrm{O}_{3}$ at $77 \mathrm{~K}$," p. 555 in Beam Solid Interactions: Physical Phenomena, ed. by James A. Knapp, Peter Borgesen, and Raymond A. Zuhr, Materials Research Socicty, Pittsburgh, Pennsylvania, 1990.

F. A. Modine, R. W. Major, S.-I. Choi, L. B. Bergman, and M. N. Silver, "Zinc Oxide Varistor Time Response," p. 176 in Advances in Varistor Technology, Ceramic Transactions, Vol. 3, ed. by Lionel M. Levinson, The American Ceramic Society, Westerville, Ohio, 1989. 
H. A. Mook, "Neutron Scattering Studies of $n(p)$ at Reactor Sources," p. 159 in Momentum Distributions, ed. by Richand N. Silver and Paul E. Sokol, Plenum Publishing Company, New York, 1989

K. is More, S. P. Withrow, T. E. Haynes, and R. A. Zuhr, "Growth of Epitaxial SiC Layers onto Onand Off-Axis 6H-SiC Substrates by Ion Beam Deposition," p. 451 in Diamond, Siticon Cartide, end Related Wide Bandgap Seniconductors, ed. by J. T. Glass, R. Messier, and N. Fujimori, Materials Researctr. Society, Pittsburgh, Pennsylvania, 1990.

N. R. Parikh, C. T. Keo, D. R. Lee, J. Muse, M. L Swanson, T. E. Haynes, R. Venkatasubramanian, and M.Timmons, "Dopant Site Location in Dual-Implanted GaP Using (111) Planar Channeling," p. 671 in Beam-Solid Interactions: Physical Phencmena, ed. by James A. Knapp. Peter Bongesen, and Raymond A. Zuhr, Materials Research Society, Pittsburgh, Peansylvania, 1990.

S. J. Pennycook, Structural and Chemical Imaging of Superconductors and Semiconductors by HighResolution STEM," p. 329 in Characterization of the Structure and Chemistry of Defects in Materials, ed. by Bennett C. Larson, Manfred Rūhle, and David N. Seidman, Materials Research Society, Pittsburgh, Pennsylvania, 1989.

S. J. Pennycook, Structural and Chemical Imaging of Superconductors and Semiconductors by HighResolution STEM," p. 39 in High-Resolucion Microscopy of Meterials, ed. by W. Krakow, F. A. Ponce, and D. J. Smith, Materials Research Society, Pittsburgh, Pennsylvania, 1989.

S. J. Pennycook, "High-Resolution Z-Contrast Imaging of Semiconductor Interfaces," p. 468 in Proceedings of the 47th Annual Meeting of the Electron Microscopy Society of America, ed. by G. W. Bailey, San Francisco Press, San. Francisco, California, 1989.

S. J. Pennycook, D. E. Jesson, and M. F. Chisholm, 'High-Resolution Imaging of Semiconductor Interfaces by Z-Contrast STEM," p. 51 in Microscopy of Semiconducting Materials 1989, ed. by A. G. Cullis and J. L. Hutchison, The Institute of Physics, Bristol, United Kingdom, 1989.

S. J. Pennycook, "Diffusion, Segregation, and Recrystallization in High-Dose Ion-Implanted Si," p. 39 in Ion Beam Processing of Adoanced Electronic Materials, ed. by N.W. Cheung, A. D. Marwick, and J. B. Roberto, Materials Rescarch Socicty, Pittsburgh, Pennsylvania, 1989.

S. J. Pennycook, M. F. Chisholm, D. E. Jesson, D. P. Norton, J. W. McCamy, and D. H. Lowndes, "Direct Imaging of the Atomic Structure and Chemistry of Defects and Interfaces by Z-Contrast STEM," p. 765 in High-Temperature Superconductors: Fundamental Properties and Nodel Meterials Processing, ed. by David K. Christen, Jagdish Narayan, and Lynn F. Schneemeyer, Materials Research Society, Pittsburgh, Pennsylvania, 1990.

S. J. Pennycouk, D. E. Jeseon, and M. F. Chisholnn, "High-Resolution Z-Contrast Imaging in the STEM," p. 394 in Electron Microscopy 1990, Proceedings of the Xilth International Congress for Eleciron Microscopy, Vol. 4, ed. by L. D. Peachey and D. B. Williams, San Francisco Press, San Francisco, California, 1990.

Stephen J. Pennycook, "High-Resolution Imaging of Materials by Z-Contrast STEM," p. 4 in Proceedings of the 27th Annual Electron Micrnseopy Colloquium, ed. by Michael L. Thompson, lowa State University, Ames, lowa, 1990. 
S. J. Pennycook, D. E. Jesson, and M. F. Chisholm, "Incoherent Imaging of Materials Structure and Composition by Z-Contrast STEM," p. 211 in High-Resolution Electron Microscopy of Defects in Materials, ed. by Robert Sinclair, David J. Smith, and Ulrich Dahmen, Materials Research Society, Pittsburgh, Pennsylvania, 1990.

D. B. Poker and W. Xia, "Optical Waveguide Fabrication by Stoichiometric Implentation of $\mathrm{Ti}$ and $O$ into LiNbO3," p. 531 in Berm-Solid Interactions: Physical Phenomera, ed. by James A. Knapp, Peter Borgesen, and Raymwnd A. Zuhr, Materials Research Society, Pittsburgh, Pennsylvania, 1990.

M. Roppaz, S. A. David, J. M. Vitek, and L A. Boatner, "Analysis of Microstructural Development in Single-Crysta: Welds," p. 147 in Intemational Trends in Welding Science and Technology, ed. by S. A. David and J. M. Vitek, American Socicty for Metals, Metals Park, Ohio, 1989.

Mark Rasolt and Zlatko Tesanovic, "A New Crossover Behavior of Type II Superconductors in Strong Magnetic Fields," p. 167 in Field Theories in Condensed Matter Physics-A Workshop, ad. by Zlatko Tesanovic, Addison-Wesley Publishing Company, Redwood City, California, 1990.

Roberto Rojas and Francisco Claro, "Excitations in Small Spheri al Particles," p. 167 in V Simposio Chiceno de Fisice, Proceedings of the Vth Chilean Physirs Symposium, Santiago, Chile, December 18-20, 1986.

B. C. Sales, E. Sonder, and B. C. Chakoumakos, The Effects of Oxygen and Strontium Vacancies on

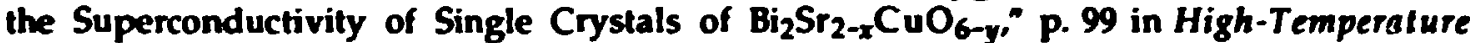
Superconductors: Fundamental Properties and Novel Materials Processing, ed. by David K. Christen, Jagdish Narayan, and Lynn F. Schneemeyer, Materials Research Society, Pittsburgh, Pennsylvania, 1990.

S. T. Sekula, R. Feenstra, J. R. Thompson, Y. C. Kim, D. K. Christen, H. R. Kerchner, H. A. Deeds, and L. A. Boatner, "Static and Time Dependent Magnetization Studies of $\mathrm{Y}_{1} \mathrm{Ba}_{2} \mathrm{Cu}_{3} \mathrm{O}_{2}$ Thin Films," p. 385 in Adoances in Cryogenic Engincering, Vol. 36A, Plenum Publishing Company, New York, 1990.

T. P. Sjoreen, O. W. Holland, M. K. El-Ghor, and C. W. White, “Damage Formation in Semiconductors During MeV Ion Implantation," p. 593 in Processing and Characterization of Materials Using Ion Beams, ed. by L. E. Rehn, J. E. Greene, and F. A. Smidt, Materials Rescarch Society, Piltsburgh, Pennsylvania, 1989.

P. S. Sklad, J. C. McCallum, S. J. Pennycook, C. J. McHargue, C. W. White, and A. Perez, "Microstructural Characterization of $\alpha \cdot \mathrm{Al}_{2} \mathrm{O}_{3}$ Implanted with Iron," p. 119 in Characterizaticn of the Structure and Chemistry of Defects in Malerials, ed. by Bennett $C$. Larson, Manfred Rühle, and David N. Seidman, Materials Rescarch Society, Pittsburgh, Pennsylvania, 1989.

D. A. Smith, D. R. Clarke, M. F. Chisholm, P. E. Batson, and E. Olsson, "Transport Critical Curreuts and Microstncli're of Yttrium Barium Cuprate," p. 32 in Electron Microscopy 1990, Proceedings of the XIIth International Congress for Electron Microscopy, Vol. 4, ed. by L. D. Peachey and D. B. Williams, San Francisco Press, San Franciscn, California, 1990. 
E. Sonder, B. C. Chakoumakos, and B. C. Sales, Oxidation and Reduction of $\mathrm{Bi}_{2} \mathrm{Sr}_{2} \mathrm{CuO}_{6-y}$ Crystals," p. 217 in High-Temperature Superconductors: Fundamental Properties and Novel Materials Processing, ed. by David K. Christen, Jagdish Narayan, and Lynn F. Schneemeyer, Materials Research Society, Pittsburgh, Pennsylvania, 1990.

J. R. Thompson, D. K. Christen, H. A. Deeds, Y. C. Kim, J. Brynestad, S. T. Sekula, J. Budai, and J. G. Ossandon, "Magnetic Penetration Depth and Intragrain Critical Current Density in GrainAligned $\mathrm{Tl}_{2} \mathrm{Ca}_{2} \mathrm{Ba}_{2} \mathrm{Cu}_{3} \mathrm{O}_{10}$ Superconductor," p. 1065 in High-Temperature Superconductors: Fundamental Properties and Nood Materials Processing, ed. by David K. Christen, Jagdish Narayan, and Lynn F. Schneemeyer, Materials Research Society, Pittsburgh, Pennsylvania, 1990.

P. Tiwari, S. Sharan, R. K. Singh, J. Narayan, and O. W. Holland, In Situ Pulsed-Laser Deposition of High- $T_{c} \mathrm{YB}_{2} \mathrm{Cu}_{3} \mathrm{O}_{3}$ Superoonducting Thin Films on (100) LaANO 3 Substrates," p. 451 in HighTempenture Superconductors: Fundanental Properties and Novd Materials Processing, ed. by David K. Christen, Jagdish Narayan, and Lynn F. Schneemeyer, Materials Research Society, Pittsburgh, Pennsylvania, 1990.

P. E. A. Turchi, F. J. Pinski, R. H. Howell, A. L Wachs, M. J. Fluss, D. D. Johnson, G. M. Stocks, D.M. Nicholson, and W.Schweika, "Ordering Mechanism in Ni-Cr Alloys: Theory and Experiment," p. 231 in Neutron Scattering for Materials Science, ed. by S. M. Shapiro, S. C. Moss, and J. D. Jorgensen, Materials Research Society, Pittsburgh, Pennsylvania, 1990.

L. J. van IJzendoom, G.F.A. van de Walle, A. A. van Gorkum, A. M. L. Theunissen, R. A. van den

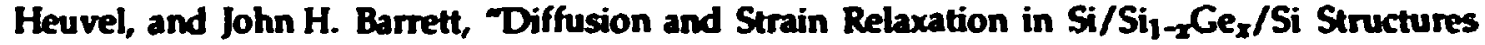
Studied with Rutherford Backscattering Spectrometry," P 127 in Acceleretors in Applied Resenrch and Technology, ed. by K. Bethge, F. Rauch, and P. Misaelides, North-Holland, Amsterdam, 1990.

J. C. Wang and J. B. Bates, Hopping Model for the Non-Debye Dielectric Response in lonic Crystals," p. 57 in Solid State lonics, ed. by Cholamabbas Nazri, Robert A. Huggins, Duward F. Shriver, and P. T. Wu, Materials Research Society, Pittsburgh, Pennsylvania, 1989.

J. C. Wang, "Model for Lithium Intercalation into TiS," p. 548 in Solid State lonics-89, ed. by S. Hoshino, North-Holland, Amsterdam, 1990.

R. A. Weeks, H. Hosono, R. A. Zuhr, R. Magruder, and H. Mogul, "Correlation Betwcen Optical Absorption and Refractive Index of Silica and Silicate Glasses Implanted with Copper," p. 115 in Optical Materials: Processing and Science, ed. by David B. Poker and Camen Ortiz, Materials Research Society, Pittsburgh. Pennsylvania, 1989.

C. Whichard, R. A. Weeks, and R. A. Zuhr, "Comparison of the Magnetic Properties of Iron and Manganese Ion-Implanted Silica," p. 271 in Proceedings of the XVth International Congress on Cless, Vol. 2B, Nauks.' '. ingrad, USSR, 1989.

G. D. Wignall and F. S. Bates, "Isolope-Driven Critical Scattering and Phase Separatiun in Mixtures of Deuterated and Protonated Polvmers," p. 17 in International Conference on Polymer Research by Neutron Scaltering, The Taniguchi Foundation, Kyolo, Japan, 1989.

G. D. Wignall, "Neutron Scattering from Polymers," p. 645 in Concise Encyclopedia of Polymer Science, ed. by J. Kroschwitz, Wiley-Interscience, New York, 1990. 
J. M. Williams, Beam Ion Implantations," p. 45 in Concise Encyclopedia of Medical and Dental Materials, ed. by David Williams, Pergamon Press, Oxford, 1990.

S. P. Withrow, O. W. Holland, and S. J. Pennycook, Ton Beam Annealing of Ga-Implanted Si," p. 191 in Beam Processing and Leser Chamistry, ed. by I. W. Boyd and E. Rimini, Elsevier Science Publishers, Amstendam, 1989.

S. P. Withrow, O. W. Holland, S. J. Pennycook, J. Pankove, and A. Mascarenhas, "Ion Beam Annealing of Si Co-implanted with $G$ and As," p. 143 in Beam-Solid Interactions: Physical Phenomena, ed. by James A. Knapp, Peter Bergesen, and Raymond A. Zuhr, Materials Research Society, Pittsburgh, Pennsylvania, 1990.

R. A. Zuhr, S. J. Pennycook, T. E Haynes, and O. W. Holland, Metal Silicides Formed by Direet lon Beam Deposition," p. 47 in Processing and Characterization of Materials Using lon Beams, ed. by L. E. Rehn, J.E. Greene, and F. A. Smidt, Materials Research Society, Pittsburgh, Pennsylvania, 1989.

\section{REPORTS ISSUED}

John D. Axe and John B. Hayter, Proceedings of the Oak Ridge National Laboratory/Brookhaven National Laboratory Workshop on Neutron Instrumentation at High-Flux Reactors, Oak Ridge National Laboratory, CONF-8906311 (July 1990).

R. L. Chaplin, H. R. Kerchner, C. E. Klabunde, and R. R. Coltman, Jr., Stored Energy in Fusion Magnet Material's Irradiated at Low Temperatures, ORNL/TM-11217 (August 1989).

K. G. Huang, B. M. Ocko, D. Gibbs, A. Sandy, S. G. J. Mochrie, and D. M. Zehner, X-Ray Diffraction Studies of Au(111) Surfaces," p. 495 in National Synchrotron Light Source Annual Report 1989, Brookhaven National Laboratory, BNL-52218 (April 1990).

B. M. Ocko, Doon Gibbs, K. G. Huang, S. G. J. Mochrie, and D. M. Zehner, "Au(100) Surface and Subsurface Ordering," p. 499 in Netional Synchrotron Light Source Annual Report 1989, Brookhaven National Laboratory, BNL-52218 (April 1990).

Xioha Pan, D. Zehner, R. Bartynski, and E. Ward Plummet, "Angle-Resolved Photoemission Study of the $\mathrm{H}_{2}$ Chemisorption on $\mathrm{Ta}(100), "$ p. 206 in National Synchrotron Light Source Annual Report 1989, Brookhaven National Laboratory, BNL-52218 (April 1990).

S. J. Pennycook, "Inner-Shell Excitation by Channeled Electrons," p. 101 in The 11th Wermer Brandt Workshop on Charged Particle Penetration Phenomena, Oak Ridge National Laboratory, CONF-8804141 (September 1989).

C. C. Tsai, L. A. Berry, S. M. Gorbatkin, H. H. Hasclton, J. B. Roberto, D. E. Schechter, and W. L. Stirling, Potential Applications of an Electron Cyclotron Resonance Multicusp Plasma Source, ORNL/TM-11442 (March 1990).

R. F. Wiod, "Photovoltaic Technology," p. 109 in Energy Technology R\&D: What Could Make a Difference?" ORNL-6541/V2/P2 (December 1989). 
D. M. Zehner, G. R. Grizalsk, G. W. Ownby, and David Heskett, Surface Core-Level Spectroscopy from HFC(100) and HFC(111)," p. 210 in National Symchrotron Light Source Annual Report 1989, Brookhaven National Laboratory, BNL-52215 (April 1990).

\section{PATENTS GRANTED}

L. A. Boatner and B.C. Sales, Lead-Iron Phosphote Glass as a Containment Medium for the Disposal of High-Leved Nuclear Wastes, U.S. Patent No. 4,847,008 (July 11, 1989).

L. A. Boatner and B. C. Sales, Nood Lead-Irom Phosphate Glass, US. Patent No. 4,847,219 July 11, 1989\%.

Ch. Buchal and B. R. Appletion, Fabrication of Optical Waveguides by Ion Implantation Doping, U.S. Patent No. 4,810,816 (June 20, 1989).

Orin W. Holland, Dariush Fathy, and Clark W. White, Method for Enhancing Growth of $\mathrm{SiO}_{2}$ in Si by the Implantation of Germanium, US. Patent No. 4,920,076 (April 24, 1990).

Raymond A. Zuhr and Orin W. Holland, Method for Forming Metallic Silicide Films on Silicon Substrates by Ion Beam Deposition, U.S. Patert No. 4,908,334 (March 13, 1990).

\section{THESES}

Young C. Kim, "Anisotropies in the Magnetic Properties of Aligned High-T ${ }_{c}$ Superconducting $\mathrm{YBa}_{2}\left(\mathrm{Cu}_{1-\mathrm{x}} \mathrm{Fe}_{2}\right)_{3} \mathrm{O}_{2}$ Powder Composites," Ph.D. Thesis, The University of Tennessee, Knoxville, Tennessee, 1989.

Janet Rankin, "Ion Beam Damage and Recovery Effects in Three Perovskite-Type Oxides: Calcium Titanate, Strontium Titanate, and Potassium Tantalate," Ph.D. Thesis, Mascachusetts Institute of Technology, Cambridge, Maseachusetts, 1989.

\section{PAPERS PRESENTED AT TECHNICAL MEETINGS}

Low-Energy Ion Beame-5 Conference, University of Surrey, Guildford, United Kingdom, April 3-5, 1989:

S. P. Withrow, K. More, R. A. Zuhr, and T. E. Haynes, "Ion Beam Deposition of 3C:SiC Layer onto $6 \mathrm{H}: \mathrm{SiC}^{\prime \prime}$

International Symposium on Quantum Chemistry, Solid State Theory, and Molecular Dynamics, St. Auguatine, Florida, April 1-8, 1989:

Janos J. Ladik, Peter Saalfrank, and Richard F. Wood, "Theoretical Considerations of High-Tc Superconductivity" 
International Workshop on Ion Beam Modification and Processing in High-T, Superconductors, Oxford, England, April 10-12, 198\%.

S. J. Pennycook, M. F. Chisholm, R. Feenstra, D. Mashbum, and J. C. McCallum, Electron Micruscopy of Defects and Interfaces in High-T, Superconductors" (inviled paper)

Sixth Oxford Conference on Microscopy of Semicunducting Interfaces, Oxford, England, April 10-13, 190\%.

S. J. Pennycook and M. F. Chisholm, "Atomic Resolution Z-Contrast Imaging of Semiconductor Interfaces"

ACS Symposium on Polymers in Reatricted Geometry, Dalles, Texas, April 12-14, 1909:

X. Quan, S. H. Lee, I. Gancarz, J. T. Koberstein, G. D. Wigmall, and F. C. Wilson, "Homopolymers Confined in Block Copolymer Lamellae"

American Chemical Society Symposium Honoring L Mandelkern for the Applied Polymer Science Award, Dallas, Texas, April 13, 1999:

G. D. Wignall, Neutron Scattering Studies of the Crystallization of Polymers" (invited paper)

Intermational Conference on Metallurgical Coaturgs, San Diego, California, April 17-21, 1999:

J. M. Williams, C. M. Egert, J. R. McNeely, and R. A. Buchanan, "lon Beam Processing for Advanced Metallic Materials"

American Ceramic Society Ninety-Fira Annual Meeting, Indianapolis, Indiana, fopril 23-27, 1999:

M. M. Abraham and L. A. Boatner, "EPR Analysis of Texturing Effects in YPO, Ceramics"

J. B. Bates, N. J. Dudney, Y. T. Chu, and C. Luck, "Deposition of Electrolyte and Cathode Thin Films by Magnetron Sputtering"

R. Feenstra, L. A. Boatner, J. D. Budai, D. K. Christen, M. F. Chisholm, and M. D. Calloway, "KTaO $-A$ New Substrate for the Epitaxial Crowth of $\mathrm{Y}_{1} \mathrm{Ba}_{2} \mathrm{Cu}_{3} \mathrm{O}_{7}-\mathrm{Thin}$ Films"

S. Q. Fu, W. K. Lee, A. S. Nowick, L. A. Boatner, and M. M. Abraham, Trotons in AcceptorDoped $\mathrm{KTaO}_{3}$ Crystals"

J. Rankin, J. C. MCCallum, L. A. Boatnet, and C. W. White, "TEM, RBS, and TRR Studies of the Effect of Water Vapor on Epitaxial Regrowth in $\mathrm{SrTiO}_{3}$ and $\mathrm{CaTiO}_{3}$ "

B. C. Sales, J. O. Ramey, L. A. Boatner, and J. C. MCCallum, "Structural Inequivalence of the Ion-Damage-Produced Amorphous State and the Class State in Lead Pyrophosphate" 
Materials Research Society Meeting, San Diego, California, April 24-29, 1999.

B. C. Chakoumakos, J. D. Budai, B. C. Sales, and Edward Sonder, Variation of Superlattice Structure of the $\mathrm{Bi}_{2} \mathrm{Sr}_{2-x} \mathrm{CuO}_{6}$ Superconductor with Composition and Thermal History" (invited paper)

Y. Chen, V. M. Orera, R. Conzalez, R. T. Williams, G. P. Williams, Jr., and G. H. Rosenblatt, The Effect of Hydrogen on the Charge States of Oxygen Vacancies in Thermochemically Reduced Alkaline Earth Oxides"

M. K. E-Ghor, S. J. Pennycook, and R. A. Zuhr, "Extended Defect Removal Following Thermal Annealing of Shallow As Implants into Silicon"

O. W. Hollanl, M. K. E-Ghor, and T. P. Sjoreen, Influence of Pre-Existing Damage on Defect Production During MeV Ion Irradiation of Si"

5. J. Pennycook, "SPE Growth and Dopant Activation in High-Dose lon-Implanted Silicon"

D. B. Poker, "Cakculation of Ion Channeling Directions in Low-Symmetry Optical Materials"

C. W. White, D. K. Thomas, P.R. Ashley, W. S. C. Chang, and C. Buchal, Ton Implantation of Ti into LiNbO3: Fabrication of Waveguides and Simple Modulators"

175th Meeting of the Electrochemical Society, Los Angeles, California, May 7-12, 1999:

S. H. Li", T. Kaplan, and L. J. Gray, "Fractal Models for the AC Response of Rough ElectrodeElectrolyte Interfaces"

Tweaty-Fifth Annual Symposium of the American Vacuum Society-New Mexico Chapter, Alboquerque, New Medio, May 9-11, 1909:

J. R. Noonan and H. L. Davis, "Surface Structure by LEED" (invited paper)

Thind Workd Conference on Neutron Radiography, Osaka, Japan, May 14-18, 1999:

H. G. Smith, J. B. Davidson, and R. A. W. Zlems, "New Neutron-Sensitive TV Image Intensifiet System"

Forty-Second Annual Conference of the Society for Imaging Science and Technology, Boston, Masaxchusetts, May 14-19, 1909:

Nancy J. Dudney, "Enhanced lonic Conduction in Silver Halide Composites" (invited paper) 
The Ninth Annual TVC-AVS Symposium and Equipment Exhibition, Oak ikidge, Tennessee, May 15-18, 190\%:

Arthur P. Baddorf and J. F. Wendelken, Initial Stages in Oxidation of Cu(110)" (invited paper)

John Budai, Ron Feenstra, and Lynn Boatner, "X-Ray Characterization of Epitaxial $\mathrm{Y}_{1} \mathrm{Ba}_{2} \mathrm{Cu}_{3} \mathrm{O}_{2}$ Thin Films on (001) $\mathrm{SrTH}_{3}$ and $\mathrm{KTaO}_{3}$ Substrates" (invited paper)

D. Eres, D. H. Lowndes, J. Z. Tischler, T. E. Haynes, and M. F. Chisholm, Deposition of Crystalline and Amorphous Germanium Films irom Digermane ( $\left.\mathrm{Ce}_{2} \mathrm{H}_{6}\right)^{-}$(invited paper)

T. E Haynes, R. A. Zuhr, S. P. Withrow, S. J. Pennycook, and K. L More, "Gruwth of Epitaxial Compound Thin Films Using Alemating Low-Energy lon Beams" (invited paper)

G. E. Jellison, Jr., Two-Channel Polarization Modulation Ellipsometry as an Altemative to Rotating Analyzer Enlipsometry for Thin-Film Analysis" (invited paper)

National Energy Software Center Installation Representatives' Meeting Argonne, IIlinois, May 16-17, 1969:

Mark T. Robinson, "MARLOWE: A Simulation of Atomic Collision Cascades in Crystalline Solids in the Binary Collision Approximation" (invited paper)

1969 Oak Ridze ASM International Educational Symposium on Advances in Materials Processing, Oak Ridge, Tennessere, May 19, 1909:

J. M. Williams, "Recent Trends in Surface Modification of Materials" (invited paper)

First International Symposium on Electrochemical Impedance Spectroscopy, Bombannes, France, May 22-26, 1909:

S. H. Liu, T. Kaplan, and L. I. Gray, Theory of the AC Response of Rough Electrode-Electrolyte Interfaces" (invited paper)

European Materials Research Society Mecting, Straboure. France, May 30-June 2, $199 \%$

j. P. Withrow, O. W. Holland, and S. J. Pennyconk, "Ion Beam Annealing of Ca-Implanted Si"

Intemational Workshop on Neutron Scattering Instrumentation, Oak Ridge, Tennessee, June 5-7, 198\%:

J. B. Hayter, "The Advanced Neutron Source" (inviied paper) 
Twelfith Symposium on Ion Sources and Ion-Accisted Technolosy, Tolyo, Japan, June 5-7, 1999.

T. E. Haynes, R. A. Zuhr, S. J. Pennycook, and B. C. Larson, Mon Beam Deposition of Epitaxial Germanium and Gallium Arsenide Layers" (invited paper)

Sixth Conversation in the Discipline Biomolecular Sterodymamics, Albany, New York, June 6-10, 1989:

Jocl M. Harp, Edwand C Uberbacher, Arthur E Roberson, and Gerard J. Bunick, Precise Reconstitution of Nucleosome Core Particles with Defined Sequence DNAs"

Tenth University Conference on Glass, Pennsylvania State University, University Park, Pennogiveniz, June 7-9, 1909:

R. H. Magruder III, S. H. Morgan, R. A. Weeks, and R. A. Zuhr, "Effects of Ion Implantation on Intermediate Range Order: IR Spectra of Silica"

Fifth International Conference on Radiation Effects in Insulators, Hamilton, Oatario, Canada, June 19-23, 1909:

B. C. Chakoumakos, J. C. McCallum, L. A. Boatner, W. C. Oliver, G. R. Lumpkin, and R. C. Ewing. "Microhardness in Natural Zircon as a Function of a-decay Dose Compared with Synthetic Zircon Damaged by Po lon implantation"

C. C. Farlow and L. A. Boatner, "Effects of Ion Irradiation on $\mathrm{MgF}_{2}$ "

D. L. Joslin, C. J. McHargue, and J. M. Williams, The Hard Elastic Modulus of Ion-Implanted Silicon Carbide"

J. C. McCallum, J. Rankin, C. W. White, and L. A. Boatner, Time-Resolved Reflectivity Measurements in lon-Implanted Crystailine Ceramic Oxides"

J. C. McCallum, C. W. White, P. S. Sklad, and C. J. McHargue, "Annealing Environment Effects in Solid-Phase-Epitaxial Regrowth of Fe-Implanted $\mathrm{Al}_{2} \mathrm{O}_{3}$ "

1999 Eiectronic Materials Conference, Cambridge, Massachusetts, June 21-23, 1989

L. S. Darken, P. Sangsingkeow, and G. E. Je!lison, Jr., "Hole Capture at Acceptors in P-Type Cermanium"

Ion Bean Analysis Conference, Kingston, Ontario, Canada, June 26-30, 1999:

John H. Barrett, "Methods of Channeling Simulation" (invited paper) 
Fifventi International Congress on Glass, Leningrad, USSR, July 2-7, 1909:

G. Whichard, R. A. Weeks, and R. A. Zuhr, "Comparison of the Magnetic Properties of Iron and Manganese Ion-lmplanted Silica"

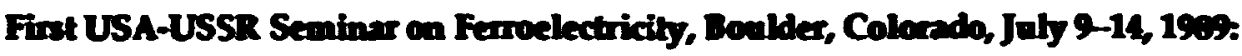

J. P. Solkolof, L. L. Chase, D. Ryts, and L. A. Boatner, Direct Observation of Relaxation Modes in KIN Using Inelastic Light Scattering" (invited paper)

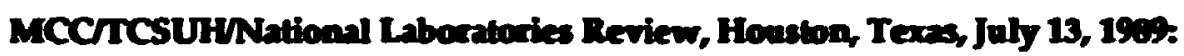

Doughas H. Lowndes, Tighlights of Oxide Supenconductor Thin-Film Reseanch at Oak Ridge National Laboratory" (inviled paper)

International Conference en Materials and Mechanisms of Supercondectivity: High-Temperature Semperconductors, Strafard, California, Juby 23-28, 1999.

D. K. Christen, C. E. Kabunde, J. R. Thompson, H. R. Kerchner, S. T. Sekula, R. Feenstra, and J. D. Budai, Transport Critical Currents in Epitaxial $\mathrm{Y}_{1} \mathrm{Ba}_{2} \mathrm{Cu}_{3} \mathrm{O}_{7}$ Thin Films" (invited paper)

R. Feenstra, L. A. Bos.tner, J. D. Budai, S. J. Pennycook, M. F. Chisholm, M. D. Galloway, D. K. Christen, J. R. Thompson, H. R. Kerchner, and S. T. Sekula, "Critical Currents and Epitaxy of Y123 Films on Doped and Undoped $\mathrm{KTaO}_{3}$

H. R. Kerchner, R. Feenstra, J. O. Thomson, J. R. Thompson, Y. C. Kim, D. K. Christen, and S. T. Sekula, Magnetic AC Response of $\mathrm{YBa}_{2} \mathrm{Cu}_{3} \mathrm{O}_{x}$ Films"

Cryozenic Engineering Conference and International Cryogenic Materials Conference, Los Angelex, California, July 24-28, 199\%:

S. T. Sekula, R. Feenstra, J. R. Thompson, Y. C Kim, D. K. Christen, H. R. Kerchner, and L. A. Boatrer, "Static and Time Dependent Magnetization Studies of $\mathrm{Y}_{1} \mathrm{Ba}_{2} \mathrm{Cu}_{3} \mathrm{O}_{7}$ Thin Films"

Shoteenth International Conference on the Physics of Electroaic and Atomic Collislons, New York, New York, July 26-Auguat 1, 1909:

F. W. Meyer, C. C. Havener, K. J. Snowdon, and D. M. Zehner, "Electron Spectroscopy of Multicharged lon-Surface Interactions at keV Energies"

Annual Meeting of the Electron Microscopy Society of America, San Antonio, Texas, August 6-11, 198\%:

M. F. Chisholm and S. J. Pennycook, "High-Resolution Z-Contrast Imaging of $\mathrm{YBa}_{2} \mathrm{Cu}_{3} \mathrm{O}_{7-8}$ Grain Boundaries" 
S. J. Pennycook, "High-Resolution Z-Contrast Imaging of Semiconductor Interfaces"

SPIE Thirty-Third Annual International Technical Symposium on Optical and Optrelectronic Applied Science and ingineering, San Diego, California, August 6-11, 196\%:

G. E. Jellison, Jr., "Accurate Caiibration of a Photo-Elastic Modulator in a Polarization Modulation Ellipsometry Experiment" (invited paper)

Condon Conference on X-Ray Physics, New London, New Hampshire, August 7-11, 1999.

J. Z. Tischler, B. C. Larson, and G. E. Ice, Resonant Nuclear Scattering of Synchrotron Radiation from Epitaxial Crystals of ${ }^{57} \mathrm{Fe}_{2} \mathrm{O}_{3}$ "

Gordon Conference on Dynamics of Gas-Surface Interactions, Andover, New Hampshire, Auguat 7-11, 198:

Arthur P. Baddorf and John F. Wendelken, "Unexpected Characteristics of a Molecular Intermediate in Dissociative Adsorption of $\mathrm{O}_{2}$ on $\mathrm{Cu}(110)^{\prime \prime}$

Thirteenth International Conference on Atomic Collisions in Solids, Aarhus, Denmark, August 7-11, 1989.

M. T. Robinson, "The Temporal Development of Collision Cascades in the Binary Cullision Approximation"

Seventh Intsmational Meeting on Ferroelectricity, Saarbracken, Germany, Aagust 20-September 9, 2989:

S. Jandl, P. Grenier, and L. A. Boatner, "Raman and Fluorescence Spectra of Ferroelectric Micro Regions in $\mathrm{KTaO}_{3}$ "

First European Conference on Accelerators in Applied Research Technology, Frankfurt, Germany, September 4-9, 199\%:

L. J. van IJzendoorn, G. F. A. van de Walle, A. A. van Corkum, A. M. L. Theunissen, R. A. van den Heuvel, and John H. Barrett, "Diffusion and Strain Relaxation in $\mathrm{Si}_{/} \mathrm{Si}_{1-x} \mathrm{Ge}_{x} / \mathrm{Si}$ Structures Studied with Rutherford Backscattering Spectrometry"

American Chemical Society Symposium on the Crystalline State, Miami Beach, Florida, September 10-15, 1989:

G. D. Wignall, "Neutron Scattering Studies of the Crystallization of Polymers" (iıvited paper) 
Third Annual Conference on Superconductivity and Applications, Buffalo, New York, September 19-21, 19:9:

D. B. Geohegan and D. N. Mashbum, Studies of Neutral and Ion Transport Ablation of 1:2:3 Superconductors by Optical Absorption Spectroscopy" (invited paper)

S. H. 'iu, Theory of Strongly Fluctuating Superconductivity" (invited paper)

Alabama Materials Research Conference, Funtoville, Alabama, September 20-21, 1909:

J. B. Roberto, "Advanced Materials Processing Using Ion Beams and Lasers" (invited paper)

Actinides 89 Conference, Tahkent, USSR, September 24-29, 1999:

N. Edelstein, W. Kott, D. Piehler, G. Shalimoff, D. Ball, M. M. Abraham, C. B. Finch, and L. A. Loatner, "Optical and EPR Spectra of Trivalent Actinides Diluted in LuPO,"

Symposium on Physical Aging in Polymers, 18th Conference of the North American Thermal Analyais Society, San Diezo, California, September 25-29, 199\%:

B. C. Sales, "Structural Relaxation Dynamics of Phosphate Glasces: The Effects of Network Topology on the Glass Transition" (invited paper)

European Conference on High-T, Thin Films and Single Crystals, Ustron, Poland, September $30-$ October 4, 1909:

S. T. Sekula, "Static and Dynamic Magnetization Properties of $\mathrm{Y}_{2} \mathrm{Ba}_{2} \mathrm{Cu}_{3} \mathrm{O}_{2}$ Thin Films" (invited paper)

ASM International Materials Week-Textures in Nonmetallic Materials Symposium, Indianapolis, Indiana, October 2-5, 1999:

L. A. Boatner, J. L. Boldú O., and M. M. Abraham, "EPR Analysis of Texturing Effects in Oxide and Phosphate Ceramics"

NATO Advanced Research Workshop on Dynamics of Magnetic Fluctuations in High-Temperature Superconductors, Crete, Greece, October 10-15, 199:

H. A. Mook, G. Aeppli, S. M. Hayden, Z. Fisk, and D. Rytz, Neutron Scattering Measurements of the Magnetic Excitations of High-Temperature Superconducting Materials" (invited paper) 
Twenty-First International Thermal Conductivity Conference, Lexington, Kentucky, October 15-18, 1989.

Sune Pettersson, "On the Choice of Trial Function in Theoretical Calculations of the Thermal Conductivity"

One-Hundred and Seventy-Sixth Annual Meeting of the Electrochemical Society, Follywood, Flocida, October 15-20, 159\%:

S. J. Pennycook, Precipitation, Segregation, and Enhanced Diffusion of Implanted Impurities in $\mathrm{Si}^{\text {" }}$ (invited paper)

Third Conference on Cryatal Growth, ACCG/East-3, Atlantic City, New Jersey, October 18-20, 1999:

L. A. Boatner, R. Feenstra, J. D. Budai, D. K. Christen, and M. D. Galloway, "Potassium Tantalate Single-Crystal Substrates for the Epitaxial Growth of High-Temperature Superconducting Films"

American Vacuuni Society Thirty-Sixth National Symposium and Topical Conference, Boston, Maseachusetts, October 23-27, 1909:

Arthur P. Baddorf and John F. Wendelken, "High Coverages of Oxygen on Cu(110)"

H. L. Davis and J. R. Noonan, "Search for Near Perfect" Agreement in LEED Analysis"

Steven M. Gorbatkin, L A. Berry, and J. B. Roberto, Behavior of Ar and $\mathrm{N}_{2}$ Plasmas Formed in a Mirror Field ECR Microwave lon Source"

K. G. Huang, Doon Gibbs, B. M. Ocko, A. Sandy, S. G. J. Mochrie, and D. M. Zehner, X-Ray Surface Diffraction Studies of Au(111)"

S.-C. Lui, D. M. Zehner, G. R. Gruzalski, and D. R. Heskett, "Core Level Photoemiscion Study of the $\mathrm{TaC}(111)$ Surface"

J. R. Noonan and H. L. Davis, "Confirmation of an Exception to the 'General Rule' of Surface Relaxations" $\mathrm{Au}(100)^{*}$

B. M. Ocko, Doon Gibbs, D. M. Zehner, and S. G. J. Mochrie, "Surface X-Ray Scattering from

K. B. Ray, E. W. Plummer, and H. L. Davis, "A Large Interlayer Expansion for Be(0001)"

C. C. Tsai, L. A. Berry, S. M. Corbatkin, H. H. Haselton, J. B. Roberto, and W. L. Stirling, "Potential Applications of ORNL ECR Multicusp Plasmatron"

I. F. Wendelken, "An Angle-Resolved LEED Study of the Atomic Step Structure of NiAl and NiAl(111)" 
ASTeX ECR User Group Meeting Dodton, Masenchusetts, October 24, $190 \%$

S. M. Corbatkin, Contamination by Sputtering in a Mirror Field ECR Microwave lon Source" (invited paper)

Workshop on Research and Technical Transfer of Ion Implantation Technolosy for Specialty Materiak, Knoxville, Tennessee, Odwober 26-20, 15\%:

J. M. Wiliams, C. M. Egert, R A. Buchanan, D. K. Thomas, W. C. Oliver, and R. H. Staunion, Ton Implantation Research at Oak Ridge National Laboratory" (invited paper)

MCCICSUSHational Laboratories Review, Anstin, Texas, October 31, 1309:

Ron Feenstra, Update of Recent Results on YBCO Thin-Film Research at Oak Ridge National Laboratory" (invited paper)

Seventh International Conference on Solid State Ionics, Hakone, Japan, November 5-9, 1909.

J. C. Wang "Model for Lithium Intercalation into TSS"

Tanizuchi Coaference on Polymer Research by Neutron Scattering, Kyoto, Japan, November 7-9, 1909:

G. D. Wignall and F. S. Bates, Msotope Driven Critical Scattering and Phase Separation in Mixtures of Deuterated and Protonated Polymers" (invited paper)

American Thysical Society, Sontheastern Section Meeting. Tuscaloosa, Alabama, November 9-11, 1909: paper)

D. M. Zehner, "Surface Structure of Alloys" [Bull. Am. Phys. Soc. 34, 2359 (1989)] (invited

L. S. Cain, G. J. Pogatshnik, Y. Chen, and M. Kokta, "Color Centers Produced by Reduction of Undoped and Doped LMA" (Bull. Am. Phys. Soc. 34, 2356 (1989))

Thirty-Fisst Annual Meeting of the American Physical Society, Division of Plasma Physics, Anthein, Califomia, November 13-17, 199\%:

C. C. Tsai, L. A. Berry, S. M. Corbatkin, H. H. Haselton, J. B. Roberto, and D. E. Schechter, "Potential Applications of ORNL Multicusp Plasmatron"

Workehop on Applied Ion Implantution, St. Louls, Miscouri, Noverniber 16-17, 1999 :

J. M. Williams, C. M. Egert, and T. C. Kollie, "Ion Implantation for Corrosion Inhibition of Uranium" (invited paper) 
Materials Reseach Society Fall Meeting, Rocton, Massachusetts, November 27-December 2, 1909:

Brvan. C. Cinakoumakos, Brian C. Sales, and Edward Sonder," Physical Properties of $\mathrm{Bi}_{2} \mathrm{Sr}_{2} \mathrm{CuO}_{6}$, the Semiconducting Phase. Structurally Distinct from the $\mathrm{N}=1 \mathrm{Bi}-\mathrm{Cluprate}$ Superconductor"

M. F. Chisholm, S. J. Pennycook, and D. E. Jesson, "Atomic Structure and Chemistry of Si/Ge Interfaces Determined by Z-Contrast STEM"

D. K. Christen, C. E. Kiabunde, R. Feenstra, D. H. Lowndes, D. Norton, H. R. Kerchner, J. R. Thompson, S. T. Sekula, J. D. Budai, L A. Boatner, J. Narayan, and R. Singh, Electrical Transport Dissipetion: Effects in Epitaxial $\mathrm{Y}_{1} \mathrm{Ba}_{2} \mathrm{Cu}_{3} \mathrm{O}_{7-x}$ Thin Films"

M. K. E-Ghor, S. J. Pennycook, and R. A. Zuhr, "Fomation of Deffect-Free Shallow Junctions by Low-Temperature Rapid Thernal Annealing"

M. K. E-Ghor, O. W. Holland, C. W. White, and S. 1. Pennycook, Structural Characterization of Damage in Si(100) Produced by MeV Si+ Ion Implantation and Annealing"

Djula Eres, Douglas H. Lowndes, J. Z. Tischler, T. E. Haynes, and M. F. Chisholm, Rapid Heteroepitaxial Growth of Ge Films oy Pulsed Supersonic Free Jet Chemical Beam Epitaxy"

R. Feenstra, J. D. Budai, S. J. Pennycook, M. F. Chisholm, M. D. Galloway, D. K. Christen, D. P. Norton, J.W. McCamy, D. H. Lowndes, and L. A. Boatner, "Epitaxial and Superconducting Properties of $\mathrm{Y}_{1} \mathrm{Ba}_{2} \mathrm{Cu}_{3} \mathrm{O}_{7-\pi}$ Films on Five Perovskite Substrates"

D. B. Geohegan and D. N. Mashbum, "Characterization of Ground State Neutral Ion Transport During Laser Ablation of 1:2-3 Superconductors by Transient Optical Absorption Spectroscopy"

John B. Hayter, "The Advanced Neutron Source" (invited paper)

David E. Jesson, S. J. Pennycook, and M. F. Chisholm, "Simulation and Quantification of HighResolution Z-Contrast Imaging of Semiconductor Interfaces"

H. R. Kerchner, R. Feenstra, J. O. Thomson, J. R. Thompson, D. K. Christen, S. T. Sekula, and L. A. Boatner, "Magnetic Flux Pinning in $\mathrm{YBa}_{2} \mathrm{Cu}_{3} \mathrm{O}_{x}$ Films"

Douglas H. Lowndes, D. P. Norton, J. W. McCamy, Ron Feenstra, I. D. Budai, D. K. Christen, E. Jones, and D. B. Poker, "In Situ Growth of High-Quality Epitaxial $\mathrm{YBa}_{2} \mathrm{Cu}_{3} \mathrm{O}_{2}$ Films at Moderate Substrate Temperature and Over Large Areas by Pulsed-Laser Ablation"

I. W. McCamy, D. P. Norton, Douglas H. Lowndes, L. A. Boatnr, and J. D. Budai, "In Situ Crowth of Epitaxial Superconducting $\mathrm{YBa}_{2} \mathrm{Cu}_{3} \mathrm{O}_{x}$ Films on Insulating, Semiconducting, and Ferroelectric $\mathrm{KTaO}_{3}$ by Pulsed-Laser Ablation"

K. L. More, S. P. Withrow, T. E. Haynes, and R. A. Zuhr, "Growth of Epitaxial SiC Layers onto On- and Off-Axis 6H-SiC Substrates by Ion Beam Deposition"

N. R. Parikh, C. T. Tao, D. R. Lee, J. Muse, M. L. Swanson, T. E. Haynes, R. Venkatasubramanian, and M. Timmons, "Dopant Site Location in Dual-Implanted GaP Using (111) Planar Channeling" 
S. J. Pennycooix, M. F. Chisholm, D. E. Jessen, R. Feenstra, D. Mashburn, !. W. McCamy, and D. H. Lowndes, "Atomic Scale Characterization of Defects and Interfaces by Z-Contrast STEM" (invited paper)

C. B. Poker and $W$. Xia, Optical Wavequide Fabrication by Stoichiometric Implantation of $\mathrm{Ti}$ and $\mathrm{O}$ inco $\mathrm{LiNbO}_{3}$

B. C. Sales, B. C. Chakoumakos, and Edward Sonder, The Effects of Oxygen and Strontium Vecancies on the Superconductivity of Single Crystals of $\mathrm{Bi}_{2} \mathrm{Sr}_{2-x} \mathrm{CuO}_{6-y}$

A. K. Singh, C. Lee, J. Narayan, and L A. Boatnet, "In Situ Laser Depo.-stion of $\mathrm{YBa}_{2} \mathrm{Cu}_{3} \mathrm{O}_{7}-8$ and $\mathrm{H}_{2} \mathrm{~B}_{2} \mathrm{Cu}_{3} \mathrm{O}_{7} \rightarrow \mathrm{High}-\mathrm{T}_{6}$ Superconducting Thin Films on $\mathrm{Si}(100)$ and $\mathrm{KTaO}_{3}(100)^{-\prime}$

Edward Sonder, B. C. Chakoumakos, and B. C. Sales, Oxidation and Reduction Kinetics of $\mathrm{Bi}_{2} \mathrm{Sr}_{2} \mathrm{CuO}_{6}=$

J. R. Thompson, H. A. Deeds, Y. C. Kim, J. Brynestad, D. K. Christen, S. T. Sekula, H. R. Kerchner, and J.D. Budai, Magnetic Penetration Depths of Aligned Composites of the HighTemperature Superconductor $\mathrm{TH}_{2} \mathrm{Ca}_{2} \mathrm{Ba}_{2} \mathrm{Cu}_{3} \mathrm{O}_{10+8}$ "

S. P. Withrow, O. W. Holland, S. J. Pennycook, and J. Pankove, "Ion Beam Annealing of Si Co-Implanted with Ga and As"

Thinty-Fourth Annual Conference on Magnetion and Magnetic Materials, Boston, Massachusetts, Novenber 20-December 1, 190\%:

J. A. Fernandez-Baca, J. J. Rhyne, G. E. Fish, M. Hennion, and B. Hernion, "Spin Waves in Amorphous Feygin $\mathrm{Z}_{10}{ }^{*}$ (invited paper)

J. A. Fernandez-Baca, R. M. Nicklow, and J. J. Rhyne, "Magnetic Excitations in Thulium Metal"

The Univessity of Miami Workshop on Electronic Structure and Mechanisms for High-Temperature Superconductivity, Miami, Florida, January 3-9, 19s0.

Douglas H. Lowndes, David P. Norton, and J. D. Budai, "Superconductivity and Experimental Tests of Hole Filling in Epitaxial High-T $T_{c}$ Superconductor-Semiconductor Superlaltices" (invited paper)

Wockshop on Modem Theory of Solids, Brasilia, Brazil, January 8-19, 1990.

S. H. Liu, "Theory of Heavy Fermion Systems" (four invited papers)

C. D. Mahan, "lonic Solids" (four invited papers)

Chaos Workshop, Oak Ridge National Laboratory, Oak Ridge, Tennescee, January 10-11, 1990.

Theodore Kaplan, S. H. Liv, and L. J. Cray, "Ceometric and Dynamic Properties of Detcrministic Sand Piles" (invited paper) 
Anmeal Meeting of the Frysical Society of the Republic of China Triwan, February 5-6, 1590

S. H. Liu, Interlayer Pairing: Possible New Type of Superconductivity in Layered Materials" (invited paper)

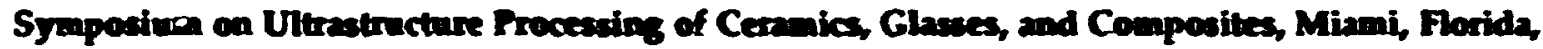
February 20-23, 15s0:

Daie W. Schnefer, L. Jian, C-C. Sun, D. MoCarthy, C.Y. Jiang, Y.-P. Ning J. E. Mark, and Steve Spooner, The Structure of Silica-Polysiloxane Composites and its Effects on Their Elastomeric Properties"

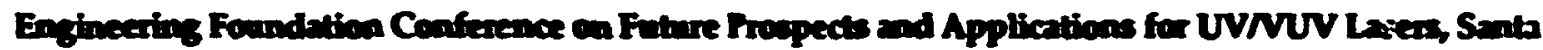
Babara, Californian, February 25-March 2, 19\%:

D. H. Lowndes, UV Growth of Thin Films and Superiattices" (invited paper)

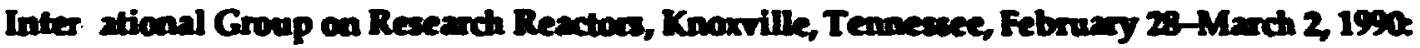

J. B. Hayter, "Worldwide Facility User Plans" (invited paper)

American Physical Society Meeting, Anmeim, Callfornia, Marsh 16-20, 1990:

Bill R. Appleton, Facilities, Resources, and International Aspects" (Bull. Am. Phys. Soc. 35, 320 (1990)] (Invited papet)

A. P. Baddorf, Nitrogen Coverages on Cu(110\%" [Bull. Am. Phys. Sac. 35, 589 (1990)]

Xiang-Xin Bi, P. C. EkJund, J. G. Zhang, Mark Mostoller, and P. Metcalf, "Optical Phonons and Phonon Dispersion in $\mathrm{La}_{2-x} \mathrm{Sr}_{x} \mathrm{NiO}_{4}$ " (Bull. Am. Phys. Sö. 35, 783 (1990)]

J. D. Budai, R. Feenstra, L. A. Boatner, D. H. Lowndes, and D. P. Norton, X-Ray Diffraction Determination of In-Plane Epitaxy for YBaCuO Thin Films on Perovskite Substratcs" [Bull. Am. Phys. Soc. 35, 679 (1990)]

Y. Chen, M. M. Abraham, G. J. Pogatshnik, L. S. Cain, and M. R. Kokta, Thermochemical Reduction of Tb-Doped Lanthanum Magnesium Aluminate Crystals" [Bull. Am. Phys. Soc. 35, 424 (1990)]

D. K. Christen, C. E. Klabunde, H. R. Kerchner, J. R. Thompson, S. T. Sekula, R. Feenstra, and D. H. Lowndes, "Effects of Flux Pinning and Thermally Activated Dissipation in Epitaxial $\mathrm{Y}_{1} \mathrm{Ba}_{2} \mathrm{Cu}_{3} \mathrm{O}_{7-x}$ Thin Films" [Bull. Am. Phys. Soc. 35, 394 (1990)]

J. F. Cooke and J. A. Blackman, "Effect of Wave Function Overlap Terms on the ltinerant Electron Theory of Magnetism" [Bull. Am. Phys. Soc. 35, 265 (1990)]

H. L. Davis, D. M. Zehner, D. v. Cemunden, B. Dötsch, and K. Müllet, "Constituent Segregation and Multilayer Relaxation of the MogsRe $e_{15}(100)$ Surface" [Bull. Am. Phys. Soc. 35, 296 (1990)] 
J. A. Fernandez-Bace, R. M. Nicklow, and J. j. Rhyne, Study of the Magnetic Excitations of Thulium Metal' [Bull. Am. Phys. Soc. 35, 795 (1990)]

K. Flensberg and Mats Jonson, "Correlation and Charging Effects in Small Tunnel Junctions" [Bull. Am. Phys. Soc. 35, 218 (1990)]

G. Grübel, J. D. Axe, D. Gibbs, G. H. Lander, and H. G. Smith, Tock-In Transf smations in a-Uranium and Their Description in Terms of a Landau-Type Theory" [Bull. Am. Phys. Soc. 35, 193 (1990)]

G. R. Gruzal-ki, S.C. Lui, and D. M. Zehner, Work Function Changes of $\mathrm{TaC}_{\mathrm{x}}$ and $\mathrm{HFC}_{\mathrm{x}}$ Associated with thanges in $x^{\prime \prime}$ [Bull. Am. Phys. Soc. 35, 602 (1990)]

K. G. Huang, L. Gibbs, B. M. Ocko, A. R. Sandy, S. G. J. Mochrie, and D. M. Zehner, X-Ray Scattering Study of the Au(111) Surface" [Bull. Ara. Phys. Soc. 35, 252 (1990)]

5. Jandl, P. Dufour, M. Banville, R. Lacasse, and L. A. Boatner, Infrared Study of Ferroelectric Microdomains in $\mathrm{KTaO}_{3}{ }^{-}$[Bull. Am. Phys. Soc. 35, 725 (1990)]

Mats Jonson and L I. Glazman, The Globally Adiabatic Regime in Quantum Ballistic Transport" [Bull. Am. Phys. Soc. 35, 492 (1990)]

H. R. Kerchner, J. O. Thomson, D. K. Christen, and R. Feenstra, Mnterpretation of the Magnetic AC Response of $\mathrm{YBa}_{2} \mathrm{Cu}_{3} \mathrm{O}_{7}$ Thin Films" [Bull. Am. Phys. Soc. 35, 338 (1990)]

R. A. Klemm and S. H. Liu, "Intra- Versus Interlayer Pairing in the Copper Oxide Superconductors" [Bull. A.m. Phys. Soc. 35, 291 (1990)]

S. H. Liu, T. Kaplan, and L. J. Gray, "Ceometric and Dynamic Properties of Deterministic Sandpiles" [Bull. Am. Phys. Soc. 35, 308 (1990)]

F. A. Modine and B. A. Sanborn, "A Modified Parallel Resistance Model of Resistivity Saturation" [Bull. Am. Phys. Soc. 35, 737 (1990)]

J. R. Noonan, "Surface Structure, Composition, and Ordering of Binary Alloys" [Bull. Am. Phys. Soc. 35, 615 (1990)] (invited paper)

S. J. Pennycook, "High-Resolution Incoherent Imaging of Crystals by Z-Contrast STEM" [Bull. Am. Phys. Soc. 35, 705 (1990)] (invited paper)

Sune Pettersson and G. D. Mahan, "A Theoretical Calculation of the Kapitza Resistance Between Crystals with Different Lattice Constants" [Bull. Am. Phys. Soc. 35, 330 (1990)]

Barbara Sanbom and G. D. Mahan, "A Calculation of Minority Carrier Transport in Heavily Doped Silicon" [Bull. Am. Phys. Soc. 35, 517 (1990)]

H. G. Smith, R. Berliner, J. Trivisonno, and J. Jorgensen, "Pressure Effects on the Martensitic Transformation in Sodium" [Bull. Am. Phys. Soc. 35, 575 (1990)]

M. D. Stiles, D. R. Penn, Mats Jonson, and S. M. Girvin, "Coulomb Blockade Effects in Transmission Lines" [Bull. Am. Phys. Soc. 35, 218 (1990)] 
I. R. Thompson, H. A. Deeds, D. K. Christen, S. T. Sekul, H. R. Kerchner, R. K. Williams, J. Brynestad, and D. M. Kroeger, "Are High-T, Materials Weak Coupling Superconductors?" IBull. Am. Phys. Soc. 35, 785 (1990)]

J. Z. Tischler, B. C. Larson, and G. E. Ice, Mössbaver Bandwidth X-Ray Monochromator Using an Epitaxial Crystal of ${ }^{57} \mathrm{Fe}_{2} \mathrm{O}_{3}{ }^{\prime \prime}$ (Eull. Am. Phys. Soc. 35, 765 (1990)]

X. M. Wang, L. A. Knauss, J. Toulouse, and L. A. Boatner, "Dielectric-Non-Linearity and Spontaneous Polarization of KTN in the Diffuse Transition Range" [Bull. Am. Phys. Soc. 35, 470 (1990)]

R. F. Wood, P. Saalfrank, and J. Ladik, "DODS Cluster Calculations for La $\mathrm{CuO}_{4}$ and $\mathrm{YBa}_{2} \mathrm{Cu}_{3} \mathrm{O}$ " [Bull. Am. Phys. Sac. 35, 479 (1990)]

Ji-Guang Zhang, Xiang-Xin Bi, E. McRae, P. C. Eklund, B. C. Sales, and Mark Mostoller, "Optical Conductivity of $\mathrm{Nd}_{2-x} \mathrm{Ce}_{2} \mathrm{CuO}_{4}$ " [Bull. Am. Phys. Coc. 35, 814 (1990)]

Center for Materials Processing, The University of Tennessee, Annual Review Meeting, March 22-23, 1990:

D. H. Lowndes, Epitaxial $\mathrm{YBa}_{2} \mathrm{Cu}_{3} \mathrm{O}_{7-x} / \mathrm{PrBa}_{2} \mathrm{Cu}_{3} \mathrm{O}_{7-x}$ Supen, thices: Growth and Properties of Multilayered Crystalline Materials Incorporating Ultrathin Superconducting Layers" (invited paper)

SPIE's 1990 Symposium on Advances in Semiconductors and Superconductors: Physics Toward Device Applicieicens, San Diego, Callfornia, March 17-21, 1990.

S. J. Pennycook, "Atomic Scale Imaging of the Structure and Chemistry of Semiconductor Materials" (invited paper)

Special Operations Expo 90, Idaho Falls, Idaho, March 20-22, 1990:

J. B. Bates, "Solid State Micropower Sources" (invited paper)

American Crystallographic Ascociation 1990 Annual Meeting, New Orieans, Louisiana, April 8-13, 1990.

John B. Hayter, "The Advanced Neutron Source"

John B. Hayter, "Small-Angle Scattering from Ferrofluids"

Materials Research Society Spring Meeting, San Francisco, California, April 16-21, 1990:

J. B. Bates, N. J. Dudncy, C. F. Luck, M. D. Galloway, B. C. Sales, and J. D. Robertson, "LithiumIon Conducting $(1-x) \mathrm{Li}_{4} \mathrm{SiO}_{4}: x \mathrm{Li}_{3} \mathrm{PO}_{4}$ Amorphous Electrolyte Thin Films" 
D. K. Christen, "Critical Currents and Flux Motion in Epitaxial Thin Films of $\mathrm{Y}_{1} \mathrm{Ba}_{2} \mathrm{Cu}_{3} \mathrm{O}_{7-x}$ " (invited paper)

Nancy J. Dudney, Thin-Film $\mathrm{CaF}_{2}-\mathrm{Al}_{2} \mathrm{O}_{3}$ Composites"

D. B. Geohegan and D. N. Mashburn, Spectroscopic and Ion Probe Characterization of the Laser-Ablation Transport Process During Thin-Film Growth: $\mathrm{Y}_{1} \mathrm{Ba}_{2} \mathrm{Cu}_{3} \mathrm{O}_{2}$ and Other Multicomponent Targets"

D. E. Jesson, S. J. Pennycook, and M. F. Chisholm, “Cohumn by Cohumn' Compositional Mapping at Semiconductor Interfaces Using Z-Contrast STEM"

Douglas H. Lowndes, D. P. Norton, J. D. Budai, S. J. Pennycook, D. K. Christen, B. C. Sales, and R. Feenstra, "Superconductivity in Nonsymmetric Epitaxial $\mathrm{YBa}_{2} \mathrm{Cu}_{3} \mathrm{O}_{7-8} / \mathrm{PrBa}_{2} \mathrm{Cu}_{3} \mathrm{O}_{7-8}$ Superattices Grown by Pulsed-Laser Ablation"

S. J. Pennycook, M. F. Chisholm, and D. E. Jesson, Direct Imaging of Interface Structure and Composition in Samionnductors and Superconductors" (invited paper)

J. R. Thompson, D. K. Christen, R. K. Williams, H. A. Deeds, D. M. Kroeger, S. T. Sekula, and J. G. Ossandon, Intragrain Critical Density and Magnetic Penetration Depth of $\mathrm{Y}_{1} \mathrm{Ba}_{2} \mathrm{Cu}_{4} \mathrm{O}_{8}$ (Y-124) High-Temperature Superconductor"

International Meeting on Analytical Versus Numerical Treatments of Models in High-T Superconductivity, Orsay, France, April 17-27, 1990: paper)

M. Rasolt, "Self-Induced Gauge Fields in the CuO Plane of High-T ${ }_{c}$ Perovskites" (invited Ninety-Second Annual Meeting of the American Ceramic Society, Dalla, Texa, April 22-26, 1990:

J. B. Bates, N. J. Dudney, C. F. Luck, M. D. Galloway, B. C. Sales, and J. D. Robertson, "(1-x) Li4 $\mathrm{SiO}_{4}: \mathrm{xLi}_{3} \mathrm{PO}_{4}$ Amorphous Electrolyte Thin Films"

N. J. Dudney, J. B. Bates, and C. F. Luck, Effect of Target Fabrication on the Composition of $\mathrm{Li}_{4} \mathrm{SiO}_{4}$-Li33 $\mathrm{PO}_{4}$ Films from RF Magnetron Sputtering"

Nancy J. Dudney, J. B. Bates, C. F. Luck, and S. J. Pennycook, "Thin-Film Ceramic Nanocomposites"

G. R. Gruzalski, Electron Spectroscopic Studies of Bulk Transition-Metal Carbides and Their Stoichiometric Surfaces" (invited paper)

R. W. Major, F. A. Modine, S.-1. Choi, L. B. Bergman, and M. N. Silver, Tolarization Currents in Varistors"

F. A. Modine, "Pulse Response Characteristics of ZnO Varistors"

F. A. Modine, "Optical and Electrical Properties of Transition-Metal Carbides" 
J. Rankin, J. C MCCallum, and L A Boatner, The Effect of $\mathrm{H}_{2} \mathrm{O}$ on Epitaxial Regrowth in lonBeam-Amorphised Layers of SrTOO

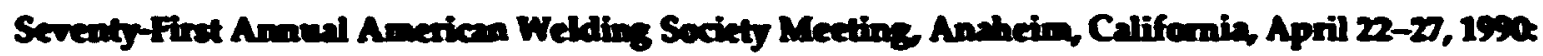

J. M. Vitek, S. A. David, M. R. Rappaz, and L A. Boatner, Evaluation of Weld Pool Shapes Using Single-Crystal Welds

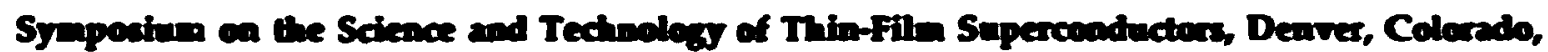
April 30-Mags 4 isen:

David P. Norton, Dougles H. Lowndes, D. K. Christen, E. D. Jones, J. D. Buda, Thomas D. Ketchams, Dell St. Julien, K. W. Lay, and J. E. Tkaczyk, Y-BaCuO Thin Films Grown on SingleCrystal and Polycrystalline Yttria-Stabilized Zirconia-

David P. Norton, Douglas H. Lowndes, J. D. Bucai, S. J. Pennycook, B. C Sales, and D.K. Christen, "Superconductivity in Single Unit Cell $\mathrm{YBaz}_{2} \mathrm{Cu}_{3} \mathrm{O}_{-2} / \mathrm{PrB}_{2} \mathrm{Cu}_{3} \mathrm{O}_{7-2}$ Supertaltices"

SoTAFOCS XI, Moatreal, Quebec, Canade, My G-11, 19\%,

S. M. Gulwadi, M. V. Reo, D. S. Simmons, O. W. Holland, and H. B. Dietrich, Fe Implantation in Inacosanas

ICMCs0 Topical Confereace: High-Tenperahure Supercoaductors, Garmisch-Partenkirchen, Cermany, Mas's-11, 19\%

W. F. Huang, S. H. Lu, and Z J. Xu, Positron Annihilation Studies of Polycrystalline YBCO'

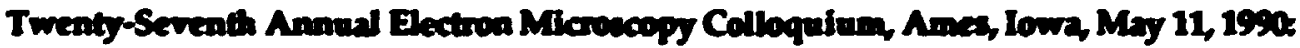

Stephen J. Pennycook, "High-Resolution Imaging of Materials by 2-Contrast STEM" (invited paper)

Symposiom on Small-Angle Neutron Seattering: Applications in Materials Science and Biolozy. Mevaster Univercits, Ontarlo, Canada, Mry 12, 19\%:

J. B. Hayter, "SANS Studies of Submicron Structures" (invited paper) paper)

C. D. Wignall, "Small-Angle Scattering Neutron Studies of Synthetic Polymers" (invited

DOE/DMS Discusilon Meeting on Neutron Scattering, Gatdinburz. Tennewee, May 14-16, 19s0.

J. B. Hayter, "Status of the Advanced Neutron Source" (invited paper) 
The Thind Conference on Froatiess of Electron Mierescopy in Materials Science, Oak Brook, Illinois, Mas 20-24, 1500:

S. J. Pennycook, High-Resolution 2-Contrast Imaging in the STEM" (invited paper)

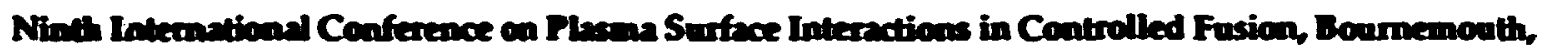

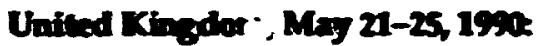

R. Siegele, J. Roth, B. M. U. Scherzer, and S. P. Withrow, Trapping of D in SiC and Damage Due to Implantation"

Conference on Medem Analyais Techniques in Materials Science and Belated Vacumm Technobsy.

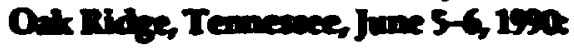

O. W. Holland, Materials Characterization by Rutherford Backscattering" (invited paper)

S. J. Pennycook, "Alomic Imaging Using Transmiscion Electron Microscopy" (invited paper)

Fifticth Anaual Conference on Fhysical Electronics, Gaitheriburg, Maryland, June 11-13, 19s0.

R. A. Bartynski, E. Jensen, S. Yang, S. L. Hulbert, C. C Kao, M. Weinert, and D. M. Zehner, Surface Electronic Structure and Interatomic Auger Transitions on TaC(111) Observed by Auger Photoelectron Coincidence Spectroscopy"

American Nudear Sockty $19 \% 0$ Anual Meeting, Nahhville, Tennescee, June 11-14, 1990:

J. B. Hayter, "Applications of the Advanced Neutron Source" (inviled paper)

Fifth International Confererice on Metalloganic Vapor Phase Epitaxy, Aachen, Germany, June 10-21, 1930.

N. H. Karam, A. Mastrovito, V. Haven, K. Lsmail, S. J. Pennycook, and H. I. Smith, Patterning and Overgrowth of Nanostructure Quantum Well Wire Arrays by LP-MOVPE"

International Discuscion Meeting on Relaxations in Complex Systems, Crete, Greece, June 18-29, 1990:

J. B. Bates, J. C. Wang and Y. T. Chu, Interface and Bulk Relaxation in Ionic Conductors" (invited paper)

P. J. Bray Symposium, Providence, Rhode Island, June 24-26, 1990.

C. E. Jellison, fr., Two-Channel Polarization Modulation Ellipsometry: A Unique Analysis Tool for Thin-film and Materials Characterization" (invited paper) 
1900 National Meeting on Figh-Temperature Supercondectivity, Taiwan, June 26-28, 1990:

W. F. Huang Z J. Xu, S. H. Liu, and M. K. Wu, Electron Momentum Redistrabution in YBCO Superconductors'

SPIE's 1950 Intermational Symposium en Optical and Optoelectronic Applied Science and

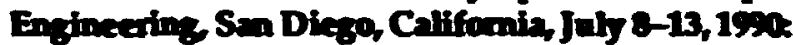

B. C Larson and J. Z Tischler, Time-Resolved Techniques: An Overview" (invited paper)

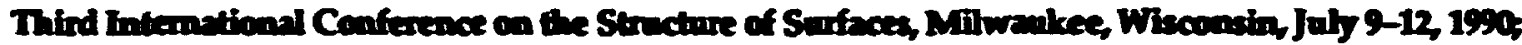

H. L Devis, D. M. Zehner, D. v. Gemunden, B. Dötsch, and K. Müller, Layer-by-Layer Segregation and Multilayer Rehation of the (100) Surface of the Random Alloy MousRe15"

H. L. Davis, J. E. Houston, and D. G. ONeill, Multilayer Relaxation of the Ru(0001) Surface and Changes Induced by Monolayer Coverage of Cu: Stucture of a Simple Metellic Interface"

D. M. Zehner, D. Gibbs, K G. Huang, B. M. Odzo, S. G. J. Mochrie, and A. R. Sandy, Structure and Phases of the Au(001), (111), and (110) Surfaces: X-Pay Scattering Measurements

Fifteenth International Coaference on $X-R$ ary and Inner-Shell Proceses, Knoxville, Tenaensee, Juby 9-13, 1950:

T. P. Rosecel, J. P. Young C. R. Vane, R. A. Zuhr, and R. S. Peterson, Tligh-Resolution HeavyIon-lnduced X-Ray Satellite Emiscion Study of Subsurface Moieties"

Eighth American Conference on Cystal Growth, Vail, Colorado, July 15-20, 19s0:

M. Rappaz, S. A. David, J. M. Vilek, and L. A. Boatner, Dendritic Microstructure Formation in Single-Crystal Welds" (invited paper)

SEMATECH-DOE National Laboratory Meeting, Austin, Texas, July 16-17, 1990:

C. E. Jellison, Jr., Thin-Film Measurement Using 2-Channel Polarization Modulation Ellipsometry" (invited paper)

Fifteenth International Congress and General Ascembly of the Intemational Union of Cryctallogophy, Bordeau, France, July 19-28, 1990:

B. C. Larson, M. D. Galloway, J Z. Tischler, and G. E. Ice, "Characterization of Precipitates Using High-Order X-Ray Diffuse Scattering" [Acta Cryst. A, Suppl. 16, C-404 (1990)]

C. W. Bartges, ! D. Budai, and E. Ryba, "A Patterson Analysis of the Structure of Icosahedral $\mathrm{Al}_{6} \mathrm{CuLi}_{3}$ Using Synchrotron Radiation Data" [Acta Cryst. Suppl. A 16, C-390 (1990)] 
H. G. Smith, R. Berliner, J. Trivisonno, and J. Jorgensen, Pressure Effects on the Martensitic Transformation in Sodium," [Acta Cryst. Suppl. A 46, C-310 (1990)]

Conference on the Physics of Simple Metal Surfaces and Thin Overlayers, Philadelphia, Pennogitrania, July 23-24, 1950:

H. L Davis, "Large Interlayer Expansion in the Be(0001) Surface"

Incernational Satilite Conference of the International Union of Crgstallographers XVth Congress and Cenerl Amembly, Grenoble, France, Jubly 30-Angust 1, 19;0.

R. Berliner, H. G. Smith, J. Copley, and J. Trivisonno, Neutron Diffraction Studies of Defect Structures Following the Martensitic Transformation in Single-Crystal Sodium Metal"

!. B. Davidsen, H. G. Smith, and R. A. Willems, "A Neutron-Sensitive Image-Intensifier Television System"

Conference on Complementary Applications of Diffaction by Neutroas and by X-Ray Synchrotron Radiation, Ape driner, France, Jubly 30-Auguat 1, 19s0:

H. Ohno, Y. Morii, S. Funahashi, M. lizumi, H. R. Child, R. M. Nicklow, and R. M. Moon, Real-Time Neutron and X-Ray Diffraction Studies of Phase Transition of Pure Metastable Tetragonal $\mathrm{ZO}_{2}$

Eighteenth Internatioan Meeting on Small-Angle Scattering, Leuven, Belgium, August 6-9, 1990.

G. D. Wignall, "Instrumental Resolution Effects in Small-Angle Scattering" (invited paper)

Twelfth International Congress for Electroa Microscopy, Seattle, Washington, August 12-18, 1990:

Y. L Chen, R. W. Cappenter, and T. P. Spreen, "Precipitation in Silicon Implanted by Oxygen and Carbon Plus Oxygen"

M. F. Chisholm and S. J. Pennycook, M.ïsfit Accommodation in Epitaxial Ge Films on Si"

D. E. Jescon and S. J. Pennycook, "Atomic Imaging of Crystals Using Large-Angle Electron Scattering in STEM"

S. J. Pennycook, D. E. Jesson, and M. F. Chisholm, "High-Resolution Z-Contrast Imaging in the STEM* (invited paper)

D. A. Smith, D. R. Clarke, M. F. Chisholm, P. E. Jatson, and E. Olson, "Transport Critical Cuments and Microstructure of Ytrium Barium Cuprate" (invited paper) 
Fifth International Conference on Solid Films and Surfaces, Providence, Rhode Island, Angust 13-17, 1990:

D. M. Zehner, G. R. Gruzalski, S.C. Lui, and D. R. Heskett, Structure and Termination of the (100), (110), and (111) Surfaces of TaC"

J.-K. Zuo and J. F. Wendelken, "High-Resolution Low-Energy Electron Diffraction Study of $(\sqrt{3} \times \sqrt{3})$ R30'-Ag Domain Growth on Si(111) Surface"

Nineteenth International Conference on Low-Temperature Physics, Brighton, England, Angust 15-22, 1990

J. R. Thompson, D. K. Christen, Yangren Sun, B. C. Chakoumakos, B. C. Sales, it. R. Kerchner, J. G. Ossandon, and E. Sonder, "Evidence of Weak Pair Coupling in the Penetration Depth of BiBased High- $T_{c}$ Superconductors"

International Congress on Membranes and Membrane Processes, Chicago, Illinois, August 20-24, 1990:

V. A. Hackley, M. A. Anderson, and S. Spooner, "Small-Angle X-Ray Scattering Investigation of Porous $\mathrm{TiO}_{2}$ Ceramic Membranes" (invited paper)

ACS Symposium on Light X-Ray, Neutron Scattering, and Reflectivity from Polymers, Washington, D.C. August 26-31, 1990.

T. Kyu, M. H. Cho, J. S. Lin, K. Saijo, and T. Hashimoto, Time-Resolved SAXS and WAXD Studies on Premelting During Annealing of Gelatin-Crystallized Ultrahigh Molecular Weight Polyethylene Films"

J. Rosedale, F. S. Bates, and G. D. Wignall, "Experimental and Theoretical Investigations of Isotope Effects in SANS Studies of Polymers"

Working Party in Electrochemistry, Trieste, Italy, August 27-September 7, 1990:

S. H. Liu, T. Kaplan, and L. J. Gray, "Fractal Electrodes: Interplay of Physics and Geometry" (invited paper)

Conference on Lattice Defects in Insulating Materials, Groningen, the Netherlands, September 3-7, 197\%:

V. M. Oreta, M. L. Sanjuan, and Y. Chen, "The [H-Ca+ $]$ Defect in Thermochemically Reduced CaO: Static and Dynamical EPR Study"

V. M. Orera, R. I. Merino, Y. Chen, R. Cases, and P. J. Alonso, "Intrinsic Defects in Stabilized Zirconia Produced by Thermoreduction or Irradiation" 
First Intemational Conference on F-Elements, Louvain, Belgium, September 4-7, 1990:

J. C. Gacon, M. Bouazaoui, B. Jacquier, L. A. Boatner, and M. M. Abraham, "Polarization Dependence of the 7F0-5D6 Two-Photon Transition in $\mathrm{Sm}^{2+}$ - and $\mathrm{Eu}^{3+}$ - Doped Materials"

Seventh International Conference on Ion Beam Modification of Materials, Knorville, Tennessee, September 9-14, 1990:

Y. Chen, M. M. Abraham, and D. F. Pedraza, "Radiation Damage in $\mathrm{Al}_{2} \mathrm{O}_{3}$ Crystals Implanted with 4 MeV Fe' Ions"

A. Golanski, W. H. Christie, M. D. Galloway, J. L. Park, S. J. Pennycook, D. B. Poker, J. L. Moore, H. E. Harmon, and C.W. White, "Ion-Beam-Induced Diffusion and Crystallization in High-Dose Er-Implanted Si"

T. E. Haynes and O. W. Holland, "Dose Rate Effects on Damage Accumulation in Si+-Implanted Gallium Arsenide"

O. W. Holland, C. W. White, J. D. Budai, and M. K. El-Ghor, "Ion-Induced Damage and Amorphization in Si" (invited paper)

R. H. Magruder III, R. A. Zuhr, and R. A. Weeks, "Dose Rate Dependence of the Optical Absorption of Cu-Implanted Silica"

C. J. McHargue, A. Perez, and J. C. McCallum, The Chemical State of Iron Ions Implanted into Silicon Carbide"

Bent Nielsen, O. W. Holiand, T. C. Leung, and K. G. Lynn, "Defects in MeV Ion-Implanted Si "robed with Positrons"

Janet Rankin, Lynn A. Baatner, and Jeffrey C. McCallum, "The Effects of Ambient Water Vapor on the Epitaxial Regrowth Processes in lon-Beam-Amorphized Layers on Single-Crystal $\mathrm{SrTiO}_{3}$ "

B. C. Sales, J. C. McCallum, and L. A. Boatnet, "Structural Differences Between the Glass State and Ion-Implanted Layers on Lead Pyrophosphate"

P. S. Sklad, C. J. McHargue, J. C. McCallum, L. Romana, and C. W. White, "The Effect of Postimplantation Annealing on the Microstructure of $\mathrm{Al}_{2} \mathrm{O}_{3}$ Implanted with Iron at $-185^{\circ} \mathrm{C}$ "

M. L. Swanson, J. D. Hunn, N. R. Parikh, R. A. Zuhr, W. R. Lennard, and Th. Wichert, "Implantation Doping of Diamond"

J. M. Williams, A. Conzales, J. Quintana, R. A. Buchanan, I-S. Lee, J. Brodkin, F. C. Burns, R. J. Culbertson, W. Franzen, M. Levy, and J. R. Treglio, "Ion Implantation for Corrosion Inhibition of Aluminum Alloys in Saline Media"

Catherine E. Vallet and C. W. White, "Examples of Application of Ion Implantalion to Electrochemical Studies"

W. Xia, C. Cozzolino, J. Zhang, C. W. Tu, P. K. L. Yu, S. S. Lau, and D. B. Poker, "Ion Mixing of Semiconductor Superlattices" 
R. A. Zuhr, T. E. Haynes, M. D. Galloway, S. Tanaka, A. Yamada, and I. Yamada, "Formation of Aluminum Films on Silicon by Ion Beam Deposition: A Comparison with lonized Cluster Beam Deposition"

Twenty-Fifth Colloque Ampere, Stuttgat, Cermany, September 9-14, 1990:

M. M. Abraham, J. M. Baker, B. Bleaney, A. A. Jenkins, P. M. Martineau, and J. Z Pfeffer, Magnetic Resonance of Probe $\mathrm{Yb}^{3+}$ and $\mathrm{Er}^{3+}$ lons in the Antiferromagnetic Phase of $\mathrm{DyPO}_{4}$ "

Sixth International Conference on Vibrations at Surfaces, Shelter Island, New York, Seplember 10-14, 19s:

Arthur P. Baddorf and E. W. Plummer, Surface Anharmonicity: Temperature Dependence of Phonon Energies on Cu(110)

New Yodk State Institute on Superconductivity's Fourth Annual Conference en Superconductivity and Applications, Buffalo, New York, September 18-20, 1990.

D. K. Christen, "Orientation-Dependent Critical Currents in $\mathrm{Y}_{1} \mathrm{Ba}_{2} \mathrm{Cu}_{3} \mathrm{O}_{7-x}$ Epitaxial Thin Films: Evidence for Intrinsic Flux Pinning" (invited paper)

Third Annual U.S.-Japan Workshop on Supercoaductors, Buffalo, New Yark, September 20-21, 1990.

D. K. Christen, "Limitations on Critical Current by Thermally Activated Processes" (invited paper)

1990 Applied Superconductivity Conference, Snowmass Village, Colorado, September 24-28, 1990,

H. R. Kerchner, J. O. Thomson, J. R. Thompson, J. Ossandon, D. Norton, D. H. Lowndes, R. Feenstra, and D.K. Christen, "Magnetic Hysteresis and AC Response of In Situ Grown $\mathrm{YBa}_{2} \mathrm{Cu}_{3} \mathrm{O}$ Superlattice Thin Films" 


\section{Seminars}

\section{SOLID STATE DIVISION SEMINARS AT ORNL}

During this period H. L. Davis served es Seminar Chairman. The following seminars were held:

Optical Properties of High-T, Cuprates, P. C. Ekhund, Univensity of Kentuchy, Lexington, $K_{y}$.

Static Coulomb Model of High-Tenperature Superconductivity," D. Mattis, University of Utah, Salt Lake City, Utah

Fnhanced Epitaxial Growth in the Vacuum of Spece: The Merger of Thin-Film Semiconductor and Thin-Fim Superconductor Technologies," A. Ignatiev, University of Houston, Houston, Tex.

"Perspective on the Theories of High-Temperature Supenconductivity," M. L. Cohen, University of California, Berkeley, Calif.

"Laser-Induced Pyrolysis of Triethylgallium on GaAs(100) and Its Application to Selected Area Growth," J. A. McCauley, ATKT Bell Laboratories, Murray Hill, N.J.

Methods of Waveguide Production in $\mathrm{LNbO}_{3}$ and $\mathrm{KNbO}_{3}$ " T. Bremer, University of Osnabruck, Oanabruck, Germany

In Situ Preparation of YBCO Superconducting Thin Films Using Pure Ozone Vapor Oxidation," D. Berkeley, University of Minnesota, Minneapolis, Minn.

"Phenomenological Model of Copper Oxide Superconductors," R. A. Kemm, Ames Laboratory, Ames, lowa

"Growth Mode of MBE-Deposited Molybdenum on Silicon," A. Shapiro, University of Arizona, Tempe, Ariz.

"Ion-Induced Electron Emisaion from Materials," W. Hoter, Forschungazentrum, Jülich, Cermany

"Top-Seeded Solution Growth of $\mathrm{BaTiO}_{3}, \mathrm{KNbO}_{3}$, and $\mathrm{KTN}$ for Photoreflective and Nonlinear Optical Applications," D. Rytz, Hughes Research Laboratory, Malibu, Calif.

"Measurement of Phonon Dispersion Curves by Inelastic X-Ray Scattering" B. Dornea, Institut Lave-Langevin, Grenoble, France

Positron Annihilation Studies of the Electronic Structure of High- $T_{c}$ Superconductors and Related Materials," A. L. Wachs, Lawrence Livermore National Laboratory, Livermore, Calif. 
Pulsed-Laser Deposition of High-T, and Superconducting Thin Films: Theory and In Situ Processing, R. K. Singh, North Carolin State University, Raleigh, N.C.

"Critical Currents in $\mathrm{YBa}_{2} \mathrm{Cu}_{3} \mathrm{O}_{3}$ s Thin Films," L. Allen, Naval Research Laboratory, Washington, D.C.

On the Electroreduction of Thionyl Chloride," J. J. Sonith, Department of Energy, Washington, D.C.

The Theory and Use of Resonant Nuclear Reaction Depth Profiling in Solid State Physics," I. Vickridge, University of Paris VI, Paris, France

In Situ Diagnostics of Superconductor and Diamond Film Deposition Proceses," P. W. Morrison, Jr., Advanced Fuel Research, Inc., Enst Hartford, Conn.

"Exciton Model of High-T, Y123 Superconductors Based on Band Structure Cakculations," T. C. Collins, The University of Tennessee, Knoxville, Tenn.

Recent Development in the Percolation Theory of Phase Transitions," C. K. Hu, Academia Sinica, Taipei, Taiwan

"Ton Damage in B1 Phase Superconductors and a Displacerless Regenerative Cryocooler," J. Elis, Alabama Cryogenic Engineering Inc., Huntsville, Ala.

Recent Progress in the Statistical Mechanics of Phase Coarsening, M. E. Glicksman, Rensselaer Polytechnic Institute, Troy, N.Y.

"Creation Dynamics and Spatial Correlation Effects in Annealing Kinetics of Defects in IonImplanted Amorphous $\mathrm{SiO}_{2}$ " A. Colanski, Centre National d'Etudes des Telecommunications, Grenoble, France

Neutron Scattering Studies of Liquid Arsenic and Liquid Sulfur," R. Bellissent, Laboratoire Lén Brillouin, Saclay, France

Neutron Scattering Studies of the Magnetic Excitations in Thulium," J. A. Fernandez-Baca, Solid State Division, ORNL

"Dissipative Flux Motion in the High-Temperature Superconductors," T. T. M. Palstra, AT\&T Bell Laboratories, Murray Hill, N.J.

"Recent Optical Investigations in Ag Halides by Picosecond Spectroscopy," W. von der Osten, University of Paderbom, Paderborn, Cermany

"Impurity Levels and Excitons in Quantum Well Systems," J. J. Quinn, The University of Tennessee, Knoxville, Tenn.

"Mutlicomponent Oxide Thin Films Including Superconductors and Ferroelectrics," A. Kingon, North Carolina State University, Raleigh, N.C.

"Magnetic Excitations in $\mathrm{La}_{2} \mathrm{CuO}_{4}$ " S. Hayden, Institut Laue-Langevin, Grenoble, France 
Threshold-Level Laser Ablation of Solid Targets: Product Kinetic Energy Distribution," H. Halvä̈an, Aerospace Corp., Los Angeles, Calif.

Do the High-Temperature Superconductors Have an Anisotropic Gap? R. A. Kemm, Ames Laboratory, Ames, lowa

Focused lon Beam Technology and Its Applications," J. Melingailis, MIT, Cambridge, Mass.

Mondous (M = Cu, Ni, Co) Phase Separation and Superconductivity Resulting from Excess Oxygen," B. Dabrowski, Argonne National Laboratory, Argonne, $\mathbf{m}$.

"Spin-Polaron Theory of High-T, Superconductivity," R. F. Wood, Solid State Division, ORNL

Zone Boundary Collection States in Simple Metals," L. E. Otiveira, Universidad de Flummence, Rio de Janeiro, Brazil

Resistance Fluctuations in Small Samples," S. Washburn, IBM Thomas J. Watson Research Center, Yorktown Heights, N.Y.

Fractional Statistics," G. C. Conright, Indiana University, Bloomington, Ind.

Some Reseanch Activities at the University of Tsukuba: (1) Electronic Structure of Epitaxial $\mathrm{SiO}_{2} / \mathrm{Si}$ Interfaces and (2) Atomic Layer Epitaxy of Si, T. Motooka, University of Tsukuba, Tsukcuba, Japan

Ton Beam Research at Hitachi," K. Miyake, Hitachi Research Laboratories, Hitachi City, Japan

"Epitaxial Deposition of Al on Si by Ionized Cluster Beam Deposition," I. Yamada, Kyoto University, Kyoto, Japan

"Some Recent Experiments of Surface Studies by Nonlinear Optics," Y. R. Shen, University of Califomia-Berkeley, Berkeley, Calif.'

"Selective Area Epitaxy of GaAs on Si by Atomic Layer Epitaxy," N. H. Karam, SPIRE Corporation, Bedford, Mass.

"Optical Studies of Semiconductor Microstructures," B. MoCombe, State University of New York at Buffalo, Buffalo, N.Y.

"Semiconductors, Surfaces, and Superlattices," L. C. Feldman, ATkT Bell Laboratories, Murray Hill, N.J.

"Modem Optical Analyais of Exotic Solids," S. Perkowitz, Emory University, Atlanta, Ga.

"Quantum Smearing of the Coulomb Blockade," L. I. Glazman, Institute of Microelectronics Technology and High-Purity Materials, USSR Academy of Sciences, Chernogolvka, USSR

"Oxide Nonlinear Conductors," L. M. Levinson, Ceneral Electric Corporate R\&D, Schenectady, N.Y.

"The Magnetic Phase Diagram of High-Temperature Superconductors," A. Malozemoff, IBM T. J. Watson Research Center, Yorktown Heights, N.Y. 
Tntralayer Versus Interhyer Pairing in the Copper Oxide Superconductors," R. A. Klemm, Solid State Division, ORNL

Neutron Scattering Determination of Phonon Dispersion in High-T, Cuprates," W. Reichandt, Kemforschungseentrum, Karksuhe, Cermany

The Cold Neutron Source at Kyoto University Reseanch Reactor," H. Yamaoka, Kyoto University. Kyoto, Japan

Monte Carlo Calculations of Correlation Functions for Heisenberg Antiferromagnets," S. Liang, University of Mlinois, Urbana, 111.

Universality in a Triangular Antiferromagnet," T. Mason, McMaster University, Hamilton, Ontario, Carseda

"Growth and Properties of Epitaxial YBCO-PrBCO Superlattices Containing Ultrathin Superconducting Layers," D. H. Lowndes, Solid State Division, ORNL

Tectron Microscopy Studies of Structural Imperfections in Oxide Superconductors," K. Zandberger, Delft University of Technology, the Netherlands

"Optical Matter: Crystallization and Binding in Intense Optical Fields," G. A. Golovchenko, Harvard University, Cambridge, Mass.

"Solid State Device R\&D at Martin Marietta Laboratories," J.-M. Chen, Martin Marietta Laboratories, Baltimore, Md.'

Ton Beam Deposition Modification and Synthesis of Thin Films," J. J. Cuomo, IBM T. J. Watson Research Center, Yorktown Heights, N.Y.

Tsaves and Flux Creep in Type-II Superconductors," S. N. Coppersmith, AT\&T Bell Laboratories, Murray Hill, N.J.

"Thin-Film Characterization Using Scanning-Acoustic Microscopy," G. M. Crean, National Microelectronics Research Centre, University College, Cork, Ireland

"Universal Resistance at the Superconductor-Insulator Transition," S. M. Girvin, Indiana University, Bloomington, Ind.

"Photoemission Imaging, Diffraction, and Holography of Metastable Ultrathin Films," B. Tonner, University of Wisconsin, Milwaukee, Wis.

"Growth and Stability of Thin Films," M. F. Chisholm, Solid State Division, ORNL

"Corrections to Fresnel Optics at Surfaces," W. L. Schaich, Indiana University, Bloomington, Ind.

"Basic Phenomena and Deposition Techniques for the Synthesis of Multicomponent and Multilayered Films for Advanced Technologies," O. Auciello, Microelectronics Center of North Carolina, Research Triangle Park, N.C.

"Breakdown of the Spin-Wave Model in a Heisenberg Ferromagnet," R. S. Fishman, North Dakota State University, Fargo, N. Dak. 
Quasicrystal Dynamics," R. Stinchcombe, Oxford University, Oxfond, United Kingdom

Dynamic Percolntion Theory: Carrier Transport in a Dynamically Evolving Environment," A. Nitean, Tel Aviv University, Tel Aviv, Isreel

"Surface Studies Using Time-of-Flight Neutron Reflectometry," W. A. Hamilton, Los Alamos National Laboratory, Los Alamos, N. Mex.

Variations in Vertical Vibrations at the Verge of Vacurum: A Study of Anharmonicity in Surface Phonons," A. P. Baddurf, Solid State Division, ORNL

"Negative Ion Resonances in Electron Scattering by Oriented Molecules on Surfaces," R. E. Palmer, Cavendish Laboralory, Cambridge, England

Neutron Scattering Investigation of the Orientation Disorder in the Plastic Gamma-Phase of Solid Oxygen," Fredric Durstetter, Laboratoire Leon Brillouin, Saclay, France

"Physics of Laser Ablation of Superconductors, Metals, and Metal Oxides," L Lynds, United Technologies Research Center, East Hartford, Conn.

MOCVD Growth of Novel Materials," A. Erbil, Georgia Institute of Technology, Attanta, Ga.

"Ion Surface Interactions and Surface Spectroscopy During Si-Ge Epitaxial Growth," H. A. Atwater, California Institute of Technology, Pasadena, Calif.

"Growth, Modification, and Superconducting Properties of Epitaxial $\mathrm{YBa}_{2} \mathrm{Cu}_{3} \mathrm{O}_{7-x}$ Films," R. Feenstra, Solid State Division, ORNL

1Metals and Ceramics Division and Solid State Division Joint Seminar.

\section{LECTURES AND SEMINARS BY DIVISION MEMBERS}

A. P. Baddorf-University of Rhode Island, Providence, Rhode Island, Variations in Vertical Vibrations at the Verge of Vacuum: A Study of Anharmonicity in Surface Phonons"

J. B. Bates-Instrumentation and Controls Division, Oak Ridge National Laboratory, Oak Ridge, Tennessee, "Ceramic Thin Films and Thin-Film Devices"

L. A. Boatner-Texas Tech University, Lubbock, Texas, "The Characterization and Formation of Textared Nanophase Ceramics"

J. D. Budai-Michigan Technological University, Houghton, Michigan, "Structural Studies of YBaCuO Thin Films on (001) SrTiO and $\mathrm{KTaO}_{3}{ }^{\prime \prime}$ 
D. K. Christen-University of Louisville, Louisville, Kentucky, Critical Currents and Dissipation in High-T, Superconducting Thin Films"; IBM T. J. Watson Research Center, Yorktown Heights, New York, "Orientation-Dependent Critical Currents in $\mathrm{Y}_{1} \mathrm{Ba}_{2} \mathrm{Cu}_{3} \mathrm{O}_{7-x}$ Epitaxial Thin Films: Evidence for Intrinsic Fux Pinning"; University of Louisville, Louisville, Kentucky, Magnetic and Transport Studies of High-Temperature Superconductors"

J. F. Cooke-Rutherford Appleton Laboratory, Oxfordshire, United Kingdom, Spin Excitations in Magnetic Systems"

H. L. Davis-Sandia National Laboratories, Albuquerque, New Mexico, LEED Studies of the Relaxation of Metallic Surfaces-From Cu(100) to NiA(111) and Beyond"

J. A. Fernandez-Bacn-Paul Scherrer Institut, Villigen, Switzerland, Neutron Scattering Study of the Magnetic Excitations"; Florida Institute of Technology, Melbourne, Florida, Neutron Scattering Studies of Magnetic Excitations in Solids"

T. E Haynes-Alabama A\&M University, Normal, Alabama, Ton Beam Deposition of Compound Semiconductors"; Japan Atomic Energy Research Institute, Tokai-mura, Japan, "Material Modification by Low-Energy Ion Beam Deposition"; Hitachi Research Laboratory, Hitachi-shi, Japan, "Low-Energy Ion Beam Deposition of Epitaxial Semiconductor Layers"; Osaka University, Osaka, Japan, "Recent Activities in lon-Solid Interactions at Oak Ridge National Laboratory"

J. B. Hayter-Engineering Physics and Mathematics Division, Oak Ridge National Laboratory, Oak Ridge, Tennessee, "Applications of Cold Neutrons"; Institut Laue-Langevin, Grenoble, France; Forschungzentrum, Jülich, Cermany; Laboratoire Lén-Brillouin, Saclay, France, The Advanced Neutron Source"; University of Rhode Island, Kingston, Rhode Island, Small-Angle Neutron Scattering from Submicron Structures"

G. E. Jellison, Jr.-University of Alabama, Huntsville, Alabama, Twn-r nannel Polarization Ellipsometry: A Unique Analysis Tool for Thin-Film and Materials Char.' _cerization"

T. Kaplan-Alabama A\&M University, Huntsville, Alabama, "Dynamics of Sand Piles"

H. R. Kerchner-Pellissippi State Technical Community College, Knoxville, Tennessee, Lenoir City High School, Lenoir City, Tennessee, and Webb School of Knoxville, Knoxville, Tennessee, "Superconductivity"; Christian Academy of Knoxville, Knoxville, Tennessee, and Lenoir City High School, Lenoir City, Tennessee, "Cryogenics"

B. C. Larson-Virginia Polytechnic Institute and State University, Blacksburg, Virginia, Tnvestigation of Precipitates and Dislocation Loops in Materials Using X-Ray Diffraction"

S. H. Liu-Exxon Research and Engineering Company, Annandale, New Jersey, "Geometric and Dynamic Properties of Deterministic Sand Piles"

D. H. Lowndes-Harvard University, Cambridge, Massachusetts, "Rapid Heteroepitaxial Film Growth: The Roles of Kinetic Processes and Kinetic Energy"; Alabama A\&M University, Normal, Alabama, "Fabrication and Characterization of High-Te Superconducting Thin Films" 
G. D. Mahan-Norwegian Technical University, Trondheim, Norway, Transport in Semiconductors" (six lectures); Texas Inotruments Corporation, Austin, Texas, Hot Electrons in Semiconductors"; Michigan Stak University, East Lanwing, Michigan; Physics Division, Oak Ridge National Laboratory, Oak Ridge, Tennessee; Centro Alonvico, Bariloche, Argentina; International Centre Condensed Matter Physics, Brasilia, Brazil; Institute for Spectroscopy, Moscow, U.SS.R.; Leveder Institute, Moscow, USSR; Kunchatov Institute, Moscow, USSR; Clemson University, Clemson, South Carolina; and University of Toronlo, Toronto, Canada, Band Width of Metals"

D. N. Mashburn-The University of Tennessee, Knoxville, Tennesce, Use of Laser Ablation in Prepartion of Thin Films of High-Temperature Superconductors and Other Materialso

F. A. Modine-University of Richmond, Richmond, Virginia, Electrical Properties of TransitionMetal Carbides"

R. M. Nictdow-University of South Carolina, Columbia, South Carolina, Neutron Diffraction Studies of Nuclear Magnetic Order in Cystals"

D. P. Norton-Louisiana Stale University, Baton Rouge, Louisiana, In Situ Thin-Film Growth of YBazCug $\mathrm{O}_{2}$ Superconductors by Laser Ablation"

S. J. Pennycook-University of Bristol, Bristol, United Kingdom, Z-Contrast STEM Imaging of Superconductors and Semiconductors"; Erizona State University, Tempe, Arizona, ThighResolution Imaging of Superconductors and Semiconductors by Z-Contrast Imaging"; IBM T. J. Watson Research Center, Yorktown Heights, New York; Rutgers University, New Brunswick, New Jersey; and North Carolina State University, Raleigh, North Carolina, Thigh-Resolution Imaging of Superconductors and Semiconducters by Z-Contrast STEM"

D. B. Poker-Alabama A\&M University, Normal, Alabama, "Optical Waveguide Formation by Ion Implantation of $\mathrm{Ti}$ into LiNbO3"; Massachusetts Institute of Technology, Cambridge, Massachusetts, Ton Processing of Materials: Ion Implantation and Annealing of Ceramics and Optical Materials"

M. Rasolt-IBM, Yorktown Heights, New York, "Another Look at Chiral Symmetry States in High-Temperature Superconductors"; Mount Allison University, Sachville, Canada, High-T, Superconductivity: Theory and Experiment" and "An Examination of High-T ${ }_{c}$ Superconductivity"; Dalhousie University, Halifax, Canada, "Density Functional Theory in Strong Magnetic Fields"; University of Missouri, Columbia, Miscouri, Dissipation Due to a Valley Wave' Channel in the Quantum Hall Effect of a Multivalley Semiconductor"

J. B. Roberto-SEMATECH, Austin, Texas, "Semiconductor-Related Research Activities in the Solid State Division"

T. P. Sjoreen-Alabama A\&M University, Normal, Alabama, MeV Ion Implantation of Materials"

J. R. Thompson-Forschungzentrum Jülich, Jülich, Germany; Kemforschungszentrum, Karlsruhe, Cermany; and Kamerlingh Onnes Laboratorium, University of Leiden, the Netherlands, "Studies of the Magnetic Penetration Depth of High-T Compounds: Evidence for Weak Coupling Superconductivity"; University of Louisville, Louisville, Kentucky, Magnetic and Transport Studies of High-Temperature Superconductors"

J. 2. Tischlet-Ohio University, Athens, Ohio, "Resonant Nuclear Brag8 Scattering" 
G. D. Wignall-Eastman Kodak Company, Kingsport, Tennessee, Small-Angle Facilities and Polymer Research at Oak Ridge"; Xerox Research Center, Mississauga, Ontario, Canada; University of Toronto, Toronto, Canada; and University of Waterbo, Ontario, Canada, SmallAngle Scattering Neutron Studies of Synthetic Polymers"; Eastman Chemical Company, Oak Ridge, Tennessee, Small-Angie Neutron Scattering from Polymers"; Japan Alomic Energy Research Institute, Tokai, Japan, Why So Much Time and Money to Study the Origin of Q-Space?; PostConference Course on Theory and Practice of Small-Angle Scattering, Leuven Betgium, Instrumental Aspects of Small-Angle-Neutron Scattering"

J. M. Williams-Alabama A\&M University, Normal, Alabama, Beam Ion Implantation in Biomaterials"

D. M. Zehner-University of Rhode Island, Kingston, Rhode Island, Ceometric Surface Structure of Ordered and Random Alloys"

R. A. Zuhr-Vanderbilt University, Nashville, Tennessee, Formation of Thin Films by Direct lon Beam Deposition" 


\section{Scientific Activities, Awards, and Honors}

M. M. Abraham

A. P. Baddorf

J. B. Bates

L. A. Boatiner

J. D. Budai
Visiting Fellow, St. Catherine's College, Oxford University, Oxford, United Kingdom

Member, Executive Board, Tennescee Valley Chapter, American Vecuum Society, 1909-1990

Vice-President, Tennessee Valley Chapter, American Vacuum Society, 1990-1991

Lecture, ORAU Travding Lecture Program, 1988-1989

Co-Organizer, Symposium on Inorganic Solid Electrolytes and Electrodes, 17oth Meeting of the Electrochemical Society (to be held in 1990)

Member, Search Committee for ORNL Solid State Division Director

Chairman, Gordon Research Conference on Solid State lonics, July 1909

Member, International Scientific Committee, Seventh International Conference on Solid State Ionics, Hakone, Japan, November 1909

Recipient, 1990 Intermational Metallographic Contest, Optical Microscopy - First in Class Award

Recipient, Martin Marietta Energy Systems Significant Event Award, 1999

Recipient, Martin Marietta Energy Systems Publication Award, $1 \% 9$

Co-Chaiman, Program Committee, Eighth American Conference on Crystal Growth, Vail, Coloredo, Juby 1990

Chairman, ORNL-Universidad Nacional Autonoma de Mexico Joint Steering Committee

Member, Excecutive Committee, American Association for Cystal Growth

Chaiman, Miembership Committee, Materials Research Society, 1909

Session Chaiman, American Physical Society Meeting, Anaheim, Californit, March 1990

Member, ORNL Showcase Talk Selection Committee, 1990-1991

Sescion Chairman, Materials Research Society Symposium, Boston, Masachusetts, November 1989

Recipient, Martin Marietta Energy Systems Technical Achievement Award, 1990

Lecturer, Soclety of Physics Students Speakers Program 
J. W. Cable

B. C. Chakoumakas

Y. Chen

D. K. Christen

J. F. Cooke
Visiting Scientist, Laboratoire Léon Brillouin, Saclay, France, October 1909-April 1990

Participant, Refinement Round-Robin sponsored by the Commission On Powder Diffraction, International Union of Crystallography, 1990

Volunteer Abstractor, Mineralogical Abstrects, 1989

Sescion Chairman, F. Donald Bloss Symposium, Virginia Technological Institute, Backsburg, Virginia, 1990

Traveling Lecturer, Onk Ridge Associated Universities, 1990-1991

Adjunct Professor, North Carolina State University, Raleigh, North Carolina

Member, Review Committee for the Australian Government Grant Scheme, 1999

Detailee, DOE/OBES/DMS, Washington, D.C., 1990-1991

Co-Chairman, Materials Physics Topical Group on Optical Materials, American Physical Society Meeting. Cincinnati, Ohio (to be held in 1991)

Session Chairman, Europhysical Conference on Lattice Defects in Insulating Materials, Groningen, The Netherlands, 1990

Co-Chairman, Symposium on Synthesis and Processing in Ceramics: Scientific Issues, Materials Research Society, Anaheim, California (to be held in 1991)

Co-Chairman, DOE Research Assistance Task Force Meeting on Dielectric Breakdown in Insulating Ceramics Enhanced by a High-Radiation Field (to be held in 1991)

Adjunct Professor, Department of Physics, The University of Tennessee, Knoxville, Tennessee

Recipient, Martin Marietta Energy Systems Significant Event Awand, 1989

Co-Organizer, Symposium on High-Temperature Superconductors: Fundamental Properties and Novel Materials Processing, Materials Research Society, Boston, Massachusetts, November 1989

Co-Editor, High-Temperature Superconductors: Fundamental Properties and Nooel Materials Processing, Materials Research Society, Pittsburgh, Pennsylvania, 1990

Session Chairman, Conference on the Science and Technology of Thin-Film Superconductors, Denver, Colorado, 1990

Co-Organizer, DOE/DMS Discussion Meeting on Theory Applied to Materials, Gatlinburg, Tennessee, November 1989

Member, Organizing Committee, and Session Chairman, American Physical Society, Anaheim, California, March 1990

Member, Advisory Committee, lournal of Physics, Condensed Matter

Co-Organizer, Workshop on Theory and Modeling, Southeast Regional Conference on Materiais Science \& Engineering for the 1990's, Gainesville, Florida, 1990 
Participan, DOE/DUS Discussion Meeting on Neutron Scattering, May 1990

Traveling Lecturer, Onk Ridge Associated Universities, 1988-1989

H. L. Davis

N. J. Dixdney

J. A. Fernar sez-Baca

D. B. Geohegan

S. M. Corbatkin
Honored as one of 25 "Accomplished Graduates" at Bowting Green State University's Spring Commencement 100,000th Greduate Celebration, Bowling Green, Ohio, 1989

President, Tennessee Valley Chapter of the American Vacuum Society 1988-1989

Member, Selection Committee, Morton M. Traum Award, 37th Amual Symposium of the American Vacuum Society, Toronto, Ontario, Canada, 1990

Participant, DOE/DMS Discussion Meeting on Theory Applied to Materials, Gatinburg Tennescee, 1989

Past President, Tennesiee Valley Chapter of the Aorsican Vecuum Society, 1909-1990

Member, Selection Committee, Martin Marietta Energy Systems Awards for Outstanding Publications, 1990

Session Chaiman, Thind Intemational Conference on the Structure of Surfaces, Milwaukee, Wiscorsin, 1990

Participant, Southest Regional Confurence on Materials Science and Engineering for the 1990s, Gaines ville, Florida, 1990

Member, Search Committee for ORNL Solid State Division Director

Recipient, Award for Distinguished Scientific Achievement, East Tennessee Section, Aseociation of Women in Science, 1989

Associate Editor, Joumal of the American Ceramic Society

Abstractor, American Ceramic Society

Judge, Southem Appalachia Science and Engineering Fair, 1909

Traveling Lecturer, Oak Ridge Associated Universities, 1989-1990

Recipient, Martin Marietta Energy Systems Technical Achievement Award, 1990

Traveling Lecturer, Oak Ridge Associated Universities, 1990-1991

Member, Organizing Committee for Plasma Processing Session, American Physical Society Meeting, Anaheim, Califomia (to be held in 1991)

Co-Organizer, Workshop on High-Density Plasma Sources for Integrated Circuit Manufacturing. Burlingame, California, 1990

Chairman, ECR Microwave Plasma Deposition Session, 37th Annual American Vacuum Society Symposium, Toronto, Canada, 1990

Member, Plasma Science Program Committee, 38th Annual American Vacuum Society Symposium, Seattle, Washington (to be held in 1991) 
P. H. Green

T. E. Haynes

J. B. Hayter
G. E. Jellison, Jr.
T. Kaplan

Executive Secretary, Search Committee for ORNL Solid State Division Director

Conference Secretary/Treasurer, Seventh International Conference on Ion Beam Modification of Materials, Knoxville, Tennessee, September 1990

Secretary/Treasurer, DOE/DMS Discussion Meeting on Theory Applied to Materials, Gatlinburg. Tennessee, November 1989

Secretary/Treasure, DOE/DMS Discussion Meeting on Neutron Scattering, Gatimburg, Tennessee, May 1990

Recipient, Achievement Award in Periodic Activity Reports, 1990 Technical Communication Award, East Tennessee Chapter of the Society for Technical Communication

Organizer, Session on Thin Films: Advanced Growth and Characterization Techniques, Ninth Annual Tennessee Valley Chapter-American Vacuum Society Symposium, Oak Ridge, Tennessee, 1969

Co-Chairman, Program Committee, and Session Organizer, Seventh International Conference on Ion Beam Modification of Materials, Knoxville, Tennessee, September 1990

Member, Executive Board, Tennessee Valley Chapter, American Vacuum Society

Recipient, R\&D 100 Award for Transmission Polarizer for Neutron Beams, 1909

Executive Member, National Steering Committee for the Advanced Neutron Source

Member, LANSCE Advisory Committee, Los Alamos National Laboratory, Los Alamos, New Mexico, 1987-1990

Member, Liquids Editorial Board, !ournal of Physics, Condensed Matter, 1989-1990

Organizer and Co-Chairman, ORNL/BNL Workshop on Neutron Scattering Instrumentation, Oak Ridge, Tennessee, 1989

Scientific Director, Advanced Neutron Source Project, ORNL.

Session Chairman, Gordon Research Conference on Dynamics of Macromolecular and Polyelectrolyte Solutions, Oxnard, California, 1990

Session Chairman, International Group on Research Reactors, Knoxvilke, Tenncsee, 1990

Witness, Congressional Subcommittee on Energy Research and Development, Committee on Science, Space, and Technology, Washington, D.C.

Traveling Lecturet, Oak Ridge Associated Universities, 1988-1989

Member, Editorial Board, Applied Physics Communication

Member, Proposal Review Committee, Martin Marietta Energy Systems, Inc. 
B. C. Larson

S. H. Liu

D. H. Lowndes

G. D. Mahan

H. A. Mook

M. Mostoller
Chairman, Policy and Advisory Board, Cornell High Energy Synchrotron Source, Cornell University

Co-Organizer, Materials Physics Topical Group Focused Session on Time-Resolved Structural Kinetics of Materials (to be held in 1991)

Co-Editor, Charecterization of the Structure and Chemistry of Defects in Materials, Materials Research Society, Boston, Massachusetts, 1989

Member, Program Review Committee, Department of Physics, University of Missouti, Columbia, Missouri, 1990

Member, Program Committee, 12th International IEEE Conference, Engineering in Medicine and Biology Society

Co-Chairman, Symposium on Surface Chemistry and Beam-Solid Interactions, Materials Research Society, Boston, Massachusetts (to be held in December 1990)

Co-Chairman, Focused Session on Epitaxial Layers of High-T Superconductors, American Physical Society, Cincinnati, Ohio (to be held in 1991)

Member, Program Committee, Workshop on Pulsed-Laser Ablation, Oak Ridge, Tennessee (to be held in 1991)

Recipient, Martin Mariett Energy Systems Technical Achievement A ward, 1990

Recipient, Martin Marietta Energy Systems Significant Event Award, 1990

Member, Search Committee for ORNL Solid State Division Director

Member-at-Iarge, Executive Committee, Division of Condensed Matter Physics, American Physical Society, 1989-1992

Chairman, Scientific Committee, International Centre of Condensed Matter Physics, Brazil, 1989-1993

Member, Search Committee for ORNL Solid State Division Director

Member, Editorial Board, Intermational Joumal of Modern Physics $B$

Chairman, Workshop on Many Body Theory, International Center of Condensed Matter Physics, Brasilia, 1990-1991

Guest Professor, Norwegian Institute of Technology, Trondheim, Norway, March 1990

Recipient, R\&D 100 Award for Transmission Polarizer for Neutron Beams, 1989

Vice Chairman, Martin Marietta Energy Systems Corporate Fellows Council

Member, UT-ORNL Distinguished Scientists Selection Committee

Member, Search Committee for ORNL Solid State Division Director

Recipient, Martin Marietta Energy Syatems Technical Achievement Award, 1990 
R. M. Nicklow

J. R. Noonan

D. P. Norton

S. J. Pennycook

D. B. Poket

M. Rasolt

J. B. Roberto
Recipient, Martin Marietta Energy Systems Technical Achievement Award, 1990

Member, Triple-Axis Spectrometer Subcommittee, Workshop on Neutron Scattering Instrumentation at High Flux Reactors, 1989

Secretary-Treasurer, Surface Science Division, Member, Publications Committee, American Vacuum Society

Member, Subcommittee on Journals, American Institute of Physics

Member, Editorial Board, Journal of Vacuum Science and Technology

Member, Board of Directors, American Vacuum Society, 1989-1990

Co-Chairman, AVS-ASM Conference on Modern Analysis Techniques in Maberials Science, Oak Ridge, Tennessee, June 1990

Recipient, Outstanding Dissertation Award, Louisiana State University, 1989

Recipient, DOE Awand for Outstanding Scientific Accomplishment in Solid State Physics, 1999

Recipient, R\&D 100 Award for Scanning Transmission Electron Microscope (VG Microscopes HB501 UX STEM), 1990

Co-Chairman, Program Committee, and Session Organizer, Seventh International Conference on Ion Beani Modification of Materials, Knoxville, Tennessee, September 1990

Co-Editor, Proceedings of the Seventh International Conference on Ion Beam Modification of Materials, Knoxville, Tennessee (to be published in 1991)

Co-Editor, Optical Materials: Processing and Science, Materials Research Society, Pittsburgh, Pennsylvania, 1989

Co-Chairman, Symposium on Optical Materials: Processing and Science, Materials Research Society, San Diego, California, 1999

Traveling Lecturer, Oak Ridge Associated Universities, 1988-1989

Co-Organizer, International Meeting on Analytical Versus Numerical Treatments of Models in High- $T_{c}$ Superconductivity, Orsay, France, 1990

Traveling Lecturer, Oak Ridge Associated Universities, 1990-1991

First Vice President (President-Elect), Materials Research Society, 1990

Second Vice President, Materials Research Society, 1989

Organizer, Symposium on Atomic Resolution Structure and Chemistry of Surfaces, American Physical Society, Anaheim, Califomia, 1990

Member, ORNL Overhead Oversight Committee, 1990

Member, ORNL Waste Management Operations Committee, 1990

Member, ORNL Image Committee, 1990

Member, Technical Achievement Awards Committee, Martin Marietta Energy Systems, Inc., 1990 
Member, Technology Transfer Strategic Planning Group, Martin Marietta Energy Systems, Inc.

Member, Local Organizing Committee, International Conference on Ion Beam Modification of Materials, Knoxville, Tennessee, 1990

Member, Long-Range Planning Committee, Materials Research Society, 1989-1990

Co-Editor, Ion Beam Processing of Adonnced Electronic Materials, Materials Research Society, Pittsburgh, Pennsylvania

M.T. Robinson

Member, International Advisory Committee, Symposium on Fundamental Processes in Sputtering, Danish Academy of Sciences and Letters, Copenhagen (to be held in 1992)

B. C. Sales

T.P.Spreen

H. G. Smith

S. Spooner

J. R. Thompson

J. F. Wendelken

C. W. White

G. D. Nignall

M. K. Wilkinson

Executive Secretary, DOE Basic Energy Sciences Laboratory Program Panel

Adjunct Professor, School of Physics, Georgia Institute of Technology, Allanta, Georgia

Distinguished Lecturer, Ontario Center for Materials Research Member, Editorial Board, Macromolecules

Invited Expert Analyst, Chemtracts-Macromolecular Edition, Wiley and Sons

Principal Editor, Joumal of Materials Research, 1987-1990

Founding Father, International Materials Research Committee$$
\text { nology, Allanin, Ceorgio }
$$ 
Consultant to Steering Committee, U.S.-Japan Cooperative Program on Neutron Scattering

Member, ORNL Emergency Review and Advisory Committee

Member, ORNL Steering Committee, Advaroed Neutron Source

Secretary, National Steering Committee for the Advanced Neutron Source (NSCANS)

Secretary, NSCANS Executive Committee

Member, ORNL Stage II Budget Review Committee

Chairman, ORNL Research Reactor Users Committee

Chairman, Evaluation Panel for Reactor Radiation Division, National Institute of Standards and Technology

Member, Evaluation Panel for Materials Science and Engineering Laboratory, National Institute of Standards and Technology

Chairman, Search Committee for ORNL Solid State Division Director

Member, ORNL 50th Year Celebration Committee

J. M. Williams

S. P. Withrow

R. F. Wood

F. W. Young Jr.
Member, Organizing Coumittee and Program Committee, Seventh International Conference on Ion Beam Modification of Materials, Knoxville, Tennessee, September 1990

Manager, IBMM 90 EXPO, Seventh International Conference on lon Beam Modification of Materials, Knoxville, Tennessee, September 1990

Co-Chairman, AVS-ASM Symposium on Modern Analysis Techniques in Makerials Science, Oak Ridge, Tennessee, 1990

Co-Chairman, Program Committee, and Member, Organizing Committee, Seventh International Conference on Ion Beam Modification of Materials, Knoxville, Tennescee, 1990

Co-Editor, Proceadings of the Seoenth Intermational Conference on Ion Beam Modification of Materials, Knoxville, Tennessee (to be published in 1991)

Member, Corporate Fellows Advisory Committee on Science and Technology

Member, Wigner Fellowship Committee, ORNL

Member, ORNL-UT Distinguished Scientist Committee

Guest Scientist, Universitat Erlangen, Nümberg, Federal Republic of Cermany, July 1988-July 1989

Lecturer, ORAU Traveling Lecture Program, 1989-1990

Chairman, ORNL Corporate Fellows Subcommittee on Target Areas for the Director's R\&D Funds

Secretary, Division of Materials Physics, American Physical Society, 1991-1993

Member, Executive Committee, American Association for Crystal Growth

Member, Editorial Board, Topics in Crystal Growth

Member, External Affairs Committee, Publications Committee, A wards Committec, Long-Range Planning Committec, and Von Hippel Selection Committce, Materials Research Society

Examining Board, lournal of Materials Research 
Member, Materials Science Advisory Committee, Southeastern Universities Research Association

D. M. Zehner

Member, VUV Proposal Study Panel, National Synchrotron Light Source, 1908-1989

Member, User Executive Committee, National Synchrotron Light Sounce, 1999-1992

Guest Scientist, Universitat Erlangen, Nūmberg, Federal Republic of Cermeny, Juby-September 1909

R. A. Zuhr

Co-Chairman, Symposium on Beam-Solid Interactions: Physical Phenomena, Materials Research Society, Boston, Massachusetts, 1909

Co-Editor, Beam-Solid Interactions: Physical Phenomena, Materials Research Society, Pittsburgh, Pennsylvania, 1990

Member, Program and Local Committees, and Session Organizer, Seventh International Conference on lon Beam Modification of Materials, Knoxville, Tennessee, 1990

Recipient, Martin Marietta Energy Systems Certificate of Recognition, Patent Application, 1990 


\section{Personnel Changes}

\section{New Sthf Memkex}

\section{A. Scientific Staff}

R. A Kemm, Argonne National Laboratory, Agzonne, mirois

D. P. Norton, Louisiana State University, Baton Rouge, Louisiana

J. Rankin, Massachusetts Institute of Technology, Cambridge, Masoachusetts

A. L. Wachs, Lawrence Livermore National Laborabory, Livermore, California

B. Adminiotrative and Techwical Support Staff

V. E Bames, Secretary

B. E. Gray, Secretary

\section{Spff Transfers and Terminations}

A. Scientific Steff

J. H. Barrett (retirement)

C. J. Bunick (transferred to Biology Division)

C. B. Finch (retirement)

R. A. Kemm (completion of temporary employment)

D. N. Mashbum (transferred to Oak Ridge Caseous Diffusion Plant)

F. A. Modine (transferred to Central Management Office for two-year assignment)

O. S. Oen (retirement)

O. E. Schow III (retirement)

S. T. Sekula (decensed)

J.C. Wang (transferred to Energy Division)

C. K. Werner (transferred to Oak Ridge Gaseous Diffusion Plant)

B. Administratioe and Technical Suppent Staff

C. W. Boggs (transferred to Metals and Ceramics Division)

K. C. Brunson (transferred to Metals and Ceramics Division)

T. L. Collins (transferred to Metals and Ceramics Division)

E. A. Hill (voluntary resignation)

C. M. Malone (transferred to Metals and Ceramics Division)

L. S. Tyler (retirement)

D. M. Watson (transferred to Central Management Offices) 


\section{Guest Assionments}

\section{A. Scientific Staff}

G. S. Canwright, The University of Tennessee, Knoxville, Tennessee

L. de Oliveira, The University of Tennessee, Knoxville, Tennessee

B. Dötsch, Universitat Erlangen-Nümberg, Erlangen, Germany

R. S. Fishman, The University of Tennessee, Knoxville, Tennessee

K. Fiensberg. The University of Tennessee, Knoxville, Tennessee

H. O. da Frot, The University of Tennessee, Knoxville, Tennessee

A. Colanski, Centre National dEtudes des Telecommunications, Meylan, France

M. Jonson, The University of Tennessee, Knoxville, Tennessee

S. Katano, Japan Atomic Energy Research Institute, Tokai, Japan

G. D. Mahan", The University of Tennessee, Knoxville, Tennessee

P. Mazumdar, Perkin-Elmer Corporation, Danbury, Connecticut

J. L. Park, North Carolina State University, Raleigh, North Carolina

S. Pettersson, The University of Tennessee, Knoxvilie, Tennessee

A. Rojo, The University of Tennessee, Knoxville, Tennessee

B. Rudberg, The University of Tennessee, Knoxville, Tennessee

B. Sernelius, The University of Tennessee, Knoxville, Tennessee

S. Tanaka, Kyoto University, Kyoto, Japan

D. G. Tonn, Wooster College, Wooster, Ohio

Y. Tsunoda, Osaka University, Osaka, Japan

J. L. Zarestky, Ames Laboratory/lowa State University, Ames, lowa

-ORNL/UT Distinguished Scientist

B. Oak Ridge Associated Universities Postgraduate Reseanch Participation Program

M. K. El-Ghor, North Carolina State University, Raleigh, North Carolina

J. D. Gresset, University of North Texas, Denton, Texas

D. E. Jesson, University of Bristol, Bristol, United Kingdom

S. C. Lui, University of Pennsylvania, Philadelphia, Pennsylvania

J. C. McCallum, University of Melboume, Melbourne, Australia

L. Romano, Universite Claude Bernard, Lyon, France

J. K. Zuo, Renssealear Polytechnic Institute, Troy, New York

\section{Graduate Students}

H. A. Deeds, The University of Tennessee, Knoxville, Tennessee

E. C. Jones, The University of Tenneseee, Knoxville, Tennessee

Y. C. Kim, The University of Tennessee, Knoxville, Tennessee

I. W. McCamy, The University of Tennessee, Knoxville, Tennessee

J. Ossandon, The University of Tennessee, Knoxville, Tennessee

B. Sanborn, State University of New York-Stony Brook, Stony Brook, New York

J. Sharp. The University of Tennessee, Knoxville, Tennessee

T. Swahn, The University of Tennessec, Knoxville, Tennessee

S. Zhu, The University of Tennessee, Knoxville, Tennessee 
D. Undergraduate Students

A. Baugher, Washington College, Chestertown, Maryland

R. Boekenhaves, Coe College, Cedar Ropids, Jowa

\section{Summer Assionmentis (1989)}

A. Scientific Staff

R. Triolo, University of Palermo, Palermo, Italy

B. Administrative and Techuical Support Staff

D. Cribley, U.S. Naval Academy, Annapolis, Maryland

P. S. Ebey, St. Olaf College, Northfield, Minnesota

B. E. Gray, The University of Tennescee, Knoxville, Tennessee

J.C. Lee, Massachusetts Institute of Technology, Cambridge, Maseachusetts

K. J. Platt, Wake Forest University, Winston-Salem, North Carolina

C. Oak Rilge Associated Universities-Faculty Reseanch Participants

M. J. Axiz, Harvard University, Cambridge, Maceachusetts

G. C. Farlow, Wright State University, Dayton, Ohio

D. Oak Ridge Associated Universities-Student Reseanch Participants

C. L. Albert, North Texas State University, Denton, Texas

N. Modine, Virginia Polytechnic Institute and State University, Blacksburg, Virginia

D. T. Williams, Alabama A\&M University, Huntsville, Alabama

\section{Summer Asgignments (1990)}

A. Scientific Staff

R. S. Fishman, North Dakota State University, Fargo, North Dakota

V. Homer, University of South Carolina, Columbia, South Carolina

B. S. Itchkawitz, University of Pennsylvania, Philadelphia, Pennsylvania

M. Karimi, Alabama A\&M University, Huntsville, Alabama

Y. Morii, Japan Atomic Energy Research Institute, Tokai, Japan

T. P. Molooka, University of Tsukuba, Tsukuba, Japan

M. Norton, University of Ceorga, Athens, Ceorgia

R. Triolo, University of Palermo, Palermo, Italy

B. Administrative and Technical Support Staff

D. L. Gilmore, California Institute of Technology, Pasadena, Califomia

S. Graber, The University of Tennessee, Knoxville, Tennessee

C. Oak Ridge Associated Universities-Faculty Revearch Participants

M. J. Aziz, Harvard University, Cambridge, Massachusetts 


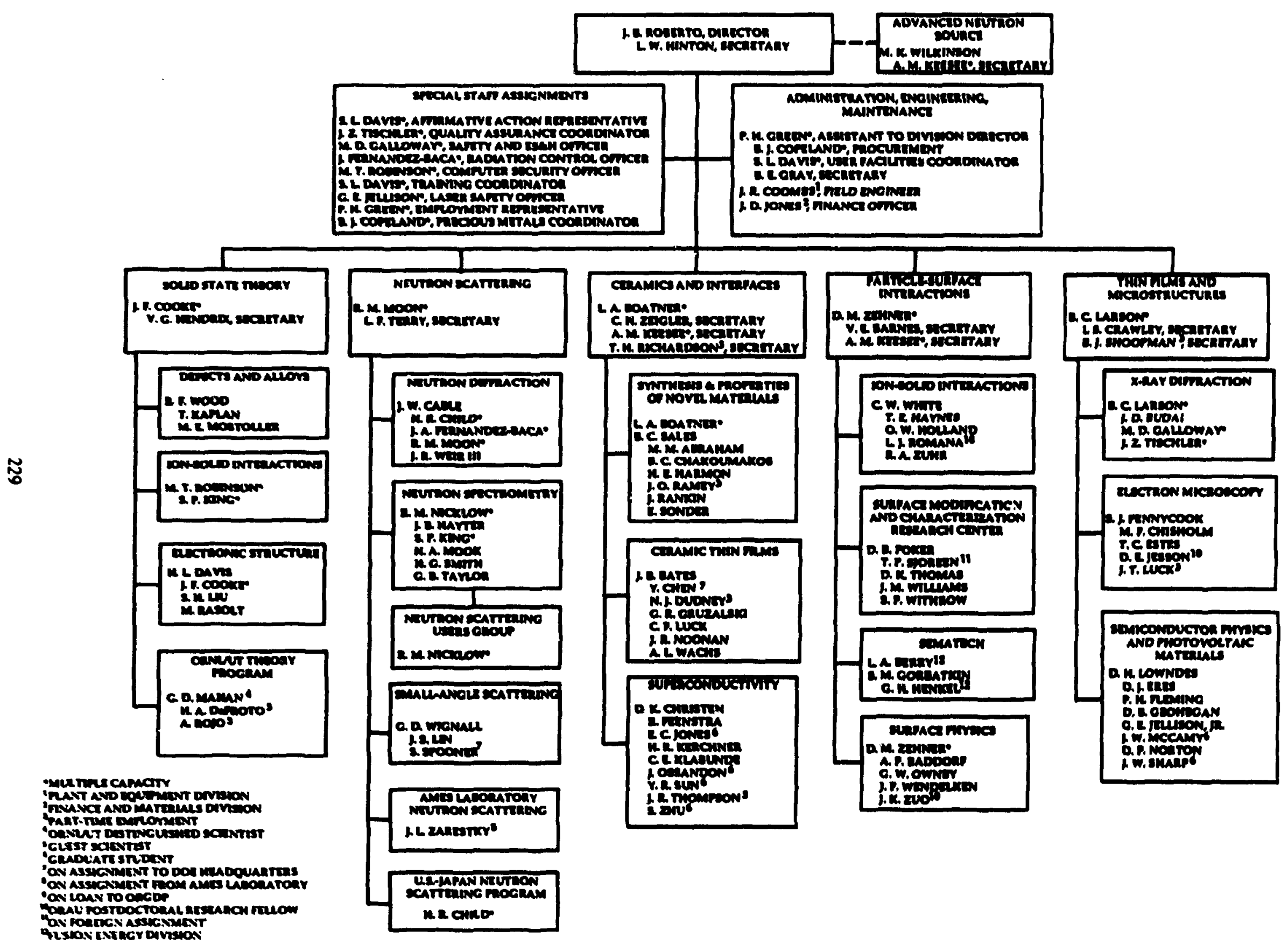




\section{Author Index}

Abdel-Roouf, M. A.-4, 21

Abraham, M. M-67, 73, 78, 83, 84, 85

Aeppli, G-41

Aliey, G. T. -86

Alondah, K.-26

Boddorf, A. P.-130, 131, 132

Banville, M.-7

Baribeau, J. M.-143, 144

Bates, F. S.25, 26

Bates, J. B.-59, 60, 61, 62, 63, 65, 66

Becter, K. - 4,93

Becker, P. C-85

Berlines, R-34, 35

Bernier, G.72

Berry, L A. 98,99

Biackman, J. A-10

Boatner, L A A-48, 53, 55, 67, 69, 70, 71, 72, 73, $81,82,83,84,85,96,96,107,155,157$

Boldú O, J. L-67

Bourke, M. A. M. 40

Brymestad, J. -58

Buchanan, R. A. $\rightarrow$-9

Budai, J. D.-16, 50, 52, 53, 54, 55, 56, 58, 90, $106,110,112,140,153,156,157$

Bums, F. C - 94

Cable, J. W. -36

Callisch, G. B. -28

Canwright, C. S.-3

Cappenter, R. W. $\rightarrow 1$

Chakoumakos, B. C-38, 41, 57, 58,69

Chang S. L-33

Chase, L L L-70

Chen, S. H. -33

Chen, $Y .-78,79,80$

Chen, Y. L $\rightarrow 91$

Child, H. R.-13

Chisholm, M. F.-117, 139, 140, 141, 142, 145, $146,147,155$

Cho, M. H. -31

Chou, Henry-15

Christen, D. K.-46, 47, 48, 49, 53, 55, 56, 58, $110,112,155$
Christie, W. H.-102

Che, Y. T. 66

Civale, L-A7

Conwry, J. G. -85

Cooke, I. F. - 4, 10

Coulombe, S- 1

Cocosolino, C-109

Culberton, R J. $\rightarrow$ 9

Darken, L. S.76

David, S. A- $10,81,82$

Davis, H. L-16, 17, 127

Dotsch, B.-16

Dubrex, W.S.-30

Dudney, N. J.-59,60, 61, 62, 63, 65, 67

Dufour, P. - n

Duribar, B. J.-157

Edelatein, N. -83, 84, 85

Edis, Taner-6

E-Chor, M. K. -90

Eres, D.-117, 118, 119, 120

Fagerburg D. R.-28

Falcer, D. D. -86

Falter, K. C. -86

Farlow, G. C. -97

Feenctra, $\mathbf{R}-46,48,49,52,53,54,55,106$, $110,113,140,149,155$

Femander-Bace, J. A.-37, 38, 39, 43

Fisk, Z- 11

Fleming, P. H. -86

Flencberg, Karsten-12, 13

Fredrickson, C. H-26

Frot, H. O.-11, 12, 13

Fu, S. Q. -73

Calloway, M. D.-54, 102, 106, 149, 156

Ceohegan, D. B.-113, 115, 116

Gibbs, Doon-128, 129

Gillon, B.-36

Colanski, A.-102, 149

Coldstone, Joyce-10

Conzales, A. -9

Conzalez, R.-79, 80

Corbatkin, S. M. $\rightarrow 98,99$ 
Gray, L J.-18, 19

Grenier, P.-72

Gruzalski, G. R-65, 133, 134

Hagen, M, -13

Harmon, H. E.-102, 149

Harvey, J. A. 11

Hashimoto, T.-31

Hayden, S. M. 11

Haynes, T. E. \&8, 100, 101 102, 117

Hayter, John B.-25

Henkel, G. H. $\rightarrow 99$

Hiil, N. W. 1

Hoghund, R. F. -93

Holden, T. M. 10

Holland, O. W. $-89,90,100,101,103,104,146$

Holteberg, F. H. -47

Houston, J. E-17

Hoang, K G-129

la, G. E-151

Jand, S-71, 72

Jellison, G. E Jr.-73, 74, 75, 76, 77,86

Jesion, D. E-135, 137, 138, 141, 143, 144, 147, 148

Jones, E C $-56,112$

Jonoon, M.-12, 13

Jorgensen, J. D. - 34, 35

Kaler, E. W.-32

$K=0$, C T. -102

Kaplan, T.-18, 19

Kerchner, H. R-16, 48, 49, 51, 53, 55, 58, 141

Ketcham, Thomas D.-112

Kine J. S. -29

Kabunde, C. E-16,53

Nemm, R A.-2

Kyu, T,-31

Lodik, J. $\rightarrow$

Larson, B. C.151, 152, 153, 156

Lu, S. S.-109

Lay, K. W.-56, 112

Lee, D. R.-102

Lee, I-S.-94

Lee, W. K.-73

Levy, M. - A

Liegener, C-M.-4

Lin, J. S. - 31, 33

Lindermer, T. B.-54

Liu, S. H. $-2,18,19$

Long M. -32

Lowndes, D. H. $-46,50,51,52,53,56,106$, $110,112,113,117,140,141,153,155$

Luck, C. F, $-59,61,63,65$

Lui, S.C.-134
Magruder, R. H. $-92,93$

Mahan, G. D.-7, 11, 14, 15

Malozemoff, A. P. $\rightarrow 7$

Martino, A.-32

Marwick, A. D. $-46,47$

MoCollum, J. C $\longrightarrow$ - 6,107

McCamy, J. W.-110, 112, 113

McElfresh, M. W. -47

Mirebeau, I-36

Mochrie, S. G. J.-128, 129

Modine, F. A.75

Mook, H A.24, 37, 38, 41

Moon, R. M-39

Morii, Y.-39

Mos, S. C-15

Mostoller, Mark-4, 8, 14, 15, 41

Mullica, D. F.-157

Mullins, D. R.126

Muse, J.-102

Minller, K.-16

Nakai, Y. -36

Narayan, J. -53

Nicklow, R. M. -13, 157

Nielsen, M.-34

Noonan, J. R-127

Norton, D. P. $-16,50,51,52,53,56,106,110$, $112,140,141,153,155$

Nortion, M. L-24

Nowick, A. S. -73

Ocko, B. M.-128

ONveil, D. G.-17

Oren, V. M. $-79,80$

Oscandon, J.-51,58

Overbury, S. H. -126

Pappert, S. A.-109

Parette, G. -36

Parikh, N. R. -102

Park, J. L-102, 149

Pennycook, S. J.-101, 102, 103, 104, 106, 135, $137,138,139,140,141,142,143,144,145$. $146,147,148,149$

Peterson, R. S. - 108

Petterseon, S. - 15

Plummer, E. W. -130

Pogatshnik, G. J. -80

Poker, D. B. $-95,109,110$

Pym, Roger-25

Quintana, J.-94

Raman, S. -39

Ramey, J. O. -96

Rankin, J.-107

Rappaz, M. $-81,82$ 
Rasolt, Mark-2, 6, 9

Rhyne, J. J. 13

Rili, R. L-33

Roberto, J. B. $-98,99$

Robertson, J. D. $60,61,62$

Robinson, G. Y.-147

Robinson, Mark T.-20

Rochelle, J. M.-86

Rojo, A. G.3

Root, J. H. -40

Rosedale, J. H.-25, 26

Rosenblatt, G. H. -80

Roseed, T. M.-108

Roth, J.-105

Rytz, D. -41

Saalfrank, P.-A

Saijo, K.-31

Salce, B. -72

Sales, B. C. $-38,41,57,58,61,68,69,73,96$

Sandy, A. R.-129

Sappenfield, E. L-157

Sass, C-28

Scherzer, B. M. U.-105

Schultz, J. M. $-\mathbf{3 0}$

Sekula, S. T. $-48,53,55,58$

Shapiro, S. M.-15

Sharp, J. W.-117, 118, 119, 120

Siegete, R.-105

Singh, $R-53$

Sioreen, T. P. $\rightarrow 1$

Smith, H. G.-34, 35

Sokoloff, J. P. -70

Sonder, E-58

Spooner, Steve-39, 40

St. Julien, Dell-112

Sun, Yangren-17,58

Swanson, M. L-102

Tang, H-Yi-24

Tangari, C. -29

Teianović, Zlatko-6, 9

Thomas, D. A. -157

Thompson, J. R.-46, 47, 48, 51, 53, 55, 58

Thomeon, J. O.-48, 49,51

Trmmons, M-102

Tischlex, J. Z.-117, 151, 152, 153

Tkaczyk, J. E.-56, 112

Toubuse, J, -71

Treglio, J. R.-94

Trivisonno, J.-34, 35

Trohidou, K. N. -10

Tu, C. W.-109

Tun, Z-43
Tung R. T. -148

Uliman, R-29

v. Ceminden, D.-16

Vane, C. R.-108

Venkatasubramanian, R-102

Vignale, G.-2

Vitek, J. M. $-81,82$

Wachs, A. L. 61,63

Wang J. C. -66

Wang, X. M.-n

Weeks, R A. $-92,93$

Wendelken, J. F.-124, 125, 126, 131

Westbrook, R. D. 86

White, C. W. $-89,90,102,149$

Wignall. G. D. $-24,25,26,28,29,30,32$

Williams, G. M. $-83,84,85$

Williams, G. P. -80

Williams, J. M. -9

Williams, R. T. -80

Withrow, S. P.-104, 105

Wood, R. F. - 4, 5, 21

Worthington, T. K. -17

Xia, W. $-95,109$

Yalisove, S. M.-148

Yang, L. -93

Young J.P.-108

Yu, P. K. L-109

Zarestky, J. L. -43

Zehnes, D. M.-16, 128, 129, 132, 133, 134

Zhang, J.-109

Zhu, S. - 16,113

Zschack, P.-153, 156

Zuhr, R. A.61, 88, 92, 93, 108

Zuo, J.-K.-124, 125 